\title{
Collective decision making in economic environments : deterministic and probabilistic approaches
}

Citation for published version (APA):

Ehlers, L. H. (2001). Collective decision making in economic environments : deterministic and probabilistic approaches. [Doctoral Thesis, Maastricht University]. Universiteit Maastricht. https://doi.org/10.26481/dis.20010511le

Document status and date:

Published: 01/01/2001

DOI:

10.26481/dis.20010511le

Document Version:

Publisher's PDF, also known as Version of record

\section{Please check the document version of this publication:}

- A submitted manuscript is the version of the article upon submission and before peer-review. There can be important differences between the submitted version and the official published version of record.

People interested in the research are advised to contact the author for the final version of the publication, or visit the DOI to the publisher's website.

- The final author version and the galley proof are versions of the publication after peer review.

- The final published version features the final layout of the paper including the volume, issue and page numbers.

Link to publication

\footnotetext{
General rights rights.

- You may freely distribute the URL identifying the publication in the public portal. please follow below link for the End User Agreement:

www.umlib.nl/taverne-license

Take down policy

If you believe that this document breaches copyright please contact us at:

repository@maastrichtuniversity.nl

providing details and we will investigate your claim.
}

Copyright and moral rights for the publications made accessible in the public portal are retained by the authors and/or other copyright owners and it is a condition of accessing publications that users recognise and abide by the legal requirements associated with these

- Users may download and print one copy of any publication from the public portal for the purpose of private study or research.

- You may not further distribute the material or use it for any profit-making activity or commercial gain

If the publication is distributed under the terms of Article $25 \mathrm{fa}$ of the Dutch Copyright Act, indicated by the "Taverne" license above, 


\section{Collective Decision Making in Economic Environments: Deterministic and Probabilistic Approaches}

PROEFSCHRIFT

ter verkrijging van de graad van doctor aan de Lniversiteit Maastricht, op gezag van Rector Magnificus,

Prof. Dr. A.C. Nieuwenhuijzen Kruseman, volgens het besluit van het College van Decanen, in het openbaar te verdedigen op vrijdag 11 mei 2001 om 16.00 uur.

door

Lars Hermann EHLERS 


\section{Promotor:}

Prof. dr. H.J.M. Peters

\section{Co-promotor:}

Dr. A.J.A. Storcken

\section{Beoordelingscommissie:}

Prof. dr. P.J.J. Herings (voorzilter)

Prof. dr. W.I. Thomson (University of Rochester, LSA)

Dr. A.J. Vermeulen 
Collective Decision Making in Economic Environments:

Deterministic and Probabilistic Approaches

Lars Hermann

EHLERS

PROEFSCHRIFT 


\section{Preface}

This thesis contains most of my research that I conducted during the past three years, from October 1997 until September 2000. The history of my thesis starts in July 1995. At that time I was studying mathematics at the Liniversity of Regensburg. Having followed a course in game theory in my third year, I was interested in writing a master's thesis in that field. However, Professor Bierlein, who would have been the supervisor, was retiring. He encouraged me to continue my studies at another university where I could work in game theory. I went to Aachen and met Hans Peters. Without knowing me he was so kind to give me a topic for my master's thesis in game theory. In July 1997 I received my master's degree in mathematics with first class honors from the University of Technology Aachen. Hans Peters asked me whether I would be interested in writing a Ph.D.-thesis at Maastricht Lniversity. Since I also liked the idea of living in a foreign country; I signed a contract to work for four years as an "assistent in opleiding" (AIO) at the Department of Quantitative Economics at Mastricht Lniversity from October 1st, 1997.

The topic of my thesis was "Nonmanipulable Probabilistic Decision Mechanisms". My supervisors were Hans Peters and Ton Storcken. I am grateful to them for their advice, their support, and the freedom they gave me during my research. Since the deadline for the Fourth International Meeting of the Society for Social Choice and Welfare in Vancouver was approaching, my supervisors pushed me to derive as early as possible some results that I could present in Vancouver. Fortunately, I succeeded and presented my first results at the Social Choice and Welfare Seminar on January 12, 1998. At this seminar I met two people who became important for my thesis.

The first person is Bettina Klaus. Because of her Ph.D.-defense on January 13, 1998, Hans Peters and Ton Storcken organized the seminar. I wish to thank her for her advice and suggestions, and the many people she introduced to me. Several collaborations originate from the discussions we had. I hope we will continue to work together.

The second person is William Thomson. He was a member of the committee of Bettina's Ph.D.-defense. In 1998 and 1999 I had regular e-mail correspondence with him. In 1999 he created the possibility for me to stay at the Department of Economics at Lniversity of 
Rochester as a visiting scholar. I had a very productive time in Rochester from October 1, 1999, to April 20, 2000. I am indebted to William Thomson for teaching me how to write clearly and unambiguously, and for the many comments and suggestions he gave on my papers. He deserves a lot of Belgium chocolate. Every Thursday I enjoyed his seminar with his students no time constraints, many questions, and proofs.

Many people provided me with helpful comments and suggestions on my work. I thank especially Szilvia Pápai, John Duggan, Stephen Ching, Biung-Ghi Ju, and the anonymous referees of my papers. I also thank Hervé Moulin, Peter P. Wakker, and Stef Tijs for writing several reference letters.

During the past three years I have lived in three places in Maastricht, in Rochester, and in Cham.

In Maastricht I first want to thank Dries. We have been sharing an office for almost two years and became friends. In summer 1999 we had a very pleasant trip to China. Second I want to thank Horst for his advice and for the beautiful hikes in British Columbia with Dolores and him. Third, I want to thank Hans van der Stel, Alain, Veronika, Olga, my aunt Margarete, the fellow residents of Plankstraat 13, and the members of TTV Maastricht. I ann also grateful to Philipp for the relaxing evenings we had in Aachen.

In Rochester I want to thank Kalina, Irasema, John, Larry, and the Ph.D.-students for the fun we had. I am grateful to the Netherlands Organization of Scientific Research (NWO) for the financial support during my visit.

In Chan I want to thank my friends Michael, Reinhold, Micha, Erwin, Toni, Ulli, Volker, Ralf, Karl-Heinz, and Anton for the nice hours we spent together.

My biggest gratitude is to my family. The cohesion and their mental support were essential in writing this thesis. I dedicate this thesis to my father, who passed away on August 21, 1998.

Lars H. Ehlers

Maastricht

The Netherlands

November 2000 


\section{Contents}

$\begin{array}{ll}\text { Preface } & \text { iii }\end{array}$

Notation $\quad$ xiii

1 Introduction 1

I Provision of Public Good(s) 5

2 Multiple Public Goods $\quad 7$

2.1 Introduction . . . . . . . . . . . . . . . . . 7

2.2 The Max-Extension . . . . . . . . . . . . . . . . . . . 14

2.3 Miyagawa Results . . . . . . . . . . . . . . . . . 16

2.4 Independence Axioms . . . . . . . . . . . . . . . . . . 18

2.4 .1 Definitions .......................... 18

2.4 .2 Augmented Median Solutions . . . . . . . . . . . . 20

2.4 .3 The Extreme-Peaks Solution . . . . . . . . . . . . . 22

2.4 .4 The Incompatibility . . . . . . . . . . . . . . 26

2.4 .5 Relation to Strategy-Proofness . . . . . . . . . . . . . . . 26

2.5 The Lexicographic-Extension . . . . . . . . . . . . . . . . . . . . . 27

2.6 The Replacement Principle . . . . . . . . . . . . . . . . . . . 30

2.6 .1 Replacement-Domination . . . . . . . . . . . . . 30

2.6.2 Single-Peaked Preference Rules . . . . . . . . . . . . . . 31

2.6 .3 Three Public Goods . . . . . . . . . . . . . . . 37

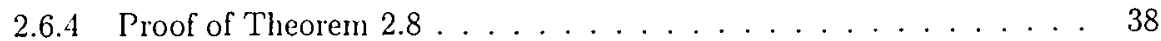

2.6 .5 Proof of Theorem $2.9 \ldots \ldots \ldots \ldots \ldots$

2.7 Population-Monotonicity . . . . . . . . . . . . . 52

2.7 .1 Population-Monotonicity . . . . . . . . . . . . 52

2.7.2 Single-Plateaued Preference Rules . . . . . . . . . . . . 53 
2.7 .3 Proof of Theorem $2.10 \ldots \ldots \ldots \ldots 5$

3 Probabilistic Rules for One Public Good 71

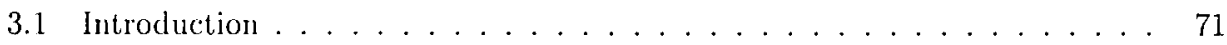

3.2 The Probabilistic Approach . . . . . . . . . . . . . . 76

3.3 Strategy-Proofness . . . . . . . . . . . . . . . . . 79

3.3 .1 Definitions ........................ 79

3.3.2 Uncompromisingness .................. 80

3.3.3 Strategy-Proofness and Peaks-Onliness . . . . . . . . . . 83

3.3.4 Strategy-Proofness and Lnanimity . . . . . . . . . . . 88

3.3 .5 Deterministic Rules . . . . . . . . . . . . . . . . . . . . . . 93

3.4 Solidarity . . . . . . . . . . . . . . . . . . 96

3.4 .1 Definitions ......................... 96

3.4 .2 Logical Relations . . . . . . . . . . . . . . . . . . . . . . . 98

3.4 .3 Target Rules. . . . . . . . . . . . . . . 103

II Assignment of Indivisibilities 109

4 Strategy-Proof House Allocation $\quad 111$

4.1 Introduction . . . . . . . . . . . . . . . . 111

4.2 The Model . . . . . . . . . . . . . . . . . . . . . . 114

4.3 A Lnique Maximal Domain . . . . . . . . . . . . . . . 115

4.4 Proof of Theorem 4.1 . . . . . . . . . . . . . . . 119

4.5 Proof of Theorem $4.2 \ldots \ldots \ldots \ldots \ldots \ldots$

5 Strategy-Proofness and Multiple Assignments 131

5.1 Introduction . . . . . . . . . . . . . . . . . . . . . . 131

5.2 The Model and Basic Properties . . . . . . . . . . . . . . . . . . . . 133

5.3 Coalitional Strategy-Proofness . . . . . . . . . . . . . . . . 135

5.4 Resource-Monotonicity . . . . . . . . . . . . . . . 142

6 Implementability in Matching Problems $\quad 147$

6.1 Introduction . . . . . . . . . . . . . . . . . . 147

6.1.1 Generalized Matching Problems . . . . . . . . . . . . . . . 147

6.1 .2 Linear Orders and the Strong Core . . . . . . . . . . . . . . . . 148

6.1.3 Weak Orders and Overview of the Results . . . . . . . . . . . . . 150

6.2 The Model . . . . . . . . . . . . . . . . . . . 152 
6.3 The Strong $T$-Core . . . . . . . . . . . . . . . . . . 153

6.4 The Lnion Strong $T$-Core . . . . . . . . . . . . 156

6.5 The Weak Core . . . . . . . . . . . . . . . . 158

6.6 Implenentability . . . . . . . . . . . . . . . . 163

6.7 How much Indifference? . . . . . . . . . . . . . . . 167

6.8 Applications . . . . . . . . . . . . . . . . 167

6.8 .1 Marriage Problems . . . . . . . . . . . . . . . . 167

6.8 .2 Objects Exchange . . . . . . . . . . . . . . 168

\section{Allocation of an Infinitely Divisible Commodity 171}

7 Resource-Monotonic Allocation $\quad 173$

7.1 Introduction . . . . . . . . . . . . . . . . . 173

7.2 The Model and Resource-Monotonicity . . . . . . . . . . . . . . . 175

7.3 Fixed-Path Rationing Methods . . . . . . . . . . . . . . . . 177

7.4 The Uniform Rule . . . . . . . . . . . . . . . . . . . 181

7.4 .1 No-Envy ........................... 182

7.4 .2 Consistency . . . . . . . . . . . . . . . 183

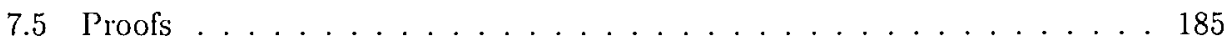

7.5 .1 Proof of Proposition $7.2 \ldots \ldots \ldots \ldots \ldots$

7.5.2 Proof of Theorem $7.2 \ldots \ldots \ldots . \ldots . \ldots . \ldots 189$

8 Strategy-Proofness and Single-Plateauedness 193

8.1 Introduction . . . . . . . . . . . . . . . . . . . . 193

8.2 The Model and Basic Properties . . . . . . . . . . . . . . . . . . . . 195

8.3 The Lniform Rule . . . . . . . . . . . . . . . . . . . . . . 198

8.3.1 Single-Peaked Preferences . . . . . . . . . . . . . . . 198

8.3.2 Single-Plateaued Preferences . . . . . . . . . . . . . . . . 199

8.3 .3 Proof of Theoren 8.6 . . . . . . . . . . . . 201

8.4 Sequential Allotment Solutions . . . . . . . . . . . . . . 204

8.4.1 Pareto-Indifferent Extensions . . . . . . . . . . . . . 204

8.4 .2 Coalitional Strategy-Proofness . . . . . . . . . . . . . . 205

8.4 .3 Proof of Theorem $8.7 \ldots \ldots \ldots 207$

9 Probabilistic Allocation and Single-Dippedness 213

9.1 Introduction . . . . . . . . . . . . . . . . . . . . . . . 213

9.2 The Model . . . . . . . . . . . . . . . . . . . 216 
9.3 Replacement-Domination . . . . . . . . . . . . . . . 222

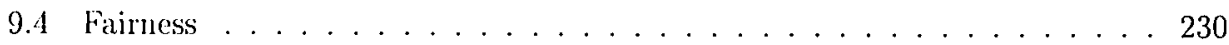

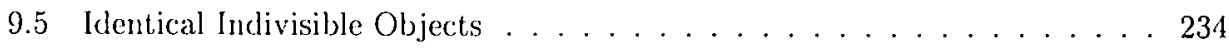

9.6 Independence . . . . . . . . . . . . . . . . . 236

9.7 Conclusion . . . . . . . . . . . . . . . . . 237

$\begin{array}{ll}\text { A Some Results from Measure Theory } & \mathbf{2 3 9}\end{array}$

$\begin{array}{ll}\text { References } & 243\end{array}$

$\begin{array}{ll}\text { Author Index } & 253\end{array}$

$\begin{array}{ll}\text { Summary in Dutch } & 255\end{array}$

$\begin{array}{lr}\text { Curriculum Vitae } & 259\end{array}$ 


\section{List of Figures}

2.1 Max-Extension: weak upper contour sets and indifference sets . . . . . . 15

2.2 Lexicographic-Extension: weak upper contour sets and indifference sets . . 28

2.3 Lexicographic-Extension: the set of Pareto-optimal alternatives . . . . . . 29

2.4 The set of equitable altematives for a single-peaked preference relation . . 33

2.5 Maximization process of a single-peaked preference rule . . . . . . . . . 34

2.6 Illustration of Case (a) in Example 2.6. . . . . . . . . . . . . . . . . . . . . . . . . . . . . . . . .

2.7 Illustration of Case (b) in Example 2.6. . . . . . . . . . . . 35

2.8 Illustration of Case (c) when $j=l$ in Example 2.6. . . . . . . . . . 36

2.9 Illustration of Case (c) when $j=l+1$ in Example 2.6. . . . . . . . 36

2.10 Illustration of Case (a) in Example 2.8. . . . . . . . . . . . 55

2.11 Illustration of Case $(\mathrm{c})$ in Example 2.8. . . . . . . . . . . . . 55

2.12 Illustration of Subcase (d.i) in Example 2.8. . . . . . . . . . . 56

2.13 Illustration of Subcase (d.ii) in Example 2.8. . . . . . . . . . . . . 56

2.14 The set of equitable alternatives for a single-plateaued preference relation . 57

2.15 Maximization process of a single-plateaued preference rule . . . . . . . 58

3.1 The distribution chosen by the probabilistic rule $\Phi^{\Delta}$ at $R . \ldots . \ldots 9$ 


\section{List of Tables}

4.1 Proof of Lemma $4.3 \ldots \ldots \ldots \ldots \ldots \ldots$

9.1 Probabilistic allocation and single-dipped preferences. . . . . . . . . . . 237

9.2 Deteministic allocation and single-dipped preferences. . . . . . . . . . . 237

9.3 Deterministic allocation and single-peaked preferences. . . . . . . . . 238 


\section{Notation}

Most of the notations that are used in the thesis are defined in the text were they appear the first time. Here, we give a short list of some notational conventions that we do not introduce in the text.

\section{Set theoretical notations}

Let $A, B$ be sets.

$A \subseteq B \quad A$ is a subset of $B$.

$A \subseteq B \quad A$ is a strict subset of $B$, i.e., $A \subseteq B$ and $A \neq B$.

$|A| \quad$ cardinality of $A$.

\section{Natural and real numbers}

$\mathbb{N} \quad$ set of natural numbers.

$\mathbb{R} \quad$ set of real numbers.

$\mathbb{R}_{+} \quad$ set of non-negative real numbers, i.e., $\mathbb{R}_{+} \equiv\{x \in \mathbb{R} \mid x \geq 0\}$,

$\overline{\mathbb{R}} \quad$ compactified real line, i.e., $\overline{\mathbb{R}} \equiv \mathbb{R} \cup\{-\infty,+\infty\}$. 
Let $a, b \in \mathbb{R}, a \leq b$.

$[a, b]$ closed interval between $a$ and $b$, i.e., $[a, b] \equiv\{x \in \mathbb{R} \mid a \leq x \leq b\}$.

$\mid a, b[$ open interval between $a$ and $b$, i.e., $] a, b[\equiv\{x \in \mathbb{R} \mid a<x<b\}$.

$\mid a, b]$ left half open interval between $a$ and $b$, i.c., $\mid a, b] \equiv\{x \in \mathbb{R} \mid a<x \leq b\}$.

$[a, b[$ right half open interval between $a$ and $b$, i.e., $|a, b| \equiv\{x \in \mathbb{R} \mid a \leq x<b\}$.

\section{Miscellaneous}

$\square$ end of a proof.

- end of a proof of a claim or an induction step that is included in another proof.

- end of an example.

$\triangleleft$ end of a remark. 


\section{Chapter 1}

\section{Introduction}

This thesis considers economic and political environments in which a group of people has to derive a common decision. Each individual has a preference relation over the possible outcomes. The only information that is available are preference relations that the individuals report. Based on the list of announced preferences the group collectively chooses an alternative.

Depending on the decision mechanisms to be used, an individual might have the possibility to strategically misrepresent his true preference relation in order to manipulate the outcome to his own advantage. When misrepresentation occurs, the common decision is not based on the right information. In particular, our choice could be inefficient with respect to the true preferences. To avoid misrepresentation we impose the following property on the decision mechanism. It is not possible for an individual to gain by announcing a false preference relation, irrespective of the preferences of the other members of the group. If a mechanism satisfies this requirement, then for each individual it is a clominant strategy to announce his true preference relation and our choice will be based on the true information. Such a mechanism is called strategy-proof.

For example, consider the election of the mayor of a city. In such a situation it makes a tremendous difference whether there are two or three candidates. In a two candidate election, majority voting is efficient and strategy-proof (May, 1952). However, when there are three candidates only mechanisms that always choose the most preferred candidate of a unique dictator are efficient and strategy-proof (Gibbard, 1973; Satterthwaite, 1975). The last result is very unsatisfactory. If we also require anonymity meaning that the same candidate should be elected when we change the names of the individuals but keep the preferences fixed, then we have an impossibility.

In proving the Gibbard-Satterthwaite Theorem we need to assume that each individual's preference relation may be any possible ranking over the three candidates. The impos- 
sibility vanishes if we structure the alternatives and restrict the preference relations of each individual. For instance, we may interpret the political spectrum as a one-dimensional continum representing the positions of the candidates. Suppose that candidate 1 is left-wing, candidate 2 is in the center, and candidate 3 is right-wing. Each individual's preference relation has a unique top position (his peak), and on either side of the peak, positions that are closer to the peak are preferred to those which are further away (Black, 1948). Such a relation is called single-peaked. The restriction of preferences does not allow the following rankings of the candiciates: 132 and 312. Excluding these preference relations is natural. A left-wing supporter does not prefer the right-wing candidate to the center candidate. When preferences are single-peaked and the number of individuals is odd, the mechanism that chooses the median of all top candidates is efficient, strategy-proof, and anonymous. In case of an even number of individuals, we may add one calibration vote. Moulin (1980) shows that medians with calibration voters are the only mechanisms satisfying the above requirements.

Part I of the thesis extends Moulin's work in two directions. The real line is the set of possible locations of a public good and each agent has a single-peaked preference relation over the set of locations. Chapter 2 considers the location of not one, but multiple public goods. The goods are identical, say two bus stops or two telephone booths, and for each good we choose a location based on the preferences of the agents. Several authors have extended Moulin's work to choosing a point in a multi-dimensional space. Cintil Miyagawa (1998a) each agent's preference satisfied certain multi-dimensional single-peakedness conditions where each dimension is interpreted as a separate political issue. Our problem can be interpreted as choosing a point in a multidimensional continuum. However, the preference relations in Miyagawa violate all single-peakedness conditions. He considers situations where each agent only consumes the good at his most preferred location. An individual compares two lists of locations via the most preferred elements relative to his single-peaked preference relation over the line.

In Chapter 3, for the location of one good, instead of choosing a unique point on the real line we allow mechanisms to be probabilistic. The interpretation is that the final location is drawn relative to the probability distribution over the real line. We extend Moulin's work and characterize the probabilistic mechanisms that satisfy efficiency, (a probabilistic version of) strategy-proofness, and anonymity. In the probabilistic setting, such a mechanism is determined by a collection of calibration distributions.

Part II focuses on the assignment of indivisibilities. For instance, consider the situation where each individual owns exactly one indivisible object. The individuals may be workers having exactly one job. Each worker is selfish, meaning that he only cares about 
his own job. When the preferences of the workers over the jobs change, the question is how to reassign the jobs among the workers. Although each individual may possess any strict ranking over the objects, the Gibbard-Satterthwaite Theorem does not apply to this problem. The mechanism that reassigns the objects according to Gale's top trading cycle algorithm is efficient, individual rational (each worker is not worse off with his new job relative to his old one), and strategy-proof. The difference is that the decisions in part I are public (given any two alternatives $x$ and $y$ and any two individuals, either of the two agents may strictly prefer $x$ to $y$ ), and the decisions in part II are private (let $x$ and $y$ be two altematives such that agent 1 receives the same object at $x$ and $y$, and agent 2 receives distinct objects at $x$ and $y$; then 1 is always indifferent between $x$ and $y$, whereas 2 either strictly prefers $x$ to $y$ or $y$ to $x$ ).

Gale's top trading cycle algorithm is not well-defined when agents are indifferent between distinct objects. Chapter 4 analyzes the assignment problem in economic environments where individuals might be indifferent between distinct jobs. In real life we cannot exclude such indifference. As basic requirements we impose efficiency and strategyproofness.

In Chapter 5 each individual receives a set of objects. Such problems arise for instance in heritage. Each heir receives a bundle of objects. Most of the literature on the assignment problem considers only environments where each agent consumes exactly one object. We show that when we require the mechanism to be strategy-proof and efficient, there is a tremendous difference whether we allow multiple assignments or not.

Chapter 6 considers generalized matching problems. Here, an assignment specifies for each agent exactly one object, but an agent might not receive any of the objects. For example, the agents are partitioned in workers and firms. Each firm has exactly one vacancy and each worker likes to work for exactly one firm. Then each worker might be assigned to a firm or to remain unemployed, and a firm might have an unfilled vacancy. Again, we do not exclude indifference between distinct objects. We weaken both efficiency and the incentive requirement strategy-proofness and search for mechanisms (possibly multi-valued) that are implementable (in Nash equilibrium).

Finally, Part III investigates the allocation of an infinitely divisible endowment among a group of agents when free disposal is not allowed. For example, we have to allocate the working time of a project among the individuals. The time that is needed to finish the project is the infinitely divisible endowment of the group. Let $T$ be this endowment. The group has to finish the project in order to be paid, i.e., disposal of the total working time $T$ is not allowed. Each individual is paid according to the time he worked for the project. In such situations each agent possesses a single-peaked preference relation over the interval 
$[0, T]$ of the possible amounts of time he has to work for the project. His peak is the optimal combination of work, payment, and leisure. Again, the Gibbard-Satterthwaite Theorem does not apply because the decisions are private. It has been shown that a certain mechanism, the uniform rule, is the only mechanism satisfying efficiency, strategy-proofness, and anonymity.

In Chapter 7 the endowment may vary. When such changes occur, we require that all agents are affected in the same direction. As a result, if they have to work less than before, then either they all weakly gain or they all weakly lose.

A preference relation over $[0, T]$ is single-plateaued when there is a closed interval such that preference is strictly increasing at the left of that interval, each amount belonging to the interval is a most preferred amount, and preference is strictly decreasing at the right of the interval. The domain of single-plateaued preferences is the biggest domain including the domain of single-peaked preferences on which we can extend the uniform rule in such a way that it satisfies efficiency, strategy-proofness, and anonymity. Chapter 8 considers the allocation problem when each agent is equipped with all single-plateaued preferences over the interval $[0, T]$.

A preference relation is single-dipped when there is a worst amount, the dip, preference is strictly decreasing left of the dip, and preference is strictly increasing right of the dip. Chapter 9 analyzes the allocation problem when agents have single-dipped preferences over $[0, T]$. If we allocate some free hours among workers for which they are not paid, then they may prefer consuming all free hours or continuing to work rather than having only a few hours off. When the mechanism is deterministic several negative results were derived for the allocation problem with single-dipped preferences. We allow mechanisms to be probabilistic and show that this feature brings about positive results.

Each chapter is self-contained and thus, the chapters can be read independently. 


\section{Part I}

\section{Provision of Public Good(s)}




\section{Chapter 2}

\section{Multiple Public Goods}

\subsection{Introduction}

This chapter, which is based on Ehlers (2000a,b,c), considers the problem of choosing multiple locations in an interval for an exogenously given number of identical public facilities. Each agent has a "single-peaked" preference relation over the interval and is allowed to select which public facility to use. An agent's preference relation is "single-peaked" if up to a certain point, his "peak", his welfare is strictly increasing, and it is strictly decreasing beyond that point. For example, a certain number of bus stops have to be located along a street. Other examples are gymnasiums, libraries, schools, telephone booths, and broadcasting news cluring a day. The planner faces the problem of choosing for each preference profile a list of locations. ${ }^{1}$

An economy is completely described by the set of feasible locations, the set of agents, their preferences, and the number of public goods. A solution is a systematic way to assign to each economy and each public facility a location. Moulin (1980) introduces this problem in the special case of one good chosen from a one-dimensional continuum and considers strategic issues. The literature that follows extends his work in several directions. Danilov (1994) and Schummer and Vohra (2001) allow the set of feasible locations to be a tree, and Border and Jordan (1983), and Zhou (1991) model it as a multi-dimensional continuum. Ching and Thomson (1999) study population changes, and Thomson (1993), Barberà and Jackson (1994), Ching (1997), and Berga and Serizawa (2000) preference changes. However, until Miyagawa (1998a,b,c,d) there was no axiomatic study of the problem of locating multiple public goods. Recently, other authors study related problems. Barberà and Beviá (1999), and Bogomolnaia and Nicoló (1999) consider the location of public facilities when the agents are not free to choose the good they consume. Each agent is assigned to one of

\footnotetext{
${ }^{1}$ In the latter example the planner has to choose a time schedule.
} 
the goods. Konishi, Le Breton, and Weber (1998) search for equilibria in multiple public goods models with money.

Hotelling (1929) considers two competing businesses choosing where to locate on a street. He assumes that the businesses are identical and each individual patronizes only the one that is closest to where he lives.

Miyagawa (1998a) studies this model from a normative perspective and identifies rules on the basis of desirable properties. For instance, a state government faces the problem of choosing two locations where to build two identical public facilities. Agents have singlepeaked preferences on some interval of possible locations. An alternative specifies for each of the two public goods a location. Such a list is an option set and each agent compares two option sets by comparing their best elements according to his preference relation over locations. We call this extension of single-peaked preferences from the set of possible locations to the set of alternatives its max-extension. The first half of Chapter 2 is devoted to this extension.

In a further study Moulin (1984) considers the problem of locating one public good, when the closed interval actually available may vary. We interpret the interval as an additional constraint that needs to be taken into consideration, e.g., the city government deciding in which section of the street bus stops need to be built. Moulin (1984) is the first author to apply the ideas of "independence of irrelevant alternatives" to a public good model. We are particularly interested in the following two independence axioms. Nash's $I I A$ states that given a profile and an interval, if the interval shrinks in such a way that the previous choice remains feasible, then the solution chooses the same location. Arrow's IIA is the requirement that the choice only depends on the restrictions of preferences to the interval. Moulin asks the question of what solutions satisfy Pareto-optimality; anonymity, Nash's IIA, Arrow's IIA, and interval continuity (small changes in the feasible set should not affect the outcome too much). It turns out that each solution satisfying those properties chooses for each profile and each interval the point that is closest to the median of $n-1$ predetermined points and the $n$ most preferred locations of the agents. Lsing results due to Ching (1997), we generalize his characterization. We drop Pareto-optimality and anonymity, and show that the considerably larger class of "augmented median solutions" are the only solutions satisfying Nash's IIA, Arrow's IIA, and interval continuity.

Section 2.4 adapts the setup of Moulin to the model of Miyagawa. A solution chooses for each set of locations (a closed interval) and each preference profile an alternative consisting of locations belonging to this set. Our main contribution of Section 2.4 is to understand the implications of these independence axioms for the provision of multiple public goods. For two public goods, the following solution plays an important role in Miyagawa (1998a). 
It is the solution that always selects the two locations in an interval that are closest to the extreme peaks of the reported profile. This solution, called the extreme peaks solution, satisfies Nash's IIA and Arrow's IIA. If we require Pareto-optimality and interval continuity additionally, then this combination of axions only allows for the extreme peaks solution.

If the stronger Nash independence requirement each single location satisfies the Nash requirement is imposed, then already Pareto-optimality and interval continuity are only satisfied by one solution. Again this solution is the extreme peaks solution. We also show that for more than two public goods, Pareto-optimality and interval continuity are incompatible.

Furthermore, we uncover an interesting relation between our results and results on decision rules with fixed sets of feasible alternatives. Ching (1997) and Miyagawa (1998a) study strategy-proofness (telling the truth is a weakly dominant strategy) and preference continuity (small changes in preferences only allow small changes in the option set). Since our classes of solutions are the same as these authors characterize, we obtain that under Pareto-optimality, independently of the number of public goods, Nash's IIA, Arrow's IIA, and interval continuity are equivalent to strategy-proofness and preference continuity.

The second half of Chapter 2 contrasts the max-extension with a different extension of preferences. The town government has to locate two public facilities, say two libraries, on a street. The libraries, though identical, only have one copy of each book. Then a certain book may have been already lent out and an individual that wants to borrow this book may have to drive to his second choice library. In most cases each individual visits his most preferred library, but sometimes both. Another example is the provision of telephone booths. Given two altenatives, first an agent compares the most preferred locations of each of the two alternatives, and if there is a tie, then he compares the other locations. We call this extension of single-peaked preferences the lexicographic-extension. ${ }^{2}$ Primarily, each agent uses the facility at his most preferred location, but he might be forced to consume the facility at his second choice location because the other facility is out of use. It turns out that this feature of preferences brings about results that are radically different from Miyagawa (1998b,c).

As a basic requirement we impose Pareto-optimality meaning that the rule chooses for each preference profile an efficient alternative. In our model, Pareto-optimality is weaker than in Miyagawa (1998a). If the smallest and the greatest peak of a preference profile are distinct, then each alternative which is efficient for the max-extension is also efficient

\footnotetext{
${ }^{2}$ Dutta and Massó (1997) study two-sided matching when workers have lexicographic preferences. Each worker first compares firms and second co-workers.
} 
for the lexicographic-extension (Section 2.5). ${ }^{3}$ This is "good news" meaning that there are many possibilities for a rule to select efficient alternatives. We might hope that if we require rules to satisfy additional properties, then there are many rules satisfying these requirements. We show that this will be the case.

Section 2.6 studies the following notion of faimess. If the environment of an economy changes, then the welfares of all agents who are not responsible for this change are affected in the same direction: either all weakly gain or all weakly lose. As a variable parameter of an economy which may change over time, we consider preferences. Solidarity applied to such situations says that when the preference relation of an agent changes, then the welfares of all other agents are affected in the same direction. This replacement principle is called welfare-domination under preference-replacement, or simply replacement-domination.

Moulin (1987) introduces replacement-domination in the context of binary choice with quasi-linear preferences. He calls it "agreement". Among others, Barberà, Jackson, and Neme (1997), Sprumont and Zhou (1999), and Thomson $(1997,1998)$ study the "replacement principle" in different settings, e.g., in private good economies with single-peaked preferences, in classical exchange economies, and in economies with indivisible goods and monetary transfers. A review of the literature is by Thomson (1999a).

For different models of public good economies the rules satisfying Pareto-optimality and replacement-domination have been identified. For the provision of one public good, Thomson (1993), and Ehlcrs and Klaus (2000a) characterize the class of rules satisfying these properties on closed intervals. Each of these rules is determined by a unique point, called the target point. A target rule chooses for each preference profile the efficient location that is closest to the target point. Vohra (1999) characterizes the same class of rules on tree networks. For two pure public goods and the max-extension, Miyagawa (1998b) shows that only the left-peaks rule and the right-peaks rule (for more details see Section 2.3) satisfy Pareto-optimality and replacement-domination.

In all these public good models Pareto-optimality and replacement-domination imply strategy-proofness (telling the truth is a weakly dominant strategy), anonymity (the rule is symmetric), and peaks-onliness (the rule depends only on the peaks). We will show that these implications also hold in our model. Furthermore, our results and all the results derived in the above papers remain true when we consider a stronger version of replacement-domination. They also apply to situations when the preferences of any group of

\footnotetext{
${ }^{3}$ In the rare case when the smallest peak and the greatest peak are identical, there is only one efficient alternative for the lexicographic-extension whereas there are infinitely many efficient allocations for the max-extension.
} 
agents may change.

The class of rules satisfying Pareto-optimality and replacement-domination in the models of the papers above is narrow. ${ }^{4}$ Each location in the closed interval, respectively, on the tree, corresponds to a unique target rule and vice versa. For two pure public goods and the max-extension, Miyagawa (1998b) shows that there are only two rules satisfying Pareto-optinality and replacement-domination. However, by slightly changing the indifference relations and considering the lexicographic-extension of preferences, for economies with two public goods Pareto-optimality and replacement-domination admit a big class of rules.

Each rule satisfying Pareto-optimality and replacement-domination is described by means of a continuous and single-peaked binary relation over the set of locations. We interpret this relation to be the preference relation of the social planner, denoted by $R_{s o c}$. An altemative is $R_{\text {soc }}$-equitable if society is indifferent between its two locations, meaning that the two locations are "equal" in terms of society's preference. For each preference profile, the rule maximizes the welfare of the social planner on the set of $R_{\text {soc }}$-equitable alternatives subject to Pareto-optimality. If the peak of the planner does not belong to the peaks interval ${ }^{5}$ of the reported profile, then there is a unique alternative maximizing the planner's welfare subject to Pareto-optimality. It is either the alternative with both locations on the smallest peak or the alternative with both locations on the greatest peak. If the peak of the planner's relation belongs to the peaks interval of the reported profile, then the rule selects the following alternative maximizing the planner's welfare subject to Pareto-optimality: the first location is smaller than or equal to the planner's peak and the second location is greater than or equal to the planner's peak. We call these rules single-peaked preference rules and show that they are the only rules satisfying Pareto-optimality and replacement-domination. Interestingly, the above procedure links a single-peaked preference relation and a rule via a concept of equity.

We also consider the provision of three public goods. The result for two public goods does not extend to that case. For three public goods only two rules satisfy Pareto-optimality and replacement-domination, the smallest-peak rule and the greatest-peak rule. The smallest-peak rule chooses all three locations to be the smallest reported peak. The greatest-peak rule chooses all three locations to be the greatest reported peak. By locating one more facility, the single-peaked preference rules are restricted to only two rules. This

\footnotetext{
${ }^{4}$ It seems to be a general feature of this property that in any model any class of rules characterized by replacement-domination and certain other properties is restricted. The review of Thomson (1999a) supports this statement.

${ }^{5}$ The peaks interval of a profile is the interval between the smallest peak and the greatest peak of this profile.
} 
result is similar to that of Miyagawa (1998b).

In Section 2.7 we consider another property that pertains to changes in the environment. We apply the idea of solidarity among agents to changes in population. We require that the consequences of changes in population are shared by all agents that are present before and after. The welfares of all these agents should be affected in the same direction: as a result they all weakly gain or they all weakly lose. This solidarity property is called population-monotonicity.

Thonson (1983a) introduces population-monotonicity in the context of bargaining. It is further studied in various models: in cooperative games with transferable utility (Sprumont, 1990; Hokari, 1999), in classical exchange economies (Moulin, 1990; Sprumont and Zhou, 1999; Kin, 1999), and in private good economies with single-peaked preferences (Thomson, 1995; Klaus, Peters, and Storcken, 1997a). For a survey of the literature on population-monotonicity we refer to Thomson (1999b). For the provision of one public good, Ching and Thomson (1999) and Klaus (1999a) characterize the class of target rules by Pareto-optimality and population-monotonicity. For the location of two public goods and the max-extension of preferences, Miyagawa (1998c) identifies a certain class of rules and shows that these rules are characterized by Pareto-optimality and population-monotonicity.

For the location of two public goods and the lexicographic-extension, we identify the class of rules satisfying Pareto-optimality and population-monotonicity. A preference relation is single-plateaued when there is a closed interval of most preferred locations (called the plateau), preference is strictly increasing up to the left-endpoint of this interval and decreasing beyond the right-endpoint of the interval. Each rule in this class is based on a continuous single-plateaued binary relation, which we interpret as the preference relation of the government or the social planner. A rule based on such a relation is defined as follows. If the peaks interval of the profile contains the planner's plateau and no peak of the profile belongs to the planner's plateau, then the rule selects for the first good the peak of the peaks profile that is best for the planner. The rule selects as other location the point that is indifferent to this peak according to the planner's preference in such a way that the following holds: (i) when the location of the first good is smaller than the left-endpoint of the planner's relation, the other location is greater than the right-endpoint of the planner's relation; and (ii) when the location of the first good is greater than the right-endpoint of the planner's relation, the other location is smaller than the left-endpoint of the planner's relation. In all other cases, the rule chooses the locations by "two target rules" having as target points the left-endpoint and the right-endpoint of the planner's plateau, respectively. Our main result characterizes single-plateaued preference rules by 
the combination of Pareto-optimality and population-monotonicity.

Our result is radically different from the corresponding characterization of Miyagawa (1998c). When agents compare alternatives by the max-extension, he characterizes a certain complex class of rules by Pareto-optimality and population-monotonicity. There is only one single-plateaued preference rule which belongs to his class. It is the extreme-peaks rule which selects for each profile the smallest and the greatest peak. Miyagawa (1998c) also shows that the extreme-peaks rule is the only rule satisfying Pareto-optimality, population-monotonicity, and continuity (small preference changes cause only smooth changes of the chosen alternative). In our model continuity does not refine our class of rules. Each single-plateaued preference rule is continuous.

Single-plateaued preference rules satisfy also other properties of interest. They are coalitional strategy-proof (a group of agents cannot gain by jointly misrepresenting their preferences), anonymous (the rule does not depend on the names of the agents), and peaks-only (the chosen alternative depends only on the peaks). These properties are all implications of Pareto-optimality and population-monotonicity. In Miyagawa (1998c), Pareto-optimality and population-monotonicity imply coalitional strategy-proofness and anonymity, but not peaks-onliness.

Chapter 2 is organized as follows. Section 2.2 presents the general model and the maxextension. Section 2.3 reviews some of the results by Miyagawa $(1998 a, b, c)$.

Section 2.4 applies the idea of independence of irrelevant alternatives to the model of Miyagawa. Subsection 2.4.1 adapts the setup of Moulin to the model of Miyagawa. Subsection 2.4.2 extends the result of Moulin (1984) for one public good. Subsection 2.4.3 contains the characterizations of the extreme peaks solution, and Subsection 2.4.4 the incompatibility for more than two public goods. Subsection 2.4 .5 relates our results to certain results on strategy-proofness.

Section 2.5 introduces the lexicographic-extension of preferences over locations to preferences over alternatives. We also compare Pareto-optimality relative to the max-extension and the lexicographic-extension.

Section 2.6 is devoted to the replacement principle. In Subsection 2.6.1 we introduce replacement-domination and show some general implications. In Subsection 2.6.2 for two public goods we define single-peaked preference rules and characterize these rules by Pareto-optimality and replacement-domination. Subsection 2.6 .3 contains the result for three public goods. The proofs are relegated to Subsections 2.6.4 and 2.6.5.

In Section 2.7 we study population-monotonicity. In Subsection 2.7.1 we introduce population-monotonicity and establish some logical relations. In Subsection 2.7.2 we define single-plateaued preference rules and characterize these rules by Pareto-optimality and 
population-monotonicity. Subsection 2.7 .3 contains the proof of the characterization.

\subsection{The Max-Extension}

We consider the problem of choosing locations in the unit interval $[0,1]$ for identical public goods. There is a population $\mathbb{P}$ of "potential" agents, indexed by $i \in \mathbb{P} \subseteq \mathbb{N}$ where $\mathbb{N}$ denotes the set of natural numbers. The population $\mathbb{P}$ can be either finite or infinite. Let $\mathcal{P}$ denote the class of non-empty and finite subsets of $\mathbb{P}$. Each agent $i \in \mathbb{P}$ is equipped with a continuous and single-peaked preference relation $R_{i}$ over $[0,1]$. Let $I_{i}$ denote the indifference relation associated with $R_{i}$, and $P_{i}$ the corresponding strict preference relation. Single-peakedness means that there exists a location, called the peak of $R_{i}$ and denoted by $p\left(R_{i}\right)$, such that for all $x, y \in[0,1]$, if $x<y \leq p\left(R_{i}\right)$ or $x>y \geq p\left(R_{i}\right)$, then $y P_{i} x$. Let $\mathcal{R}$ denote the set of all continuous and single-peaked preferences over $[0,1]$. Given $N \in \mathcal{P}$, let $\mathcal{R}^{N}$ denote the set of (preference) profiles $R \equiv\left(R_{i}\right)_{i \in N}$ such that for all $i \in N$, $R_{i} \in \mathcal{R}$. Given $S \subseteq N$ and $R \in \mathcal{R}^{N}$, let $R_{S}$ denote the restriction $\left(R_{i}\right)_{i \in S}$ of $R$ to $S$. Given $N, N^{\prime} \in \mathcal{P}$ such that $N \cap N^{\prime}=\emptyset, R \in \mathcal{R}^{N}$, and $R^{\prime} \in \mathcal{R}^{N^{\prime}}$, let $\left(R, R^{\prime}\right)$ denote the profile $R^{\prime \prime} \in \mathcal{R}^{N \cup N^{\prime}}$ such that for all $i \in N, R_{i}^{\prime \prime}=R_{i}$, and for all $i \in N^{\prime}, R_{i}^{\prime \prime}=R_{i}^{\prime}$. Given $N \in \mathcal{P}$ and $R \in \mathcal{R}^{N}$, let $\underline{p}(R) \equiv \min \left\{p\left(R_{i}\right) \mid i \in N\right\}$ and $\bar{p}(R) \equiv \max \left\{p\left(R_{i}\right) \mid i \in N\right\}$. We call the interval $[\underline{p}(R), \bar{p}(R)]$ the peaks interval of $R$.

There is a fixed number, denoted by $m \in \mathbb{N}$, of identical public facilities. This number is exogenously given. Note that we do not exclude the case $m=1$. For each of the facilities we have to select a location in the interval $[0,1]$. Let $M \equiv\{1, \ldots, m\}$. The order in which we locate the facilities is irrelevant. An alternative is a $m$-tuple $x=\left(x_{1}, \ldots, x_{m}\right)$ such that $0 \leq x_{1} \leq x_{2} \leq \cdots \leq x_{m} \leq 1$. Let $[0,1]^{M}$ denote the set of all alternatives. We allow the possibility that for some $k, k^{\prime} \in M, x_{k}=x_{k^{\prime}}$.

Preferences are defined over $[0,1]$. When $m \geq 2$ the set of alternatives and the set of locations are distinct. In this and the following two sections we consider the location of pure public goods. Agents consume the facilities without rivalry and each agent uses only the good located at his best point. An example is the location of bus stops. Lsing this motivation we extend preferences from locations to alternatives as follows. Given two altenatives, an agent strictly prefers the first alternative to the second if he strictly prefers the best location of the first alternative to the best location of the second relative to his single-peaked preference relation over locations. We think of each alternative as an option set from which agents can freely select, and assume that each agent compares two alternatives by comparing the locations that he prefers. 


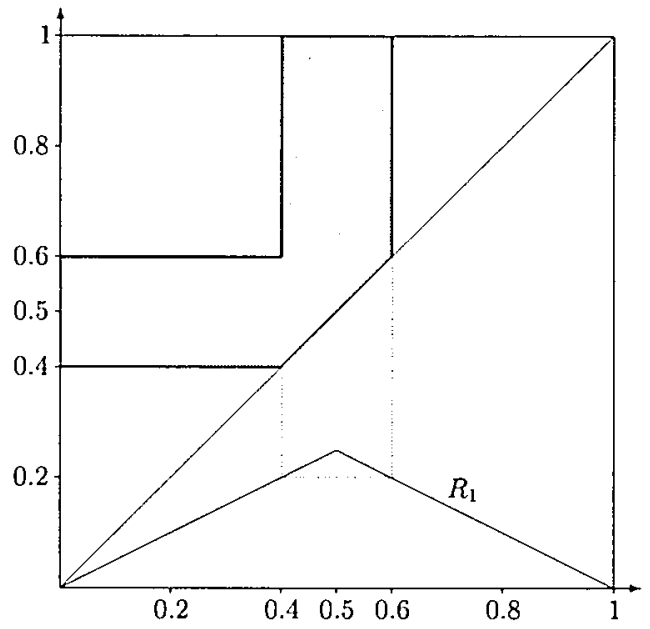

Figure 2.1: The preference $R_{1}$ is symmetric around the peak 0.5 . The closed set of the shaded area is the weak upper contour set at $(0.2,0.6)$ when we extend $R_{1}$ maximally. Furthermore, the four thicker line segments $[0.0 .4] \times\{0.4\},[0,0.4] \times\{0.6\},\{0.4\} \times[0.6,1]$, and $\{0.6\} \times[0.6,1]$ is the set of all alternatives in $[0,1]^{M}$ that are indifferent to $(0.2,0.6)$.

Max-Extension of Preferences, $R_{i}^{\max }$ : Let $i \in \mathbb{P}$ and $R_{i} \in \mathcal{R}$. Given two alternatives $x, y \in[0,1]^{M}, x$ is maximally strictly preferred to $y, x P_{i}^{\max } y$, if and only if for some $\bar{k} \in M$ and all $k \in M, x_{\bar{k}} P_{i} y_{k}$. Furthermore, $x$ is maximally indifferent to $y, x I_{i}^{\max } y$, if and only if for some $\bar{k}, \tilde{k} \in M$ and all $k \in M, x_{\bar{k}} R_{i} y_{k}$ and $y_{\bar{k}} R_{i} x_{k}$.

When we extend preferences maximally, weak upper contour sets ${ }^{6}$ are closed but not convex. Furthermore, in general, indifference sets consist of four line segments. Figure 2.1 illustrates this fact for $M=\{1,2\}$.

A (decision) rule is a mapping $\varphi$ that associates with each $N \in \mathcal{P}$ and each $R \in \mathcal{R}^{N}$ an alternative, denoted by $\varphi(R)=\left(\varphi_{1}(R) \ldots, \varphi_{m}(R)\right)$. In other words a rule selects for each profile and each facility a location.

The first requirement says that for each preference profile the chosen alternative camnot be changed in such a way that no agent is worse off and some agent is better off. Given $N \in \mathcal{P}$ and $R \in \mathcal{R}^{N}$, let $E\left(R^{\max }\right)$ denote the set of Pareto-optimal (or efficient) alternatives

\footnotetext{
${ }^{6}$ Given $i \in \mathbb{P}, R_{i} \in \mathcal{R}$, and $x \in[0,1]^{M}$, the set $B\left(x, R_{i}\right)=\left\{y \in[0,1]^{M} \mid y R_{i}^{m a x} x\right\}$ is the weak upper contour set of $x$ at $R_{i}^{\max }$.
} 
for $R$ when preferences are extended maximally. Formally, $E\left(R^{\max }\right) \equiv\left\{y \in[0,1]^{M} \mid\right.$ for all $x \in[0,1]^{M}$, if for some $i \in N, x P_{i}^{\max } y$, then for some $\left.j \in N, y P_{j}^{\max } x\right\}$.

Pareto-Optimality: For all $N \in \mathcal{P}$ and all $R \in \mathcal{R}^{N}, \varphi(R) \in E\left(R^{\max }\right)$.

When $n \geq 2$, for Pareto-optimality it is necessary but not sufficient that for each public good the selected location belongs to $[p(R), \bar{p}(R)]$. When $m=1$ for Pareto-optimality it is also sufficient that for each profile the chosen location belongs to the peaks interval of that profile.

Remark 2.1 If all agents report the same peak, then an altenative is Pareto-optimal if and only if one good is located at the common peak. This also means that the other goods may be located on some arbitrary points.

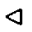

\subsection{Miyagawa's Results}

Throughout Section 2.3 let $M \equiv\{1,2\}$. By Remark 2.1, a rule may locate one facility arbitrarily if all agents report the same peak. For all characterizations of Section 2.3 we restrict the domain to all profiles having at least two distinct peaks. Given $N \in \mathcal{P}$, let $\mathcal{V}(N) \subsetneq \mathcal{R}^{N}$ denote the set of profiles with at least two distinct peaks. Formally, $\mathcal{V}(N) \equiv\left\{R \in \mathcal{R}^{N} \mid \underline{p}(R) \neq \bar{p}(R)\right\}$.

We give a short overview of some of the results derived by Miyagawa (1998a,b,c). We briefly describe each property and state his characterizations. The properties are discussed in more detail in later sections.

The first one says that agents can never gain by misreporting their preferences. The second one requires that small changes in preferences do not affect the option set too much. Here, $[0,1]^{M}$ is a subset of $\mathbb{R}^{M}$ and endowed with the induced Euclidean topology.

Strategy-Proofness: For all $N \in \mathcal{P}$, all $i \in N$, and all $R, \bar{R} \in \mathcal{R}^{N}$ such that $R_{N \backslash\{i\}}=$ $\bar{R}_{N \backslash\{i\}}: \varphi(R) R_{i}^{\max } \varphi(\bar{R})$.

Preference Continuity: For all $N \in \mathcal{P}$, all $i \in N$, all $R \in \mathcal{R}^{N}$, and all sequences $\left(R_{i}^{l}\right)_{t \in \mathbb{N}}$ such that for all $t \in \mathbb{N}, R_{i}^{\ell} \in \mathcal{R}:$ if $R_{i}^{l} \rightarrow R_{i}$, then $\varphi\left(R_{i}^{l}, R_{N \backslash\{i\}}\right) \rightarrow \varphi(R)$.

It turns out that the following rule is important for these two requirements together with Pareto-optimality. It is the rule that chooses for each profile the smallest and the 
greatest peak.

Extreme-Peaks Rule, $\varphi^{E}:$ For all $N \in \mathcal{P}$ and all $R \in \mathcal{R}^{N}, \varphi^{E}(R) \equiv(\underline{p}(R), \bar{p}(R))$.

Theorem 2.1 (Miyagawa, 1998a) Let $N \in \mathcal{P}$. On the domain $\mathcal{V}(N)$ the extremepeaks rule is the only rule satisfying Pareto-optimality, strategy-proofness, and preference continuity.

The next property says that if the preference of some agent changes, then, as a result, either all the remaining agents lose or they all gain. ${ }^{7}$

Replacement-Domination: For all $N \in \mathcal{P}$, all $j \in N$, and all $R, \bar{R} \in \mathcal{R}^{N}$ such that $R_{N \backslash\{j\}}=\bar{R}_{N \backslash\{j\}}$ : either [for all $i \in N \backslash\{j\}, \varphi(R) R_{i}^{\text {max }} \varphi(\bar{R})$ ] or [for all $i \in N \backslash\{j\}$, $\left.\varphi(\bar{R}) R_{i}^{\max } \varphi(R)\right]$.

The following two rules satisfy replacement-domination. One is the rule that chooses for each profile the two smallest distinct peaks, and the other is the rule that chooses for each profile the two greatest distinct peaks.

Left-Peaks Rule, $\varphi^{L}$ : For all $N \in \mathcal{P}$ and all $R \in \mathcal{R}^{N}$, if $\underline{p}(R)=\bar{p}(R)$, then $\varphi^{L}(R) \equiv$ $(\underline{p}(R), \underline{p}(R))$, and otherwise, $\varphi^{L}(R) \equiv\left(\underline{p}(R), \min \left\{p\left(R_{j}\right) \mid j \in \bar{N}\right.\right.$ and $\left.\left.\underline{p}(R)<p\left(R_{j}\right)\right\}\right)$.

Right-Peaks Rule, $\varphi^{C}$ : For all $N \in \mathcal{P}$ and all $R \in \mathcal{R}^{N}$, if $\underline{p}(R)=\bar{p}(R)$, then $\varphi^{C}(R) \equiv$ $(\bar{p}(R), \bar{p}(R))$, and otherwise, $\varphi^{C}(R) \equiv\left(\max \left\{p\left(R_{j}\right) \mid j \in N\right.\right.$ and $\left.\left.p\left(R_{j}\right)<\bar{p}(R)\right\}, \bar{p}(R)\right)$.

It turns out that for populations with at least four agents these two rules are the only ones satisfying in addition Pareto-optimality.

Theorem 2.2 (Miyagawa, 1998b) Let $N \in \mathcal{P}$ be such that $|N| \geq 4$. On the domain $\mathcal{V}(N)$ the left-peaks rule and the right-peaks rule are the only rules satisfying Paretooptimality and replacement-domination.

Remark 2.2 Let $N \in \mathcal{P}$ be such that $|N|=3$. Consider a rule that chooses two distinct peaks for all profiles with at least two distinct peaks. Such a rule satisfies Pareto-optimality and replacement-domination since a preference change affects at most one agent.

\footnotetext{
${ }^{7}$ When defining a solidarity property, we write that either statement $A$ or statement $B$ holds. However, then we do not exclude the possibility that both $A$ and $B$ hold.
} 
The next property says that if some agents leave, then the welfares of the agents who did not leave are affected in the same direction: as a result of the leave either they all lose or they all gain.

Population-Monotonicity: For all $N, N^{\prime} \in \mathcal{P}$ such that $N \subseteq N^{\prime}$ and all $R \in \mathcal{R}^{N^{\prime}}$ : either [for all $i \in N, \varphi\left(R_{N}\right) R_{i}^{\max } \varphi(R)$ ] or [for all $i \in N, \varphi(R) R_{i}^{\max } \varphi\left(R_{N}\right)$ ].

The following characterization of the extreme-peaks rule uses population-monotonicity.

Theorem 2.3 (Miyagawa, 1998c) Let $\mathbb{P}=\mathbb{N}$. On the domain $\cup_{N \in \mathcal{P}} \mathcal{V}(N)$ the extremepeaks rule is the only rule satisfying Pareto-optimality, population-monotonicity, and preference continuity.

On the general domain $\mathcal{R}^{N}$ the above characterizations have to be reformulated in the following way. For example, a rule satisfies Pareto-optimality, strategy-proofness, and preference continuity if and only if it is the extreme peaks rule on the domain $\mathcal{V}(N)$ and for each profile where the peaks of all agents coincide, the rule chooses one location to be the unanimous peak. Similar reformulations apply to Theorems 2.2 and 2.3. This is due to Remark 2.1 and the fact that for each preference relation an alternative where one location is the peak of this relation is most preferred among all altematives relative to the max-extension of this relation.

\subsection{Independence Axioms}

\subsubsection{Definitions}

Throughout Section 2.4 we keep the set of agents fixed. Let $n \in \mathbb{N}$ and $N \equiv\{1, \ldots, n\}$ be the set of agents. We allow for changes in the set of locations. First, we introduce some notation and adapt some definitions of Section 2.2 to include such changes in our model.

A feasible set of locations is a closed interval $[a, b] \subseteq[0,1]$. Let $\mathcal{B} \equiv\{[a, b] \mid 0 \leq a \leq$ $b \leq 1\}$ denote the set of all such intervals. Given $B \in \mathcal{B}$ and $x \in[0,1]$, let $\operatorname{proj}_{B}(x)$ denote the closest point to $x$ in $B$. Let $B^{M}=\left\{x \in[0,1]^{M} \mid\right.$ for all $\left.k \in M, x_{k} \in B\right\}$ denote the altematives where each location belongs to $B$. Given $B \in \mathcal{B}, R \in \mathcal{R}^{N}$, and $i \in N$, let $R_{i} \mid B$ denote the restriction of $R_{i}$ to $B$ and $R \mid B \equiv\left(R_{i} \mid B\right)_{i \in N}$. A solution is a mapping $\psi$ associating with every profile $R \in \mathcal{R}^{N}$ and every interval $B \in \mathcal{B}$ an alternative $\psi(R, B) \in[0,1]^{M}$ such that for all $k \in M, \psi_{k}(R, B) \in B$. When $m=1$, then $\psi(R, B)$ denotes the location of the unique public good. Note that a rule defined as in Section 2.2 
does not depend on the set of locations. We consider solutions only in Section 2.4. All other sections deal with rules.

We are interested in identifying solutions satisfying certain axions. The first requirement is that for each feasible interval and each chosen alternative $y$, there does not exist another altemative $x$ consisting of locations taken from this interval such that all agents weakly prefer $x$ to $y$ and some agent strictly prefers $x$ to $y$. The axiom is called Pareto-optimality. Note that we have to redefine this property since a solution depends on the set of locations. If $m=1$, then Pareto-optimality is equivalent to the requirement that the solution chooses for all $R \in \mathcal{R}^{N}$ and all $B \in \mathcal{B}$ a location belonging to $\left[\operatorname{proj}_{B}(\underline{p}(R)), \operatorname{proj}_{B}(\bar{p}(R))\right]$. If $m \geq 2$, it is necessary for Pareto-optimality but not sufficient that the option set be a subset of $\left[\operatorname{proj}_{B}(\underline{p}(R)), \operatorname{proj}_{B}(\bar{p}(R))\right]$.

Pareto-Optimality: For all $R \in \mathcal{R}^{N}$ and all $B \in \mathcal{B}$ : there exists no $x \in B^{M}$ such that for all $i \in N, x R_{i}^{\text {max }} \psi(R, B)$, and for some $j \in N, x P_{j}^{\max } \psi(R, B)$.

Note that with respect to our extension, under Pareto-optimality, the case $m \geq n$ is trivial. Then we can locate one public good at each peak. We will always assume that $m \leq n-1$.

The first independence axiom requires that given a fixed preference profile and a feasible interval, if the feasible interval shrinks in such a way that the previous choice remains feasible, then both choices are the same. We use the three letters IIA for the abbreviation of the phrase "Independence of Irrelevant Alternatives."

Nash's IIA: For all $R \in \mathcal{R}^{N}$ and all $B, B^{\prime} \in \mathcal{B}$ such that $B \subseteq B^{\prime}$ : if $\psi\left(R, B^{\prime}\right) \in B^{M}$, then $\psi(R, B)=\psi\left(R, B^{\prime}\right)$.

Next is the requirement that for each feasible interval and for each pair of preference profiles that coincide on the set of alternatives that consist of locations belonging to that interval, the social choice is the same. Note that two preference profiles restricted to a feasible interval coincide if and only if their extensions restricted to the alternatives that consist of locations belonging to the feasible interval coincide. It is straightforward to prove that $R|B=\bar{R}| B$ if and only if $R^{\max }\left|B^{M}=\vec{R}^{\max }\right| B^{M}$ by considering alternatives $(x, \ldots, x), x \in B$.

Arrow's IIA: For all $R, \bar{R} \in \mathcal{R}^{N}$ and all $B \in \mathcal{B}$ : if $R|B=\tilde{R}| B$, then $\psi(R, B)=\psi(\bar{R}, B)$. 
The last requirement is that a small change in the feasible interval does not affect the choice too much. In our formal definition $\mathcal{B}$ and $[0,1]^{M}$ are identified with subsets of $\mathbb{R}^{2}$ and $\mathbb{R}^{M}$, respectively, and endowed with the induced Euclidean topologies.

Interval Continuity: For all $R \in \mathcal{R}^{N}: \not,(R, B)$ is continuous with respect to $B \in \mathcal{B}$.

Our first lemma says that under Nash's IIA and interval continuity, for each preference profile, if the lower boundary point of the feasible interval increases, then the following holds: (i) when the smallest point of the initial option set belongs to the new interval, then the smallest points of both choices are the same; and (ii) otherwise, the smallest point of the new option set is the lower boundary point of the new interval. A parallel statement holds for the greatest point of the option set when the upper boundary point of the feasible interval decreases.

Lemma 2.1 Let $\psi$ be a solution satisfying Nash's IIA and interval continuity. Let $R \in$ $\mathcal{R}^{N}$ and $B \equiv[a, b] \in \mathcal{B}$. Then for all $x \in[a, b], \psi_{1}(R,[x, b])=\operatorname{proj}_{[x, b]}\left(\psi_{1}(R, B)\right)$ and $\psi_{m}(R,[a, x])=\operatorname{proj}_{[a, x]}\left(\psi_{m}(R, B)\right)$.

Proof. Let $R \in \mathcal{R}^{N}, B \equiv[a, b] \in \mathcal{B}$; and $x \in[a, b]$. We show that

$$
\psi_{1}(R,[x, b])=\operatorname{proj}_{[x, b]}\left(\psi_{1}(R, B)\right)
$$

If $x \leq \psi_{1}(R, B)$, then Nash's IIA implies (2.1). If $x>\psi_{1}(R, B)$ and

$$
\operatorname{proj}_{[x, b]}\left(\psi_{1}(R, B)\right)=x<\psi_{1}(R,[x, b])
$$

then by interval continuity there exists $\left.x^{\prime} \in\right] a, x\left[\right.$ such that $\left.\psi_{1}\left(R,\left[x^{\prime}, b\right]\right) \in\right] x, \psi_{1}(R,[x, b])[$, which contradicts Nash's IIA. Hence, (2.1) holds. Similarly it follows that $\psi_{m}(R,[a, x])=$ $\operatorname{proj}_{[a, x]}\left(\psi_{m}(R, B)\right)$.

\subsubsection{Augmented Median Solutions}

For the problem of locating one public good, Moulin (1984) characterizes the class of solutions satisfying anonymity (the solution is symmetric in its arguments) and the four axioms introduced in Subsection 2.4.1. Using the results of Ching (1997) we obtain a simple generalization of Moulin's result. We drop Pareto-optimality and anonymity, and show that the augmented median solutions are the only solutions satisfying Nash's $I I A$, Arrow's $I I A$, and interval continuity. 
Augmented Median Solution, $\psi^{\alpha}$ : Let $\alpha \equiv\left(\alpha_{S}\right)_{S \subseteq N}$ be such that for all $S \subseteq N$, $\alpha_{S} \in[0,1]$, and for all $N \supseteq S \supseteq S^{\prime}, \alpha_{S} \leq \alpha_{S^{\prime}}$. For all $B \in \mathcal{B}$ and all $R \in \mathcal{R}^{N}$ such that $p\left(R_{i_{1}}\right) \leq p\left(R_{i_{2}}\right) \leq \cdots \leq p\left(R_{i_{n}}\right)$, let

$$
\psi^{\alpha}(R, B) \equiv \operatorname{proj}_{B}\left(\operatorname{med}\left(p\left(R_{1}\right), \ldots, p\left(R_{n}\right), \alpha_{\emptyset}, \alpha_{\left\{i_{1}\right\}}, \alpha_{\left\{i_{1}, i_{2}\right\}}, \ldots, \alpha_{N}\right)\right) .
$$

Theorem 2.4 Let $m=1$. The augmented median solutions are the only solutions satisfying Nash's IIA, Arrow's IIA, and interval continuity.

We prove Theorem 2.4 in two steps. First, we show that Nash's IIA, Arrow's IIA, and interval continuity imply uncompromisingness. ${ }^{8}$ Ching (1997) characterizes the class of augmented median solutions by uncompromisingness. Second, we use his result to provide a simple proof of Theorem 2.4 .

Uncompromisingness: For all $j \in N$, all $R, R^{\prime} \in \mathcal{R}^{N}$ such that $R_{N \backslash\{j\}}=R_{N \backslash\{j\}}^{\prime}$, and all $B \in \mathcal{B}$ : if $\left[\psi(R, B)<p\left(R_{j}\right)\right.$ and $\left.\psi(R, B) \leq p\left(R_{j}^{\prime}\right)\right]$ or $\left[\psi(R, B)>p\left(R_{j}\right)\right.$ and $\left.\psi(R, B) \geq p\left(R_{j}^{\prime}\right)\right]$, then $\psi(R, B)=\psi\left(R^{\prime}, B\right)$.

Lemma 2.2 Let $\psi$ be a solution satisfying Nash's IIA, Arrow's IIA, and interval continuity. Then $\psi$ satisfies uncompromisingness.

Proof. Let $j \in N$ and $R, R^{\prime} \in \mathcal{R}^{N}$ be such that $R_{N \backslash\{j\}}=R_{N \backslash\{j\}}^{\prime}$. Let $B=[a, b] \in \mathcal{B}$ and, without loss of generality, assume that $\psi(R, B)<p\left(R_{j}\right)$ and $\psi(R, B) \leq p\left(R_{j}^{\prime}\right)$. In order to prove uncompromisingness, we have to show that

$$
\psi(R, B)=\psi\left(R^{\prime}, B\right)
$$

If $b \leq \min \left\{p\left(R_{j}\right), p\left(R_{j}^{\prime}\right)\right\}$, then Arrow's IIA implies (2.2). Let $\left\{p\left(R_{j}\right), p\left(R_{j}^{\prime}\right)\right\} \cap[0, b[\neq \emptyset$. Thus, $\psi(R, B)<b$.

Claim 1: If $p\left(R_{j}^{\prime}\right)>\psi(R, B)$, then (2.2) holds.

Proof of Claim 1: Without loss of generality, we assume that $\psi(R, B)<p\left(R_{j}^{\prime}\right) \leq p\left(R_{j}\right)$. Nash's IIA inplies $\psi(R, B)=\psi\left(R,\left[a, p\left(R_{j}^{\prime}\right)\right]\right)$. Since $p\left(R_{j}^{\prime}\right) \leq p\left(R_{j}\right)$, it follows that $R\left\|\left[a, p\left(R_{j}^{\prime}\right)\right]=R^{\prime}\right\|\left[a, p\left(R_{j}^{\prime}\right)\right]$. Thus, by Arrow's $I I A, \psi\left(R,\left[a, p\left(R_{j}^{\prime}\right)\right]\right)=\psi\left(R^{\prime},\left[a, p\left(R_{j}^{\prime}\right)\right]\right)$. By Lemma 2.1, $\psi\left(R^{\prime},\left[a, p\left(R_{j}^{\prime}\right)\right]\right)=\operatorname{proj}_{\left[a, p\left(R_{j}^{\prime}\right)\right]}\left(\psi\left(R^{\prime}, B\right)\right)$. Hence, $\psi\left(R^{\prime},\left[a, p\left(R_{j}^{\prime}\right)\right]\right)=\psi\left(R^{\prime}, B\right)$ and $\psi\left(R^{\prime}, B\right)=\psi(R, B)$.

To conclude we have to show (2.2) for $p\left(R_{j}^{\prime}\right)=\psi(R, B)$. By Nash's IIA and Arrow's IIA, $\psi(R, B)=\psi\left(R,\left[a, p\left(R_{j}^{\prime}\right)\right]\right)=\psi\left(R^{\prime},\left[a, p\left(R_{j}^{\prime}\right)\right]\right)$. Thus, by Lemma $2.1, p\left(R_{j}^{\prime}\right) \leq \psi\left(R^{\prime}, B\right)$. If

\footnotetext{
${ }^{8}$ Border and Jordan (1983) introduce uncompromisingness in this model.
} 
$p\left(R_{j}^{\prime}\right)<\psi\left(R^{\prime}, B\right)$, then by adapting the proof of Claim 1 we can show that for all $R^{\prime \prime} \in \mathcal{R}^{N}$ such that $R_{N \backslash\{j\}}^{\prime \prime}=R_{N \backslash\{j\}}^{\prime}$ and $p\left(R_{j}^{\prime \prime}\right)<\psi\left(R^{\prime}, B\right)$, we have $\psi\left(R^{\prime \prime}, B\right)=\psi\left(R^{\prime}, B\right)$. Because ]$p\left(R_{j}^{\prime}\right), \psi\left(R^{\prime}, B\right)[\neq \emptyset$, the previous fact contradicts Claim 1.

Proof of Theorem 2.4. It is straightforward to check that augmented median solutions satisfy the properties listed in Theorem 2.4 . Conversely, let $\psi$ be a solution satisfying these properties. By Lemma 2.2, $\psi$ satisfies uncompromisingness. By the results of Ching (1997, Theorem and Lemma 2), for all $B \in \mathcal{B}$ there exists a list of parameters $\alpha^{B} \equiv\left(\alpha_{S}^{B}\right)_{S \subseteq N}$ such that for all $S \subseteq N, \alpha_{S}^{B} \in B, N \supseteq S \supseteq S^{\prime}$ implies $\alpha_{S}^{B} \leq \alpha_{S^{\prime}}^{B}$, and for all $R \in \mathcal{R}^{N}$, $\psi(R, B)=\psi^{\alpha^{\prime \prime}}(R, B)$. It suffices to show that for all $B \in \mathcal{B}$ and all $S \subseteq N$,

$$
\alpha_{S}^{B}=\operatorname{proj}_{B}\left(\alpha_{S}^{\mid 0,1]}\right)
$$

Let $B \equiv[a, b] \in \mathcal{B}$ and $S \subseteq N$. Let $R \in \mathcal{R}^{N}$ be such that for all $i \in S, p\left(R_{i}\right)=0$, and for all $j \in N \backslash S, p\left(R_{j}\right)=1$. Then $\psi(R, B)=\psi^{\alpha^{b}}(R, B)=\alpha_{S}^{B}$ and $\psi(R,[0,1])=$ $\psi^{\alpha^{[0,1]}}(R,[0,1])=\alpha_{S}^{[0,1]}$. If $\alpha_{S}^{[0,1]} \in B$, then by Nash's $I I A \alpha_{S}^{B}=\alpha_{S}^{[0,1]}$. Without loss of generality, suppose that $b<\alpha_{S}^{[0,1]}$. By Nash's IIA, $\psi(R,[a, 1])=\alpha_{S}^{[0,1]}$. By Lemma 2.1, $\psi(R, B)=\operatorname{proj}_{B}(\psi(R,[a, 1]))=\operatorname{proj}_{B}\left(\alpha_{S}^{[0,1]}\right)$. Thus, (2.3) holds and $\psi$ is an augmented median solution.

\subsubsection{The Extreme-Peaks Solution}

This subsection studies the problem of locating two public facilities. It turns out that the following solution plays an important role. It is the solution that always chooses the two feasible points that are closest to the extreme peaks of the profile.

Extreme-Peaks Solution, $\psi^{E}$ : For all $R \in \mathcal{R}^{N}$ and all $B \in \mathcal{B}, \psi^{E}(R, B) \equiv\left(\operatorname{proj}_{\mathcal{B}}(\underline{p}(R))\right.$, $\left.\operatorname{proj}_{B}(\bar{p}(R))\right)$.

It is straightforward to verify that the extreme-peaks solution satisfies Pareto-optimality, Nash's IIA, Arrow's IIA, and interval continuity. Our next result is that this solution is the only one satisfying this combination of axioms.

Theorem 2.5 Let $m=2$. The extreme-peaks solution is the only solution satisfying Pareto-optimality, Nash's IIA, Arrow's IIA, and interval continuity.

Proof. Let $\psi$ be a solution satisfying the properties listed in Theorem 2.5. Suppose that $\psi \neq \psi^{E}$. Then there exists $R \in \mathcal{R}^{N}$ and $B \equiv[a, b] \in \mathcal{B}$ such that

$$
\psi(R, B) \neq \psi^{E}(R, B)
$$


We distinguish two cases.

Case 1: $\operatorname{proj}_{B}(\underline{p}(R))=\operatorname{proj}_{B}(\bar{p}(R))$.

By definition, $\psi^{E}(R, B)=\left(\operatorname{proj}_{B}(\underline{p}(R)), \operatorname{proj}_{B}(\underline{p}(R))\right)$. By Pareto-optimality, $\operatorname{proj}_{B}(\underline{p}(R)) \in\left\{\psi_{1}(R, B), \psi_{2}(R, B)\right\}$. Without loss of generality, suppose that $\psi_{2}(R, B)=$ $\operatorname{proj}_{B}(\underline{p}(R))<1$. Thus, $\psi_{1}(R, B)<\operatorname{proj}_{B}(\underline{p}(R)) .{ }^{9} \operatorname{By} \operatorname{Nash}^{\prime} \operatorname{IIA}, \psi\left(R,\left[a, \operatorname{proj}_{B}(\underline{p}(R))\right]\right)=$ $\psi(R, B)$. Let $R^{\prime} \in \mathcal{R}^{N}$ be such that $p\left(R_{1}^{\prime}\right)=\operatorname{proj}_{B}(p(R)), p\left(R_{2}^{\prime}\right)=1$, and for all $i \in N \backslash\{1,2\}, R_{i}^{\prime}=R_{2}^{\prime}$. By Arrow's IIA,

$$
\psi\left(R^{\prime},\left[a, \operatorname{proj}_{B}(\underline{p}(R))\right]\right)=\psi\left(R,\left[a, \operatorname{proj}_{B}(\underline{p}(R))\right]\right)=\left(\psi_{1}(R, B), \operatorname{proj}_{B}(\underline{p}(R))\right)
$$

By Pareto-optimality, for all $\left.\varepsilon \in] 0,1-p\left(R_{1}^{\prime}\right)\right]$,

$$
\psi\left(R^{\prime},\left[a, \operatorname{proj}_{B}(\underline{p}(R))+\varepsilon\right]\right)=\left(\operatorname{proj}_{B}(\underline{p}(R)), \operatorname{proj}_{B}(\underline{p}(R))+\varepsilon\right)
$$

which contradicts interval continuity. Hence, Case 1 camnot occur.

Case 2: $\operatorname{proj}_{B}(\underline{p}(R)) \neq \operatorname{proj}_{B}(\bar{p}(R))$.

By Pareto-optimality, $\underline{p}(R) \leq \psi_{1}(R, B)$ and $\psi_{2}(R, B) \leq \bar{p}(R)$. By definition, $a \leq$ $\psi_{1}(R, B)$ and $\psi_{2}(R, B) \leq b$. Hence, $\max \{\underline{p}(R), a\} \leq \psi_{1}(R, B)$ and $\psi_{2}(R, B) \leq \min \{\bar{p}(R), b\}$. By the definition of the extreme-peaks solution,

$$
\psi^{E}(R, B)=(\max \{\underline{p}(R), a\}, \min \{\bar{p}(R), b\}) .
$$

Thus, (2.4) implies $\max \{\underline{p}(R), a\}<\psi_{1}(R, B)$ or $\psi_{2}(R, B)<\min \{\bar{p}(R), b\}$. Without loss of generality, suppose that

$$
\psi_{2}(R, B)<\min \{\bar{p}(R), b\}
$$

Let $a^{\prime} \equiv \psi_{1}(R, B), b^{\prime} \equiv \min \left\{\bar{p}(R), \frac{1}{2}\left(b+\psi_{2}(R, B)\right)\right\}$, and $B^{\prime} \equiv\left[a^{\prime}, b^{\prime}\right]$. By Nash's IIA and (2.5), $\psi(R, B)=\psi\left(R, B^{\prime}\right)$. Note that $b^{\prime}<1$. Let $\bar{R} \in \mathcal{R}^{N}$ be such that for all $i \in N$ :

- if $p\left(R_{i}\right) \leq a^{\prime}$, then $\bar{R}_{i} \equiv R_{i}$;

- if $\left.p\left(R_{i}\right) \in\right] a^{\prime}, b^{\prime}\left[\right.$, then $\bar{R}_{i}\left|B^{\prime} \equiv R_{i}\right| B^{\prime}, a^{\prime} \bar{P}_{i} 1$, and $\bar{R}_{i}$ is continuous on $\left[b^{\prime}, 1\right] ;$ and

- if $p\left(R_{i}\right) \geq b^{\prime}$, then $p\left(\bar{R}_{i}\right) \equiv 1$.

By definition of $\bar{R}, \bar{R}\left|B^{\prime}=R\right| B^{\prime}$. Hence, by Arrow's IIA, $\psi\left(\bar{R}, B^{\prime}\right)=\psi\left(R, B^{\prime}\right)$. By Lemma $2.1, \psi_{2}\left(\tilde{R}, B^{\prime}\right)=\operatorname{proj}_{B^{\prime}}\left(\psi_{2}\left(\bar{R},\left[a^{\prime}, 1\right]\right)\right)$. Since $\psi_{2}\left(\bar{R}, B^{\prime}\right)<b^{\prime}$, it follows that $\psi_{2}\left(\bar{R}, B^{\prime}\right)=$

\footnotetext{
${ }^{9}$ If $\xi_{2}(R, B)=1$, then by interval continuity and Lemma 2.1 , there exists $\varepsilon>0$ such that $\psi_{1}(R,[a, 1-$ $\varepsilon])<1-\varepsilon$ and $\psi \%_{2}(R,[a, 1-\varepsilon])=1-\varepsilon$.
} 
$\psi_{2}\left(\bar{R},\left[a^{\prime}, 1\right]\right)$. Thus, by Nash's IIA and Lemma $2.1, \psi\left(\bar{R}, B^{\prime}\right)=\psi\left(\bar{R},\left[a^{\prime}, 1\right]\right)$. Let $S \equiv\{i \in$ $\left.N \mid p\left(\bar{R}_{i}\right)=1\right\}$.

Let $\bar{a} \equiv \max \left\{p\left(\bar{R}_{i}\right) \mid i \in N\right.$ and $\left.p\left(\bar{R}_{i}\right)<1\right\}$. By construction of $\bar{R}$, Pareto-optimality, and $\psi_{2}\left(\bar{R}, B^{\prime}\right)<1$, we have $a^{\prime}<\bar{a}<b^{\prime}$. By Pareto-optimality and the definition of $\bar{a}$, $\psi(\bar{R},[\bar{a}, 1])=(\bar{a}, 1)$. By construction of $\bar{R}$, there exists $\varepsilon>0$ such that $1-\varepsilon>b^{\prime}$ and for all $i \in N \backslash S, a^{\prime} \bar{P}_{i}(1-\varepsilon)$. Since $\psi_{2}\left(\bar{R},\left[a^{\prime}, 1\right]\right)<b^{\prime}$ and $\psi_{2}(\bar{R},[\bar{a}, 1])=1$, by interval continuity, for some $\left.a_{\varepsilon} \in\right] a^{\prime}, \bar{a}\left[, \psi_{2}\left(\bar{R},\left[a_{\varepsilon}, 1\right]\right)=1-\varepsilon\right.$. By Lemma 2.1, $\psi_{1}\left(\bar{R},\left[a_{\varepsilon}, 1\right]\right)=a_{\varepsilon}$. Since for all $i \in N \backslash S, a^{\prime} \bar{P}_{i}(1-\varepsilon)$, and $\bar{p}(\bar{R})=1$, it follows that for all $i \in N \backslash S,\left(a_{\varepsilon}, 1\right) \bar{R}_{i}^{\text {max }} \psi\left(\bar{R},\left[a_{\varepsilon}, 1\right]\right)$, and for all $j \in S,\left(a_{\varepsilon}, 1\right) \bar{P}_{j}^{\text {max }} \psi\left(\bar{R},\left\{a_{\varepsilon}, 1\right]\right)$. Hence, $\psi$ violates Pareto-optimality and Case 2 cannot occur.

In Theorem 2.5 Arrow's IIA can be dropped if we impose a stronger version of Nash's $I I A$. This version says that if one point is chosen for a certain interval, then this point also has to be an option for all subintervals containing this point. The requirement can be described as a "consistency" condition.

Strong Nash's IIA: For all $R \in \mathcal{R}^{N}$ and all $B, B^{\prime} \in \mathcal{B}$ such that $B \subseteq B^{\prime}:\left\{\psi_{1}\left(R, B^{\prime}\right)\right.$, $\left.\psi_{2}\left(R, B^{\prime}\right)\right\} \cap B \subseteq\left\{\psi_{1}(R, B), \psi_{2}(R, B)\right\}$.

Theorem 2.6 Let $m=2$. The extreme-peaks solution is the only solution satisfying Pareto-optimality, strong Nash's IIA, and interval continuity.

Proof. Let $\psi$ be a solution satisfying the properties listed in Theorem 2.6. Claims 1 and 2 below state that for each profile $R \in \mathcal{R}^{N}$ there exist two points, $\underline{x}(R)$ and $\bar{x}(R)$, such that for all intervals the points closest to $\underline{x}(R)$ and $\bar{x}(R)$ are chosen for that profile. Claim 3 is that for all $R \in \mathcal{R}^{N}, \underline{x}(R)=\underline{p}(R)$ and $\bar{x}(R)=\bar{p}(R)$, i.e., $\psi=\psi^{E}$.

Claim 1: Let $R \in \mathcal{R}^{N}$ and $B, B^{\prime} \in \mathcal{B}$ be such that $B \subseteq B^{\prime}$. Then

$$
\psi(R, B)=\left(\operatorname{proj}_{B}\left(\psi_{1}\left(R, B^{\prime}\right)\right), \operatorname{proj}_{B}\left(\psi_{2}\left(R, B^{\prime}\right)\right)\right)
$$

Proof of Claim 1: Let $B \equiv[a, b]$ and $B^{\prime} \equiv\left[a^{\prime}, b^{\prime}\right]$. If $\left\{\psi_{1}\left(R, B^{\prime}\right), \psi_{2}\left(R, B^{\prime}\right)\right\} \cap B=\emptyset$, then the proof of Lemma 2.1 yields $(2.6)$. If $\left\{\psi_{1}\left(R, B^{\prime}\right), \psi_{2}\left(R, B^{\prime}\right)\right\} \cap B=\left\{\psi_{1}\left(R, B^{\prime}\right), \psi_{2}\left(R, B^{\prime}\right)\right\}$, then Nash's IIA implies (2.6). Suppose that $\left|\left\{\psi_{1}\left(R, B^{\prime}\right), \psi_{2}\left(R, B^{\prime}\right)\right\} \cap B\right|=1$ and $\psi_{1}\left(R, B^{\prime}\right)$ $\neq \psi_{2}\left(R, B^{\prime}\right)$. Without loss of generality, suppose that $\psi_{1}\left(R, B^{\prime}\right) \in B$. By Nash's IIA, $\psi\left(R,\left[a, b^{\prime}\right]\right)=\psi\left(R, B^{\prime}\right)$. By strong Nash's $I I A, \psi_{1}(R, B)=\psi_{1}\left(R,\left[a, b^{\prime}\right]\right)$, and by Lemma $2.1, \psi_{2}(R,[a, b])=\operatorname{proj}_{[a, b]}\left(\psi_{2}\left(R,\left[a, b^{\prime}\right]\right)\right)$. Hence, (2.6) holds.

Claim 2: Let $R \in \mathcal{R}^{N}$. Then there exist two points, $\underline{x}(R)$ and $\bar{x}(R)$, such that for all $B \in \mathcal{B}, \psi(R, B)=\left(\operatorname{proj}_{B}(\underline{x}(R)), \operatorname{proj}_{B}(\bar{x}(R))\right)$. 
Proof of Claim 2: Let $\underline{x}(R) \equiv \psi_{1}(R,[0,1])$ and $\bar{x}(R) \equiv \psi_{2}(R,[0,1])$. By Claim 1, the desired conclusion follows.

Claim 3: For all $R \in \mathcal{R}^{N}, \underline{x}(R)=\underline{p}(R)$ and $\bar{x}(R)=\bar{p}(R)$.

Proof of Claim 3: Suppose that there exists $R \in \mathcal{R}^{N}$ such that $\underline{x}(R) \neq \underline{p}(R)$. By Pareto-optimality, $\underline{p}(R)<\underline{x}(R)$. By Claim $2, \psi(R,[0, \underline{x}(R)])=(\underline{x}(R), \underline{x}(R))$. But then $(\underline{p}(R), \underline{x}(R))$ Pareto dominates $(\underline{x}(R), \underline{x}(R))$, a contradiction. Similarly it follows that $\bar{x}(R)=\bar{p}(R)$.

The following four examples of solutions establish the independence of the axioms in Theorems 2.5 and 2.6 .

Example 2.1 For all $R \in \mathcal{R}^{N}$ and all $B \in \mathcal{B}, \psi(R, B) \equiv\left(\operatorname{proj}_{B}(0), \operatorname{proj}_{B}(1)\right)$. The solution $\psi$ satisfies strong Nash's IIA, Arrow's IIA, and interval continuity, but not Paretooptimality.

Example 2.2 Let $N \equiv\{1,2,3,4\}$. For all $B \equiv[a, b] \in \mathcal{B}$, let $\alpha(B) \equiv \min \left\{\max \left\{\frac{1}{8}-\right.\right.$ $\left.a, 0\}, \max \left\{b-\frac{7}{8}, 0\right\}\right\}$. Let $R \in \mathcal{R}^{N}$. Without loss of generality, we assume $p\left(R_{1}\right) \leq$ $p\left(R_{2}\right) \leq p\left(R_{3}\right) \leq p\left(R_{1}\right)$. If $\left(p\left(R_{1}\right), p\left(R_{2}\right), p\left(R_{3}\right), p\left(R_{1}\right)\right)=\left(\frac{1}{4}, \frac{3}{8}, \frac{5}{8}, \frac{3}{4}\right), p\left(R_{1}\right) P_{2} p\left(R_{3}\right)$, and $p\left(R_{1}\right) P_{3} p\left(R_{2}\right)$, then for all $B \in \mathcal{B}$,

$$
\breve{\psi}(R, B) \equiv\left(\operatorname{proj}_{B}(\underline{p}(R)+\alpha(B)), \operatorname{proj}_{B}(\bar{p}(R)-\alpha(B))\right) \text {. }
$$

Otherwise, for all $B \in \mathcal{B}, \breve{\psi}(R, B) \equiv \psi^{E}(R, B)$. The solution $\breve{\psi}$ satisfies Pareto-optimality, Arrow's IIA, and interval continuity, but not Nash's IIA.

Example 2.3 Let $N \equiv\{1,2,3,4\}$. Let $\bar{R} \in \mathcal{R}^{N}$ be such that $\left(p\left(\bar{R}_{1}\right), p\left(\bar{R}_{2}\right), p\left(\bar{R}_{3}\right), p\left(\bar{R}_{4}\right)\right)$ $=\left(\frac{1}{4}, \frac{1}{2}, \frac{3}{4}, 1\right)$, and $p\left(\bar{R}_{4}\right) \bar{P}_{3} p\left(\bar{R}_{2}\right)$. For all $B \equiv[a, b] \in \mathcal{B}, \bar{\psi}_{1}(\bar{R}, B) \equiv \operatorname{proj}_{B}(\underline{p}(\bar{R}))$ and

$$
\bar{\psi}_{2}(\bar{R}, B) \equiv \operatorname{proj}_{B}\left(p\left(\bar{R}_{3}\right)+\max \left\{a-p\left(\vec{R}_{1}\right), 0\right\}\right) .
$$

For all $R \in \mathcal{R}^{N} \backslash\{\bar{R}\}$ and all $B \in \mathcal{B}, \bar{\psi}(R, B) \equiv \psi^{E}(R, B)$. The solution $\bar{\psi}$ satisfies Paretooptimality, Nash's IIA, and interval continuity, but neither Arrow's IIA nor strong Nash's IIA.

Example 2.4 The left peaks solution $\psi^{L}$ is defined as follows. For all $R \in \mathcal{R}^{N}$ and all $B \equiv[a, b] \in \mathcal{B}, \psi_{1}^{L}(R, B) \equiv \operatorname{proj}_{B}(\underline{p}(R))$ and

$$
\psi_{2}^{L}(R, B) \equiv\left\{\begin{array}{l}
\operatorname{proj}_{B}(\underline{p}(R)) \text { if } \operatorname{proj}_{B}(\underline{p}(R))=\operatorname{proj}_{B}(\bar{p}(R)) \\
\min \left\{\operatorname{proj}_{B}\left(p\left(R_{i}\right)\right) \mid p\left(R_{i}\right)>\operatorname{proj}_{B}(\underline{p}(R))\right\} \text { otherwise. }
\end{array}\right.
$$

The solution $\psi^{L}$ satisfies Pareto-optinality, strong Nash's IIA, and Arrow's IIA, but not interval continuity. Note that $\psi^{L}$ satisfies continuity with respect to the intervals where the lower bound is fixed. 


\subsubsection{The Incompatibility}

Theorem 2.6 does not involve any requirement on preference changes similar to Arrow's IIA. Interval continuity is quite strong, not only for the location of two public facilities but also for the case of one public good (see Lemma 1 in Moulin (1984)).

Indeed, when we have to locate more than two public facilities, Pareto-optimality and interval continuity are incompatible. Therefore, neither independence axiom is compatible with these requirements.

Theorem 2.7 Let $m \geq 3$. Then there exists no solution satisfying Pareto-optimality and interval continuity.

Proof. Suppose that $\psi$ satisfies Pareto-optimality and interval continuity. Let $R \in \mathcal{R}$ be such that

$$
0=p\left(R_{1}\right)<p\left(R_{2}\right)<\cdots<p\left(R_{m}\right)<p\left(R_{m+1}\right)=\cdots=p\left(R_{n}\right)=1,
$$

$O P_{2} p\left(R_{3}\right), R_{2}$ is continuous, $1 P_{m} p\left(R_{m-1}\right)$, and $R_{m}$ is continuous. By Pareto-optimality, for all $k \in M, \psi_{k}\left(R,\left[0, p\left(R_{m}\right)\right]\right)=p\left(R_{k}\right)$. By Pareto-optimality, for all $b \in\left[p\left(R_{m}\right), 1\right]$, $\psi_{m}(R,[0, b]) \in\left[p\left(R_{m}\right), b\right]$. Suppose that for some $\left.\left.\hat{b} \in\right] p\left(R_{m}\right), 1\right], \psi_{m-1}(R,[0, \hat{b}])>p\left(R_{m-1}\right)$. Thus, by Pareto-optimality, $\psi_{m-1}(R,[0, \hat{b}]) P_{m} \psi_{m}(R,[0, \hat{b}])$. Since $R_{m}$ is continuous; $1 P_{m} p\left(R_{m-1}\right)$, and by interval continuity, for some $\left.\tilde{b} \in\right] 0, \hat{b}\left[, \psi_{m-1}(R,[0, \tilde{b}])>p\left(R_{m-1}\right)\right.$ and $1 P_{m} \psi_{m-1}(R,[0, \tilde{b}])$, which contradicts Pareto-optimality. Thus, for all $b \in\left[p\left(R_{m}\right), 1\right]$, $\psi_{m-1}(R,[0, b])=p\left(R_{m-1}\right)$, and by Pareto-optimality, for all $k \in\{1, \ldots, m-1\}, \psi_{k}(R,[0, b])$ $=p\left(R_{k}\right)$. In particular;

$$
\psi_{1}(R,[0,1])=0 \text { and } \psi_{2}(R,[0,1])=p\left(R_{2}\right) .
$$

Similarly, by Pareto-optimality, for all $k \in M, \psi_{k}\left(R,\left[p\left(R_{2}\right), 1\right]\right)=p\left(R_{k+1}\right)$. By Paretooptimality, for all $a \in\left[0, p\left(R_{2}\right)\right], \psi_{1}(R,[a, 1]) \in\left[a, p\left(R_{2}\right)\right]$. Similarly, since $R_{2}$ is continuous, $0 P_{2} p\left(R_{3}\right)$, and by interval continuity and Pareto-optimality, for all $k \in\{2, \ldots, m\}$, $\psi_{k}(R,[0, b])=p\left(R_{k+1}\right)$. In particular, $\psi_{1}(R,[0,1]) \in\left[0, p\left(R_{1}\right)\right]$ and $\psi_{2}(R,[0,1])=p\left(R_{3}\right)$, which contradicts $(2.7)$.

\subsubsection{Relation to Strategy-Proofness}

There is an interesting connection between our results and certain results on strategyproofness. Before we explain this connection, we introduce additional notation. Recall that a rule $\varphi$ assigns to each preference profile $R \in \mathcal{R}^{N}$ an alternative $\varphi(R) \in[0,1]^{M}$. Such a rule does not depend on the set of feasible intervals. Let $\varphi$ be a rule and $\psi$ a 
solution. A pair consists of a decision rule and a solution. The pair $(\varphi, \psi)$ is called corresponding if and only if for all $R \in \mathcal{R}^{N}$ and all $B \in \mathcal{B}, \psi(R, B)=\operatorname{proj}_{B}(\varphi(R))$. Note that if $(\varphi, \psi)$ is corresponding, then for all $R \in \mathcal{R}^{N}, \varphi(R)=\psi(R,[0,1])$.

Given an augmented median solution $\psi^{\alpha}$, the corresponding augmented median rule $\varphi^{\alpha}$ is defined as follows: for all $R \in \mathcal{R}, \varphi^{\alpha}(R) \equiv \psi^{\alpha}(R,[0,1])$. In particular, $\left(\varphi^{\alpha}, \psi^{\alpha}\right)$ is a corresponding pair. Obviously, for such a pair the following holds: $\varphi^{\alpha}$ is Paretooptimal if and only if $\psi^{\alpha}$ is Pareto-optimal. Ching (1997, Theorem) characterizes the class of augmented median rules by strategy-proofness and preference continuity. Miyagawa (1998a) shows that the extreme-peaks rule is the only rule satisfying Pareto-optimality, strategy-proofness, and preference continuity. Note that the extreme-peaks rule and the extreme-peaks solution is a corresponding pair. Moreover, we can adapt the proof of Theorem 2.7 to show that for more than two public goods, Pareto-optimality and preference continuity are not compatible. ${ }^{10}$ Therefore, by Theorems $2.4,2.5$, and 2.7 , we obtain the following general corollary.

Corollary 2.1 Let $m \in\{1, \ldots, n-1\}$ and $(\varphi, \psi)$ be a corresponding pair. Under Paretooptimality, the solution $\psi$ satisfies Nash's IIA, Arrow's IIA, and interval continuity if and only if the rule $\varphi$ satisfies strategy-proofness and preference continuity.

\subsection{The Lexicographic-Extension}

We extend preferences from the set of locations to the set of alternatives lexicographically. Each agent compares two altematives via the lexicographic preference relation over $[0,1]^{M}$ incluced by his single-peaked preference relation over $[0,1]$.

Lexicographic-Extension of Preferences: Let $i \in \mathbb{P}$ and $R_{i} \in \mathcal{R}$. Given two alternatives $x, y \in[0,1]^{M}$ and two permutations $\tau, \rho$ of $M$ such that $x_{\tau(1)} R_{i} x_{\tau(2)} R_{i} \ldots R_{i} x_{\tau(m)}$ and $y_{\rho(1)} R_{i} y_{\rho(2)} R_{i} \ldots R_{i} y_{\rho(m)}, x$ is lexicographically strictly preferred to $y$ if and only if there exists $h \in M$ such that for all $k<h, x_{\tau(k)} I_{i} y_{\rho(k)}$ and $x_{\tau(h)} P_{i} y_{\rho(h)}$. Furthermore, $x$ is lexicographically indifferent to $y$ if and only if for all $k \in M, x_{\tau(k)} I_{i} y_{\rho(k)}$.

Abusing notation, we use the same symbols to denote preferences over possible locations and lexicographic preferences over alternatives. When we extend preferences lexicographically, weak upper contour sets are neither closed nor open, and as for the max-extension

\footnotetext{
${ }^{10}$ Simply consider $R \in R^{N^{\prime}}$ such that $0<p\left(R_{1}\right)=p\left(R_{2}\right)<p\left(R_{3}\right)<\cdots<p\left(R_{m}\right)=\cdots=p\left(R_{n}\right)<1$ and apply similar arguments as in the proof of Theorem 2.7 by using preference continuity.
} 


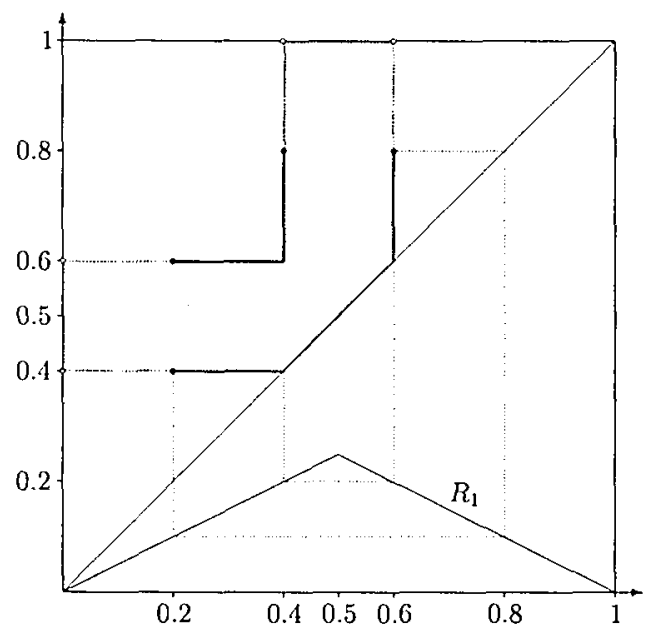

Figure 2.2: The preference $R_{1}$ is symmetric around the peak 0.5 . The shaded area is the weak upper contour set at $(0.2,0.6)$ when we extend $R_{1}$ lexicographically. Note that the dotted line segments $\{0,0.2[\times\{0.4\},[0,0.2[\times\{0.6\},\{0.4\} \times] 0.8,1]$, and $\{0.6\} \times] 0.8,1]$ do not belong to this upper contour set. Furthermore, the four bullet points $(0.2,0.4),(0.2,0.6)$, $(0.4,0.8)$, and $(0.6,0.8)$ are all alternatives in $[0,1]^{M}$ that are indifferent to $(0.2,0.6)$.

they are non-convex. Furthermore, indifference sets only contain a finite number of alternatives. Figure 2.2 illustrates this fact for $M=\{1,2\}$.

Remark 2.3 Compare Figures 2.1 and 2.2. The closure of the weak upper contour set of $R_{1}$ at $(0.2,0.6)$ is the weak upper contour set of $R_{1}^{\max }$ at $(0.2,0.6)$. It is easy to see that this is true for all alternatives $x \in[0,1]^{M}$ such that $p\left(R_{1}\right) \notin\left\{x_{1}, x_{2}\right\}$. If $p\left(R_{1}\right) \in\left\{x_{1}, x_{2}\right\}$; then the indifference set of $R_{1}^{\max }$ consists of the two line segments $[0,0.5] \times\{0.5\}$ and $\{0.5\} \times[0.5,1]$. If $x_{1}=0.5$, then the weak upper contour set of the lexicographic $R_{1}$ at $x$ consists of the two line segments $\left[1-x_{2}, 0.5\right] \times\{0.5\}$ and $\{0.5\} \times\left[0.5, x_{2}\right]$. As we will show, this "slight" change in weak upper contour sets and indifference sets brings about conclusions that are radically different from those in Miyagawa (1998b).

Again, Pareto-optimality says that for each preference profile the chosen alternative camnot be changed in such a way that no agent is worse off and some agent is better off relative to his lexicographic preference relation. Given $N \in \mathcal{P}, S \subseteq N$, and $R \in \mathcal{R}^{N}$, let $E\left(R_{S}\right)$ denote the set of Pareto-optimal (or efficient) alternatives for $R_{S}$. Formally, 


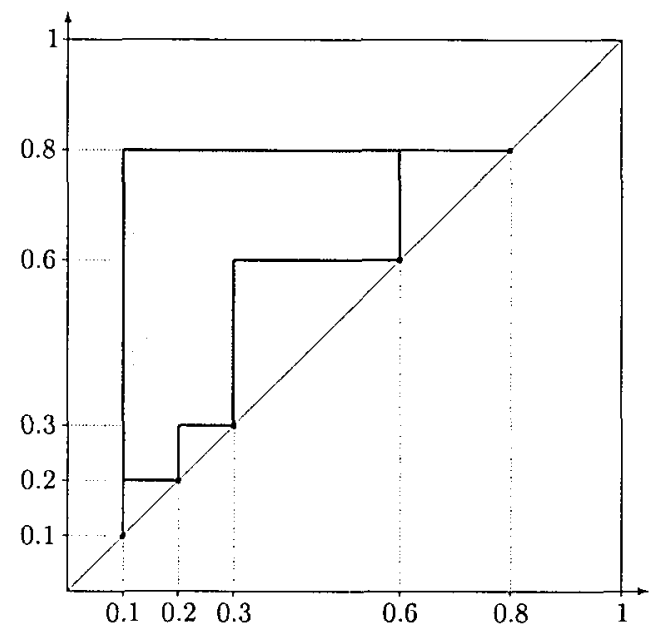

Figure 2.3: The set of Pareto-optimal alternatives for a preference profile $R \in \mathcal{R}^{N}$ such that $\left\{p\left(R_{i}\right) \mid i \in N\right\}=\{0.1,0.2,0.3,0.6,0.8\}$. The set $E(R)$ consists of the closed shaded area and the two line segments $\{0.1\} \times[0.1,0.2]$ and $[0.6,0.8] \times\{0.8\}$.

$E\left(R_{S}\right) \equiv\left\{y \in[0,1]^{M} \mid\right.$ for all $x \in[0,1]^{M}$, if for some $i \in S, x P_{i} y$, then for some $j \in S$, $\left.y P_{j} x\right\}$.

Pareto-Optimality: For all $N \in \mathcal{P}$ and all $R \in \mathcal{R}^{N}: \varphi(R) \in E(R)$.

When $m \geq 2$, for Pareto-optimality to hold it is not sufficient that for each public good the selected location belongs to $[\underline{p}(R), \bar{p}(R)]$. For every chosen alternative it is necessary that each closed interval having as two endpoints two selected locations contains at least one peak. The straightforward proof is left to the reader.

Lemma 2.3 Let $\varphi$ be a rule. Then $\varphi$ satisfies Pareto-optimality if and only if for all $N \in \mathcal{P}$ and all $R \in \mathcal{R}^{N}$ the following holds: (i) for all $k \in M, \varphi_{k}(R) \in[\underline{p}(R), \bar{p}(R)]$, and (ii) for all $k \in\{1, \ldots, m-1\}$, there exists $i \in N$ such that $p\left(R_{i}\right) \in\left[\varphi_{k}(R), \varphi_{k+1}(R)\right]$.

By Lemma 2.3, the set of efficient alternatives depends only on the peaks of the profile. Figure 2.3 shows an example of such a set for $M=\{1,2\}$.

Remark 2.4 Miyagawa (1998a,b) describe $E\left(R^{\max }\right)$. For the lexicographic-extension of preferences, Pareto-optimality is weaker than for the max-extension. For all $N \in \mathcal{P}$ and all 
$R \in \mathcal{R}^{N}$ such that $p(R)<\bar{p}(R), E\left(R^{\max }\right) \subseteq E(R)$. Generally the set $E(R)$ is considerably larger than $E\left(R^{\max }\right)$. For example, let $R \in \mathcal{R}^{N}$ be such that $\left\{p\left(R_{i}\right) \mid i \in N\right\}=\{0,1\}$. Then $E\left(R^{\max }\right)=\{(0,1)\} \subseteq([0,1] \times\{1\}) \cup(\{0\} \times[0,1])=E(R)$.

We also introduce the following two properties. Anonymity means that the rule is symmetric in its arguments across populations of equal size. Peaks-onliness means that a rule only depends on the peaks profile.

Given $N, N^{\prime} \in \mathcal{P}, R \in \mathcal{R}^{N}$, and a bijection $\sigma: N \rightarrow N^{\prime}$, let $\sigma(R) \equiv\left(R_{\sigma(i)}\right)_{i \in N} \in \mathcal{R}^{N^{\prime}}$ denote the renamed profile $R$ according to $\sigma$ on $N^{\prime}$. Note that this definition does not rule out the case $N=N^{\prime}$.

Anonymity: For all $N, N^{\prime} \in \mathcal{P}$, all $R \in \mathcal{R}^{N}$, and all bijections $\sigma: N \rightarrow N^{\prime}: \varphi(R)=$ $\varphi(\sigma(R))$.

Peaks-Onliness: For all $N \in \mathcal{P}$ and all $R, R^{\prime} \in \mathcal{R}^{N}$ : if for all $i \in N, p\left(R_{i}\right)=p\left(R_{i}^{\prime}\right)$, then $\varphi(R)=\varphi\left(R^{\prime}\right)$.

Finally, we prove that for any two efficient alternatives, if all agents are indifferent between them, then the two alternatives are the same.

Lemma 2.4 For all $N \in \mathcal{P}$, all $S \subseteq N$, all $R \in \mathcal{R}^{N}$, and all $x, y \in E\left(R_{S}\right)$, if for all $i \in S, x I_{i} y$, then $x=y$.

Proof. Let $j \in S$ be such that $p\left(R_{j}\right)=\min _{i \in S} p\left(R_{i}\right)$. Since $x, y \in E\left(R_{S}\right), p\left(R_{j}\right) \leq x_{1} \leq$ $x_{2} \leq \cdots \leq x_{m}$ and $p\left(R_{j}\right) \leq y_{1} \leq y_{2} \leq \cdots \leq y_{m}$. Because $x I_{j} y$, it follows that for all $k \in M, x_{k}=y_{k}$. Hence, $x=y$.

\subsection{The Replacement Principle}

\subsubsection{Replacement-Domination}

Throughout Section 2.6 we keep the set of agents fixed. Let $N \equiv\{1, \ldots, n\}$ denote the set of agents.

The solidarity property we discuss is replacement-domination. It requires that when the preference relation of some agent changes, the welfares of all other agents are affected in the same direction. It is the same principle as in Section 2.3 applied to the lexicographicextension of preferences. 
Replacement-Domination: For all $j \in N$, and all $R, \bar{R} \in \mathcal{R}^{N}$ such that $R_{N \backslash\{j\}}=$ $\bar{R}_{N \backslash\{j\}}$ : either [for all $\left.i \in N \backslash\{j\}, \varphi(R) R_{i} \varphi(\bar{R})\right]$ or [for all $\left.i \in N \backslash\{j\}, \varphi(\bar{R}) R_{i} \varphi(R)\right]$.

We state some implications of Pareto-optimality and replacement-domination. These implications are very general and not specific to our model. They hold also in the public good models of Thomson (1993) and Vohra (1999), and in our model when use the maxextension.

First, if the preference relation of some agent changes and the choices of the rule at the initial and new profile are Pareto-optimal for the profile consisting of the remaining agents' preferences, then the same alternative is chosen for both profiles.

Lemma 2.5 Let $\varphi$ be a rule satisfying Pareto-optimality and replacement-domination. Let $j \in N$ and $R, \bar{R} \in \mathcal{R}^{N}$ be such that $R_{N \backslash\{j\}}=\vec{R}_{N \backslash\{j\}}$. If $\varphi(R), \varphi(\bar{R}) \in E\left(R_{N \backslash\{j\}}\right)$, then $\varphi(R)=\varphi(\bar{R})$.

Proof. By replacement-domination, [for all $\left.i \in N \backslash\{j\}, \varphi(R) R_{i} \varphi(\vec{R})\right]$ or [for all $i \in$ $\left.N \backslash\{j\}, \varphi(\bar{R}) R_{i} \varphi(R)\right]$. Since $\varphi(R), \varphi(\bar{R}) \in E\left(R_{N \backslash\{j\}}\right)$, it follows that for all $i \in N \backslash\{j\}$ : $\varphi(R) I_{i} \varphi(\bar{R})$. Hence, by Lemma $2.4, \varphi(R)=\varphi(\bar{R})$.

Second, if the preference relation of some agent changes and all Pareto-optimal alternatives at the new profile are also efficient for the profile consisting of the remaining agents' preferences, then all these agents weakly prefer the alternative chosen by the rule for the new profile to the initially chosen alternative.

Lemma 2.6 Let $\varphi$ be a rule satisfying Pareto-optimality and replacement-domination. Let $j \in N$ and $R, \bar{R} \in \mathcal{R}^{N}$ be such that $R_{N \backslash\{j\}}=\tilde{R}_{N \backslash\{j\}}$. If $E(\bar{R})=E\left(R_{N \backslash\{j\}}\right)$, then for all $i \in N \backslash\{j\}, \varphi(\bar{R}) R_{i} \varphi(R)$.

Proof. By replacement-domination, either [for all $i \in N \backslash\{j\}, \varphi(\bar{R}) R_{i} \varphi(R)$ ] or [for all $i \in N \backslash\{j\}, \varphi(R) R_{i} \varphi(\bar{R})$. Suppose that the assertion of Lemma 2.6 does not hold. Thus, for all $i \in N \backslash\{j\}, \varphi(R) R_{i} \varphi(\bar{R})$, and for some $t \in N \backslash\{j\}, \varphi(R) P_{\imath} \varphi(\bar{R})$. Because $E(\bar{R})=$ $E\left(R_{N \backslash\{j\}}\right), \varphi(\bar{R}) \in E\left(R_{N \backslash\{j\}}\right)$. The previous two facts constitute a contradiction.

\subsubsection{Single-Peaked Preference Rules}

Throughout Subsection 2.6 .2 we consider the problem of providing two public goods, i.e., $M=\{1,2\}$. Miyagawa (1998b) shows that when $n \geq 4$ and we extend preferences from $[0,1]$ to alternatives maximally, only the left-peaks rule and the right-peaks rule satisfy 
Pareto-optimality and replacement-domination. When agents compare altematives lexicographically, then, by Lemma 2.3 , the left-peaks rule and the right-peaks rule satisfy Pareto-optimality. However, both rules violate replacement-domination in this case.

Example 2.5 Let $n \geq 3$ and $R \in \mathcal{R}^{N}$ be such that $p\left(R_{1}\right)=0, p\left(R_{2}\right)=\frac{1}{2}, p\left(R_{3}\right)=1$, and for all $i \in N \backslash\{1,2,3\}, p\left(R_{i}\right) \in\{0,1\}$. Let $\bar{R} \in \mathcal{R}^{N}$ be such that $\bar{R}_{N \backslash\{2\}}=R_{N \backslash\{2\}}$ and $p\left(\bar{R}_{2}\right)=\frac{2}{3}$. Then $\varphi^{L}(R)=\left(0, \frac{1}{2}\right)$ and $\varphi^{L}(\bar{R})=\left(0, \frac{2}{3}\right)$. In particular, $\varphi^{L}(R) P_{1} \varphi^{L}(\bar{R})$ and $\varphi^{L}(\bar{R}) P_{3} \varphi^{L}(R)$. Thus, the left-peaks rule violates replacement-domination. Similarly, the right-peaks rule violates replacement-domination.

A "constant" rule selecting for each preference profile the same alternative satisfies replacement-domination, but not Pareto-optimality. Therefore, in our model Paretooptimality and replacement-domination are independent.

Each rule satisfying Pareto-optimality and replacement-domination is described by a continuous and single-peaked preference relation over $[0,1]$. We interpret this relation to be the preference relation of the social plamner. Let $R_{\text {soc }} \in \mathcal{R}$ denote this relation. An alternative $x \in[0,1]^{M}$ is $R_{\text {soc }}$ equitable if the society is indifferent between its two locations, i.e., $x_{1} I_{s o c} x_{2}$. Let $A\left(R_{s o c}\right) \equiv\left\{x \in[0,1]^{M} \mid x_{1} I_{s o c} x_{2}\right\}$ be the set of $R_{\text {soc }}$-equitable alternatives. For each preference profile, the rule maximizes the welfare of the social planner on the set of $R_{s o c}$-equitable alternatives subject to Pareto-optimality. Formally, for all $R \in \mathcal{R}^{N}$ the rule selects an alternative in $\operatorname{Best}\left(R_{\text {soc }}, A\left(R_{\text {soc }}\right) \cap E(R)\right) \equiv\{y \in$ $A\left(R_{\text {soc }}\right) \cap E(R) \mid$ for all $\left.x \in A\left(R_{\text {soc }}\right) \cap E(R), y_{1} R_{\text {soc }} x_{1}\right\}$. If the peak of the plammer does not belong to the interval having as two endpoints the smallest peak and the greatest peak of the reported profile, then the latter set contains exactly one altemative. It is either the alternative having as both locations the smallest peak or the alternative having as both locations the greatest peak. If the peak of the planner's relation belongs to the peaks interval of the reported profile, then the rule selects the following alternative in the latter set: the first location is smaller than or equal to the planner's peak and the second location is greater than or equal to the planner's peak. We call these rules single-peaked preference rules. Figure 2.4 illustrates an example of a set of $R_{s o c}$-equitable alternatives and Figure 2.5 the welfare maximization for a particular profile.

Here is an example of such a rule when the social planner has a continuous and symmetric single-peaked preference relation with peak $\frac{1}{3}$.

Example 2.6 We represent the symmetric single-peaked preference relation with peak $\frac{1}{3}$ by its corresponding indifference map. Let $f:\left[0, \frac{2}{3}\right] \rightarrow\left[0, \frac{2}{3}\right]$ be such that for all $x \in\left[0, \frac{2}{3}\right], f(x)=\frac{2}{3}-x$. Then $f\left(\frac{1}{3}\right)=\frac{1}{3}$. For all $R \in \mathcal{R}^{N}$, we define the rule $\phi^{f}$ as follows: (a) if $\underline{p}(R)>\frac{1}{3}$, then $\phi^{f}(R)=(\underline{p}(R), \underline{p}(R))$ (Figure 2.6); (b) if $\bar{p}(R)<\frac{1}{3}$, then 


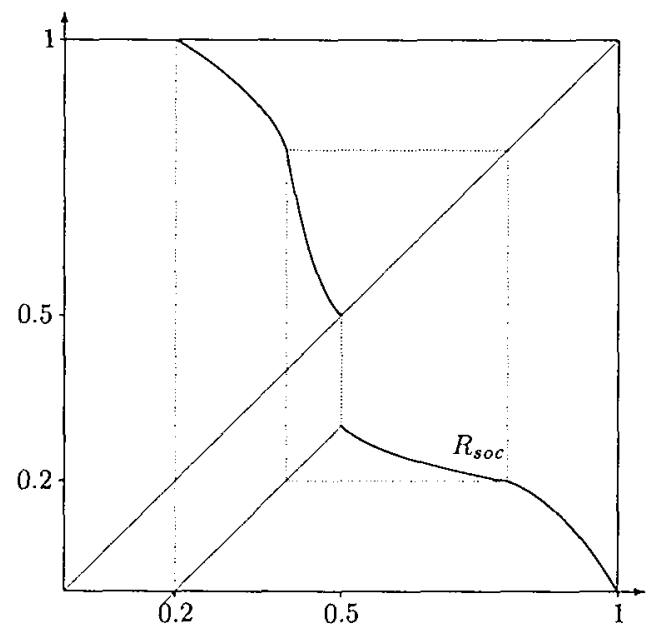

Figure 2.4: The set $R_{\text {soc }}$-equitable alternatives. The set $A\left(R_{\text {soc }}\right)$ consists of the diagonal and the path from $(0.2,1)$ to $(0.5,0.5)$. Notice that we can choose the numerical representation of $R_{s o c}$ to be linear on the set $\left[0, p\left(R_{s o c}\right)\right]$.

$\phi^{f}(R)=(\bar{p}(R), \bar{p}(R))$ (Figure 2.7); and (c) if $\frac{1}{3} \in[\underline{p}(R), \bar{p}(R)]$ and $j \in\{t \in N \mid$ for all $\left.i \in N,\left|p\left(R_{i}\right)-\frac{1}{3}\right| \geq\left|p\left(R_{i}\right)-\frac{1}{3}\right|\right\}$, then $\phi^{f}(R) \in\left\{\left(p\left(R_{j}\right), f\left(p\left(R_{j}\right)\right)\right),\left(f\left(p\left(R_{j}\right)\right), p\left(R_{j}\right)\right)\right\}$. When $p\left(R_{1}\right) \leq \ldots \leq p\left(R_{l}\right) \leq \frac{1}{3} \leq p\left(R_{l+1}\right) \leq \ldots \leq p\left(R_{n}\right)$, Figure 2.8 illustrates Case (c) for $j=l$ and Figure 2.9 for $j=l+1$.

Before we formally define our rules, we introduce an equivalent representation of a continuous and single-peaked preference relation over $[0,1]$.

Let $R_{0} \in \mathcal{R}$. Then $0 R_{0} 1$ or $1 P_{0} 0$. Suppose that $0 R_{0} 1$. Since $R_{0}$ is continuous, for some $b \in\left[p\left(R_{0}\right), 1\right], 0 I_{0} b$. For all $x \in[0, b]$, let $f(x) \in[0, b]$ be such that $x I_{0} f(x)$ and the following holds: (i) when $x \leq p\left(R_{0}\right), f(x) \geq p\left(R_{0}\right)$, and (ii) when $x \geq p\left(R_{0}\right), f(x) \leq p\left(R_{0}\right)$. Because $R_{0}$ is continuous, it follows that $f$ is continuous. Therefore, with $R_{0}$ we associate a unique function $f:[0, b] \rightarrow[0, b]$ such that $f$ is continuous, $f=f^{-1}$ (this follows from $I_{0}$ being a symmetric preference relation), and $f$ is strictly decreasing (this follows from single-peakedness of $\left.R_{0}\right)$. In particular, $f$ possesses as a unique fixed point $p\left(R_{0}\right)$, i.e., $f\left(p\left(R_{0}\right)\right)=p\left(R_{0}\right)$. Furthermore, associated with such a function is a unique single-peaked preference relation over $[0,1]$. If $1 P_{0} 0$, then for some $b \in\left\{p\left(R_{0}\right), 1\right], b I_{0} 1$. Analogously as above, then we associate with $R_{0}$ a unique function $f:[b, 1] \rightarrow[b, 1]$ that is continuous, $f=f^{-1}$, and strictly decreasing. 


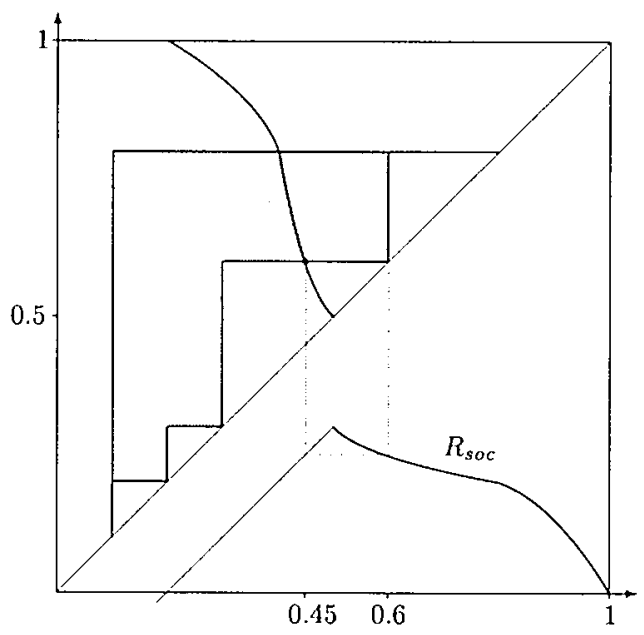

Figure 2.5: Given a preference profile $R \in \mathcal{R}^{N}$ such that $\left\{p\left(R_{i}\right) \mid i \in N\right\}=$ $\{0.1,0.2,0.3,0.6,0.8\}$, the rule maximizes the welfare on the set of $R_{s o c}$-equitable alternatives subject to Pareto-optimality and chooses the dotted alternative $(0.45,0.6)$.

Let $f:[0, b] \rightarrow[0, b]$ (or, alternatively, $f:[b, 1] \rightarrow[b, 1]$ ) be a continuous strictly decreasing function such that $f(0)=b(f(b)=1)$ and $f=f^{-1}$ ( $f$ is symmetric). Let $a$ denote the unique fixed point of $f$ and $\mathcal{F}$ the set of all such functions.

Single-Peaked Preference Rule, $\phi^{f}:$ Given $f \in \mathcal{F}$, the single-peaked preference rule $\phi^{f}$ based on $f$ is defined as follows. For all $R \in \mathcal{R}^{N}$ such that $p\left(R_{i_{1}}\right) \leq \cdots \leq p\left(R_{i_{n}}\right)$,

- if $a \notin[\underline{p}(R), \bar{p}(R)]$, then

$$
\phi^{f}(R) \equiv \begin{cases}(\underline{p}(R), \underline{p}(R)) & \text { when } a<\underline{p}(R) \\ (\bar{p}(R), \bar{p}(R)) & \text { when } \bar{p}(R)<a\end{cases}
$$

- if $p\left(R_{i_{l}}\right) \leq a \leq p\left(R_{i_{l+1}}\right)$, then

$$
\phi^{f}(R) \equiv\left\{\begin{array}{cl}
\left(p\left(R_{i_{l}}\right), f\left(p\left(R_{i_{l}}\right)\right)\right) & \text { when } f\left(p\left(R_{i_{l}}\right)\right) \leq p\left(R_{i_{l+1}}\right) \\
\left(f\left(p\left(R_{i_{l+1}}\right)\right), p\left(R_{i_{l+1}}\right)\right) & \text { otherwise. }
\end{array}\right.
$$

Theorem 2.8 below says that if $N$ contains at least 3 agents, then every decision rule satisfying Pareto-optimality and replacement-domination is a single-peaked preference rule. 


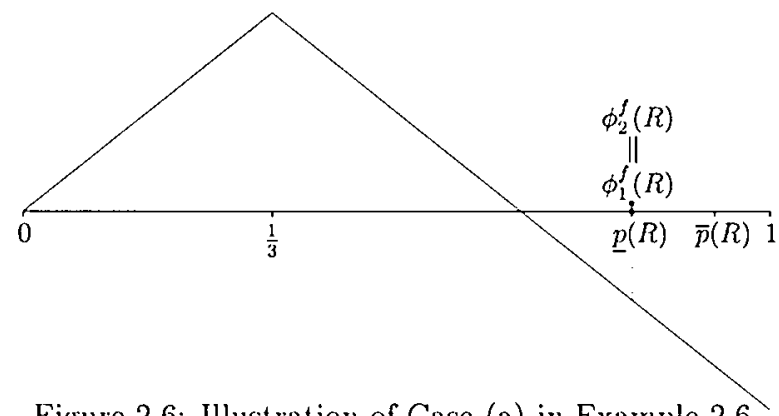

Figure 2.6: Illustration of Case (a) in Example 2.6.

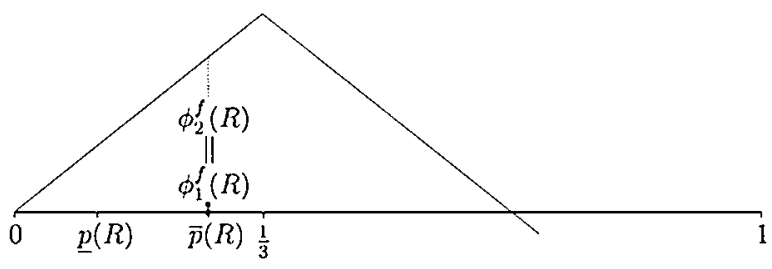

Figure 2.7: Illustration of Case (b) in Example 2.6.

Subsection 2.6.4 contains the proof of Theorem 2.8. Note that when $N$ contains only two agents, replacement-domination has no bite.

Theorem 2.8 Let $m=2$ and $n \geq 3$. Then the single-peaked preference rules are the only rules satisfying Pareto-optimality and replacement-domination.

Remark 2.5 In Miyagawa (1998b) we have to distinguish two cases. If $N$ contains at least four agents, then the left-peaks rule and the right-peaks rule are the only rules satisfying Pareto-optimality and replacement-domination. If $N$ contains three agents, then any rule choosing for each profile two distinct peaks satisfies Pareto-optimality and replacementdomination. In Theorem 2.8, there is no distinction between these two cases.

$\triangleleft$

In our model Pareto-optimality and replacement-domination imply anonymity. They also imply coalitional strategy-proofness (no group of agents can gain by jointly misrepresenting their true preferences), as the careful reader may check. Therefore, other rules than 


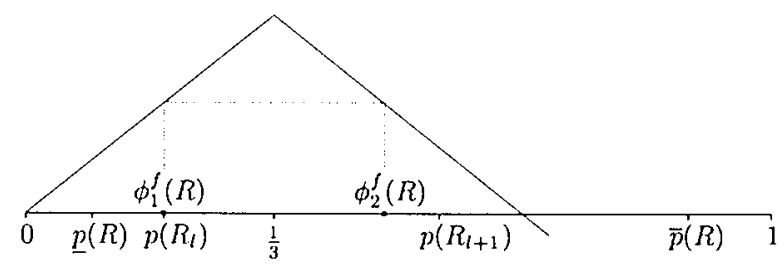

Figure 2.8: Illustration of Case (c) when $j=l$ in Example 2.6.

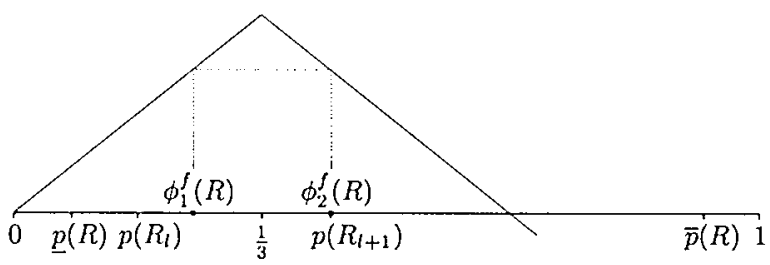

Figure 2.9: Illustration of Case (c) when $j=l+1$ in Example 2.6.

rules choosing for each public good the corresponding location according to some median operation may satisfy strategy-proofness and additional axioms. We do not require the above properties, they are implied by Pareto-optimality and replacement-domination.

In Theorem 2.8 it is not possible to weaken Pareto-optimality to unanimity. Unanimity requires that when all agents have the same preference, the rule locates both public goods at the common peak. The following example establishes the previous fact.

Example 2.7 Let $c \in[0,1]$. For all $R \in \mathcal{R}^{N}$, if $\underline{p}(R)=\bar{p}(R)$, then $\tilde{\varphi}_{1}(R) \equiv \tilde{\varphi}_{2}(R) \equiv \underline{p}(R)$, and otherwise, when $c \leq \underline{p}(R), \tilde{\varphi}(R) \equiv(c, \underline{p}(R))$, and when $\underline{p}(R)<c, \tilde{\varphi}(R) \equiv(\underline{p}(R), c)$. The rule $\dot{\varphi}$ satisfies unanimity and replacement-domination, but not Pareto-optimality. $\circ$ 


\subsubsection{Three Public Goods}

In this subsection we consider the provision of three public goods, i.e., $M=\{1,2,3\}$. The result of Miyagawa (1998b) generalizes to this case as follows: ${ }^{11}$ If $n \geq 5$, then a rule satisfies Pareto-optimality and replacement-domination with respect to the max-extension if and only if either for all profiles the three distinct smallest peaks are chosen, or for all profiles the three distinct greatest peaks are chosen.

In our model these rules "correspond" to the smallest peak rule and the greatest-peak rule. The smallest-peak rule always locates all public goods at the smallest peak, and the greatest-peak rule always at the greatest peak.

Smallest-Peak Rule, $\underline{\phi}:$ For all $R \in \mathcal{R}^{N}$ and all $k \in M, \underline{\phi}_{k}(R) \equiv \underline{p}(R)$.

Greatest-Peak Rule, $\bar{\phi}:$ For all $R \in \mathcal{R}^{N}$ and all $k \in M, \bar{\phi}_{k}(R) \equiv \bar{p}(R)$.

For two public goods, $\phi$ is the single-peaked preference rule when the peak of the social planner is at 0 , and $\bar{\phi}$ is the rule when the peak of the social planner is at 1 . The corresponding functions $\underline{f}, \bar{f} \in \mathcal{F}$ are given by $\underline{f}:[0,0] \rightarrow[0,0]$ and $\bar{f}:[1,1] \rightarrow[1,1]$. Then for two public goods, $\phi=\phi^{\mathcal{L}}$ and $\bar{\phi}=\phi^{\bar{J}}$.

Theorem 2.8 does not generalize to the provision of three public goods. In that case, if $N$ contains at least five agents, then Pareto-optimality and replacement-domination admit only the smallest peak rule and the greatest-peak rule. By providing one more good, the single-peaked preference rules are restricted to only two rules.

Theorem 2.9 Let $m=3$ and $n \geq 5$. Then the smallest-peak rule and the greatest-peak rule are the only rules satisfying Pareto-optimality and replacement-domination.

Remark 2.6 Our two rules and Miyagawa's rules have the following features in common. For all preference profiles, the left-peaks rule chooses the unique left-most Pareto-optimal alternative relative to the max-extension, and the smallest-peak rule chooses the unique left-most Pareto-optimal alternative relative to the lexicographic-extension. By this we mean that for all $R \in \mathcal{R}^{N}$, for all $x \in E\left(R^{\max }\right) \backslash\left\{\varphi^{L}(R)\right\}, L_{1}(R) \leq x_{1}$ and $L_{2}(R)<$ $x_{2}$, and for all $y \in E(R) \backslash\{\underline{\phi}(R)\}, \underline{\phi}_{1}(R) \leq y_{1}$ and $\underline{\phi}_{2}(R)<y_{2}$. Similarly, the rightpeaks rule and the greatest-peak rule choose always the unique right-most Pareto-optimal alternative relative to each extension. All these rules choose only the extreme Paretooptimal alternatives.

\footnotetext{
${ }^{11}$ Personal communication with E.Miyagawa at the Fourth International Meeting of the Society for Social Choice and Welfare, 1998, Vancouver, BC, Can.
} 
Section 2.6.5 contains the proof of Theorem 2.9. It is an open question whether in Theorem 2.9 the restriction $n \geq 5$ is tight or not. For the characterization of Miyagawa (1998b) the restriction $n \geq 5$ is tight.

\subsubsection{Proof of Theorem 2.8}

Throughout Subsection 2.6.4 let $n \geq 3, M=\{1,2\}$, and $\varphi$ be a rule satisfying Pareto-optimality and replacement-domination.

Successive applications of Lemma 2.5 yield the following lemma.

Lemma 2.7 Let $R \in \mathcal{R}^{N}$ be such that $\left|\left\{p\left(R_{i}\right) \mid i \in N\right\}\right|=2$. For all $\bar{R} \in \mathcal{R}^{N}$, if $\left\{p\left(\bar{R}_{i}\right) \mid i \in N\right\}=\left\{p\left(R_{i}\right) \mid i \in N\right\}$, then $\varphi(\bar{R})=\varphi(R)$.

The next lemma says that for any preference profile, the open interval having as endpoints the two chosen locations contains no peak.

Lemma 2.8 For all $R \in \mathcal{R}^{N}$ and all $\left.i \in N, p\left(R_{i}\right) \notin\right] \varphi_{1}(R), \varphi_{2}(R)[$.

Proof. Suppose that for some $R \in \mathcal{R}^{N}$ and some $j \in N$,

$$
\left.p\left(R_{j}\right) \in\right] \varphi_{1}(R), \varphi_{2}(R) \mid
$$

Without loss of generality, we suppose that $j \notin\{1,2\}, p\left(R_{1}\right)=\underline{p}(R)$, and $p\left(R_{2}\right)=\bar{p}(R)$. By successive applications of Lemma 2.5 we may assume that for all $i \in N \backslash\{1,2, j\}$, $p\left(R_{i}\right)=p\left(R_{j}\right)$.

Let $\bar{R} \in \mathcal{R}^{N}$ be such that $\bar{R}_{N \backslash\{2\}}=R_{N \backslash\{2\}}$ and $\bar{R}_{2}=R_{j}$. Thus, $E(\bar{R})=E\left(R_{N \backslash\{2\}}\right)$. By Lemma 2.6, for all $i \in N \backslash\{2\}$,

$$
\varphi(\bar{R}) R_{i} \varphi(R)
$$

Claim 1: $\varphi_{2}(\bar{R})=\bar{p}(\bar{R})=p\left(R_{j}\right)$.

Proof of Clairn 1. Suppose that

$$
\varphi_{2}(\bar{R})<p\left(R_{j}\right)
$$

Let $R_{j}^{\prime} \in \mathcal{R}$ be such that $\bar{p}(R) P_{j}^{\prime} \varphi_{2}(\bar{R})$ and $p\left(R_{j}^{\prime}\right)=p\left(R_{j}\right)$, and let $\bar{R}^{\prime}=\left(\bar{R}_{N \backslash\{j\}}, R_{j}^{\prime}\right)$. By Lemma 2.7,

$$
\varphi\left(\bar{R}^{\prime}\right)=\varphi(\bar{R}) .
$$


Let $R^{\prime}=\left(R_{N \backslash\{j\}}, R_{j}^{\prime}\right)$. If $p\left(R_{j}\right) \in\left\{\varphi_{1}\left(R^{\prime}\right), \varphi_{2}\left(R^{\prime}\right)\right\}$, then by Lemma $2.6, \varphi_{2}\left(\bar{R}^{\prime}\right)=$ $p\left(R_{j}\right)$, which contradicts $(2.10)$ and $(2.11)$. Hence, by (2.8), Pareto-optimality and replacement-domination, ${ }^{12}$

$$
\varphi_{1}\left(R^{\prime}\right)<p\left(R_{j}\right)<\varphi_{2}\left(R^{\prime}\right)
$$

Thus, since $\bar{p}(R) P_{j}^{\prime} \varphi_{2}(\bar{R})$ and by using (2.11), we obtain $\varphi\left(R^{\prime}\right) P_{j}^{\prime} \varphi\left(\bar{R}^{\prime}\right)$, which contradicts Lemma 2.6.

Let $\tilde{R} \in \mathcal{R}^{N}$ be such that $\tilde{R}_{N \backslash\{1\}}=R_{N \backslash\{1\}}$ and $\tilde{R}_{1}=R_{j}$. By the same arguments as in Claim 1 it follows that $\varphi_{1}(\tilde{R})=\underline{p}(\tilde{R})=p\left(R_{j}\right)$. To summarize, Claim 1 , the previous fact, replacement-domination, and (2.8) imply that

$$
\varphi_{1}(\tilde{R})=p\left(R_{j}\right), p\left(R_{j}\right)<\varphi_{2}(\tilde{R})
$$

and

$$
\varphi_{1}(\bar{R})<p\left(R_{j}\right), \varphi_{2}(\bar{R})=p\left(R_{j}\right)
$$

Let $\bar{R}^{1} \in \mathcal{R}^{N}$ be such that $p\left(\bar{R}_{1}^{1}\right)=\underline{p}(\bar{R})$, for all $i \in N \backslash\{1\}, p\left(\bar{R}_{i}^{1}\right)=p\left(R_{j}\right), \varphi_{1}(\bar{R}) \bar{P}_{2}^{1} \varphi_{2}(\tilde{R})$, and $\varphi_{2}(\tilde{R}) \bar{P}_{3}^{1} \varphi_{1}(\tilde{R})$. Also, let $\bar{R}^{2} \in \mathcal{R}^{N}$ be such that $\vec{R}_{N \backslash\{1\}}^{2}=\bar{R}_{N \backslash\{1\}}^{1}$ and $p\left(\bar{R}_{1}^{2}\right)=\bar{p}(\tilde{R})$. By Lemma 2.7,

$$
\varphi\left(\bar{R}^{1}\right)=\varphi(\bar{R}) \text { and } \varphi\left(\bar{R}^{2}\right)=\varphi(\tilde{R}) .
$$

By definition of $\bar{R}_{2}^{1}$ and $\bar{R}_{3}^{1}, \varphi(\vec{R}) \bar{P}_{2}^{1} \varphi(\tilde{R})$ and $\varphi(\tilde{R}) \bar{P}_{3}^{1} \varphi(\bar{R})$. Since $\bar{R}_{N \backslash\{1\}}^{1}=\bar{R}_{N \backslash\{1\}}^{2}$ and (2.12), the previous relations contradict replacement-domination.

Next, we establish some properties of $\varphi$. First we show that $\varphi$ satisfies anonymity, second that $\varphi$ satisfies peaks-onliness, and third that $\varphi$ satisfies a certain invariance property relative to the peaks.

Lemma $2.9 \varphi$ satisfies anonymity.

Proof. Let $R \in \mathcal{R}^{N}$ and $\sigma: N \rightarrow N$ be a permutation. By Lemma 2.8, for all $i \in N$,

$$
p\left(R_{i}\right) \in\left[\underline{p}(R), \varphi_{1}(R)\right] \cup\left[\varphi_{2}(R), \bar{p}(R)\right]
$$

We distinguish two cases.

Case 1: $|N| \geq 4$.

\footnotetext{
${ }^{12}$ Note that when $N=\{1,2,3\}$ we cannot conclude $\varphi\left(R^{\prime}\right)=\varphi(R)$.
} 
Without loss of generality, we suppose that $p\left(R_{1}\right)=p(R)$ and $p\left(R_{2}\right)=\bar{p}(R)$. Thus, for some $j \in N \backslash\{1,2\}, \varphi(R) \in E\left(R_{N \backslash\{j\}}\right)$. Let $\widetilde{R} \in \mathcal{R}^{N}$ be such that $\bar{R}_{N \backslash\{j\}}=R_{N \backslash\{j\}}$ and $\bar{R}_{j}=R_{1}$. By Lemma 2.5, $\varphi(\bar{R})=\varphi(R)$. Successive applications of Lemma 2.5 yield $\varphi(\sigma(\bar{R}))=\varphi(\bar{R})$. The previous two facts imply

$$
\varphi(\sigma(\bar{R}))=\varphi(R)
$$

Next, we determine $\varphi(\sigma(R))$. Note that $\sigma(R)_{N \backslash\{\sigma(j)\}}=\sigma(\vec{R})_{N \backslash\{\sigma(j)\}}$. By replacementdomination, there are two possible cases.

If for all $i \in N \backslash\{\sigma(j)\}, \varphi(\sigma(\bar{R})) \bar{R}_{i} \varphi(\sigma(R))$, then (2.13) and (2.14) imply $\varphi_{1}(\sigma(R)) \geq$ $\varphi_{1}(\sigma(\bar{R}))$ and $\varphi_{2}(\sigma(R)) \leq \varphi_{2}(\sigma(\bar{R}))$. Thus, $\varphi(\sigma(R)) \in E\left(\bar{R}_{N \backslash\{\sigma(j)\}}\right)$. Hence, by Lemma 2.5, $\varphi(\sigma(R))=\varphi(\sigma(\bar{R}))$. Thus, by $(2.14), \varphi(\sigma(R))=\varphi(R)$, the desired conclusion.

If $\varphi(\sigma(R)) \neq \varphi(\sigma(\bar{R}))$ and for all $i \in N \backslash\{\sigma(j)\}, \varphi(\sigma(R)) \bar{R}_{i} \varphi(\sigma(\bar{R}))$, then $\varphi_{1}(\sigma(R))<$ $\varphi_{1}(\sigma(\bar{R}))$ and $\varphi_{2}(\sigma(\bar{R}))<\varphi_{2}(\sigma(R))$. Since $\varphi(\sigma(\bar{R})) \in E(\sigma(\bar{R}))$, it follows that for some $t \in$ $N \backslash\{\sigma(j)\}, p\left(\sigma(\bar{R})_{\ell}\right) \in\left[\varphi_{1}(\sigma(\bar{R})), \varphi_{2}(\sigma(\vec{R}))\right]$. Hence, the above inequalities and $p\left(\sigma(\bar{R})_{\ell}\right)=$ $p\left(\sigma(R)_{l}\right)$ imply $\left.p\left(\sigma(R)_{l}\right) \in\right] \varphi_{1}(\sigma(R)), \varphi_{2}(\sigma(R))[$, which contradicts Lemma 2.8. Hence, $\varphi(\sigma(R))=\varphi(\sigma(\bar{R}))$. Thus, by $(2.14), \varphi(\sigma(R))=\varphi(R)$, the desired conclusion.

Case 2: $N=\{1,2,3\}$.

If $\left|\left\{p\left(R_{i}\right) \mid i \in N\right\}\right| \in\{1,2\}$, then the conclusion follows from Lemma 2.7. Suppose that $p\left(R_{1}\right)<p\left(R_{2}\right)<p\left(R_{3}\right)$. By Lemma 2.8 , either $\varphi_{1}(R), \varphi_{2}(R) \in\left[p\left(R_{1}\right), p\left(R_{2}\right)\right]$ or $\varphi_{1}(R), \varphi_{2}(R) \in\left[p\left(R_{2}\right), p\left(R_{3}\right)\right]$. Without loss of generality, we suppose that

$$
\varphi_{1}(R), \varphi_{2}(R) \in\left[p\left(R_{1}\right), p\left(R_{2}\right)\right]
$$

Let $\bar{R}=\left(R_{1}, R_{2}, R_{2}\right)$. By Lemma 2.5, $\varphi(\bar{R})=\varphi(R)$. By Lemma 2.7, $\varphi(\sigma(\tilde{R}))=\varphi(\bar{R})$. Thus, by the two previous facts,

$$
\varphi(\sigma(\bar{R}))=\varphi(R)
$$

Next, we determine $\varphi(\sigma(R))$. If $\varphi_{1}(\sigma(R)), \varphi_{2}(\sigma(R)) \in\left[p\left(R_{1}\right), p\left(R_{2}\right)\right]$, then $\varphi(\sigma(R)) \in$ $E\left(R_{1}, R_{2}\right)$. Hence, by Lemma $2.5, \varphi(\sigma(R))=\varphi(\sigma(\bar{R}))$. By $(2.15), \varphi(\sigma(R))=\varphi(R)$, which is the desired conclusion.

Suppose that $\varphi_{2}(\sigma(R))>p\left(R_{2}\right)$. Thus, by Lemma $2.8, \varphi_{1}(\sigma(R)) \geq p\left(R_{2}\right)$. Let $\tilde{R}=$ $\left(R_{2}, R_{2}, R_{3}\right)$. Thus, by Lemma $2.5, \varphi(\tilde{R})=\varphi(\sigma(R))$. We distinguish three subcases.

Subcase 2.1: $\varphi_{1}(R)=\varphi_{2}(R)=p\left(R_{2}\right)$.

By Lemma 2.5, $\varphi(\tilde{R})=\varphi(R)$. By Lemma $2.7 \varphi(\tilde{R})=\varphi(\sigma(R))$. The previous two equalities contradict $\varphi(R) \neq \varphi(\sigma(R))$.

Subcase 2.2: $\varphi_{2}(R)<p\left(R_{2}\right)<\varphi_{1}(\sigma(R))$. 
Then $\varphi(\bar{R})=\varphi(R)$ and $\varphi(\tilde{R})=\varphi(\sigma(R))$. Using the same arguments as in the proof of Lemma 2.8 we derive a contradiction to replacement-domination.

Subcase 2.3: $\varphi_{2}(R)=p\left(R_{2}\right)$ (or $p\left(R_{2}\right)=\varphi_{1}(\sigma(R))$ ).

By Subcase 2.1, $\varphi_{1}(R)<p\left(R_{2}\right)$. By Lemma 2.6, $\varphi(\tilde{R}) R_{2} \varphi(R)$. Thus, $\varphi_{1}(\tilde{R})=p\left(R_{2}\right)$. By Lemma 2.7, $\varphi(\tilde{R})=\varphi(\sigma(R))$. Thus, $p\left(R_{2}\right)<\varphi_{2}(\tilde{R})$. Lsing the same arguments as in the proof of Lemma 2.8 we derive a contradiction to replacement-domination.

Lemma $2.10 \varphi$ satisfies peaks-onliness.

Proof. By Lemma 2.9, $\varphi$ satisfies anonymity. Let $j \in N$ and $R, \bar{R} \in \mathcal{R}^{N}$ be such that $R_{N \backslash\{j\}}=\bar{R}_{N \backslash\{j\}}$ and $p\left(R_{j}\right)=p\left(\bar{R}_{j}\right)$. To prove peaks-onliness, it suffices to show that $\varphi(R)=\varphi(\bar{R})$. We distinguish two cases.

Case 1: $|N| \geq 4$.

Without loss of generality, we suppose that $p\left(R_{1}\right)=\underline{p}(R)$ and $p\left(R_{2}\right)=\bar{p}(R)$. Thus, for some $t \in N \backslash\{1,2\}, \varphi(R) \in E\left(R_{N \backslash\{\ell\}}\right)$. We consider two subcases.

Subcase 1.1: $t=j$.

Let $\tilde{R} \in \mathcal{R}^{N}$ be such that $\tilde{R}_{N \backslash\{j\}}=R_{N \backslash\{j\}}$ and $\tilde{R}_{j}=R_{1}$. By Lemma $2.5, \varphi(\tilde{R})=$ $\varphi(R)$. Using the same arguments as in the proof of Case 1 in Lemma 2.9, it follows that $\varphi(\bar{R})=\varphi(\tilde{R})$. Hence, $\varphi(\bar{R})=\varphi(R)$, the desired conclusion.

Subcase 1.2: $t \neq j$.

Let $\tilde{R} \in \mathcal{R}^{N}$ be such that $\tilde{R}_{N \backslash\{t\}}=R_{N \backslash\{t\}}$ and $\tilde{R}_{t}=\bar{R}_{j}$. By Lemma 2.5, $\varphi(\tilde{R})=\varphi(R)$. Let $\sigma: N \rightarrow N$ be the permutation such that for all $i \in N \backslash\{j, t\}, \sigma(i)=i, \sigma(j)=t$, and $\sigma(t)=j$. By anonymity, $\varphi(\sigma(\tilde{R}))=\varphi(\tilde{R})$. Lsing the same arguments as in Case 1 of Lemma 2.9, it follows that $\varphi(\bar{R})=\varphi(\sigma(\tilde{R}))$. Hence, $\varphi(\bar{R})=\varphi(R)$, the desired conclusion.

Case 2: $N=\{1,2,3\}$.

Similar to Case 1, the conclusion follows from the proof of Case 2 in Lemma 2.9.

Lemma 2.11 For all $j \in N$ and all $R, R^{\prime} \in \mathcal{R}^{N}$ such that $R_{N \backslash\{j\}}=R_{N \backslash\{j\}}^{\prime}$, if $p\left(R_{j}\right), p\left(R_{j}^{\prime}\right)$ $<\varphi_{1}(R)$ or $\varphi_{2}(R)<p\left(R_{j}\right), p\left(R_{j}^{\prime}\right)$, then $\varphi\left(R^{\prime}\right)=\varphi(R)$.

Proof. By Lemma 2.10, $\varphi$ satisfies peaks-onliness. We prove Lemma 2.11 only for the first case. Without loss of generality, we suppose that $p\left(R_{j}\right) \leq p\left(R_{j}^{\prime}\right)<\varphi_{1}(R)$, and by anonymity, $p\left(R_{1}\right) \leq p\left(R_{2}\right) \leq \cdots \leq p\left(R_{n}\right)$. If $p\left(R_{j}\right)=p\left(R_{j}^{\prime}\right)$, then the conclusion follows from peaks-onliness. Let $p\left(R_{j}\right)<p\left(R_{j}^{\prime}\right)$.

Case 1: For some $l \in N \backslash\{j\}, p\left(R_{l}\right)<\varphi_{1}(R)$. 
By Pareto-optimality and Lemma 2.8, for some $t \in N \backslash\{j, l\}, p\left(R_{\ell}\right) \in\left\{\varphi_{1}(R), \varphi_{2}(R)\right\}$. If for all $i \in N \backslash\{j\}, \varphi\left(R^{\prime}\right) R_{i} \varphi(R)$, then $p\left(R_{l}\right) \in\left\{\varphi_{1}\left(R^{\prime}\right), \varphi_{2}\left(R^{\prime}\right)\right\}$. Replacement-domination implies $\varphi\left(R^{\prime}\right)=\varphi(R)$, the desired conclusion.

If for all $i \in N \backslash\{j\}, \varphi(R) R_{i} \varphi\left(R^{\prime}\right)$, then by peaks-onliness, replacement-domination, and $p\left(R_{l}\right)<\varphi_{1}(R), p\left(R_{l}\right)<\varphi_{1}(R) \leq \varphi_{1}\left(R^{\prime}\right)$. Since $p\left(R_{j}^{\prime}\right)<\varphi_{1}(R)$, Pareto-optimality and the previous fact imply $\varphi\left(R^{\prime}\right) \in E\left(R_{N \backslash\{j\}}\right)$. Hence, $\varphi(R), \varphi\left(R^{\prime}\right) \in E\left(R_{N \backslash\{j\}}\right)$ and by Lemma $2.5, \varphi\left(R^{\prime}\right)=\varphi(R)$, the desired conclusion.

Case 2: For all $i \in N \backslash\{j\}, p\left(R_{i}\right) \in\left[\varphi_{1}(R), \bar{p}(R)\right]$.

By Lemma 2.8, for all $i \in N \backslash\{j\}, p\left(R_{i}\right) \in\left\{\varphi_{1}(R)\right\} \cup\left[\varphi_{2}(R), \bar{p}(R)\right]$. We consider two subcases.

\section{Subcase 2.J: $|N| \geq 4$.}

Then for some $t \in N \backslash\{j\}, \varphi(R) \in E\left(R_{N \backslash\{\}}\right)$ and $\bar{p}\left(R_{N \backslash\{\ell\}}\right)=\bar{p}(R)$. Let $\bar{R} \in \mathcal{R}^{N}$ be such that $\bar{R}_{N \backslash\{t\}}=R_{N \backslash\{t\}}$ and $p\left(\bar{R}_{t}\right)=\frac{1}{2}\left(\varphi_{1}(R)+p\left(R_{j}^{\prime}\right)\right)$. Pareto-optimality, replacement-domination, and Lemma 2.5 imply $\varphi(\bar{R})=\varphi(R)$. Let $\bar{R}^{\prime} \in \mathcal{R}^{N}$ be such that $\bar{R}_{N \backslash\{t\}}^{\prime}=R_{N \backslash\{\}}^{\prime}$ and $\bar{R}_{t}^{\prime}=\bar{R}_{t}$. By Case 1, $\varphi\left(\bar{R}^{\prime}\right)=\varphi(\bar{R})$. By Pareto-optimality, replacement-domination, and Lemma 2.5, $\varphi\left(\bar{R}^{\prime}\right)=\varphi\left(R^{\prime}\right)$. Hence, $\varphi\left(R^{\prime}\right)=\varphi(R)$, the desired conclusion.

Subcase 2.2: $N=\{1,2,3\}$.

Hence, $j=1$. We distinguish three subcases.

Subcase 2.2.1: $\varphi_{2}(R)=p\left(R_{2}\right)$.

Let $\bar{R} \in \mathcal{R}^{N}$ be such that $\vec{R}_{N \backslash\{3\}}=R_{N \backslash\{3\}}$ and $p\left(\bar{R}_{3}\right)=\frac{1}{2}\left(\varphi_{1}(R)+p\left(R_{1}^{\prime}\right)\right)$. Pareto-optimality, replacement-domination, and Lemma 2.5 imply $\varphi(\bar{R})=\varphi(R)$. Let $\bar{R}^{\prime}=$ $\left(R_{1}^{\prime}, \bar{R}_{2}, \bar{R}_{3}\right)$. By Case $1, \varphi\left(\bar{R}^{\prime}\right)=\varphi(\bar{R})$.

If $\varphi_{2}\left(R^{\prime}\right) \leq p\left(R_{2}\right)$, then $\varphi\left(R^{\prime}\right) \in E\left(\bar{R}_{N \backslash\{3\}}^{\prime}\right)$. Thus, $\varphi\left(\bar{R}^{\prime}\right), \varphi\left(R^{\prime}\right) \in E\left(\bar{R}_{N \backslash\{3\}}^{\prime}\right)$ and by Lemma 2.5, $\varphi\left(R^{\prime}\right)=\varphi\left(\bar{R}^{\prime}\right)$. Hence, $\varphi\left(R^{\prime}\right)=\varphi(R)$, the desired conclusion.

If $\varphi_{2}\left(R^{\prime}\right)>p\left(R_{2}\right)$, then by Lemma $2.8, \varphi_{1}\left(R^{\prime}\right) \geq p\left(R_{2}\right)$. Thus, $\varphi\left(\bar{R}^{\prime}\right) \bar{P}_{1}^{\prime} \varphi\left(R^{\prime}\right)$. By replacement-domination, $\varphi\left(\bar{R}^{\prime}\right) \tilde{R}_{2}^{\prime} \varphi\left(R^{\prime}\right)$. Thus, by peaks-onliness, $\varphi_{1}\left(R^{\prime}\right)>p\left(R_{2}\right)$. Hence, by $\varphi\left(\bar{R}^{\prime}\right)=\varphi(R), \varphi(R) P_{2} \varphi\left(R^{\prime}\right)$ and $\varphi\left(R^{\prime}\right) P_{3} \varphi(R)$, which contradicts replacement-domination. Thus, subcase $\varphi_{2}\left(R^{\prime}\right)>p\left(R_{2}\right)$ cannot occur.

Subcase 2.2.2: $\varphi_{1}(R)=p\left(R_{2}\right)<\varphi_{2}(R)=p\left(R_{3}\right)$.

Let $\bar{R} \in \mathcal{R}^{N}$ be such that $\bar{R}_{N \backslash\{2\}}=R_{N \backslash\{2\}}$ and $p\left(\bar{R}_{2}\right)=\frac{1}{2}\left(\varphi_{1}(R)+p\left(R_{1}^{\prime}\right)\right)$. Pareto-optimality, replacement-domination, and Lemma 2.5 imply $\varphi(\bar{R})=\varphi(R)$. Let $\vec{R}^{\prime}=$ $\left(R_{1}^{\prime}, \bar{R}_{2}, \bar{R}_{3}\right)$. By Case $1, \varphi\left(\bar{R}^{\prime}\right)=\varphi(\bar{R})$.

If $\varphi_{2}\left(R^{\prime}\right)=p\left(R_{3}\right)$, then $\varphi\left(R^{\prime}\right) \in E\left(\bar{R}_{N \backslash\{2\}}^{\prime}\right)$. Thus, $\varphi\left(\bar{R}^{\prime}\right), \varphi\left(R^{\prime}\right) \in E\left(\bar{R}_{N \backslash\{2\}}^{\prime}\right)$ and by Lemma 2.5, $\varphi\left(R^{\prime}\right)=\varphi\left(\bar{R}^{\prime}\right)$. Hence, $\varphi\left(R^{\prime}\right)=\varphi(R)$, the desired conclusion. 
If $\varphi_{2}\left(R^{\prime}\right)<p\left(R_{3}\right)$, then $\varphi\left(\bar{R}^{\prime}\right) \bar{P}_{3}^{\prime} \varphi\left(R^{\prime}\right)$. By replacement-domination, $\varphi\left(\bar{R}^{\prime}\right) \bar{R}_{1}^{\prime} \varphi\left(R^{\prime}\right)$. Thus, $\varphi_{1}\left(R^{\prime}\right) \geq p\left(R_{2}\right)$. By Pareto-optimality, $\varphi_{1}\left(R^{\prime}\right)=p\left(R_{2}\right)$. Hence, by $\varphi\left(\bar{R}^{\prime}\right)=\varphi(R)$, we obtain $\varphi\left(R^{\prime}\right) P_{2} \varphi(R)$ and $\varphi(R) P_{3} \varphi\left(R^{\prime}\right)$, which contradicts replacement-domination. Thus, subcase $\varphi_{2}\left(R^{\prime}\right)<p\left(R_{3}\right)$ cannot occur.

Subcase 2.2.3: $\varphi_{1}(R)=p\left(R_{2}\right)<\varphi_{2}(R)<p\left(R_{3}\right)$.

If for all $i \in N \backslash\{1\}, \varphi\left(R^{\prime}\right) R_{i} \varphi(R)$, then it follows that $\varphi\left(R^{\prime}\right)=\varphi(R)$.

Suppose that for all $i \in N \backslash\{1\}, \varphi(R) R_{i} \varphi\left(R^{\prime}\right)$ and $\varphi\left(R^{\prime}\right) \neq \varphi(R)$. Peaks-onliness implies $\varphi_{2}\left(R^{\prime}\right)<p\left(R_{2}\right)$. By Pareto-optimality, $\varphi_{1}\left(R^{\prime}\right)=p\left(R_{1}^{\prime}\right)$. Let $\vec{R}=\left(R_{1}^{\prime}, R_{2}^{\prime}, R_{1}^{\prime}\right)$. By Lemma $2.5, \varphi(\bar{R})=\varphi\left(R^{\prime}\right)$. Thus,

$$
\varphi_{2}(\bar{R})<p\left(R_{2}\right)
$$

Let $\tilde{R}=\left(R_{1}, R_{2}, R_{1}^{\prime}\right)$. By Pareto-optimality, $\varphi(\tilde{R}) P_{1} \varphi(R)$. By replacement-domination, $\varphi(\tilde{R}) R_{2} \varphi(R)$. Thus, by peaks-onliness, $\varphi(\tilde{R})=\left(p\left(R_{2}\right), p\left(R_{2}\right)\right)$. By Lemma $2.5, \varphi(\bar{R})=$ $\varphi(\tilde{R})$. The previous two equalities contradict $(2.16)$.

We construct a function $f \in \mathcal{F}$ and show that $\varphi=\phi^{f}$. In order to define $f$ some additional notation is needed. Given $x \in[0,1]$; let $R^{x} \in \mathcal{R}^{N}$ denote a preference profile such that $p\left(R_{1}^{x}\right)=0, p\left(R_{2}^{x}\right)=x$, and for all $i \in N \backslash\{1,2\}, p\left(R_{i}^{x}\right)=1$. Pareto-optimality implies that $\varphi_{1}\left(R^{0}\right)=0$ or $\varphi_{2}\left(R^{0}\right)=1$. Without loss of generality, we suppose that $\varphi_{1}\left(R^{0}\right)=0$. The case $\varphi_{2}\left(R^{0}\right)=1$ is symmetric by interchanging the roles of $\varphi_{1}\left(R^{0}\right)$ and $\varphi_{2}\left(R^{0}\right)$. Define $b \equiv \varphi_{2}\left(R^{0}\right)$ and the function $f:[0, b] \rightarrow[0, b]$ as follows. ${ }^{13}$

Definition of $f:$ For all $x \in[0, b]$, when $x<\varphi_{2}\left(R^{x}\right), f(x) \equiv \varphi_{2}\left(R^{x}\right)$, and when $x \geq \varphi_{2}\left(R^{x}\right), f(x) \equiv \varphi_{1}\left(R^{x}\right)$.

We prove in three subsequent lemmas that $f \in \mathcal{F}$.

Lemma 2.12 For all $x \in[0, b], f(x) \in[0, b]$.

Proof. Let $x \in[0, b]$. By Lemma 2.8, for all $\left.i \in N, p\left(R_{i}^{x}\right) \notin\right] \varphi_{1}\left(R^{x}\right), \varphi_{2}\left(R^{x}\right)[$. Thus, if $\left.\varphi_{2}\left(R^{x}\right) \in \mid b, 1\right]$, then $\varphi_{1}\left(R^{x}\right) \in[x, 1]$. Hence, $\varphi\left(R^{0}\right) P_{1}^{0} \varphi\left(R^{x}\right)$ and $\varphi\left(R^{x}\right) P_{3}^{0} \varphi\left(R^{0}\right)$ in contradiction to replacement-domination. Thus, $f(x) \in[0, b]$.

Lemma 2.13 If $x<x^{\prime}$, then $f(x)>f\left(x^{\prime}\right)$. Moreover, $f=f^{-1}$.

\footnotetext{
${ }^{13}$ When $\varphi_{1}\left(R^{0}\right)>0$, we define $b \equiv \varphi_{1}\left(R^{0}\right)$ and a function $f:[b, 1] \rightarrow[b, 1]$
} 
Proof. By Lemma 2.8, Lemma 2.12, and replacement-domination, for all $x \in] 0, b[$, $f(x) \in] 0, b[$. Thus, by Lemma 2.8, Pareto-optimality, and the definition of $b$, for all $x \in[0, b]$,

$$
\varphi_{1}\left(R^{x}\right)=x \text { or } \varphi_{2}\left(R^{x}\right)=x
$$

Let $x, x^{\prime} \in[0, b]$ be such that $x<x^{\prime}$. Without loss of generality, we suppose that $\varphi_{1}\left(R^{x}\right)=$ $x$. Thus, $\varphi_{2}\left(R^{x}\right) \geq x$. If $x^{\prime}=\varphi_{2}\left(R^{x}\right)$, then Lemma 2.8 , replacement-domination and (2.17) imply $\varphi\left(R^{x}\right)=\varphi\left(R^{x^{\prime}}\right)$. Hence, by definition of $f, f\left(x^{\prime}\right)=x<x^{\prime}=f(x)$. If $\left.x^{\prime} \in\right] x, \varphi_{2}\left(R^{x}\right)\left[\right.$, then by $(2.17), x^{\prime} \in\left\{\varphi_{1}\left(R^{x^{\prime}}\right), \varphi_{2}\left(R^{x^{\prime}}\right)\right\}$. Thus, by replacement-domination, $\left.\varphi_{1}\left(R^{x^{\prime}}\right), \varphi_{2}\left(R^{x^{\prime}}\right) \in\right] x, \varphi_{2}\left(R^{x}\right)\left[\right.$ and $f\left(x^{\prime}\right)<\varphi_{2}\left(R^{x}\right)=f(x)$. If $\left.\left.x^{\prime} \in\right] \varphi_{2}\left(R^{x}\right), b\right]$, then by $(2.17), x^{\prime} \in\left\{\varphi_{1}\left(R^{x^{\prime}}\right), \varphi_{2}\left(R^{x^{\prime}}\right)\right\}$. Thus, by replacement-domination, $\varphi_{1}\left(R^{x^{\prime}}\right)<x$ and $x^{\prime}=\varphi_{2}\left(R^{x^{\prime}}\right)$. By definition of $f, f\left(x^{\prime}\right)=\varphi_{1}\left(R^{x^{\prime}}\right)<x \leq f(x)$. Hence, $f$ is strictly decreasing.

Since $f$ is strictly decreasing, it follows that $f^{-1}$ is well-defined. For the second part, let $x \in[0, b]$. By (2.17) and the definition of $f,(x, f(x))=\varphi\left(R^{x}\right)$ or $(f(x), x)=\varphi\left(R^{x}\right)$. By $(2.17), f(x) \in\left\{\varphi_{1}\left(R^{f(x)}\right), \varphi_{2}\left(R^{f(x)}\right)\right\}$. Hence, by replacement-domination, $\varphi\left(R^{f(x)}\right)=$ $\varphi\left(R^{x}\right)$. Thus, $f(x)=f^{-1}(x)$, the desired conclusion.

Lemma 2.14 The function $f$ is continuous.

Proof. It suffices to prove that $f$ is left-continuous and right-continuous. We only show that $f$ is left-continuous. Right-continuity can be shown similarly. Let $x \in[0, b]$ and $\left(x_{k}\right)_{k \in \mathbb{N}}$ be a strictly increasing sequence converging to $x$. By Lemma 2.13, $\left(f\left(x_{k}\right)\right)_{k \in \mathbb{N}}$ is a strictly decreasing sequence. Let $\bar{x} \equiv \lim _{k \rightarrow \infty} f\left(x_{k}\right)$. If $\left(f\left(x_{k}\right)\right)_{k \in N}$ does not converge to $f(x)$, then, by Lemma $2.13, f(x)<\bar{x}$. We distinguish two cases.

Case 1: $x \leq f(x)$.

Consider $R^{\frac{1}{2}\left(\int(x)+\bar{x}\right)}$. By $(2.17)$ in the proof of Lemma 2.13,

$$
\frac{1}{2}(f(x)+\bar{x}) \in\left\{\varphi_{1}\left(R^{\frac{1}{2}(f(x)+\bar{x})}\right), \varphi_{2}\left(R^{\frac{1}{2}(f(x)+\bar{x})}\right)\right\} .
$$

Thus, by replacement-domination, $\varphi_{1}\left(R^{\frac{1}{2}(f(x)+\bar{x})}\right)<x$. Hence, for some $k \in \mathbb{N}$, $x_{k} \in$ ]$\varphi_{1}\left(R^{\frac{1}{2}(f(x)+\bar{x})}\right), x\left[\right.$. Thus, $f\left(x_{k}\right)>\bar{x}>\varphi_{2}\left(R^{\frac{1}{2}(f(x)+\bar{x})}\right)$. Because $\varphi\left(R^{x_{k}}\right)=\left(x_{k}, f\left(x_{k}\right)\right)$ it follows that $\varphi\left(R^{\left.\frac{1}{2}(f(x)+\bar{x})\right)}\right) P_{1}^{x_{k}} \varphi\left(R^{x_{k}}\right)$ and $\varphi\left(R^{x_{k}}\right) P_{3}^{x_{k}} \varphi\left(R^{\left.\frac{1}{2}(f(x)+\bar{x})\right)}\right)$, which contradicts replacement-domination.

Case 2: $x>f(x)$.

Let $\varepsilon>0$ be such that $f(x)+\varepsilon<\min \{\bar{x}, x\}$. By (2.17),

$$
f(x)+\varepsilon \in\left\{\varphi_{1}\left(R^{f(x)+\varepsilon}\right), \varphi_{2}\left(R^{f(x)+\varepsilon}\right)\right\} .
$$


Thus, by replacement-domination, $\varphi_{2}\left(R^{f(x)+\varepsilon}\right)<x$. Hence, for some $k \in \mathbb{N}, x_{k} \in$ ]$\varphi_{2}\left(R^{f(x)+\varepsilon}\right), x\left[\right.$. By our choice of $\varepsilon, f\left(x_{k}\right)>f(x)+\varepsilon$. Because $\varphi\left(R^{x_{k}}\right)=\left(x_{k}, f\left(x_{k}\right)\right)$ it follows that $\varphi\left(R^{f(x)+\varepsilon}\right) P_{\mathrm{I}}^{x_{k}} \varphi\left(R^{x_{k}}\right)$ and $\varphi\left(R^{x_{k}}\right) P_{3}^{x_{k}} \varphi\left(R^{f(x)+\varepsilon}\right)$, which contradicts replacement-domination. Therefore, $f$ is left-continuous.

By Lemmas 2.12, 2.13, and 2.14, $f \in \mathcal{F}$. Let $a \in[0, b]$ be such that $f(a)=a$. The following lemma completes the proof of Theorem 2.8 .

Lemma $2.15 \varphi=\phi^{f}$.

Proof. Let $R \in \mathcal{R}^{N}$. By anonymity, we may suppose that $p\left(R_{1}\right) \leq p\left(R_{2}\right) \leq \cdots \leq p\left(R_{n}\right)$. We distinguish three cases.

Case 1: $a \in[\underline{p}(R), \bar{p}(R)]$.

Without loss of generality, we suppose that for some $t \in N, \phi^{f}(R)=\left(p\left(R_{t}\right), f\left(p\left(R_{t}\right)\right)\right)$. Let $\bar{R} \in \mathcal{R}^{N}$ be such that $\bar{R}_{t}=R_{t}$ and for all $i \in N \backslash\{t\}$,

- when $p\left(R_{i}\right) \leq p\left(R_{i}\right), p\left(\bar{R}_{i}\right)=0$, and

- when $p\left(R_{i}\right)>p\left(R_{i}\right), p\left(\bar{R}_{i}\right)=1$.

By anonymity, peaks-onliness, and construction of $f$,

$$
\varphi(\bar{R})=\varphi\left(R^{p\left(R_{t}\right)}\right)=\dot{\phi}^{J}(R)=\left(p\left(R_{t}\right), f\left(p\left(R_{t}\right)\right)\right)
$$

Note that by construction and Lemma 2.8 , for all $i \in N \backslash\{t\}, p\left(R_{i}\right), p\left(\bar{R}_{i}\right) \leq p\left(R_{i}\right)$ or $p\left(R_{i}\right), p\left(\bar{R}_{i}\right) \geq f\left(p\left(R_{t}\right)\right)$. By successive applications of Lemma 2.5, Lemma 2.11, and replacement-domination, it follows that $\varphi(R)=\varphi(\bar{R})$. Thus, by the above equalities, $\varphi(R)=\phi^{f}(R)$, the desired conclusion

Case 2: $\bar{p}(R)<a$.

Let $\bar{R} \in \mathcal{R}^{N}$ be such that $p\left(\bar{R}_{2}\right)=1$ and $\bar{R}_{N \backslash\{2\}}=R_{N \backslash\{2\}}$. By Case $1, \varphi(\bar{R})=$ $(\bar{p}(R), f(\bar{p}(R)))$. Let $\bar{R}^{\prime} \in \mathcal{R}^{N}$ be such that $p\left(\bar{R}_{2}^{\prime}\right)=p\left(\bar{R}_{1}\right)$ and $\bar{R}_{N \backslash\{2\}}^{\prime}=\bar{R}_{N \backslash\{2\}}$. By Lemma 2.6, for all $i \in N \backslash\{2\} ; \varphi\left(\bar{R}^{\prime}\right) \bar{R}_{i} \varphi(\bar{R})$. Thus, by peaks-onliness, $\varphi\left(\bar{R}^{\prime}\right)=$ $(\bar{p}(\bar{R}), \bar{p}(\bar{R}))$. Thus, Lemma 2.5 or Lemma 2.11 implies

$$
\varphi(R)=\varphi\left(\bar{R}^{\prime}\right)=(\bar{p}(R), \bar{p}(R))=\phi^{\jmath}(R)
$$

the desired conclusion.

Case 3: $a<\underline{p}(R)$. 
If $\underline{p}(R) \in] a, b]$, then the same proof of Case 2 yields the desired conclusion. Thus, let $\underline{p}(R) \in] b, 1]$. Let $\bar{R} \in \mathcal{R}^{N}$ be such that $p\left(\bar{R}_{n-1}\right)=b$ and $\bar{R}_{N \backslash\{n-1\}}=R_{N \backslash\{n-1\}}$. By the previous fact, $\varphi(\vec{R})=(b, b)$. Since $b<\underline{p}(R)$, Pareto-optimality implies $\varphi(R) \bar{R}_{n} \varphi(\bar{R})$. Thus, by replacement-domination and peaks-onliness, $\varphi_{1}(R)=\underline{p}(R)$. Suppose that $\varphi_{2}(R)>$ $\underline{p}(R)$. Let $R^{\prime} \in \mathcal{R}^{N}$ be such that $p\left(R_{n-1}^{\prime}\right)=\frac{1}{2}\left(\underline{p}(R)+\varphi_{2}(R)\right)$ and $R_{N \backslash\{n-1\}}^{\prime}=R_{N \backslash\{n-1\}}$. By the previous argument, $\varphi_{1}\left(R^{\prime}\right)=\underline{p}(R)$. Hence, by Lemma $2.8, \varphi_{2}\left(R^{\prime}\right) \leq p\left(R_{n-1}^{\prime}\right)<\varphi(R)$. Thus, $\varphi\left(R^{\prime}\right) P_{1} \varphi(R)$ and $\varphi(R) P_{n} \varphi\left(R^{\prime}\right)$ in contradiction to replacement-domination. Therefore,

$$
\varphi(R)=(\underline{p}(R), \underline{p}(R))=\phi^{f}(R)
$$

the desired conclusion.

\subsubsection{Proof of Theorem 2.9}

Throughout Subsection 2.6.5 let $m=3, n \geq 5$, and $\varphi$ be a rule satisfying Pareto-optimality and replacenent-domination.

The following lemma follows from successive applications of Lemma 2.5 .

Lemma 2.16 Let $R \in \mathcal{R}^{N}$ be such that $\left|\left\{p\left(R_{i}\right) \mid i \in N\right\}\right| \leq 4$. For all $\bar{R} \in \mathcal{R}^{N}$, if $\left\{p\left(\bar{R}_{i}\right) \mid i \in N\right\}=\left\{p\left(R_{i}\right) \mid i \in N\right\}$, then $\varphi(\vec{R})=\varphi(R)$.

Our first step is similar to the proof of Theorem 2.8. We show in two lemmas that for any preference profile, the open interval having as endpoints the smallest and the greatest assigned location contains no peak.

Lemma 2.17 For all $R \in \mathcal{R}^{N}$ and all $\left.i \in N, p\left(R_{i}\right) \notin\right] \varphi_{1}(R), \varphi_{2}(R)[\cup] \varphi_{2}(R), \varphi_{3}(R)[$.

Proof. Suppose that for some $R \in \mathcal{R}^{N}$ and some $\left.j \in N, p\left(R_{j}\right) \in\right] \varphi_{1}(R), \varphi_{2}(R)[\cup$ $\int \varphi_{2}(R), \varphi_{3}(R)[$. Without loss of generality, we suppose that

$$
\left.p\left(R_{j}\right) \in\right] \varphi_{1}(R), \varphi_{2}(R)[
$$

Because $n \geq 5$, there exists $t \in N \backslash\{j\}$ such that $\underline{p}\left(R_{N \backslash\{t\}}\right)=\underline{p}(R), \bar{p}\left(R_{N \backslash\{t\}}\right)=\bar{p}(R)$, and $\varphi(R) \in E\left(R_{N \backslash\{t\}}\right)$. Let $R^{\prime} \in \mathcal{R}^{N}$ be such that $R_{N \backslash\{\}}^{\prime}=R_{N \backslash\{t\}}$ and $p\left(R_{t}^{\prime}\right)=\varphi_{3}(R)$. We show that $\varphi\left(R^{\prime}\right)=\varphi(R)$. By replacement-domination, there are two cases.

If for all $i \in N \backslash\{t\}, \varphi(R) R_{i} \varphi\left(R^{\prime}\right)$, then $\varphi\left(R^{\prime}\right) \in E\left(R_{N \backslash\{t\}}\right)$. Hence, by Lemma 2.5, $\varphi\left(R^{\prime}\right)=\varphi(R)$.

If for all $i \in N \backslash\{t\}, \varphi\left(R^{\prime}\right) R_{i} \varphi(R)$, then $\varphi_{1}\left(R^{\prime}\right) \leq \varphi_{1}(R)$ and $\varphi_{3}(R) \leq \varphi_{3}\left(R^{\prime}\right)$. Suppose that $\varphi\left(R^{\prime}\right) \neq \varphi(R)$. Then $\varphi_{1}\left(R^{\prime}\right)<\varphi_{1}(R)$ or $\varphi_{3}(R)<\varphi_{3}\left(R^{\prime}\right)$. By $(2.18)$, $\varphi_{1}\left(R^{\prime}\right)<\varphi_{1}(R)$ 
implies $\varphi_{2}\left(R^{\prime}\right)<\varphi_{2}(R)$ and $\varphi_{3}(R)<\varphi_{3}\left(R^{\prime}\right)$. By our choice of $t, \varphi(R) \in E\left(R_{N \backslash\{\ell\}}\right)$. Thus, for some $l \in N \backslash\{t\}, p\left(R_{l}\right) \in\left[\varphi_{2}(R), \varphi_{3}(R)\right]$ and $\varphi(R) P_{l} \varphi\left(R^{\prime}\right)$, which contradicts replacement-domination. Thus, this case cannot occur. When $\varphi_{3}(R)<\varphi_{3}\left(R^{\prime}\right)$, similar arguments yield a contradiction to replacement-domination.

Without loss of generality, we suppose that $p\left(R_{1}^{\prime}\right)=\underline{p}\left(R^{\prime}\right), p\left(R_{2}^{\prime}\right)=\varphi_{3}\left(R^{\prime}\right)$. Let $l \notin\{1,2, j\}$. Let $R^{\prime \prime} \in \mathcal{R}^{N}$ be such that $R_{N \backslash\{\}}^{\prime \prime}=R_{N \backslash\{l\}}^{\prime}$ and $p\left(R_{l}^{\prime \prime}\right)=\varphi_{1}\left(R^{\prime}\right)$. Using the above arguments it follows that $\varphi\left(R^{\prime \prime}\right)=\varphi\left(R^{\prime}\right)$.

Let $\bar{R} \in \mathcal{R}^{N}$ be such that $\bar{R}_{1}=R_{l}^{\prime \prime}, \vec{R}_{2}=R_{2}^{\prime \prime}$, and for all $i \in N \backslash\{1,2\}, \bar{R}_{i}=R_{j}^{\prime \prime}$. Successive applications of Lemma 2.5 imply $\varphi(\bar{R})=\varphi\left(R^{\prime \prime}\right)$. Let $\left.x \in\right] \varphi_{1}(\bar{R}), p\left(\bar{R}_{3}\right)[$ be such that $x \bar{P}_{3} \varphi_{2}(\bar{R})$. Let $\bar{R}^{x} \in \mathcal{R}^{N}$ be such that $\bar{R}_{N \backslash\{1\}}^{x}=\bar{R}_{N \backslash\{1\}}$ and $p\left(\vec{R}_{1}^{x}\right)=x$. We show that $\varphi\left(\bar{R}^{x}\right)=\varphi(\bar{R})$.

If $\varphi\left(\bar{R}^{x}\right) \in E\left(\bar{R}_{N \backslash\{4\}}\right)$, then Lemma 2.5 implies $\varphi\left(\bar{R}^{x}\right)=\varphi(\bar{R})$. Suppose that $\varphi\left(\bar{R}^{x}\right) \notin$ $E\left(\tilde{R}_{N \backslash\{1\}}\right)$. Hence, by Pareto-optimality, $\left.p\left(\bar{R}_{4}^{x}\right) \in\left[\varphi_{1}\left(\bar{R}^{x}\right), \varphi_{2}\left(\bar{R}^{x}\right)\right] \subseteq\right] p\left(\bar{R}_{1}\right), p\left(\bar{R}_{3}\right)[$ or $\left.p\left(\bar{R}_{4}^{x}\right) \in\left[\varphi_{2}\left(\bar{R}^{x}\right), \varphi_{3}\left(\bar{R}^{x}\right)\right] \subseteq\right] p\left(\bar{R}_{1}\right), p\left(\bar{R}_{3}\right)\left[\right.$. In both cases, by our choice of $p\left(\bar{R}_{4}^{x}\right)$, $\varphi\left(\bar{R}^{x}\right) \bar{P}_{3} \varphi(\bar{R})$. Then by replacement-domination, for all $i \in N \backslash\{4\}, \varphi\left(\bar{R}^{x}\right) \bar{R}_{i} \varphi(\bar{R})$. Hence, $\varphi_{3}\left(\bar{R}^{x}\right)=\varphi_{3}(\bar{R})$ and $\varphi_{2}\left(\bar{R}^{x}\right)<p\left(\bar{R}_{3}\right)<\varphi_{2}(\bar{R})$. Thus, $\varphi(\bar{R}) \bar{P}_{2} \varphi\left(\bar{R}^{x}\right)$, which contradicts replacement-domination.

Hence, $\varphi\left(\bar{R}^{x}\right)=\varphi(\bar{R})$. To summarize,

$$
\left.\varphi_{1}\left(\bar{R}^{x}\right)=\underline{p}(\bar{R}), p\left(\bar{R}_{3}\right) \in\right] \varphi_{1}\left(\ddot{R}^{x}\right), \varphi_{2}\left(\bar{R}^{x}\right)\left[, \text { and } \varphi_{3}\left(\bar{R}^{x}\right)=\bar{p}(\vec{R}) .\right.
$$

Note that $p\left(\bar{R}_{3}^{x}\right)=p\left(\bar{R}_{5}^{x}\right)$ and $\left|\left\{p\left(\bar{R}_{i}^{x}\right) \mid i \in N\right\}\right| \leq 4$. By Lemma 2.16, we may assume that

$$
\varphi_{2}\left(\bar{R}^{x}\right) \bar{P}_{\overline{\mathrm{j}}}^{x} p\left(\bar{R}_{\mathrm{i}}^{x}\right)
$$

Let $\tilde{R}^{x} \in \mathcal{R}^{N}$ be such that $\tilde{R}_{N \backslash\{1\}}^{x}=\bar{R}_{N \backslash\{1\}}^{x}$ and $\tilde{R}_{1}^{x}=\bar{R}_{3}^{x}$. By Lemma 2.6, for all $i \in N \backslash\{1\}$,

$$
\varphi\left(\tilde{R}^{x}\right) \bar{R}_{i}^{x} \varphi\left(\bar{R}^{x}\right)
$$

Thus, $\varphi_{3}\left(\tilde{R}^{x}\right)=\varphi_{3}\left(\bar{R}^{x}\right)$. By (2.19) and using the same argument as in Lemma 2.8, we obtain $\varphi_{1}\left(\tilde{R}^{x}\right)=\underline{p}\left(\tilde{R}^{x}\right)=x$. Thus, by $(2.20)$ and $(2.21), \varphi_{2}\left(\tilde{R}^{x}\right)=\varphi_{2}\left(\bar{R}^{x}\right)$. To summarize,

$$
\varphi_{1}\left(\tilde{R}^{x}\right)=x, \varphi_{2}\left(\tilde{R}^{x}\right)=\varphi_{2}\left(\tilde{R}^{x}\right), \text { and } \varphi_{3}\left(\tilde{R}^{x}\right)=\bar{p}(\bar{R}) .
$$

Let $\hat{R}^{x} \in \mathcal{R}^{N}$ be such that $\hat{R}_{N \backslash\{2\}}^{x}=\bar{R}_{N \backslash\{2\}}^{x}$ and $\hat{R}_{2}^{x}=\bar{R}_{3}^{x}$. By Lemma 2.6, for all $i \in N \backslash\{2\}, \varphi\left(\hat{R}^{x}\right) \bar{R}_{i}^{x} \varphi\left(\vec{R}^{x}\right)$. Thus, $\varphi_{1}\left(\hat{R}^{x}\right)=p(\bar{R})$. By (2.19) and using the same argument as in Lemma 2.8 , we obtain $\varphi_{3}\left(\hat{R}^{x}\right)=\bar{p}\left(\hat{R}^{x}\right)=p\left(\bar{R}_{3}\right)$. By Pareto-optimality, $\varphi_{2}\left(\hat{R}^{x}\right) \in$ $\left[\underline{p}\left(\hat{R}^{x}\right), \bar{p}\left(\hat{R}^{x}\right)\right]$. We show that without loss of generality we may assume that

$$
\varphi_{2}\left(\hat{R}^{x}\right) \neq x
$$


If $\varphi_{2}\left(\hat{R}^{x}\right)=x$, then

$$
\varphi_{1}\left(\hat{R}^{x}\right)=\underline{p}(\bar{R}), \varphi_{2}\left(\hat{R}^{x}\right)=x, \text { and } \varphi_{3}\left(\hat{R}^{x}\right)=p\left(\bar{R}_{3}\right)
$$

Let $y=\frac{1}{2}\left(x+p\left(\bar{R}_{3}\right)\right)$ and consider the profiles $\bar{R}^{y}$ and $\hat{R}^{y}$ which we define in the same way as $\tilde{R}^{x}$ and $\hat{R}^{x}$. L Ling the same arguments as for $\bar{R}^{x}$ and $\hat{R}^{x},(2.19)$ holds for $\bar{R}^{y}$, and the first and last equality of (2.24) hold for $\hat{R}^{y}$. If $\varphi_{2}\left(\hat{R}^{y}\right)=y$, then the previous fact and (2.24) together with Lemma 2.16 contradict replacement-domination. Hence, $\varphi_{2}\left(\hat{R}^{y}\right) \neq y$ and instead of $x$ we use $y$ for the definitions of $\bar{R}^{x}, \tilde{R}^{x}$, and $\hat{R}^{x}$.

We assume that (2.23) holds. Then

$$
\varphi_{1}\left(\hat{R}^{x}\right)=\underline{p}(\bar{R}), \varphi_{2}\left(\hat{R}^{x}\right) \neq x, \text { and } \varphi_{3}\left(\hat{R}^{x}\right)=p\left(\vec{R}_{3}\right)
$$

Let $\sigma: N \rightarrow N$ be the permutation such that for all $i \in N \backslash\{1,2\}, \sigma(i)=i, \sigma(1)=2$, and $\sigma(2)=1$. By Lemma 2.16, $\varphi\left(\sigma\left(\tilde{R}^{x}\right)\right)=\varphi\left(\tilde{R}^{x}\right)$. By the previous fact, (2.22), and (2.25), $\varphi\left(\sigma\left(\tilde{R}^{x}\right)\right) \hat{P}_{1}^{x} \varphi\left(\hat{R}^{x}\right)$ and $\varphi\left(\hat{R}^{x}\right) \hat{P}_{3}^{x} \varphi\left(\sigma\left(\tilde{R}^{x}\right)\right)$. Since $\sigma\left(\tilde{R}^{x}\right)_{N \backslash\{1\}}=\hat{R}_{N \backslash\{1\}}^{x}$, the previous relations contradict replacement-domination.

Lemma 2.18 For all $R \in \mathcal{R}^{N}$ and all $\left.i \in N, p\left(R_{i}\right) \notin\right] \varphi_{1}(R), \varphi_{3}(R)[$.

Proof. Suppose that for some $R \in \mathcal{R}^{N}$ and some $\left.j \in N, p\left(R_{j}\right) \in\right] \varphi_{1}(R), \varphi_{3}(R)[$. By Lemma $2.17, p\left(R_{j}\right)=\varphi_{2}(R)$. Without loss of generality, we suppose that $p\left(R_{1}\right)=\underline{p}(R)$ and $p\left(R_{2}\right)=\bar{p}(R)$. Let $\bar{R} \in \mathcal{R}^{N}$ be such that $\bar{R}_{\{1,2, j\}}=R_{\{1,2, j\}}$ and for all $i \in N \backslash\{1,2, j\}$, $\breve{R}_{i}=R_{j}$. Successive applications of Lemma 2.7 yicld $\varphi(\bar{R})=\varphi(R)$. Let $\bar{R}^{1} \in \mathcal{R}^{N}$ be such that $\bar{R}_{N \backslash\{2\}}^{1}=\bar{R}_{N \backslash\{2\}}$ and $\bar{R}_{2}^{1}=\bar{R}_{j}$, and $\bar{R}^{2} \in \mathcal{R}^{N}$ be such that $\bar{R}_{N \backslash\{1\}}^{2}=\bar{R}_{N \backslash\{1\}}^{1}$ and $\vec{R}_{1}^{2}=\bar{R}_{2}$. Lsing the same arguments as in the proof of Lemma 2.8 we can show that

$$
\varphi_{1}\left(\bar{R}^{1}\right)<\varphi_{2}\left(\bar{R}^{1}\right)=\varphi_{3}\left(\bar{R}^{1}\right)=p\left(\bar{R}^{j}\right)
$$

and

$$
p\left(\bar{R}_{j}\right)=\varphi_{1}\left(\bar{R}^{2}\right)=\varphi_{2}\left(\bar{R}^{2}\right)<\varphi_{3}\left(\bar{R}^{2}\right)=p\left(\bar{R}^{j}\right)
$$

By Lemma 2.16 and the two previous facts, the same argument as in the proof of Lemma 2.8 yields a contradiction to replacement-domination.

Next, we show that for any preference profile the assigned locations of all public goods are equal.

Lemma 2.19 For all $R \in \mathcal{R}^{N},\left|\left\{\varphi_{k}(R) \mid k \in M\right\}\right|=1$. 
Proof. Suppose that for some $R \in \mathcal{R}^{N},\left|\left\{\varphi_{k}(R) \mid k \in M\right\}\right| \geq 2$. Without loss of generality, we suppose that $p\left(R_{1}\right)=\underline{p}(R)$ and $p\left(R_{2}\right)=\bar{p}(R)$. We consider four cases.

Case 1: For some $j \in N, \underline{p}(R)<\varphi_{1}(R)<\varphi_{2}(R)=\varphi_{3}(R)=p\left(R_{j}\right)<\bar{p}(R)$ and for all $i \in N, p\left(R_{i}\right) \neq \varphi_{1}(R)$.

Let $\bar{R} \in \mathcal{R}^{N}$ be such that $\bar{R}_{\{1,2, j\}}=R_{\{1,2, j\}}$ and for all $i \in N \backslash\{1,2, j\}, \bar{R}_{i}=R_{1}$. Successive applications of Lemma 2.5 yield $\varphi(\bar{R})=\varphi(R)$. Given $x \in] \varphi_{1}(R), \varphi_{2}(R)[$, let $\bar{R}^{x} \in \mathcal{R}^{N}$ be such that $\bar{R}_{N \backslash\{j\}}^{x}=\bar{R}_{N \backslash\{j\}}$ and $p\left(\bar{R}_{j}^{x}\right)=x$. We prove the following claim.

Claim 1: For all $x \in] \varphi_{1}(\bar{R}), \varphi_{2}(\bar{R})\left[,\left|\left\{k \in M \mid \varphi_{k}\left(\bar{R}^{x}\right)=x\right\}\right| \geq 2\right.$.

Proof of Claim 1: Let $x \in] \varphi_{1}(\bar{R}), \varphi_{2}(\bar{R})\left[\right.$. By Lemmas 2.17 and $2.18, \varphi_{3}\left(\bar{R}^{x}\right) \leq x$ or $\varphi_{1}\left(\bar{R}^{x}\right) \geq x$.

If $\varphi_{3}\left(\bar{R}^{x}\right) \leq x$, then $\varphi(\bar{R}) \bar{P}_{2} \varphi\left(\bar{R}^{x}\right)$. Thus, by replacement-domination, $\varphi_{1}\left(\bar{R}^{x}\right)>\varphi_{1}(\bar{R})$. Hence, by Pareto-optimality, $\left|\left\{k \in M \mid \varphi_{k}\left(\bar{R}^{x}\right)=x\right\}\right| \geq 2$.

If $\varphi_{1}\left(\bar{R}^{x}\right) \geq x$, then $\varphi(\bar{R}) \bar{P}_{1} \varphi\left(\bar{R}^{x}\right)$. Thus, by replacement-domination, $\varphi_{3}\left(\bar{R}^{x}\right) \leq \varphi_{3}(\bar{R})$. Hence, by Pareto-optimality, $\left|\left\{k \in M \mid \varphi_{k}\left(\bar{R}^{x}\right)=x\right\}\right| \geq 2$.

Claim 1 and replacement-domination imply that for all $x \in] \varphi_{1}(\bar{R}), \varphi_{2}(\bar{R})[$,

$$
\left.\left.\varphi_{1}\left(\bar{R}^{x}\right), \varphi_{2}\left(\bar{R}^{x}\right), \varphi_{3}\left(\bar{R}^{x}\right) \in\right] \varphi_{1}(\bar{R}), \varphi_{3}(\bar{R})\right]
$$

Claim 2: For some $y \in] \varphi_{1}(\bar{R}), \varphi_{2}(\bar{R})\left[, \varphi_{1}\left(\bar{R}^{y}\right)<\varphi_{2}\left(\bar{R}^{y}\right)=\varphi_{3}\left(\bar{R}^{y}\right)=y\right.$.

Proof of Claim 2: Suppose that for all $x \in] \varphi_{1}(\bar{R}), \varphi_{2}(\bar{R})[$,

$$
\varphi_{1}\left(\bar{R}^{x}\right) \geq x
$$

By Claim 1 and replacement-domination, for some $\tilde{x} \in] \varphi_{1}(\bar{R}), \varphi_{2}(\bar{R})\left[, \varphi_{3}\left(\bar{R}^{\bar{x}}\right)<\varphi_{2}(\bar{R})\right.$. Let $\bar{x}=\frac{1}{2}\left(\varphi_{3}\left(\bar{R}^{\bar{x}}\right)+\varphi_{2}(\vec{R})\right)$. By $(2.27), \varphi\left(\bar{R}^{\bar{x}}\right) \bar{P}_{1} \varphi\left(\bar{R}^{\bar{x}}\right)$ and $\varphi\left(\bar{R}^{\bar{x}}\right) \bar{P}_{2} \varphi\left(\bar{R}^{\bar{x}}\right)$, which contradicts replacement-domination.

Claim 2 guarantees the existence of $y$. Let $z=\varphi_{1}\left(\bar{R}^{y}\right)$. By $(2.26), z>\varphi_{1}(\bar{R})$. Consider $\varphi\left(\bar{R}^{y}\right)$ and $\varphi\left(\bar{R}^{z}\right)$. By Claim $1,\left|\left\{k \in M \mid \varphi_{k}\left(\bar{R}^{z}\right)=z\right\}\right| \geq 2$. Thus, $\varphi\left(\bar{R}^{z}\right) \bar{P}_{1} \varphi\left(\bar{R}^{y}\right)$. By replacement-domination, $\varphi\left(\bar{R}^{z}\right) \bar{R}_{2} \varphi\left(\bar{R}^{z}\right)$. Hence;

$$
\varphi_{1}\left(\vec{R}^{z}\right)=\varphi_{2}\left(\bar{R}^{z}\right)=z<y<\varphi_{3}\left(\tilde{R}^{z}\right)
$$

Let $w=\frac{1}{2}\left(y+\varphi_{3}\left(\bar{R}^{z}\right)\right)$. Consider $\varphi\left(\bar{R}^{y}\right)$ and $\varphi\left(\bar{R}^{w}\right)$. By Claim 1, $\mid\left\{k \in M \mid \varphi_{k}\left(\bar{R}^{w}\right)=\right.$ $w\} \mid \geq 2$. Thus, $\varphi\left(\bar{R}^{w}\right) \bar{P}_{2} \varphi\left(\vec{R}^{y}\right)$. By replacement-domination, $\varphi\left(\bar{R}^{w}\right) \bar{R}_{1} \varphi\left(\bar{R}^{y}\right)$. Hence;

$$
\varphi_{1}\left(\bar{R}^{w}\right)<z<\varphi_{2}\left(\bar{R}^{w}\right)=\varphi_{3}\left(\bar{R}^{w}\right)=u
$$

By our choice of $w,(2.28)$, and (2.29), it follows that $\varphi\left(\bar{R}^{w}\right) \bar{P}_{1} \varphi\left(\bar{R}^{z}\right)$ and $\varphi\left(\bar{R}^{z}\right) \bar{P}_{2} \varphi\left(\bar{R}^{w}\right)$, which contradicts replacement-domination. Hence, Case 1 cannot occur. 
Case 2: For some $j \in N, \underline{p}(R)<\varphi_{1}(R)=\varphi_{2}(R)=p\left(R_{j}\right)<\varphi_{3}(R)<\bar{p}(R)$ and for all $i \in N, p\left(R_{i}\right) \neq \varphi_{3}(R)$.

Case 2 is analogous to Case 1.

Case 3: $\left.\varphi_{2}(R) \in\right] \varphi_{1}(R), \varphi_{3}(R) \mid$.

By Lemmas 2.17 and 2.18, for all $i \in N, p\left(R_{i}\right) \in\left[\underline{p}(R), \varphi_{1}(R)\right] \cup\left[\varphi_{3}(R), \bar{p}(R)\right]$. Thus, by Pareto-optimality, for some $j, l \in N, p\left(R_{j}\right)=\varphi_{1}(R)$ and $p\left(R_{l}\right)=\varphi_{3}(R)$. Let $\bar{R} \in \mathcal{R}^{N}$ be such that $\bar{R}_{1}=R_{j}, \bar{R}_{2}=R_{l}$, and for all $i \in N \backslash\{1,2\}, \bar{R}_{i}=R_{l}$. Successive applications of Lemmas 2.5 and 2.16 yield $\varphi(\bar{R})=\varphi(R)$. Let $\bar{R}^{\prime} \in \mathcal{R}^{N}$ be such that $\bar{R}_{N \backslash\{3\}}^{\prime}=\bar{R}_{N \backslash\{3\}}$ and $p\left(\bar{R}_{3}^{\prime}\right)=\varphi_{2}(\bar{R})$. By Lemmas 2.17 and $2.18, \varphi_{1}\left(\bar{R}^{\prime}\right) \geq p\left(\bar{R}_{3}^{\prime}\right)$ or $\varphi_{3}\left(\bar{R}^{\prime}\right) \leq p\left(\bar{R}_{3}^{\prime}\right)$. Without loss of generality, we suppose that $\varphi_{3}\left(\bar{R}^{\prime}\right) \leq p\left(\bar{R}_{3}^{\prime}\right)$. By replacement-domination, $\varphi_{1}\left(\bar{R}^{\prime}\right)>\underline{p}\left(\bar{R}^{\prime}\right)$. Since Cases 1 and 2 cannot occur, we obtain

$$
\varphi_{1}\left(\bar{R}^{\prime}\right)=\varphi_{2}\left(\bar{R}^{\prime}\right)=\varphi_{3}\left(\bar{R}^{\prime}\right)=p\left(\bar{R}_{3}^{\prime}\right)
$$

Let $\tilde{R} \in \mathcal{R}^{N}$ be such that $\tilde{R}_{N \backslash\{3\}}=\vec{R}_{N \backslash\{3\}}$ and $p\left(\tilde{R}_{3}\right)=\frac{1}{2}\left(\varphi_{2}(\bar{R})+\varphi_{3}(\bar{R})\right)$. By Lemmas 2.17 and $2.18, \varphi_{1}(\tilde{R}) \geq p\left(\tilde{R}_{3}\right)$ or $\varphi_{3}(\tilde{R}) \leq p\left(\tilde{R}_{3}\right)$. By replacement-domination and (2.30), $\varphi_{3}(\tilde{R}) \leq p\left(\tilde{R}_{3}\right)$ and $\varphi_{1}(\tilde{R})<\varphi_{2}(\bar{R})$. Since Cases 1 and 2 cannot occur, Pareto-optimality and replacement-domination imply

$$
\varphi_{1}(\tilde{R})=\underline{p}(\tilde{R})<\varphi_{2}(\tilde{R})=\varphi_{3}(\tilde{R})=p\left(\tilde{R}_{3}\right) .
$$

Let $\hat{R} \in \mathcal{R}^{N}$ be such that $\hat{R}_{N \backslash\{3\}}=\bar{R}_{N \backslash\{3\}}$ and $p\left(\hat{R}_{3}\right)=\frac{1}{2}\left(\varphi_{1}(\bar{R})+\varphi_{2}(\bar{R})\right)$. Symmetric arguments as above yield

$$
p\left(\hat{R}_{3}\right)=\varphi_{1}(\hat{R})=\varphi_{2}(\hat{R})<\varphi_{3}(\hat{R})=\bar{p}(\hat{R}) .
$$

By (2.31) and (2.32), $\varphi(\tilde{R}) \bar{P}_{1} \varphi(\hat{R})$ and $\varphi(\hat{R}) \bar{P}_{2} \varphi(\tilde{R})$, which contradicts replacement-domination. Hence, Case 3 cannot occur.

Case 4: $\left|\left\{\varphi_{k}(R) \mid k \in M\right\}\right|=2$ and for some $j, l \in N, p\left(R_{j}\right)=\varphi_{\mathrm{I}}(R)$ and $p\left(R_{l}\right)=\varphi_{3}(R)$.

Without loss of generality, we suppose that $p\left(R_{j}\right)=\varphi_{1}(R)=\varphi_{2}(R)$ and $p\left(R_{l}\right)=\varphi_{3}(R)$. Let $\bar{R} \in \mathcal{R}^{N}$ be such that $\bar{R}_{1}=R_{j}, \bar{R}_{2}=R_{l}$, and for all $i \in N \backslash\{1,2\}, \bar{R}_{i}=R_{l}$. Successive applications of Lemmas 2.5 and 2.16 yield $\varphi(\ddot{R})=\varphi(R)$.

Let $\bar{R}^{\prime} \in \mathcal{R}^{N}$ be such that $\bar{R}_{N \backslash\{3\}}^{\prime}=\bar{R}_{N \backslash\{3\}}$ and $p\left(\bar{R}_{3}^{\prime}\right)=\frac{1}{2}\left(\varphi_{1}(\bar{R})+\varphi_{3}(\bar{R})\right)$. By Lemmas 2.17 and 2.18, $\varphi_{1}\left(\bar{R}^{\prime}\right) \geq p\left(\bar{R}_{3}^{\prime}\right)$ or $\varphi_{3}\left(\bar{R}^{\prime}\right) \leq p\left(\vec{R}_{3}^{\prime}\right)$. Without loss of generality, we suppose that $\varphi_{3}\left(\bar{R}^{\prime}\right) \leq p\left(\bar{R}_{3}^{\prime}\right)$. If $\left|\left\{\varphi_{k}\left(\bar{R}^{\prime}\right) \mid k \in M\right\}\right|=1$, then choose $p\left(\bar{R}_{3}^{\prime}\right) \in$ $\int \varphi_{1}(\bar{R}), \varphi_{3}(\vec{R})\left\{\backslash\left\{\frac{1}{2}\left(\varphi_{1}(\bar{R})+\varphi_{3}(\bar{R})\right)\right\}\right.$. Since Cases 1,2 , and 3 cannot occur, replacementdomination inplies $\varphi_{1}\left(\bar{R}^{\prime}\right)=\underline{p}\left(\bar{R}^{\prime}\right)$ and $\varphi_{2}\left(\bar{R}^{\prime}\right)=\varphi_{3}\left(\bar{R}^{\prime}\right)=p\left(\bar{R}_{3}^{\prime}\right)$. Let $\bar{R}^{\prime \prime} \in \mathcal{R}^{N}$ be such that $\bar{R}_{N \backslash\{4\}}^{\prime \prime}=\bar{R}_{N \backslash\{4\}}^{\prime}$ and $p\left(\bar{R}_{1}^{\prime \prime}\right)=\frac{1}{2}\left(\varphi_{1}\left(\bar{R}^{\prime}\right)+\varphi_{3}\left(\bar{R}^{\prime}\right)\right)$. By replacement-domination, for all $i \in N \backslash\{4\}, \varphi\left(\tilde{R}^{\prime}\right) \bar{R}_{i}^{\prime} \varphi\left(\bar{R}^{\prime \prime}\right)$. If $\left\{\left\{\varphi_{k}\left(\bar{R}^{\prime \prime}\right) \mid k \in M\right\} \mid=1\right.$, then choose 
$\left.p\left(\bar{R}_{1}^{\prime \prime}\right) \in\right] \varphi_{1}\left(\bar{R}^{\prime}\right), \varphi_{3}\left(\bar{R}^{\prime}\right)\left\lfloor\left\{\frac{1}{2}\left(\varphi_{1}\left(\bar{R}^{\prime}\right)+\varphi_{3}\left(\bar{R}^{\prime}\right)\right)\right\}\right.$. Because Cases 1, 2, and 3 cannot occur, replacement-domination implies $\varphi_{1}\left(\bar{R}^{\prime \prime}\right)=\varphi_{2}\left(\bar{R}^{\prime \prime}\right)=p\left(\bar{R}_{1}^{\prime \prime}\right)$ and $\varphi_{3}\left(\bar{R}^{\prime \prime}\right)=p\left(\bar{R}_{3}^{\prime \prime}\right)$. Let $\tilde{R} \in \mathcal{R}^{N}$ be such that $\tilde{R}_{N \backslash\{3\}}=\tilde{R}_{N \backslash\{3\}}$ and $\tilde{R}_{3}=\bar{R}_{1}^{\prime \prime}$. By Lemma $2.5, \varphi(\tilde{R})=\varphi\left(\bar{R}^{\prime \prime}\right)$. Thus, $\underline{p}(\tilde{R})<p\left(\tilde{R}_{4}\right)=\varphi_{1}(\tilde{R})=\varphi_{2}(\tilde{R})<\varphi_{3}(\tilde{R})<\bar{p}(R)$ and for all $i \in N, p\left(\tilde{R}_{i}\right) \neq \varphi_{3}(\tilde{R})$. By Case 2, this cannot occur. Hence, Case 4 cannot occur.

The final lemma completes the proof of Theorem 2.9 .

Lemma $2.20 \varphi \in\{\underline{\phi}, \bar{\phi}\}$.

Proof. First, suppose that for some $R \in \mathcal{R}^{N}$ and $\left.k \in M, \varphi_{k}(R) \in\right] \underline{p}(R), \bar{p}(R)[$. By Lemma 2.19 and Pareto-optimality, for some $j \in N$,

$$
\varphi_{1}(R)=\varphi_{2}(R)=\varphi_{3}(R)=p\left(R_{j}\right)
$$

Without loss of generality, we suppose that $p\left(R_{1}\right)=\underline{p}(R)$ and $p\left(R_{2}\right)=\bar{p}(R)$. Let $\bar{R} \in \mathcal{R}^{N}$ be such that $\bar{R}_{\{1,2, j\}}=R_{\{1,2, j\}}$ and for all $i \in N \backslash\{1,2, j\}, \bar{R}_{i}=R_{1}$. Successive applications of Lemma 2.5 yield $\varphi(\tilde{R})=\varphi(R)$. Let $\bar{R}^{\prime} \in \mathcal{R}^{N}$ be such that $\bar{R}_{N \backslash\{j\}}^{\prime}=\bar{R}_{N \backslash\{j\}}$ and $\bar{R}_{j}^{\prime}=\bar{R}_{2}$. By Lemma 2.19, either [for all $k \in M, \varphi_{k}\left(\bar{R}^{\prime}\right)=\underline{p}\left(\bar{R}^{\prime}\right)$ ] or [for all $k \in M, \varphi_{k}\left(\bar{R}^{\prime}\right)=\bar{p}\left(\bar{R}^{\prime}\right)$ ]. Both cases yield a contradiction to replacement-domination. Hence, for all $R \in \mathcal{R}^{N}$,

$$
\left\{\varphi_{k}(R) \mid k \in M\right\} \in\{\{\underline{p}(R)\},\{\bar{p}(R)\}\} .
$$

Let $R^{o} \in \mathcal{R}^{N}$ be such that $p\left(R_{1}^{o}\right)=0$ and for all $i \in N \backslash\{1\}, p\left(R_{i}^{o}\right)=1$. By (2.33), without loss of generality, we suppose that for all $k \in M, \varphi_{k}\left(R^{o}\right)=0$. We show that $\varphi=\phi .{ }^{14}$

Let $R \in \mathcal{R}^{N}$. We show that $\varphi(R)=\underline{\phi}(R)$. Let $\bar{R} \in \mathcal{R}^{N}$ be such that $p\left(\bar{R}_{1}\right)=\underline{p}(R)$ and for all $i \in N \backslash\{1\}, p\left(\bar{R}_{i}\right)=\bar{p}(R)$. By (2.33) and successive applications of Lemmas 2.5 and 2.16 yield

$$
\varphi(\bar{R})=\varphi(R)
$$

Let $\tilde{R} \in \mathcal{R}^{N}$ be such that $\tilde{R}_{N \backslash\{1\}}=\bar{R}_{N \backslash\{1\}}$ and $\tilde{R}_{1}=R_{1}^{o}$. Successive applications of Lemma 2.5, replacement-domination, and (2.33) yield

$$
\varphi(\tilde{R})=\varphi\left(R^{o}\right)
$$

Let $\tilde{R}^{\prime} \in \mathcal{R}^{N}$ be such that $\tilde{R}_{N \backslash\{2\}}^{\prime}=\tilde{R}_{N \backslash\{2\}}$ and $\tilde{R}_{2}^{\prime}=\bar{R}_{1}$. By replacement-domination and (2.33),

$$
\varphi\left(\tilde{R}^{\prime}\right)=\varphi(\tilde{R}) .
$$

\footnotetext{
${ }^{14}$ When for all $k \in M, \varphi_{k}\left(R^{\circ}\right)=1$ we would show that $\varphi=\bar{\phi}$.
} 
If $p\left(\tilde{R}_{2}^{\prime}\right)=0$, then Lemma 2.16 implies $\varphi\left(\tilde{R}^{\prime}\right)=\varphi(\bar{R})$. Thus, by $(2.34)$, for all $k \in M$, $\varphi_{k}(R)=\underline{p}(R)$. Hence, $\varphi(R)=\underline{\phi}(R)$, the desired conclusion.

If $p\left(\tilde{R}_{2}^{\prime}\right)>0$, then by Lemma 2.16 , we may assume that $0 \tilde{P}_{2}^{\prime} \bar{p}\left(\tilde{R}^{\prime}\right)$. Let $\tilde{R}^{\prime \prime} \in \mathcal{R}^{N}$ be such that $\tilde{R}_{N \backslash\{1\}}^{\prime \prime}=\tilde{R}_{N \backslash\{1\}}^{\prime}$ and $\tilde{R}_{1}^{\prime \prime}=\tilde{R}_{3}^{\prime}$. By replacement-domination, (2.33), (2.35), (2.36), and our choice of $\tilde{R}_{2}^{\prime}$, for all $k \in M, \varphi_{k}\left(\tilde{R}^{\prime \prime}\right)=p\left(\tilde{R}^{\prime \prime}\right)$. By Lemma 2.16, $\varphi\left(\tilde{R}^{\prime \prime}\right)=\varphi(\bar{R})$. Thus, by (2.34), for all $k \in M, \varphi_{k}(R)=\underline{p}(R)$. Hence, $\varphi(R)=\underline{\phi}(R)$, the desired conclusion.

\subsection{Population-Monotonicity}

\subsubsection{Population-Monotonicity}

Throughout Section 2.7 let $M=\{1,2\}$. We consider changes in the population. When a group of agents leaves or comes in, the rule may choose different alternatives before and after the population change. If the agents that are present before and after are not responsible for this change, then it would be unfair if a change in population affected their welfares in different directions. We require that the consequences of population changes are shared by all agents that are present before and after the change in population. The welfares of all these agents should be affected in the same direction: as a result they all weakly gain or they all weakly lose. This solidarity property is called population-monotonicity.

Population-Monotonicity: For all $N, N^{\prime} \in \mathcal{P}$ such that $N \subseteq N^{\prime}$ and all $R \in \mathcal{R}^{N^{\prime}}$ : either [for all $i \in N, \varphi\left(R_{N}\right) R_{i} \varphi(R)$ ] or [for all $i \in N, \varphi(R) R_{i} \varphi\left(R_{N}\right)$ ].

Instead of all possible population changes we consider only the departure or arrival of one agent.

One-Person Population-Monotonicity: For all $N \in \mathcal{P}$, all $j \in \mathbb{P} \backslash N$, and all $R \in$ $\mathcal{R}^{N \cup\{j\}}$ : either [for all $\left.i \in N, \varphi\left(R_{N}\right) R_{i} \varphi(R)\right]$ or [for all $i \in N, \varphi(R) R_{i} \varphi\left(R_{N}\right)$ ].

Note that population-monotonicity implies one-person population-monotonicity, but in general the reverse implication does not hold. The following lemma states that under Pareto-optimality and one-person population-monotonicity, if one agent leaves, then all agents that remain present weakly gain as a result of the departure. Therefore, whenever a group of agents leaves the agents that are present after weakly benefit.

Lemma 2.21 Let $\varphi$ be a rule satisfying Pareto-optimality and one-person populationmonotonicity. Then for all $N \in \mathcal{P}$, all $j \in \mathbb{P} \backslash N$, and all $R \in \mathcal{R}^{N \cup\{j\}}$, we have for all 
$i \in N, \varphi\left(R_{N}\right) R_{i} \varphi(R)$

Proof. By one-person population-monotonicity, either [for all $i \in N, \varphi(R) R_{i} \varphi\left(R_{N}\right)$ ] or [for all $i \in N, \varphi\left(R_{N}\right) R_{i} \varphi(R)$ ]. Suppose that for some $l \in N, \varphi(R) P_{l} \varphi\left(R_{N}\right)$. Thus, for all $i \in N, \varphi(R) R_{i} \varphi\left(R_{N}\right)$. Hence, $\varphi\left(R_{N}\right) \notin E\left(R_{N}\right)$, which contradicts Pareto-optinality.

The following lemma is a useful tool in the proof of our main result. It says that under Pareto-optimality and population-monotonicity, if one agent departs and the initially chosen alternative is efficient for the subeconomy, then the rule assigns to the initial and the subeconomy the same alternative. The lemma does not rely on the specific structure of our model. The implication is also true in Ching and Thomson (1999), Ehlers and Klaus (2000a), Klaus (1999a), and Miyagawa (1998c).

Lemma 2.22 Let $\varphi$ be a rule satisfying Pareto-optimality and population-monotonicity. Then for all $N \in \mathcal{P}$, all $j \in \mathbb{P} \backslash N$, and all $R \in \mathcal{R}^{N \cup\{j\}}$, if $\varphi(R) \in E\left(R_{N}\right)$, then $\varphi\left(R_{N}\right)=$ $\varphi(R)$.

Proof. By Lemma 2.21, for all $i \in N, \varphi\left(R_{N}\right) R_{i} \varphi(R)$. Since $\varphi(R) \in E\left(R_{N}\right)$, it follows that for all $i \in N, \varphi\left(R_{N}\right) I_{i} \varphi(R)$. Because $\underline{p}\left(R_{N}\right)=\underline{p}(R)$ or $\bar{p}\left(R_{N}\right)=\bar{p}(R)$, Paretooptimality and the previous fact imply that $\varphi\left(R_{N}\right)=\varphi(R)$.

\subsubsection{Single-Plateaued Preference Rules}

We characterize the class of rules satisfying Pareto-optimality and population-monotonicity. Each rule belonging to this class is defined by a continuous binary relation over $[0,1]$ which we interpret as the preference relation of the (social) planner. The planner's domain of preferences is an extension of the domain of single-peaked preferences. Instead of having a unique most preferred location in $[0,1]$, a preference relation in this domain may have a whole segment of most preferred locations which are all indifferent to each other. We describe those preferences in two ways. The first one is the usual definition and the second will be used in the definition of our rules.

Let $R_{0}$ be a continuous preference relation over [0,1]. Then $R_{0}$ is called single-plateaued when there exist two numbers $\underline{a}, \bar{a} \in[0,1]$ such that $\underline{a} \leq \bar{a}$ and for all $x, y \in[0,1]$, (i) if $x<y \leq \underline{a}$ or $x>y \geq \bar{a}$, then $y P_{0} x$; and (ii) if $x, y \in[\underline{a}, \bar{a}]$, then $x I_{0} y$. We call the interval $[\underline{a}, \bar{a}]$ the plateau of $R_{0}, \underline{a}$ the left-endpoint of the plateau of $R_{0}$, and $\bar{a}$ the right-endpoint. Note that $\underline{a}=\bar{a}$ implies that $R_{0}$ is single-peaked.

Alternatively, $R_{0}$ can be described by its corresponding indifference map $g$ which we define as follows. Since $R_{0}$ is single-plateaued over $[0,1]$, we have $0 R_{0} 1$ or $1 P_{0} 0$. 
(i) Let $0 R_{0} 1$. By continuity and single-plateauedness of $R_{0}$ there exists a unique $b \in[0,1]$ such that $b I_{0} 0$ and for all $\left.\left.x \in\right] b, 1\right], b P_{0} x$. Let $g:[0, \underline{a}] \rightarrow[\bar{a}, b]$ be the function such that for all $x \in[0, \underline{a}], g(x) \in[\bar{a}, b]$ and $g(x) I_{0} x$. By continuity and singleplateanedness of $R_{0}, g$ is uniquely defined, strictly decreasing, continuous, $g(0)=b$, and $g(\underline{a})=g(\bar{a})$.

(ii) Let $1 P_{0} 0$. By continuity and single-plateauedness of $R_{0}$ there exists a unique $\left.\left.b \in\right] 0,1\right]$ such that $b I_{0} 1$ and for all $x \in\left[0, b\left[, b P_{0} x\right.\right.$. Let $g:[b, \underline{a}] \rightarrow[\bar{a}, 1]$ be the function such that for all $x \in[b, \underline{a}], g(x) \in[\bar{a}, 1]$ and $g(x) I_{0} x$. By continuity and singleplateauedness of $R_{0}, g$ is uniquely defined, strictly decreasing, continuous, $g(b)=1$, and $g(\underline{a})=g(\bar{a})$.

By $\mathcal{G}$ we denote all functions defined as in (i) or (ii). Note that each function in $\mathcal{G}$ corresponds to a unique single-plateaued preference relation over $[0,1]$ and vice versa.

Each rule satisfying Pareto-optimality and population-monotonicity is completely defined by a single-plateaued preference relation over $[0,1]$. We interpret the single-plateaued preference to be a preference relation of the social planner who provides the two public goods. For each preference profile the rule based on the single-plateaued preference relation locates the two public goods as follows:

(I) If the peaks interval of the profile contains the planner's plateau and no peak of the profile belongs to the planner's plateau, then the rule selects for the first good the peak of the peaks profile that is best for the planner. The rule selects as other location the unique distinct point that is indifferent to this peak according to the planner's preference relation. Note that in (I), the chosen locations belong to different sides of the planner's plateau.

(II) In all other cases, the rule provides the first good at the location in $[\underline{p}(R), \bar{p}(R)]$ that is closest to the left-endpoint of the planner's plateau, and the second good at the location in $[\underline{p}(R), \bar{p}(R)]$ that is closest to the right-endpoint of the planner's relation.

We call these rules single-plateaued preference rules. Here is an example when the planner's plateau is the interval $\left[\frac{1}{5}, \frac{2}{5}\right]$. Let med denote the median operator for three numbers, i.e., for all $x, y, z \in[0,1]$, if $x \leq y \leq z$, then $\operatorname{med}(x, y, z)=y$.

Example 2.8 The social planner has a single-plateaued preference relation over $[0,1]$ with plateau $\left[\frac{1}{5}, \frac{2}{5}\right]$. The indifference map $g:\left[0, \frac{1}{5}\right] \rightarrow\left[\frac{2}{5}, \frac{4}{5}\right]$ is such that for all $x \in\left[0, \frac{1}{5}\right]$, $g(x)=\frac{4}{5}-2 x$. We define the rule $\phi^{g}$ as follows. For all $N \in \mathcal{P}$ and all $R \in \mathcal{R}^{N}$ :

(a) $\underline{p}(R) \geq \frac{2}{5}$. Then $\phi^{g}(R) \equiv(\underline{p}(R), \underline{p}(R))$. 


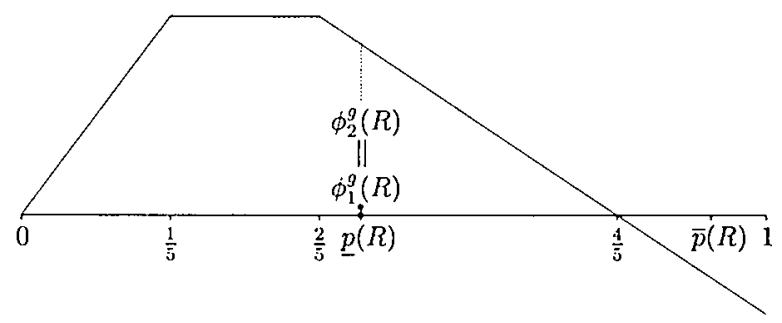

Figure 2.10: Illustration of Case (a) in Example 2.8.

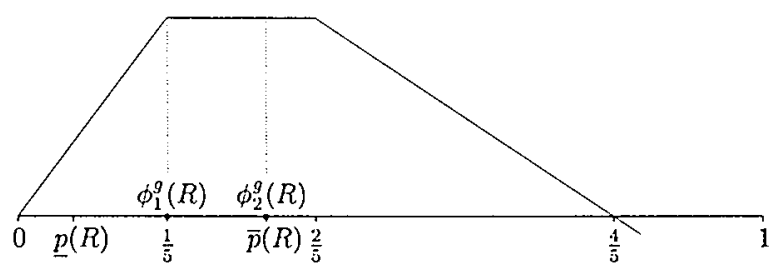

Figure 2.11: Illustration of Case (c) in Example 2.8.

(b) $\bar{p}(R) \leq \frac{1}{\bar{s}}$. Then $\phi^{g}(R) \equiv(\bar{p}(R), \bar{p}(R))$.

(c) For some $i \in N, p\left(R_{i}\right) \in\left[\frac{1}{5}, \frac{2}{5}\right]$. Then $\dot{\phi}^{g}(R) \equiv\left(\operatorname{med}\left(\underline{p}(R), \frac{1}{5}, \bar{p}(R)\right), \operatorname{med}\left(\underline{p}(R), \frac{2}{5}, \bar{p}(R)\right)\right)$.

(d) $\left[\frac{1}{5}, \frac{2}{5}\right] \subseteq[\underline{p}(R), \bar{p}(R)]$ and for all $i \in N, p\left(R_{i}\right) \notin\left[\frac{1}{5}, \frac{2}{5}\right]$. Choose $j \in N$ such that for all $i \in N$ : (i) if $p\left(R_{j}\right)<\frac{1}{5}$, then $p\left(R_{i}\right) \leq p\left(R_{j}\right)$ or $g\left(p\left(R_{j}\right)\right) \leq p\left(R_{i}\right)$; and (ii) if $p\left(R_{j}\right)>\frac{2}{5}$, then $p\left(R_{i}\right) \geq p\left(R_{j}\right)$ or $g^{-1}\left(p\left(R_{j}\right)\right) \geq p\left(R_{i}\right)$. In Subcase (d.i), $\phi^{g}(R) \equiv\left(p\left(R_{j}\right), g\left(p\left(R_{j}\right)\right)\right)$ and in Subcase $(\mathrm{d} . \mathrm{ii}), \phi^{g}(R) \equiv\left(g^{-1}\left(p\left(R_{j}\right)\right), p\left(R_{j}\right)\right)$.

Here, Cases (a), (b), and (c) correspond to (II), and Case (d) to (I). Let $N \in \mathcal{P}$ and $R \in \mathcal{R}^{N}$ be such that for all $i \in N, p\left(R_{i}\right) \in\{\underline{p}(R), \bar{p}(R)\}$. Below we illustrate $\dot{\phi}^{g}(R)$ for cases (a), (c), and (d). Figure 2.10 illustrates Case (a), Figure 2.11 Case (c), Figure 2.12 Subcase (d.i), and Figure 2.13 Subcase (d.ii).

Now we formally define single-plateaued preference rules.

Single-Plateaued Preference Rule, $\phi^{g}:$ Given $g \in \mathcal{G}$, the rule $\phi^{g}$ is defined as follows. For all $N \in \mathcal{P}$ and all $R \in \mathcal{R}^{N}$, 


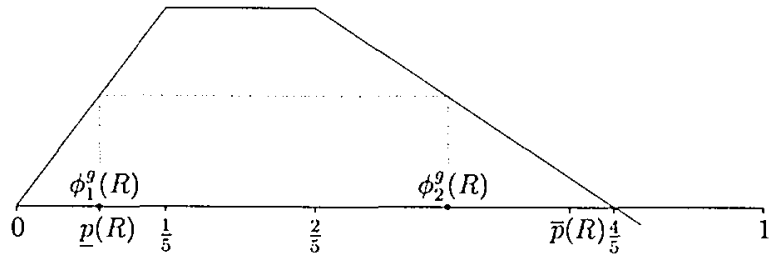

Figure 2.12: Illustration of Subcase (d.i) in Example 2.8.

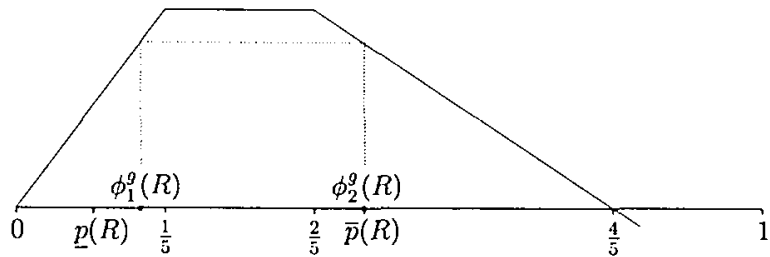

Figure 2.13: Illustration of Subcase (d.ii) in Example 2.8.

(I) if $[\underline{a}, \bar{a}] \subseteq[\underline{p}(R), \bar{p}(R)]$ and for all $i \in N, p\left(R_{i}\right) \notin[\underline{a}, \bar{a}]$, then let $j_{1}, j_{2} \in N$ be such that $p\left(R_{j_{1}}\right)<\underline{a}, p\left(R_{j_{2}}\right)>\bar{a}$, and for all $i \in N, p\left(R_{i}\right) \in\left[0, p\left(R_{j_{1}}\right)\right] \cup\left[p\left(R_{j_{2}}\right), 1\right]$. Then

$$
\phi^{g}(R) \equiv\left\{\begin{array}{cl}
\left(p\left(R_{j_{1}}\right), g\left(p\left(R_{j_{1}}\right)\right)\right) & \text { if } g\left(p\left(R_{j_{1}}\right)\right) \leq p\left(R_{j_{2}}\right) \\
\left(g^{-1}\left(p\left(R_{j_{2}}\right)\right), p\left(R_{j_{2}}\right)\right) & \text { if } p\left(R_{j_{1}}\right) \leq g^{-1}\left(p\left(R_{j_{2}}\right)\right)
\end{array}\right.
$$

(II) otherwise,

$$
\phi^{g}(R) \equiv(\operatorname{med}(\underline{p}(R), \underline{a}, \bar{p}(R)), \operatorname{med}(\underline{p}(R), \bar{u}, \bar{p}(R)))
$$

Similarly as for single-peaked preference rules, the alternatives chosen by a singleplateaued preference rule can be described via a concept of equity relative to its singleplateaued preference relation. Let $R_{\text {soc }}$ be the single-plateaued preference relation of the society. By $A\left(R_{s o c}\right)$ we denote the set of all $R_{\text {soc }}$-equitable alternatives, i.e., $A\left(R_{\text {soc }}\right)=$ $\left\{x \in[0,1]^{M} \mid x_{1} I_{s o c} x_{2}\right\}$. Figure 2.14 below illustrates an example of such a set.

Then for each profile, the rule based on $R_{\text {soc }}$ maximizes society's welfare on the set $A\left(R_{\text {soc }}\right)$ subject to Pareto-optimality. Formally, for all $R \in \mathcal{R}^{N}$, the rule selects an alterna- 


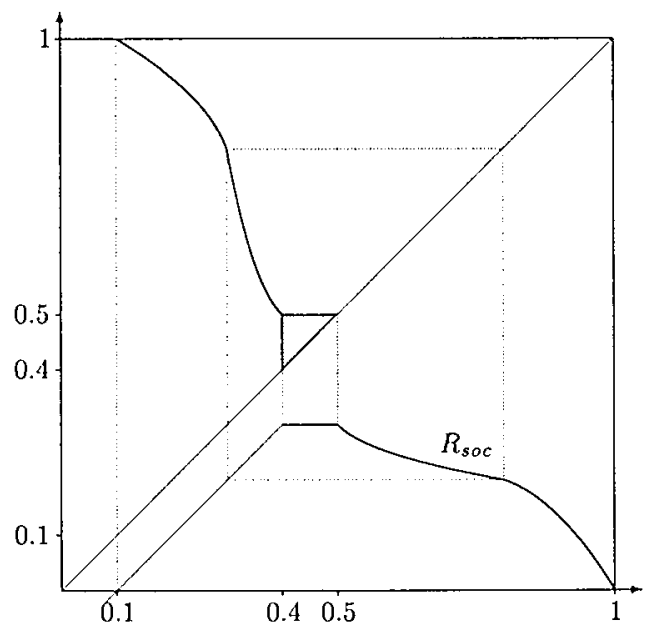

Figure 2.14: The set $R_{\text {soc }}$-equitable alternatives. The planner's plateau is $[0.4,0.5]$. The set $A\left(R_{s o c}\right)$ consists of the shaded area, the diagonal, and the path from $(0.1,1)$ to $(0.4,0.5)$. Notice that we can choose the numerical representation of $R_{\text {soc }}$ to be linear on the set $[0,0.4]$.

tive in $\operatorname{Best}\left(A\left(R_{\text {soc }}\right) \cap E(R)\right)=\left\{y \in A\left(R_{\text {soc }}\right) \cap E(R) \mid\right.$ for all $\left.x \in A\left(R_{\text {soc }}\right) \cap E(R), y_{1} R_{\text {soc }} x_{1}\right\}$ such that (I) and (II) are satisfied. Figure 2.15 below illustrates the previous fact.

It is easy to see that each single-plateaued preference rule satisfies Pareto-optimality and population-monotonicity. The class of these rules contains some interesting subclasses.

A subclass consists of the single-plateaved preference rules where the social planner has a single-peaked preference relation on $[0,1]$. Then the planner has the same characteristics as an agent. These are exactly the single-peaked preference rules characterized in Theorem 2.8 .

When we locate one public good, a "target rule" chooses for each profile the efficient location that is closest to an a priori fixed target point. Ching and Thomson (1999) characterize the class of target rules by Pareto-optimality and population-monotonicity. Target rules extend straightforwardly to the provision of two public goods. Simply provide each of the two public goods by a target rule. However, if both target points belong to ]0,1[, then these rules violate Pareto-optimality. These rules satisfy Pareto-optimality only if one of the target points is 0 or 1 . We obtain them as a subclass of the single-plateaued preference rules. 


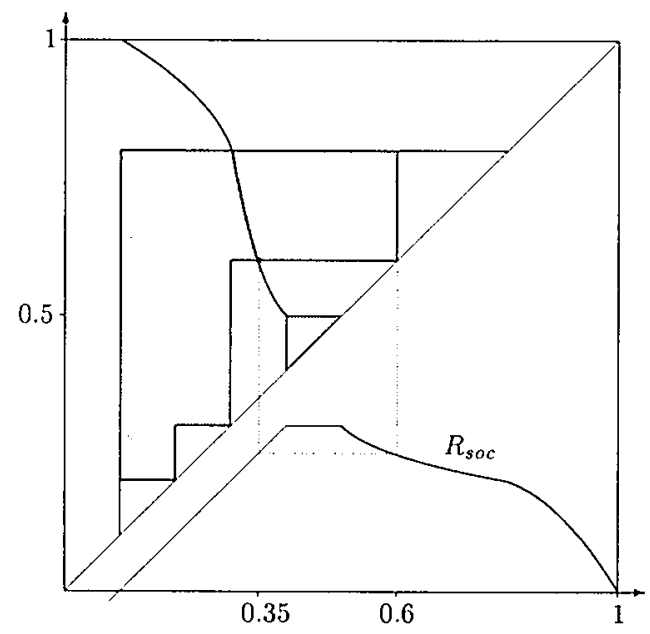

Figure 2.15: Given a preference profile $R \in \mathcal{R}^{N}$ such that $\left\{p\left(R_{i}\right) \mid i \in N\right\}=$ $\{0.1,0.2,0.3,0.6,0.8\}$, the rule maximizes the welfare on the set of $R_{\text {soc-equitable alter- }}$ natives subject to Pareto-optimality. The alternatives $(0.35,0.6)$ and $(0.6,0.6)$ maximize the welfare on $A\left(R_{\text {soc }}\right) \cap E(R)$. By (I), the rule chooses the dotted alternative $(0.35,0.6)$.

Example 2.9 (Turget rules) Let $z \in[0,1]$. Then for all $N \in \mathbb{P}$ and all $R \in \mathcal{R}^{N}$, $\underline{\phi^{z}}(R) \equiv(\underline{p}(R), \operatorname{med}(\underline{p}(R), z, \bar{p}(R)))$ and $\bar{\phi}^{z}(R) \equiv(\operatorname{med}(\underline{p}(R), z, \bar{p}(R)), \bar{p}(R))$. The rules $\underline{\phi}^{z}$ and $\bar{\phi}^{z}$ are single-plateaued preference rules for which the planner's plateau is $[0, z]$ and $[z, 1]$, respectively.

Remark 2.7 In (II) of the definition of single-plateaued preference rules, the location of the goods are chosen by two target rules having as target points the left-endpoint and right-endpoint of the planner's plateau.

By our main result, if $\mathbb{P}$ consists of at least 4 agents, then for each rule satisfying Paretooptinality and population-monotonicity there exists a single-plateaued preference relation such that the rule is the single-plateaued preference rule corresponding to this relation. Pareto-optimality and population-monotonicity characterize the class of single-plateaued preference rules. Subsection 2.7.3 contains the proof of Theorem 2.10.

Theorem 2.10 Let $|\mathbb{P}| \geq 4$. The single-plateaued preference rules are the only rules satisfying Pareto-optimality and population-monotonicity. 
By Theorem 2.10, in our model Pareto-optimality and population-monotonicity imply anonymity. They also imply coalitional strategy-proofness (no group of agents can gain by jointly misrepresenting their true preferences), as the careful reader may check. The above implications also hold in Miyagawa (1998c). However, there are two other important differences between Theorem 2.10 and Miyagawa (1998c) which we describe below.

Remark 2.8 By Theorem 2.10, if we extend preferences from $[0,1]$ to $[0,1]^{M}$ lexicographically, then Pareto-optimality and population-monotonicity imply peaks-onliness. By Miyagawa (1998c), this implication does not hold when we extend preferences from $[0,1]$ to $[0,1]^{M}$ maximally.

Remark 2.9 By Theorem 2.10, if we extend preferences from $[0,1]$ to $[0,1]^{M}$ lexicographically, then Pareto-optimality and population-monotonicity imply preference continuity. In Miyagawa (1998c), only the extreme-peaks rule satisfies Pareto-optinality, populationmonotonicity, and preference continuity. This rule is the single-plateaued preference rule for which the planner's plateau is $[0,1]$. Henceforth, preference continuity does not bring about a reduction of the class of single-plateaued preference rules whereas it does in Miyagawa (1998c).

By Remark 2.9, only the extreme-peaks rule of the complex class of rules characterized by Pareto-optimality and population-monotonicity in Miyagawa (1998c) belongs to the class of single-plateaued preference rules. Therefore, if the domain of each agent consists of the lexicographically and maximally extended preferences, then Pareto-optimality and population-monotonicity characterize the extreme-peaks rule. His and our characterization are completely different because we extend preferences from $[0,1]$ to $[0,1]^{M}$ differently.

In Theorem 2.10 the restriction $|\mathbb{P}| \geq 4$ is tight. When $|\mathbb{P}|=3$, the following rule does not belong to the class of single-plateaued preference rules. Note that in Miyagawa (1998c) the set of potential agents is infinite.

Example 2.10 Let $\mathbb{P}=\{1,2,3\}, N \in \mathcal{P}$, and $R \in \mathcal{R}^{N}$. If $\left|\left\{p\left(R_{i}\right) \mid i \in N\right\}\right| \leq 2$, then $\tilde{\varphi}_{1}(R) \equiv \underline{p}(R)$ and $\tilde{\varphi}_{2}(R) \equiv \bar{p}(R)$. If $p\left(R_{i_{1}}\right)<p\left(R_{i_{2}}\right)<p\left(R_{i_{3}}\right)$, then $\tilde{\varphi}_{1}(R) \equiv$ $\frac{1}{2}\left(\underline{p}(R)+p\left(R_{i_{2}}\right)\right)$ and $\tilde{\varphi}_{2}(R) \equiv \frac{1}{2}\left(p\left(R_{i_{2}}\right)+\bar{p}(R)\right)$. The rule $\tilde{\varphi}$ satisfies Pareto-optimality and population-monotonicity, but violates preference continuity. Hence, there is no $g \in \mathcal{G}$ such that $\tilde{\varphi}=\phi^{g}$.

\subsubsection{Proof of Theorem 2.10}

Throughout this subsection let $\varphi$ be a rule satisfying Pareto-optimality and populationmonotonicity. Theorem 2.10 requires $|\mathbb{P}| \geq 4$. First we prove Theorem 2.10 for $|\mathbb{P}|=4$. At the end of this subsection we outline the proof for $|\mathbb{P}|>4$. Let $\mathbb{P}=\{1,2,3,4\}$. 
Before constructing the planner's single-plateaued preference relation, we show some useful implications of Pareto-optimality and population-monotonicity.

Lemma 2.23 Let $N \in \mathcal{P}, j \in \mathbb{P}$, and $R \in \mathcal{R}^{N \cup\{j\}}$ be such that $[\underline{p}(R), \bar{p}(R)]=\left[\underline{p}\left(R_{N}\right), \bar{p}\left(R_{N}\right)\right]$. If $\varphi\left(R_{N}\right) \neq \varphi(R)$, then for all $i \in N, \varphi\left(R_{N}\right) P_{i} \varphi(R)$.

Proof. Let $\varphi\left(R_{N}\right) \neq \varphi(R)$ and $1,2 \in N$ be such that $p\left(R_{1}\right)=\underline{p}(R)$ and $p\left(R_{2}\right)=\bar{p}(R)$. By Lemma 2.21, for all $i \in N$,

$$
\varphi\left(R_{N}\right) R_{i} \varphi(R)
$$

Since $\varphi\left(R_{N}\right) \neq \varphi(R)$, applying $(2.37)$ for $i \in\{1,2\}$ yields $\varphi_{1}\left(R_{N}\right)<\varphi_{1}(R)$ and $\varphi_{2}(R)<$ $\varphi_{2}\left(R_{N}\right)$. Thus, by $(2.37)$, for all $i \in N, p\left(R_{i}\right) \notin\left[\varphi_{1}(R), \varphi_{2}(R)\right]$. Therefore, by (2.37), for all $i \in N$,

$$
\varphi\left(R_{N}\right) P_{i} \varphi(R)
$$

the desired conclusion.

Lemma $2.24 \varphi$ satisfies peaks-onliness.

Proof. Let $N \in \mathcal{P}, l \in N$, and $R, \bar{R} \in \mathcal{R}^{N}$ be such that $R_{N \backslash\{l\}}=\bar{R}_{N \backslash\{l\}}$ and $p\left(R_{l}\right)=$ $p\left(\bar{R}_{l}\right)$. To prove peaks-onliness, it suffices to show that $\varphi(R)=\varphi(\bar{R})$. We distinguish two cases.

Case 1: $N \neq \mathbb{P}$.

Let $j \in \mathbb{P} \backslash N$ and $R^{\prime}=\left(R, R_{l}\right) \in \mathcal{R}^{N \cup\{j\}}$, i.e., $R_{j}^{\prime}=R_{l}$. Since $\varphi\left(R^{\prime}\right) \in E\left(R_{N}^{\prime}\right)$, Lemma 2.22 and the definition of $R^{\prime}$ imply

$$
\varphi\left(R^{\prime}\right)=\varphi\left(R_{N}^{\prime}\right)=\varphi(R) .
$$

Similarly,

$$
\varphi\left(R^{\prime}\right)=\varphi\left(R_{(N \cup\{j\}) \backslash\{l\}}^{\prime}\right)
$$

Let $\vec{R}^{\prime}=\left(\bar{R}, R_{l}\right) \in \mathcal{R}^{N \cup\{j\}}$, i.e., $\bar{R}_{j}^{\prime}=R_{l}$. Since $p\left(R_{l}\right)=p\left(\bar{R}_{l}\right)$, it follows that $\varphi\left(\bar{R}^{\prime}\right) \in$ $E(\bar{R}) \cap E\left(\bar{R}_{(N \cup\{j\}) \backslash\{l\}}^{\prime}\right)$. Thus, by Lemma 2.22 ,

$$
\varphi(\bar{R})=\varphi\left(\bar{R}^{\prime}\right)=\varphi\left(\bar{R}_{(N \cup\{j\}) \backslash\{l\}}^{\prime}\right) .
$$

Since $\bar{R}_{(N \cup\{j\}) \backslash\{l\}}^{\prime}=R_{(N \cup\{j\}) \backslash\{l\}}^{\prime},(2.38),(2.39)$, and (2.40) imply $\varphi(R)=\varphi(\bar{R})$, the desired conclusion for Case 1. 
Case 2: $N=\mathbb{P}$.

Thus, $N=\{1,2,3,4\}$. Without loss of generality, we suppose that $p\left(R_{1}\right)=\underline{p}(R)$, $p\left(R_{2}\right)=\bar{p}(R)$, and $l \in N \backslash\{1\}$. Hence, $\varphi(R) \in E\left(R_{\{1,2,3\}}\right)$ or $\varphi(R) \in E\left(R_{\{1,2,1\}}\right)$. Without loss of generality, we suppose that $\varphi(R) \in E\left(R_{\{1,2,3\}}\right)$. Hence, by Lemma $2.22, \varphi\left(R_{\{1,2,3\}}\right)=$ $\varphi(R)$. By Case $1, \varphi\left(R_{\{1,2,3\}}\right)=\varphi\left(\bar{R}_{\{1,2,3\}}\right)$. Suppose that $\varphi(\bar{R}) \neq \varphi\left(\bar{R}_{\{1,2,3\}}\right)=\varphi(R)$. Then by Lemma 2.23 ,

$$
\varphi(R) P_{1} \varphi(\bar{R})
$$

If $\varphi(\bar{R}) \in E\left(\bar{R}_{\{1,2,3\}}\right)$, then by Lemma $2.22, \varphi(\bar{R})=\varphi\left(\bar{R}_{\{1,2,3\}}\right)$, in contradiction to $\varphi(\bar{R}) \neq \varphi(R)$. Hence, $\varphi(\bar{R}) \in E\left(\bar{R}_{\{1,2,4\}}\right)$. Thus, Lemma 2.22 and Case 1 together imply $\varphi\left(R_{\{1,2,1\}}\right)=\varphi\left(\bar{R}_{\{1,2,1\}}\right)=\varphi(\bar{R})$. Since $\varphi(R) \neq \varphi(\bar{R})$, Lemma 2.23 implies

$$
\varphi(\bar{R}) P_{1} \varphi(R)
$$

Now, (2.41) and (2.42) together constitute a contradiction.

Lemma $2.25 \varphi$ satisfies anonymity.

Proof. Let $N, N^{\prime} \in \mathcal{P}, R \in \mathcal{R}^{N}$ and $\sigma: N \rightarrow N^{\prime}$ be a bijection. We show that $\varphi(R)=\varphi(\sigma(R))$. We distinguish two cases.

Case 1: $N \neq \mathbb{P}$.

Then by arguments similar to those used in the proof of Case 1 of Lemma 2.24, it is easy to show that $\varphi(R)=\varphi(\sigma(R))$.

Case 2: $N=\mathbb{P}$.

Let $p\left(R_{1}\right)=p(R)$ and $p\left(R_{2}\right)=\bar{p}(R)$. Without loss of generality, we suppose that $\varphi(R) \in E\left(R_{\{1,2,3\}}\right)$. Thus, by Lemma 2.22,

$$
\varphi(R)=\varphi\left(R_{\{1,2,3\}}\right)
$$

Note that $p\left(R_{1}\right)=\underline{p}(\sigma(R))$ and $p\left(R_{2}\right)=\bar{p}(\sigma(R))$. Thus, for some $j \in\{3,4\}, \varphi(\sigma(R)) \in$ $E\left(R_{\{\sigma(1), \sigma(2), \sigma(j)\}}\right)$. Hence, by Lemma 2.22 ,

$$
\varphi(\sigma(R))=\varphi\left(R_{\{\sigma(1), \sigma(2), \sigma(j)\}}\right)
$$

If $j=3$, then Case 1 implies $\varphi\left(R_{\{1,2,3\}}\right)=\varphi\left(R_{\{\sigma(1), \sigma(2), \sigma(3)\}}\right)$. Then (2.43) and (2.44) yield $\varphi(R)=\varphi(\sigma(R))$, the desired conclusion.

Let $j=4$. If $\varphi(R) \neq \varphi(\sigma(R))$, then by Case $1, \varphi\left(R_{\{1,2,3\}}\right)=\varphi\left(R_{\{\sigma(1), \sigma(2), \sigma(3)\}}\right)$. Thus, (2.43) implies $\varphi(R) P_{1} \varphi(\sigma(R))$. By Case $1, \varphi\left(R_{\{1,2,4\}}\right)=\varphi\left(R_{\{\sigma(1), \sigma(2), \sigma(4)\}}\right)$, and by (2.44), $\varphi(\sigma(R)) P_{1} \varphi(R)$. The previous two relations are in contradiction. 


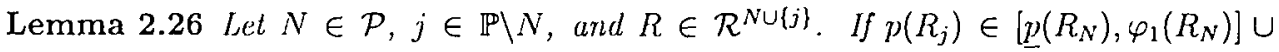
$\left[\varphi_{2}\left(R_{N}\right), \bar{p}\left(R_{N}\right)\right]$, then $\varphi(R)=\varphi\left(R_{N}\right)$.

Proof. Let $1,2 \in N, p\left(R_{1}\right)=\underline{p}\left(R_{N}\right)$, and $p\left(R_{2}\right)=\bar{p}\left(R_{N}\right)$. Assume that $\varphi(R) \neq \varphi\left(R_{N}\right)$. By Lemma 2.23, $\varphi\left(R_{N}\right) P_{1} \varphi(R)$ and $\varphi\left(R_{N}\right) P_{2} \varphi(R)$. Thus, $\varphi_{1}\left(R_{N}\right)<\varphi_{1}(R)$ and $\varphi_{2}(R)<$ $\varphi_{2}\left(R_{N}\right)$. Since $p\left(R_{j}\right) \in\left[\underline{p}\left(R_{N}\right), \varphi_{1}\left(R_{N}\right)\right] \cup\left[\varphi_{2}\left(R_{N}\right), \bar{p}\left(R_{N}\right)\right]$, by Pareto-optimality there exists $i \in N$ such that $p\left(R_{i}\right) \in\left[\varphi_{1}(R), \varphi_{2}(R)\right]$. Hence, $\varphi(R) P_{i} \varphi\left(R_{N}\right)$, which contradicts Lemma 2.23. Therefore, $\varphi(R)=\varphi\left(R_{N}\right)$.

Next, we construct a function $g \in \mathcal{G}$ and show that $\varphi=\phi^{g}$. Before we have to make some preparations.

Let $\tilde{R} \in \mathcal{R}^{\{1,2\}}$ be such that $p\left(\tilde{R}_{1}\right)=0$ and $p\left(\tilde{R}_{2}\right)=1$. By Pareto-optimality, $\varphi_{1}(\tilde{R})=0$ or $\varphi_{2}(\tilde{R})=1$. Without loss of generality, we suppose that

$$
\varphi_{1}(\tilde{R})=0 .
$$

Define $b \equiv \varphi_{2}(\tilde{R})$. Let $R_{3}^{x} \in \mathcal{R}$ be such that $p\left(R_{3}^{x}\right)=x$ and $R^{x}=\left(\tilde{R}, R_{3}^{x}\right)$. Let

$$
\underline{M} \equiv\left\{\varphi_{1}\left(R^{x}\right) \mid x \in[0, b]\right\}
$$

and

$$
\bar{M} \equiv\left\{\varphi_{2}\left(R^{x}\right) \mid x \in[0, b]\right\} .
$$

Define $\underline{a} \equiv \sup \underline{M}$ and $\bar{a} \equiv \inf \bar{M}$. We will construct a function $g:[0, \underline{a}] \rightarrow[\bar{a}, b]{ }^{15}$

Lemma 2.27 For all $x \in \underline{M}, \varphi_{1}\left(R^{x}\right)=x$, and for all $x \in \bar{M}, \varphi_{2}\left(R^{x}\right)=x$.

Proof. We show only the first statement. Let $x \in \underline{M}$. Then there exists $\bar{x} \in[0, b]$ such that $\varphi_{1}\left(R^{\bar{x}}\right)=x$. Let $R_{4}^{x} \in \mathcal{R}$ be such that $p\left(R_{4}^{x}\right)=x$. By Lemma 2.26, $\varphi\left(R^{\bar{x}}, R_{4}^{x}\right)=$ $\varphi\left(R^{\tilde{x}}\right)$. Hence,

$$
\varphi\left(R^{\bar{x}}\right)=\varphi\left(R^{\bar{x}}, R_{4}^{x}\right)=\varphi\left(\tilde{R}, R_{4}^{x}\right)=\varphi\left(R^{x}\right),
$$

where the second equality follows from Lemma 2.22 , and the third from anonymity. Thus, $\varphi_{1}\left(R^{x}\right)=x$, the desired conclusion.

Lemma $2.28 \underline{a} \leq \bar{a}$.

\footnotetext{
${ }^{15}$ If $\varphi_{1}(\tilde{R})>0$ and $\varphi_{2}(\tilde{R})=1$, then set $b \equiv \varphi_{1}(\bar{R}), \underline{M} \equiv\left\{\varphi_{1}\left(R^{x}\right) \mid x \in[b, 1]\right\}$, and $\bar{M} \equiv\left\{\varphi_{2}\left(R^{x}\right) \mid x \in\right.$ $[b, 1]\}$. Define $\underline{a} \equiv \sup \underline{M}$ and $\bar{a} \equiv \inf \bar{M}$. In that case we would construct a function $g:[b, \underline{a}] \rightarrow[\bar{a}, 1]$.
} 
Proof. Assume that $\underline{u}>\bar{a}$. Then there exist $\bar{x} \in \underline{M}$ and $\tilde{x} \in \bar{M}$ such that $\bar{x}>\bar{x}$. By Lemma 2.27,

$$
\varphi_{1}\left(R^{\tilde{x}}\right)=\bar{x} \leq \varphi_{2}\left(R^{\bar{x}}\right)
$$

and

$$
\varphi_{2}\left(R^{\tilde{x}}\right)=\tilde{x} \geq \varphi_{1}\left(R^{\tilde{x}}\right)
$$

Let $R_{4}^{\tilde{x}} \in \mathcal{R}$ be such that $p\left(R_{4}^{\tilde{x}}\right)=\tilde{x}$ and $\bar{R}=\left(R^{\bar{x}}, R_{1}^{\tilde{x}}\right)$. By Lemma 2.26,

$$
\varphi(\bar{R})=\varphi\left(R^{\bar{x}}\right)
$$

By anonymity, $\varphi\left(\bar{R}_{\{1,2,4\}}\right)=\varphi\left(R^{\bar{x}}\right)$. Hence, by (2.46), (2.47), (2.48), and $\bar{x}>\tilde{x}$, $\varphi\left(\bar{R}_{)} \tilde{P}_{2} \varphi\left(\bar{R}_{\{1,2,4\}}\right)\right.$, which contradicts Lemma 2.21 .

Lemma $2.29 \underline{M}=[0, \underline{a}]$ and $\vec{M}=[\bar{a}, b]$.

Proof. First, we show that $\underline{a} \in \underline{M}$. Suppose otherwise. Since $\underline{a} \notin \underline{M}$; it follows that $\varphi_{1}\left(R^{\underline{a}}\right)<\underline{a}$. By Lemma 2.28, $\varphi_{2}\left(R^{\underline{a}}\right) \geq \underline{a}$. Since $\underline{a}=\sup \underline{M}$, there exists $y \in \underline{M}$ such that $\varphi_{1}\left(R^{\underline{a}}\right)<y<\underline{a}$. By Lemma 2.27,

$$
\varphi_{1}\left(R^{y}\right)=y
$$

Let $R_{1}^{\underline{a}} \in \mathcal{R}$ be such that $p\left(R_{4}^{\underline{a}}\right)=\underline{a}$ and $\bar{R}=\left(R^{y}, R_{4}^{\underline{a}}\right)$. By Lemma 2.21, $\varphi\left(R^{y}\right) \tilde{R}_{1} \varphi(\bar{R})$. Hence, by (2.49), $y \leq \varphi_{1}(\bar{R})$. Thus,

$$
\varphi_{1}\left(R^{\underline{a}}\right)<y \leq \varphi_{1}(\bar{R}) \leq \underline{a}
$$

where the last inequality follows from Pareto-optimality and population-monotonicity. By Lemmas 2.22 and 2.28, $\underline{a} \leq \varphi_{2}(\bar{R})$. Hence, $\varphi(\vec{R}) \in E\left(R^{\underline{a}}\right)$. Thus, anonymity and Lemma 2.22 imply $\varphi\left(R^{\underline{a}}\right)=\varphi(\bar{R})$. By our choice of $y, \varphi_{1}\left(R^{\underline{a}}\right)<y$, which contradicts the previous fact and (2.50). It follows that $\underline{a} \in \underline{M}$.

Analogously, it can be shown that $\bar{a} \in \bar{M}$. Next, we show that $\underline{M}$ is connected. Suppose that there exist $y, z \in[0,1]$ such that $0<y<z, y \notin \underline{M}$ and $z \in \underline{M}$. By Lemma 2.27, $\varphi_{1}\left(R^{z}\right)=z$. Let $R_{4}^{y} \in \mathcal{R}$ be such that $p\left(R_{4}^{y}\right)=y$ and $\bar{R}=\left(R^{z}, R_{4}^{y}\right)$. By Lemma 2.26, $\varphi(\bar{R})=\varphi\left(R^{z}\right)$. By anonymity, $\varphi\left(\bar{R}_{\{1,2,1\}}\right)=\varphi\left(R^{y}\right)$.

We show that $\varphi_{2}(\bar{R})<1$. Suppose that $\varphi(\bar{R})=(z, 1)$. By applying Lemma 2.22 twice, it follows that $\varphi(\bar{R})=\varphi\left(\bar{R}_{\{1,2,3\}}\right)=\varphi\left(\bar{R}_{\{1,2\}}\right)$. Hence, $\varphi_{1}\left(\bar{R}_{\{1,2\}}\right)=\varphi_{1}(\bar{R})>0$, which contradicts assumption $(2.45)$. 
Hence, $\varphi_{2}(\bar{R})<1$. Then by Pareto-optimality, $\varphi\left(R^{y}\right) \neq \varphi(\bar{R})$. By Lemma 2.23, for all $i \in\{1,2,4\}, \varphi\left(R^{y}\right) P_{i}^{y} \varphi(\bar{R})$. Since $y \notin \underline{M}$, it follows that $\varphi_{1}\left(R^{y}\right) \neq y$. If $\varphi_{1}\left(R^{y}\right)>y$, then by Pareto-optimality, $\varphi_{2}\left(R^{y}\right)=1$. Hence, by Lemma $2.22,0<\varphi_{1}\left(R^{y}\right)=\varphi_{1}(\tilde{R})$, which contradicts assumption (2.45). Suppose that $\varphi_{1}\left(R^{y}\right)<y$. Note that by Lemma 2.28 ,

$$
y<z \leq \underline{a} \leq \varphi_{2}(\bar{R}), \varphi_{2}\left(R^{y}\right)
$$

Then by peaks-onliness, we could choose $R_{4}^{y} \in \mathcal{R}$ such that $z P_{4}^{y} \varphi_{1}\left(R^{y}\right)$. Hence, $\varphi(\ddot{R}) I_{1}^{y} \varphi\left(R^{y}\right)$, which contradicts Lemma 2.21. Thus, $\underline{M}$ is connected. Since $\underline{a} \in \underline{M}$ and $\varphi_{1}\left(R^{0}\right)=0$, it follows that $\underline{M}=[0, \underline{a}]$.

Analogously, it can be shown that $\bar{M}$ is connected. It remains to show that $b=\max \bar{M}$. If for some $x \in[0, b], \varphi_{2}\left(R^{x}\right)>b$, then $\varphi\left(R^{x}\right) \tilde{P}_{2} \varphi(\tilde{R})$, which contradicts Lemma 2.21 . Hence, $\bar{M}=[\bar{a}, b]$.

Now, we are ready to define the function $g$.

Definition of $g:[0, \underline{a}] \rightarrow[\bar{a}, b]:$ For all $x \in[0, \underline{a}], g(x) \equiv \varphi_{2}\left(R^{x}\right)$

Lemma 2.29 guarantees that $g$ is well-defined. We show that $g \in \mathcal{G}$.

Lemma $2.30 g \in \mathcal{G}$

Proof. By definition it follows that $g(0)=b$. First, we show that $g$ is strictly decreasing. Let $y, z \in[0, \underline{a}]$ be such that $y<z$. By Lemma 2.27 and the definition of $g$,

$$
\varphi\left(R^{y}\right)=(y, g(y)) \text { and } \varphi\left(R^{z}\right)=(z, g(z))
$$

Let $\bar{R}=\left(R^{z}, R_{4}^{y}\right)$. By Lemma $2.26, \varphi(\bar{R})=\varphi\left(R^{z}\right)=(z, g(z))$. By anonymity; $\varphi\left(\bar{R}_{\{1,2,4\}}\right)=$ $\varphi\left(R^{y}\right)$. Since $\varphi\left(R^{y}\right) \neq \varphi(\vec{R})$, Lemma 2.23 implies $\varphi\left(R^{y}\right) \tilde{P}_{2} \varphi(\bar{R})$. Hence, $g(y)>g(z)$ and $g$ is strictly decreasing.

It remains to prove that $g$ is continuous. This is done in three steps.

Claim 1: For all $x \in[\underline{a}, \bar{a}], \varphi\left(R^{x}\right)=(\underline{a}, \bar{a})$.

Proof of Claim 1: First, we show that $\varphi\left(R^{\underline{a}}\right)=(\underline{a}, \bar{a})$. By Lemma 2.27, $\varphi_{1}\left(R^{\underline{a}}\right)=\underline{a}$. Assume that $\varphi_{2}\left(R^{\underline{a}}\right)>\bar{a}$. By Lemma $2.27, \varphi_{2}\left(R^{\bar{a}}\right)=\bar{a}$ and $\varphi_{1}\left(R^{\bar{a}}\right) \leq \underline{a}$. Let $\bar{R}=\left(R^{\underline{a}}, R_{1}^{\bar{a}}\right)$. By Lemma $2.21, \varphi\left(R^{\underline{a}}\right) \tilde{R}_{1} \varphi(\bar{R})$. Hence, $\underline{a} \leq \varphi_{1}(\bar{R})$.

If $\underline{a}<\varphi_{1}(\bar{R})$, then by Lemma 2.26 and anonymity, $\varphi(\bar{R})=\varphi\left(\bar{R}_{\{1,2,4\}}\right)=\varphi\left(R^{\bar{a}}\right)$. Thus, $\underline{a}<\varphi_{1}\left(R^{\bar{a}}\right)$, which contradicts $\underline{a}=\sup \underline{M}$. 
Thus, $\varphi_{1}(\bar{R})=\underline{a}$. Analogously it can be shown that $\varphi_{2}(\bar{R})=\bar{a}$. Hence, $\varphi(\bar{R})=(\underline{a}, \bar{a})$. Since $\varphi_{2}\left(R^{\underline{a}}\right)>\bar{a}$, it follows that $\varphi(\bar{R}) P_{3}^{\underline{a}} \varphi\left(R^{\underline{a}}\right)$, which contradicts Lemma 2.21. Hence, $\varphi\left(R^{\underline{a}}\right)=(\underline{a}, \bar{a})$.

Let $x \in[\underline{a}, \bar{a}]$ and $R_{1}^{x} \in \mathcal{R}$ be such that $p\left(R_{1}^{x}\right)=x$. Since $\varphi\left(R^{a}\right)=(\underline{a}, \bar{a}), \underline{a}=\max \underline{M}$, and $\bar{a}=\min \bar{M}$, Lemmas 2.21 and 2.22 imply $\varphi\left(R^{\underline{a}}, R_{\mathrm{f}}^{x}\right)=(\underline{a}, \bar{a})$. By Lemma 2.22 and anonymity,

$$
\varphi\left(R^{x}\right)=\varphi\left(R^{\underline{a}}, R_{4}^{x}\right)=(\underline{a}, \bar{a})
$$

the desired conclusion.

Claim 2: $[\bar{a}, b]=\{g(x) \mid x \in[0, \underline{a}]\}$.

Proof of Claim 2: By definition of $g$ and Lemma 2.29, $\{g(x) \mid x \in[0, \underline{a}]\} \subseteq[\bar{a}, b]$. Let $y \in[\bar{a}, b]$. By Lemmas 2.27 and $2.29, \varphi_{2}\left(R^{y}\right)=y$ and $\varphi_{1}\left(R^{y}\right) \in[0, \underline{a}]$. Let $R_{1}^{\varphi_{1}\left(R^{y}\right)} \in \mathcal{R}$ be such that $p\left(R_{1}^{\varphi_{1}\left(R^{v}\right)}\right)=\varphi_{1}\left(R^{y}\right)$, and $\bar{R}=\left(R^{y}, R_{1}^{\varphi_{1}\left(R^{y}\right)}\right)$. By Lemma $2.26, \varphi(\bar{R})=\varphi\left(R^{y}\right)$. By Lemma 2.22 and anonymity, $\varphi(\bar{R})=\varphi\left(\bar{R}_{\{1,2,4\}}\right)=\varphi\left(R^{\varphi_{1}\left(R^{y}\right)}\right)$. Hence, $y \in\{g(x) \mid x \in$ $[0, \underline{a}]\}$, the desired conclusion.

Claim 3: For all $x \in] \bar{a}, b], \varphi\left(R^{x}\right)=\left(g^{-1}(x), x\right)$.

Proof of Claim 3: Let $x \in] \bar{a}, b]$. Since $g$ is strictly decreasing and by Claim $2, g^{-1}(x)$ is well-defined. Thus, $\varphi\left(R^{g^{-1}(x)}\right)=\left(g^{-1}(x), x\right)$. Let $R_{1}^{x} \in \mathcal{R}$ be such that $p\left(R_{1}^{x}\right)=x$. By Lemma 2.26, $\varphi\left(R^{g^{-1}(x)}, R_{4}^{x}\right)=\left(g^{-1}(x), x\right)$. By Lemma 2.22 and anonymity,

$$
\varphi\left(R^{x}\right)=\varphi\left(R^{g^{-1}(x)}, R_{4}^{x}\right)=\left(g^{-1}(x), x\right)
$$

the desired conclusion.

By Claim 2, $[\bar{a}, b]=\{g(x) \mid x \in[0, \underline{a}]\}$. Because $g$ is strictly decreasing, Claims 1 and 3 imply that $g$ is continuous. Therefore, $g \in \mathcal{G}$.

The final lemma completes the proof of Theorem 2.10 for $|\mathbb{P}|=4$.

Lemma $2.31 \varphi=\phi^{g}$.

Proof. Let $N \in \mathcal{P}$ and $R \in \mathcal{R}^{N}$. We show that $\varphi(R)=\phi^{g}(R)$. We distinguish two cases.

Case 1: $N \neq \mathbb{P}$.

If $|N|=1$, then the conclusion follows from Pareto-optimality. If $|N|=2$, then for $i \in N$ and $j \in \mathbb{P} \backslash N$ consider $\left(R, R_{i}\right) \in \mathcal{R}^{N \cup\{j\}}$. Lemma 2.22 implies $\varphi(R)=\varphi\left(R, R_{i}\right)$, and by the definition of $\phi^{g}, \phi^{g}(R)=\dot{\phi}^{g}\left(R, R_{i}\right)$. Hence, to prove Case 1 it suffices to show that for all $N \in \mathcal{P}$ such that $|N|=3$ and all $R \in \mathcal{R}^{N}, \varphi(R)=\phi^{g}(R)$. 
Let $|N|=3$. By anonymity we may suppose that $N=\{1,2,3\}, p\left(R_{1}\right)=\underline{p}(R)$, and $p\left(R_{2}\right)=\bar{p}(R)$. We consider four subcases.

Subcase 1.1: $[\underline{a}, \bar{a}] \subseteq\left[p\left(R_{1}\right), p\left(R_{2}\right)\right]$ and for all $\left.i \in N, p\left(R_{i}\right) \notin\right] \underline{a}, \bar{a}[$.

Hence, $\underline{p}(R) \leq \underline{a}$ and $\bar{a} \leq \bar{p}(R)$. Let $j \in N$ be such that $\phi^{g}(R) \in\left\{\left(p\left(R_{j}\right), g\left(p\left(R_{j}\right)\right)\right)\right.$; $\left.\left(g^{-1}\left(p\left(R_{j}\right)\right), p\left(R_{j}\right)\right)\right\}$. By Claim 3 of Lemma 2.30 and the definition of $g$ and $\phi^{?}$,

$$
\varphi\left(R^{p\left(R_{j}\right)}\right)=\dot{\phi}^{g}\left(R^{p\left(R_{j}\right)}\right)=\phi^{g}(R)
$$

Moreover,

$$
\varphi\left(R^{p\left(R_{j}\right)}\right)=\varphi\left(R^{p\left(R_{j}\right)}, R_{1}\right)=\varphi\left(R_{\{2,3\}}^{p\left(R_{j}\right)}, R_{1}\right)
$$

where the first equality follows from the choice of $j,(2.51)$, and Lemma 2.26, and the second from Lemma 2.22. The same arguments yield $\varphi\left(R_{\{2,3\}}^{p\left(R_{j}\right)}, R_{1}\right)=\varphi\left(R_{3}^{p\left(R_{j}\right)}, R_{1}, R_{2}\right)$. Thus, by (2.51) and (2.52),

$$
\phi^{q}(R)=\varphi\left(R_{3}^{p\left(R_{j}\right)}, R_{1}, R_{2}\right)
$$

If it is possible to choose $j=3$, then by peaks-onliness we could choose $R_{3}^{p\left(R_{3}\right)} \in \mathcal{R}$ such that $R_{3}^{p\left(R_{3}\right)}=R_{3}$. Thus, by anonymity, $\phi^{g}(R)=\varphi\left(R_{3}^{p\left(R_{3}\right)}, R_{1}, R_{2}\right)=\varphi(R)$, the desired conclusion.

Thus, suppose that only $j=1$ or $j=2$ are the possible choices. Without loss of generality, let $j=1$. Then $\left.\left.p\left(R_{3}\right) \in\right] g\left(p\left(R_{1}\right)\right), \bar{p}(R)\right]$. Thus,

$$
\phi^{g}(R)=\varphi\left(R_{3}^{p\left(R_{1}\right)}, R_{1}, R_{2}\right)=\varphi\left(R_{3}^{p\left(R_{1}\right)}, R_{1}, R_{2}, R_{3}\right)=\varphi(R)
$$

where the first equality follows from (2.53), the second from Lemma 2.26, and the third from Lemma 2.22 and anonymity. This completes the proof of Subcase 1.1.

Subcase 1.2: For some $\left.j \in N, p\left(R_{j}\right) \in\right] \underline{a}, \bar{a}[$.

First, we show that if $[\underline{a}, \bar{a}] \subseteq[\underline{p}(R), \bar{p}(R)]$, then

$$
\varphi(R)=(\underline{a}, \bar{a})
$$

Since $[\underline{a}, \bar{a}] \subseteq[\underline{p}(R), \bar{p}(R)]$, it follows that $\left.p\left(R_{3}\right) \in\right] \underline{a}, \bar{a}[$. By Claim 1 in the proof of Lemma 2.30, $\varphi\left(R^{p\left(R_{3}\right)}\right)=(\underline{a}, \bar{a})$. Applying Lemmas 2.26 and 2.22 twice as in Subcase 1.1 yields

$$
\varphi\left(R^{p\left(R_{3}\right)}\right)=\varphi\left(R_{\{2,3\}}^{p\left(R_{3}\right)}, R_{1}\right)=\varphi\left(R_{3}^{p\left(R_{3}\right)}, R_{1}, R_{2}\right)=\varphi(R)
$$

where the last equality follows from anonymity and peaks-onliness. Hence, $\varphi(R)=(\underline{a}, \vec{a})=$ $(\operatorname{med}(\underline{p}(R), \underline{a}, \bar{p}(R)), \operatorname{med}(\underline{p}(R), \bar{a}, \bar{p}(R)))=\phi^{g}(R)$, the desired conclusion. 
Now, suppose that $\bar{p}(R)<\bar{a}$ and $\varphi_{2}(R)<\bar{p}(R)$. Let $\left(R, R_{1}^{\bar{a}}\right) \in \mathcal{R}^{\mathrm{P}}$ be such that $p\left(R_{i}^{\bar{a}}\right)=\bar{a}$. By Lemma 2.21, $\varphi(R) R_{2} \varphi\left(R, R_{1}^{\bar{a}}\right)$. Thus, by peaks-onliness and Lemma 2.22,

$$
\varphi_{2}\left(R_{\{1,3\}}, R_{\hat{1}}^{\bar{a}}\right)=\varphi_{2}\left(R, R_{4}^{\vec{a}}\right) \leq \varphi_{2}(R)<\bar{a} .
$$

If $p\left(R_{1}\right) \leq \underline{a}$, then $[\underline{a}, \bar{a}] \subseteq\left[\underline{p}\left(R_{\{1,3\}}, R_{4}^{\vec{a}}\right), \bar{p}\left(R_{\{1,3\}}, R_{1}^{\bar{a}}\right)\right]$. Thus, (2.54) together with anonymity implies $\varphi_{2}\left(R_{\{1,3\}}, R_{4}^{\bar{a}}\right)=\bar{a}$, which contradicts $(2.55)$. Hence, $\varphi_{2}(R)=\bar{p}(R)=$ $\operatorname{med}(\underline{p}(R), \bar{a}, \bar{p}(R))=\phi_{2}^{g}(R)$.

If $p\left(R_{1}\right)>\underline{a}$, then let $\left(R_{\{1,3\}}, R_{2}^{\underline{a}}, R_{4}^{\bar{a}}\right) \in \mathcal{R}^{\mathrm{P}}$ be such that $p\left(R_{2}^{\underline{a}}\right)=\underline{a}$. By Lemma 2.21 , $\varphi\left(R_{\{1,3\}}, R_{4}^{\bar{a}}\right) R_{4}^{\bar{a}} \varphi\left(R_{\{1,3\}}, R_{2}^{\underline{a}}, R_{4}^{\bar{a}}\right)$. Thus, by peaks-onliness,

$$
\varphi_{2}\left(R_{\{1,3\}}, R_{2}^{a}, R_{4}^{\bar{a}}\right) \leq \varphi_{2}\left(R_{\{1,3\}}, R_{1}^{\bar{a}}\right)<\bar{a} .
$$

Then $\varphi\left(R_{\{1,3\}}, R_{2}^{\underline{a}}, R_{4}^{\bar{a}}\right)$ is Pareto-optimal for $\left(R_{1}, R_{2}^{\frac{a}{2}}, R_{4}^{\bar{a}}\right)$ or for $\left(R_{3}, R_{2}^{\underline{a}}, R_{4}^{\bar{a}}\right)$. Let $\varphi\left(R_{\{1,3\}}, R_{2}^{\underline{a}}, R_{1}^{\bar{a}}\right)$ be Pareto-optimal for $\left(R_{1}, R_{2}^{a}, R_{4}^{\bar{a}}\right)$. Thus, by Lemma 2.22 and $(2.56)$,

$$
\varphi_{2}\left(R_{1}, R_{2}^{\underline{a}}, R_{4}^{\bar{a}}\right)=\varphi_{2}\left(R_{\{1,3\}}, R_{2}^{\frac{a}{2}}, R_{4}^{\bar{a}}\right)<\bar{a} .
$$

Since $[\underline{a}, \bar{a}]=\left[\underline{p}\left(R_{1}, R_{2}^{\underline{a}}, R_{1}^{\bar{a}}\right), \bar{p}\left(R_{1}, R_{2}^{\underline{a}}, R_{4}^{\bar{a}}\right)\right]$, the previous inequality contradicts $(2.54)$. Hence, we have shown that if $\bar{p}(R)<\bar{a}$, then

$$
\varphi_{2}(R)=\bar{p}(R)=\operatorname{med}(\underline{p}(R), \bar{a}, \bar{p}(R))=\phi_{2}^{g}(R) .
$$

Similar arguments yield $\varphi_{1}(R)=\underline{p}(R)=\operatorname{med}(\underline{p}(R), \underline{a}, \bar{p}(R))=\phi_{1}^{g}(R)$ when $\underline{a}<\underline{p}(R)$.

It remains to show that $\varphi_{1}(R)=\underline{a}$ when $\underline{a}<\bar{p}(R)<\bar{a}$ and $\underline{p}(R) \leq \underline{a}$. (To show that $\varphi_{2}(R)=\bar{a}$ when $\underline{a}<\underline{p}(R)<\bar{a}$ and $\bar{a} \leq \bar{p}(R)$ is analogous.) By $(2.57), \varphi(R) \in E\left(R_{\{1,2\}}\right)$. Thus, by Lemma $2.22, \varphi(R)=\varphi\left(R_{\{1,2\}}\right)$. Let $\left(R_{\{1,2\}}, R_{3}^{\underline{a}}, R_{4}^{\bar{a}}\right) \in \mathcal{R}^{\mathrm{P}}$ be such that $p\left(R_{3}^{\underline{a}}\right)=\underline{a}$ and $p\left(R_{4}^{\bar{a}}\right)=\bar{a}$. By $(2.54)$ and Lemma 2.22, $\varphi\left(R_{\{1,2\}}, R_{3}^{\underline{a}}, R_{i}^{\bar{a}}\right)=(\underline{a}, \bar{a})$. Thus, by peaksonliness and Lemma 2.21, $\varphi\left(R_{\{1,2\}}, R_{3}^{\underline{a}}\right)=(\underline{a}, \bar{p}(R))$. Since $(\underline{a}, \bar{p}(R)) \in E\left(R_{\{1,2\}}\right)$, Lemma 2.22 implies $\varphi\left(R_{\{1,2\}}\right)=(\underline{a}, \bar{p}(R))$, the desired conclusion.

Hence, $\varphi_{1}(R)=\underline{a}=\operatorname{med}(\underline{p}(R), \underline{a}, \bar{p}(R))=\phi_{1}^{g}(R)$. This completes the proof of Subcase 1.2 .

Subcase 1.3: $\bar{p}(R) \leq \underline{a}$.

We show that $\varphi(R)=(\bar{p}(R), \bar{p}(R))$. Consider $R^{p\left(R_{2}\right)}$. By peaks-onliness we may suppose that $R_{3}^{p\left(R_{2}\right)}=R_{2}$. By Lemmas 2.26 and 2.22 ,

$$
\varphi\left(R^{p\left(R_{2}\right)}\right)=\varphi\left(R^{p\left(R_{2}\right)}, R_{1}\right)=\varphi\left(R_{\{2,3\}}^{p\left(R_{2}\right)}, R_{1}\right)
$$

Let $R_{4}^{1} \in \mathcal{R}$ be such that $p\left(R_{1}^{1}\right)=1$. Then

$$
\varphi\left(R_{\{2,3\}}^{p\left(R_{2}\right)}, R_{1}\right)=\varphi\left(R_{\{2,3\}}^{p\left(R_{2}\right)}, R_{1}, R_{1}^{1}\right)=\varphi\left(R_{3}^{p\left(R_{2}\right)}, R_{1}, R_{1}^{1}\right)=\varphi\left(R, R_{4}^{1}\right)
$$


where the first equality follows from Lemma 2.26, the second from Lemma 2.22 , and the third from Lemma 2.26 and anonymity. Thus, by the previous equality and (2.58), $\varphi\left(R, R_{1}^{1}\right)=\varphi\left(R^{p\left(R_{2}\right)}\right)$. Hence, the definition of $g$ and Lemma 2.22 imply $\varphi\left(R, R_{1}^{1}\right)=$ $\left(p\left(R_{2}\right), g\left(p\left(R_{2}\right)\right)\right)$. Now, we calculate $\varphi(R)$.

If $p\left(R_{2}\right)=g\left(p\left(R_{2}\right)\right)$, then $p\left(R_{2}\right)=g\left(p\left(R_{2}\right)\right)=\underline{a}$. Thus, by Lemma 2.21, $\varphi(R)=(\underline{a}, \underline{a})$, the desired conclusion.

If $p\left(R_{2}\right)<g\left(p\left(R_{2}\right)\right)$, then Pareto-optimality implies $\varphi(R) \neq \varphi\left(R, R_{4}^{1}\right)$. Hence, by Lemma 2.23, $\varphi(R) P_{2} \varphi\left(R, R_{1}^{1}\right)$. Thus, $\varphi_{2}(R)=p\left(R_{2}\right)=\bar{p}(R)$. Since $p\left(R_{2}\right)<g\left(p\left(R_{2}\right)\right)$. peaks-onliness, and $\varphi(R) P_{2} \varphi\left(R, R_{4}^{1}\right)$, it follows that $\varphi_{1}(R)=p\left(R_{2}\right)=\bar{p}(R)$. To summarize,

$$
\varphi(R)=(\bar{p}(R), \bar{p}(R))=(\operatorname{med}(\underline{p}(R), \underline{a}, \bar{p}(R)), \operatorname{med}(\underline{p}(R), \bar{a}, \bar{p}(R)))=\phi^{g}(R) .
$$

Subcase 1.4: $\bar{a} \leq \underline{p}(R)$.

If $\underline{p}(R) \in[\bar{a}, b]$, then similar to Subcase 1.3 it can be shown that $\varphi(R)=(\underline{p}(R), \underline{p}(R))$. Let $b<1$ and $\underline{p}(R) \in] b, 1]$. Let $\left(R_{\{1,2\}}, R_{4}^{b}\right) \in \mathcal{R}^{\{1,2,4\}}$ be such that $p\left(R_{4}^{b}\right)=b$. Hence, $\varphi\left(R_{\{1,2\}}, R_{4}^{b}\right)=(b, b)$. By Lemma 2.26, $\varphi\left(R, R_{4}^{b}\right)=(b, b)$. Pareto-optimality, peaksonliness, and Lemma 2.21 imply $\varphi_{1}(R)=\underline{p}(R)$ and $\varphi_{2}(R) \in\left[\underline{p}(R), p\left(R_{3}\right)\right]$.

Suppose that $\varphi_{2}(R)>\underline{p}(R)$. Let $z \equiv \frac{1}{2}\left(\underline{p}(R)+\varphi_{2}(R)\right)$. Let $\left(R_{\{1,2\}}, R_{1}^{z}\right) \in \mathcal{R}^{\{1,2,4\}}$ be such that $p\left(R_{i}^{z}\right)=z$. The same arguments as before yield $\varphi_{1}\left(R_{\{1,2\}}, R_{1}^{z}\right)=\underline{p}(R)$ and $\varphi_{2}\left(R_{\{1,2\}}, R_{4}^{z}\right) \in[\underline{p}(R), z]$. Thus,

$$
\varphi\left(R_{\{1,2\}}, R_{4}^{z}\right)=\varphi\left(R_{\{1,2\}}\right)=\varphi(R)
$$

where the first equality follows from Lemma 2.22, and the second from Lemma 2.26. Thus, $\varphi_{2}(R) \leq z$, which contradicts our choice of $z$.

To summarize,

$$
\varphi(R)=(\underline{p}(R), \underline{p}(R))=(\operatorname{med}(\underline{p}(R), \underline{a}, \bar{p}(R)), \operatorname{med}(\underline{p}(R), \bar{a}, \bar{p}(R)))=\phi^{g}(R)
$$

Case 2: $N=\mathbb{P}$.

Without loss of generality, we suppose that $p\left(R_{1}\right)=\underline{p}(R)$ and $p\left(R_{2}\right)=\bar{p}(R)$. Then $\varphi(R) \in E\left(R_{\{1,2,3\}}\right)$ or $\varphi(R) \in E\left(R_{\{1,2,4\}}\right)$. Without loss of generality, we suppose that $\varphi(R) \in E\left(R_{\{1,2,3\}}\right)$. Thus, by Lemma 2.22 ,

$$
\varphi(R)=\varphi\left(R_{\{1,2,3\}}\right)
$$

Case 1 implies $\varphi\left(R_{\{1,2,3\}}\right)=\phi^{g}\left(R_{\{1,2,3\}}\right)$. If $p\left(R_{4}\right) \in\left[\underline{p}(R), \varphi_{1}\left(R_{\{1,2,3\}}\right)\right] \cup\left[\varphi_{2}\left(R_{\{1,2,3\}}\right), \bar{p}(R)\right]$, then Lemma 2.26 and the definition of $\phi^{3}$ imply

$$
\varphi(R)=\varphi\left(R_{\{1,2,3\}}\right)=\phi^{g}\left(R_{\{1,2,3\}}\right)=\phi^{g}(R)
$$


the desired conclusion.

Suppose that $\left.p\left(R_{4}\right) \in\right] \varphi_{1}(R), \varphi_{2}(R)[$. Then the same arguments as above and Case 1 imply $\varphi(R)=\varphi\left(R_{\{1,2,1\}}\right)=\phi^{g}\left(R_{\{1,2,1\}}\right)$. If $\left.p\left(R_{3}\right) \notin\right] \varphi_{1}(R), \varphi_{2}(R)[$, then by interchanging the roles of 4 and 3 implies $\varphi(R)=\phi^{g}(R)$. Let $\left.p\left(R_{3}\right), p\left(R_{4}\right) \in\right] \varphi_{1}(R), \varphi_{2}(R)[$. Then by the definition of $\phi^{g}$,

$$
\phi^{g}\left(R_{\{1,2,3\}}\right)=(\operatorname{med}(\underline{p}(R), \underline{a}, \bar{p}(R)), \operatorname{med}(\underline{p}(R), \underline{a}, \bar{p}(R)))=\phi^{g}\left(R_{\{1,2,4\}}\right)
$$

Hence, $\phi^{9}(R)=\phi^{g}\left(R_{\{1,2,3\}}\right)=\varphi\left(R_{\{1,2,3\}}\right)=\varphi(R)$, which is the desired conclusion.

We have shown Theorem 2.10 for $\mathbb{P}=\{1,2,3,4\}$. Finally, we outline the proof of Theorem 2.10 for $|\mathbb{P}|>4$.

It is straightforward to generalize the proofs of Lemmas $2.23,2.24,2.25$, and 2.26 to $|\mathbb{P}|>4$. Thus, by Lemma $2.25, \varphi$ satisfies anonymity.

Let $\bar{N} \in \mathcal{P}$ be such that $|\bar{N}|=4$. Then the above proof shows that there exists $g_{\bar{N}} \in \mathcal{G}$ such that for all $N \in\{S \in \mathcal{P} \mid S \subseteq \bar{N}\}$ and all $R \in \mathcal{R}^{N}, \varphi(R)=\varphi^{g_{N}}(R)$. Anonymity implies that for all $\bar{N}^{\prime} \in \mathcal{P}$ such that $\left|\bar{N}^{\prime}\right|=4, g_{\bar{N}}=g_{\bar{N}^{\prime}}$. Thus, $g \equiv g_{\bar{N}}$ is well-defined.

We show that $\varphi=\phi^{9}$. Let $N \in \mathcal{P}$ and $R \in \mathcal{R}^{N}$. If $|N| \leq 4$, then the above argument shows that $\varphi(R)=\phi^{g}(R)$. If $|N| \geq 5$, then there exists $\bar{N} \subseteq N$ such that $|\bar{N}|=4, \varphi(R) \in$ $E\left(R_{\bar{N}}\right), \underline{p}(R)=\underline{p}\left(R_{\bar{N}}\right)$, and $\bar{p}(R)=\bar{p}\left(R_{\bar{N}}\right)$. Successive application of Lemma 2.22 yields $\varphi(R)=\varphi\left(R_{\bar{N}}\right)$. Since $|\vec{N}|=4$, it follows that $\varphi\left(R_{\bar{N}}\right)=\phi^{g}\left(R_{\bar{N}}\right)$. Then the same argument in the proof of Case 2 of Lemma 2.31 shows that $\phi^{g}(R)=\phi^{g}\left(R_{\bar{N}}\right)=\varphi\left(R_{\bar{N}}\right)=\varphi(R)$. Thus, $\varphi=\phi^{g}$ which completes the proof of Theorem 2.10 . 


\section{Chapter 3}

\section{Probabilistic Rules for One Public Good}

\subsection{Introduction}

Consider the following elementary collective decision problem. There are two alternatives $a$ and $b$ and two agents 1 and 2. Agent 1 prefers $a$ to $b$, whereas agrent 2 prefers $b$ to $a$. Exactly one alternative has to be chosen. The only, at least ex ante, fair solution to this problem seems to be to give each alternative an equal chance of being chosen.

Somewhat surprisingly, the study of such probabilistic rules in the theory of social choice has been rather limited. To a considerable extent, this may be due to the fact that under the requirement of strategy-proofness which means that it never pays to lie about one's true preferences, a strong but natural condition in collective decision making where individual preferences are private information the existence of nondictatorial probabilistic rules is not obvious. In fact, the solution suggested for the simple problem above is a so-called random dictatorship where each agent has an equal chance of being the dictator. The example shows that a random dictatorship may be the most obvious solution. Random dictatorships came up in the first studies on strategy-proof probabilistic rules by Gibbard $(1977,78)$, in the classical model of social choice with all preferences admissible.

This leaves the question open whether in a more restricted context probabilistic rules other than random dictatorships can be strategy-proof. For the deterministic setting the impossibility (i.e., dictatorship) results of Gibbard (1973) and Satterthwaite (1975) can be avoided by restricting the set of preferences and at the same time adapting the domain of alternatives. In particular, Moulin (1980) characterizes classes of deterministic rules on the real line that are nondictatorial and strategy-proof with respect to single-peaked preferences. These results have later been extended in several directions by many authors. 
Also in the framework that Moulin (1980) considers deterninistic rules may be unsatisfactory. Suppose there are 201 agents, 100 of which have their peak at a whereas the other 101 agents have their peak at $b, b \neq a$. Then the median rule without phantom voters picks the alternative $b$ where a lottery between $a$ and $b$ seems to be more fair, at least ex ante.

In the present chapter, which is based on Ehlers, Peters, and Storcken (1999) and Ehlers and Klaus (2000a), we adopt the Moulin framework but consider probabilistic rules. Such a rule assigns to every profile of reported individual preferences a probability distribution over the real line. The interpretation is that the final outcome can be drawn from this distribution, as in the simple example above. Altenatively, given a profile, the number assigned by the scheme to a (measurable) subset of the real line can be interpreted as the probability that the final outcome will be in this set at this profile.

The first property that we impose is strategy-proofness. In order to formulate this condition the preferences of the agents have to be extended to probability distributions. This will be done as follows. Given a single-peaked preference, an agent (weakly) prefers one probability distribution over another if the former assigns at least as much probability to any upper contour set of the preference as the latter. Here, an upper contour set is an interval around the peak of the preference consisting of those points that are weakly preferred to a given outcome. In other words, preference over probability distributions is defined in terms of first degree stochastic dominance. A probabilistic rule is strategy-proof if honest reporting always results in a probability distribution that (weakly) dominates, in the sense just described, any probability distribution brought about by lying. This condition - which is analogous to the one adopted by Gibbard $(1977,1978)$. is quite strong; in particular it implies comparability in the sense of stochastic dominance between the probability distributions induced by the scheme in case of honest and false reporting by any agent. It is, however, a natural condition in a framework where the mechanism can only use ordinal information about the agents' preferences because by the familiar characterization of first degree stochastic dominance it implies that no agent can gain by lying independent of any possible von Neumann-Morgenstern representation of his preference. Moreover, it still admits a large class of probabilistic rules.

The first important result is that every strategy-proof probabilistic rule that is peaksonly (the rule depends only on the peaks of the reported preferences) is characterized by $2^{n}$ (the number of subsets of agents) fixed probability distributions. These fixed probability distributions play a role similar to the fixed ballots (phantom voters) in the deterministic setting of Moulin (1980), but the actual description of the probabilistic rule is considerably more complicated. We call these rules fixed probabilistic ballots rules. A random dictator- 
ship is one example of such a rule. Another example for two agents is the following. Fix a distribution, for instance a normal distribution, on the real line. Take the two reported peaks of the agents, transfer all the weight of the distribution left from the lower peak to this peak, transfer all the weight right from the higher peak to the higher peak, and between the peaks just take the distribution originally fixed.

Adding anonymity (the names of the agents do not matter) reduces the number of fixed probabilistic ballots to $n+1$ (the number of different cardinalities of subsets of agents).

Second, it is shown that under strategy-proofness, peaks-onliness is implied by the very weak and natural unanimity property: if all agents report the same preference, then the rule attaches probability one to the peak of that common preference.

The results of Moulin (1980) for deterministic rules follow from our results for probabilistic rules, and even in a stronger fashion, because the peaks-onliness condition can be dispensed with. This is also proved directly in Barberà and Jackson (1994) and Ching (1997).

As hinted above the strategy-proofness condition can also be fornulated in terms of utility representations of preferences. If the condition depends not only on the preferences but also on the specific utility representation then, in general, it becomes weaker, hence less demanding. Some references for the cardinal case are Freixas (1984) and Barberà, Bogomolnaia, and Van der Stel (1998).

The results described so far are concerned with strategic questions. The other part of Chapter 3 focuses on changes in the environment. We consider situations where the agents whose characteristics remained fixed are not responsible for such changes. The decisions made by the social planner, say the government, are typically different before and after the change. Such situations include the allocation of resources before and after a growth in population and the compulsory duration of schooling before and after the change of the interests of some members of the society. Solidarity means that in such situations the welfares of the agents whose characteristics did not change be affected in the same direction: as a result of the change either they all (weakly) benefit or they all (weakly) lose. Solidarity ensures that changes of the decisions made by the social planner are "fair" in the following sense: there cannot be a pair of agents whose characteristics did not change such that one of them strictly benefits whereas the other strictly loses.

We apply the idea of solidarity to situations when the population of agents varies or the preference relations of some agents change. If some agents leave, then as a result either all remaining agents (weakly) gain or they all (weakly) lose. This requirement is called population-monotonicity (Thomson, 1983a). Alternatively, if the preference relations of some agents change, then as a result either all the agents whose preference relations did 
not change (weakly) gain or they all (weakly) lose. This requirement is called replacementdomination (Moulin, 1987). The idea of solidarity has been applied to various contexts. Thomson (1983a) studied the idea in bargaining, Chichilnisky and Thomson (1987), Kim (1999), and Sprumont and Zhou (1999) in classical exchange economies, Thomson (1997), Chun (1999), and Klaus (2000) in private good economies with single-peaked preferences, Sprumont (1991) and Hokari (1999) in cooperative games with transferable utility, and Miyagawa (1998b,c) for the provision of multiple public goods.

The idea of solidarity has been also applied to the problem of choosing the level of a public good along a one-dimensional continuum. A rule assigns to every economy (consisting of a set of agents and a preference profile) a level. Thomson (1993) and Ching and Thomson (1999) show that if a rule satisfies Pareto-optimality and either of the two solidarity properties described above, then it must be a so-called target rule. Such a rule is determined by its target (level). If the target is Pareto-optimal, then the target is chosen by the rule. If the target is not Pareto-optimal, then the Pareto-optimal level that is closest to the target is chosen by the rule. The target that essentially determines any target rule might be the "status quo" of the present level of the public good, or alternatively it may be a target level for the public good that is imposed by the social planner.

For the probabilistic approach the main results are the following. First, we show that Pareto-optimality and either of the two solidarity properties imply anonymity and strategy-proofness. Second, we show that deterministic target rules are the only probabilistic rules satisfying Pareto-optimality and replacement-domination. Surprisingly, even if we allow rules to be probabilistic, then still only deterministic rules satisfy these two requirements. Further, we extend deterministic target rules to the probabilistic setting. Given a (fixed) target distribution, a probabilistic target rule assigns to every economy the following distribution: in the interior of the interval having as endpoints the smallest peak level and the greatest peak level the target probability distribution is applied; all probability outside that interval is "projected" to the smallest peak level and the greatest peak level, respectively. Third, we show that the probabilistic target rules are the only probabilistic rules satisfying Pareto-optimality and population-monotonicity. Therefore, a probabilistic rule that satisfies must be of the "target type," using a target probability distribution or a target level.

Samuelson and Zeckhauser (1988) prove that in many situations individuals disproportionally stick to the status quo. In other words, a "target bias" with the target equal to the status quo is present in many decisions. Our main results imply that in public good economies Pareto-optimality and solidarity imply such a target bias. Target rules with the target equal to the status quo are useful in economic situations when agents have veto 
power over changes in the status quo, as this might be the case for risky undertakings. A practical advantage of target rules is that they are simple and can be implemented easily and quickly. Furthermore, they are strategy-proof and to some extent fair if they use "fair" target probability distributions; e.g., the uniform probability distribution. On the other hand, since target rules are almost constant (apart from ruling out probabilities on inefficient levels) one can interpret our (and previous) results concerning target rules as impossibility results.

Some other references from the literature on probabilistic social choice mechanisms are Barberà and Sonnenschein (1978), Dutta (1980), Bandyopadhyay, Deb, and Pattanaik (1982), and Pattanaik and Peleg (1986) - but this list is not exhaustive. Recently, probabilistic mechanisms in private goods contexts have been studied by Sasaki (1997), Ehlers (1998), Abdulkadiroğlu and Sönmę (1998), Crès and Moulin (1998a,b), and Bogomolnaia and Moulin (1999). The first reference concerns a probabilistic extension of the uniform rule for the division problem with single-peaked preferences and identical indivisible objects. The second reference considers private good economies with single-dipped preferences. The other references deal with random assignment of heterogeneous indivisible objects.

The extension of the present one-dimensional framework to more dimensions can be found in Dutta, Peters, and Sen (1999), where it is shown that strategy-proofness and unanimity imply random dictatorship if all strictly convex single-peaked preferences are admissible.

This chapter is organized as follows. Section 3.2 presents the probabilistic nodel including the ordinal extension of preferences and Pareto-optimality. Section 3.3 is devoted to strategy-proofness. In Subsection 3.3.1 we formulate strategy-proofness and two other requirements. In Subsection 3.3.2 we show that strategy-proofness and peaks-onliness are equivalent to the so-called uncompromisingness property, introduced by Border and Jordan (1983) for the deterministic model, and extended here to the probabilistic case. Subsection 3.3.3 contains the characterization of all strategy-proof and peaks-only probabilistic rules. Subsection 3.3.4 extends the analysis to include unanimity instead of peaks-onliness. Subsection 3.3.5 discusses the implications of our results for the deterministic case.

Section 3.4 is devoted to solidarity properties. Subsection 3.4.1 introduces the population-monotonicity and replacement-domination. Subsection 3.4.2 presents relations among strategy-proofness, anonymity, and the solidarity properties. Subsection 3.4 .3 introduces deterministic and probabilistic target rules and presents the characterizations of these classes. 


\subsection{The Probabilistic Approach}

We consider the problem of choosing a level of a public good on the real line $\mathbb{R}^{1}$ Let $\mathbb{N}$ denote the set of natural numbers. There is a population $\mathbb{P} \subseteq \mathbb{N}$ of "potential" agents. The population $\mathbb{P}$ can be either finite or infinite. Let $\mathcal{P}$ denote the class of non-empty and finite subsets. Each agent $i \in \mathbb{P}$ is equipped with a "single-peaked" preference relation $R_{i}$ over $\mathbb{R}$. Single-peakedness of $R_{i}$ means that there exists a real number $p\left(R_{i}\right) \in \mathbb{R}$, called the peak of $R_{i}$, with the following property: for all $x, y \in \mathbb{R}$, if $x<y \leq p\left(R_{i}\right)$ or $x>y \geq p\left(R_{i}\right)$, then $y P_{i} x$. As usual, $x R_{i} y$ means " $x$ is weakly preferred to $y$ ", and $x P_{i} y$ means " $x$ is strictly preferred to $y$ ". Let $\mathcal{R}$ denote the class of all single-peaked preference relations over $\mathbb{R}$. Given $x \in \mathbb{R}$ and $R_{i} \in \mathcal{R}$, the weak upper contour set of $x$ at $R_{i}$ is defined by $B\left(x, R_{i}\right) \equiv\left\{y \in \mathbb{R} \mid y R_{i} x\right\}$, and the strict upper contour set of $x$ at $R_{i}$ is defined by $B^{\circ}\left(x, R_{i}\right) \equiv\left\{y \in \mathbb{R} \mid y P_{i} x\right\}$. The single-peakedness of $R_{i}$ implies that these upper contour sets are intervals which are either closed, open or half open. Given $N \in \mathcal{P}$, let $\mathcal{R}^{N}$ denote the set of all (preference) profiles $R=\left(R_{i}\right)_{i \in N}$ such that for all $i \in N, R_{i} \in \mathcal{R}$. We also call $R$ an economy. Given $R \in \mathcal{R}^{N}$, let $p(R) \equiv\left(p\left(R_{i}\right)\right)_{i \in N}$ denote the profile of peaks at $R, \underline{p}(R) \equiv \min _{i \in N} p\left(R_{i}\right), \bar{p}(R) \equiv \max _{i \in N} p\left(R_{i}\right)$, and $E(R) \equiv[\underline{p}(R), \bar{p}(R)]$. We call $E(R)$ the peaks interval of $R$. Given $S \subseteq N$, let $R_{S} \equiv\left(R_{i}\right)_{i \in S}$ denote the profile $R$ restricted to $S$. Given $N \in \mathcal{P}, i \in N$, and $R, \bar{R} \in \mathcal{R}^{N}$, profiles $R$ and $\bar{R}$ are called $i$-deviations if $R_{N \backslash\{i\}}=\bar{R}_{N \backslash\{i\}}$.

A deterministic (decision) rule $\varphi$ is a function that selects for every $N \in \mathcal{P}$ and every $R \in \mathcal{R}^{N}$ a level in $\mathbb{R}$, denoted by $\varphi(R)$. We extend the original analysis of deterministic rules by considering "probabilistic" rules. A probabilistic (decision) rule $\Phi$ is a function that selects for every $N \in \mathcal{P}$ and every $R \in \mathcal{R}^{N}$ a (probability) distribution over $\mathbb{R}$, denoted by $\Phi(R)$. For all (measurable) subsets $X$ of $\mathbb{R}$, the number $\Phi(R)(X)$, which the distribution $\Phi(R)$ assigns to $X$, is the probability that the final level belongs to $X$. We consider distributions defined on the Borel $\sigma$-algebra $\mathcal{L}$. Elements of $\mathcal{L}$ are called Borel sets. Appendix A contains all results from measure theory that are used in this chapter.

A deterministic rule can be seen as a probabilistic rule that selects for every $N \in \mathcal{P}$ and every $R \in \mathcal{R}^{N}$ a distribution placing probability 1 on a single level in $\mathbb{R}$. We extend preferences over levels in $\mathbb{R}$ to preferences over distributions ordinally (Gibbard, 1977; Bogomolnaia and Moulin, 1999). This ordinal extension is based on the concept of upper contour sets.

In the probabilistic model, we extend preferences in the following way. Given a preference relation $R_{i} \in \mathcal{R}$ and two distributions $Q, Q^{\prime}$ over $\mathbb{R}, Q$ is weakly preferred to $Q^{\prime}$

\footnotetext{
${ }^{1}$ All results of Chapter 3 remain true if we restrict $\mathbb{R}$ to a closed interval $[a, b]$ or an open interval $] a, b[$, $a, b \in \mathbb{R}$.
} 
under $R_{i}$, if $Q$ assigns to each weak upper contour set of $R_{i}$ at least the probability that is assigned by $Q^{\prime}$ to this set. Abusing notation we use the same symbols to denote preferences over distributions and preferences over levels.

Ordinal Extension of Preferences: For all $R_{i} \in \mathcal{R}$ and all distributions $Q, Q^{\prime}$ over $\mathbb{R}, Q R_{i} Q^{\prime}$ if and only if

$$
\text { for all } x \in \mathbb{R}, Q\left(B\left(x, R_{i}\right)\right) \geq Q^{\prime}\left(B\left(x, R_{i}\right)\right) .
$$

Furthermore, $Q P_{i} Q^{\prime}$ if and only if

$$
Q R_{i} Q^{\prime} \text { and for some } y \in \mathbb{R}, Q\left(B\left(y, R_{i}\right)\right)>Q^{\prime}\left(B\left(y, R_{i}\right)\right) \text {. }
$$

Inequality (3.1) is a first order stochastic dominance condition; in particular it requires that the distributions $Q$ and $Q^{\prime}$ are comparable in that respect. Therefore, our extension is not complete over the set of all distributions over $\mathbb{R}$. But note that for preferences over distributions completeness is a demanding requirement.

Our first lemma shows that we can equivalently extend preferences with respect to strict upper contour sets.

Lemma 3.1 Let $R_{i} \in \mathcal{R}$ and $Q, Q^{\prime}$ be distributions over $\mathbb{R}$. Then $Q R_{i} Q^{\prime}$ if and only if

$$
\text { for all } x \in \mathbb{R}, Q\left(B^{\circ}\left(x, R_{i}\right)\right) \geq Q^{\prime}\left(B^{\circ}\left(x, R_{i}\right)\right) \text {. }
$$

Furthermore, $Q P_{i} Q^{\prime}$ if and only if

$$
Q R_{i} Q^{\prime} \text { and for some } y \in \mathbb{R}, Q\left(B^{\circ}\left(y, R_{i}\right)\right)>Q^{\prime}\left(B^{\circ}\left(y, R_{i}\right)\right) \text {. }
$$

Proof. We show only the equivalence for (3.3). For the only-if part, let $Q R_{i} Q^{\prime}$ and $x \in \mathbb{R}$. We have to show (3.3). Observe that $B^{\circ}\left(x, R_{i}\right)$ is an interval between two points $a, b \in \mathbb{R} \cup\{-\infty,+\infty\}$ and its form is $] a, b],] a, b[$ or $[a, b[$. In each case we can find a sequence $x_{1}, x_{2}, \ldots$ such that for all $k \in \mathbb{N}, B\left(x_{k}, R_{i}\right) \subseteq B\left(x_{k+1}, R_{i}\right)$ and $\cup_{k \in \mathbb{N}} B\left(x_{k}, R_{i}\right)=B^{o}\left(x, R_{i}\right)$. By Theorem A.1,

$$
Q\left(B^{o}\left(x, R_{i}\right)\right)=\lim _{k \rightarrow \infty} Q\left(B\left(x_{k}, R_{i}\right)\right)
$$

and

$$
Q^{\prime}\left(B^{o}\left(x, R_{i}\right)\right)=\lim _{k \rightarrow \infty} Q^{\prime}\left(B\left(x_{k}, R_{i}\right)\right)
$$

Since $Q R_{i} Q^{\prime}$, we obtain that for all $k \in \mathbb{N}$,

$$
Q\left(B\left(x_{k}, R_{i}\right)\right) \geq Q^{\prime}\left(B\left(x_{k}, R_{i}\right)\right) .
$$


Combining these inequalities with the equalities above yields (3.3).

The if-part follows in a similar way.

From now on, we use $[(3.1)$ and (3.2)] or $[(3.3)$ and $(3.4)]$ for our ordinal extension of preferences.

The first property we discuss says that for each profile, there does not exist a distribution that all agents weakly prefer to the distribution assigned by the probabilistic rule to this profile with strict preference for some agent.

Let $N \in \mathcal{P}$ and $Q, Q^{\prime}$ be distributions over $\mathbb{R}$. If for all $i \in N, Q R_{i} Q^{\prime}$ and for some $j \in N, Q P_{j} Q^{\prime}$, then we call $Q$ a Pareto improvement of $Q^{\prime}$.

Pareto-Optimality: For all $N \in \mathcal{P}$ and all $R \in \mathcal{R}^{N}$ : there exists no Pareto improvement of $\Phi(R)$.

Remark 3.1 A deterministic rule satisfies Pareto-optimality if and only if for all $N \in \mathcal{P}$ and all $R \in R^{N}, \varphi(R) \in E(R)$. Therefore, we call $E(R)$ the Pareto set of $R$.

We show that a probabilistic rule satisfies Pareto-optimality if and only if it only selects distributions that place for every profile probability 1 on its Pareto set. Therefore, in our model, ex-post efficiency is equivalent to Pareto-optimality.

Lemma 3.2 Let $\Phi$ be a probabilistic rule. Then $\Phi$ satisfies Pareto-optimality if and only if for all $N \in \mathcal{P}$ and all $R \in \mathcal{R}^{N}, \Phi(R)(E(R))=1$.

Proof. For the only-if part, suppose that $\Phi$ satisfies Pareto-optimality. Assume that there exist $N \in \mathcal{P}$ and $R \in \mathcal{R}^{N}$ such that $\Phi(R)([\underline{p}(R), \bar{p}(R)])<1$. Without loss of generality, suppose that $\Phi(R)(]-\infty, \underline{p}(R)[)>0$. Let $j \in N$ be such that $p\left(R_{j}\right)=\underline{p}(R)$. Define $Q$ by $Q(\{\underline{p}(R)\}) \equiv \Phi(R)(]-\infty, \underline{p}(R)])$ and $Q$ equal to $\Phi(R)$ on $] \underline{p}(R),+\infty[$. Then for all $i \in N, Q R_{i} \Phi(R)$. Since $Q(\{\underline{p}(R)\})>\Phi(R)(\{\underline{p}(R)\})$ and $p\left(R_{j}\right)=\underline{p}(R)$, it follows that $Q P_{j} \Phi(R)$, which contradicts Pareto-optimality.

For the if-part, let $N \in \mathcal{P}, R \in \mathcal{R}^{N}$, and $\Phi(R)([\underline{p}(R), \bar{p}(R)])=1$. Without loss of generality, suppose that $N=\{1, \ldots, n\}$ and $p\left(R_{1}\right) \leq \cdots \leq p\left(R_{n}\right)$. Let $Q$ be a distribution over $\mathbb{R}$ such that for all $i \in N, Q R_{i} \Phi(R)$. We show $Q=\Phi(R)$. Since $B\left(\bar{p}(R), R_{1}\right) \cap B\left(\underline{p}(R), R_{n}\right)=[\underline{p}(R), \bar{p}(R)]$ and $\Phi(R)([\underline{p}(R), \bar{p}(R)])=1$, we have

$$
Q([\underline{p}(R), \bar{p}(R)])=1 \text {. }
$$

Next, we prove that for all $x \in[\underline{p}(R), \bar{p}(R)]$,

$$
Q([\underline{p}(R), x])=\Phi(R)([\underline{p}(R), x]) .
$$


Let $x \in[\underline{p}(R), \bar{p}(R)]$. By $(3.5), p\left(R_{1}\right)=\underline{p}(R)$, and $Q R_{1} \Phi(R)$,

$$
Q\left(\left[p\left(R_{1}\right), x\right]\right)=Q\left(B\left(x, R_{1}\right)\right) \geq \Phi(R)\left(B\left(x, R_{1}\right)\right)=\Phi(R)\left(\left[p\left(R_{1}\right), x\right]\right) .
$$

By Lemma 3.1, (3.5), $p\left(R_{n}\right)=\bar{p}(R)$, and $Q R_{n} \Phi(R)$,

$$
\left.\left.\left.\left.Q(] x, p\left(R_{n}\right)\right]\right)=Q\left(B^{o}\left(x, R_{n}\right)\right) \geq \Phi(R)\left(B^{o}\left(x, R_{n}\right)\right)=\Phi(R)(] x, p\left(R_{n}\right)\right]\right) .
$$

Since $\left[p\left(R_{1}\right), x\right]$ and $\left.] x, p\left(R_{n}\right)\right]$ are disjoint, and $\left.\left.\left[p\left(R_{1}\right), x\right] \cup\right] x, p\left(R_{n}\right)\right]=[\underline{p}(R), \bar{p}(R)],(3.5)$, (3.7), and (3.8) together imply $Q\left(\left[p\left(R_{1}\right), x\right]\right)=\Phi(R)\left(\left[p\left(R_{1}\right), x\right]\right)$. Hence, by (3.6), for all ]$a, b] \subseteq[\underline{p}(R), \bar{p}(R)]$,

$$
\begin{aligned}
Q(] a, b]) & =Q([\underline{p}(R), b])-Q([\underline{p}(R), a]) \\
& =\Phi(R)([\underline{p}(R), b])-\Phi(R)([\underline{p}(R), a]) \\
& =\Phi(R)([a, b]) .
\end{aligned}
$$

Thus, by (3.5), for all $] a, b] \subseteq \mathbb{R}, Q(] a, b])=\Phi(R)(\mid a, b])$. Since the $\sigma$-algebra $\mathcal{L}$ is generated by the set of the left half open intervals $\{\mid a, b] \mid a, b \in \mathbb{R}, a \leq b\}$ (cf. Remark A.3), it follows that $Q=\Phi(R)$, the desired conclusion.

Finally, we introduce the requirement that the probabilistic rule is symmetric in its arguments. Given $N \in \mathcal{P}, R \in \mathcal{R}^{N}$, and a permutation $\sigma: N \rightarrow N$, let $\sigma(R)$ denote the profile $\left(R_{\sigma(i)}\right)_{i \in N}$.

Anonymity: For all $N \in \mathcal{P}$, all $R \in \mathcal{R}^{N}$, and all permutations $\sigma: N \rightarrow N: \Phi(R)=$ $\Phi(\sigma(R))$.

\subsection{Strategy-Proofness}

\subsubsection{Definitions}

Throughout this section we keep the set of agents fixed. Let $N=\{1, \ldots, n\}$ be the set of agents. Our main interest in this section is in strategy-proofness. This means that agents cannot gain from misrepresenting their true preferences.

Strategy-Proofness: For all $i \in N$ and all $R, \bar{R} \in \mathcal{R}^{N}$ such that $R_{N \backslash\{i\}}=\bar{R}_{N \backslash\{i\}}$ : $\Phi(R) R_{i} \Phi(\bar{R})$. 
Strategy-proofness ensures that no agent can increase the probability of any weak upper contour set by mispresenting his true preference. Observe that this notion of strategyproofness is a stochastic dominance condition; in particular it requires comparability in this respect of the distributions $\Phi(R)$ and $\Phi(\bar{R})$. It implies that for any von-NeumannMorgenstern utility function representing an agent's preference relation, his expected utility is maximal when he reports his true preference relation.

Also the following conditions play a central role in this section. The first one says that at unanimous profiles the probabilistic rule places probability one on the common peak. The second one says that the probabilistic rule only depends on the profile of peaks.

Unanimity: For all $R \in \mathcal{R}^{N}$ such that for all $i, j \in N, R_{i}=R_{j}: \Phi(R)\left(\left\{p\left(R_{1}\right)\right\}\right)=1$.

Peaks-Onliness: For all $R, \bar{R} \in \mathcal{R}^{N}$ such that $p(R)=p(\bar{R}): \Phi(R)=\Phi(\bar{R})$.

Note that Pareto-optinality implies unanimity, but not the other way around.

\subsubsection{Uncompromisingness}

In this subsection we show that strategy-proofness together with peaks-onliness is equivalent to a condition called uncompromisingness. In the deteministic setting, uncompromisingness means that after an $i$-deviation the outcome does not change whenever the changed peak remains on the same side of the outcome. Therefore, if $p\left(R_{i}\right)$ is below the outcome and $R, \bar{R}$ are $i$-deviations such that $p\left(\bar{R}_{i}\right)$ is less than or equal to that outcome, then at $R$ and $\bar{R}$ the same level is chosen. Border and Jordan (1983) show in the deterministic model that unanimity and strategy-proofness imply uncompromisingness.

We extend the definition of uncompromisingness to the probabilistic model in the following way. A probabilistic rule satisfies uncompromisingness if for all $i$-deviations the assigned probability does not change for those subsets of the real line which have not been crossed by this unilateral peak change. Therefore, if $R$ and $\bar{R}$ are $i$-deviations, then the distributions $\Phi(R)$ and $\Phi(\bar{R})$ are equal outside the closed interval $\left[\min \left\{p\left(R_{i}\right), p\left(\bar{R}_{i}\right)\right\}\right.$, $\left.\max \left\{p\left(R_{i}\right), p\left(\vec{R}_{i}\right)\right\}\right]$.

Given $a, b \in \mathbb{R}$, let $\operatorname{conv}(a, b)$ denote the convex hull of $a$ and $b$, i.e.; the closed interval with endpoints $a$ and $b$.

Uncompromisingness: For all $i \in N$, all $R, \bar{R} \in \mathcal{R}^{N}$ such that $R_{N \backslash\{i\}}=\bar{R}_{N \backslash\{i\}}$, and all $X \in \mathcal{L}$ such that $X \cap \operatorname{conv}\left(p\left(R_{i}\right), p\left(\bar{R}_{i}\right)\right)=\emptyset: \Phi(R)(X)=\Phi(\bar{R})(X)$. 
Remark 3.2 Since $\Phi(R)$ and $\Phi(\vec{R})$ are distributions, uncompromisingness implies $\Phi(R)$ $\left(\operatorname{conv}\left(p\left(R_{i}\right), p\left(\bar{R}_{i}\right)\right)\right)=\Phi(\bar{R})\left(\operatorname{conv}\left(p\left(R_{i}\right), p\left(\bar{R}_{i}\right)\right)\right)$.

In the following two lemmas we prove that uncompromisingness is equivalent to the combination of strategy-proofness and peaks-onliness.

Lemma 3.3 Let $\Phi$ be a probabilistic rule. If $\Phi$ satisfies strategy-proofness and peaksonliness, then $\Phi$ satisfies uncompromisingness.

Proof. Let $i \in N, R, \bar{R} \in \mathcal{R}^{N}$ be $i$-deviations, and $[a, b[$ be an interval such that $\left[a, b\left[\cap \operatorname{conv}\left(p\left(R_{i}\right), p\left(\bar{R}_{i}\right)\right)=\emptyset\right.\right.$. Since a finite measure is uniquely determined by the weights on such intervals (cf. Remark A.3), it suffices to prove that $\Phi(R)([a, b[)=\Phi(\bar{R})([a, b[)$. Because $\left[a, b\left[\right.\right.$ is disjoint from $\operatorname{conv}\left(p\left(R_{i}\right), p\left(\bar{R}_{i}\right)\right)$, we have to distinguish two cases.

Case 1: $a>\max \left\{p\left(R_{i}\right), p\left(\bar{R}_{i}\right)\right\}$.

Let $c<\min \left\{p\left(R_{i}\right), p\left(\bar{R}_{i}\right)\right\}$. Let $R^{\prime}, R^{\prime \prime}, \bar{R}^{\prime}, \bar{R}^{\prime \prime} \in \mathcal{R}^{N}$ be $i$-deviations from $R$ such that $\left.p\left(R_{i}^{\prime}\right)=p\left(R_{i}^{\prime \prime}\right)=p\left(R_{i}\right), p\left(\bar{R}_{i}^{\prime}\right)=p\left(\bar{R}_{i}^{\prime \prime}\right)=p\left(\bar{R}_{i}\right), B^{o}\left(a, R_{i}^{\prime}\right)=B^{o}\left(a, \bar{R}_{i}^{\prime}\right)=\right] c, a[$, and $\left.B^{o}\left(b, R_{i}^{\prime \prime}\right)=B^{o}\left(b, \bar{R}_{i}^{\prime \prime}\right)=\right] c, b[$. Then

$$
\begin{aligned}
\Phi(R)\left(B^{o}\left(a, R_{i}^{\prime}\right)\right) & =\Phi\left(R^{\prime}\right)\left(B^{o}\left(a, R_{i}^{\prime}\right)\right) \\
& \geq \Phi\left(\bar{R}^{\prime}\right)\left(B^{o}\left(a, R_{i}^{\prime}\right)\right) \\
& =\Phi(\bar{R})\left(B^{o}\left(a, R_{i}^{\prime}\right)\right)
\end{aligned}
$$

where the two equalities follow from peaks-onliness and the inequality from strategyproofness. Hence, $\Phi(R)(] c, a[) \geq \Phi(\bar{R})(] c ; a[)$. Similarly the reverse inequality holds. Therefore, $\Phi(R)(] c, a[)=\Phi(\bar{R})(] c, a[)$.

By considering $R^{\prime \prime}$ and $\bar{R}^{\prime \prime}$ we analogously deduce $\Phi(R)(] c, b[)=\Phi(\bar{R})(] c, b[)$. Thus,

$$
\begin{aligned}
\Phi(R)([a, b]) & =\Phi(R)(] c, b[)-\Phi(R)(] c, a[) \\
& =\Phi(\bar{R})(] c, b[)-\Phi(\bar{R})(] c, a[) \\
& =\Phi(\bar{R})([a, b[),
\end{aligned}
$$

which is the desired result.

Case 2: $b \leq \min \left\{p\left(R_{i}\right), p\left(\bar{R}_{i}\right)\right\}$.

If $b<\min \left\{p\left(R_{i}\right), p\left(\bar{R}_{i}\right)\right\}$, then similar to Case 1 it follows that $\Phi(R)([a, b[)=\Phi(\bar{R})([a, b[)$. If $b=\min \left\{p\left(R_{i}\right), p\left(\bar{R}_{i}\right)\right\}$, then similar to the proof of Lemma 3.1 we use the previous fact and Theorem A.1 to show $\Phi(R)\left(\left[a, \min \left\{p\left(R_{i}\right), p\left(\bar{R}_{i}\right)\right\}[)=\Phi(\bar{R})\left(\left[a, \min \left\{p\left(R_{i}\right), p\left(\bar{R}_{i}\right)\right\}[)\right.\right.\right.\right.$.

Lemma 3.4 Let $\Phi$ be a probabilistic rule. If $\Phi$ satisfies uncompromisingness, then $\Phi$ satisfies strategy-proofness and peaks-onliness. 
Proof. It is straightforward to show that uncompromisingness implies peaks-onliness. In order to show strategy-proofness, let $i \in N$ and $R, \bar{R} \in \mathcal{R}^{N}$ be such that $R_{N \backslash\{i\}}=$ $\bar{R}_{N \backslash\{i\}}$. Without loss of generality, we suppose that $p\left(\bar{R}_{i}\right)<p\left(R_{i}\right)$. We show that for all $x \in\left[p\left(\bar{R}_{i}\right), p\left(R_{i}\right)[\right.$

$$
\left.\left.\left.\left.\Phi(R)(] x, p\left(R_{i}\right)\right]\right) \geq \Phi(\vec{R})(] x, p\left(R_{i}\right)\right]\right)
$$

and for all $\left.x \in] p\left(\bar{R}_{i}\right), p\left(R_{i}\right)\right]$

$$
\Phi(R)\left(\left[x, p\left(R_{i}\right)\right]\right) \geq \Phi(\bar{R})\left(\left[x, p\left(R_{i}\right)\right]\right) .
$$

First, we prove (3.9). Let $x \in\left[p\left(\bar{R}_{i}\right), p\left(R_{i}\right)\left[\right.\right.$ and $y \in\left[p\left(\bar{R}_{i}\right), p\left(R_{i}\right)[\right.$ be such that $x<y$. Let $R^{\prime} \in \mathcal{R}$ be an $i$-deviation of $R$ such that $p\left(R_{i}^{\prime}\right)=y$. Then

$$
\begin{aligned}
\left.\left.\Phi(R)(] x, p\left(R_{i}\right)\right]\right) & \left.=\Phi\left(R^{\prime}\right)\left(\mid x, p\left(R_{i}\right)\right]\right) \\
& \left.\left.\geq \Phi\left(R^{\prime}\right)(] y, p\left(R_{i}\right)\right]\right) \\
& \left.\left.=\Phi(\bar{R})(] y, p\left(R_{i}\right)\right]\right) .
\end{aligned}
$$

where the first equality follows from Remark 3.2 and uncompromisingness, and the second from uncompromisingness. Thus, for all $\left.y \in] x, p\left(R_{i}\right)\left[\Phi(R)(] x, p\left(R_{i}\right)\right]\right) \geq \Phi(\vec{R})\left(\left[y, p\left(R_{i}\right)\right]\right)$. Hence, similar to the proof of Lemma 3.1, Theorem A.1 yields (3.9).

To show (3.10), by interchanging the roles of $R$ and $\tilde{R}$ it follows that for all $x \in$ $\left.\mid p\left(\bar{R}_{i}\right), p\left(R_{i}\right)\right]$,

$$
\Phi(\bar{R})\left(\left[p\left(\bar{R}_{i}\right), x[) \geq \Phi(R)\left(\left[p\left(\bar{R}_{i}\right), x[) .\right.\right.\right.\right.
$$

Hence, Remark 3.2 yields (3.10).

By (3.9), (3.10), and uncompromisingness, for all $z \in \mathbb{R}$,

$$
\Phi(R)\left(B\left(z, R_{i}\right)\right) \geq \Phi(\bar{R})\left(B\left(z, R_{i}\right)\right) .
$$

This is the strategy-proofness requirement for $\Phi$ at $R$ and $\bar{R}$.

Lemmas 3.3 and 3.4 yield the following corollary.

Corollary 3.1 The combination of strategy-proofness and peaks-onliness is equivalent to uncompromisingness.

Corollary 3.1 is the important tool for the proofs in the following subsection. In Subsection 3.3 .4 we show that Corollary 3.1 remains valid in the deterministic model. 


\subsubsection{Strategy-Proofness and Peaks-Onliness}

In this subsection we give a complete description of the probabilistic rules satisfying strategy-proofness and peaks-onliness in terms of fixed probability distributions. More precisely, such a probabilistic rule determines for each subset $S$ of $N$ a fixed probabilistic "ballot" over $\overline{\mathbb{R}} \equiv \mathbb{R} \cup\{-\infty,+\infty\}$. It is obtained by taking the limits of the probabilistic rule where the peaks of the preferences of the agents in $S$ converge to $-\infty$ and the peaks of the preferences of the agents in $N \backslash S$ to $+\infty$. As probability can vanish to $-\infty$ or $+\infty$ these distributions are defined on the compactified real line. ${ }^{2}$

To describe how, conversely, these fixed probability distributions determine the probabilistic rule some additional notation is needed. This notation will be used throughout the rest of Chapter 3.

Given $R \in \mathcal{R}^{N}$, let $p^{1}(R), \ldots, p^{k}(R), k \leq n$, denote the different peaks at $R$ such that for all $l \in\{1, \ldots, k-1\}, p^{l}(R)<p^{l+1}(R)$. Thus, $p^{1}(R)=p(R), p^{k}(R)=\bar{p}(R)$, and $\left\{p^{l}(R) \mid l \in\{1, \ldots, k\}\right\}=\left\{p\left(R_{i}\right) \mid i \in N\right\}$. Let $S_{l}$ denote the set of agents whose peaks at profile $R$ are lower than or equal to $p^{l}(R)$. Thus, $S_{1} \subsetneq S_{2} \subsetneq \cdots \subsetneq S_{k}$ and $S_{k}=N$. Let $S_{0} \equiv \emptyset, p^{0}(R) \equiv-\infty$, and $p^{k+1}(R) \equiv+\infty$.

For all $S \in 2^{N}$, let $D_{S}$ be a probability distribution over $\overline{\mathbb{R}}$. We call $\Delta \equiv\left(D_{S}\right)_{S \in 2^{N}}$ a collection of fixed probabilistic ballots if the following holds:

(i) $D_{\emptyset}(\{-\infty\})=0$;

(ii) $D_{N}(\{+\infty\})=0$; and

(iii) for all $S, T \subseteq N$ and all $x \in \mathbb{R}$,

$$
D_{S \cup T}([-\infty, x])-D_{S}([-\infty, x[) \geq 0
$$

Inequality (3.11) will ensure the nomnegativity of a probabilistic rule defined on the basis of a collection of fixed probabilistic ballots. Given $X \in \mathcal{L}$, let $1_{X}$ denote the indicator function of the set $X$.

Fixed-Probabilistic-Ballots Rule, $\Phi^{\Delta}$ : Given a collection $\Delta=\left(D_{S}\right)_{S \in 2^{N}}$ of fixed probabilistic ballots, the fixed-probabilistic-ballots rule $\Phi^{\Delta}$ based on $\Delta$ is defined as follows.

\footnotetext{
${ }^{2}$ If the set of alternatives is restricted to a closed interval, then the endpoints of this interval play the same roles as $-\infty$ and $+\infty$.
} 
For all $R \in \mathcal{R}^{N}$ and all $X \in \mathcal{L}$,

$$
\begin{aligned}
\Phi^{\Delta}(R)(X) & \equiv \sum_{l=0}^{k} D_{S_{l}}(X \cap] p^{l}(R), p^{l+1}(R)[) \\
& +\sum_{l=1}^{k} 1_{X}\left(p^{l}(R)\right)\left(D_{S_{l}}\left(\left[-\infty, p^{l}(R)\right]\right)-D_{S_{l-l}}\left(\left[-\infty, p^{l}(R)[)\right) .\right.\right.
\end{aligned}
$$

To get some grasp on formula (3.12), consider an arbitrary profile $R \in \mathcal{R}^{N}$. The distribution $\Phi^{\Delta}(R)$ is equal to the distribution $D_{\emptyset}$ on the interval ] $-\infty, \underline{p}(R)$ [. For all $l \in\{1, \ldots, k-1\}$, the distribution $\Phi^{\Delta}(R)$ is equal to $D_{S_{l}}$ on the interval $] p^{l}(R), p^{l+1}(R)[$. On the interval $] \bar{p}(R),+\infty$ [, the distribution $\Phi^{\Delta}(R)$ is equal to $D_{N}$. The probabilities of the peaks at $R$ are determined in the following way: for all $l \in\{1, \ldots, k\}$ the weight placed by $\Phi^{\Delta}(R)$ on $p^{l}(R)$ is equal to the probability placed by $D_{S_{l}}$ on the interval $\left[-\infty, p^{l}(R)\right]$ minus the probability placed by $D_{S_{l-1}}$ on the half open interval $\left[-\infty, p^{l}(R)[\right.$. Condition (3.11) ensures the nonnegativity of $\Phi^{\Delta}(R)$. Note that the interval in the first term in (3.11) is closed but the interval in the second term is half open. The $\sigma$-additivity of $\Phi^{\Delta}(R)$ follows immediately from definition (3.12) and the fact that the $D_{S}, S \in 2^{N}$, are distributions. To show that $\Phi^{\Delta}(R)$ is a probability distribution over $\mathbb{R}$, we prove that $\Phi^{\Delta}(R)(\mathbb{R})=1$. We have

$$
\begin{aligned}
\Phi^{\Delta}(R)(\mathbb{R})= & \sum_{l=0}^{k} D_{S_{l}}(] p^{l}(R), p^{l+1}(R)[) \\
& +\sum_{l=1}^{k}\left(D_{S_{l}}\left(\left[-\infty, p^{l}(R)\right]\right)-D_{S_{l-1}}\left(\left[-\infty, p^{l}(R)[)\right)\right.\right. \\
= & D_{\emptyset}(]-\infty, p^{1}(R)[)-D_{\emptyset}\left(\left[-\infty, p^{1}(R)[)\right.\right. \\
& +\sum_{l=1}^{k-1}\left(D_{S_{l}}\left(\left[-\infty, p^{l}(R)\right]\right)+D_{S_{l}}(] p^{l}(R), p^{l+1}(R)[)-D_{S_{l}}\left(\left[-\infty, p^{l+1}(R)[)\right)\right.\right. \\
& +D_{N}\left(\left[-\infty, p^{k}(R)\right]\right)+D_{N}(] p^{k}(R),+\infty[) \\
= & D_{N}([-\infty,+\infty[) \\
= & 1,
\end{aligned}
$$

where the third equality follows from the fact that $D_{\emptyset}(\{-\infty\})=0$, and the fourth from the fact that $D_{N}(\{+\infty\})=0$. Hence, $\Phi^{\Delta}(R)$ is a probability distribution over $\mathbb{R}$, the desired conclusion.

In order to illustrate these definitions we include two examples. The first one is a random dictatorship formulated in terms of fixed probability distributions based on formula (3.12). Obviously, the class of rules that can be defined with the aid of (3.11) and (3.12) is much bigger. 
Example 3.1 Let $\alpha \in \mathbb{R}^{N}$ be a vector of weights, i.e., for all $i \in N, \alpha_{i} \geq 0$, and $\sum_{j=1}^{n} \alpha_{j}=1$. For all $S \subseteq N$, let $D_{S}$ be the probability distribution over $\overline{\mathbb{R}}$ putting weight $\sum_{i \in S} \alpha_{i}$ on $-\infty$ and the remaining weight on $+\infty$ (where the empty summation is defined to be equal to 0 ). Then (3.12) defines the random dictatorship where agent $i$ has weight $\alpha_{i}$.

Example 3.2 Let $N=\{1,2\}$. Let $D_{\emptyset}$ be the uniform distribution over the interval $[3,4]$, and $D_{\{1\}}, D_{\{2\}}$, and $D_{N}$ be uniform distributions over the intervals $[2,3],[1,2]$, and $[0,1]$, respectively. Then (3.11) is satisfied and $\Delta \equiv\left(D_{0}, D_{\{1\}}, D_{\{2\}}, D_{N}\right)$ is a collection of fixed probabilistic ballots. For example, let $R \in \mathcal{R}^{N}$ be such that $p\left(R_{1}\right)=1$ and $p\left(R_{2}\right)=3$. Then, by (3.12), $\Phi^{\Delta}(R)=\Phi^{\Delta}\left(R_{1}, R_{2}\right)=D_{\{1\}}$ and $\Phi^{\Delta}\left(R_{2}, R_{1}\right)=D_{\{2\}}$.

The following theorem states that each strategy-proof and peaks-only probabilistic rule is determined by $2^{n}$ fixed probability distributions as in (3.12).

Theorem 3.1 The fixed-probabilistic-ballots rules are the only probabilistic rules satisfying strategy-proofness and peaks-onliness. Moreover, the collection of fixed probabilistic ballots is uniquely determined for each fixed-probabilistic-ballots rule.

Proof. Note that, by definition, fixed-probabilistic-ballots rules are peaks-only. Strategyproofness follows easily with the aid of Corollary 3.1. The proof of this is left to the reader.

In proving the converse statement, let $\Phi$ be a probabilistic rule satisfying strategyproofness and peaks-onliness. The proof consists of two steps. In the first step we construct the probability distributions $D_{S}$, and in the second we prove the validity of (3.12).

Step 1: Construction of the probability distributions $D_{S}$.

Given $x \in \mathbb{R}$, let $R^{x} \in \mathcal{R}^{N}$ be such that for all $i \in N, p\left(R_{i}^{x}\right)=x$. Let $S \in 2^{N}$. For each $A \in \mathcal{L}$ define

$$
D_{S}(A) \equiv \lim _{x \rightarrow+\infty} \Phi\left(R_{S}^{-x}, R_{N \backslash S}^{x}\right)(A \cap]-x, x[) .
$$

By Corollary 3.1, $\Phi$ satisfies uncompromisingness. Therefore, the numbers $\Phi\left(R_{S}^{-x}, R_{N \backslash S}^{x}\right)(A \cap]-x, x[)$ are nondecreasing in $x$. Furthermore, they are bounded by 1 , so that the limit in (3.13) exists and is smaller than or equal to 1 . Theorem A.2 implies that $D_{S}$ is a measure on the measurable space $(\mathbb{R}, \mathcal{L})$.

We additionally define the values of $D_{S}$ on $-\infty$ and $+\infty$, as follows. First, define for all $A \in \mathcal{L}$

$$
D_{S}^{-}(A) \equiv \lim _{x \rightarrow+\infty} \Phi\left(R_{S}^{-x}, R_{N \backslash S}^{1}\right)(A \cap]-x,+\infty[)
$$


and

$$
D_{S}^{+}(A) \equiv \lim _{x \rightarrow+\infty} \Phi\left(R_{S}^{-1}, R_{N \backslash S}^{x}\right)(A \cap]-\infty, x[)
$$

Hence, the agents in $N \backslash S$ (respectively, in $S$ ) are arbitrarily kept at 1 (respectively, -1 ). By uncompromisingness, these limits exist. Now define

$$
D_{S}(\{-\infty\}) \equiv 1-D_{S}^{-}(\mathbb{R})
$$

and

$$
D_{S}(\{+\infty\}) \equiv 1-D_{S}^{+}(\mathbb{R})
$$

It remains to show that $D_{S}$ is a probability distribution on the measurable space $(\overline{\mathbb{R}}, \overline{\mathcal{L}})$, cf. Remark A.4. It suffices to show that

$$
D_{S}(\overline{\mathbb{R}})=1-D_{S}^{-}(\mathbb{R})+1-D_{S}^{+}(\mathbb{R})+D_{S}(\mathbb{R})=1
$$

By uncompromisingness, $D_{S}(]-\infty, 1[)=D_{S}^{-}(]-\infty, 1[)$ and $D_{S}\left(\left[1,+\infty[)=D_{S}^{+}([1,+\infty[)\right.\right.$. Therefore, (3.14) reduces to

$$
2-D_{S}^{-}\left(\left[1,+\infty[)-D_{S}^{+}(]-\infty, 1[)=1\right.\right.
$$

Note that $\Phi\left(R_{S}^{-1}, R_{N \backslash S}^{1}\right)(\mathbb{R})=1$. By uncompromisingness, $D_{S}^{-}([1,+\infty[)=$ $\Phi\left(R_{S}^{-1}, R_{N \backslash S}^{1}\right)\left(\left[1,+\infty[)\right.\right.$ and $D_{S}^{+}(]-\infty, 1[)=\Phi\left(R_{S}^{-1}, R_{N \backslash S}^{1}\right)(]-\infty, 1[)$. Hence, (3.15) holds and therefore, (3.14). This completes Step 1.

Step 2: Validity of (3.12).

Let $R \in \mathcal{R}^{N}$ and $j \in\{0, \ldots, k\}$. First, we show that $\Phi(R)$ coincides with $D_{S_{j}}$ on $\left|p^{j}(R), p^{j+1}(R)\right|$. Let $x \in \mathbb{R}$ be such that $x>\bar{p}(R)$ and $-x<\underline{p}(R)$. By uncompromisingness and successive replacement of the preferences in $R$ by those in $\left(R_{S_{j}}^{-x}, R_{N \backslash S_{j}}^{x}\right)$ it follows that $\Phi(R)$ and $\Phi\left(R_{S_{j}}^{-x}, R_{N \backslash S_{j}}^{x}\right)$ coincide on $] p^{j}(R), p^{j+1}(R)[$. By uncompromisingness, $\Phi\left(R_{S_{j}}^{-x}, R_{N \backslash S_{j}}^{x}\right)$ is constant on $] p^{j}(R), p^{j+1}(R)[$ when $x$ converges to $+\infty$. This limit is exactly the distribution $D_{S_{j}}$ as defined in (3.13). Hence, $\Phi(R)$ and $D_{S_{j}}$ coincide on ]$p^{j}(R), p^{j+1}(R)[$. Thus, the first part of $(3.12)$ is valid.

It remains to determine the weights that $\Phi(R)$ places on the peaks of $R$. Let $p^{j}(R)$ be one of the peaks. Consider the probabilistic rule $\bar{\Phi}$ on $\mathcal{R}^{N \backslash\left(S_{j} \backslash S_{j-1}\right)}$ defined by for all $\vec{R} \in \mathcal{R}^{N \backslash\left(S_{j} \backslash S_{j-1}\right)}, \bar{\Phi}(\bar{R}) \equiv \Phi\left(\bar{R}, R_{S_{j} \backslash S_{j-1}}\right)$. Note that for all $i \in S_{j} \backslash S_{j-1}, p\left(R_{i}\right)=p^{j}(R)$. Because $\Phi$ satisfies strategy-proofness and peaks-onliness, $\bar{\Phi}$ satisfies strategy-proofness and peaks-onliness. Now, similar to $D_{S_{j-1}}$, we can define $\bar{D}_{S_{j-1}}$. Then, by the definition 
of these fixed distributions and uncompromisingness,

$$
\begin{aligned}
\bar{D}_{S_{-1}}\left(\left[-\infty, p^{j}(R)\right]\right) & \left.\left.=1-\bar{D}_{S_{j-1}}(] p^{j}(R),+\infty\right]\right) \\
& \left.=1-D_{S_{j}}\left(\mid p^{j}(R),+\infty\right]\right) \\
& =D_{S_{j}}\left(\left[-\infty, p^{j}(R)\right]\right)
\end{aligned}
$$

Also by uncompromisingness, $\bar{D}_{S_{j-1}}\left(\left[-\infty, p^{j}(R)[)=D_{S_{j-1}}\left(\left[-\infty, p^{j}(R)[)\right.\right.\right.\right.$. Further,

$$
\begin{aligned}
\Phi(R)\left(\left\{p^{j}(R)\right\}\right) & =\Phi\left(R_{N \backslash\left(S_{j} \backslash S_{j-1}\right)}, R_{S_{j} \backslash S_{j-1}}\right)\left(\left\{p^{j}(R)\right\}\right) \\
& =\bar{\Phi}\left(R_{N \backslash\left(S_{j} \backslash S_{j-1}\right)}\right)\left(\left\{p^{j}(R)\right\}\right) \\
& =\bar{D}_{S_{j-1}}\left(\left\{p^{j}(R)\right\}\right),
\end{aligned}
$$

where the last equality follows from the first part of the proof. Thus,

$$
\begin{aligned}
\Phi(R)\left(\left\{p^{j}(R)\right\}\right) & =\bar{D}_{S_{j-1}}\left(\left\{p^{j}(R)\right\}\right) \\
& =\bar{D}_{S_{j-1}}\left(\left[-\infty, p^{j}(R)\right]\right)-\bar{D}_{S_{j-1}}\left(\left[-\infty, p^{j}(R)[)\right.\right. \\
& =D_{S_{j}}\left(\left[-\infty, p^{j}(R)\right]\right)-D_{S_{j-1}}\left(\left[-\infty, p^{j}(R)[),\right.\right.
\end{aligned}
$$

the desired conclusion. Hence, (3.12) holds and the proof is complete.

Moulin (1980) characterizes all anonymous, strategy-proof, and peaks-only deterministic rules. For each profile, such a rule chooses the median of $2 n+1$ "mumbers": $n$ reported peaks and $n+1$ additional fixed ballots ("phantom" peaks). Moulin's result follows easily from his characterization of all strategy-proof and peaks-only deterministic rules. Similarly, a characterization of strategy-proof and peaks-only probabilistic rules which are in addition anonymous can be derived from Theorem 3.1, as follows.

Let $\Phi$ be an anonymous, strategy-proof; and peaks-only probabilistic rule. Then, by Theorem 3.1, $\Phi$ is characterized by $2^{n}$ fixed probabilistic ballots. Consider the calibration of these distributions in the proof of Theorem 3.1. For $S \in 2^{N}$, the distribution $D_{S}$ is the limit of $\Phi$ when the peaks of $S$ converge to $-\infty$ and the peaks of $N \backslash S$ to $+\infty$. Therefore, for all $T \in 2^{N}$ such that $|S|=|T|$, anonymity implies $D_{S}=D_{T}$. Thus, these limits depend only on the cardinality of the set $S$, and $|S| \in\{0,1, \ldots, n\}$. This yields the following corollary.

Corollary 3.2 Let $\Phi$ be a probabilistic rule. Then $\Phi$ satisfies anonymity, strategy-proofness, and peaks-onliness, if and only if there exists a unique collection $\Delta$ of fixed probabilistic ballots such that for all $S, T \in 2^{N}$, if $|S|=|T|$, then $D_{S}=D_{T}$, and $\Phi=\Phi^{\Delta}$. 
In a characterization result the properties that are used should be independent. Here, independence holds for Theorem 3.1 and Corollary 3.2. This follows from the fact that the probabilistic setting is a generalization of the deterninistic one, as will be shown later. Finally, we show how to modify Example 3.2 such that the probabilistic rule satisfies anonymity.

Example 3.3 (Example 3.2 continued.) Since $\Phi^{\Delta}\left(R_{1}, R_{2}\right)=D_{\{1\}} \neq D_{\{2\}}=\Phi^{\Delta}\left(R_{2}, R_{1}\right)$, the probabilistic rule $\Phi^{\Delta}$ violates anonymity. Let $\Delta^{\prime} \equiv\left(D_{\emptyset}, D_{\{1\}}, D_{\{1\}}, D_{N}\right)$. Then $\Phi^{\Delta^{\prime}}\left(R_{1}, R_{2}\right)=D_{\{1\}}=\Phi^{\Delta^{\prime}}\left(R_{2}, R_{1}\right)$ and $\Phi^{\Delta^{\prime}}$ satisfies anonymity.

\subsubsection{Strategy-Proofness and Unanimity}

In this subsection we show that unanimity and strategy-proofness imply Pareto-optimality and peaks-onliness. As a result, the only-if part of Theorem 3.1 remains true if we replace peaks-onliness by unanimity.

Proposition 3.1 Unanimity and strategy-proofness imply Pareto-optimality.

Proof. Let $\Phi$ be a probabilistic rule satisfying unanimity and strategy-proofness. Let $R \in \mathcal{R}^{N}$. By Lemma 3.2, we have to show $\Phi(R)([\underline{p}(R), \bar{p}(R)])=1$. We prove the assertion by induction on the number of different peaks of $R$.

Induction basis: $k=1$.

Suppose that all peaks at $R$ coincide, i.e., for all $i, j \in N, p\left(R_{i}\right)=p\left(R_{j}\right)$. Since for all $i \in N, B\left(p\left(R_{i}\right), R_{i}\right)=\left\{p\left(R_{i}\right)\right\}$, unanimity and strategy-proofness imply $\Phi(R)\left(\left\{p\left(R_{1}\right)\right\}\right)=$ 1 , the desired conclusion.

Induction step: $k>1$.

Assume that $\Phi$ is Pareto-optimal for all profiles which have less than $k>1$ different peaks. Let $R \in \mathcal{R}^{N}$ be such that $R$ has $k$ different peaks. Order set $S_{1}$, the agents who have their peaks on $\underline{p}(R)$, as follows:

$$
B\left(\bar{p}(R), R_{i_{1}}\right) \supseteq B\left(\bar{p}(R), R_{i_{2}}\right) \supseteq \cdots \supseteq B\left(\bar{p}(R), R_{i_{m}}\right),
$$

where $S_{1}=\left\{i_{1}, i_{2}, \ldots, i_{m}\right\}$. Note that ordering (3.16) is possible because the reported preferences are strict on each side of the peak. Therefore, for all $j \in S_{1}$ and some $a_{j} \in$ $[-\infty, \underline{p}(R)], B\left(\bar{p}(R), R_{j}\right)=\left[a_{j}, \bar{p}(R)\right]$ or $\left.\left.B\left(\bar{p}(R), R_{j}\right)=\right] a_{j}, \bar{p}(R)\right]$. Thus, $a_{i_{1}} \leq a_{i_{2}} \leq \ldots \leq$ 
$a_{i_{m}}$. Then

$$
\begin{aligned}
\Phi(R)\left(B\left(\bar{p}(R), R_{i_{1}}\right)\right) & \geq \Phi\left(R_{N \backslash\left\{i_{1}\right\}}, R_{i_{1}}^{\bar{p}(R)}\right)\left(B\left(\bar{p}(R), R_{i_{1}}\right)\right) \\
& \geq \Phi\left(R_{N \backslash\left\{i_{1}\right\}}, R_{i_{1}}^{\bar{p}(R)}\right)\left(B\left(\bar{p}(R), R_{i_{2}}\right)\right) \\
& \geq \Phi\left(R_{N \backslash\left\{i_{1}, i_{2}\right\}}, R_{\left\{i_{1}, i_{2}\right\}}^{\bar{p}(R)}\right)\left(B\left(\bar{p}(R), R_{i_{2}}\right)\right) \\
& \vdots \\
& \geq \Phi\left(R_{N \backslash S_{1}}, R_{S_{1}}^{\bar{p}(R)}\right)\left(B\left(\bar{p}(R), R_{i_{m}}\right)\right),
\end{aligned}
$$

where the first, the third, ... inequalities follow from strategy-proofness, the second, the fourth, ... from (3.16) and monotonicity of distributions (cf. Remark A.2). Since $\left(R_{N \backslash S_{1}}, R_{S_{1}}^{\vec{p}(R)}\right)$ has $k-1$ different peaks, it follows by our induction assumption that $\Phi\left(R_{N \backslash S_{1}}, R_{S_{1}}^{\bar{p}(R)}\right)$ is Pareto-optimal. Hence, by the above inequalities and $B\left(\bar{p}(R), R_{i_{m}}\right) \supseteq$ $[\underline{p}(R), \bar{p}(R)]$

$$
\Phi(R)\left(B\left(\bar{p}(R), R_{i_{1}}\right)\right) \geq \Phi\left(R_{N \backslash S_{1}}, R_{S_{1}}^{\bar{p}(R)}\right)\left(B\left(\bar{p}(R), R_{i_{m}}\right)\right)=1
$$

Since $\Phi(R)$ is a probability distribution, we obtain $\Phi(R)(]-\infty, \bar{p}(R)])=1$. Similarly, it follows that $\Phi(R)([\underline{p}(R),+\infty[)=1$. Thus, by the two previous facts, $\Phi(R)([\underline{p}(R), \bar{p}(R)])=1$, the desired conclusion.

An immediate consequence is that for strategy-proof probabilistic rules, unanimity and Pareto-optimality are equivalent. Next, we show that unanimity and strategy-proofness imply peaks-onliness. First, we need the following lemma.

Lemma 3.5 Let $\Phi$ be a probabilistic rule satisfying unanimity and strategy-proofness. Let $i \in N$ and $R, \bar{R} \in \mathcal{R}$ be $i$-deviations such that either $p\left(R_{i}\right)=p\left(\bar{R}_{i}\right)=\underline{p}(R)$ or $p\left(R_{i}\right)=p\left(\tilde{R}_{i}\right)=\bar{p}(R)$. Then $\Phi(R)=\Phi(\bar{R})$.

Proof. By Proposition 3.1, $\Phi$ satisfies Pareto-optimality. Let $\{a, b[\subseteq[\underline{p}(R), \bar{p}(R)]$. By Pareto-optimality and because $\mathcal{L}$ is generated by all right half open intervals, it is sufficient to prove that $\Phi(R)([a, b])=\Phi(\bar{R})([a, b[)$ (cf. Remark A.3).

Case 1: $p\left(R_{i}\right)=\underline{p}(R)$.

Then

$$
\begin{aligned}
\Phi(R)([\underline{p}(R), a[) & =\Phi(R)\left(B^{o}\left(a, R_{i}\right)\right) \\
& \geq \Phi(\bar{R})\left(B^{o}\left(a, R_{i}\right)\right) \\
& =\Phi(\bar{R})([\underline{p}(R), a])
\end{aligned}
$$


where the equalities follow from Pareto-optimality and the inequality from strategy-proofness. Similarly, $\Phi(\bar{R})([\underline{p}(R), a[) \geq \Phi(R)([\underline{p}(R), a[)$. Thus, $\Phi(\bar{R})([\underline{p}(R), a[)=\Phi(R)([\underline{p}(R), a[)$ and similarly, $\Phi(\tilde{R})([\underline{p}(R), b[)=\Phi(R)([\underline{p}(R), b[)$. Hence, $\Phi(\bar{R})([a, b[)=\Phi(R)([a, b[)$.

Case 2: $p\left(R_{i}\right)=\bar{p}(R)$.

This case is analogous to Case 1.

Now we are able to prove the main result of this subsection.

Proposition 3.2 Unanimity and strategy-proofness imply peaks-onliness.

Proof. By Proposition 3.1, $\Phi$ satisfies Pareto-optimality. Let $j \in N$ and $R, R^{\prime} \in \mathcal{R}^{N}$ be $j$-deviations such that $p\left(R_{j}\right)=p\left(R_{j}^{\prime}\right)$. We have to show that $\Phi(R)=\Phi\left(R^{\prime}\right)$. By Remark A.3 it is sufficient to show that for all $x \in[\underline{p}(R), \bar{p}(R)]$,

$$
\Phi(R)([\underline{p}(R), x])=\Phi\left(R^{\prime}\right)([\underline{p}(R), x]) .
$$

If $\underline{p}(R)=\bar{p}(R)$, then Pareto-optimality implies $\Phi(R)=\Phi\left(R^{\prime}\right)$. Thus, let $k \geq 2$ and for some $m \in\{1, \ldots, k-1\}, x \in\left[p^{m}(R), p^{m+1}(R)\right]$.

First, we show (3.17) for $x \in] p^{m}(R), p^{m+1}(R)\left[\right.$. Let $\tilde{R} \in \mathcal{R}^{N}$ be such that for all $i \in N$ :

- if $p\left(R_{i}\right)<p^{m}(R)$, then $p\left(\vec{R}_{i}\right) \equiv p^{m}(R)$ and $B\left(x, \bar{R}_{i}\right) \equiv B\left(x, R_{i}\right)$,

- if $p\left(R_{i}\right) \in\left\{p^{m}(R), p^{m+1}(R)\right\}$, then $\bar{R}_{i} \equiv R_{i}$,

- if $p\left(R_{i}\right)>p^{m+1}(R)$, then $p\left(\vec{R}_{i}\right) \equiv p^{m+1}(R)$ and $B^{o}\left(x, \vec{R}_{i}\right) \equiv B^{o}\left(x, R_{i}\right)$.

We show that

$$
\Phi(R)([\underline{p}(R), x])=\Phi(\bar{R})([\underline{p}(R), x])
$$

We have for all $i \in S_{1}$,

$$
\begin{aligned}
\Phi(R)([\underline{p}(R), x]) & =\Phi(R)\left(B\left(x, R_{i}\right)\right) \\
& \geq \Phi\left(R_{N \backslash\{i\}}, \bar{R}_{i}\right)\left(B\left(x, R_{i}\right)\right) \\
& =\Phi\left(R_{N \backslash\{i\}}, \bar{R}_{i}\right)\left(B\left(x, \tilde{R}_{i}\right)\right) \\
& \geq \Phi(R)\left(B\left(x, \bar{R}_{i}\right)\right) \\
& =\Phi(R)([\underline{p}(R), x]),
\end{aligned}
$$

where the first and the last equalities follow from Pareto-optimality, the second from the choice of $\bar{R}_{i}$, and the inequalities from strategy-proofness. Thus, by Pareto-optimality, for all $i \in S_{1}$,

$$
\Phi(R)([\underline{p}(R), x])=\Phi\left(R_{N \backslash\{i\}}, \bar{R}_{i}\right)([\underline{p}(R), x]) .
$$


Therefore, by successively replacing for all $i \in S_{1}$ the relation $R_{i}$ by $\tilde{R}_{i}$,

$$
\Phi(R)([\underline{p}(R), x])=\Phi\left(R_{N \backslash S_{1}}, \bar{R}_{S_{1}}\right)([\underline{p}(R), x]) .
$$

By repeating the above arguments for $\left(R_{N \backslash S_{l}}, \bar{R}_{S_{l}}\right)$ and $\left(R_{N \backslash S_{l+1}}, \bar{R}_{S_{l+1}}\right), l \in\{1, \ldots, m-2\}$, we obtain that

$$
\Phi\left(R_{N \backslash S_{l}}, \bar{R}_{S_{l}}\right)([\underline{p}(R), x])=\Phi\left(R_{N \backslash S_{l+1}}, \vec{R}_{S_{l+1}}\right)([\underline{p}(R), x]) .
$$

Hence, $\Phi(R)([\underline{p}(R), x])=\Phi\left(R_{N \backslash S_{m-1}}, \tilde{R}_{S_{m-1}}\right)([\underline{p}(R), x])$. Using the same arguments as above, we can show that $\left.\left.\left.\left.\Phi\left(R_{N \backslash S_{m-1}}, \bar{R}_{S_{m-1}}\right)(] x, \bar{p}(R)\right]\right)=\Phi(\bar{R})(] x, \bar{p}(R)\right]\right)$. Hence, Paretooptimality yields (3.18).

With the analogous definition of $\bar{R}^{\prime}$ for $R^{\prime}$ we find

$$
\Phi\left(R^{\prime}\right)([\underline{p}(R), x])=\Phi\left(\bar{R}^{\prime}\right)([\underline{p}(R), x]) .
$$

Note that the peak profiles of $\bar{R}$ and $\bar{R}^{\prime}$ coincide and these profiles only have the two different peaks $p^{m}(R)$ and $p^{m+1}(R)$. Thus, by repeated application of Lemma $3.5, \Phi(\bar{R})=$ $\Phi\left(\bar{R}^{\prime}\right)$. Hence, by (3.18) and (3.19), (3.17) holds for all $\left.x \in\right] p^{m}(R), p^{m+1}(R)[$.

If $x \in\left\{p^{m}(R), p^{m+1}(R)\right\}$, then, similarly as in the proof of Lemma 3.1, Theorem A.1 can be used to show (3.17).

Propositions 3.1 and 3.2 enable us to characterize all unanimous, anonymous, and strategy-proof probabilistic rules. Let $Q^{+\infty}$ and $Q^{-\infty}$ denote the probability distributions placing probability 1 on $+\infty$ and $-\infty$, respectively. If $\Phi$ is a unanimous, anonymous, and strategy-proof probabilistic rule, then $\Phi$ is Pareto-optimal and peaks-only. Thus, by Corollary $3.2, \Phi$ is characterized by $n+1$ fixed probability distributions. Consider the determination of these distributions in the proof of Theorem 3.1. Since $\Phi$ is unanimous, it follows that that $D_{\emptyset}$ and $D_{N}$ are the one-point distributions on $+\infty$ and $-\infty$, respectively. Therefore, $D_{\emptyset}=Q^{+\infty}$ and $D_{N}=Q^{-\infty}$. The following corollary summarizes this.

Corollary 3.3 Let $\Phi$ be a probabilistic rule. Then $\Phi$ satisfies unanimity, anonymity, and strategy-proofness if and only if there exists a unique collection of fixed probabilistic ballots $\triangle$ such that $D_{\emptyset}=Q^{+\infty}, D_{N}=Q^{-\infty}$, for all $S, T \in 2^{N}$, if $|S|=|T|$, then $D_{S}=D_{T}$, and $\Phi=\Phi^{\Delta}$.

Remark 3.3 Observe that in the situation of Corollary 3.3, the distributions $D_{\emptyset}$ and $D_{N}$ do not play an active role and can be dropped from the description. Consequently, a unanimous, anonymous, and strategy-proof probabilistic rule is determined by $n-1$ fixed probability distributions. 


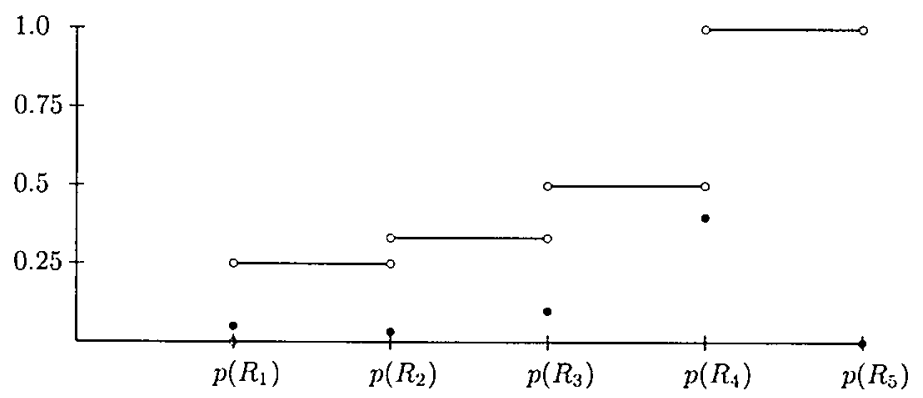

Figure 3.1: The distribution chosen by the probabilistic rule $\Phi^{\Delta}$ at $R$.

Note that by Corollary 3.3, Examples 3.2 and 3.3 violate unanimity. The following is an example of a nontrivial probabilistic rule satisfying unanimity, anonymity and strategyproofness.

Example 3.4 Let $N \equiv\{1, \ldots, 5\}$. We fix four probability distributions $D_{1}, \ldots, D_{4}$ as follows. For all $k \in\{1, \ldots, 4\}, D_{k}$ distributes $\frac{1}{5-k}$ uniformly over $\left[0,1\left[\right.\right.$ and $D_{k}(\{1\}) \equiv$ $1-\frac{1}{5-k}$. Let $D_{0}(\{1\}) \equiv 1$ and $D_{\tilde{j}}(\{0\}) \equiv 1$. Let $\Delta \equiv\left(D_{|S|}\right)_{S \in 2^{N}}$. Let $R \in \mathcal{R}^{N}$ be such that for all $i \in N, p\left(R_{i}\right)=\frac{i}{5}$. Then, using formula (3.12), $\Phi^{\Delta}$ chooses the following distribution.

The probabilities that are placed on the peaks by are $\Phi^{\Delta}(R)\left(\left\{p\left(R_{1}\right)\right\}\right)=\frac{1}{20}, \Phi^{\Delta}(R)$ $\left(\left\{p\left(R_{2}\right)\right\}\right)=\frac{1}{30}, \Phi^{\Delta}(R)\left(\left\{p\left(R_{3}\right)\right\}\right)=\frac{1}{10}, \Phi^{\Delta}(R)\left(\left\{p\left(R_{4}\right)\right\}\right)=\frac{2}{5}$, and $\Phi^{\Delta}(R)\left(\left\{p\left(R_{5}\right)\right\}\right)=0$. In addition, $\Phi^{\Delta}(R)$ distributes uniformly probability $\frac{1}{20}$ over the interval $] p\left(R_{1}\right): p\left(R_{2}\right)\left[: \frac{1}{15}\right.$ over the interval $] p\left(R_{2}\right), p\left(R_{3}\right)\left[, \frac{1}{10}\right.$ over the interval $] p\left(R_{3}\right), p\left(R_{1}\right)\left[\right.$, and $\frac{1}{5}$ over the interval ]$p\left(R_{4}\right), p\left(R_{5}\right)[$. See Figure 3.1 .

A subclass of fixed-probabilistic-ballots rules may be obtained as follows. For $n$ agents, fix $n-1$ probability distributions over $\overline{\mathbb{R}}$ and draw fixed ballots (in the sense of Moulin (1980)) according to these distributions. Next, take the median of these ballots and the peaks of the reported preferences. By using uncompromisingness, it is straightforward to check that such a probabilistic rule is unanimous, anonymous, and strategy-proof, and thus belongs to the class described in Corollary 3.3. Except for the case $n=2$, however, the class of probabilistic rules described in Corollary 3.3 contains rules that cannot be obtained as randomization of Moulin's fixed ballots ("phantom voter") rules.

For $N=\{1,2\}$, let $D_{\emptyset}$ put probability 1 on $+\infty$, let $D_{1} \equiv D_{\{1\}}=D_{\{2\}}$, let $D_{N}$ put probability 1 on $-\infty$, and $\Delta \equiv\left(D_{\emptyset}, D_{\{1\}}, D_{\{2\}}, D_{N}\right)$. The corresponding rule $\Phi^{\Delta}$ is the general form of a unanimous, anonymous, and strategy-proof rule, see Corollary 3.3 . If 
the agents report peaks $a$ and $b$ with $a<b$, then the resulting probability distribution coincides with $D_{1}$ between $a$ and $b$, and puts $D_{1}([-\infty, a])$ on $a$, and $D_{1}([b,+\infty])$ on $b$. Alternatively, taking the median of $a, b$, and a number $\lambda$ drawn according to $D_{1}$ results in exactly the same rule. Therefore, this rule can be interpreted as randomizing between the fixed ballot rules of Moulin.

For $N=\{1,2,3\}$, Corollary 3.3 implies that any unanimous, anonymous, and strategyproof rule is a fixed-probabilistic-ballots rule with $D_{\emptyset}$ and $D_{N}$ as above, and with probabilistic ballots $D_{1}$ and $D_{2}$ for one- and two-person coalitions, respectively. Let $\hat{D}_{1}$ and $\hat{D}_{2}$ be the distribution functions corresponding to $D_{1}$ and $D_{2}$, respectively. That is, for all $x \in \mathbb{R}, \hat{D}_{1}(x) \equiv D_{1}([-\infty, x])$ and $\hat{D}_{2}(x) \equiv D_{2}([-\infty, x])$. Let $a, b, c \in \mathbb{R}$ be such that $a<b<c$ and $R \in \mathcal{R}^{N}$ be such that $p(R)=(a, b, c)$. The fixed-probabilistic-ballots rule generates the distribution function

$$
\hat{\Phi}^{\Delta}(R)(x)= \begin{cases}0 & \text { if } x<a \\ \hat{D}_{1}(x) & \text { if } a \leq x<b \\ \hat{D}_{2}(x) & \text { if } b \leq x<c ; \text { and } \\ 1 & \text { if } x \geq c .\end{cases}
$$

In order to obtain the same result by drawing fixed ballots $\lambda_{1}$ and $\lambda_{2}$ according to distribution functions $\hat{Q}_{1}$ and $\hat{Q}_{2}$ we should have for all $x \in \mathbb{R}$ that

$$
\begin{aligned}
& \hat{D}_{1}(x)=\operatorname{Prob}\left\{\max \left\{\lambda_{1}, \lambda_{2}\right\} \leq x\right\} \\
& \hat{D}_{2}(x)=\operatorname{Prob}\left\{\min \left\{\lambda_{1}, \lambda_{2}\right\} \leq x\right\},
\end{aligned}
$$

which implies that $\hat{Q}_{1}$ and $\hat{Q}_{2}$ should satisfy for all $x \in \mathbb{R}$ :

$$
\hat{D}_{1}(x)=\hat{Q}_{1}(x) \hat{Q}_{2}(x), \hat{D}_{2}(x)=\hat{Q}_{1}(x)+\hat{Q}_{2}(x)-\hat{Q}_{1}(x) \hat{Q}_{2}(x) .
$$

Therefore, for all $x \in \mathbb{R}, \hat{Q}_{1}(x)$ and $\hat{Q}_{2}(x)$ must be the roots of the polynomial $X^{2}-\left(\hat{D}_{1}(x)+\hat{D}_{2}(x)\right) X+\hat{D}_{1}(x)$. Hence, the discriminant of this polynomial must be nonnegative, which implies $\left(\hat{D}_{1}(x)+\hat{D}_{2}(x)\right)^{2} \geq 4 \hat{D}_{1}(x)$. It is easy to see that this inequality does not need to hold, e.g., if $0<\hat{D}_{1}(x)=\hat{D}_{2}(x)<1$.

\subsubsection{Deterministic Rules}

A deterministic rule $\varphi$ chooses for each profile $R \in \mathcal{R}^{N}$ a level $\varphi(R) \in \mathbb{R}$. With each deterministic rule $\varphi$ we associate the corresponding probabilistic rule $\Phi^{\varphi}$ such that for each $R \in \mathcal{R}^{N}, \Phi^{\varphi}(R)$ places probability 1 on the point $\varphi(R)$. Conversely; with a one-point probabilistic rule $\Phi$, i.e., a probabilistic rule that places for each profile probability 1 on 
a single point, we associate the deterministic rule $\varphi^{\Phi}$ in the obvious way. The properties unanimity, Pareto-optimality, anonymity, strategy-proofness, and peaks-onliness, when applied to one-point probabilistic rules, are equivalent to the corresponding properties for deterministic rules. Therefore, they do not need separate introduction.

The literature derives several formulas for the description of the class of deterministic rules satisfying strategy-proofness and peaks-onliness. There is the minmax formula in Moulin (1980), the maxmin formula in Border and Jordan (1983), or the formula for the augmented median solution in Ching (1997) (see Subsection 2.4.2). We will now consider the implications of the expressions (3.11) and (3.12) for deterministic rules, and show that these indeed give the same result. Suppose that $\Phi$ is a one-point probabilistic rule meaning that for each $R \in \mathcal{R}^{N}, \Phi(R)$ places probability one on a unique point in $\mathbb{R}$. By equation (3.12) and the fact that $\Phi$ is deterministic, for all $S \in 2^{N}$ the probabilistic ballot $D_{S}$ must be a one-point distribution over $\overline{\mathbb{R}}$. For every $S \in 2^{N}$, let $a_{S} \in \overline{\mathbb{R}}$ denote the point on which $D_{S}$ places probability 1 . Since $D_{\emptyset}(\{-\infty\})=0$ and $D_{N}(\{+\infty\})=0$, we obtain $a_{\emptyset}>-\infty$ and $a_{N}<+\infty$. Condition (3.11) implies that for all $S, T \subseteq N$

$$
a_{S \cup T} \leq a_{S}
$$

(If $a_{S}<a_{S \cup T}$, then $D_{S \cup T}\left(\left[-\infty, \frac{1}{2}\left(a_{S \cup T}+a_{S}\right)\right]\right)-D_{S}\left(\left[-\infty, \frac{1}{2}\left(a_{S \cup T}+a_{S}\right)[)=-1\right.\right.$, which contradicts (3.11).) For all $R \in \mathcal{R}^{N}$ and all $X \in \mathcal{L}$, equation (3.12) yields

$$
\begin{aligned}
\Phi(R)(X) \equiv & \sum_{l=0}^{k} 1_{X \cap] p^{l}(R), p^{l+1}(R)[}\left(a_{S_{l}}\right) \\
& +\sum_{l=1}^{k} 1_{X}\left(p^{l}(R)\right)\left(1_{\left[-\infty, p^{l}(R)\right]}\left(a_{S_{l}}\right)-1_{\left[-\infty, p^{l}(R)[\right.}\left(a_{S_{l-1}}\right)\right)
\end{aligned}
$$

which implies

$$
\varphi^{\Phi}(R)=\sum_{l=0}^{k} a_{S_{l}} 1_{\left[p^{l}(R), p^{l+1}(R)[\right.}\left(a_{S_{l}}\right)+\sum_{l=1}^{k} p^{l}(R) 1_{\left[a_{S_{l}}, a_{S_{l-1}}[\right.}\left(p^{l}(R)\right)
$$

Now consider the collection of points $p^{1}(R), \ldots, p^{k}(R)$ and $a_{S_{0}}, \ldots, a_{S_{k}}$ such that both $p^{l}(R)$ and $a_{S_{l}}$ are taken with multiplicity equal to the number of agents in $S_{l} \backslash S_{l-1}$. According to the minmax formula of Moulin (1980) the median of these points should be chosen at the profile $R$. The assignment of $\varphi^{\Phi}$ in (3.21) does exactly that. If $a_{S_{l}}$ is the median, then $p^{l}(R) \leq a_{S_{l}}<p^{l+1}(R)$ and thus $\varphi^{\Phi}(R)=a_{S_{l}}$. If $p^{l}(R)$ is the median, then $a_{S_{l}} \leq p^{l}(R)<a_{S_{l-1}}$ and thus $\varphi^{\Phi}(R)=p^{l}(R)$. Conversely; if $\varphi^{\Phi}(p)=a_{S_{l}}$ then $p^{l}(R) \leq a_{S_{l}}<p^{l+1}(R)$ and $a_{S_{l}}$ is the median, and if $\varphi^{\Phi}(R)=p^{l}(R)$ then $a_{S_{l}} \leq p^{l}(R)<a_{S_{l-1}}$ and $p^{l}(R)$ is the median. 
Let us now consider the relationship between an implication of our results for the deterministic case and those in the literature, notably Moulin (1980). One result there characterizes all anonymous, strategy-proof, Pareto-optimal, and peaks-only deterministic rules. These assign the median of the peaks of the reported profile and $n-1$ fixed ballots. Because all our results apply to one-point probabilistic rules, they also apply to deterministic rules. In particular, Corollary 3.3 and Remark 3.3 imply the following result which is a strengthening of the main result in Moulin (1980) for preferences that are strict on either side of the peak. Also Ching (1997) proves this result in the deterministic setting.

Theorem 3.2 Let $\varphi$ be a deterministic rule. Then $\varphi$ is unanimous, anonymous, and strategy-proof if and only if there exist $n-1$ fixed ballots such that for each $R \in \mathcal{R}^{N}, \varphi$ chooses the median of the peaks at $R$ and the $n-1$ fixed ballots. Alternatively, $\varphi$ can be described by (3.21) such that $a_{\emptyset}=+\infty, a_{N}=-\infty$, and for $S, T \in 2^{N}$, if $|S|=|T|$, then $a_{S}=a_{T}$. Moreover, these fixed ballots are uniquely determined.

Let $\varphi$ be a deterministic rule. Then $\varphi$ satisfies uncompromisingness if for all $i \in N$ and all $R, \bar{R} \in \mathcal{R}^{N}$, if $R$ and $\vec{R}$ are $i$-deviations and either $p\left(R_{i}\right)<\varphi(R)$ and $p\left(\bar{R}_{i}\right) \leq \varphi(R)$ or $p\left(R_{i}\right)>\varphi(R)$ and $p\left(\tilde{R}_{i}\right) \geq \varphi(\bar{R})$, then $\varphi(\bar{R})=\varphi(R)$.

Lemma 3.6 A deterministic rule satisfies uncompromisingness if and only if the associated one-point probabilistic rule satisfies uncompromisingness.

Proof. The only-if part is left to the reader. For the if-part, let $\Phi$ be a one-point probabilistic rule. We show that $\varphi^{\Phi}$ satisfies uncompromisingness. Let $i \in N$ and $R, \bar{R} \in$ $\mathcal{R}^{N}$ be $i$-deviations. Let $x \in \mathbb{R}$ be such that $\Phi(R)(\{x\})=1$. Suppose $p\left(R_{i}\right)<x$. Thus, $\varphi^{\Phi}(R)=x$. If $p\left(\bar{R}_{i}\right)<x$, then, by uncompromisingness, $\Phi(\bar{R})$ places probability 1 on $x$. Thus, $\varphi^{\Phi}(\bar{R})=x$.

Suppose $p\left(\bar{R}_{i}\right)=x$. By uncompromisingness, $\Phi(\bar{R})\left(\left[p\left(R_{i}\right), x\right]\right)=\Phi(R)\left(\left[p\left(R_{i}\right), x\right]\right)=1$. Since $\Phi(\bar{R})$ is a one-point distribution, there exists $y \in\left[p\left(R_{i}\right), x\right]$ such that $\Phi(\bar{R})$ puts weight 1 on $y$. Therefore, $\varphi^{\Phi}(\bar{R})=y$. It suffices to show $y=x$. If $y<x$, then let $R^{\prime} \in \mathcal{R}$ be an $i$-deviation from $R$ such that $p\left(R_{i}^{\prime}\right)=y$. Like above it follows that $\Phi\left(R^{\prime}\right)$ is the one-point probability measure on $x$, i.e., $\Phi\left(R^{\prime}\right)(\{y\})=0$. But then agent $i$ can increase this probability by deviating to $\vec{R}_{i}$, contradicting strategy-proofness. Thus, $y=x$ and $\varphi^{\Phi}(\bar{R})=x$, the desired conclusion.

Lemma 3.6 and Corollary 3.1 imply that a deterministic rule satisfies strategy-proofness and peaks-onliness if and only if it satisfies uncompromisingness. Ching (1997) also shows this result. 
The following example establishes the independence of unaninity from all the other properties.

Example 3.5 Let $N \equiv\{1\}$ and for all $R \in \mathcal{R}^{N}$,

$$
\varphi(R) \equiv\left\{\begin{array}{cl}
-1 & \text { if } p\left(R_{1}\right)<-1 \\
p\left(R_{1}\right) & \text { if } p\left(R_{1}\right) \in[-1,1] \\
1 & \text { if } p\left(R_{1}\right)>1
\end{array}\right.
$$

It is straightforward to check that $\varphi$ satisfies anonymity, strategy-proofness, peaks-onliness, and uncompromisingness, but not unanimity.

The unanimity requirement can be seen, basically, as a condition on the range of the rule. In the deterministic framework and in the presence of strategy-proofness it can be replaced by surjectivity of the rule or by a direct condition on the range. The latter is employed in Barberà and Jackson (1994, Theorem 3). In the probabilistic setting, however, it is not clear what an alternative appropriate range condition is.

In the deterministic setting, Ching shows that in the presence of strategy-proofness, peaks-onliness and continuity are equivalent. Continuity means that for a given preference profile, for each agent small changes in his preference relation only cause small changes of the point chosen by the deterministic rule. In the probabilistic setting there are several definitions of "small changes" in probability distributions (for example relative to the weak convergence topology). We leave this and the above point for further research.

\subsection{Solidarity}

\subsubsection{Definitions}

Throughout this section the population is variable. We assume that $\mathbb{P}$ contains at least four agents and $\mathcal{P}$ is the class of all non-empty and finite subsets of $\mathbb{P}$ with cardinality greater than or equal to three.

In applications the environment of an economy may change. When such a change occurs, the decisions made by a rule before and after change in the enviromment may be different. If the agents whose "parameters" are the same before and after the change are not responsible for this change, then it would be unfair if a change in the environment affected their welfares in different directions. A solidarity property requires that the consequences of such changes are shared by all agents whose characteristics are the same before and after the change. The welfares of all these agents should be affected in the same direction: as a result they all weakly gain or they all weakly lose. 
First, we apply the idea of solidarity to population changes. We require that when a group of agents leaves or comes in, the welfares of all agents who are present before and after the change in population are affected in the same direction. Instead of considering all possible changes in population we only consider the arrival or departure of one agent.

Population-Monotonicity: For all $N \in \mathcal{P}$; all $j \in \mathbb{P} \backslash N$, and all $R \in \mathcal{R}^{N \cup\{j\}}$, either [for all $i \in N, \Phi\left(R_{N}\right) R_{i} \Phi(R)$ ] or [for all $i \in N, \Phi(R) R_{i} \Phi\left(R_{N}\right)$ ].

Thomson (1983a) introduces population-monotonicity in the context of bargaining. Our notion of population-monotonicity is due to Chun (1986) who calls it "solidarity". For a survey on population-monotonicity we refer to Thomson (1995).

The following lemma is analogous to Lemma 2.21. It states that under Pareto-optimality and population-monotonicity, if an agent leaves, then all agents that remain present after weakly gain as a result of the departure. We omit the obvious proof.

Lemma 3.7 Let $\Phi$ be a probabilistic rule sutisfying Pareto-optimality and populationmonotonicity. Then for all $N \in \mathcal{P}$, all $j \in \mathbb{P} \backslash N$, and all $R \in \mathcal{R}^{N \cup\{j\}}$, we have for all $i \in N, \Phi\left(R_{N}\right) R_{i} \Phi(R)$.

Second, we apply the idea of solidarity to changes in preference relations. When the preference relation of an agent changes, then the welfares of all agents whose preference relations remained the same should be affected in the same direction. Either they all weakly gain or they all weakly lose as a result of the change of an agent's preference relation.

Replacement-Domination: For all $N \in \mathcal{P}$, all $j \in N$, and all $R, \bar{R} \in \mathcal{R}^{N}$ such that $R_{N \backslash\{j\}}=\bar{R}_{N \backslash\{j\}}$, either [for all $i \in N \backslash\{j\}, \Phi(R) R_{i} \Phi(\bar{R})$ ] or [for all $i \in N \backslash\{j\}$, $\left.\Phi(\bar{R}) R_{i} \Phi(R)\right]$.

Moulin (1987) introduces replacement-domination in the context of binary choice with quasi-linear preferences. Thomson $(1993,1997)$ calls it welfare-domination under preferencereplacement and studies its implications in different settings, e.g., in public and private good economies with single-peaked preferences. For a survey, we refer to Thomson (1999a).

It will turn out that replacement-domination is a strong requirement. We also propose a weakening of replacement-domination. This condition only requires solidarity when the Pareto set before the change of an agent's preference relation is either a subset or a superset of the Pareto set after the change. We call these changes one-sided because we restrict changes in the Pareto set to changes of "one of its sides". This property does not require 
solidarity if the peak level of the agent whose preference relation changes is the unique smallest peak level at the initial profile, and his new peak level is the unique greatest peak level at the new profile, or vice versa.

One-Sided Replacement-Domination: For all $N \in \mathcal{P}$, all $j \in N$, and all $R, \bar{R} \in R^{N}$ such that $R_{N \backslash\{j\}}=\bar{R}_{N \backslash\{j\}}$ and $E(R) \subseteq E(\bar{R})$ or $E(R) \supseteq E(\bar{R})$, either [for all $i \in N \backslash\{j\}$, $\left.\Phi(R) R_{i} \Phi(\bar{R})\right]$ or [for all $i \in N \backslash\{j\}, \Phi(\bar{R}) R_{i} \Phi(R)$ ].

For allocation problems with single-peaked preferences, Thomson (1997) proves that Pareto-optimality, replacement-domination, and certain equity properties are incompatible. This incompatibility only occurs if the unilateral change of the preference profile is "too disruptive". Here, "too disruptive" means changing an economy from excess demand to excess supply or vice versu. Thomson (1997) introduces one-sided replacementdomination in private good economies with single-peaked preferences. One-sidedness in both moclels boils down to the fact that the Pareto set of the original economy is either a subset or a superset of the Pareto set of the economy after the deviation.

Proposition 3.1 and Corollary 3.3 yield the following characterization of all probabilistic rules satisfying Pareto-optimality, anonymity, and strategy-proofness. Here, it is straightforward to adjust the definition of strategy-proofness to the variable population setting.

Theorem 3.3 Let $\Phi$ be a probabilistic rule. Then $\Phi$ satisfies Pareto-optimality, anonymity, and strategy-proofness if and only if for all $N \in \mathcal{P}$, there exist $|N|-1$ fixed distributions over $\overline{\mathbb{R}}$, denoted by $D_{1}^{N}, \ldots, D_{|N|-1}^{N}$, such that (i) for all $l \in\{2, \ldots,|N|-1\}$ and all $x \in \mathbb{R}, D_{l}^{N}([-\infty, x]) \geq D_{l-1}^{N}\left(\left[-\infty, x[)\right.\right.$ and (ii) for all $R \in \mathcal{R}^{N}$, all $X \in \mathcal{L}, D_{0}^{N} \equiv Q^{+\infty}$, and $D_{|N|}^{N} \equiv Q^{-\infty}$,

$$
\begin{aligned}
\Phi(R)(X) & =\sum_{l=1}^{k-1} D_{l}^{N}(X \cap] p^{l}(R), p^{l+1}(R)[) \\
& +\sum_{l=1}^{k} 1_{X}\left(p^{l}(R)\right)\left(D_{l}^{N}\left(\left[-\infty, p^{l}(R)\right]\right)-D_{l-1}^{N}\left(\left[-\infty, p^{l}(R)[)\right) .\right.\right.
\end{aligned}
$$

Moreover, all $D_{l}^{N}$ are uniquely determined.

\subsubsection{Logical Relations}

In this subsection we investigate the relations among strategy-proofness, anonymity, and the solidarity properties defined in Subsection 3.4.1. We have introduced one-sided re- 
placement domination mainly as an auxiliary requirement. Lnder Pareto-optimality, we show the following relations among the three solidarity properties.

population-monotonicity

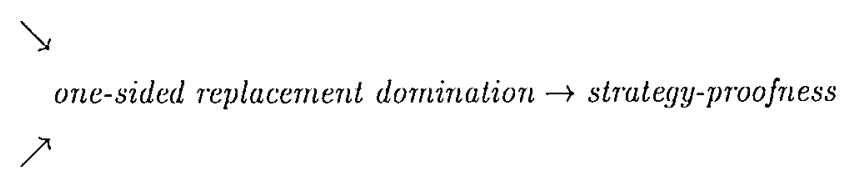

replacenent-domination

Therefore, one-sided replacement-domination allows us to show in one step that Paretooptimality and either soliclarity property imply strategy-proofness. We also show that Pareto-optimality and one-sided replacement-domination imply anonymity.

We prove these results in several steps. The first lemma will be a useful tool.

Lemma 3.8 For all $N \in \mathcal{P}$, all $R \in \mathcal{R}^{N}$, all $i, j \in N$ such that $p\left(R_{i}\right) \leq p\left(R_{j}\right)$, and all distributions $Q, Q^{\prime}$ over $\mathbb{R}$, if $Q R_{i} Q^{\prime}, Q R_{j} Q^{\prime}$, and $Q\left(\left[p\left(R_{i}\right), p\left(R_{j}\right)\right]\right)=1=Q^{\prime}\left(\left[p\left(R_{i}\right), p\left(R_{j}\right)\right]\right)$, then $Q=Q^{\prime}$.

Proof. Let $x \in\left[p\left(R_{i}\right), p\left(R_{j}\right)\right]$. Then, $Q\left(\left[p\left(R_{i}\right), p\left(R_{j}\right)\right]\right)=1=Q^{\prime}\left(\left[p\left(R_{i}\right), p\left(R_{j}\right)\right]\right)$ and $Q R_{i} Q^{\prime}$ imply $Q\left(\left[p\left(R_{i}\right), x\right]\right)=Q\left(B\left(x, R_{i}\right)\right) \geq Q^{\prime}\left(B\left(x, R_{i}\right)\right)=Q^{\prime}\left(\left[p\left(R_{i}\right), x\right]\right)$. Similarly, $Q R_{j} Q^{\prime}$ implies $\left.\left.\left.Q(] x, p\left(R_{j}\right)\right]\right) \geq Q^{\prime}(] x, p\left(R_{j}\right)\right]$. Hence, all inequalities are equalities because $Q\left(\left[p\left(R_{i}\right), p\left(R_{j}\right)\right]\right)=1=Q^{\prime}\left(\left[p\left(R_{i}\right), p\left(R_{j}\right)\right]\right)$. Therefore, for all intervals $\left.] a, b\right] \subseteq \mathbb{R}$; $\left.\left.Q(] a, b])=Q^{\prime}(] a, b\right]\right)$. Because the $\sigma$-algebra $\mathcal{L}$ is generated by those intervals, it follows that $Q=Q^{\prime}$.

Applying Pareto-optimality, population-monotonicity, and Lemma 3.8 yields the following.

Lemma 3.9 Let $\Phi$ be a probabilistic rule satisfying Pareto-optimality and populationmonotonicity. For all $N \in \mathcal{P}$, all $j \in \mathbb{P} \backslash N$, and all $R \in \mathcal{R}^{N \cup\{j\}}$, if $E\left(R_{N}\right)=E(R)$, then $\Phi\left(R_{N}\right)=\Phi(R)$

Successive application of Lemma 3.9 yields the following.

Lemma 3.10 Let $\Phi$ be a probabilistic rule satisfying Pareto-optimality and populationmonotonicity. Then $\Phi$ satisfies anonymity.

Next, we prove that Pareto-optimality and population-monotonicity imply one-sided replacement-domination. 
Proposition 3.3 Pareto-optimality and population-monotonicity imply one-sided replacement-domination.

Proof. By Lemma 3.10, $\Phi$ satisfies anonymity. Let $N \in \mathcal{P}, j \in N$, and $R, \tilde{R} \in \mathcal{R}^{N}$ be such that $R_{N \backslash\{j\}}=\bar{R}_{N \backslash\{j\}}$ and $E(R) \subseteq E(\bar{R})$. Without loss of generality, we suppose that $\underline{p}(R)=\underline{p}(\vec{R})$. We show that for all $i \in N \backslash\{j\}$,

$$
\Phi(R) R_{i} \Phi(\vec{R})
$$

which proves one-sided replacement-domination. We distinguish two cases.

Case 1: $\mathbb{P} \backslash N \neq \emptyset$.

Let $k \in \mathbb{P} \backslash N$ and $\tilde{R} \in \mathcal{R}^{N \cup\{k\}}$ be such that $\tilde{R}_{N}=R$ and $\tilde{R}_{k}=\bar{R}_{j}$. By Lemma 3.7, for all $i \in N, \Phi(R) R_{i} \Phi(\tilde{R})$. By Lemma 3.9 and $E(R) \subseteq E(\bar{R}), \Phi\left(\tilde{R}_{(N \cup\{k\}) \backslash\{j\}}\right)=\Phi(\tilde{R})$. By anonymity, $\Phi\left(\tilde{R}_{(N \cup\{k\}) \backslash\{j\}}\right)=\Phi(\tilde{R})$. The previous three facts imply (3.23).

Case 2: $N=\mathbb{P}$.

If $E(R)=E(\bar{R})$, then Lemma 3.9 and Case 1 imply (3.23). Let $E(R) \subsetneq E(\bar{R})$ and suppose that $p\left(R_{j}\right)<\bar{p}(R)$. By Lemma 3.9, $\Phi\left(R_{N \backslash\{j\}}\right)=\Phi(R)$. Let $R^{\prime} \in \mathcal{R}^{N}$ be such that $R_{N \backslash\{j\}}^{\prime}=R_{N \backslash\{j\}}$ and $p\left(R_{j}^{\prime}\right)=\bar{p}\left(R_{N \backslash\{j\}}\right)$. By Lemma 3.9 and the previous fact, $\Phi\left(R^{\prime}\right)=\Phi\left(R_{N \backslash\{j\}}\right)=\Phi(R)$. Therefore, without loss of generality, we may assume that $p\left(R_{j}\right)=\bar{p}(R)$.

Because $|\mathbb{P}| \geq 4, \underline{p}(R)=\underline{p}(\bar{R}), p\left(R_{j}\right)=\bar{p}(R)$, and $p\left(\bar{R}_{j}\right)=\bar{p}(\bar{R})$, there exist $l, k \in N$ such that $E\left(R_{N \backslash\{k\}}\right)=E(R)=E\left(R_{N \backslash\{l\}}\right)$ and $E\left(\bar{R}_{N \backslash\{k\}}\right)=E(\bar{R})=E\left(\bar{R}_{N \backslash\{l\}}\right)$. By Lemma 3.9, $\Phi\left(R_{N \backslash\{k\}}\right)=\Phi(R)$ and $\Phi\left(\bar{R}_{N \backslash\{k\}}\right)=\Phi(\bar{R})$. Thus, by Case 1, for all $i \in$ $N \backslash\{j, k\}$,

$$
\Phi(R) R_{i} \Phi(\bar{R})
$$

Similarly, by considering $N \backslash\{l\}$, it follows that for all $i \in N \backslash\{j, l\}, \Phi(R) R_{i} \Phi(\bar{R})$. The previous fact and (3.24) imply (3.23).

Applying Pareto-optimality, one-sided replacement-domination, and Lemma 3.8 yields the following.

Lemma 3.11 Let $\Phi$ be a probabilistic rule satisfying Pareto-optimality and one-sided replacement-domination. For all $N \in \mathcal{P}$, all $j \in N$, and all $R, \bar{R} \in \mathcal{R}^{N}$ such that $R_{N \backslash\{j\}}=\bar{R}_{N \backslash\{j\}}$, if $E(R)=E(\bar{R})$, then $\Phi(R)=\Phi(\bar{R})$.

Successive application of Lemma 3.11 yields the following. 
Lemma 3.12 Let $\Phi$ be a probabilistic rule satisfying Pareto-optimality and one-sided replacement-domination. Then $\Phi$ satisfies anonymity.

The following implication of Pareto-optimality and one-sided replacement-domination is useful later.

Lemma 3.13 Let $\Phi$ be a probabilistic rule satisfying Pareto-optimality and one-sided replacement-domination. For all $N \in \mathcal{P}$, all $j \in N$, and all $R, \bar{R} \in \mathcal{R}^{N}$ such that $R_{N \backslash\{j\}}=\bar{R}_{N \backslash\{j\}}$, if $E(R) \subsetneq E(\bar{R})$, then for all $i \in N \backslash\{j\}, \Phi(R) R_{i} \Phi(\bar{R})$.

Proof. Without loss of generality, we suppose that $\underline{p}(R)=\underline{p}(\vec{R})$. Assume, by contradiction, that for all $i \in N \backslash\{j\}, \Phi(\bar{R}) R_{i} \Phi(R)$, and for some $l \in N \backslash\{j\}, \Phi(\bar{R}) P_{l} \Phi(R)$. Thus, for some $x \in \mathbb{R}, \Phi(\bar{R})\left(B\left(x, R_{l}\right)\right)>\Phi(R)\left(B\left(x, R_{l}\right)\right)$. Without loss of generality, we suppose that $B\left(x, R_{l}\right)=[y, x]$. Hence,

$$
\Phi(\bar{R})([y, x])>\Phi(R)([y, x])
$$

Since $E(R) \subsetneq E(\bar{R})$, we have $\underline{p}\left(R_{N \backslash j\}}\right)=\underline{p}(R)$ and $p\left(\bar{R}_{j}\right)=\bar{p}(\bar{R})$. Let $k \in N \backslash\{j\}$ be such that $p\left(R_{k}\right)=\underline{p}(R)$. We show that

$$
\Phi(\bar{R}) P_{k} \Phi(R)
$$

If $y \leq \underline{p}(R)$, then by Pareto-optimality, $p\left(R_{k}\right)=\underline{p}(R)=\underline{p}(\bar{R})$, and (3.25), it follows that $\Phi(\bar{R})\left(\bar{B}\left(x, R_{k}\right)\right)>\Phi(R)\left(B\left(x, R_{k}\right)\right)$. The previous fact and $\Phi(\bar{R}) R_{k} \Phi(R)$ imply $(3.26)$.

If $\underline{p}(R)<y$, then

$$
\Phi(\bar{R})\left(\left[\underline{p}(R), y[)=\Phi(\bar{R})\left(B^{o}\left(y, R_{k}\right)\right) \geq \Phi(R)\left(B^{o}\left(y, R_{k}\right)\right)=\Phi(R)([\underline{p}(R), y[),\right.\right.
$$

where the equalities follow from Pareto-optimality, and the inequality from $\Phi(\bar{R}) R_{k} \Phi(R)$. Therefore, $\Phi(\bar{R})([\underline{p}(R), y[) \geq \Phi(R)([\underline{p}(R), y[)$. This and $(3.25)$ yields $\Phi(\bar{R})([\underline{p}(R), x])>$ $\Phi(R)([\underline{p}(R), x])$. So, by Pareto-optimality and $p\left(R_{k}\right)=\underline{p}(R)=\underline{p}(\bar{R}), \Phi(\bar{R})\left(B\left(x, R_{k}\right)\right)>$ $\Phi(R)\left(B\left(x, R_{k}\right)\right)$. The previous fact and $\Phi(\bar{R}) R_{k} \Phi(R)$ imply $(3.26)$.

Let $h \in N \backslash\{k, j\}$ and $R^{\prime} \in \mathcal{R}^{N}$ be such that $R_{N \backslash\{h\}}^{\prime}=R_{N \backslash h\}}$ and $p\left(R_{h}^{\prime}\right)=\bar{p}(R)$. Let $\bar{R}^{\prime} \in \mathcal{R}^{N}$ be such that $\bar{R}_{N \backslash\{h\}}^{\prime}=\bar{R}_{N \backslash\{h\}}$ and $\ddot{R}_{h}^{\prime}=R_{h}^{\prime}$. By Lemma 3.11, $\Phi\left(R^{\prime}\right)=\Phi(R)$ and $\Phi\left(\bar{R}^{\prime}\right)=\Phi(\bar{R})$. Since $R_{k}^{\prime}=R_{k}=\bar{R}_{k}^{\prime},(3.26)$ and the previous two equalities imply

$$
\Phi\left(\bar{R}^{\prime}\right) P_{k} \Phi\left(R^{\prime}\right)
$$

Then, $\Phi\left(\bar{R}^{\prime}\right)\left(B\left(\bar{p}\left(R^{\prime}\right), R_{k}\right)\right) \geq \Phi\left(R^{\prime}\right)\left(B\left(\bar{p}\left(R^{\prime}\right), R_{k}\right)\right)=\Phi\left(R^{\prime}\right)\left(\left[\underline{p}\left(R^{\prime}\right), \bar{p}\left(R^{\prime}\right)\right]\right)=1$, where the inequality follows from (3.27), and the equalities from Pareto-optimality. Hence, 
$\Phi\left(\bar{R}^{\prime}\right)\left(\left[\underline{p}\left(R^{\prime}\right), \bar{p}\left(R^{\prime}\right)\right]\right)=1$. Thus, one-sided replacement-domination and (3.27) yield $\Phi\left(\bar{R}^{\prime}\right) P_{k} \Phi\left(R^{\prime}\right)$ and $\Phi\left(\bar{R}^{\prime}\right) R_{h} \Phi\left(R^{\prime}\right)$. Because $\left[p\left(R_{k}\right), p\left(R_{h}\right)\right]=\left[p\left(R^{\prime}\right), \bar{p}\left(R^{\prime}\right)\right]$, the three previous facts and Lemma $3.8 \mathrm{imply} \Phi\left(\bar{R}^{\prime}\right)=\Phi\left(R^{\prime}\right)$. Hence, $\Phi\left(R^{\prime}\right) R_{k} \Phi\left(\bar{R}^{\prime}\right)$, which contradicts (3.27). Therefore, assumption (3.25) was wrong and it follows that for all $i \in N \backslash\{j\}$, $\Phi(R) R_{i} \Phi(\vec{R})$.

Next, we show that Pareto-optimality and one-sided replacement-domination imply strategy-proofness.

Proposition 3.4 Pareto-optimality and one-sided replacement-domination imply anonymity and strategy-proofness.

Proof. By Lemma 3.12, $\Phi$ satisfies anonymity. Let $N \in \mathcal{P}, j \in N$, and $R, \bar{R} \in \mathcal{R}^{N}$ be such that $R_{N \backslash\{j\}}=\bar{R}_{N \backslash\{j\}}$. We have to show that $\Phi(R) R_{j} \Phi(\bar{R})$. We consider four cases.

Case 1: $E(R)=E(\bar{R})$.

By Lemma 3.11, $\Phi(R)=\Phi(\bar{R})$. Thus, $\Phi(R) R_{j} \Phi(\tilde{R})$, the desired conclusion.

Case 2: $E(R) \subseteq E(\bar{R})$.

Without loss of generality, we suppose that $\underline{p}(R)=\underline{p}(\bar{R})$. Let $k \in N \backslash\{j\}$ be such that $p\left(R_{k}\right)=\underline{p}(R)$, and $l \in N \backslash\{k, j\}$. Let $R^{\prime} \in \mathcal{R}^{N}$ be such that $R_{N \backslash\{l\}}^{\prime}=R_{N \backslash\{l\}}$ and $R_{l}^{\prime}=\bar{R}_{j}$. Thus, $E\left(R^{\prime}\right)=E(\bar{R})$ and $E(R) \subseteq E\left(R^{\prime}\right)$. By Lemma 3.13, for all $i \in N \backslash\{l\}, \Phi(R) R_{i} \Phi\left(R^{\prime}\right)$. In particular, $\Phi(R) R_{j} \Phi\left(R^{\prime}\right)$. By anonymity and Lemma 3.11, $\Phi\left(R^{\prime}\right)=\Phi(\bar{R})$. Thus, $\Phi(R) R_{j} \Phi(\bar{R})$, the desired conclusion.

Case 3: $E(R) \supsetneq E(\bar{R})$.

Without loss of generality, we suppose that $\underline{p}(R)=\underline{p}(\bar{R})$. Let $k \in N \backslash\{j\}$ be such that $p\left(R_{k}\right)=\underline{p}(R)$. By Lemma 3.13, $\Phi(\bar{R}) R_{k} \Phi(R)$. Thus, for all $x \in[\underline{p}(\bar{R}), \bar{p}(\bar{R})]$,

$$
\Phi(\bar{R})([\underline{p}(\bar{R}), x]) \geq \Phi(R)([\underline{p}(\bar{R}), x]) .
$$

By Pareto-optimality, $\Phi(R)([\underline{p}(R), \bar{p}(R)])=1$. Because $\underline{p}(R)=\underline{p}(\bar{R})$ and $\bar{p}(\bar{R})<\bar{p}(R)$, (3.28) implies that for all $x \in[\underline{p}(R), \bar{p}(R)], \Phi(R)(] x, \bar{p}(R)]) \geq \Phi(\bar{R})(] x, \bar{p}(R)])$. Hence, by Pareto-optimality and $p\left(R_{j}\right)=\bar{p}(R)$, for all $x \in[\underline{p}(R), \bar{p}(R)], \Phi(R)\left(B^{o}\left(x, R_{j}\right)\right) \geq$ $\Phi(\bar{R})\left(B^{\circ}\left(x, R_{j}\right)\right)$. Thus, $\Phi(R) R_{j} \Phi(\bar{R})$, the desired conclusion.

Case 4: $E(R) \backslash E(\bar{R}) \neq \emptyset$ and $E(\bar{R}) \backslash E(R) \neq \emptyset$.

Without loss of generality, we suppose that $p\left(R_{j}\right)>\bar{p}\left(R_{N \backslash\{j\}}\right)$. Thus, $p\left(\bar{R}_{j}\right)<$ $\underline{p}\left(R_{N \backslash\{j\}}\right)$. Let $R^{\prime} \in \mathcal{R}^{N}$ be such that $R_{N \backslash\{j\}}^{\prime}=R_{N \backslash\{j\}}$ and $p\left(R_{j}^{\prime}\right)=\bar{p}\left(R_{N \backslash\{j\}}\right)$. By Case 3, $\Phi(R) R_{j} \Phi\left(R^{\prime}\right)$. By Case 2, $\Phi\left(R^{\prime}\right) R_{j}^{\prime} \Phi(\bar{R})$. By Pareto-optimality, for all $x \in\left[\underline{p}\left(R^{\prime}\right), \bar{p}\left(R^{\prime}\right)\right]$; $\Phi\left(R^{\prime}\right)\left(B\left(x, R_{j}^{\prime}\right)\right)=\Phi\left(R^{\prime}\right)\left(\left[x, p\left(R_{j}^{\prime}\right)\right]\right)=\Phi\left(R^{\prime}\right)\left(B\left(x, R_{j}\right)\right)$ and $\Phi(\bar{R})\left(\bar{B}\left(x, R_{j}^{\prime}\right)\right)=$ 
$\Phi(\bar{R})\left(\left[x, p\left(R_{j}^{\prime}\right)\right]\right)=\Phi(\bar{R})\left(B\left(x, R_{j}\right)\right)$. Therefore, it follows that for all $x \in[\underline{p}(R), \bar{p}(R)]$, $\Phi(R)\left(B\left(x, R_{j}\right)\right) \geq \Phi(\bar{R})\left(B\left(x, R_{j}\right)\right)$. Hence, $\Phi(R) R_{j} \Phi(\bar{R})$, the desired conclusion.

\subsubsection{Target Rules}

The following classes of "target rules" play the central role in the sequel. A deterministic target rule is determined by its target level. If the target level is Pareto-optimal, then the rule chooses the target level. If the target level is not Pareto-optimal, then the rule chooses the Pareto-optimal level that is closest to the target level.

Let med denote the median operator; i.e., if $x \leq y \leq z$, then $\operatorname{med}(x, y, z) \equiv y$. Since for all $N \in \mathcal{P}$ and all $R \in \mathcal{R}^{N}, \underline{p}(R), \bar{p}(R) \in \mathbb{R}$, it follows that for all $a \in \overline{\mathbb{R}}$; $\operatorname{med}(\underline{p}(R), a, \bar{p}(R)) \in \mathbb{R}$.

Deterministic Target Rules, $\varphi^{a}$ : Given $a \in \overline{\mathbb{R}}$, the deterministic target rule $\varphi^{a}$ based on target level $a$ is defined as follows. For all $N \in \mathcal{P}$ and all $R \in \mathcal{R}^{N}, \varphi^{a}(R) \equiv$ $\operatorname{med}(\underline{p}(R), a, \bar{p}(R))$.

Given $N \in \mathcal{P}$, we say that a probabilistic rule $\Phi$ is a deterministic target rule on the domain $\mathcal{R}^{N}$, if there exists $a^{N} \in \overline{\mathbb{R}}$ such that for all $R \in \mathcal{R}^{N}, \Phi(R)\left(\left\{\varphi^{a^{N}}(R)\right\}\right)=1$.

In many public choice decision processes, target oriented decisions where the target equals the status quo are prevailing; for instance if all participants have veto power over changes in the status quo.

A probabilistic target rule is determined by its target distribution. In the interior of the Pareto set, the target distribution is applied. Probability outside the Pareto set is projected to the closest level belonging to the Pareto set.

Probabilistic Target Rules, $\Phi^{Q}:$ Given distribution $Q$ over $\overline{\mathbb{R}}$, the probabilistic target rule $\Phi^{Q}$ based on target distribution $Q$ is defined as follows. For all $N \in \mathcal{P}$ and all $R \in \mathcal{R}^{N}$, if $\underline{p}(R) \neq \bar{p}(R)$, then for all $X \in \mathcal{L}$,

$$
\begin{aligned}
\Phi^{Q}(R)(X)= & 1_{X}(\underline{p}(R)) Q([-\infty, \underline{p}(R)])+Q(X \cap] \underline{p}(R), \bar{p}(R)[) \\
& +1_{X}(\bar{p}(R)) Q([\bar{p}(R),+\infty])
\end{aligned}
$$

and if $p(R)=\bar{p}(R)$, then for all $X \in \mathcal{L}$,

$$
\Phi^{Q}(R)(X)=1_{X}(\underline{p}(R))
$$

It is easy to check that all probabilistic target rules satisfy Pareto-optimality and population-monotonicity. If the target distribution assigns probability 1 to a single point 
$a \in \overline{\mathbb{R}}$, then the probabilistic target rule $\Phi^{Q}$ is identical to the deterministic target rule $\varphi^{a}$.

Remark 3.4 All probabilistic target rules satisfy Pareto-optimality, strategy-proofness, and anonymity. Probabilistic target rules are a special subclass of the class of rules as characterized in Theorem 3.3: a probabilistic rule as described in Theorem 3.3 is a probabilistic target rule if and only if all fixed probabilistic ballots are the same, i.e., for all $N, M \in \mathcal{P}, D_{1}^{N}=\cdots=D_{|N|-1}^{N}=D_{1}^{M}=\cdots=D_{|M|-1}^{M}$.

Thomson (1993) shows that all deterministic target rules satisfy replacement-domination. However, the following example shows that not all probabilistic target rules satisfy replacement-domination.

Example 3.6 Let $Q$ be the distribution over $\overline{\mathbb{R}}$ placing probability $\frac{1}{2}$ on $-\infty$ and $\frac{1}{2}$ on $+\infty$. The resulting probabilistic target rule $\Phi^{Q}$ is determined as follows. For all $N \in \mathcal{P}$, all $R \in \mathcal{R}^{N}$, and all $X \in \mathcal{L}$,

$$
\Phi^{Q}(R)(X)=\frac{1}{2} 1_{X}(\underline{p}(R))+\frac{1}{2} 1_{X}(\bar{p}(R)) .
$$

We show that $\Phi^{Q}$ violates replacement-domination. Let $N=\{1,2,3\}$ and $R \in \mathcal{R}^{N}$ be such that $\left(p\left(R_{1}\right), p\left(R_{2}\right), p\left(R_{3}\right)\right)=(0,1,2)$. Let $\bar{R}_{3} \in \mathcal{R}$ be such that $p\left(\bar{R}_{3}\right)=-1$ and $\ddot{R}=\left(R_{1}, R_{2}, \bar{R}_{3}\right)$. Then, $\bar{R}_{N \backslash\{3\}}=R_{N \backslash\{3\}}$, and by definition of $\Phi^{Q}$,

$$
\Phi^{Q}(R)(\{0\})=\frac{1}{2}>0=\Phi^{Q}(\widetilde{R})(\{0\})
$$

and

$$
\Phi^{Q}(R)(\{1\})=0<\frac{1}{2}=\Phi^{Q}(\bar{R})(\{1\}) .
$$

Since $p\left(R_{1}\right)=0$ and $p\left(R_{2}\right)=1$, this contradicts replacement-domination.

Given $N \in \mathcal{P}$, we show that the class of probabilistic target rules that is restricted to the domain $\mathcal{R}^{N}$ is characterized by Pareto-optimality and one-sided replacement-domination on $\mathcal{R}^{N}$. The proof uses the fact that Pareto-optimality and one-sided replacementdomination imply anonymity and strategy-proofness. Then we apply Theorem 3.3 and Remark 3.4 to deduce that a rule satisfying these requirements must be a probabilistic target rule.

Theorem 3.4 Let $\Phi$ be a probabilistic rule and $N \in \mathcal{P}$. Then $\Phi$ satisfies Pareto-optimality and one-sided replacement-domination on $\mathcal{R}^{N}$ if and only if there exists a distribution $Q^{N}$ over $\overline{\mathbb{R}}$ such that for all $R \in \mathcal{R}^{N}, \Phi(R)=\Phi^{Q^{N}}(R)$. 
Proof. Let $\Phi$ be a probabilistic rule satisfying Pareto-optimality and one-sided replacement-domination and $N \in \mathcal{P}$. By Proposition 3.4 and Lemma 3.12, $\Phi$ satisfies strategy-proofness and anonymity on $\mathcal{R}^{N}$. Without loss of generality, we assume that $N=\{1, \ldots, n\}$. Therefore, by Theorem $3.3, \Phi$ is characterized on $\mathcal{R}^{N}$ by $(n-1)$ fixed distributions $D_{1}^{N}, \ldots, D_{n-1}^{N}$ over $\overline{\mathbb{R}}$. By Remark 3.4 it is sufficient to show that $D_{1}^{N}=\cdots=D_{n-1}^{N} \equiv Q^{N}$.

Suppose that there exist some $l, t \in\{1, \ldots, n-1\}$ such that $D_{l}^{N} \neq D_{t}^{N}$. Since the Borel $\sigma$-algebra on $\mathbb{R} \cup\{-\infty,+\infty\}$ is generated by all intervals $[x,+\infty], x \in \mathbb{R}$ (cf. Remark A.4), there exists $y \in \mathbb{R}$ such that $D_{l}^{N}([y,+\infty]) \neq D_{l}^{N}([y,+\infty])$ (cf. Remark A.3). Without loss of generality, we assume that $l<t$. Let $R_{1}, R_{n} \in \mathcal{R}$ be such that $p\left(R_{1}\right)=y-1$ and $p\left(R_{n}\right)=y$. Let $R \in \mathcal{R}^{N}$ be such that for all $i \in\{1, \ldots, l\}, R_{i}=R_{1}$, and for all $i \in\{l+1, \ldots, n\}, R_{i}=R_{n}$. Let $\bar{R} \in \mathcal{R}^{N}$ be such that for all $i \in\{1, \ldots, t\}, \bar{R}_{i}=R_{1}$, and for all $i \in\{t+1, \ldots, n\}, \bar{R}_{i}=R_{n}$. By Theorem 3.3, equality (3.22),

$$
\Phi(R)(\{y\})=D_{i}^{N}([y,+\infty]) \neq D_{i}^{N}([y,+\infty])=\Phi(\bar{R})(\{y\})
$$

Notice that $\bar{R}$ can be obtained from changing successively $(t-l)$ agents' preference relations from $R_{1}$ to $R_{n}$. Since at these unilateral deviations the Pareto set does not change, Lemma 3.12 implies $\Phi(R)=\Phi(\bar{R})$, which contradicts $(3.29)$.

The next result states that Pareto-optimality and replacement-domination essentially characterize the class of deterministic target rules.

Theorem 3.5 A probabilistic rule satisfies Pareto-optimality and replacement-domination if and only if for all $N \in \mathcal{P}$, it is a deterministic target rule on the domain $\mathcal{R}^{N}$.

Theorem 3.4 and the following proposition yield the proof of Theorem 3.5. We show that if a probabilistic target rule satisfies replacement-domination, then it must be a deterministic rule.

Proposition 3.5 Let $N \in \mathcal{P}$ and $Q$ be a distribution over $\overline{\mathbb{R}}$. If $\Phi^{Q}$ satisfies replacementdomination on $\mathcal{R}^{N}$, then $Q$ places probability 1 on a single point in $\overline{\mathbb{R}}$.

Proof. Suppose that $Q$ does not place probability 1 on a single level in $\overline{\mathbb{R}}$. Then there exist $y, x \in \mathbb{R}$ such that

$$
y<x, Q([-\infty, y])>0, \text { and } Q([x,+\infty])>0
$$

We show that $\Phi^{Q}$ violates replacement-domination on $\mathcal{R}^{N}$. 
Without loss of generality, we assume that $\{1,2,3\} \subseteq N$. Let $R \in \mathcal{R}^{N}$ be such that $p\left(R_{1}\right)=\frac{1}{2}(y+x), p\left(R_{2}\right)=\frac{1}{4} y+\frac{3}{4} x, p\left(R_{3}\right)=x$, and for all $i \in N \backslash\{1,2,3\}, R_{i}=R_{1}$. Let $\bar{R} \in \mathcal{R}^{N}$ be such that $\bar{R}_{N \backslash\{3\}}=R_{N \backslash\{3\}}$ and $p\left(\bar{R}_{3}\right)=y$. By definition of $\Phi^{Q}$ and (3.30),

$$
\Phi^{Q}(R)\left(\left\{\frac{1}{4} y+\frac{3}{4} x\right\}\right)=Q\left(\left\{\frac{1}{4} y+\frac{3}{4} x\right\}\right)<Q\left(\left[\frac{1}{4} y+\frac{3}{4} x,+\infty\right]\right)=\Phi^{Q}(\bar{R})\left(\left\{\frac{1}{4} y+\frac{3}{4} x\right\}\right)
$$

and

$$
\Phi^{Q}(R)\left(\left\{\frac{1}{2}(y+x)\right\}\right)=Q\left(\left[-\infty, \frac{1}{2}(y+x)\right]\right)>Q\left(\left\{\frac{1}{2}(y+x)\right\}\right)=\Phi^{Q}(\bar{R})\left(\left\{\frac{1}{2}(y+x)\right\}\right) .
$$

Since $p\left(R_{1}\right)=\frac{1}{2}(y+x)$ and $p\left(R_{2}\right)=\frac{1}{4} y+\frac{3}{4} x$, the previous two inequalities contradict replacement-domination.

Pareto-optimality and population-monotonicity characterize the class of probabilistic target rules. The proof uses the fact that these two requirements imply one-sided replacement-domination. ${ }^{3}$

Theorem 3.6 A probabilistic rule satisfies Pareto-optimality and population-monotonicity if and only if it is a probabilistic target rule.

Proof. Let $\Phi$ be a probabilistic rule satisfying Pareto-optimality and population-monotonicity. By Proposition 3.3, $\Phi$ satisfies one-sided replacement-domination. Hence, by Theorem 3.4, for each $N \in \mathcal{P}$ there exists a distribution $Q^{N}$ over $\overline{\mathbb{R}}$ such that for all $R \in \mathcal{R}^{N}, \Phi(R)=\Phi^{Q^{N}}(R)$.

We show that for all $N, M \in \mathcal{P}, Q^{N}=Q^{M}$. Let $x \in \mathbb{R}, R^{1} \in \mathcal{R}^{N}, R^{2} \in \mathcal{R}^{M}$, and $R^{3} \in \mathcal{R}^{N \cup M}$ be such that $R_{N}^{3}=R^{1}, R_{M}^{3}=R^{2}$, and $E\left(R^{1}\right)=E\left(R^{2}\right)=E\left(R^{3}\right)=[x-1, x]$.

Successive application of Lemma 3.9 yields $\Phi\left(R^{3}\right)=\Phi\left(R_{N}^{3}\right)=\Phi\left(R^{1}\right)$ and $\Phi\left(R^{3}\right)=$ $\Phi\left(R_{M}^{3}\right)=\Phi\left(R^{2}\right)$. Thus, $\Phi\left(R^{1}\right)=\Phi\left(R^{2}\right)$. Therefore,

$$
\begin{aligned}
Q^{N}([x,+\infty]) & =\Phi^{Q^{N}}\left(R^{1}\right)(\{x\})=\Phi\left(R^{1}\right)(\{x\})=\Phi\left(R^{2}\right)(\{x\}) \\
& =\Phi^{Q^{M}}\left(R^{2}\right)(\{x\})=Q^{M}([x,+\infty]),
\end{aligned}
$$

where the first and the last equality follow from (3.22). Thus, $Q^{N}([x,+\infty])=Q^{M}([x,+\infty])$. Since $x$ was arbitrary, it follows that for all $x \in \mathbb{R}, Q^{N}([x,+\infty])=Q^{M}([x,+\infty])$. Because the Borel $\sigma$-algebra $\overline{\mathcal{L}}$ on $\overline{\mathbb{R}}$ is generated by all intervals $[x,+\infty], x \in \mathbb{R}$, it follows that $Q^{N}=Q^{M}$. Hence, $\Phi=\Phi^{Q}$ where $Q \equiv Q^{N}=Q^{M}$.

\footnotetext{
${ }^{3}$ Theorem 3.6 remains valid if $|P| \geq 3$ and $\mathcal{P}$ contains all non-empty subsets of $\mathbb{P}$.
} 
Interestingly, our proofs provide an alternative way to show the results of Thomson (1993) and Ching and Thomson (1999).

By Corollary 3.3, Theorem 3.3 remains valid when Pareto-optimality is replaced by unanimity. The following example shows that we cannot weaken Pareto-optimality to unanimity in Theorems 3.5 and 3.6.

Example 3.7 Let $a \in \mathbb{R}$. We define the deterministic rule $\Psi^{a}$ as follows. For all $N \in \mathcal{P}$ and all $R \in \mathcal{R}^{N}$,

$$
\Psi^{a}(R) \equiv \begin{cases}\underline{p}(R) & \text { if for all } i, j \in N, R_{i}=R_{j} \\ a & \text { otherwise }\end{cases}
$$

The deterministic rule $\Psi^{a}$ satisfies unanimity, population-monotonicity, replacement-domination, but not Pareto-optimality.

Example 3.7 and the following example establish the independence in Theorems 3.5 and 3.6 .

Example 3.8 We define the deterministic rule $\Theta$ as follows. For all $N \in \mathcal{P}$ and all $R \in \mathcal{R}^{N}$,

$$
\Theta(R) \equiv p\left(R_{\min \{i \mid i \in N\}}\right)
$$

The deterministic rule $\Theta$ satisfies Pareto-optimality, but neither population-monotonicity nor replacement-domination. 


\section{Part II}

\section{Assignment of Indivisibilities}




\section{Chapter 4}

\section{Coalitional Strategy-Proof House Allocation}

\subsection{Introduction}

This chapter, which is based on Ehlers (2000d), studies the problem of allocating indivisible objects (for example, jobs, houses, etc.) among a group of agents when monetary compensations are not possible and each agent receives at most one object. In most of the literature on house allocation, agents are assumed to possess asymmetric, also called strict, preferences over the set of houses. ${ }^{1}$ In applications we cannot rule out that agents may be indifferent between various objects. There are several reasons why this might occur. For example, agents might have little information about the objects. Then we expect that they might not have the ability to distinguish among all objects. It is important to understand this case. This is the purpose of our study.

Specifically, we search for maximal domains on which (Pareto) efficiency (only efficient allocations are chosen) and coalitional strategy-proofness (no group of agents benefits from misrepresenting their true preferences) are compatible. We identify such a maximal domain, and characterize the allocation rules satisfying these properties.

When agents have strict preferences over the set of houses, the classes of allocation rules satisfying efficiency, coalitional strategy-proofness, and some additional requirements have been characterized in earlier literature. Pápai (2000a) defines a certain class of "hierarchical exchange rules" and shows that they are the only rules satisfying additionally a certain condition of "reallocation-proofness". Svensson (1999) shows that only serial dictatorships additionally satisfy neutrality (the rule is independent of the names of the objects).

\footnotetext{
${ }^{1}$ Recent studies in house allocation with strict preferences are Abdulkadiroğlu and Sönmez $(1998,1999)$, Ergin (2000), and Miyagawa (2001).
} 
However, a complete characterization of all rules satisfying efficiency and coalitional strategy-proofness is missing.

It turns out, as we show, that if agents may have any semi-strict preference relation, which means that they may be indifferent between houses, then efficiency and coalitional strategy-proofness are not compatible. We also show that there is a unique maximal domain containing all strict preferences on which these two properties are compatible. It is a subdomain of the domain of semi-strict preferences. A preference relation is in this domain whenever, beginning from the best house, it is a strict descending ranking of houses to a certain house and then indifference holds over this certain house and all remaining houses. Therefore, we conclude that one cannot go much beyond strict preferences if one insists on efficiency and coalitional strategy-proofness.

Barberà, Sonnenschein, and Zhou (1991) established the first maximal domain result in a public good model. Further results were derived by Serizawa (1995). These papers study the question whether, when a certain rule is accepted, because it satisfies strategy-proofness and perhaps some other properties of interest on some domains, what is a maximal domain on which it can be extended in a way such that the extended rule still satisfies these properties. We do not start from a specific rule on the domain of strict preferences. We are interested instead in identifying maximal domains on which rules exist that satisfy efficiency and coalitional strategy-proofness.

Closely related to our study is a paper by Ching and Serizawa (1998). ${ }^{2}$ For the problem of allocating an infinitely divisible endowment they show that the single-plateaued domain is the unique maximal domain (i) that includes all single-peaked preferences and (ii) for which efficiency, equal treatment of equals, and strategy-proofness are compatible. Instead of having a single best consumption (his peak) an agent may possess a whole segment of best consumptions (his plateau). A similar relation holds between the domain of strict preferences and our maximal domain. Instead of a single worst house, there may be a whole set of worst houses which are equivalent to each other. However, in their study the collective endowment may vary, whereas we keep the set of objects fixed.

Finally, we characterize the class of rules satisfying efficiency and coalitional strategyproofness on the unique maximal domain. It turns out that the following class of rules satisfies these properties. Each rule partitions the set of agents into pairs and singletons; specifies a partition of the set of houses for each pair, and orders these sets. For each profile of preferences the allocation chosen by the rule is efficient and satisfies the following. If the first set relative to the order is a singleton, then that agent receives one of

\footnotetext{
${ }^{2}$ A recent reference is also Berga and Serizawa (2000) who determine a maximal domain for the strategyproof provision of one public good in an interval.
} 
his most preferred houses (he is a dictator). If the first set consists of two agents, then their allotments result from an efficient house exchange relative to the specified partition of the houses (pairwise exchange). Then the allotments of the agents belonging to the second set relative to the order satisfy the same properties with respect to the remaining houses, and so on. We call these rules mixed dictator-pairwise-exchange rules. Henceforth, imposing efficiency and coalitional strategy-proofness on the unique maximal domain restricts the class of hierarchical exchange rules considerably and the result of Pápai (2000a) does not extend. Note that in her characterization, on the domain of strict preferences Pápai required reallocation-proofness in addition to these properties. We do not impose reallocation-proofness.

In house exchange markets each agent owns initially one house. If the agents have strict preferences over the set of houses, then the strong core from endowments is always non-empty (Shapley and Scarf, 1974). It turns out that the strong core from endowments contains exactly one allocation, which is the unique competitive allocation (Roth and Postlewaite, 1977). Moreover, the rule selecting for each profile the unique strong core allocation is the only rule satisfying individual rationality (each agent weakly prefers his allotment to his endowment), efficiency, and strategy-proofness (Ma, 1994). Wako (1991) shows that the uniqueness of the strong core disappears when agents may be indifferent between various objects. Still the strong core from endowments is always a subset (possibly strict) of the set of competitive allocations. However, the strong core may be empty. Sönmez (1999) proves in a more general setting that an exchange rule satisfies individual rationality, efficiency, and strategy-proofness if and only if the strong core from endowments is essentially single-valued. On our maximal domain, given any endowment profile, the strong core from this endowment profile may be empty. ${ }^{3}$

When multiple indivisible objects have to be assigned, on different domains Pápai (2001) and Ehlers and Klaus (2000b) characterize the classes of all rules satisfying efficiency and coalitional strategy-proofness.

The organization of Chapter 4 is as follows. Section 4.2 introduces the model. Section 4.3 presents the results. Sections 4.4 and 4.5 contain the proofs.

\footnotetext{
${ }^{3}$ Wako (1991) proved this fact on the domain of semi-strict preferences by using preferences which are not in our maximal domain. On our maximal domain, consider three agents 1,2 , and 3 who own houses $h_{1}$, $h_{2}$, and $h_{3}$, respectively. If 1 is indifferent between all houses, and house $h_{1}$ is the unique most preferred house of agents 2 and 3 , then the strong core is empty.
} 


\subsection{The Model}

Let $n \geq 3$. There are $n$ houses and $n$ agents, denoted by $H=\left\{h_{1}, \ldots, h_{n}\right\}$ and $N=$ $\{1,2, \ldots, n\}$, respectively. "Each agent $i \in N$ is equipped with a preference relation $R_{i}$ over $H$. Of particular interest, we do not require preference relations to be reflexive, complete, or transitive. By $I_{i}$ we denote the indifference relation associated with $R_{i}$ (for all $h, h^{\prime} \in H, h I_{i} h^{\prime}$ if and only if $h R_{i} h^{\prime}$ and $h^{\prime} R_{i} h$ ), and by $P_{0}$ the corresponding strict preference relation (for all $h, h^{\prime} \in H, h P_{i} h^{\prime}$ if and only if $h R_{i} h^{\prime}$ and not $h^{\prime} R_{i} h$ ). By $\mathcal{C}$ we denote the set of all preference relations over $H$ and by $\mathcal{C}^{N}$ the set of (preference) profiles $R \equiv\left(R_{i}\right)_{i \in N}$ such that for all $i \in N, R_{i} \in \mathcal{C}$. Given $R \in \mathcal{C}^{N}$ and $M \subseteq N$, let $R_{M}$ denote the restriction $\left(R_{i}\right)_{i \in M}$ of $R$ to $M$.

Since the set of houses and the set of agents are fixed, $\mathcal{C}^{N}$ completely describes the class of (house) allocation problems. A (feasible) allocation is a list $x=\left(x_{i}\right)_{i \in N}$ such that for all $i \in N, x_{i} \in H$, and no house is assigned to more than one agent. We denote by $X$ the set of all allocations. An allocation rule $\varphi$, or a rule for short, is a function that assigns to every $R \in \mathcal{C}^{N}$ an allocation, denoted by $\varphi(R)$. Given $i \in N$, we call $\varphi_{i}(R)$ the allotment of agent $i$ (at $R$ ).

We are interested in rules that select (Pareto) efficient allocations. An allocation is efficient at $R \in \mathcal{C}^{N}$ if there is no other allocation that all agents weakly prefer, and at least one agent strictly prefers. If all agents have the same complete but non-transitive preference relation, then the set of efficient allocations is empty. Therefore, since we consider arbitrary domains, we only require efficiency whenever the set of efficient allocations is non-cmpty.

For all $R \in \mathcal{C}^{N}$, let $E(R) \equiv\left\{x \in X \mid\right.$ there is no $y \in X$ such that for all $i \in N, y_{i} R_{i} x_{i}$ and for some $\left.j \in N, y_{j} P_{j} x_{j}\right\}$ be the set of (Pareto) efficient allocations at $R$.

Efficiency: For all $R \in \mathcal{C}^{N}$ : if $E(R) \neq \emptyset$, then $\varphi(R) \in E(R)$.

We are interested in coalitional strategy-proofness: no group of agents ever benefits from jointly misrepresenting their preferences. This is the standard definition. It turns out that for our results to hold the following weaker property is sufficient: If one agent mispresents his preference, then (i) he should not benefit from it, and (ii) if he weakly prefers his new allotment to his initial allotment, then no other agent should strictly prefer his new allotment to his initial allotment. ${ }^{5}$

\footnotetext{
${ }^{4}$ If there are more houses than agents, then the preferences could have an arbitrary structure over the last houses. If there are less houses than agents, then by adding null houses our analysis extends in a straightforward way.

${ }^{5}$ Note that our definition is still weaker than requiring coalitional strategy-proofness for all coalitions
} 
Coalitional Strategy-Proofness: For all $R \in \mathcal{C}^{N}$, all $i \in N$, and all $R^{\prime} \in \mathcal{C}^{N}$ such that $R_{N \backslash\{i\}}^{\prime}=R_{N \backslash\{i\}}:$ (i) $\varphi_{i}(R) R_{i} \varphi_{i}\left(R^{\prime}\right)$, and (ii) if $\varphi_{i}\left(R^{\prime}\right) R_{i} \varphi_{i}(R)$, then there exists no $j \in N \backslash\{i\}$ such that $\varphi_{j}\left(R^{\prime}\right) P_{j} \varphi_{j}(R)$.

Given a domain $\mathcal{R}^{N} \subseteq \mathcal{C}^{N}$, we say that a rule satisfies coalitional strategy-proofness on $\mathcal{R}^{N}$ when the above definition holds for all profiles belonging to $\mathcal{R}^{N}$. We show that equivalently we could have imposed the standard definition of coalitional strategy-proofness.

Remark 4.1 The definition of coalitional strategy-proofness requires that if an agent deviates, then by (i), he has to be able to compare his initial allotment and his new allotment. This definition will allow us to derive reflexivity and completeness of the preferences. If we changed the definition to "there exists no $R^{\prime}$ such that (i) $\varphi_{i}\left(R^{\prime}\right) P_{i} \varphi_{i}(R) \ldots$ " neither reflexivity nor completeness could be derived. For example, if a preference relation makes no comparisons, then condition (i) is always met. However, most of the literature uses the same definition of strategy-proofness as we do, even if an agent may not be able to compare any two altematives (in our model an alternative is an allocation). With this definition we will derive certain properties of the preference relations that are usually imposed a priori. If we would have required that preference relations are reflexive and complete, then all our results remain true with the altemative definition of strategy-proofness.

$\triangleleft$

\subsection{A Unique Maximal Domain}

We are interested in identifying domains for which efficiency and coalitional strategy-proofness are compatible, and in finding largest such domains. This motivates the following definition.

A domain $\mathcal{R}^{N} \subseteq \mathcal{C}^{N}$ is maximal for a list of properties if (i) there is a rule satisfying these properties on $\mathcal{R}^{N}$; and (ii) for all $\tilde{\mathcal{R}}^{N}$ such that $\mathcal{R}^{N} \subseteq \tilde{\mathcal{R}}^{N} \subseteq \mathcal{C}^{N}$ and $\mathcal{R}^{N} \neq \tilde{\mathcal{R}}^{N}$ there is no rule satisfying them on $\tilde{\mathcal{R}}^{N}$.

Before we state the result we introduce three domains of preferences which are important in our study.

For $R_{0} \in \mathcal{C}$, reflexivity means that for all $h \in H, h R_{0} h$, and completeness means that for all $h, h^{\prime} \in H, h R_{0} h^{\prime}$ or $h^{\prime} R_{0} h$. Let $\mathcal{W}$ denote the set of all semi-strict preferences over $H$, i.e., preferences in $\mathcal{C}$ that are reflexive, complete, and transitive (for all $h, h^{\prime}, h^{\prime \prime} \in H$, $h R_{0} h^{\prime}$ and $h^{\prime} R_{0} h^{\prime \prime}$ imply $\left.h R_{0} h^{\prime \prime}\right)$. Given $R_{0} \in \mathcal{W}$ and $H^{\prime} \subseteq H$, let $B\left(H^{\prime}, R_{0}\right) \equiv\left\{h^{\prime} \in\right.$ $H^{\prime} \mid$ for all $\left.h \in H^{\prime}, h^{\prime} R_{0} h\right\}$ be the set of most preferred houses in $H^{\prime}$ according to $R_{0}$. Let with cardinality smaller than or equal to 2 . 
$\mathcal{S}$ denote the set of all strict preferences over $H$, i.e., preferences in $\mathcal{W}$ that are asymmetric (for all $h, h^{\prime} \in H, h R_{0} h^{\prime}$ and $h^{\prime} R_{0} h$ imply $h=h^{\prime}$ ). Let

$$
\overline{\mathcal{S}} \equiv\left\{R_{0} \in \mathcal{W} \mid \text { for all } h, h^{\prime}, h^{\prime \prime} \in H, h \neq h^{\prime}, h I_{0} h^{\prime} \text {, and } h^{\prime} R_{0} h^{\prime \prime} \text { imply } h^{\prime} I_{0} h^{\prime \prime}\right\}
$$

Preferences in $\overline{\mathcal{S}}$ are described as follows. A preference is in this domain whenever, beginning from the best house, it is a strict descending ranking to a certain house and then indifference holds over this certain house and the remaining houses. This feature of indifference can be interpreted as reflecting the existence of an outside option. If all of the houses that an agent strictly prefers to his outside option already have been allocated to other agents, then it does not matter to him which of the remaining houses he will receive since he will then exercise his outside option.

Theorem 4.1 The domain $\overline{\mathcal{S}}^{N}$ is the unique maximal domain $\mathcal{R}^{N}$ such that

(i) $\mathcal{S}^{N} \subseteq \mathcal{R}^{N} \subseteq \mathcal{C}^{N}$ and

(ii) on $\mathcal{R}^{N}$ efficiency and coalitional strategy-proofness are compatible.

By Theorem 4.1 there exist rules that satisfy efficiency and coalitional strategy-proofness on $\overline{\mathcal{S}}^{N}$. Here is a class of such rules. First, specify a partition of the set of agents into pairs and singletons, order these sets, and for each pair in the partition, specify a partition of the set of houses. For each profile of preferences the allocation chosen by the rule is efficient and satisfies the following. If the first set relative to the order is a singleton, then that agent receives one of his most preferred houses (he is a dictator). If the first set consists of two agents, then their allotments result from an efficient house exchange relative to the specified partition of the remaining houses (pairwise exchange). Then the allotments of the agents belonging to the second set relative to the order satisfy the same properties with respect to the remaining houses, and so on. We call these rules mixed dictator-pairwise-exchange rules.

We call a list $\mathcal{T} \equiv\left(T_{1}, T_{2}, \ldots, T_{m}\right), m \in\{1, \ldots, n\}$, an "ordered partition of $N^{\prime \prime}$, if for all $l \in\{1, \ldots, m\}, T_{l} \neq \emptyset,\left|T_{l}\right| \in\{1,2\}, \cup_{l=1}^{m} T_{l}=N$, and for all $l, l^{\prime} \in\{1, \ldots, m\}$ such that $l \neq l^{\prime}, T_{l} \cap T_{l^{\prime}}=\emptyset$. Given $\mathcal{T}$, we call $\mathcal{H} \equiv\left(\left\{H_{i}^{T_{l}} \mid i \in T_{l}\right\}\right)_{l \in\{1, \ldots, m\}}$ a "house partition structure for $\mathcal{T}^{\prime \prime}$, when for all $l \in\{1, \ldots, m\}, \cup_{i \in T_{l}} H_{i}^{T_{l}}=H$, and if $T_{l}=\{i, j\}$, then $H_{i}^{T_{l}} \neq$ $\emptyset \neq H_{j}^{T_{l}}$ and $H_{i}^{T_{l}} \cap H_{j}^{T_{l}}=\emptyset$. Given $x \in X$ and $l \in\{1, \ldots, m\}$, let $H^{l}(x) \equiv \cup_{k \in \cup_{t=1}^{l} T_{t}}\left\{x_{k}\right\}$ be the set of houses that the agents belonging to the first $l$ sets of the ordered partition $\mathcal{T}$ receive at $x$ (with the convention $\left.H^{0}(x) \equiv \emptyset\right)$. Given $(\mathcal{T}, \mathcal{H})$ and $R \in \overrightarrow{\mathcal{S}}^{N}$, we define the set of allocations $Y^{(\mathcal{T}, \mathcal{H})}(R)$ as follows. For all $x \in X, x \in Y^{(\mathcal{T}, \mathcal{H})}(R)$ if and only if $x \in E(R)$ and for all $l \in\{1, \ldots, m\}$, 
(i) when $T_{l}=\{i\}, x_{i} \in B\left(H \backslash H^{l-1}(x), R_{i}\right)$,

(ii) when $T_{l}=\{i, j\}, i \neq j$,

○ if $B\left(H \backslash H^{l-1}(x), R_{i}\right) \subseteq H_{i}^{T_{l}}$, then $x_{i} \in B\left(H \backslash H^{l-1}(x), R_{i}\right)$ and $x_{j} \in B\left(H \backslash\left(H^{l-1}(x) \cup\right.\right.$ $\left.\left.\left\{x_{i}\right\}\right), R_{j}\right)$, and

o otherwise, $x_{j} \in B\left(H \backslash H^{l-1}(x), R_{j}\right)$ and $x_{i} \in B\left(H \backslash\left(H^{l-1}(x) \cup\left\{x_{j}\right\}\right), R_{i}\right)$.

Note that in (ii) of the above definition it does not matter how we order $i$ and $j$.

Mixed Dictator-Pairwise-Exchange Rules: Let $\mathcal{T}=\left(T_{1}, \ldots, T_{n}\right)$ be an ordered partition of $N$ and $\mathcal{H}$ be a house partition structure for $\mathcal{T}$. A rule $\varphi$ is a mixed dictatorpairwise-exchange relative to $(\mathcal{T}, \mathcal{H})$ if for all $R \in \overline{\mathcal{S}}^{N}, \varphi(R) \in Y^{(\mathcal{T}, \mathcal{H})}(R)$.

Example 4.1 Let $N \equiv\{1,2,3,4,5,6,7\}, \mathcal{T} \equiv(\{1\},\{2,6\},\{3,4\},\{5,7\})$, and $\mathcal{H} \equiv$ $\left(\{H\},\left\{\left\{h_{4}\right\}, H \backslash\left\{h_{4}\right\}\right\},\left\{\left\{h_{2}\right\}, H \backslash\left\{h_{2}\right\}\right\},\left\{\left\{h_{5}\right\}, H \backslash\left\{h_{5}\right\}\right\}\right)$. Let $R \in \overrightarrow{\mathcal{S}}^{N}$ be such that

\begin{tabular}{|c|c|c|c|c|c|c|}
\hline$R_{1}$ & $R_{2}$ & $R_{3}$ & $R_{1}$ & $R_{5}$ & $R_{6}$ & $R_{7}$ \\
\hline$\underline{h_{3}}$ & $h_{3}$ & $h_{2}$ & $h_{4}$ & $h_{3}$ & $\underline{h_{1}}$ & $h_{5}$ \\
\hline$h_{5}$ & $\underline{h_{2}}$ & $h_{4}$ & $h_{3}$ & $h_{4}$ & $h_{1}$ & $\underline{h_{7}}$ \\
\hline$h_{1}$ & $h_{6}$ & $h_{3}$ & $h_{2}$ & $\underline{h_{5}}$ & $h_{2}$ & $h_{2}$ \\
\hline$h_{2}$ & $h_{5}$ & $\underline{h_{1}}, h_{5}, \underline{h_{6}}, h_{7}$ & $\underline{h_{1}}, h_{\overline{5}}, \underline{h_{0}}, h_{7}$ & $h_{7}$ & $h_{7}$ & $h_{6}$ \\
\hline$h_{4}$ & $h_{1}, h_{1}, h_{7}$ & & & $h_{2}$ & $h_{3}$ & $h_{3}$ \\
\hline$h_{6}$ & & & & $h_{6}, h_{1}$ & $h_{\overline{5}}, h_{6}$ & \\
\hline$h_{7}$ & & & & & & \\
\hline
\end{tabular}

For example, in the above table we have $h_{2} P_{3} h_{4} P_{3} h_{3} P_{3} h_{1} I_{3} h_{5} I_{3} h_{6} I_{3} h_{7}$. We describe the allocations belonging to $Y^{(\mathcal{T}, \mathcal{H})}(R)$. Let $x \in E(R)$. If $x \in Y^{(\mathcal{T}, \mathcal{H})}(R)$, then the following has to hold. Since the top ranked set of $\mathcal{T}$ is $\{1\}$, the first step is a dictator step and agent 1 receives the underlined house $h_{3}$. We have $H^{\mathrm{l}}(x)=\left\{h_{3}\right\}$. The second-ranked set of $\mathcal{T}$ is $\{2,6\}$ corresponding to a pairwise exchange. Among the remaining houses, $h_{2}$ is top-ranked by agent 2 's relation and $h_{4}$ by 6 's relation. Respectively, these houses belong to each others specified partition of the remaining houses at the second step $\left(\left\{h_{1}\right\}\right.$ for 2 and $H \backslash\left\{h_{3}, h_{4}\right\}$ for 6 ). Therefore, after swapping houses 2 receives $h_{2}$, and 6 receives $h_{1}$. We have $H^{2}(x)=\left\{h_{2}, h_{3}, h_{4}\right\}$. The third-ranked set of $\mathcal{T}$ is $\{3,4\}$. For both of them the remaining houses $H \backslash H^{2}(x)$ are "worst". They exercise their outside options. Since $h_{5} P_{5} h_{7} P_{5} h_{1} R_{5} h_{6}$ and $h_{5} P_{7} h_{7} P_{7} h_{6} P_{7} h_{1}$, efficiency implies $\left\{x_{3}, x_{4}\right\}=\left\{h_{1}, h_{6}\right\}$. We have $H^{3}(x)=\left\{h_{1}, h_{2}, h_{3}, h_{4}, h_{6}\right\}$. The fourth-ranked set of $\mathcal{T}$ is $\{5,7\}$ corresponding to a pairwise exchange. Among the remaining houses, 5 owns $h_{5}$ and 7 owns $h_{7}$. Since agent 5 strictly prefers $h_{5}$ to $h_{7}$, he receives $h_{5}$ and 7 receives $h_{7}$. 
Hence, we have $Y^{(\mathcal{T}, \mathcal{H})}(R)=\left\{\left(h_{3}, h_{2}, h_{1}, h_{6}, h_{5}, h_{4}, h_{7}\right),\left(h_{3}, h_{2}, h_{6}, h_{1}, h_{5}, h_{4}, h_{7}\right)\right\}$. If $\varphi$ is a mixed dictator-pairwise-exchange rule relative to $(\mathcal{T}, \mathcal{H})$, then $\varphi(R) \in Y^{(\mathcal{T}, \mathcal{H})}(R)$. 。

Remark 4.2 The set $Y^{(\mathcal{T}, \mathcal{H})}(R)$ has the following feature. For all $i \in N$, if for some $x, y \in Y^{(\mathcal{T}, \mathcal{H})}(R), x_{i} \neq y_{i}$, then $i$ exercises his outside option of $R_{i}$ at all allocations belonging to $Y^{(\mathcal{T}, \mathcal{H})}(R)$.

Mixed dictator-pairwise-exchange rules satisfy efficiency and coalitional strategy-proofness on the unique maximal domain. On the domain of strict preferences these rules are a subclass of the "hierarchical exchange rules" introduced by Pápai (2000a). One polar case of a hierarchical exchange rule is serial dictatorship. Given a fixed vector of endowments (each agent owns a unique house), the other polar case is the rule that chooses for each profile of strict preferences the unique strong core allocation obtained by applying Gale's top trading cycle algorithm to the house exchange market. ${ }^{6}$ We onit the details and refer the interested reader to Pápai (2000a).

If a mixed dictator-pairwise-exchange rule partitions the set of agents into singletons, then it is a serial dictatorship. When calculating the allocations of $Y^{(\mathcal{T}, \mathcal{H})}(R)$ at each step at most two agents own houses and trade. It even turns out that efficiency and coalitional strategy-proofness characterize mixed dictator-pairwise exchange rules on the unique maximal domain. Therefore, on the domain $\overline{\mathcal{S}}^{N}$ these two requirements restrict the class of hierarchical exchange rules considerably. In particular on the unique maximal domain it is not possible that simultaneously three or more agents own at least one house and they "trade" 7 if we insist on efficiency and coalitional strategy-proofness.

Theorem 4.2 On the domain $\overline{\mathcal{S}}^{N}$, mixed dictator-pairwise-exchange rules are the only rules satisfying efficiency and coalitional strategy-proofness.

Remark 4.3 On the domain $\mathcal{S}^{N}$, our definition of coalitional strategy-proofness is equivalent to the conjunction of strategy-proofness (statement (i)) and non-bossiness (if the deviating agent is indifferent between his initial allotment and his new allotment, then each of the other agents is indifferent between his initial allotment and his new allotment). Note that the class of mixed dictator-pairwise-exchange rules violates non-bossiness on the domain $\overline{\mathcal{S}}^{N}$.

Finally we discuss the robustness of Theorem 4.1. If we drop efficiency or weaken coalitional strategy-proofness to weak coalitional strategy-proofness (it is not possible that all

\footnotetext{
${ }^{6}$ A house exchange market consists of a vector of endowments and a profile of strict preferences.

${ }^{7}$ Note that Gale's top trading cycle algorithm is not well-defined on $\overline{\mathcal{S}}^{N}$.
} 
agents who manipulate gain strictly), then on larger domains the weaker properties are compatible.

Example 4.2 Let $x \in X$. For all $R \in \mathcal{W}^{N}, \varphi^{x}(R) \equiv x$. The rule $\varphi^{x}$ satisfies coalitional strategy-proofness on $\mathcal{W}^{N}$, but not efficiency.

Example 4.3 Let $N \equiv\{1,2,3\}$ and $H \equiv\left\{h_{1}, h_{2}, h_{3}\right\}$. For all $R \in \mathcal{W}^{N}$, if $h_{1} P_{1} h_{2}$, then

$$
\bar{\varphi}_{1}(R) \in B\left(H, R_{1}\right) \text { and } \bar{\varphi}_{2}(R) \in B\left(H \backslash\left\{\tilde{\varphi}_{1}(R)\right\}, R_{2}\right)
$$

such that $\bar{\varphi}(R)$ is efficient. If there exists more than one efficient allocation satisfying (4.1): then $\bar{\varphi}$ chooses the allocation at which agent 1 receives the house with the smallest index. Otherwise, if $h_{2} R_{1} h_{1}$, then

$$
\bar{\varphi}_{1}(R) \in B\left(H, R_{1}\right) \text { and } \bar{\varphi}_{3}(R) \in B\left(H \backslash\left\{\bar{\varphi}_{1}(R)\right\}, R_{3}\right)
$$

such that $\bar{\varphi}(R)$ is efficient. If there exists more than one efficient allocation satisfying (4.2), then $\bar{\varphi}$ chooses the allocation at which agent 1 receives the house with the smallest index. The rule $\bar{\varphi}$ satisfies efficiency and weak coalitional strategy-proofness on $\mathcal{W}^{N}$, but not coalitional strategy-proofness. To see that $\bar{\varphi}$ satisfies weak coalitional strategy-proofness, note that agent 1 never strictly gains by manipulation.

Note that in Example 4.3 the chosen allocation may depend on agent 1's preference over houses that are irrelevant for him.

\subsection{Proof of Theorem 4.1}

For the proof of Theorem 4.1, first we show that any maximal domain containing all strict preferences, on which efficiency and coalitional strategy-proofness are compatible, is a subdomain of $\tilde{\mathcal{S}}^{N}$.

Let $\mathcal{S}^{N} \subseteq \mathcal{R}^{N} \subseteq \mathcal{C}^{N}$ be a maximal domain on which efficiency and coalitional strategyproofness are compatible. Let $\varphi$ be a rule satisfying these properties on $\mathcal{R}^{N}$. First we show that $\mathcal{R}$ only contains semi-strict preferences. Thus, for efficient and coalitional strategyproof rules, we need not to impose a priori reflexivity, completeness, and transitivity of preferences.

Lemma $4.1 \mathcal{R} \subseteq \mathcal{W}$. 
Proof. Let $R_{0} \in \mathcal{R}$. We show reflexivity, completeness, and transitivity of $R_{0}$.

(I) Reflexivity: For all $h \in H, h R_{0} h$.

Let $h \in H$ and $\tilde{R} \in \mathcal{R}^{N}$ be such that for all $i \in N, \bar{R}_{i}=R_{0}$. Since $|H|=|N|$, then for some $j \in N, \varphi_{j}(\bar{R})=h$. Let $\bar{R}_{j}^{\prime} \in \mathcal{S}$ be such that $B\left(H, \bar{R}_{j}^{\prime}\right)=\{h\}$ and $\vec{R}^{\prime} \equiv\left(\bar{R}_{N \backslash\{j\}}, \bar{R}_{j}^{\prime}\right)$. By strategy-proofness, $\varphi_{j}\left(\bar{R}^{\prime}\right)=h$. Thus, by strategy-proofness and the choice of $\bar{R}_{j}^{\prime}$, $\varphi_{j}(\bar{R}) \bar{R}_{j} \varphi_{j}\left(\bar{R}^{\prime}\right)$. Hence, $h R_{0} h$.

(II) Completeness: For all $h, h^{\prime} \in H, h R_{0} h^{\prime}$ or $h^{\prime} R_{0} h$.

Let $h, h^{\prime} \in H$ and $\bar{R} \in \mathcal{R}^{N}$ be such that for all $i \in N, \bar{R}_{i}=R_{0}$. Let $i \in N$ be such that $\varphi_{i}(\tilde{R}) \notin\left\{h, h^{\prime}\right\} ;$ say, $i=1$. Let $\bar{R}_{1}^{1} \in \mathcal{S}$ be such that $B\left(H, \bar{R}_{1}^{1}\right)=\left\{\varphi_{1}(\bar{R})\right\}$ and $\bar{R}^{1} \equiv\left(\bar{R}_{1}^{1}, \bar{R}_{N \backslash\{1\}}\right)$. By strategy-proofness, $\varphi_{1}\left(\bar{R}^{1}\right)=\varphi_{1}(\bar{R})$. Let $i \in N \backslash\{1\}$ be such that $\varphi_{i}\left(\bar{R}^{1}\right) \notin\left\{h, h^{\prime}\right\}$; say, $i=2$. Let $\bar{R}_{2}^{2} \in \mathcal{S}$ be such that $B\left(H, \bar{R}_{2}^{2}\right)=\left\{\varphi_{2}\left(\bar{R}^{1}\right)\right\}$ and $\bar{R}^{2} \equiv\left(\bar{R}_{N \backslash\{2\}}^{1}, \bar{R}_{2}^{2}\right)$. By strategy-proofness, $\varphi_{2}\left(\bar{R}^{2}\right)=\varphi_{2}\left(\bar{R}^{1}\right)$. Thus, by reflexivity of $R_{0}$ and coalitional strategy-proofness, $\varphi_{1}\left(\bar{R}^{2}\right)=\varphi_{1}\left(\bar{R}^{1}\right)$. Now, by induction there exists $\bar{R}^{n-2} \in \mathcal{R}^{N}$ such that (after possibly exchanging the indices) for all $i \in\{1, \ldots, n-2\}$, $\bar{R}_{i}^{n-2} \in \mathcal{S}$ and $B\left(H, \bar{R}_{i}^{n-2}\right)=\left\{\varphi_{i}\left(\bar{R}^{n-2}\right)\right\}, \bar{R}_{n-1}^{n-2}=R_{0}, \bar{R}_{n}^{n-2}=R_{0}, \varphi_{n-1}\left(\bar{R}^{n-2}\right)=h$, and $\varphi_{n}\left(\bar{R}^{n-2}\right)=h^{\prime}$. Let $\bar{R}_{n-1}^{n-1} \in \mathcal{S}$ be such that $B\left(H, \bar{R}_{n-1}^{n-1}\right)=\left\{h^{\prime}\right\}$ and $B\left(H \backslash\left\{h^{\prime}\right\}, \bar{R}_{n-1}^{n-1}\right)=$ $\{h\}$, and $\bar{R}^{n-1} \equiv\left(\bar{R}_{N \backslash\{n-1\}}^{n-2}, \vec{R}_{n-1}^{n-1}\right)$. By strategy-proofness, $\varphi_{n-1}\left(\bar{R}^{n-1}\right) \in\left\{h, h^{\prime}\right\}$.

If $\varphi_{n-1}\left(\bar{R}^{n-1}\right)=h^{\prime}$, then by strategy-proofness, $\varphi_{n-1}\left(\bar{R}^{n-2}\right) \bar{R}_{n-1}^{n-2} \varphi_{n-1}\left(\bar{R}^{n-1}\right)$. Hence, $h R_{0} h^{\prime}$, the desired conclusion.

If $\varphi_{n-1}\left(\bar{R}^{n-1}\right)=h$, then by reflexivity of $R_{0}$ and coalitional strategy-proofness, for all $i \in N \backslash\{n\}, \varphi_{i}\left(\bar{R}^{n-1}\right)=\varphi_{i}\left(\bar{R}^{n-2}\right)$. Thus, $\varphi_{n}\left(\vec{R}^{n-1}\right)=h^{\prime}$. Let $\bar{R}_{n}^{n} \in \mathcal{S}$ be such that $B\left(H, \bar{R}_{n}^{n}\right)=\{h\}$ and $B\left(H \backslash\{h\}, \bar{R}_{n-1}^{n-1}\right)=\left\{h^{\prime}\right\}$, and $\bar{R}^{n} \equiv\left(\bar{R}_{N \backslash\{n\}}^{n-1}, \ddot{R}_{n}^{n}\right)$. Let $x \in X$ be such that for all $i \in N,\left\{x_{i}\right\}=B\left(H, \bar{R}_{i}^{n}\right)$. By construction, $E\left(\bar{R}^{n}\right)=\{x\}$. By efficiency, $\varphi\left(\bar{R}^{n}\right)=x$. Thus, $\varphi_{n}\left(\bar{R}^{n}\right)=h$ and by strategy-proofness, $\varphi_{n}\left(\bar{R}^{n-1}\right) \bar{R}_{n}^{n-1} \varphi_{n}\left(\bar{R}^{n}\right)$. Hence, $h^{\prime} R_{0} h$, the desired conclusion.

(III) Transitivity: For all $h, h^{\prime}, h^{\prime \prime} \in H$, if $h R_{0} h^{\prime}$ and $h^{\prime} R_{0} h^{\prime \prime}$, then $h R_{0} h^{\prime \prime}$.

Assume that $R_{0} \in \mathcal{R} \backslash \mathcal{W}$. Since $R_{0} \in \mathcal{R} \backslash \mathcal{W}$ and $R_{0}$ is complete, there exist $h_{1}, h_{2}, h_{3} \in$ $H$ such that $h_{1} R_{0} h_{2}, h_{2} R_{0} h_{3}$, and $h_{3} P_{0} h_{1}$. Using the same construction as in the proof of completeness of $R_{0}$ and starting from the profile $\left(R_{0}\right)_{i \in N}$, there exists $\tilde{R} \in \mathcal{R}^{N}$ such that (after possibly exchanging the indices) for all $i \in\{1,2,3\}, \tilde{R}_{i}=R_{0}$ and $\varphi_{i}(\tilde{R})=h_{i}$, and for all $i \in N \backslash\{1,2,3\}, \tilde{R}_{i} \in \mathcal{S}, B\left(H, \tilde{R}_{i}\right)=\left\{\varphi_{i}(\tilde{R})\right\}$, and for all $h \in H \backslash\left\{h_{1}, h_{2}, h_{3}\right\}$,

$$
h \tilde{P}_{i} h_{1}, h \tilde{P}_{i} h_{2} \text {, and } h \tilde{P}_{i} h_{3}
$$

Let $\tilde{R}_{1}^{\prime} \in \mathcal{S}$ be such that $B\left(H, \tilde{R}_{1}^{\prime}\right)=\left\{h_{3}\right\}, B\left(H \backslash\left\{h_{3}\right\}, \tilde{R}_{1}^{\prime}\right)=\left\{h_{1}\right\}$, and $B\left(H \backslash\left\{h_{1}\right.\right.$, $\left.\left.h_{3}\right\}, \tilde{R}_{1}^{\prime}\right)=\left\{h_{2}\right\}$, and $\tilde{R}^{\prime} \equiv\left(\tilde{R}_{1}^{\prime}, \tilde{R}_{N \backslash\{1\}}\right)$. By applying strategy-proofness twice, $\varphi_{1}\left(\tilde{R}^{\prime}\right)=$ 
$h_{1}$. By coalitional strategy-proofness, for all $i \in N \backslash\{1,2,3\}, \varphi_{i}\left(\tilde{R}^{\prime}\right)=\varphi_{i}(\tilde{R})$. Without loss of generality, we suppose that $\varphi_{2}\left(\tilde{R}^{\prime}\right)=h_{2}$ and $\varphi_{3}\left(\tilde{R}^{\prime}\right)=h_{3}$.

Let $\tilde{R}_{2}^{\prime \prime} \in \mathcal{S}$ be such that $B\left(H, \tilde{R}_{2}^{\prime \prime}\right)=\left\{h_{1}\right\}$ and $B\left(H \backslash\left\{h_{1}\right\}, \tilde{R}_{2}^{\prime \prime}\right)=\left\{h_{2}\right\}$, and $\tilde{R}^{\prime \prime} \equiv$ $\left(\tilde{R}_{2}^{\prime \prime}, \tilde{R}_{N \backslash\{2\}}^{\prime}\right)$. By strategy-proofness, $\varphi_{2}\left(\tilde{R}^{\prime \prime}\right) \in\left\{h_{1}, h_{2}\right\}$. Let $x \in X$ be such that for all $i \in N \backslash\{3\},\left\{x_{i}\right\}=B\left(H, \tilde{R}_{i}^{\prime \prime}\right)$. Note that $x \in E\left(\tilde{R}^{\prime \prime}\right)$. Hence, $E\left(\tilde{R}^{\prime \prime}\right) \neq \emptyset$ and we can apply efficiency. We consider two cases.

Case 1: $\varphi_{2}\left(\tilde{R}^{\prime \prime}\right)=h_{1}$.

By strategy-proofness, $\varphi_{2}\left(\tilde{R}^{\prime}\right) \tilde{R}_{2}^{\prime} \varphi_{2}\left(\tilde{R}^{\prime \prime}\right)$. Since $h_{1} R_{0} h_{2}$, the previous fact implies $h_{2} I_{0} h_{1}$. By coalitional strategy-proofness and completeness of $\tilde{R}_{1}^{\prime}, \varphi_{1}\left(\tilde{R}^{\prime}\right) \tilde{R}_{1}^{\prime} \varphi_{1}\left(\tilde{R}^{\prime \prime}\right)$. Thus, since $\varphi_{2}\left(\tilde{R}^{\prime \prime}\right)=h_{1}, \varphi_{1}\left(\tilde{R}^{\prime \prime}\right) \notin\left\{h_{1}, h_{3}\right\}$. Hence, by efficiency and $(4.3)$, for all $i \in N \backslash\{1,2,3\}$, $\varphi_{i}\left(\tilde{R}^{\prime \prime}\right) \notin\left\{h_{h_{2}}, h_{3}\right\}$ (otherwise, swapping the allotments of 1 and that agent yields a Pareto improvement). Therefore,

$$
\varphi_{1}\left(\tilde{R}^{\prime \prime}\right)=h_{2} \text { and } \varphi_{3}\left(\tilde{R}^{\prime \prime}\right)=h_{3}
$$

Let $\tilde{R}_{3}^{\prime \prime \prime} \in \mathcal{S}$ be such that $B\left(H, \tilde{R}_{3}^{\prime \prime \prime}\right)=\left\{h_{2}\right\}$ and $B\left(H \backslash\left\{h_{2}\right\}, \tilde{R}_{3}^{\prime \prime \prime}\right)=\left\{h_{3}\right\}$, and $\tilde{R}^{\prime \prime \prime} \equiv$ $\left(\tilde{R}_{3}^{\prime \prime \prime}, \tilde{R}_{N \backslash\{3\}}^{\prime \prime}\right)$. Let $y \in X$ be such that for all $i \in N,\left\{y_{i}\right\}=B\left(H, \tilde{R}_{i}^{\prime \prime \prime}\right)$. Note that $E\left(\tilde{R}^{\prime \prime \prime}\right)=\{y\}$. Therefore, by efficiency, $\varphi\left(\tilde{R}^{\prime \prime \prime}\right)=y$. By strategy-proofness, $\varphi_{3}\left(\tilde{R}^{\prime \prime}\right) \tilde{R}_{3}^{\prime \prime} y_{3}$. By $y_{3}=h_{2}, \tilde{R}_{3}^{\prime \prime}=R_{0}, h_{2} R_{0} h_{3}$, and (4.4), we have $\varphi_{3}\left(\tilde{R}^{\prime \prime}\right) \tilde{I}_{3}^{\prime \prime} y_{3}$. By $y_{1}=h_{3}, h_{3} \tilde{P}_{1}^{\prime \prime} h_{2}$, and (4.4), we have $\varphi_{1}\left(\tilde{R}^{\prime \prime \prime}\right) \tilde{P}_{1}^{\prime \prime} \varphi_{1}\left(\tilde{R}^{\prime \prime}\right)$. The previous two facts contradict coalitional strategyproofness. Thus, Case 1 cannot occur.

Case 2: $\varphi_{2}\left(\tilde{R}^{\prime \prime}\right)=h_{2}$.

By coalitional strategy-proofness, for all $i \in N \backslash\{3\}, \varphi_{i}\left(\tilde{R}^{\prime \prime}\right)=\varphi_{i}\left(\tilde{R}^{\prime}\right)$. Thus, $\varphi_{3}\left(\tilde{R}^{\prime \prime}\right)=$ $h_{3}$. Considering $\tilde{R}^{\prime \prime \prime}$ as in the second part of Case 1 and using the same arguments yields a contradiction to coalitional strategy-proofness for Case 2. Thus, Case 2 cannot occur.

Next, we show that $\varphi$ satisfies (Maskin-)monotonicity on $\mathcal{S}^{N}$. Monotonicity means that if for a given profile, all the agents' allotments move up in their preferences, then the same allocation is chosen at the new profile.

Monotonicity: For all $R, \bar{R} \in \mathcal{S}^{N}$ : if for all $i \in N$ and all $h \in H, \varphi_{i}(R) R_{i} h$ implies $\varphi_{i}(R) \bar{R}_{i} h$, then $\varphi(R)=\varphi(\bar{R})$.

The proof of the following lemma is standard.

Lemma $4.2^{8} \varphi$ satisfies monotonicity on $\mathcal{S}^{N}$.

\footnotetext{
${ }^{8}$ Note that by efficiency, $\varphi$ violates monotonicity on $\mathcal{W}^{N}$.
} 
Now we are able to prove the first part of Theorem 4.1 .

\section{Lemma $4.3 \mathcal{R} \subseteq \overline{\mathcal{S}}$.}

Proof. ${ }^{9}$ By Lemma 4.1, $\mathcal{R} \subseteq \mathcal{W}$. Hence, for all $R \in \mathcal{R}^{N}, E(R) \neq \emptyset$. Suppose that $\mathcal{R} \backslash \overline{\mathcal{S}} \neq \emptyset$. Let $R_{0} \in \mathcal{R} \backslash \overline{\mathcal{S}}$. Because $R_{0} \in \mathcal{W}$, then without loss of generality, we may assume that $h_{1} R_{0} h_{2} R_{0} h_{3} R_{0} \cdots R_{0} h_{n}$. Since $R_{0} \in \mathcal{R} \backslash \overline{\mathcal{S}}$ and $|N| \geq 3$, there exists $k \in N \backslash\{n-1, n\}$ such that $h_{k} I_{0} h_{k+1}$ and $h_{k+1} P_{0} h_{n}$. Without loss of generality, we suppose that $k=1$, i.e., ${ }^{10}$

$$
h_{1} I_{0} h_{2} \text { and } h_{1} P_{0} h_{n}
$$

Let $R \in \mathcal{S}^{N}$ be such that for all $i \in N, h_{1} P_{i} h_{2} P_{i} \cdots P_{i} h_{n}$. Since $|H|=|N|$, without loss of generality, we suppose that for all $i \in N, \varphi_{i}(R)=h_{i}$. Let $\bar{R} \in \mathcal{S}^{N}$ be such that for all $i \in N \backslash\{1,2, n\}, B\left(H, \bar{R}_{i}\right)=\left\{\varphi_{i}(R)\right\}, \bar{R}_{1}=R_{1}$, and for all $h \in H \backslash\left\{h_{1}, h_{2}, h_{n}\right\}$,

$$
\begin{aligned}
& h_{1} \bar{P}_{2} h_{2} \bar{P}_{2} h_{n} \bar{P}_{2} h, \\
& h_{2} \bar{P}_{n} h_{n} \bar{P}_{n} h_{1} \bar{P}_{n} h .
\end{aligned}
$$

By Lemma 4.2, $\varphi$ satisfies monotonicity on $\mathcal{S}^{N}$. Thus,

$$
\varphi(\bar{R})=\varphi(R) .
$$

Let $\bar{R}^{\prime}=\left(R_{0}, \bar{R}_{N \backslash\{1\}}\right)$. Strategy-proofness together with (4.5) and (4.6) imply $\varphi_{1}\left(\bar{R}^{\prime}\right) \bar{I}_{1}^{\prime} h_{1}$ and $\varphi_{1}\left(\bar{R}^{\prime}\right) \neq h_{n}$. Thus, $\bar{R}_{N \backslash\{1\}} \in \mathcal{S}^{N \backslash\{1\}}$, coalitional strategy-proofness, and (4.6) imply that for all $i \in N \backslash\{1,2, n\}, \varphi_{i}\left(\bar{R}^{\prime}\right)=\varphi_{i}(\bar{R})$. Again, by coalitional strategy-proofness, $\varphi_{2}\left(\bar{R}^{\prime}\right) \bar{R}_{2} h_{2}$. Hence, (4.5) and efficiency imply $\varphi_{1}\left(\vec{R}^{\prime}\right)=h_{2}, \varphi_{2}\left(\bar{R}^{\prime}\right)=h_{1}$, and $\varphi_{n}\left(\vec{R}^{\prime}\right)=h_{n}$. Let $\bar{R}^{\prime \prime} \in \mathcal{R}^{N}$ be such that $\bar{R}_{N \backslash\{2\}}^{\prime \prime}=\bar{R}_{N \backslash\{2\}}^{\prime}$, and for all $h \in H \backslash\left\{h_{1}, h_{2}, h_{n}\right\}$, $h_{1} \bar{P}_{2}^{\prime \prime} h_{n} \bar{P}_{2}^{\prime \prime} h_{2} \bar{P}_{2}^{\prime \prime} h$. By strategy-proofness, $\varphi_{2}\left(\tilde{R}^{\prime \prime}\right)=\varphi_{2}\left(\vec{R}^{\prime}\right)$. Thus, by coalitional strategyproofness and $\bar{R}_{N \backslash\{1,2\}}^{\prime} \in \mathcal{S}^{N \backslash\{1,2\}}, \varphi\left(\bar{R}^{\prime \prime}\right)=\varphi\left(\bar{R}^{\prime}\right)$. Hence,

$$
\varphi_{1}\left(\bar{R}^{\prime \prime}\right)=h_{2}, \varphi_{2}\left(\bar{R}^{\prime \prime}\right)=h_{1} \text {, and } \varphi_{n}\left(\bar{R}^{\prime \prime}\right)=h_{n} .
$$

Let $\tilde{R} \in \mathcal{S}^{N}$ be such that $\tilde{R}_{N \backslash\{2\}}=\bar{R}_{N \backslash\{2\}}$ and for all $h \in H \backslash\left\{h_{1}, h_{2}, h_{n}\right\}, h_{1} \tilde{P}_{2} h_{n} \tilde{P}_{2} h_{2} \tilde{P}_{2} h$. Strategy-proofness and (4.6) imply

$$
\varphi_{2}(\tilde{R}) \in\left\{h_{2}, h_{n}\right\}
$$

If $\varphi_{2}(\tilde{R})=h_{2}$, then coalitional strategy-proofness and $\bar{R}_{N \backslash\{2\}} \in \mathcal{S}^{N \backslash\{2\}}$ imply $\varphi(\tilde{R})=$ $\varphi(\bar{R})$. Let $x \in X$ be such that for all $i \in N \backslash\{2, n\} ; x_{i}=\varphi_{i}(\tilde{R}), x_{2}=h_{n}$ and $x_{n}=h_{2}$. Then, for all $i \in N, x_{i} \tilde{R}_{i} \varphi_{i}(\tilde{R})$ and $x_{2} \tilde{P}_{2} \varphi_{2}(\tilde{R})$. This contradicts efficiency.

\footnotetext{
${ }^{9}$ Table 4.1 illustrates the proof of Lemma 4.3.

${ }^{10}$ If $k>1$, then the same proof yields the assertion.
} 


\begin{tabular}{|c|c|c|c|c|c|c|}
\hline $\bar{R}_{1}$ & $\bar{R}_{2}$ & $\bar{R}_{n}$ & \multirow{4}{*}{$\rightarrow$} & $\vec{R}_{1}^{\prime}$ & $\bar{R}_{2}^{\prime}$ & $\bar{R}_{n}^{\prime}$ \\
\hline$\underline{h_{1}}$ & $h_{1}$ & $h_{2}$ & & $h_{1}, \underline{h_{2}}$ & $\underline{h_{1}}$ & $h_{l_{2}}$ \\
\hline$h_{2}$ & $\underline{h_{2}}$ & $\underline{h_{n}}$ & & $h_{n}$ & $h_{2}$ & $\underline{h_{n}}$ \\
\hline$h_{n}$ & $h_{n}$ & $h_{1}$ & & & $h_{n}$ & $h_{1}$ \\
\hline \multicolumn{3}{|c|}{$\downarrow$} & \multirow{5}{*}{$\leftarrow$} & \\
\hline$\tilde{R}_{1}$ & $\tilde{R}_{2}$ & $\tilde{R}_{n}$ & & $\bar{R}_{1}^{\prime \prime}$ & $\bar{R}_{2}^{\prime \prime}$ & $\bar{R}_{n}^{\prime \prime}$ \\
\hline$\underline{h_{1}}$ & $h_{1}$ & $\underline{h_{2}}$ & & $h_{1}, \underline{h_{2}}$ & $\underline{h_{1}}$ & $h_{2}$ \\
\hline$h_{2}$ & $\underline{h_{n l}}$ & $h_{n}$ & & $h_{n}$ & $h_{n}$ & $\underline{h_{n}}$ \\
\hline$h_{n}$ & $h_{2}$ & $h_{1}$ & & & $h_{2}$ & $h_{1}$ \\
\hline
\end{tabular}

Table 4.1: Proof of Lemma 4.3: underlined houses are allocated to those agents at the corresponding profiles.

Hence, by $(4.8), \varphi_{2}(\tilde{R})=h_{n}$. Since for all $i \in N \backslash\{2\},\left\{x_{i}\right\}=B\left(H, \tilde{R}_{i}\right)$, efficiency implies $\varphi(\tilde{R})=x$. Note that $\tilde{R}_{N \backslash\{1\}}=\bar{R}_{N \backslash\{1\}}^{\prime \prime}$ and $\tilde{R}_{1}^{\prime \prime}=R_{0}$. By $(4.7)$ and $\varphi(\tilde{R})=x$,

$$
\varphi_{1}(\tilde{R}) \bar{I}_{1}^{\prime \prime} \varphi_{1}\left(\bar{R}^{\prime \prime}\right) \text { and } \varphi_{n}(\tilde{R}) \ddot{P}_{n}^{\prime \prime} \varphi_{n}\left(\bar{R}^{\prime \prime}\right)
$$

This contradicts coalitional strategy-proofness. Thus, $R_{0} \notin \mathcal{R}$ and $\mathcal{R} \subseteq \overline{\mathcal{S}}$.

To prove the second part of Theorem 4.1, we show that the class of mixed dictatorpairwise-exchange rules satisfies efficiency and coalitional strategy-proofness on $\overline{\mathcal{S}}^{N}$.

By definition, they satisfy efficiency. We show that they satisfy the standard definition of coalitional strategy-proofness, i.e., if a coalition misrepresent their true preferences and each member weakly prefers his new allotment to his initial allotment, then no member benefits.

Lemma 4.4 The class of mixed dictutor-pairwise-exchange rules satisfies coalitional strategy-proofness on $\overrightarrow{\mathcal{S}}^{N}$.

Proof. Let $\mathcal{T} \equiv\left(T_{1}, \ldots, T_{m}\right)$ be an ordered partition of $N$ and $\mathcal{H}$ be a house partition structure for $\mathcal{T}$. Let $\tilde{\varphi}$ be a mixed dictator-pairwise-exchange rule relative to $(\mathcal{T}, \mathcal{H})$. Without loss of generality; we suppose that for all $l \in\{1, \ldots, m-1\}$, if $i \in T_{l}$ and $j \in T_{l+1}$, then $i<j$. For all $R \in \overline{\mathcal{S}}^{N}, \bar{\varphi}(R) \in Y^{(\mathcal{T}, \mathcal{H})}(R)$ and efficiency yield the following. For all $j \in N$, if $j \in T_{l}$, then for all $i \in \cup_{t=1}^{l} T_{t}$,

$$
\tilde{\varphi}_{i}(R) P_{j} \tilde{\varphi}_{j}(R) \text { inplies } \tilde{\varphi}_{i}(R) P_{i} \tilde{\varphi}_{j}(R)
$$


Let $M \subseteq N, R, \bar{R} \in \overline{\mathcal{S}}^{N}$ be such that $R_{N \backslash M}=\bar{R}_{N \backslash M}$, and for all $i \in M, \tilde{\varphi}_{i}(\bar{R}) R_{i} \tilde{\varphi}_{i}(R)$. To show coalitional strategy-proofness, it suffices to show that for all $i \in M$,

$$
\tilde{\varphi}_{i}(\bar{R}) I_{i} \tilde{\varphi}_{i}(R)
$$

Let $M^{\prime} \equiv\left\{i \in N \mid \varphi_{i}(\bar{R}) R_{i} \varphi_{i}(R)\right\}$. Let $M^{\prime}=\left\{i_{1}, i_{2}, \ldots, i_{s}\right\}$ be such that $i_{1}<i_{2}<\cdots<i_{s}$. Suppose that $\tilde{\varphi}$ violates coalitional strategy-proofness at $R$ and $\bar{R}$, i.e., there exists $k \in$ $\{1, \ldots, s\}$ such that $\tilde{\varphi}_{i_{k}}(\bar{R}) P_{i_{k}} \tilde{\varphi}_{i_{k}}(R)$. Let $k$ be minimal. Thus, for all $i \in\left\{i_{1}, \ldots, i_{k-1}\right\}$, $\tilde{\varphi}_{i}(\bar{R}) I_{i} \tilde{\varphi}_{i}(R)$.

Let $i_{k} \in T_{l_{k}}$. Hence, by $\tilde{\varphi}(R) \in Y^{(\mathcal{T}, \mathcal{H})}(R)$, for some $j \in \cup_{l=1}^{l_{k}} T_{l}$,

$$
\tilde{\varphi}_{j}(R)=\tilde{\varphi}_{i_{k}}(\bar{R})
$$

Let $j \in T_{l_{j}}$. First, suppose that $j \in T_{l_{k}}$. Then by $(4.9), \tilde{\varphi}_{j}(R) P_{j} \tilde{\varphi}_{i_{k}}(R)$. If $j \notin M^{\prime}$, then $\tilde{R}_{j}=R_{j}$ and $\tilde{\varphi}_{j}(R) P_{j} \tilde{\varphi}_{j}(\bar{R})$. Since $\tilde{\varphi}_{j}(R) \in H \backslash H^{l_{k}-1}(\tilde{\varphi}(R))$ and $\tilde{\varphi}_{j}(R) P_{i_{k}} \tilde{\varphi}_{i_{k}}(R)$, we have $\tilde{\varphi}_{j}(R) \in H_{j}^{T_{l_{k}}}$. Thus, $\tilde{\varphi}_{j}(R) \in H \backslash H^{l_{k}-1}(\tilde{\varphi}(\bar{R}))$ implies $\tilde{\varphi}_{j}(\bar{R}) R_{j} \tilde{\varphi}_{j}(R)$, a contradiction to $j \notin M^{\prime}$. If $j \in M^{\prime}$, then (4.11), $\tilde{\varphi}(R) \in E(R)$, and $R_{j} \in \overline{\mathcal{S}}$ imply $\tilde{\varphi}_{j}(\bar{R}) P_{j} \tilde{\varphi}_{j}(R)$. Thus, for some $j^{\prime} \in \cup_{t=1}^{l_{k}-1} T_{l}, \tilde{\varphi}_{j^{\prime}}(R)=\tilde{\varphi}_{j}(\bar{R})$ and the argument below applies for $j$ in the role of $i_{k}$.

Second, suppose that $j \in \cup_{l=1}^{l_{k}-1} T_{\ell}$. By definition of $M^{\prime}$ and our choice of $k, \tilde{\varphi}_{j}(R) R_{j} \tilde{\varphi}_{j}(\bar{R})$. If $j \in M^{\prime}$, then $\tilde{\varphi}_{j}(R) I_{j} \tilde{\varphi}_{j}(\bar{R})$. Thus, by $(4.9)$ and $R_{j} \in \bar{S}, \tilde{\varphi}_{j}(\bar{R})=\tilde{\varphi}_{j}(R)$, a contradiction to (4.11). If $j \notin M^{\prime}$, then $\bar{R}_{j}=R_{j}$. By $\tilde{\varphi}_{j}(R) \in H \backslash H^{l_{k}-1}(\tilde{\varphi}(\tilde{R}))$. (4.9), and $l_{j}<l_{k}$, $\tilde{\varphi}_{j}(\bar{R}) R_{j} \tilde{\varphi}_{j}(R)$, which contradicts $j \notin M^{\prime}$. Thus, (4.10) holds.

\subsection{Proof of Theorem 4.2}

Mixed dictator-pairwise-exchange rules satisfy efficiency, and by Lemma 4.4 coalitional strategy-proofness. In proving the converse statement of Theorem 4.2 the following preference relation plays a key role. It is the relation, denoted by $R_{0}^{I} \in \overline{\mathcal{S}}$, for which all houses are indifferent, i.e., for all $h, h^{\prime} \in H, h I_{0}^{I} h^{\prime}$. First, we show on the domain $\left(\mathcal{S} \cup\left\{R_{0}^{I}\right\}\right)^{N}$ that if a rule satisfies efficiency and coalitional strategy-proofness, then it is mixed dictator-pairwise-exchange rule. Second, we show that if a rule is a mixed dictator-pairwise-exchange rule on the domain $\left(\mathcal{S} \cup\left\{R_{0}^{I}\right\}\right)^{N}$, then it is a mixed dictatorpairwise-exchange rule on the domain $\tilde{\mathcal{S}}^{N}$.

Let $\varphi$ be a rule satisfying efficiency and coalitional strategy-proofness on $\left(\mathcal{S} \cup\left\{R_{0}^{I}\right\}\right)^{N}$. First we construct for all houses $h \in H$ a permutation $\sigma^{h}$.

Let $\bar{h} \in H$ and $\vec{R} \in \mathcal{S}^{N}$ be such that for all $i \in N, B\left(H, \bar{R}_{i}\right)=\{\bar{h}\}$. By efficiency, for some $j \in N, \varphi_{j}(\bar{R})=\bar{h}$. Without loss of generality; we suppose that $j=1$. We prove that at any profile agent 1 weakly prefers his allotment to $\bar{h}_{h}$. 
Lemma 4.5 For all $R \in\left(\mathcal{S} \cup\left\{R_{0}^{I}\right\}\right)^{N}, \varphi_{1}(R) R_{1} \bar{h}$.

Proof. Let $R \in\left(S \cup\left\{R_{0}^{I}\right\}\right)^{N}$. First, we show that for all $S \subseteq N \backslash\{1\}$,

$$
\varphi_{1}\left(R_{S}, \tilde{R}_{N \backslash S}\right)=\bar{h}
$$

We prove (4.12) by induction on $|S|$. For $t \in\{2, \ldots, n\}$, let $R^{t} \equiv\left(R_{\{2, \ldots, t\}}, \bar{R}_{N \backslash\{2, \ldots, t\}}\right)$.

Induction basis: (4.12) holds when $|S|=1$.

Proof: Without loss of generality, suppose $S=\{2\}$. Let $\bar{R}^{\prime} \equiv\left(\tilde{R}_{N \backslash\{2\}}, R_{0}^{I}\right)$. By definition of $R_{0}^{I}$, for all $h \in H$,

$$
h \vec{I}_{2}^{\prime} \varphi_{2}\left(\vec{R}^{\prime}\right)
$$

Since $B\left(H, \bar{R}_{1}\right)=\{\bar{h}\}$, coalitional strategy-proofness and (4.13) imply

$$
\varphi_{1}\left(\bar{R}^{\prime}\right)=\bar{h}
$$

Let $R^{2} \equiv\left(R_{2}, \bar{R}_{N \backslash\{2\}}\right)$. If $\varphi_{1}\left(R^{2}\right) \neq \bar{h}$, then, by efficiency, for some $j \in N \backslash\{1\}, \varphi_{j}\left(R^{2}\right)=\bar{h}$. Since $\bar{h} \bar{P}_{2} \varphi_{2}(\bar{R})$, strategy-proofness implies $j>2$. Thus, by (4.14), $\bar{h} \bar{P}_{j}^{\prime} \varphi_{j}\left(\bar{R}^{\prime}\right)$. By (4.13), this contradicts coalitional strategy-proofness. Thus, $\varphi_{1}\left(R^{2}\right)=\varphi_{1}\left(R_{2}, \bar{R}_{N \backslash\{2\}}\right)=\bar{h}$ and the induction basis holds.

By induction, (4.12) holds for all $S \subseteq N \backslash\{1\}$ such that $|S| \leq t-1$.

Induction step: (4.12) holds when $|S|=t$.

Proof: Without loss of generality, suppose $S=\{2,3, \ldots, t+1\}$. By induction,

$$
\varphi_{1}\left(R^{\ell}\right)=\bar{h} .
$$

Let $\tilde{R} \equiv\left(R_{N \backslash\{t+1\}}^{t}, R_{0}^{I}\right)$. Applying the same arguments as above yields $\varphi_{1}(\tilde{R})=\bar{h}$. Since $\bar{h}_{2} P_{t+1}^{t} \varphi_{t+1}\left(R^{l}\right)$, strategy-proofness implies $\varphi_{t+1}\left(R^{t+1}\right) \neq \bar{h}$. By efficiency, for some $j \in N \backslash\{t+1\}, \varphi_{j}\left(R^{t+1}\right)=\bar{h}$. Assume that $j \neq 1$. Since $R_{N \backslash\{t+1\}}^{\iota}=\tilde{R}_{N \backslash\{t+1\}}=R_{N \backslash\{t+1\}}^{t+1}$, coalitional strategy-proofness implies $\varphi_{j}(\tilde{R}) R_{j}^{t+1} \bar{h}$. Hence, $j \in S \backslash\{t+1\}$. Consider $\left(R_{S \backslash\{j\}}, \bar{R}_{N \backslash(S \backslash\{j\})}\right)$. By strategy-proofness,

$$
\varphi_{j}\left(R_{S \backslash\{j\}}, \bar{R}_{N \backslash(S \backslash\{j\})}\right)=\bar{h} .
$$

Because $|S \backslash\{j\}| \leq t-1$, this contradicts our induction assumption. Hence, $\varphi_{1}\left(R^{t+1}\right)=\bar{h} . \diamond$

We proved (4.12). Applying (4.12) for $S=N \backslash\{1\}$ yields $\varphi_{1}\left(R^{n}\right)=\varphi_{1}\left(R_{N \backslash\{1\}}, \bar{R}_{1}\right)=\bar{h}$. Thus, by strategy-proofness, $\varphi_{1}(R) R_{1} \bar{h}$, the desired conclusion. 
Define $\sigma^{h}(1) \equiv 1$. Consider $\left(R_{0}^{I}, \bar{R}_{N \backslash\{1\}}\right)$. Then for some $j \in N \backslash\{1\}, \varphi_{j}\left(R_{0}^{I}, \bar{R}_{N \backslash\{1\}}\right)=$ $\vec{h}$. Say $j=2$. Then we define $\sigma^{\bar{h}}(2) \equiv 2$.

Now, considering $\left(R_{0}^{I}, R_{0}^{I}, \bar{R}_{N \backslash\{1,2\}}\right)$ gives us $\sigma^{\bar{h}}(3)$, and inductively we define a permutation $\sigma^{\bar{h}}: N \rightarrow N$. In particular, using the same procedure we define for all $h \in H$ a permutation $\sigma^{h}: N \rightarrow N$. The following lemma generalizes Lemma 4.5.

Lemma 4.6 For all $h \in H$, all $k \in N$, and all $R \in\left(\mathcal{S} \cup\left\{R_{0}^{I}\right\}\right)^{N}$, if for all $t \in\{1, \ldots, k-$ $1\}, \varphi_{\sigma^{h}(l)}(R) \neq h$, then $\varphi_{\sigma^{h}(k)}(R) R_{\sigma^{h}(k)} h$.

Proof. If $k=1$, then the assertions of Lemma 4.5 and Lemma 4.6 are the same. Thus, let $k \in N \backslash\{1\}$ and $\bar{h} \in H$. Without loss of generality, we suppose that for all $i \in\{1, \ldots, k\}$, $\sigma^{\bar{h}}(i)=i$. Let $S=\{1, \ldots, k-1\}$. By the definition of $\sigma^{\bar{h}}(k), \varphi_{k}\left(\left(R_{0}^{I}\right)_{i \in S}, \bar{R}_{N \backslash S}\right)=\bar{h}$. Then, using effiency and the same arguments as in Lemma 4.5 it can be shown that for all $R_{N \backslash(S \cup\{k\})} \in\left(\mathcal{S} \cup\left\{R_{0}^{I}\right\}\right)^{N \backslash(S \cup\{k\}),}$

$$
\varphi_{k}\left(\left(R_{0}^{I}\right)_{i \in S}, \bar{R}_{k}, R_{N \backslash(S \cup\{k\})}\right)=\bar{h} .
$$

Now, let $R \in\left(\mathcal{S} \cup\left\{R_{0}^{I}\right\}\right)^{N}$ and suppose that for all $i \in S$,

$$
\varphi_{i}(R) \neq \bar{h}
$$

Let $T^{I} \equiv\left\{i \in N \mid R_{i}=R_{0}^{I}\right\}$. If $k \in T^{I}$, then the desired assertion is trivial. If for some $j \in T^{I}, \varphi_{j}(R)=\bar{h}$, then by efficiency, $\varphi_{k}(R) R_{k} \bar{h}$, the desired conclusion. Thus, let $R_{k} \in \mathcal{S}$ and for all $i \in T^{I}, \varphi_{i}(R) \neq \bar{h}$. Hence, by (4.17), for some $l \in N \backslash\left(S \cup T^{I}\right)$,

$$
\varphi_{l}(R)=\bar{h}
$$

We transform $R$ to a profile $\tilde{R} \in \mathcal{S}^{N}$ without changing the allotments of $k$ and $l$. Let $j \in T^{I}$. Let $R_{j}^{\prime} \in \mathcal{S}$ be such that $B\left(H, R_{j}^{\prime}\right)=\left\{\varphi_{j}(R)\right\}$ and let $R^{\prime} \equiv\left(R_{N \backslash\{j\}}, R_{j}^{\prime}\right)$. Strategyproofness implies $\varphi_{j}\left(R^{\prime}\right)=\varphi_{j}(R)$. Since $\varphi_{j}\left(R^{\prime}\right) I_{j} \varphi_{j}(R)$ and $\varphi_{j}\left(R^{\prime}\right) I_{j}^{\prime} \varphi_{j}(R)$, applying coalitional strategy-proofness twice yields that for all $i \in N \backslash T^{I}$,

$$
\varphi_{i}\left(R^{\prime}\right)=\varphi_{i}(R)
$$

In a similar way we replace stepwise the preferences of all agents in $T^{I} \backslash\{j\}$ by strict preferences. This yields a profile $\tilde{R} \in \mathcal{S}^{N}$ such that for all $i \in N \backslash T^{I}$,

$$
\tilde{R}_{i}=R_{i} \text { and } \varphi_{i}(\tilde{R})=\varphi_{i}(R) .
$$

In particular, $\varphi_{k}(\tilde{R})=\varphi_{k}(R)$ and $\varphi_{l}(\tilde{R})=\bar{h}$. Let $\tilde{R}^{\prime} \in \mathcal{S}^{N}$ be such that

- for all $i \in S, B\left(H, \tilde{R}_{i}^{\prime}\right)=\left\{\varphi_{i}(\tilde{R})\right\}$, 
- for all $i \in N \backslash(S \cup\{k\}), B\left(H, \tilde{R}_{i}^{\prime}\right)=\left\{\varphi_{i}(\tilde{R})\right\}$, and for all $h \in H \backslash\left\{\varphi_{1}(\tilde{R}), \ldots, \varphi_{k-1}(\tilde{R})\right\}$ and all $t \in S, h \tilde{P}_{i}^{\prime} \varphi_{l}(\tilde{R})$, and

- for all $h \in H \backslash\left\{\bar{h}, \varphi_{k}(\tilde{R})\right\}, \bar{h} \tilde{P}_{k}^{\prime} h$ and $\varphi_{k}(\tilde{R}) \tilde{P}_{k}^{\prime} h$; for all $h \in H \backslash\left\{\varphi_{\mathrm{I}}(\tilde{R}), \ldots, \varphi_{k-1}(\tilde{R})\right\}$ and all $t \in S, h \tilde{P}_{k}^{\prime} \varphi_{l}(\tilde{R})$; and $B\left(H, \tilde{R}_{k}^{\prime}\right)=B\left(\left\{\vec{h}, \varphi_{k}(\tilde{R})\right\}, R_{k}\right)$.

By Lemma 4.2, $\varphi$ satisfies monotonicity on $\mathcal{S}^{N}$. Hence,

$$
\varphi\left(\tilde{R}^{\prime}\right)=\varphi(\tilde{R})
$$

By (4.16),

$$
\varphi_{k}\left(\left(R_{0}^{I}\right)_{i \in S}, \bar{R}_{k}, \tilde{R}_{N \backslash(S \cup\{k\})}^{\prime}\right)=\bar{h}
$$

By strategy-proofness, we may assume that for all $h \in H \backslash\left\{\varphi_{1}(\tilde{R}), \ldots, \varphi_{k-1}(\tilde{R})\right\}$ and all $t \in S, h \bar{P}_{k} \varphi_{t}\left(\tilde{R}^{\prime}\right)$. Let $R^{o} \equiv\left(\left(R_{0}^{I}\right)_{i \in S}, \bar{R}_{k}, \tilde{R}_{N \backslash(S \cup\{k\})}^{\prime}\right)$. Thus, by construction of $\tilde{R}^{\prime}$ and efficiency,

$$
\left\{\varphi_{1}\left(R^{o}\right), \ldots, \varphi_{k-1}\left(R^{o}\right)\right\}=\left\{\varphi_{1}\left(\tilde{R}^{\prime}\right), \ldots, \varphi_{k-1}\left(\tilde{R}^{\prime}\right)\right\}
$$

Given $t \in S$, let $R^{t} \equiv\left(\tilde{R}_{\{1, \ldots, t\}}^{\prime},\left(R_{0}^{I}\right)_{S \backslash\{1, \ldots, t\}}, \bar{R}_{k}, \tilde{R}_{N \backslash(S \cup\{k\})}^{\prime}\right)$.

Consider $R^{1}$. By construction and efficiency; $\varphi_{1}\left(R^{1}\right)=\varphi_{1}\left(\tilde{R}^{\prime}\right)$. Since $\varphi_{1}\left(R^{o}\right) I_{1}^{o} \varphi_{1}\left(R^{1}\right)$, coalitional strategy-proofness implies for all $j \in N \backslash S$,

$$
\varphi_{j}\left(R^{o}\right) R_{j}^{o} \varphi_{j}\left(R^{1}\right)
$$

Efficiency and the construction of $\tilde{R}^{\prime}$ and $\bar{R}_{k}$ imply

$$
\left\{\varphi_{1}\left(R^{1}\right), \ldots, \varphi_{k-1}\left(R^{1}\right)\right\}=\left\{\varphi_{1}\left(R^{o}\right), \ldots, \varphi_{k-1}\left(R^{o}\right)\right\}
$$

Thus, by $R_{N \backslash S}^{1} \in \mathcal{S}^{N \backslash S}$, (4.22), (4.23), and efficiency, the previous fact implies that for all $j \in N \backslash S, \varphi_{j}\left(R^{1}\right)=\varphi_{j}\left(R^{o}\right)$. In particular $\varphi_{k}\left(R^{1}\right)=\bar{h}$. Consider $R^{2}$. By efficiency, $\varphi_{1}\left(R^{2}\right)=\varphi_{1}\left(\tilde{R}^{\prime}\right)$ and $\varphi_{2}\left(R^{2}\right)=\varphi_{2}\left(\tilde{R}^{\prime}\right)$. Cising the same arguments as before yields $\varphi_{k}\left(R^{2}\right)=\bar{h}$. By induction it follows that $\varphi_{k}\left(R^{k-1}\right)=\bar{h}$. Note that $R^{k-1}=\left(\tilde{R}_{N \backslash\{k\}}^{\prime}, \bar{R}_{k}\right)$.

Thus, by strategy-proofness, $\varphi_{k}\left(\tilde{R}^{\prime}\right) \tilde{R}_{k}^{\prime} \tilde{h}$. By $(4.19)$ and $(4.20), \varphi_{k}\left(\tilde{R}^{\prime}\right)=\varphi_{k}(R)$. Hence, by construction of $\tilde{R}_{k}^{\prime}$, the previous fact implies $\varphi_{k}(R) R_{k} \bar{h}$, the desired conclusion.

The collection $\sigma^{h}, h \in H$, yields a table. Given $k \in N$, the vector $\left(\sigma^{h}(k)\right)_{h \in I}$ is a row of this table. Next we prove that the first row contains at most two agents.

Lemma $4.7\left|\left\{\sigma^{h}(1) \mid h \in H\right\}\right| \leq 2$. 
Proof. Suppose that $\left|\left\{\sigma^{h}(1) \mid h \in H\right\}\right| \geq 3$ and let $h_{1}, h_{2}, h_{3} \in H$. For all $i \in\{1,2,3\}$, let $\sigma^{h_{i}}(1)=i$. Let $R \in\left(\mathcal{S} \cup\left\{R_{0}^{I}\right\}\right)^{N}$ be such that for all $i \in N \backslash\{2,3\}, R_{i}=R_{0}^{I}$, $B\left(H, R_{2}\right)=\left\{h_{1}\right\}=B\left(H, R_{3}\right), B\left(H \backslash\left\{h_{1}\right\}, R_{2}\right)=\left\{h_{2}\right\}$, and $B\left(H \backslash\left\{h_{1}\right\}, R_{3}\right)=\left\{h_{3}\right\}$. By efficiency, either $\varphi_{2}(R)=h_{1}$ or $\varphi_{3}(R)=h_{1}$. Let $\varphi_{2}(R)=h_{1}$. Let $R^{\prime} \in\left(\mathcal{S} \cup\left\{R_{0}^{I}\right\}\right)^{N}$ be such that $R_{N \backslash\{1\}}^{\prime}=R_{N \backslash\{1\}}, B\left(H, R_{1}^{\prime}\right)=\left\{h_{3}\right\}$, and $B\left(H \backslash\left\{h_{3}\right\}, R_{1}^{\prime}\right)=\left\{h_{1}\right\}$. Lemma 4.6 and efficiency imply $\varphi_{1}\left(R^{\prime}\right)=h_{3}$ and $\varphi_{3}\left(R^{\prime}\right)=h_{1}$, which contradicts coalitional strategyproofness.

Lemma 4.8If $\left|\left\{\sigma^{h}(1) \mid h \in H\right\}\right|=2$, then $\left\{\sigma^{h}(2) \mid h \in H\right\}=\left\{\sigma^{h}(1) \mid h \in H\right\}$.

Proof. Let $h_{1}, h_{2} \in H, \sigma^{h_{1}}(1)=1$, and $\sigma^{h_{2}}(1)=2$. Suppose that $\sigma^{h_{2}}(2)=3$. Consider $R \in\left(\mathcal{S} \cup\left\{R_{0}^{I}\right\}\right)^{N}$ such that for all $i \in N \backslash\{1,3\}, R_{i}=R_{0}^{I}, B\left(H, R_{1}\right)=\left\{h_{2}\right\}=B\left(H, R_{3}\right)$; $B\left(H \backslash\left\{h_{2}\right\}, R_{1}\right)=h_{1}$, and $B\left(H \backslash\left\{h_{2}\right\}, R_{3}\right)=h_{3}$. By Lemma 4.6 and efficiency, $\varphi_{1}(R)=h_{1}$ and $\varphi_{3}(R)=h_{2}$. Let $R^{\prime} \in\left(\mathcal{S} \cup\left\{R_{0}^{I}\right\}\right)^{N}$ be such that $R_{N \backslash\{2\}}^{\prime}=R_{N \backslash\{2\}}, B\left(H, R_{2}^{\prime}\right)=\left\{h_{1}\right\}$; and $B\left(H \backslash\left\{h_{1}\right\}, R_{2}^{\prime}\right)=h_{2}$. Hence, by Lemma 4.6 and efficiency, $\varphi_{1}\left(R^{\prime}\right)=h_{2}$ and $\varphi_{2}\left(R^{\prime}\right)=$ $h_{1}$, which contradicts coalitional strategy-proofness.

We define an ordered partition $\mathcal{T}$ of $N$ and a house partition structure $\mathcal{H}$ for $\mathcal{T}$, and show that $\varphi$ is a mixed dictator-pairwise-exchange rule relative to $(\mathcal{T}, \mathcal{H})$.

Define $T_{1} \equiv\left\{\sigma^{h}(1) \mid h \in H\right\}$. If for some $l, \sum_{t=1}^{l}\left|T_{l}\right|<|N|$, then define $T_{l+1} \equiv$ $\left\{\sigma^{h}\left(1+\sum_{t=1}^{l}\left|T_{l}\right|\right) \mid h \in H\right\}$. For some $m \in N, \sum_{t=1}^{m}\left|T_{l}\right| \geq|N|$. Let $\mathcal{T} \equiv\left(T_{1}, \ldots, T_{m}\right)$. For all $l \in\{1, \ldots, m\}$, when $i \in T_{l}$, we define $H_{i}^{T_{i}} \equiv\left\{h \in H \mid \sigma^{h}\left(1+\sum_{t=1}^{l-1}\left|T_{\ell}\right|\right)=i\right\}$. Let $\mathcal{H} \equiv\left(\left\{H_{i}^{T_{i}} \mid i \in T_{l}\right\}\right)_{l \in\{1, \ldots, m\}}$.

Lemma 4.9 On the domain $\left(\mathcal{S} \cup\left\{R_{0}^{I}\right\}\right)^{N}, \varphi$ is a mixed dictator-pairwise-exchange rule relative to $(\mathcal{T}, \mathcal{H})$.

Proof. Let $R \in\left(\mathcal{S} \cup\left\{R_{0}^{I}\right\}\right)^{N}$. By Lemmas 4.7 and $4.8,\left|T_{1}\right| \in\{1,2\}$. If $\left|T_{1}\right|=1$, say $T_{1}=\{1\}$, then $H_{1}^{T_{1}}=H$ and, by Lemma 4.6, $\varphi_{1}(R) \in B\left(H, R_{1}\right)$. When $\left|T_{1}\right|=2$, say $T_{1}=\{1,2\}$, then by definition, $H_{1}^{T_{1}} \neq \emptyset \neq H_{2}^{T_{1}}$ and $H_{1}^{T_{1}} \cap H_{2}^{T_{1}}=\emptyset$, and by Lemma 4.7, $H_{1}^{T_{1}} \cup H_{2}^{T_{1}}=H$. The following holds. If $B\left(H, R_{1}\right) \subseteq B\left(H_{1}^{T_{1}}, R_{1}\right)$, then, by Lemma 4.6, $\varphi_{1}(R) \in B\left(H, R_{1}\right)$, and by Lemmas 4.6 and $4.8, \varphi_{2}(R) \in B\left(H \backslash\left\{\varphi_{1}(R)\right\}, R_{2}\right)$. Otherwise, by Lemma 4.6 and efficiency, $\varphi_{2}(R) \in B\left(H, R_{2}\right)$ and $\varphi_{1}(R) \in B\left(H \backslash\left\{\varphi_{2}(R)\right\}, R_{1}\right)$. Consider $T_{2}$. Adapting the proofs of Lemmas 4.7 and 4.8 yields the same assertions, i.e., $\left|T_{2}\right| \leq 2$, and if $\left|T_{2}\right|=2$, then $T_{2}=\left\{\sigma^{h}\left(2+\left|T_{1}\right|\right) \mid h \in H\right\}$. By using the same arguments as above, the same conclusions hold for $T_{2}$. By induction it follows that $\varphi(R) \in Y^{(\mathcal{T}, \mathcal{H})}(R)$. Note that adapting Lemmas 4.7 and 4.8 to the sets $T_{l}, l \in\{1, \ldots, m\}$, implies that $\mathcal{T}$ is an ordered partition of $N$ and $\mathcal{H}$ is a house partition structure for $\mathcal{T}$. 
Our final lemma shows Theorem 4.2. In proving the lemma we need additional notation.

Given $R_{0} \in \overline{\mathcal{S}}$, let $W\left(H, R_{0}\right) \equiv\left\{h \in H \mid\right.$ for all $\left.h^{\prime} \in H, h^{\prime} R_{0} h\right\}$ be the set of worst louses in $H$ according to $R_{0}$. Given $R_{0}, R_{0}^{\prime} \in \bar{S}$ and $H^{\prime} \subseteq H$, we write $R_{0}\left|H^{\prime}=R_{0}^{\prime}\right| H^{\prime}$ if and only if for all $h, h^{\prime} \in H^{\prime}, h R_{0} h^{\prime} \Leftrightarrow h R_{0}^{\prime} h^{\prime}$.

Lemma 4.10 Let $\varphi$ be a rule defined on the domain $\overline{\mathcal{S}}^{N}$. If $\varphi$ satisfies efficiency and coalitional strategy-proofness, then $\varphi$ is a mixed dictator-pairwise-exchange rule.

Proof. By Lemma 4.9, $\varphi$ is a mixed dictator-pairwise-exchange rule relative to some $(\mathcal{T}, \mathcal{H})$ on the domain $\left(\mathcal{S} \cup\left\{R_{0}^{I}\right\}\right)^{N}$. Let $R \in \overline{\mathcal{S}}^{N}$. We have to show that $\varphi(R) \in Y^{(\mathcal{T}, \mathcal{H})}(R)$. Let $M \subseteq N$ be such that for all $i \in M, R_{i} \notin \mathcal{S} \cup\left\{R_{0}^{I}\right\}$, and for all $i \in N \backslash M, R_{i} \in \mathcal{S} \cup\left\{R_{0}^{I}\right\}$. Let $\bar{N} \equiv\left\{i \in N \mid \varphi_{i}(R) \in W\left(H, R_{i}\right)\right\}$ and

$$
\bar{H} \equiv \cap_{i \in \bar{N}} W\left(H, R_{i}\right) .
$$

By Pareto-optimality, for all $i \in \bar{N}, \varphi_{i}(R) \in \bar{H}$. Let $\bar{R}_{M \backslash \bar{N}} \in \mathcal{S}^{M \backslash \bar{N}}$ be such that for all $i \in M \backslash \bar{N}, \bar{R}_{i}\left|H \backslash W\left(H, R_{i}\right)=R_{i}\right| H \backslash W\left(H, R_{i}\right)$ and for all $h \in H \backslash W\left(H, R_{i}\right)$ and all $h^{\prime} \in$ $W\left(H, R_{i}\right), h \vec{P}_{i} h^{\prime}$. Let $j \in M \backslash \bar{N}$. By construction and strategy-proofness, $\varphi_{j}\left(\bar{R}_{j}, R_{N \backslash\{j\}}\right)=$ $\varphi_{j}(R)$. Thus, by applying (ii) of coalitional strategy-proofness twice, for all $i \in N \backslash\{j\}$, $\varphi_{i}\left(\bar{R}_{j}, R_{N \backslash\{j\}}\right) I_{i} \varphi_{i}(R)$. Hence, by Pareto-optimality and the two previous facts, for all $i \in N \backslash \bar{N}, \varphi_{i}(R)=\varphi_{i}\left(\bar{R}_{j}, R_{N \backslash\{j\}}\right)$, and for all $i \in \bar{N}, \varphi_{i}\left(\bar{R}_{j}, R_{N \backslash\{j\}}\right) \in \bar{H}$. Let $l \in M \backslash(\bar{N} \cup$ $\{j\})$. The same argumentation as above yields for all $i \in N \backslash \bar{N}, \varphi_{i}\left(\tilde{R}_{j}, \bar{R}_{l}, R_{N \backslash\{j, l\}}\right)=$ $\varphi_{i}\left(\vec{R}_{j}, R_{N \backslash\{j\}}\right)$ and for all $i \in \bar{N}, \varphi_{i}\left(\bar{R}_{j}, \vec{R}_{l}, R_{N \backslash\{j, l\}}\right) \in \bar{H}$. By induction it follows that for all $i \in N \backslash \bar{N}, \varphi_{i}\left(\bar{R}_{M \backslash \bar{N}}, R_{N \backslash(M \backslash \bar{N})}\right)=\varphi_{i}(R)$, and for all $i \in \bar{N}, \varphi_{i}\left(\bar{R}_{M \backslash \bar{N}}, R_{N \backslash(M \backslash N)}\right) \in \bar{H}$.

Let $j \in M \cap \bar{N}$ and $\bar{R}_{j} \in \mathcal{S}$ be such that $\bar{R}_{j}\left|H \backslash W\left(H, R_{j}\right)=R_{j}\right| H \backslash W\left(H, R_{j}\right)$ and for all $h \in H \backslash W\left(H, R_{j}\right)$ and all $h^{\prime} \in W\left(H, R_{j}\right), h \bar{P}_{j} \varphi_{j}\left(\bar{R}_{M \backslash \bar{N}}, R_{N \backslash(M \backslash \bar{N})}\right) \bar{R}_{j} h^{\prime}$. By strategyproofness, $\varphi_{j}\left(\bar{R}_{M \backslash(\bar{N} \backslash\{j\})}, R_{N \backslash(M \backslash(\bar{N} \backslash\{j\}))}\right)=\varphi_{j}\left(\bar{R}_{M \backslash \bar{N}}, R_{N \backslash(M \backslash \bar{N})}\right)$. Thus, by coalitional strategy-proofness, for all $i \in N \backslash \bar{N}, \varphi_{i}\left(\bar{R}_{M \backslash(\bar{N} \backslash\{j\})}, R_{N \backslash(M \backslash(\bar{N} \backslash\{j\}))}\right)=\varphi_{i}\left(\tilde{R}_{M \backslash \bar{N}}, R_{N \backslash(M \backslash \bar{N})}\right)$ and for all $i \in \bar{N}, \varphi_{i}\left(\bar{R}_{M \backslash(\bar{N} \backslash\{j\})}, R_{N \backslash(M \backslash(\bar{N} \backslash\{j\}))}\right) \in \bar{H}$. Then let $l \in(M \cap \bar{N}) \backslash\{j\}$ and $\bar{R}_{l} \in \mathcal{S}$ be such that $\vec{R}_{l}\left|H \backslash W\left(H, R_{l}\right)=R_{l}\right| H \backslash W\left(H, R_{l}\right)$ and for all $h \in H \backslash W\left(H, R_{l}\right)$ and all $h^{\prime} \in W\left(H, R_{l}\right), h \bar{P}_{l} \varphi_{l}\left(\bar{R}_{M \backslash(\bar{N} \backslash\{j\})}, R_{N \backslash(M \backslash(\bar{N} \backslash\{j\}))}\right) \bar{R}_{l} h^{\prime}$. By induction and using the same arguments as above we obtain a profile $\bar{R}_{M} \in \mathcal{S}^{M}$ such that for all $i \in N \backslash \vec{N}$, $\varphi_{i}\left(\bar{R}_{M}, R_{N \backslash M}\right)=\varphi_{i}(R)$, and for all $i \in \bar{N}, \varphi_{i}\left(\bar{R}_{M}, R_{N \backslash M}\right) \in \bar{H}$, and if $i \in M \cap \bar{N}$, then $\bar{R}_{i} \in \mathcal{S}$ is such that for all $h \in W\left(H, R_{i}\right)$,

$$
\varphi_{i}\left(\bar{R}_{M}, R_{N \backslash M}\right) \bar{R}_{i} h .
$$

Since $\left(\bar{R}_{M}, R_{N \backslash M}\right) \in\left(\mathcal{S} \cup\left\{R_{0}^{I}\right\}\right)^{N}$ and $\varphi$ is a mixed dictator-pairwise-exchange rule on the domain $\left(\mathcal{S} \cup\left\{R_{0}^{I}\right\}\right)^{N}$, we have $\varphi\left(\bar{R}_{M}, R_{N \backslash M}\right) \in Y^{(\mathcal{T}, \mathcal{H})}\left(\bar{R}_{M}, R_{N \backslash M}\right)$. Let $\bar{R} \equiv$ 
$\left(\bar{R}_{M}, R_{N \backslash M}\right)$. Thus, it follows that for all $i \in N$, if $i \in T_{l}$ and for some $j \in N$, $\varphi_{j}\left(\bar{R}_{M}, R_{N \backslash M}\right) \bar{P}_{i} \varphi_{i}\left(\bar{R}_{M}, R_{N \backslash M}\right)$, then $j \in \cup_{l=1}^{l} T_{\imath}$ and $\varphi_{j}\left(\bar{R}_{M}, R_{N \backslash M}\right) \bar{P}_{j} \varphi_{i}\left(\bar{R}_{M}, R_{N \backslash M}\right)$.

Hence, if $i \in M \cap \bar{N}$ in the previous statement, then (4.24) implies $j \in N \backslash \bar{N}$. Since for all $i \in N \backslash \bar{N}, \varphi_{i}(R)=\varphi_{i}\left(\bar{R}_{M}, R_{N \backslash M}\right)$, and for all $i \in \bar{N}, \varphi_{i}(R), \varphi_{i}\left(\tilde{R}_{M}, R_{N \backslash M}\right) \in \bar{H}$, it follows that $\varphi\left(\bar{R}_{M}, R_{N \backslash M}\right) \in E(R)$. Now it is straightforward that the previous facts, the construction of $\bar{R}_{M}$, and (4.24) imply $\varphi\left(\bar{R}_{M}, R_{N \backslash M}\right) \in Y^{(\mathcal{T}, \mathcal{H})}(R)$. Since for all $i \in N$, $\varphi\left(\bar{R}_{M}, R_{N \backslash M}\right) I_{i} \varphi_{i}(R)$, we obtain $\varphi(R) \in Y^{(\mathcal{T}, \mathcal{H})}(R)$, the desired conclusion. 


\section{Chapter 5}

\section{Coalitional Strategy-Proof and Resource-Monotonic Solutions for Multiple Assignment Problems}

\subsection{Introduction}

This chapter, which is based on Ehlers and Klaus (2000b), considers the problem of assigning indivisible objects to a set of agents when monetary transfers are not possible. Most of the literature considers situations where each agent receives exactly one object. Such problems arise when we have to assign jobs to workers, or apartments to students. For the assignment problem (with or without property rights) where each agent may receive at most one object and monetary transfers are not allowed, rules satisfying desirable properties were recently studied; see for instance Ehlers, Klaus, and Pápai (2000), Ergin (2000), Ma (1994), Miyagawa (2001), Pápai (2000a), and Svensson (1994,1999).

We depart from the above papers and consider a multiple assignment problem. Each agent receives a set of objects and monetary transfers are not possible. As an example, one may think of a heritage consisting of indivisible objects (e.g., furniture and household items) that has to be distributed among the heirs (e.g., the children of the deceased), respecting the wish that the objects should not be sold but allocated. Since agents may receive sets of objects, there are several interesting preference domains one could consider. Pápai $(2000 b, 2001)$ studies the multiple assignment problem on the domain of strict preferences and on monotonic preference domains (on the monotonic preference domain any set of objects is strictly preferred to all of its proper subsets and on the quantity-monotonic preference domain receiving more objects is always preferred to receiving less objects). The strict preference domain includes preference relations where receiving all objects is 
strictly preferred to receiving nothing, but receiving nothing is preferred to receiving some but not all objects.

We consider the same domains of preference relations as in Klaus and Miyagawa (1999): the general domain of strict preferences, additive and strict preferences, and responsive, separable, and strict preferences. A preference relation is separable if object $x$ is a "good" (receiving $x$ is preferred to receiving nothing), then for each set of objects not containing $x$, he prefers receiving this set and $x$ to receiving only this set. This property excludes the possibility that $x$ becomes a "bad object" if an agent consumes it with a certain set of objects. The domain of separable preferences is a natural domain for heritage problems.

We search for solutions (or assignment rules) that satisfy "desirable" properties from an economic and social point of view. Most of the literature concerned with this "axiomatic" approach to assignment problems is for models which also include the possibility of monetary transfers; for the class of multiple assignment problems see Bevia (1998) and Tadenuma (1996). Surprisingly, the results for the multiple assignment problems we study here and assignment problems where each agent may receive at most one object are very different. Whereas in the latter case natural trading mechanisms (e.g., the core) do satisfy many desirable properties, this is not the case for multiple assignment problems. The results we obtain might be interpreted as negative results since, depending on the preference domain, either incompatibility of the properties result or the set of allocation rules is narrowed down to sequential or serial dictatorship rules only. However, a practical advantage of serial (sequential) dictatorships is that they are simple and can be implemented easily and quickly. Furthermore, they are efficient, strategy-proof, and satisfy other appealing properties discussed below. They can be considered "fair" as well when the ordering of the agents is fairly determined; for instance by queuing, seniority, or randomization (Abdulkadiroğlu and Sönmez, 1998,1999; Bogomolnaia and Moulin, 1999).

We briefly discuss the organization of this chapter and the results. In Section 5.2 we introduce the model and two basic properties. First of all, we impose (Pareto) efficiency meaning that the rule chooses for each profile an efficient assignment. Second, in order to eliminate profitable misrepresentation of only privately known preferences, we impose strategy-proofness (no agent ever can gain by misrepresenting his preferences).

In Section 5.3 we consider the stronger non-manipulation property coalitional strategyproofness (no group of agents ever can gain by jointly misrepresenting their preferences). Our main result shows that efficiency and coalitional strategy-proofness only allow for sequential dictatorships; i.e., there exists a first dictator who always chooses his best set of objects. Depending on the first dictator's choice, a second dictator is determined who again chooses his best subset of the remaining objects. Depending on the choices of the 
previous dictators, a third dictator is determined etc.. Pápai (1998) shows the same result on the domain of strict preferences. However, her proof uses preference relations where receiving all objects is strictly preferred to receiving nothing, but receiving nothing is preferred to receiving some but not all objects. Her and our proof are completely different. Furthermore, it is surprising that her result even holds on the domain of separable preference. This means also that we are able to give a full characterization of efficient and coalitional strategy-proof rules for the multiple assignment problem. When each agent receives exactly one object it is an open question how the rules satisfying Pareto-optimality and coalitional strategy-proofness look like.

In Section 5.4 we consider the multiple assignment problem for variable sets of indivisible objects. Resource-monotonicity describes the effect of a change in the available resource on the welfare of the agents. A rule satisfies resource-monotonicity, if after such a change either all agents (weakly) lose together or all (weakly) gain together. On the domain of responsive, separable, and strict (or additive and strict) preferences, the solidarity property resource-monotonicity is compatible with both efficiency and coalitional strategyproofness. All three properties together characterize the class of serial dictatorships. On the domain of strict preferences, efficiency, strategy-proofness, and resource-monotonicity are not compatible.

\subsection{The Model and Basic Properties}

We consider the same multiple assignment model as Pápai (1998) and Klaus and Miyagawa (1999). Let $K \equiv\left\{x_{1}, \ldots, x_{k}\right\}$ denote the finite set of objects, and $N \equiv\{1, \ldots, n\}$ the finite set of agents. We always assume that $|K|=k \geq 2$ and $|N|=n \geq 2$. Let $2^{K}$ denote the set of all subsets of $K$ including the empty set. Each agent $i \in N$ has a preference relation $R_{i}$ over $2^{K}$. Let $P_{i}$ denote the strict preference relation associated with $R_{i}$. A preference relation $R_{i}$ is strict if for all distinct subsets $S, S^{\prime} \in 2^{K}$, we have either $S P_{i} S^{\prime}$ or $S^{\prime} P_{i} S$. Thus, $S R_{i} S^{\prime}$ implies that either $S P_{i} S^{\prime}$ or $S=S^{\prime}$. We further assume that $R_{i}$ is responsive and separable. For subsets of $K$ consisting of exactly one object we often omit the brackets and write $x$ instead of $\{x\}$.

A preference relation is responsive if, for any two sets that differ only in one object, the set containing the more preferred object is preferred to the other: for all $S \subseteq K$ and all $x, y \in K \backslash S$,

$$
x P_{i} y \Leftrightarrow S \cup\{x\} P_{i} S \cup\{y\}
$$

A preference relation is separable if $\{x\}$ is preferred to nothing if and only if for all 
$S \in 2^{K}$ not containing $x, S \cup\{x\}$ is preferred to $S$ : for all $S \subseteq K$ and all $x \in K \backslash S$,

$$
x P_{i} \emptyset \Leftrightarrow S \cup\{x\} P_{i} S
$$

Together with strictness and completeness of preferences this implies that for all $S \subseteq K$ and all $x \in K \backslash S$,

$$
\emptyset P_{i} x \Leftrightarrow S P_{i} S \cup\{x\}
$$

Roth (1985) introduced responsiveness of preference relations for college admission problems. For the notion of separability we use here, we refer to Barberà, Sonnenschein, and Zhou (1991).

Let $\mathcal{R}$ be the set of responsive, separable, and strict preference relations over $2^{K}{ }^{1}$ Let $\mathcal{S}$ denote the class of strict preference relations over $2^{K}$, and $\mathcal{A}$ the class of strict and additive preference relations over $2^{K}{ }^{2}$ Note that $\mathcal{A} \subsetneq \mathcal{R} \subsetneq \mathcal{S}$. If not otherwise stated, we assume that preferences are responsive, separable, and strict; that is, $\mathcal{R}$ is the default preference domain. In what follows, all definitions using the preference domain $\mathcal{R}$ also apply to the preference domains $\mathcal{A}$ and $\mathcal{S}$.

A preference profile is a list $R=\left(R_{1}, R_{2}, \ldots, R_{n}\right)$ where for all $i \in N, R_{i} \in \mathcal{R}$. Let $\mathcal{R}^{N}$ denote the set of preference profiles. Since, for the time being, the set of agents and the set of objects are fixed, $\mathcal{R}^{N}$ completely describes the set of multiple assignment problems under consideration.

Given $m \leq k$ and an ordered collection $\left\{y_{1}, y_{2}, \ldots, y_{m}\right\} \subseteq K$ of objects, we denote by $L\left(y_{1}, y_{2}, \ldots, y_{m}\right)$ the class of preference relations $R_{i} \in \mathcal{R}$ such that $y_{1} P_{i} y_{2} P_{i} \cdots P_{i} y_{m} P_{i} \emptyset$ and for all $y \in I \backslash\left\{y_{1}, \ldots, y_{m}\right\}, \emptyset P_{i} y$. Note that then responsiveness and separability imply $\left\{y_{1}, y_{3}\right\} P_{i}\left\{y_{2}, y_{3}\right\} P_{i} \emptyset P_{i} y_{m+1}$ and so on. Furthermore, let $L(\emptyset)$ be the set of preference relations $R_{i} \in \mathcal{R}$ such that for all $y \in K, \emptyset P_{i} y$. The set $L(\emptyset)$ contains the preference relations where each object is conceived to be a "bad".

Given $R_{i} \in \mathcal{R}$, let $B\left(R_{i}\right) \equiv\left\{x \in K \mid x P_{i} \emptyset\right\}$ be the set of objects that are each preferred to $\emptyset$. Objects belonging to $B\left(R_{i}\right)$ are conceived to be "goods". Separability of $R_{i}$ implies that $B\left(R_{i}\right)$ is most preferred at $R_{i}$ in $2^{K}$, i.e., for all $S \in 2^{K}, B\left(R_{i}\right) R_{i} S$.

An assignment is a list $\left(S_{1}, \ldots, S_{n}\right)$ such that for all $i \in N, S_{i} \subseteq K$, and for all $i, j \in N$ such that $i \neq j, S_{i} \cap S_{j}=\emptyset$. The set $S_{i}$ is the set of objects assigned to agent $i$. We do not

\footnotetext{
${ }^{1}$ All results that we establish for the domain of responsive, separable, and strict preferences also remain true on the domain of separable and strict preferences.

${ }^{2} \mathrm{~A}$ preference relation $R$ is additive if there exists a function $u: K \rightarrow \mathbb{R}$ such that for all $S, S^{\prime} \in 2^{K}$,

$$
S R_{i} S^{\prime} \Leftrightarrow \sum_{k \in S} u(k) \geq \sum_{k \in S^{\prime}} u(k)
$$
}

with the convention $\sum_{k \in \emptyset} u(k)=0$. 
rule out the possibility that $S_{i}$ is empty. The second condition simply says that no two agents receive the same object. The union of all $S_{i}$ 's may be a strict subset of $K$, which means that we allow free disposal.

An assignment rule, or rule for short, is a function $\varphi$ that associates with each preference profile $R \in \mathcal{R}^{N}$ an assignment $\varphi(R)=\left(S_{i}\right)_{i \in N}$. Let $\varphi_{i}(R)$ denote the set of objects assigned to agent $i$ at $R$. We are interested in rules satisfying the following properties.

A rule $\varphi$ is (Pareto) efficient if it chooses for each profile an efficient assignment.

Efficiency: For all $R \in \mathcal{R}^{N}$ : there is no assignment $\left(S_{i}\right)_{i \in N}$ such that for all $i \in N$, $S_{i} R_{i} \varphi_{i}(R)$, with strict preference holding for some $j \in N$.

It is straightforward to check that separability of preference relations and free disposal imply the following.

Lemma 5.1 If a rule $\varphi$ is efficient, then for all $R \in \mathcal{R}^{N}$

(i) for all $i \in N, \varphi_{i}(R) \subseteq B\left(R_{i}\right)$ and

(ii) $\cup_{i \in N} \varphi_{i}(R)=\cup_{i \in N} B\left(R_{i}\right)$.

It is easy to see that the converse of Lemma 5.1 is wrong. ${ }^{3}$ The following notation will be useful later on. Given $R \in \mathcal{R}^{N}$ and $M \subseteq N$, let $R_{M}$ denote the profile $\left(R_{i}\right)_{i \in M}$. It is the restriction of $R$ to the set of agents $M$. Given $i, j \in N$, we also use the notation $R_{-i} \equiv R_{N \backslash\{i\}}$ and $R_{-i, j} \equiv R_{N \backslash\{i, j\}}$.

A rule $\varphi$ is strategy-proof if no agent ever benefits from misrepresenting his preferences. In game theoretical terms, a rule is strategy-proof if in its associated direct revelation game form, it is a weakly dominant strategy for each agent to announce his true preference relation.

Strategy-Proofness: For all $R \in \mathcal{R}^{N}$, all $i \in N$, and all $R_{i}^{\prime} \in \mathcal{R}: \varphi_{i}(R) R_{i} \varphi_{i}\left(R_{i}^{\prime}, R_{-i}\right)$

\subsection{Coalitional Strategy-Proofness}

This section investigates a stronger nonmanipulation condition than strategy-proofness. A rule $\varphi$ satisfies coalitional strategy-proofness if no coalition of agents ever benefits from misrepresenting their preferences.

\footnotetext{
${ }^{3}$ For example, let $n \equiv 2, k \equiv 2, R_{1} \in L\left(x_{1}, x_{2}\right)$, and $R_{2} \in L\left(x_{2}, x_{1}\right)$. The assignment $\left(x_{2}, x_{1}\right)$ satisfies (i) and (ii) of Lemma 5.1 but is Pareto dominated by $\left(x_{1}, x_{2}\right)$.
} 
Coalitional Strategy-Proofness: For all $R \in \mathcal{R}^{N}$ and all $M \subseteq N$ : there exists no $R^{\prime} \in \mathcal{R}^{N}$ such that $R_{N \backslash M}^{\prime}=R_{N \backslash M}$ and for all $i \in M, \varphi_{i}\left(R^{\prime}\right) R_{i} \varphi_{i}(R)$ with strict preference holding for some $i \in M$.

Coalitional strategy-proofness excludes "bossy" behavior; that is, by unilaterally changing his preferences, one of the agents cannot influence the allocation of some other agent without changing his own allocation. This concept of non-bossiness was introduced by Satterthwaite and Sonnenschein (1981).

Non-Bossiness: For all $R \in \mathcal{R}^{N}$, all $i \in N$, and all $R_{i}^{\prime} \in \mathcal{R}$ : if $\varphi_{i}(R)=\varphi_{i}\left(R_{i}^{\prime}, R_{-i}\right)$, then $\varphi(R)=\varphi\left(R_{i}^{\prime}, R_{-i}\right)$.

The following lemma is straightforward.

Lemma 5.2 If a rule $\varphi$ satisfies coalitional strategy-proofness, then it satisfies strategyproofness and non-bossiness.

The main result is a characterization of the class of efficient and coalitional strategyproof rules. A rule satisfying these properties is a "sequential dictatorship." A first dictator chooses his most preferred set of objects. Depending on the first dictator's choice, a second dictator is determined who again chooses his most preferred subset of the remaining objects. Depending on the choices of the previous dictators, a third dictator is determined and so on. In order to formalize the class of sequential dictatorships we need some additional notation.

A permutation $\pi$ on $N$ is a bijective function $\pi: N \rightarrow N$. Let $\Pi^{N}$ denote the set of all permutations on $N$. Given $\pi \in \Pi^{N}$ and $i \in N$, we interpret agent $\pi(i)$ to be the $i$ th "dictator".

Sequential Dictatorship: For all $R \in \mathcal{R}^{N}$, there is a fixed $\pi_{R} \in \Pi^{N}$ such that

$$
\begin{aligned}
\varphi_{\pi_{R}(1)}(R) & =B\left(R_{\pi_{R}(1)}\right) \\
\varphi_{\pi_{R}(2)}(R) & =B\left(R_{\pi_{R}(2)}\right) \backslash B\left(R_{\pi_{R}(1)}\right) \\
\varphi_{\pi_{R}(3)}(R) & =B\left(R_{\pi_{R}(3)}\right) \backslash\left[B\left(R_{\pi_{R}(1)}\right) \cup B\left(R_{\pi_{R}(2)}\right)\right] \\
& \vdots \\
\varphi_{\pi_{R}(n)}(R) & =B\left(R_{\pi_{R}(n)}\right) \backslash\left[\cup_{i=\pi_{R}(1)}^{\pi_{R}(n-1)} B\left(R_{i}\right)\right] .
\end{aligned}
$$

We call agent $\pi_{R}(1)$ the first dictator at $R$, agent $\pi_{R}(2)$ the second dictator at $R$, etc.. 
For all $R, \bar{R} \in \mathcal{R}^{N}$ and $\pi_{R}, \pi_{\bar{R}} \in \Pi^{N}$ as specified above, the following two conditions must be satisfied:

(i) $\pi_{R}(1)=\pi_{n}(1)$.

(ii) Let $m \in\{1, \ldots, n-1\}$. If for all $i \in\{1, \ldots, m\}, \pi_{R}(i)=\pi_{\bar{R}}(i)$ and $\varphi_{\pi_{R}(i)}(R)=$ $\varphi_{\pi_{i k}(i)}(\bar{R})$, then $\pi_{R}(m+1)=\pi_{R}(m+1)$.

Note that, by (i), for any sequential dictatorship there exists a unique first dictator. In (ii) we formalize that the choice of the next dictator who is allowed to choose his most preferred set of objects from the remaining objects only depends on the previous dictators and their individual choices and not on the exact preferences of the previous dictators or the remaining agents.

A subclass of sequential dictatorships are serial dictatorships. Here, the choice of the next dictator does not depend on the sets of objects chosen by the first dictators. Formally, a rule $\varphi$ is a serial dictatorship if $\varphi$ is a sequential dictatorship and there exists $\bar{\pi} \in \Pi^{N}$ such that for all $R \in \mathcal{R}^{N}, \pi_{R}=\bar{\pi}$. Instead of $\varphi$ then we write $\varphi^{\bar{\pi}}$.

Theorem 5.1 The following statements are equivalent.

(a) $\varphi$ is a sequential dictatorship.

(b) $\varphi$ satisfies efficiency and coalitional strategy-proofness.

(c) $\varphi$ satisfies efficiency, strategy-proofness, and non-bossiness.

\section{Proof.}

(a) implies (b). It is straightforward to check that sequential dictatorships satisfy efficiency and coalitional strategy-proofness.

(b) implies (c). By Lemma 5.2, coalitional strategy-proofness implies strategy-proofness and non-bossiness.

(c) implies (a). Let $\varphi$ be a rule satisfying efficiency, strategy-proofness, and nonbossiness. We show in four steps that $\varphi$ is a sequential dictatorship.

Step 1. We show that there exists $j \in N$ such that for all $R \in \mathcal{R}^{N}$, if for all $i \in N$, $B\left(R_{i}\right)=K$, then

$$
\varphi_{j}(R)=K
$$

Let $R \in \mathcal{R}^{N}$ be such that for all $i \in N, B\left(R_{i}\right)=K$. Suppose that for all $i \in N$, $\varphi_{i}(R) \neq K$. By efficiency and Lemma 5.1, $\cup_{i \in N} \varphi_{i}(R)=K$. Without loss of generality, we suppose that $x_{1} \in \varphi_{1}(R) \neq \emptyset$ and $x_{2} \in \varphi_{2}(R) \neq \emptyset$.

Let $\left(R_{1}, R_{2}, R_{-1,2}^{\prime}\right) \in \mathcal{R}^{N}$ be such that for all $i \in N \backslash\{1,2\}, B\left(R_{i}^{\prime}\right)=\varphi_{i}(R)$. If we change $R$ stepwise to $\left(R_{1}, R_{2}, R_{-1,2}^{\prime}\right)$, then strategy-proofness and non-bossiness imply that 
$\varphi\left(R_{1}, R_{2}, R_{-1,2}^{\prime}\right)=\varphi(R)$. In particular, $x_{1} \in \varphi_{1}\left(R_{1}, R_{2}, R_{-1,2}^{\prime}\right)$ and $x_{2} \in \varphi_{2}\left(R_{1}, R_{2}, R_{-1,2}^{\prime}\right)$. Let $R_{2}^{\prime} \in \mathcal{R}$ be such that $B\left(R_{2}^{\prime}\right)=\varphi_{2}\left(R_{1}, R_{2}, R_{-1,2}^{\prime}\right) \cup\left\{x_{1}\right\}$ and for all $y \in \varphi_{2}\left(R_{1}, R_{2}, R_{-1,2}^{\prime}\right)$, $y P_{2}^{\prime} x_{1}$. By strategy-proofness and separability of $R_{2}^{\prime}, \varphi_{2}\left(R_{1}, R_{-1}^{\prime}\right)=\varphi_{2}\left(R_{1}, R_{2}, R_{-1,2}^{\prime}\right)$. By non-bossiness, $\varphi\left(R_{1}, R_{-1}^{\prime}\right)=\varphi\left(R_{1}, R_{2}, R_{-1,2}^{\prime}\right)$. Let $R_{1}^{\prime} \in \mathcal{R}$ be such that $B\left(R_{1}^{\prime}\right)=$ $\varphi_{1}\left(R_{1}, R_{-1}^{\prime}\right) \cup\left\{x_{2}\right\}$ and for all $y \in \varphi_{1}\left(R_{1}, R_{-1}^{\prime}\right), y P_{1}^{\prime} x_{2}$. Similarly as above it follows that $\varphi\left(R^{\prime}\right)=\varphi\left(R_{1}, R_{-1}^{\prime}\right)$.

If $\varphi_{1}\left(R^{\prime}\right)=\left\{x_{1}\right\}$, then let $\bar{R}_{1}^{\prime}=R_{1}^{\prime}$. Suppose that $\left|\varphi_{1}\left(R^{\prime}\right)\right| \geq 2$. Let $\bar{R}_{1}^{\prime} \in L\left(x_{1}, x_{2}\right)$ be such that $\varphi_{1}\left(R^{\prime}\right) \bar{P}_{1}^{\prime} \emptyset$. By efficiency, $\varphi_{1}\left(\bar{R}_{1}^{\prime}, R_{-1}^{\prime}\right) \subseteq\left\{x_{1}, x_{2}\right\}$. By strategy-proofness and construction of $\bar{R}_{1}^{\prime},\left|\varphi_{1}\left(\bar{R}_{1}^{\prime}, R_{-1}^{\prime}\right)\right| \geq 1$. Thus, by the three previous facts, efficiency, $x_{1} \bar{P}_{1}^{\prime} x_{2}$, and $x_{2} P_{2}^{\prime} x_{1}$, we have $\varphi_{1}\left(\bar{R}_{1}^{\prime}, R_{-1}^{\prime}\right)=\left\{x_{1}\right\}$ or $\varphi_{1}\left(\bar{R}_{1}^{\prime}, R_{-1}^{\prime}\right)=\left\{x_{1}, x_{2}\right\}$.

Suppose that $\varphi_{1}\left(\bar{R}_{1}^{\prime}, R_{-1}^{\prime}\right)=\left\{x_{1}, x_{2}\right\}$. Let $R_{1}^{\prime \prime} \in \mathcal{R}$ be such that $B\left(R_{1}^{\prime \prime}\right)=\varphi_{1}\left(R^{\prime}\right) \cup\left\{x_{2}\right\}$, $\left\{x_{1}, x_{2}\right\} P_{1}^{\prime \prime} \varphi_{1}\left(R^{\prime}\right)$, and $x_{1} P_{1}^{\prime \prime} x_{2}$. By efficiency, $\varphi_{1}\left(R^{\prime}\right) \backslash\left\{x_{1}\right\} \subseteq \varphi_{1}\left(R_{1}^{\prime \prime}, R_{-1}^{\prime}\right)$. By strategyproofness and construction of $R_{1}^{\prime \prime},\left|\varphi_{1}\left(R_{1}^{\prime \prime}, R_{-1}^{\prime}\right)\right|>\left|\varphi_{1}\left(R^{\prime}\right)\right|-1$. Thus, by efficiency, $x_{1} P_{1}^{\prime \prime} x_{2}$, and $x_{2} P_{2}^{\prime} x_{1}$, we have $x_{1} \in \varphi_{1}\left(R_{1}^{\prime \prime}, R_{-1}^{\prime}\right)$. Hence, by strategy-proofness and construction of $R_{1}^{\prime \prime}, \varphi_{1}\left(R_{1}^{\prime \prime}, R_{-1}^{\prime}\right)=\varphi_{1}\left(R^{\prime}\right) \cup\left\{x_{2}\right\}$. But then $\varphi_{1}\left(R_{1}^{\prime \prime}, R_{-1}^{\prime}\right) P_{1}^{\prime} \varphi_{1}\left(R^{\prime}\right)$, a contradiction to strategy-proofness.

Thus, we have $\varphi_{1}\left(\bar{R}_{1}^{\prime}, R_{-1}^{\prime}\right)=\left\{x_{1}\right\}$. By efficiency, for all $i \in N \backslash\{1\}, \varphi_{i}\left(\bar{R}_{1}^{\prime}, R_{-1}^{\prime}\right)=$ $\varphi_{i}\left(R^{\prime}\right)$. If $\varphi_{2}\left(\bar{R}_{1}^{\prime}, R_{-1}^{\prime}\right)=\left\{x_{2}\right\}$, then let $\bar{R}_{2}^{\prime}=R_{2}^{\prime}$. Suppose that $\left|\varphi_{2}\left(\bar{R}_{1}^{\prime}, R_{-1}^{\prime}\right)\right| \geq 2$. Let $\bar{R}_{2}^{\prime} \in L\left(x_{2}, x_{1}\right)$ be such that $\varphi_{2}\left(\bar{R}_{1}^{\prime}, R_{-1}^{\prime}\right) \bar{P}_{2}^{\prime} \emptyset$. Similarly as above it follows that $\varphi_{2}\left(\bar{R}_{1}^{\prime}, \bar{R}_{2}^{\prime}, R_{-1,2}^{\prime}\right)=\left\{x_{2}\right\}$ and for all $i \in N \backslash\{2\}, \varphi_{i}\left(\bar{R}_{1}^{\prime}, \bar{R}_{2}^{\prime}, R_{-1,2}^{\prime}\right)=\varphi_{i}\left(\bar{R}_{1}^{\prime}, R_{-1}^{\prime}\right)$.

Let $\bar{R}^{\prime} \equiv\left(\bar{R}_{1}^{\prime}, \bar{R}_{2}^{\prime}, R_{-1,2}^{\prime}\right)$. Hence, $\bar{R}_{1}^{\prime} \in L\left(x_{1}, x_{2}\right), \bar{R}_{2}^{\prime} \in L\left(x_{2}, x_{1}\right)$,

$$
\varphi_{1}\left(\bar{R}^{\prime}\right)=\left\{x_{1}\right\}, \text { and } \varphi_{2}\left(\bar{R}^{\prime}\right)=\left\{x_{2}\right\}
$$

Let $\tilde{R} \in \mathcal{R}^{N}$ be such that $B\left(\tilde{R}_{1}\right)=B\left(\tilde{R}_{2}\right)=\left\{x_{1}\right\},\left\{x_{1}, x_{2}\right\} \tilde{P}_{1} \emptyset,\left\{x_{1}, x_{2}\right\} \tilde{P}_{2} \emptyset$, and $\tilde{R}_{-1,2}=R_{-1,2}^{\prime}$. By efficiency, $\varphi_{1}(\tilde{R})=\left\{x_{1}\right\}$ or $\varphi_{2}(\tilde{R})=\left\{x_{1}\right\}$. Without loss of generality, suppose that $\varphi_{1}(\tilde{R})=\left\{x_{1}\right\}$ and $\varphi_{2}(\tilde{R})=\emptyset$. Let $\tilde{R}_{1}^{\prime} \in L\left(x_{1}, x_{2}\right)$, i.e., $x_{1} \tilde{P}_{1}^{\prime} x_{2}$. By efficiency and strategy-proofness, $\varphi_{1}\left(\tilde{R}_{1}^{\prime}, \tilde{R}_{-1}\right)=\left\{x_{1}, x_{2}\right\}$ and $\varphi_{2}\left(\tilde{R}_{1}^{\prime}, \tilde{R}_{-1}\right)=\emptyset$. Let $\tilde{R}_{1}^{\prime \prime} \in L\left(x_{2}, x_{1}\right)$, i.e., $x_{2} \tilde{P}_{1}^{\prime \prime} x_{1}$. By efficiency and strategy-proofness, $\varphi_{1}\left(\tilde{R}_{1}^{\prime \prime}, \tilde{R}_{-1}\right)=\left\{x_{1}, x_{2}\right\}$ and $\varphi_{2}\left(\tilde{R}_{1}^{\prime \prime}, \tilde{R}_{-1}\right)=\emptyset$. Let $\tilde{R}_{2}^{\prime} \in L\left(x_{1}, x_{2}\right)$, i.e., $x_{1} \tilde{P}_{2}^{\prime} x_{2}$. By efficiency, strategy-proofness, and $x_{1} \tilde{P}_{2}\left\{x_{1}, x_{2}\right\} \tilde{P}_{2} \emptyset, x_{1} \notin \varphi_{2}\left(\tilde{R}_{1}^{\prime \prime}, \tilde{R}_{2}^{\prime}, \tilde{R}_{-1,2}\right)$. Thus, by efficiency, $x_{1} \in \varphi_{1}\left(\tilde{R}_{1}^{\prime \prime}, \tilde{R}_{2}^{\prime}, \tilde{R}_{-1,2}\right)$. Hence, again by efficiency, $x_{2} \tilde{P}_{1}^{\prime \prime} x_{1}$, and $x_{1} \tilde{P}_{2}^{\prime} x_{2}$, we have $\left\{x_{2}\right\} \neq \varphi_{2}\left(\tilde{R}_{1}^{\prime \prime}, \tilde{R}_{2}^{\prime}, \tilde{R}_{-1,2}\right)$. Thus, $\varphi_{1}\left(\tilde{R}_{1}^{\prime \prime}, \tilde{R}_{2}^{\prime}, \tilde{R}_{-1,2}\right)=\left\{x_{1}, x_{2}\right\}$ and $\varphi_{2}\left(\tilde{R}_{1}^{\prime \prime}, \tilde{R}_{2}^{\prime}, \tilde{R}_{-1,2}\right)=\emptyset$. Applying twice strategy-proofness and since $\tilde{R}_{-1,2}=\vec{R}_{-1,2}^{\prime}$, it follows that $\varphi_{1}\left(\bar{R}^{\prime}\right)=\left\{x_{1}, x_{2}\right\}$ and $\varphi_{2}\left(\tilde{R}^{\prime}\right)=\emptyset$, a contradiction to (5.2). Hence, without loss of generality, let $\varphi_{1}(R)=K$. Now the conclusion of Step 1 follows together with strategy-proofness and non-bossiness.

Step 2. We prove that for all $R \in \mathcal{R}^{N}, \varphi_{1}(R)=B\left(R_{1}\right)$. 
Let $R^{1} \in \mathcal{R}^{N}$ be such that for all $i \in N, B\left(R_{i}^{1}\right)=K$. By Step 1, $\varphi_{1}\left(R^{1}\right)=B\left(R_{1}^{1}\right)=K$. Let $R^{2}=\left(R_{1}^{1}, R_{-1}\right) \in \mathcal{R}^{N}$. If we change profile $R^{1}$ stepwise to profile $R^{2}$, then it follows by strategy-proofness and non-bossiness that $\varphi_{1}\left(R^{2}\right)=K$ and for all $i \in N \backslash\{1\}, \varphi_{i}\left(R^{2}\right)=\emptyset$.

Let $R^{3}=\left(\bar{R}_{1}, R_{-1}\right) \in \mathcal{R}^{N}$ be such that $B\left(\bar{R}_{1}\right)=B\left(R_{1}\right)$ and for all $S \subsetneq B\left(R_{1}\right)$, $K \bar{P}_{1} S .{ }^{4}$ Thus, by efficiency and strategy-proofness, $\varphi_{1}\left(R^{3}\right)=B\left(\bar{R}_{1}\right)$. Finally, by strategyproofness, $\varphi_{1}(R)=B\left(R_{1}\right)$. Hence, without loss of generality, agent 1 is the (unique) first dictator.

Step 3. We show that for all $R \in \mathcal{R}^{N}$, there exists $\pi_{R} \in \Pi^{N}$ such that

$$
\begin{aligned}
\varphi_{\pi_{R}(1)}(R) & =B\left(R_{\pi_{R}(1)}\right), \\
\varphi_{\pi_{n}(2)}(R) & =B\left(R_{\pi_{R}(2)}\right) \backslash B\left(R_{\pi_{R}(1)}\right), \\
\varphi_{\pi_{R}(3)}(R) & =B\left(R_{\pi_{R}(3)}\right) \backslash\left[B\left(R_{\pi_{R}(1)}\right) \cup B\left(R_{\pi_{R}(2)}\right)\right], \\
& \vdots \\
\varphi_{\pi_{R}(n)}(R) & =B\left(R_{\pi_{R}(n)}\right) \backslash\left[\cup_{i=\pi_{R}(1)}^{\pi_{R}(n-1)} B\left(R_{i}\right)\right] .
\end{aligned}
$$

Let $R \in \mathcal{R}^{N}$ and set $\pi_{R}(1)=1$. Since $\varphi_{1}(R)=B\left(R_{1}\right)$, it follows for all $i \in N \backslash\{1\}$ that $\varphi_{i}(R) \subseteq B\left(R_{i}\right) \backslash B\left(R_{1}\right)$. Keeping $R_{1}$ fixed and varying the preferences of all remaining agents, similarly as in Steps 1 and 2, we can prove that there must exist a (unique) dictator $\pi_{R}(2)$ over the remaining set of objects $K \backslash B\left(R_{1}\right)$. Thus, $\varphi_{\pi_{H^{(2)}}}(R)=B\left(R_{\pi_{\boldsymbol{R}}(2)}\right) \backslash B\left(R_{1}\right)$. Hence, using Steps 1 and 2 sequentially, we can derive some $\pi_{R} \in \Pi^{N}$ such that (5.3) holds.

Step 4. Finally, we show that for all $R \in \mathcal{R}^{N}$ we can choose $\pi_{R} \in \Pi^{N}$ such that (5.3) holds and for all $R, \bar{R} \in \mathcal{R}^{N}$ and $\pi_{R}, \pi_{\bar{R}} \in \Pi^{N}$ the following additional conditions are satisfied:

(i) $\pi_{R}(1)=\pi_{\bar{R}}(1)$

(ii) Let $m \in\{1, \ldots, n-1\}$. If for all $i \in\{1, \ldots, m\}, \pi_{R}(i)=\pi_{\bar{R}}(i)$ and $\varphi_{\pi_{R}(i)}(R)=$ $\varphi_{\pi_{\bar{R}}(i)}(\bar{R})$, then $\pi_{R}(m+1)=\pi_{\bar{R}}(m+1)$.

Let $R \in \mathcal{R}^{N}$. By Step 2, agent 1 is the first dictator. Let $\pi_{R}(1) \equiv 1$ and recursively define $\pi_{R}$ as follows:

Let $m \in\{1, \ldots, n-1\}$. If $\cup_{i=\pi_{R}(1)}^{\pi_{R}(m)} B\left(R_{i}\right)=K$, then $\pi_{R}(m+1) \equiv \min \left(N \backslash\left\{\pi_{R}(1), \ldots\right.\right.$, $\left.\left.\pi_{R}(m)\right\}\right)$. Otherwise, if $\cup_{i=\pi_{H^{(1)}}}^{\pi_{R}(m)} B\left(R_{i}\right) \neq K$, then by Step 2 and 3 there exists a unique dictator in $N \backslash\left\{\pi_{R}(1), \ldots, \pi_{R}(m)\right\}$ over the remaining objects $K \backslash \cup_{i=\pi_{R}(1)}^{\pi_{h}(m)} B\left(R_{i}\right)$. Let $j \in N \backslash\left\{\pi_{R}(1), \ldots, \pi_{R}(m)\right\}$ be this dictator. Define $\pi_{R}(m+1) \equiv j$.

By definition and Step 2, (5.3) and (i) hold. In order to complete the proof, we show (ii) by induction on $m$.

\footnotetext{
${ }^{4}$ Note that $\bar{R}_{1}$ can be chosen to be additive.
} 
Induction Basis $m=1$ : By (i) and Step $3, \pi_{R}(1)=\pi_{R}(1)=1$. We show that if $\varphi_{1}(R)=\varphi_{1}(\bar{R})$, then $\pi_{R}(2)=\pi_{\bar{R}}(2)$.

If $\varphi_{1}(R)=\varphi_{1}(\bar{R})=K$, then $B\left(R_{1}\right)=B\left(\bar{R}_{1}\right)=K$. By definition $\pi_{R}(2)=\pi_{\bar{R}}(2)=2$, the desired conclusion.

Let $\varphi_{1}(R)=\varphi_{1}(\tilde{R}) \neq K$. Let $R^{\prime}, \bar{R}^{\prime} \in \mathcal{R}^{N}$ be such that $R_{1}^{\prime}=R_{1}, \bar{R}_{1}^{\prime}=\bar{R}_{1}$, and for all $i \in N \backslash\{1\}, B\left(R_{i}^{\prime}\right)=B\left(\bar{R}_{i}^{\prime}\right)=K$ and $R_{i}^{\prime}=\bar{R}_{i}^{\prime}$. By $(\mathrm{i}), \pi_{R^{\prime}}(1)=\pi_{\bar{R}^{\prime}}(1)=1$. Hence,

$$
\varphi_{1}\left(R^{\prime}\right)=B\left(R_{1}^{\prime}\right)=B\left(\bar{R}_{1}^{\prime}\right)=\varphi_{1}\left(\bar{R}^{\prime}\right)
$$

By definition and Step 3 it follows that $\pi_{R}(2)=\pi_{R^{\prime}}(2)$ and $\pi_{\bar{R}}(2)=\pi_{\bar{R}^{\prime}}(2)$. Hence,

$$
\varphi_{\pi_{l}(2)}\left(R^{\prime}\right)=\varphi_{\pi_{\bar{R}}(2)}\left(\bar{R}^{\prime}\right)=K \backslash \varphi_{1}\left(R^{\prime}\right)
$$

Recall that $\bar{R}^{\prime}=\left(\bar{R}_{1}^{\prime}, R_{-1}^{\prime},\right)$. By (5.4), strategy-proofness and non-bossiness imply $\varphi\left(\bar{R}^{\prime}\right)=$ $\varphi\left(\bar{R}_{1}^{\prime}, R_{-1}^{\prime}\right)=\varphi\left(R^{\prime}\right)$. Thus, by $(5.5), \pi_{R^{\prime}}(2)=\pi_{\bar{R}^{\prime}}(2)$. Hence, $\pi_{R}(2)=\pi_{\bar{R}}(2)$, the desired conclusion of (ii).

Induction Hypothesis: Let $m \in\{1, \ldots, n-1\}$. If for all $i \in\{1, \ldots, m-1\}, \pi_{R}(i)=\pi_{\bar{R}}(i)$ and $\varphi_{\pi_{\mu}(i)}(R)=\varphi_{\pi_{\bar{R}}(i)}(\bar{R})$, then $\pi_{R}(m)=\pi_{\bar{R}}(m)$.

Induction Step $m-1 \rightarrow m$ : Let $m \in\{1, \ldots, n-1\}$. Suppose that for all $i \in\{1, \ldots, m\}$, $\pi_{R}(i)=\pi_{\bar{R}}(i)$ and $\varphi_{\pi_{R}(i)}(R)=\varphi_{\pi_{\bar{R}}(i)}(\bar{R})$. We have to prove that $\pi_{R}(m+1)=\pi_{\bar{R}}(m+1)$.

Without loss of generality, suppose that $\pi_{R}(1)=1, \ldots, \pi_{R}(m)=m$. Hence, $\pi_{\bar{R}}(1)=$ $1, \ldots, \pi_{\bar{R}}(m)=m$. Let $M \equiv\{1, \ldots, m\}$. If $\cup_{i \in M} \varphi_{i}(R)=K$, then $\cup_{i \in M} B\left(R_{i}\right)=$ $\cup_{i \in M} B\left(\bar{R}_{i}\right)=K$. Since $N \backslash\left\{\pi_{R}(1), \ldots, \pi_{R}(m)\right\}=N \backslash\left\{\pi_{\bar{R}}(1), \ldots, \pi_{\bar{R}}(m)\right\}=N \backslash M$, by definition $\pi_{R}(m+1)=\pi_{\tilde{R}}(m+1)=m+1$, the desired conclusion.

Let $\cup_{i \in M} \varphi_{i}(R)=\cup_{i \in M} \varphi_{i}(\vec{R}) \neq K$. Let $R^{\prime}, \bar{R}^{\prime} \in \mathcal{R}^{N}$ be such that for all $i \in M$, $R_{i}^{\prime}=R_{i}, \ddot{R}_{i}^{\prime}=\bar{R}_{i}$, and for all $i \in N \backslash M, B\left(R_{i}^{\prime}\right)=B\left(\bar{R}_{i}^{\prime}\right)=K$ and $R_{i}^{\prime}=\bar{R}_{i}^{\prime}$. By our induction hypothesis, $R_{M}=R_{M}^{\prime}$, and $\bar{R}_{M}=\bar{R}_{M}^{\prime}$, we have for all $i \in M, \pi_{R}(i)=\pi_{R^{\prime}}(i)$ and $\pi_{\bar{R}}(i)=\pi_{\bar{R}^{\prime}}(i)$.

By definition and Step 3 it follows that $\pi_{R}(m+1)=\pi_{R^{\prime}}(m+1)$ and $\pi_{\bar{R}}(m+1)=$ $\pi_{\bar{R}^{\prime}}(m+1)$. Hence, for all $i \in M$,

$$
\varphi_{i}\left(R^{\prime}\right)=\varphi_{i}\left(\bar{R}^{\prime}\right)
$$

and

$$
\varphi_{\pi_{H}(m+1)}\left(R^{\prime}\right)=\varphi_{\pi_{\bar{M}}(m+1)}\left(\bar{R}^{\prime}\right)=K \backslash \cup_{i \in M} \varphi_{i}\left(R^{\prime}\right)
$$

By Step 3 and (i), $\varphi_{1}\left(R^{\prime}\right)=B\left(R_{1}^{\prime}\right)$ and $\varphi_{1}\left(\bar{R}^{\prime}\right)=B\left(\bar{R}_{1}^{\prime}\right)$. By (5.6), strategy-proofness and non-bossiness imply $\varphi\left(\bar{R}_{1}^{\prime}, R_{-1}^{\prime}\right)=\varphi\left(R^{\prime}\right)$. By Step 3 and the induction hypothesis, 
$\varphi_{2}\left(\bar{R}_{1}^{\prime}, R_{-1}^{\prime}\right)=B\left(R_{2}^{\prime}\right) \backslash B\left(R_{1}^{\prime}\right)$ and $\varphi_{2}\left(\bar{R}^{\prime}\right)=B\left(\bar{R}_{2}^{\prime}\right) \backslash B\left(\bar{R}_{1}^{\prime}\right)$. Similarly as above it follows that $\varphi\left(\bar{R}_{1}^{\prime}, \bar{R}_{2}^{\prime}, R_{-1,2}^{\prime}\right)=\varphi\left(\bar{R}_{1}^{\prime}, R_{-1}^{\prime}\right)$. Since $R_{N \backslash M}^{\prime}=\bar{R}_{N \backslash M}^{\prime}$ and (5.6) holds, by changing the preferences of agents $1, \ldots, m$ at profile $R^{\prime}$ stepwise to those at $\bar{R}^{\prime}$ it follows that $\varphi\left(R^{\prime}\right)=\varphi\left(\bar{R}^{\prime}\right)$. Thus, by $(5.7), \pi_{R^{\prime}}(m+1)=\pi_{\bar{R}^{\prime}}(m+1)$. Hence, $\pi_{R}(m+1)=\pi_{\tilde{R}}(m+1)$, the desired conclusion of (ii).

Step 4 completes the proof showing that $\varphi$ is a sequential dictatorship.

Remark 5.1 The proof of Theorem 5.1 remains valid if we restrict the preference domain $\mathcal{R}$ to the domain of additive and strict preference relations $\mathcal{A}$. By strategy-proofness, it is easy to show that Theorem 5.1 remains valid on the larger domain of strict preference relations $\mathcal{S} .^{5}$ Pápai (2001) gives a direct proof of the equivalence of (a) anc (c) as stated in Theorem 5.1 on the domain of strict preference relations $\mathcal{S}$.

Remark 5.2 When each agent receives exactly one object and all objects are preferred to receiving nothing; Svensson (1999) shows that serial dictatorships are the only rules satisfying efficiency, strategy-proofness, non-bossiness, and "neutrality" (the rule does not depend on the names of the objects). ${ }^{6}$ His result does not apply for multiple assignment problems. Lsing Theorem 5.1 these properties characterize the sequential dictatorships where the choice of the next dictator depends only on the cardinalities of the sets of objects chosen by the first dictators.

We discuss the independence of Theorem 5.1. Let $k \geq n$. Then "hierarchical exchange rules" (Pápai, 2000a) are rules that assign at every preference profile to each agent exactly one object. One member of this class is the rule where for a given initial assignment of exactly one object to each agent, say $i$ initially owns $x_{i}$, it chooses for each profile the assignment obtained from running Gale's top trading cycle algorithm. In the multiple assignment model "hierarchical exchange rules" satisfy strategy-proofness and non-bossiness but not efficiency.

Example 5.1 Let $N \equiv\{1,2,3\}$. For all $R \in \mathcal{R}^{N}$, if $B\left(R_{1}\right) \supseteq B\left(R_{2}\right)$, then $\varphi_{1}(R) \equiv$ $B\left(R_{1}\right), \varphi_{2}(R) \equiv B\left(R_{2}\right) \backslash B\left(R_{1}\right)$, and $\varphi_{3}(R) \equiv B\left(R_{3}\right) \backslash\left(B\left(R_{1}\right) \cup B\left(R_{2}\right)\right)$. Otherwise, $\varphi_{2}(R) \equiv$ $B\left(R_{2}\right), \varphi_{1}(R) \equiv B\left(R_{1}\right) \backslash B\left(R_{2}\right)$, and $\varphi_{3}(R) \equiv B\left(R_{3}\right) \backslash\left(B\left(R_{1}\right) \cup B\left(R_{2}\right)\right)$. The rule $\varphi$ satisfies non-bossiness and efficiency, but not strategy-proofness.

\footnotetext{
${ }^{5}$ On the general domain of strict preferences, the formal definition of sequential dictatorships has to be slightly adjusted.

${ }^{6}$ For example, if at a certain profile where all agents announce the same preference relation agent 1 receives the commonly most preferred object, then neutrality implies that he does so at all profiles with maximal conflict.
} 
A rule, defined similarly as sequential dictatorships, where the choice of the next dictators depends on the exact preference relation of the first dictator is efficient and strategyproof, but violates non-bossiness.

\subsection{Resource-Monotonicity}

In this section we admit variations of the set of objects to be assigned. First we extend our model and notation. We interpret $K$ to be the set of potential objects. We use $\mathcal{K}$ to denote a set of nonempty subsets of $K$ such that

(a) $K \in \mathcal{K}$ and

(b) there exists a partition $K^{1}, \ldots, K^{l}, l \geq 2$, of $K$ such that $\left\{K^{1}, \ldots, K^{l}\right\} \subseteq \mathcal{K}$ and for all $K^{\prime} \in \mathcal{K}$, there exists an index set $I \subseteq\{1, \ldots, l\}$ such that $K^{\prime}=\cup_{h \in I} K^{h}$.

Sets $K^{\prime} \in \mathcal{K}$ are called admissible. Throughout the remaining part of Chapter 5 an assignment problem consists of an admissible set of objects $K^{\prime} \in \mathcal{K}$ and a preference profile $R \in \mathcal{R}^{N}$. A rule $\varphi$ associates with each assignment problem $\left(R, K^{\prime}\right) \in \mathcal{R}^{N} \times \mathcal{K}$ an assignment $\varphi\left(R, K^{\prime}\right)$ such that $\cup_{i \in N} \varphi_{i}\left(R, K^{\prime}\right) \subseteq K^{\prime}$ and for all $i, j \in N$ such that $i \neq j, \varphi_{i}\left(R, K^{\prime}\right) \cap \varphi_{j}\left(R, K^{\prime}\right)=\emptyset$. It is straightforward to adjust the properties of rules we introduced in Sections 2 and 3 to the model at hand.

When the set of objects varies, then a natural requirement is resource-monotonicity. Conditions of resource-monotonicity have been studied by Chun and Thomson (1988), Moulin and Thomson (1988), and Thomson (1994a).

Resource-monotonicity describes the effect of a change in the available resource on the welfare of the agents. A rule satisfies resource-monotonicity, if after such a change either all agents (weakly) lose together or all (weakly) gain together.

It is easy to show that in combination with efficiency, resource-monotonicity implies the following: given some fixed preference profile and some fixed set of objects, if new additional objects are available, then this being good news all agents (weakly) gain. We use the following weaker version of resource-monotonicity.

Resource-Monotonicity: For all $R \in \mathcal{R}^{N}$ and all $K^{\prime}, K^{\prime \prime} \in \mathcal{K}$ : if $K^{\prime} \subseteq K^{\prime \prime}$, then for all $i \in N, \varphi_{i}\left(R, K^{\prime \prime}\right) R_{i} \varphi_{i}\left(R, K^{\prime}\right)$.

Our notion of resource-monotonicity is weak because we do not require that all subsets of $K$ are admissible. It is possible that $\mathcal{K}$ contains only three subsets of $K$. 
Next, we prove that in combination with efficiency and coalitional strategy-proofness only serial dictatorships satisfy resource-monotonicity. Before stating the result we extend the definition of serial dictatorships.

Serial Dictatorship: Let $\pi \in \Pi^{N}$. The serial dictatorship $\varphi^{\pi}$ with respect to $\pi$ is defined as follows: for all $\left(R, K^{\prime}\right) \in \mathcal{R}^{N} \times \mathcal{K}$,

$$
\begin{aligned}
\varphi_{\pi(1)}^{\pi}\left(R, K^{\prime}\right) & =B\left(R_{\pi(1)}\right) \cap K^{\prime} \\
\varphi_{\pi(2)}^{\pi}\left(R, K^{\prime}\right) & =\left(B\left(R_{\pi(2)}\right) \cap K^{\prime}\right) \backslash B\left(R_{\pi(1)}\right) \\
\varphi_{\pi(3)}^{\pi}\left(R, K^{\prime}\right) & =\left(B\left(R_{\pi(3)}\right) \cap K^{\prime}\right) \backslash\left[B\left(R_{\pi(1)}\right) \cup B\left(R_{\pi(2)}\right)\right], \\
& \vdots \\
\varphi_{\pi(n)}^{\pi}\left(R, K^{\prime}\right) & =\left(B\left(R_{\pi(n)}\right) \cap K^{\prime}\right) \backslash\left[\cup_{i=\pi(1)}^{\pi(n-1)} B\left(R_{i}\right)\right] .
\end{aligned}
$$

We call agent $\pi(1)$ the first dictator, agent $\pi(2)$ the second dictator, etc..

Theorem 5.2 Serial dictatorships are the only rules satisfying efficiency, coalitional strategy-proofness, and resource-monotonicity.

Proof. It is easy to check that serial dictatorships satisfy the properties listed in Theorem 5.2 .

Conversely, let $\varphi$ be a rule satisfying efficiency, coalitional strategy-proofness, and resource-monotonicity. Let $K^{\prime} \in \mathcal{K}$. Then; $\varphi\left(\cdot, K^{\prime}\right)$ satisfies efficiency and coalitional strategy-proofness on $\mathcal{R}^{N}$. By Theorem $5.1, \varphi\left(\cdot, K^{\prime}\right)$ is a sequential dictatorship. Hence, for each $R \in \mathcal{R}^{N}$ we can find $\pi_{R}^{K^{\prime}} \in \Pi^{N}$ as in the definition of a sequential dictatorship such that $\varphi\left(R, K^{\prime}\right)=\varphi^{\pi_{R}^{K^{\prime}}}\left(R, K^{\prime}\right)$. We show in three steps that $\varphi$ is a serial dictatorship. Step 1. Let $\left(R, K^{\prime}\right) \in \mathcal{R}^{N} \times \mathcal{K}$ and $\vec{m} \in N$ be such that $\cup_{i=1}^{\bar{m}-1} \varphi_{\pi_{k}^{K^{\prime}(i)}}\left(R, K^{\prime}\right) \neq K^{\prime}$. We prove by induction that for all $i \in\{1, \ldots, \bar{m}\}, \pi_{R}^{K^{\prime}}(i)=\pi_{R}^{K}(i)$.

For all $m \in\{1, \ldots, \bar{m}\}$, let $R^{m} \in \mathcal{R}^{N}$ be such that

$$
R_{\left\{\pi_{R}^{K^{\prime}}(1), \ldots, \pi_{M}^{K^{\prime}}(m-1)\right\}}^{m}=R_{\left\{\pi_{R}^{K^{\prime}}(1), \ldots, \pi_{R}^{K^{\prime}}(m-1)\right\}}
$$

and for all $i \in N \backslash\left\{\pi_{R}^{K^{\prime}}(1), \ldots, \pi_{R}^{K^{\prime}}(m-1)\right\}, B\left(R_{i}^{m}\right)=K$.

Induction Basis $m=1$ : Since $\varphi\left(\cdot, K^{\prime}\right)$ and $\varphi(\cdot, K)$ are sequential dictatorships, the first dictators are uniquely determined. Hence, $\pi_{R}^{K^{\prime}}(1)=\pi_{R^{1}}^{K^{\prime}}(1)$ and $\pi_{R}^{K}(1)=\pi_{R^{1}}^{K}(1)$. Thus, $\varphi_{\pi_{R}^{K^{\prime}(1)}}\left(R^{1}, K^{\prime}\right)=K^{\prime}$ and $\varphi_{\pi_{R}^{K}(1)}\left(R^{1}, K\right)=K$. By resource-monotonicity, $\pi_{R}^{K^{\prime}}(1)=\pi_{R}^{K}(1)$, the desired conclusion for the induction basis.

Induction Hypothesis: For all $i \in\{1, \ldots, m\}$, let $\pi_{R}^{K^{\prime}}(i)=\pi_{R}^{K}(i)$. 
Induction Step $m \rightarrow m+1$ for $m<\bar{m}$ : Since $\varphi$ is a sequential dictatorship, (ii) in the definition of sequential dictatorships implies that for all $i \in\{1, \ldots, m+1\}, \pi_{R}^{K^{\prime}}(i)=$ $\pi_{R^{m+1}}^{K^{\prime}}(i)$ and $\pi_{R}^{K}(i)=\pi_{R^{m+1}}^{K}(i)$. Since $m+1 \leq \vec{m}, \varphi_{\pi_{R}^{\kappa^{\prime \prime}}(m+1)}\left(R^{m+1}, K^{\prime}\right)=K^{\prime} \backslash \cup_{i=1}^{m}$ $\varphi_{\pi_{\mu}^{K}(i)}\left(R^{m+1}, K^{\prime}\right) \neq \emptyset$ and $\varphi_{\pi_{R}^{K}(m+1)}\left(R^{m+1}, K\right)=K \backslash \cup_{i=1}^{m} \varphi_{\pi_{R}^{K}(i)}\left(R^{m+1}, K\right) \neq \emptyset$. By the induction hypothesis and resource-monotonicity; $\pi_{R}^{K^{\prime}}(m+1)=\pi_{R}^{K}(m+1)$, the desired conclusion. This completes the proof of Step 1.

Step 2. Let $\left(R, K^{\prime}\right) \in \mathcal{R}^{N} \times \mathcal{K}$. If for all $i \in N, \varphi_{i}(R, K) \subseteq K^{\prime}$, then $\varphi\left(R, K^{\prime}\right)=\varphi(R, K)$.

By resource-monotonicity, for all $i \in N, \varphi_{i}(R, K) R_{i} \varphi_{i}\left(R, K^{\prime}\right)$. Since for all $i \in N$, $\varphi_{i}(R, K) \subseteq K^{\prime}$, efficiency implies $\varphi\left(R, K^{\prime}\right)=\varphi(R, K)$.

Step 3. We prove that there exists $\pi \in \Pi^{N}$ such that for all $\left(R, K^{\prime}\right) \in \mathcal{R}^{N} \times \mathcal{K}$, $\varphi\left(R, K^{\prime}\right)=\varphi^{\pi}\left(R, K^{\prime}\right)$.

Let $K^{1} \in \mathcal{K}$ be such that $K^{1} \neq K$. First, we show that $\varphi\left(\cdot, K^{1}\right)$ is a serial dictatorship.

Let $R^{\emptyset} \in \mathcal{R}^{N}$ be such that for all $i \in N, B\left(R_{i}^{\emptyset}\right)=\emptyset$. Define $\pi_{\emptyset}^{1} \equiv \pi_{R^{\natural}}^{K^{1}}$. Since $\varphi\left(\cdot, K^{1}\right)$ is a sequential dictatorship, $\pi_{\emptyset}^{1}$ is uniquely defined; i.e., for all $R \in \mathcal{R}^{N}, \pi_{R}^{K^{1}}(1)=\pi_{\emptyset}^{1}(1)$ and for all $m \in\{1, \ldots, n-1\}$ : if for all $i \in\{1, \ldots, m\}, \pi_{R}^{K^{1}}(i)=\pi_{\theta}^{1}(i)$ and $\varphi_{\pi_{R}{ }^{1}(i)}(R)=$ $\varphi_{\pi_{\theta}^{1}(i)}\left(R^{\emptyset}\right)=\emptyset$, then $\pi_{R}^{K^{1}}(m+1)=\pi_{\emptyset}^{1}(m+1)$. We show that $\varphi\left(\cdot, K^{1}\right)$ is a serial dictatorship with respect to $\pi_{\emptyset}^{1}$.

Let $R \in \mathcal{R}^{N}$ be such that for all $i \in N, B\left(R_{i}\right) \subseteq K \backslash K^{1}$. Then, $\cup_{i=1}^{n-1} \varphi_{\pi_{R}^{K}(i)}\left(R, K^{1}\right)=$ $\emptyset \neq K^{1}$. So, by Step $1, \pi_{R}^{K}=\pi_{\emptyset}^{1}$. Hence, $\varphi(\cdot, K)$ is a serial dictatorship on those profiles. By the assumptions on $\mathcal{K}$, there exists $K^{2} \in \mathcal{K}$ such that $K^{2} \neq \emptyset$ and $K^{1} \cap K^{2}=\emptyset$.

Let $R \in \mathcal{R}^{N}$ such that for all $i \in N, B\left(R_{i}\right) \subseteq K^{2}$. Thus, for all $i \in N, B\left(R_{i}\right) \subseteq K \backslash K^{1}$. Hence, $\varphi(R, K)=\varphi^{\pi_{b}^{1}}(R, K)$. By Step 2, $\varphi\left(R, K^{2}\right)=\varphi^{\pi_{\theta}^{1}}(R, K)$. Thus, by coalitional strategy-proofness $\varphi\left(\cdot, K^{2}\right)$ is a serial dictatorship with respect to $\pi_{\emptyset}^{1}$; i.e., for all $R \in \mathcal{R}^{N}$, $\varphi\left(R, K^{2}\right)=\varphi^{\pi \frac{1}{\theta}}\left(R, K^{2}\right)$.

Analogously we can define $\pi_{\emptyset}^{2} \equiv \pi_{R^{\natural}}^{K^{2}}$ and show that for all $R \in \mathcal{R}^{N}$ such that for all $i \in$ $N, B\left(R_{i}\right) \subseteq K \backslash K^{2}$, we have $\pi_{R}^{K}=\pi_{\emptyset}^{2}$. Moreover, the above argument shows that $\varphi\left(\cdot K^{1}\right)$ is a serial dictatorship with respect to $\pi_{0}^{2}$; i.e., for all $R \in \mathcal{R}^{N}, \varphi\left(R, K^{1}\right)=\varphi^{\pi_{a}^{2}}\left(R, K^{1}\right)$. In particular it follows that $\pi_{0}^{1}=\pi_{0}^{2}$. Let $\pi \equiv \pi_{\emptyset}^{1}$.

By our definition of $\mathcal{K}$, there exists a partition $K^{1}, \ldots, K^{l}, l \geq 2$, of $K$ such that $\left\{K^{1}, \ldots, K^{l}\right\} \subseteq \mathcal{K}$ and for all $K^{\prime} \in \mathcal{K}$ there exists an index set $I \subseteq\{1, \ldots, l\}$ such that $K^{\prime}=\cup_{h \in I} K^{h}$. So far we have shown that for all $h \in\{1, \ldots, l\}$ and all $R \in \mathcal{R}^{N}$,

$$
\varphi\left(R, K^{h}\right)=\varphi^{\pi}\left(R, K^{h}\right)
$$

Next, we show that $\varphi(\cdot, K)$ is a serial dictatorship with respect to $\pi$. Let $R \in \mathcal{R}^{N}$ and $h \in\{1, \ldots, l\}$. By $(5.8), \varphi\left(R, K^{h}\right)=\varphi^{\pi}\left(R, K^{h}\right)$. Let $\bar{m}^{h} \in N$ be maximal such that $\cup_{i=1}^{\bar{m}^{h}-1} \varphi_{\pi(i)}\left(R, K^{h}\right) \neq K^{h}$. By Step 1 , for all $i \in\left\{1, \ldots, \bar{m}^{h}\right\} ; \pi_{R}^{K}(i)=\pi(i)$. Thus, since 
$\varphi(\cdot, K)$ is a sequential dictatorship, for all $i \in\left\{1, \ldots, \bar{m}^{h}\right\}$,

$$
\varphi_{\pi(i)}\left(R, K^{h}\right) \subseteq \varphi_{\pi(i)}(R, K)
$$

Since

$$
\cup_{h=1}^{l} \cup_{i=1}^{\bar{n}^{h}} \varphi_{\pi(i)}\left(R, K^{h}\right)=\cup_{i \in N} \varphi_{i}(R, K)
$$

(5.9) implies for all $i \in N$,

$$
\varphi_{\pi(i)}(R, K)=\cup_{h=1}^{l} \varphi_{\pi(i)}\left(R, K^{h}\right) .
$$

Hence, $\varphi(R, K)=\varphi^{\pi}(R, K)$. Thus, $\varphi(\cdot, K)$ is a serial dictatorship with respect to $\pi$.

Finally, we prove that $\varphi$ is a serial dictatorship. Let $R \in \mathcal{R}^{N}$ and $K^{\prime} \in \mathcal{K}$. Let $R^{\prime} \in \mathcal{R}^{N}$ be such that for all $i \in N, B\left(R_{i}^{\prime}\right)=B\left(R_{i}\right) \cap K^{\prime}$. Coalitional strategy-proofness implies

$$
\varphi\left(R, K^{\prime}\right)=\varphi\left(R^{\prime}, K^{\prime}\right)
$$

We have proven that $\varphi\left(R^{\prime}, K\right)=\varphi^{\pi}\left(R^{\prime}, K\right)$. Since for all $i \in N, B\left(R_{i}^{\prime}\right) \subseteq K^{\prime}$, by efficiency, for all $i \in N, \varphi_{i}\left(R^{\prime}, K\right) \subseteq K^{\prime}$. Thus, Step 2 implies $\varphi\left(R^{\prime}, K^{\prime}\right)=\varphi\left(R^{\prime}, K\right)$. Note that $\varphi\left(R^{\prime}, K\right)=\varphi^{\pi}\left(R^{\prime}, K\right)=\varphi^{\pi}\left(R^{\prime}, K^{\prime}\right)$. Hence, $\varphi\left(R^{\prime}, K^{\prime}\right)=\varphi^{\pi}\left(R^{\prime}, K^{\prime}\right)$ and by $(5.10)$, $\varphi\left(R, K^{\prime}\right)=\varphi^{\pi}\left(R, K^{\prime}\right)$. This completes the proof.

Remark 5.3 The proof of Theorem 5.2 remains valid on the preference domain $\mathcal{A}$. $\triangleleft$

Similar examples as for Theorem 5.1 can be used to establish the independence of Theorem 5.2. In particular, in Theorem 5.2 coalitional strategy-proofness cannot be weakened to strategy-proofness because then the choice of the next dictators may depend on the exact preference relation of the first dictators.

The following example shows that Theorem 5.2 becomes an incompatibility on the domain of strict preferences.

Example 5.2 Let $N \equiv\{1,2\}, K \equiv\left\{x_{1}, x_{2}\right\}$, and $\mathcal{K} \equiv\left\{\left\{x_{1}\right\},\left\{x_{2}\right\}, K\right\}$. Let $\varphi$ denote the serial dictatorship with agent 1 as first dictator and agent 2 as the second dictator. Let $R=\left(R_{1}, R_{2}\right) \in \mathcal{S}^{\{1,2\}}$ be such that $\left\{x_{1}, x_{2}\right\} P_{1} \emptyset P_{1} x_{1} P_{1} x_{2}$ and $R_{2} \in L\left(x_{1}\right)$. Then $\varphi\left(R,\left\{x_{1}, x_{2}\right\}\right)=\left(\left\{x_{1}, x_{2}\right\}, \emptyset\right)$ and $\varphi\left(R,\left\{x_{1}\right\}\right)=\left(\emptyset,\left\{x_{1}\right\}\right)$. Hence, in contradiction to resource-monotonicity, $\varphi_{1}\left(R,\left\{x_{1}, x_{2}\right\}\right) P_{1} \varphi_{1}\left(R,\left\{x_{1}\right\}\right)$ and $\varphi_{2}\left(R,\left\{x_{1}\right\}\right) P_{2} \varphi_{2}\left(R,\left\{x_{1}, x_{2}\right\}\right)$. 。

Remark 5.4 The restriction of the structure of $\mathcal{K}$ is tight. If $\mathcal{K}$ does not satisfy property (b), then it is possible to find rules that are sequential dictatorships on some subsets of $K$ and that satisfy all the properties of Theorem 5.2 . 


\section{Chapter 6}

\section{Monotonic and Implementable Solutions in Generalized Matching Problems}

\subsection{Introduction}

\subsubsection{Generalized Matching Problems}

This chapter, which is based on Ehlers (2000e), considers the general problem of one-toone matching (Sönmez, 1996). There is a finite set of individuals denoted by $N$. Each agent $i \in N$ has an endowment which is represented by himself. For each agent $i \in N$ we specify a set of possible assignments $S_{i} \subseteq N$ including the option that he keeps his endownent (i.e., $i \in S_{i}$ ). Each individual has a preference relation $R_{i}$ over his possible assignments $S_{i}$. A feasible matching $\mu \in \mathcal{M}^{f}$ is a one-to-one assignment that chooses for each agent one of his possible assignments and each endowment is assigned to exactly one agent. A generalized matching problem is a triple $\left(\left(S_{i}\right)_{i \in N},\left(R_{i}\right)_{i \in N}, \mathcal{M}^{f}\right)$ consisting of the three components explained above. In such a problem monetary compensations are not possible. It is then the task of the designer to choose for each such problem the feasible matchings that are "desirable."

This formal model contains a variety of matching problems that arise in applications. It includes the marriage problems (Gale and Shapley, 1962). In such situations there are two types of individuals, usually called men $(M)$ and women $(W)$. We marry the men and women with the possibility that an individual may remain single. For each man $m$ the possible assignments are the women and himself (i.e., $S_{m}=W \cup\{m\}$ ). Marriage problems arise in many important instances: assigning workers to firms, students to colleges, 
physicians to hospitals, and so on. ${ }^{1}$

Another example that our framework includes is the exchange of indivisible objects (Shapley and Scarf, 1964), which has many real life applications. Each individual owns an object and would like to exchange his object with an object that he prefers. Each object may be assigned to each individual (i.e., $S_{i}=N$ ). Familiar problems are swapping apartments, exchanging admissions for certain universities among students, ${ }^{2}$ or simply exchanging indivisible goods in a barter market.

Marriage problems and objects exchange are the most prominent examples of our model, but it applies to many more situations. Another application is the roommate problem where we have to assign to each apartment two individuals. The marriage problem can be modified in such a way that for each worker only a subset of firms is feasible, which might be due to distance or language reasons. These restrictions, however, are not incorporated in the preference relations. We consider situations where individuals have any preference relation over all possible assignments. Otherwise we could restrict the worker's set of possible assignments by deleting some firms from it.

In all situations described so far we face the problem of selection. A solution chooses for each generalized matching problem a subset of the set of feasible matchings. The interpretation is that given a problem each matching that is chosen is "desirable" in terms of this solution. In Section 6.1.2 we discuss what solutions may be desirable or not. Throughout Chapter 6 we focus only on variations of preference profiles. The possible assignments and the set of feasible matchings remain fixed.

\subsubsection{Linear Orders and the Strong Core}

Most of the matching literature focuses on situations where each individual possesses a linear order over his assignments. That means that sufficient information is available for each agent to exclude indifference between two distinct assignments.

When preferences are linear orders, the following solution is important. It is based on the blocking relation defined as follows. A coalition $T \subseteq N$ blocks a feasible matching $\mu$ if (i) there exists another feasible matching $\mu^{\prime}$ that this coalition might bring about (the assignment of each member of $T$ at $\mu^{\prime}$ belongs to $T$ ), (ii) each member of $T$ weakly prefers his assignment at $\mu^{\prime}$ to that at $\mu$, and (iii) strict preference holds for some member of $T$. The strong core is the solution that chooses for each preference profile the feasible matchings that are maximal relative to the blocking relation.

\footnotetext{
${ }^{1}$ See Roth and Sotomayor (1990) for an introduction to marriage problems.

${ }^{2}$ This problem arises in Germany where for some fields, for example in medicine, a central agency admits each student to exactly one university.
} 
In marriage problems, the strong core is always non-empty (Gale and Shapley, 1962). The strong core selects exactly the matchings that are "divorce-proof:" no man and no woman prefers remaining single to his/her assignment and there is no pair of a man and woman such that they mutually prefer each other to their respective assignments. Therefore, in marriage problems the strong core is called the stable solution. Despite the positive existence results regarding the strong core it is very hard to find "nice" single-valued solutions. ${ }^{3}$ There does not exist a single-valued solution that is individual rational (each agent weakly prefers his assignment to remaining single, which is his endowment), Paretooptimal (the solution chooses an efficient matching), and strategy-proof (telling the truth is a weakly dominant strategy) (Roth, 1984; Alcalde and Barberà, 1994). That means when preferences are private information, individuals might misrepresent their true preferences.

In objects exchange, the strong core always consists of exactly one matching (Roth and Postlewaite, 1977). For each profile of linear orders "Gale's top trading cycle algorithm" computes this unique matching." Therefore, the strong core is a well-defined single-valued solution. Furthermore, in objects exchange the strong core solution is characterized by individual rationality, Pareto-optimality, and strategy-proofness (Ma, 1994). Recent papers give further support that the strong core and Gale's top trading cycle algorithm are the two most important concepts in objects exchange (Abdulkadiroğlu and Sönmez, 1998,99; Miyagawa, 2000; Pápai, 2000a).

Recently, it was shown that also in generalized matching problems the strong core plays the important role when we consider strategic issues. Sönmez (1999) shows in a more general environment that a single-valued solution satisfies individual rationality, Paretooptimality, and strategy-proofness if and only if for all preference profiles, the unique matching chosen by the solution belongs to the strong core and the strong core is essentially single-valued. Essentially single-valuedness means that for each preference profile; the strong core is non-empty and all agents are indifferent between any two matchings belonging to the strong core. Since only in rare cases (one of the few exceptions is objects exchange) the strong core is essentially single-valued, we could weaken the incentive requirement and ask instead the following question: given a solution, is it possible to find a game form such that for each preference profile the Nash equilibrium correspondence selects exactly the matchings that are chosen by the solution? If this is possible, then this solution is implenentable (in Nash equilibrium). Maskin (1999) shows that monotonicity is a necessary condition for a solution to be implementable. When preferences

\footnotetext{
${ }^{3}$ A single-valued solution chooses for each problem exactly one feasible matching.

${ }^{4}$ Pápai (2000a) contains a detailed description of this algorithm.
} 
are linear orders, then the strong core is implementable. Moreover, if a solution satisfies individual rationality, Pareto-optimality, and monotonicity, then it must always choose all the matchings belonging to the strong core (Kara and Sönme», 1996; Sönmez, 1996). Therefore, the strong core is the minimal solution that is implementable in generalized matching problems.

\subsubsection{Weak Orders and Overview of the Results}

In applications it is restrictive to assume that preference relations are linear orders.

"Of course there are many reasons, in practical situations of the sort modeled here, to expect that agents might not have the ability to distinguish among all alternatives facing them. Perhaps the most important such reason is that agents might have little information about the alternatives facing them." (Roth and Sotomayor, 1990, p.34)

We consider situations where agents might be indifferent between distinct assignments. Our study is one of the few exceptions that allows for the possibility of indifference. ${ }^{5}$ In this context we search for monotonic and implementable solutions. We show that this feature of preferences brings about new results that are different from the corresponding ones with linear orders. In particular we define a new solution that turns out to be important in generalized matching problems with weak orders.

Each agent's preference relation is a weak order over the set of his possible assignments. Instead of the general strong core, we allow only certain coalitions to be able to coordinate their actions. Only these coalitions may block a feasible matching. For example, in marriage problems it is natural to consider singletons and pairs consisting of a man and a woman as the only coalitions that might block a feasible matching. Given a set $\mathcal{T}$ of coalitions we consider the strong $\mathcal{T}$-core. The strong core is the special case where each coalition has the possibility to communicate. Our first result says that the strong $\mathcal{T}$-core is monotonic if and only if it is the individual rational solution. Therefore, if the strong core is monotonic, then the matching problem is trivial meaning that the only feasible matching is the matching that assigns to each agent his endowment. Contrary to when preferences are linear orders, the strong core is not implementable.

When a solution is not monotonic, it is natural to ask how to extend the solution such that monotonicity is recovered. Of course, the most desirable extension is the minimal

\footnotetext{
${ }^{5}$ Others are Wako (1984) and Sönmez (1999). But note that Sönmez (1999) excludes indifference between an agent's endowment and other assignments.
} 
one. It turns out that the following solution is the minimal monotonic extension of the strong $\mathcal{T}$-core. Given a preference profile $R$, a profile $\vec{R}$ of linear orders is a strict transformation of this profile if for all agents strict preference under $R$ is preserved at $\bar{R}$. The "union strong $\mathcal{T}$-core" always chooses the matchings that belong to the strong $\mathcal{T}$-core for some strict transformation of this profile. It turns out that for specific problems, the union strong core is a familiar solution studied in earlier literature. In marriage problems, the weakly stable solution is the minimal monotonic extension of the stable solution. In objects exchange, the competitive solution is the minimal monotonic extension of the strong core.

Furthermore, we derive the following results. Property $\alpha$ says that given a preference profile, if the endowment matching (the matching where each agent receives his endowment) is Pareto-optimal, then the solution chooses all individual rational matchings. If we impose a very weak condition on the set of feasible matchings, ${ }^{6}$ then the union strong core is the minimal solution that satisfies individual rationality, monotonicity, and property $\alpha$. If a solution satisfies these requirements, then for each preference profile it selects all the matchings that belong to the union strong core. We also show that if a solution satisfies individual rationality, Pareto-indifference (if all individuals are indifferent between two matchings, then the solution either chooses both or none of them), and monotonicity, then it must always choose the matchings belonging to the weak core. The weak core is defined in a similar way as the strong core. The difference is that a coalition $T$ strongly blocks a matching if all members of $T$ are strictly better off with the matching that they might bring about. Finally, we show that the union strong $\mathcal{T}$-core and the weak core are implementable. In particular, the weakly stable solution is implementable and in objects exchange the competitive solution is implementable. Therefore, the union strong core and the weak core are important solutions in generalized matching problems when preferences are weak orders.

We stress the fact that all proofs use only preferences at which an agent is indifferent between at most two distinct assignments. For our results to hold we do not need all weak orders. In applications we cannot exclude such indifference.

Chapter 6 is organized as follows. In Section 6.2 we define generalized matching problems and basic solutions. In Section 6.3 we consider the strong $\mathcal{T}$-core and solutions that satisfy individual rationality, Pareto-optimality, and monotonicity. In Section 6.4 we show that the union strong $\mathcal{T}$-core is the minimal monotonic extension of the strong $\mathcal{T}$-core. Section 6.5 contains the characterization of the union strong core and the weak core. In

\footnotetext{
${ }^{6}$ We explain this condition later. It is satisfied in all matching problems considered so far by the literature.
} 
Section 6.6 we prove that the weak core and the union strong $\mathcal{T}$-core are implementable. In Section 6.7 we discuss how much indifference we need for our results to hold. Finally, in Section 6.8 we apply our results to marriage problems and objects exchange.

\subsection{The Model}

First, following Sönmcz (1996) we define generalized matching problems. Second, we introduce basic solutions and properties. Third, we define some concepts of implementation.

Let $N$ denote the (finite) set of agents. Given $i \in N$, let $S_{i} \subseteq N$ denote agent $i$ 's set of possible assignments. This set includes his endowment, i.e., $i \in S_{i}$. The preference relation of each $i \in N$ is a reflexive, complete, and transitive binary relation over $S_{i}$. Such preferences are called weak orders. Let $\mathcal{R}_{i}$ be the set of all weak orders over $S_{i}$ and $\mathcal{R} \equiv \times_{i \in N} \mathcal{R}_{i}$. A (preference) profile is a list $R \equiv\left(R_{i}\right)_{i \in N} \in \mathcal{R}$. A preference relation $R_{i} \in \mathcal{R}_{i}$ is a linear order if for all $j, k \in S_{i}, j I_{i} k \Leftrightarrow j=k$. Linear orders exclude indifference between distinct assignments. Let $\mathcal{P}$ denote the set of all profiles $R \in \mathcal{R}$ such that for all $i \in N, R_{i}$ is a linear order. Since $N$ and $\left(S_{i}\right)_{i \in N}$ remain fixed, a generalized matching problem is simply a preference profile $R \in \mathcal{R}$. A matching is a bijection $\mu: N \rightarrow N$. The assignment of agent $i \in N$ at matching $\mu$ is $\mu(i)$. Given $T \subseteq N$, let $\mu(T)$ denote the set of assignments of the agents in $T$ at $\mu$, i.e., $\mu(T) \equiv\{\mu(i) \mid i \in T\}$. Let $\mathcal{M}$ denote the set of all matchings. Let $\mu^{I}$ denote the matching such that for all $i \in N, \mu^{I}(i)=i$. We specify a subset $\mathcal{M}^{J}$ of the set of all matchings as the set of feasible matchings. We always require that $\mu^{I} \in \mathcal{M}^{\delta}$ and for all $i \in N, S_{i}=\left\{\mu(i) \mid \mu \in \mathcal{M}^{\delta}\right\}$. We say that the matching problem is trivial when $\mathcal{M}^{f}=\left\{\mu^{I}\right\}$. We extend preferences over possible assignments to preferences over matchings in the following way: given $i \in N, R_{i} \in \mathcal{R}_{i}$, and $\mu, \bar{\mu} \in \mathcal{M}^{f}, \mu R_{i} \bar{\mu} \Leftrightarrow \mu(i) R_{i} \bar{\mu}(i)$. Slightly abusing notation we use the same symbols to denote preferences over possible assignments and preferences over feasible matchings.

A solution is a correspondence $\varphi: \mathcal{R} \rightarrow \mathcal{M}^{f}$ recommending for each profile a set of feasible matchings. For all $R \in \mathcal{R}$, each matching $\mu \in \varphi(R)$ is interpreted to be desirable when the profile is $R$. A solution $\varphi$ is non-empty if for all $R \in \mathcal{R}^{N}, \varphi(R) \neq \emptyset$. We allow solutions to be empty. Given two solutions $\varphi$ and $\bar{\varphi}$, let $\varphi \cap \bar{\varphi}$ denote the solution such that for all $R \in \mathcal{R},(\varphi \cap \bar{\varphi})(R) \equiv \varphi(R) \cap \bar{\varphi}(R)$. The union of two solutions is defined in a similar way. We write $\varphi \subseteq \bar{\varphi}$ if for all $R \in \mathcal{R}, \varphi(R) \subseteq \bar{\varphi}(R)$. If $\varphi \subseteq \bar{\varphi}$, then we say $\varphi$ is a subsolution of $\bar{\varphi}$ and $\bar{\varphi}$ is a supersolution of $\varphi$. The following three solutions are basic for our study. The first one chooses for each preference profile the feasible matchings such that each agent weakly prefers his assignment to his endowment. The second one chooses the feasible matchings that are efficient. The third one chooses the feasible matchings that 
are weakly efficient.

Individual Rational Solution, $\mathcal{I R}:$ For all $R \in \mathcal{R}$ and all $\mu \in \mathcal{M}^{\jmath}: \mu \in \mathcal{I R}(R) \Leftrightarrow$ for all $i \in N, \mu(i) R_{i}$.

Pareto Solution, $\mathcal{P O}:$ For all $R \in \mathcal{R}$ and all $\mu \in \mathcal{M}^{f}: \mu \in \mathcal{P O}(R) \Leftrightarrow$ there exists no $\bar{\mu} \in \mathcal{M}^{j}$ such that for all $i \in N, \bar{\mu}(i) R_{i} \mu(i)$, and for some $j \in N, \bar{\mu}(j) P_{j} \mu(j)$.

Weak Pareto Solution, $\mathcal{W P O}$ : For all $R \in \mathcal{R}$ and all $\mu \in \mathcal{M}^{\jmath}: \mu \in \mathcal{W P O}(R) \Leftrightarrow$ there exists no $\bar{\mu} \in \mathcal{M}^{f}$ such that for all $i \in N, \bar{\mu}(i) P_{i} \mu(i)$.

A solution $\varphi$ is individual rational when $\varphi \subseteq \mathcal{I R}$. It is Pareto-optimal when $\varphi \subseteq \mathcal{P O}$, and it is weak Pareto-optimal when $\varphi \subseteq \mathcal{W P O}$.

A garne form is a pair $\Gamma \equiv(X, g) \equiv\left(\times_{i \in N} X_{i}, g\right)$ where $X_{i}$ is agent $i$ 's strategy-space and $g: X \rightarrow \mathcal{M}^{f}$ is an outcome function. Given $R \in \mathcal{R}$, the pair $(\Gamma, R)$ defines a game. Let $N E(\Gamma, R)$ denote the set of pure Nash equilibria in the game $(\Gamma, R)$. A solution $\varphi$ is implementable (in Nash equilibrium) if there exists a game form $\Gamma \equiv(X, g)$ such that for all $R \in \mathcal{R}, \varphi(R)=g(N E(\Gamma, R))$.

Given $i \in N, R_{i} \in \mathcal{R}_{i}$, and $\mu \in \mathcal{M}^{f}$, let $L\left(\mu, R_{i}\right) \equiv\left\{\mu^{\prime} \in \mathcal{M}^{f} \mid \mu R_{i} \mu^{\prime}\right\}$ denote the lower contour set of $R_{i}$ at $\mu$. Given $R, R^{\prime} \in \mathcal{R}$ and $\mu \in \mathcal{M}^{j}$, the profile $R^{\prime}$ is a monotonic transformation of $R$ at $\mu$ if for all $i \in N, L\left(\mu, R_{i}\right) \subseteq L\left(\mu, R_{i}^{\prime}\right)$. Let $M T(R, \mu)$ denote the set of all monotonic transformations of $R$ at $\mu$. The following property is a necessary condition for a solution to be implementable (Maskin, 1999).

Monotonicity: For all $R \in \mathcal{R}$, all $\mu \in \varphi(R)$, and all $R^{\prime} \in M T(R, \mu): \mu \in \varphi\left(R^{\prime}\right)$.

\subsection{The Strong $\mathcal{T}$-Core}

We consider situations in which only certain coalitions can coordinate actions. A coalition structure is a set of coalitions. In particular, each individual "voluntarily" participates in the matching. Formally, a coalition structure is a set $\mathcal{T} \subseteq 2^{N} \backslash\{\emptyset\}$ such that for all $i \in N$, $\{i\} \in \mathcal{T}$. Given $R \in \mathcal{R}, T \in \mathcal{T}$, and $\mu, \bar{\mu} \in \mathcal{M}^{f}$, if

(i) $\bar{\mu}(T)=T$,

(ii) for all $i \in T, \tilde{\mu}(i) R_{i} \mu(i)$, and

(iii) for some $j \in T, \bar{\mu}(j) P_{j} \mu(j)$, 
then we say that coulition $T$ blocks $\mu$ under $R$. The following solution chooses for each preference profile the feasible matchings that are not blocked by any coalition in $\mathcal{T}$.

Strong $\mathcal{T}$-Core, $\mathcal{S C}^{\mathcal{T}}$ : For all $R \in \mathcal{R}$ and all $\mu \in \mathcal{M}^{f}: \mu \in \mathcal{S C}^{\mathcal{T}}(R) \Leftrightarrow$ there exists no $T \in \mathcal{T}$ that blocks $\mu$ under $R$.

If $\mathcal{T}=2^{N} \backslash\{\emptyset\}$, then the strong $2^{N} \backslash\{\emptyset\}$-core is what we call the "strong core". We write $\mathcal{S C}$ to denote the strong core. By adapting the proof of Sönmez (1996, Proposition 1) it follows that the strong $\mathcal{T}$-core is monotonic on $\mathcal{P}$.

The following lemma identifies a condition on the coalition structure $\mathcal{T}$ for the strong $\mathcal{T}$-core to be the individual rational solution.

Lemma 6.1 We have $S \mathcal{C}^{\mathcal{T}}=\mathcal{I R}$ if and only if the following condition holds:

(a) For all $\mu \in \mathcal{M}^{f}$ and all $T \in \mathcal{T}$, if $\mu(T)=T$, then for all $i \in T, \mu(i)=i$.

Proof. Recall that for all $i \in N,\{i\} \in \mathcal{T}$. Let (a) be true and $R \in \mathcal{R}$. Since $\mu^{I} \in \mathcal{M}^{f}$ and for all $i \in N,\{i\} \in \mathcal{T}$, it follows that $\mathcal{S C}^{\mathcal{T}}(R) \subseteq \mathcal{I R}(R)$. Let $\mu \in \mathcal{I R}(R)$. Suppose that $\mu \notin \mathcal{S C}^{\mathcal{T}}(R)$. Then there exist $\bar{\mu} \in \mathcal{M}^{f}$ and $T \in \mathcal{T}$ such that $\bar{\mu}(T)=T$, for all $i \in T, \bar{\mu}(i) R_{i} \mu(i)$, and for some $k \in T, \bar{\mu}(k) P_{k} \mu(k)$. Since $\mu \in \mathcal{I R}(R), \bar{\mu}(k) \neq h$. Thus, $\mathcal{T}$ violates (a), a contradiction. Hence, $\mathcal{S C}^{\top}(R)=\mathcal{I R}(R)$, the desired conclusion.

Let $\mathcal{S C}^{\mathcal{T}}=\mathcal{I R}$. Suppose that (a) does not hold. Then there exist $\mu \in \mathcal{M}^{f}$ and $T \in \mathcal{T}$ such that $\mu(T)=T$ and for some $k \in T, \mu(k) \neq k$. Let $R \in \mathcal{P}$ be such that for all $i \in N$ and all $j \in S_{i} \backslash\{\mu(i), i\}, \mu(i) R_{i} i R_{i} j$. Then $\mu^{I} \in \mathcal{I R}(R) \backslash \mathcal{S C}^{\top}(R)$, a contradiction.

Studies on implementation in matching problems show that the strong core is the important solution when preferences are linear orders. Kara and Sönme (1996), and Sönmez (1996) show that any solution satisfying individual rationality, Pareto-optimality, and monotonicity must be a supersolution of the strong core. ${ }^{7}$ Moreover, the strong core is implementable. It will turn out that their conclusions do not apply when preferences are weak orders. Our first result states that in general the strong $\mathcal{T}$-core is not monotonic.

Theorem 6.1 The strong $\mathcal{T}$-core is monotonic if and only if the strong $\mathcal{T}$-core is the individual rational solution.

Proof. By Lemma 6.1, the strong $\mathcal{T}$-core is the individual rational solution if and only if $\mathcal{T}$ satisfies (a).

\footnotetext{
${ }^{7}$ Tadenuma and Toda (1998) show that this conclusion does not hold in marriage problems where for each agent his endowment ("remaining single") is always his worst assignment.
} 
Suppose that (a) holds. Then the strong $\mathcal{T}$-core is the individual rational solution and monotonicity is satisfied.

Suppose that (a) does not hold. Then there exist $\mu \in \mathcal{M}^{f}$ and $T \in \mathcal{T}$ such that $\mu(T)=T$, and for some $k \in T, \mu(k) \neq k$. Since $\mu(T)=T,|T| \geq 2$. Let $R \in \mathcal{R}$ be such that for all $i \in N$,

(i) when $i=k$, for all $j \in S_{k} \backslash\{k, \mu(k)\}, \mu(k) P_{k} k P_{k} j$; and

(ii) when $i \neq k$, for all $j \in S_{i} \backslash\{i\}, i P_{i} j$.

Since $|T| \geq 2$ and $\{\{i\} \mid i \in N\} \subseteq \mathcal{T}$, it follows that $\mathcal{S C}^{\mathcal{T}}(R)=\left\{\mu^{I}\right\}$. Let $\bar{R} \in \mathcal{R}$ be such that $\bar{R}_{k}=R_{k}$ and for all $i \in N \backslash\{k\}$ and all $j \in S_{i} \backslash\{i, \mu(i)\}$,

$$
\mu(i) \bar{I}_{i} i \vec{P}_{i} j
$$

Thus, since $T \in \mathcal{T}$ and $|T| \geq 2, \mathcal{S C}^{\mathcal{T}}(\bar{R})=\{\mu\}$. Also $\tilde{R} \in M T\left(R, \mu^{I}\right)$. Therefore, the previous two conclusions yield a violation of monotonicity. Hence, the strong $\mathcal{T}$-core is not monotonic when (a) does not hold.

As a corollary of Theorem 6.1 we obtain that the strong core is monotonic only if the matching problem is trivial.

Corollary 6.1 The strong core is monotonic if and only if $\mathcal{M}^{f}=\left\{\mu^{I}\right\}$.

Proof. Let $\mu \in \mathcal{M}^{S} \backslash\left\{\mu^{I}\right\}$. Then there exists $T \in 2^{N} \backslash\{\emptyset\}$ such that $\mu(T)=T$ and for some $i \in T, \mu(i) \neq i$. Since the strong core is the strong $2^{N} \backslash\{\emptyset\}$-core, (a) in Lemma 6.1 does not hold. Therefore, $\mathcal{S C} \neq \mathcal{I R}$. Hence, by Theorem 6.1 , the strong core is not monotonic.

Next, we show that if a solution is individual rational, Pareto-optimal, and monotonic, then it only chooses matchings that the agents unanimously weakly prefer to all other matchings.

Proposition 6.1 Let $\varphi$ be a monotonic solution satisfying individual rationality and Pareto-optimality. Then for all $\mu \in \varphi(\mathcal{R})$ and all $R \in \mathcal{R}$,

$$
\mu \in \varphi(R) \text { if and only if for all } i \in N \text { and all } j \in S_{i}, \mu(i) R_{i} j \text {. }
$$

Proof. First, we show sufficiency. Let $\mu \in \varphi(\mathcal{R})$. Then for some $\bar{R} \in \mathcal{R}, \mu \in \varphi(\bar{R})$. Let $R \in \mathcal{R}$ be such that for all $i \in N$ and all $j \in S_{i}, \mu(i) R_{i} j$. Then $R \in M T(\bar{R}, \mu)$. Thus, by monotonicity, $\mu \in \varphi(R)$, which proves sufficiency.

Next, we show necessity. Suppose that for some $\mu \in \varphi(\mathcal{R})$ and some $R \in \mathcal{R}$, 
(i) $\mu \in \varphi(R)$; and

(ii) for some $k \in N$ and some $l \in S_{k} \backslash\{\mu(k)\}, l P_{k} \mu(k)$.

By individual rationality, $\mu(k) R_{k} k$. Hence, $l \neq k$. Since $S_{k}=\left\{\mu^{\prime}(k) \mid \mu^{\prime} \in \mathcal{M}^{f}\right\}$, it follows that for some $\tilde{\mu} \in \mathcal{M}^{f}, \tilde{\mu}(k)=l$. Let $\tilde{R} \in \mathcal{R}$ be such that $\tilde{R}_{k}=R_{k}$ and for all $i \in N \backslash\{k\}$ and all $j \in S_{i} \backslash\{\mu(i), \tilde{\mu}(i)\}, \mu(i) \tilde{I}_{i} \tilde{\mu}(i) \tilde{R}_{i} j$. Then $\tilde{R} \in M T(R, \mu)$. Thus, by monotonicity, $\mu \in \varphi(\tilde{R})$. But then, for all $i \in N, \tilde{\mu}(i) \tilde{R}_{i} \mu(i)$ and $\tilde{\mu}(k) \tilde{P}_{k} \mu(k)$, in violation to Paretooptinality.

Let $\overline{\mathcal{T}} \equiv\{\{i\} \mid i \in N\} \cup\{N\}$. Then the strong $\overline{\mathcal{T}}$-core is the individual rational and Pareto solution, i.e., $\mathcal{S C}^{\mathcal{T}}=\mathcal{I R} \cap \mathcal{P O}$. Proposition 6.1 implies that unless the matching problem is trivial, the individual rational and Pareto solution is not monotonic. In particular, in non-trivial problems non-empty subsolutions of the individual rational and Pareto solution are not implementable.

\subsection{The Union Strong $\mathcal{T}$-Core}

Let $R \in \mathcal{R}$ and $\bar{R} \in \mathcal{P}$. If for all $i \in N$ and all $j, k \in S_{i}, j P_{i} k \Rightarrow j \bar{P}_{i} k$, then we say that $\bar{R}$ is a strict transformation of $R$. That is, $\bar{R}$ is a profile of linear orders that preserves the strict preferences at $R$. Let $S T(R)$ denote the set of all strict transformations of $R$. Note that for all $R \in \mathcal{R}$, if $R \in \mathcal{P}$, then $S T(R)=\{R\}$.

Example 6.1 Let $N \equiv\{1,2,3\}$ and for all $i \in N, S_{i} \equiv\{1,2,3\}$. Let $R \in \mathcal{R}$ be such that

\begin{tabular}{c|c|c}
$R_{1}$ & $R_{2}$ & $R_{3}$ \\
\hline 1,3 & 1 & 2 \\
2 & 2,3 & 3 \\
& & 1
\end{tabular}

For example, in the above table $1 I_{1} 3 P_{1} 2$. Let $\tilde{R}_{1}, \tilde{R}_{1} \in \mathcal{R}_{1}$ and $\bar{R}_{2}, \tilde{R}_{2} \in \mathcal{R}_{2}$ be such that

\begin{tabular}{c|c|c|c}
$\bar{R}_{1}$ & $\tilde{R}_{1}$ & $\tilde{R}_{2}$ & $\tilde{R}_{2}$ \\
\hline 1 & 3 & 1 & 1 \\
3 & 1 & 2 & 3 \\
2 & 2 & 3 & 2
\end{tabular}

Then $S T(R)=\left\{\left(\bar{R}_{1}, \bar{R}_{2}, R_{3}\right),\left(\bar{R}_{1}, \tilde{R}_{2}, R_{3}\right),\left(\tilde{R}_{1}, \bar{R}_{2}, R_{3}\right),\left(\tilde{R}_{1}, \tilde{R}_{2}, R_{3}\right)\right\}$ 
Given $R \in \mathcal{R}^{N}$, the following solution chooses for each preference profile the union of the strong $\mathcal{T}$-cores of all strict transformations of the profile.

Union Strong $\mathcal{T}$-Core, $\Upsilon^{\mathcal{T}}$ : For all $R \in \mathcal{R}: \Upsilon^{\mathcal{T}}(R) \equiv \cup_{\bar{R} \in S T(R)} \mathcal{S C}^{\mathcal{T}}(\bar{R})$.

If $\mathcal{T}=2^{N} \backslash\{\emptyset\}$, then we simply write union strong core and $\Upsilon$. Since for all $i \in N$, $\{i\} \in \mathcal{T}$, the union strong $\mathcal{T}$-core is individual rational. In general the union strong $\mathcal{T}$-core violates Pareto-optimality.

Example 6.2 (Example 6.1 continued) Let $\mathcal{M}^{f} \equiv\left\{\mu^{I},(2,3,1),(3,1,2)\right\}$. Then $\Upsilon(R)=$ $\left\{\mu^{I},(3,1,2)\right\}$ and $\mathcal{P O}(R)=\{(3,1,2)\}$. Since $\Upsilon(R) \backslash \mathcal{P O}(R) \neq \emptyset$, the union strong core violates Pareto-optimality.

Proposition 6.2 The union strong $\mathcal{T}$-core is monotonic.

Proof. Let $R \in \mathcal{R}, \mu \in \Upsilon^{\mathcal{T}}(R)$, and $R^{\prime} \in M T(R, \mu)$. Then there exists $\bar{R} \in S T(R)$ such that $\mu \in \mathcal{S C}^{T}(\bar{R})$. Let $\bar{R}^{\prime} \in S T\left(R^{\prime}\right)$ be such that for all $i \in N$ and all $j \in S_{i} \backslash\{\mu(i)\}$, if $j I_{i}^{\prime} \mu(i)$, then $\mu(i) \bar{P}_{i}^{\prime} j$. Since $R^{\prime} \in M T(R, \mu), \vec{R}^{\prime} \in M T(\vec{R}, \mu)$. Hence, by monotonicity of $\mathcal{S C}^{\mathcal{T}}$ on $\mathcal{P}, \mu \in \mathcal{S C}^{\mathcal{T}}\left(\bar{R}^{\prime}\right)$. Hence, since $\bar{R}^{\prime} \in S T\left(R^{\prime}\right), \mu \in \Upsilon^{\mathcal{T}}\left(R^{\prime}\right)$, the desired conclusion.

When a solution is not implementable, is there a way to expand this solution such that implementability is recovered? Monotonicity is a necessary condition for implementability (Maskin, 1999). In many economic environments it is also a sufficient condition. Monotonicity is preserved under intersection and the solution choosing for each profile all feasible matchings is monotonic. Therefore, if a solution is not monotonic, then we identify the minimal way in which it has to be enlarged so as to satisfy this property (Sen, 1995). This motivates the following definition. Given a solution $\varphi$, the minimal monotonic extension is the supersolution of $\varphi$ that is monotonic and minimal in the following sense: if another supersolution of $\varphi$ is monotonic, then it contains the minimal monotonic extension of $\varphi$. Formally,

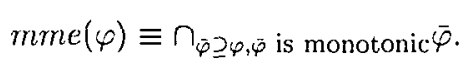

Note that the solution choosing for each preference profile all feasible matchings is monotonic. Therefore, the minimal monotonic extension of a solution is well-defined.

By Theorem 6.1, the strong $\mathcal{T}$-core violates monotonicity in general. Our next main result characterizes the minimal monotonic extension of this solution.

Theorem 6.2 The union strong $\mathcal{T}$-core is the minimal monotonic extension of the strong T-core. 
Proof. By definition, $\mathcal{S C}^{\mathcal{T}} \subseteq \Upsilon^{\mathcal{T}}$. By Proposition 6.2, $\Upsilon^{\mathcal{T}}$ is monotonic. Hence, $\Upsilon^{\mathcal{T}} \supseteq$ mme $\left(\mathcal{S C}^{\mathcal{T}}\right)$. To show the converse inclusion, let $R \in \mathcal{R}$ and $\mu \in \Upsilon^{\mathcal{T}}(R)$. Thus, for some $\bar{R} \in S T(R), \mu \in \mathcal{S C}^{\mathcal{T}}(\bar{R})$. By monotonicity of $\mathcal{S C}^{\mathcal{T}}$ on $\mathcal{P}$, we may suppose that for all $i \in N$ and all $j \in S_{i} \backslash\{\mu(i)\}$, if $j I_{i} \mu(i)$, then $\mu(i) \bar{P}_{i} j$. Since $\ddot{R} \in M T(R, \mu)$, $\mu \in \Upsilon^{\mathcal{T}}(\vec{R})$. Since $\ddot{R} \in \mathcal{P}, \Upsilon^{\mathcal{T}}(\vec{R})=\mathcal{S C}^{\mathcal{T}}(\vec{R})$. Thus, $\mu \in \operatorname{mme}\left(\mathcal{S C}^{\mathcal{T}}\right)(\bar{R})$. Since $R \in M T(R, \mu)$ and mme $\left(\mathcal{S C}^{\top}\right)$ is monotonic, it follows that $\mu \in \operatorname{mme}\left(\mathcal{S C}^{\mathcal{T}}\right)(R)$. Hence, $\Upsilon^{\top}(R) \subseteq m m e\left(\mathcal{S C}^{\top}\right)(R)$, the desired conclusion.

By Theorem 6.2, the minimal extension of the strong $\mathcal{T}$-core to satisfy monotonicity is not "much" larger than the strong $\mathcal{T}$-core. In particular, the strong $\mathcal{T}$-core and the union strong $\mathcal{T}$-core coincide on the domain $\mathcal{P}$.

\subsection{The Weak Core}

Given $R \in \mathcal{R}, T \in \mathcal{T}$, and $\mu, \bar{\mu} \in \mathcal{M}^{\jmath}$, if

(i) $\bar{\mu}(T)=T$ and

(ii) for all $i \in T, \bar{\mu}(i) P_{i} \mu(i)$,

then we say that coalition $T$ strongly blocks $\mu$ under $R$. The following solution chooses for each preference profile the feasible matchings that are not strongly blocked by a coalition in $\mathcal{T}$.

Weak $\mathcal{T}$-Core, $\mathcal{W C}^{\mathcal{T}}:$ For all $R \in \mathcal{R}$ and all $\mu \in \mathcal{M}^{\jmath}: \mu \in \mathcal{W C}^{\mathcal{T}}(R) \Leftrightarrow$ there exists no $T \in \mathcal{T}$ that strongly blocks $\mu$ under $R$.

If $\mathcal{T}=2^{N} \backslash\{\emptyset\}$, then the weak $2^{N} \backslash\{\emptyset\}$-core is what we call the "weak core". We write $\mathcal{W C}$ to denote the weak core. The weak $\mathcal{T}$-core satisfies a number of properties of interest. It always contains the matchings belonging to the strong $\mathcal{T}$-core. The following proposition strengthens this conclusion.

Proposition 6.3 The union strong $\mathcal{T}$-core is a subsolution of the weak $\mathcal{T}$-core.

Proof. Let $R \in \mathcal{R}$ and $\mu \in \Upsilon^{\mathcal{T}}(R)$. Thus, for some $\bar{R} \in S T(R), \mu \in \mathcal{S C}^{\mathcal{T}}(\vec{R})$. Let $\bar{R}^{\prime} \in S T(R)$ be such that for all $i \in N$ and all $j \in S_{i} \backslash\{\mu(i)\}$, if $j I_{i} \mu(i)$, then $\mu(i) \bar{P}_{i}^{\prime} j$. By monotonicity of $\mathcal{S C}^{T}$ on $\mathcal{P}, \mu \in \mathcal{S C}^{\top}\left(\bar{R}^{\prime}\right)$. Suppose that $\mu \notin \mathcal{W C}^{\top}(R)$. Then there exists $T \in \mathcal{T}$ and $\bar{\mu} \in \mathcal{M}^{\prime}$ such that $\bar{\mu}(T)=T$ and for all $i \in T, \bar{\mu}(i) P_{i} \mu(i)$. But 
then, by construction, for all $i \in T, \bar{\mu}(i) \bar{P}_{i}^{\prime} \mu(i)$, a contradiction to $\mu \in \mathcal{S C}^{T}\left(\tilde{R}^{\prime}\right)$. Thus, $\Upsilon^{\mathcal{T}}(R) \subseteq \mathcal{W C}^{\mathcal{T}}(R)$, the desired conclusion.

Lemma 6.2 The weak $\mathcal{T}$-core is monotonic.

Proof. Let $R \in \mathcal{R}, \mu \in \mathcal{W C}^{\top}(R)$, and $\bar{R} \in M T(R, \mu)$. Suppose that $\mu \notin \mathcal{W C}^{\mathcal{T}}(\bar{R})$. Then there exists $T \in \mathcal{T}$ and $\bar{\mu} \in \mathcal{M}^{f}$ such that $\bar{\mu}(T)=T$ and for all $i \in T, \bar{\mu}(i) \bar{P}_{i} \mu(i)$. Since for all $i \in T, L\left(\mu, R_{i}\right) \subseteq L\left(\mu, \bar{R}_{i}\right)$, we then deduce that for all $i \in T, \bar{\mu}(i) P_{i} \mu(i)$. Hence, $\mu \notin \mathcal{W C}^{\mathcal{T}}(R)$, a contradiction.

For the remaining part of this section we discuss the weak core. In general the weak core may be empty.

Example 6.3 (Roommate problem) Let $N \equiv\{1,2,3\}$, for all $i \in N, S_{i} \equiv N$, and $\mathcal{M}^{J} \equiv\left\{\mu^{I},(2,1,3),(3,2,1),(1,3,2)\right\}$. Let $R \in \mathcal{R}$ be such that

\begin{tabular}{c|c|c}
$R_{1}$ & $R_{2}$ & $R_{3}$ \\
\hline 2 & 3 & 1 \\
3 & 1 & 2 \\
1 & 2 & 3
\end{tabular}

Then $\mathcal{W C}(R)=\emptyset$.

Next, we focus on non-empty subsolutions of the individual rational and weak Pareto solution that are monotonic. In general such a solution may choose for each preference profile the endowment matching.

Example 6.4 Let $\mathcal{M}^{f} \subseteq\{\mu \in \mathcal{M} \mid$ for some $i \in N, \mu(i)=i\}$ and for all $R \in \mathcal{R}$, $\varphi(R) \equiv\left\{\mu^{I}\right\}$. The solution $\varphi$ satisfies individual rationality, weak Pareto-optimality, and monotonicity.

Note that Example 6.4 applies to marriage problems in which the number of men and women are not equal. We want to exclude solutions such as Example 6.4. Note that this solution always recommends only one matching. But even if we require that each feasible matching is chosen by the solution for some preference profile, then it may still not be a desirable solution.

Example 6.5 For all $R \in \mathcal{R}$, let

$$
\begin{aligned}
\varphi(R) \equiv & \left(\left\{\mu \in \mathcal{M}^{f} \mid \text { for all } i \in N \text { and all } j \in S_{i}, \mu(i) R_{i} j\right\} \cup\right. \\
& \left\{\mu \in \mathcal{M}^{f} \mid \text { for some } T \subseteq N \text { such that } 1 \in T, \text { (i) for all } i \in N \backslash T, \mu(i)=i,\right. \\
& \text { and (ii) for all } \left.\left.T^{\prime} \subseteq T, \mu\left(T^{\prime}\right) \neq T^{\prime}\right\}\right) \cap \mathcal{I R}(R) \cap \mathcal{W P O}(R) .
\end{aligned}
$$


The solution $\varphi$ satisfies individual rationality, weak Pareto-optimality, and monotonicity. Moreover, $\varphi(\mathcal{R})=\mathcal{M}^{f}$. In general there exists $R \in \mathcal{R}$ such that $\varphi(R) \cap \mathcal{W C}(R)=\emptyset$. $\quad$ o

Examples 6.4 and 6.5 discriminate among feasible matchings in the sense that they ignore other possibilities in many situations. The following property rules out such behaviour (Thomson, 1983b,99c; d'Aspremont and Gevers, 1977; Gevers, 1986). It says that for any two feasible matchings, if all agents are indifferent between them, then the solution either chooses both matchings or none. ${ }^{8}$

Pareto-Indifference: For all $R \in \mathcal{R}$ and all $\mu, \bar{\mu} \in \mathcal{M}^{f}$ : if for all $i \in N, \mu(i) I_{i} \bar{\mu}(i)$, then $\mu \in \varphi(R) \Leftrightarrow \bar{\mu} \in \varphi(R)$.

In general, the union strong core may violate Pareto-indifference, for example in objects exchange (Roth and Postlewaite, 1977; Subsection 6.8.2 contains their example). The weak core satisfies this condition.

Lemma 6.3 The weak core satisfies Pareto-indifference.

Proof. Let $R \in \mathcal{R}$ and $\mu \in \mathcal{W C}(R)$. Let $\bar{\mu} \in \mathcal{M}^{f}$ be such that for all $i \in N, \bar{\mu}(i) I_{i} \mu(i)$. Suppose that $\bar{\mu} \notin \mathcal{W C}(R)$. Then there exists $T \subseteq N$ and $\tilde{\mu} \in \mathcal{M}^{f}$ such that $\tilde{\mu}(T)=T$ and for all $i \in T ; \tilde{\mu}(i) P_{i} \bar{\mu}(i)$. But then for all $i \in T, \tilde{\mu}(i) P_{i} \mu(i)$, a contradiction to $\mu \in \mathcal{W C}(R)$.

We show that if a solution satisfies individual rationality, monotonicity, and Paretoindifference, then it must be a supersolution of the weak core. Before we state the theorem we have the following lemma. In this lemma and the theorem below we have to require that if the endowment matching is Paretomoptimal, then this solution is non-empty, i.e., if for some $R \in \mathcal{R}^{N}, \mu^{I} \in \mathcal{P O}(R)$, then $\varphi(R) \neq \emptyset$.

Lemma 6.4 Let $\varphi$ be a solution that is non-empty whenever the endowment matching is Pareto-optimal. If $\varphi$ satisfies individual rationality, monotonicity, and Paretoindifference, then $\varphi \supseteq \Upsilon$.

Proof. Let $R \in \mathcal{R}$ and $\mu \in \Upsilon(R)$. Thus, for some $\bar{R} \in S T(R), \mu \in \mathcal{S C}(\bar{R})$. By monotonicity of $\mathcal{S C}$ on $\mathcal{P}$ and the definition of $\Upsilon$, we may suppose that for all $i \in N$ and all $j \in S_{i} \backslash\{\mu(i)\}$, if $\mu(i) I_{i} j$, then $\mu(i) \bar{P}_{i} j$. Let $\tilde{R} \in \mathcal{P}$ be such that for all $i \in N$,

\footnotetext{
${ }^{8}$ When we aggregate each preference profile to a single "social welfare ordering", Pareto-indifference plays a key role in the so-called "welfarism theorem" (d`Aspremont and Gevers, 1977; Gevers, 1986).
} 
(i) for all $j, k \in S_{i} \backslash\{i\}, j \tilde{P}_{i} k \Leftrightarrow j \bar{P}_{i} k$;

(ii) $\mu(i) \tilde{R}_{i} i$ and for all $j \in S_{i} \backslash\{\mu(i), i\}$, either $j \tilde{P}_{i} \mu(i)$ or $i \tilde{P}_{i} j$.

Since $\tilde{R} \in M T(\bar{R}, \mu)$ and $\mathcal{S C}$ is monotonic on $\mathcal{P}$, it follows that $\mu \in \mathcal{S C}(\tilde{R})$. Next, we show the following: for all $\bar{\mu} \in \mathcal{I R}(\tilde{R})$ and all $i \in N,{ }^{9}$

$$
\mu(i) \tilde{R}_{i} \bar{\mu}(i)
$$

Suppose that $\tilde{\mu} \in \mathcal{I R}(\tilde{R})$ violates (6.1). Thus, for some $j \in N, \tilde{\mu}(j) \tilde{P}_{j} \mu(j)$. Let $T \subseteq N$ be such that (i) $j \in T$; (ii) $\tilde{\mu}(T)=T$; and (iii) for all $T^{\prime} \subsetneq T, \tilde{\mu}\left(T^{\prime}\right) \neq T^{\prime}$. Since $\tilde{\mu}$ is a matching, $T$ is uniquely defined. Hence, for all $i \in T, \tilde{\mu}(i) \neq i$. Therefore, by (ii) of the construction of $\tilde{R}$, for all $i \in T, \tilde{\mu}(i) \tilde{R}_{i} \mu(i)$. Since $j \in T, \tilde{\mu}(j) \tilde{P}_{j} \mu(j)$, and $\tilde{\mu}(T)=T$, the previous fact contradicts $\mu \in \mathcal{S C}(\tilde{R})$. Therefore, (6.1) holds.

Let $\tilde{R}^{\prime} \in \mathcal{R}$ be such that for all $i \in N$,

(i) for all $j, k \in S_{i} \backslash\{i\}, j \tilde{P}_{i}^{\prime} k \Leftrightarrow j \tilde{P}_{i} k$;

(ii) $\mu(i) \tilde{I}_{i}^{\prime} i$ and for all $j \in S_{i} \backslash\{\mu(i), i\}$, either $j \tilde{\Gamma}_{i}^{\prime} \mu(i)$ or $i \tilde{P}_{i}^{\prime} j$.

By (6.1), it follows that for all $\mu^{\prime} \in \mathcal{I R}\left(\tilde{R}^{\prime}\right), \mu^{\prime}(i) \tilde{I}_{i}^{\prime} i$. Thus, $\mu^{I} \in \mathcal{P} \mathcal{O}\left(\tilde{R}^{\prime}\right)$. Therefore, $\varphi\left(\tilde{R}^{\prime}\right) \neq \emptyset$. Note that $\tilde{R}^{\prime} \in M T(R, \mu)$. Thus, by monotonicity, $\mu \in \Upsilon\left(\tilde{R}^{\prime}\right)$. By individual rationality, Pareto-indifference, and $\varphi\left(\tilde{R}^{\prime}\right) \neq \emptyset$, we have $\varphi\left(\tilde{R}^{\prime}\right)=\mathcal{I} \mathcal{R}\left(\tilde{R}^{\prime}\right)$. Hence, $\mu \in \varphi\left(\tilde{R}^{\prime}\right)$. By monotonicity and $R \in M T\left(\tilde{R}^{\prime}, \mu\right), \mu \in \varphi(R)$, the desired conclusion.

The following property is studied in the literature: for each profile $R$, if the endowment matching is Pareto-optimal at $R$, then the solution selects all the matchings that are Pareto-indifferent to the endowment matching at $R$ (perhaps others). This condition is called property $\alpha$ (Thomson, 1987). ${ }^{10}$ Equivalently, the requirement can be stated as follows: for each profile $R$, if the endowment matching Pareto dominates all other matchings that are individual rational at $R$, then the solution selects all the matchings that are Pareto-indifferent to the endowment matching (perhaps others). Obviously, then the solution has to select all matchings that are individual rational.

Property $\alpha:$ For all $R \in \mathcal{R}:$ if for all $\mu \in \mathcal{I R}(R)$ and all $i \in N, i R_{i} \mu(i)$, then $\varphi(R) \supseteq$ $\mathcal{I R}(R)$.

In general the strong core violates property $\alpha$.

\footnotetext{
${ }^{9}$ Sönmez (1996) shows in the proof of Theorem 1 the same claim by induction.

${ }^{10}$ In classical exchange economies, if a solution satisfies individual rationality, monotonicity, and property $\alpha$, then it contains the "constrained Walrasian solution" (Thomson, 1987,99c).
} 
Example 6.6 Let $N \equiv\{1,2,3,4\}, S_{1} \equiv\{1,2\}, S_{2} \equiv S_{1}, S_{3} \equiv\{3,4\}, S_{4} \equiv S_{3}, \mathcal{M}^{f} \equiv$ $\left\{\mu^{I},(2,1,4,3)\right\}$. Let $\bar{R} \in \mathcal{P}$ be such that $2 \bar{P}_{1} 1,1 \bar{P}_{2} 2,3 \bar{P}_{3} 4$, and $4 \bar{P}_{4} 3$. Then $\mathcal{S C}(\bar{R})=\emptyset$ and $\mathcal{I R}(\bar{R})=\left\{\mu^{I}\right\}$. Therefore, the strong core violates property $\alpha$ at $\bar{R}$.

The following condition on $\mathcal{M}^{f}$ ensures that the strong core satisfies property $\alpha$. It says that given any two feasible matchings and a coalition $T$, if the assignments at both matchings for the members of $T$ belong to $T$, then there exists another feasible matching such that the assignments of the members of $T$ are the same as those at the first matching and the assignments of the members in $N \backslash T$ are the same as at the second matching.

$\left.{ }^{*}\right)$ For all $T \subseteq N$ and all $\mu, \bar{\mu} \in \mathcal{M}^{f}$, if $\mu(T)=T$ and $\bar{\mu}(T)=T$, then there exists $\tilde{\mu} \in \mathcal{M}^{f}$ such that for all $i \in T, \tilde{\mu}(i)=\mu(i)$, and for all $i \in N \backslash T, \tilde{\mu}(i)=\bar{\mu}(i)$.

We say that $\mathcal{M}^{f}$ is closed under composition if $\mathcal{M}^{f}$ satisfies condition $\left(^{*}\right)$.

Lemma 6.5 If $\mathcal{M}^{f}$ is closed under composition, then the strong core satisfies property $\alpha$.

Proof. Let $R \in \mathcal{R}$ be such that for all $\mu \in \mathcal{I R}(R)$ and all $i \in N$,

$$
i R_{i} \mu(i)
$$

Let $\bar{\mu} \in \mathcal{I R}(R)$ and suppose that $\bar{\mu} \notin \mathcal{S C}(R)$. Then there exist $T \in \mathcal{T}$ and $\tilde{\mu} \in \mathcal{M}^{f}$ such that $\tilde{\mu}(T)=T$, for all $i \in T, \tilde{\mu}(i) R_{i} \bar{\mu}(i)$, and for some $k \in T, \tilde{\mu}(k) P_{k} \bar{\mu}(k)$. Since $\mu^{I} \in \mathcal{M}^{f}$ and $\mathcal{M}^{f}$ is closed under composition, there exists $\tilde{\mu}^{\prime} \in \mathcal{M}^{f}$ such that for all $i \in T, \tilde{\mu}^{\prime}(i)=\tilde{\mu}(i)$ and for all $j \in N \backslash T, \tilde{\mu}^{\prime}(j)=j$. But then for all $i \in N, \tilde{\mu}^{\prime}(i) R_{i} i$ and $\tilde{\mu}^{\prime}(k) P_{k} k$, a contradiction to $(6.2)$.

Since $\Upsilon \supseteq \mathcal{S C}$, the union strong core satisfies property $\alpha$ whenever the strong core satisfies this requirement. In particular, when $\mathcal{M}^{f}$ satisfies $\left(^{*}\right)$, Lemma 6.4 remains true if we replace Pareto-indifference by property $\alpha$. Moreover, if a solution satisfies property $\alpha$, then it is non-empty whenever the endowment matching is Pareto-optimal. Therefore, when $\mathcal{M}^{f}$ satisfies $\left(^{*}\right)$, the union strong core is the minimal solution satisfying individual rationality, monotonicity, and property $\alpha$.

Corollary 6.2 Let $\mathcal{M}^{J}$ be closed under composition. If a solution satisfies individual rationality, monotonicity, and property $\alpha$, then it is a supersolution of the union strong core.

Theorem 6.3 If a solution is non-empty whenever the endownent matching is Paretooptimal and it satisfies individual rationality, monotonicity, and Pareto-indifference, then it is a supersolution of the weak core. 
Proof. Let $\varphi$ be a solution satisfying the properties listed in Theorem 6.3. By Lemmas 6.2 and 6.3, the weak core satisfies monotonicity and Pareto-indifference. Suppose that $\varphi \nsupseteq \mathcal{W C}$. Then there exists $R \in \mathcal{R}$ such that $\mathcal{W C}(R) \backslash \varphi(R) \neq \emptyset$. Let $\mu \in \mathcal{W C}(R) \backslash \varphi(R)$. Let $\tilde{R} \in \mathcal{R}$ be such that for all $i \in N$,

(i) for all $j \in S_{i}$ and all $k \in S_{i} \backslash\left\{\mu^{\prime}(i) \mid \mu^{\prime} \in L\left(\mu, R_{i}\right)\right\}, k \tilde{R}_{i} j \Leftrightarrow k R_{i} j$; and

(ii) for all $j \in\left\{\mu^{\prime}(i) \mid \mu^{\prime} \in L\left(\mu, R_{i}\right)\right\} \backslash\{i, \mu(i)\}, i \tilde{I}_{i} \mu(i) \tilde{\Gamma}_{i} j$.

By monotonicity, $R \in M T(\tilde{R}, \mu)$, and $\tilde{R} \in M T(R, \mu)$,

$$
\mu \in \mathcal{W C}(\tilde{R}) \backslash \varphi(\tilde{R})
$$

By Proposition 6.3 and Lemma 6.4,

$$
\Upsilon(\tilde{R}) \subseteq \mathcal{W C}(\tilde{R}) \text { and } \Upsilon(\tilde{R}) \subseteq \varphi(\tilde{R})
$$

Let $\bar{R} \in S T(\tilde{R})$ be such that for all $j \in\left\{\mu^{\prime}(i) \mid \mu^{\prime} \in L\left(\mu, R_{i}\right)\right\} \backslash\{i\}, i \vec{P}_{i} j$. By (6.3) and (6.4), $\mu \notin \Upsilon(\tilde{R})$. Thus, by Pareto-indifference, $\mu^{I} \notin \varphi(\tilde{R})$. Hence, by $(6.4), \mu^{I} \notin \mathcal{S C}(\vec{R})$. Thus, there exists $T \subseteq N$ and $\bar{\mu} \in \mathcal{M}^{f}$ such that $\bar{\mu}(T)=T$, for all $i \in T, \bar{\mu}(i) \bar{R}_{i} i$, and for some $k \in T, \bar{\mu}(k) \tilde{P}_{k} k$. Let $T^{\prime} \subseteq T$ be such that $k \in T^{\prime}$ and for all $i \in T^{\prime} ; \bar{\mu}(i) \neq i$. Since $\bar{\mu}$ is a matching, $T^{\prime}$ is uniquely defined and $\bar{\mu}\left(T^{\prime}\right)=T^{\prime}$. Thus, for all $i \in T^{\prime}, \bar{\mu}(i) \bar{P}_{i} i$. By our choice of $\bar{R} \in S T(\tilde{R})$ and the definition of $\tilde{R}$, for all $i \in T^{\prime}, \bar{\mu}(i) \tilde{P}_{i} \mu(i)$. Since $\bar{\mu}\left(T^{\prime}\right)=T^{\prime}$, $\mu \notin \mathcal{W C}(\tilde{R})$, a contradiction to (6.3).

Therefore, if we are interested in individual rational and Pareto-indifferent solutions that are implementable, then we are limited to the weak core and its supersolutions. Moreover, by Theorem 6.2 and Proposition 6.3, the weak core is the minimal monotonic and Pareto-indifferent extension of the strong core.

\subsection{Implementability}

Let $\varphi$ be a solution, $i \in N, M \subseteq \mathcal{M}^{\text {f }}$, and $\mu \in M$. Matching $\mu$ is essential for agent $i$ in $M$ for $\varphi$, if there exists $R \in \mathcal{R}$ such that $L\left(\mu, R_{i}\right) \subseteq M$ and $\mu \in \varphi(R)$. Let $\operatorname{Ess}_{i}(M, \varphi)$ denote the set of essential matchings for $i$ in $M$ for $\varphi$. A solution $\varphi$ satisfies essential monotonicity when for all $R, \tilde{R} \in \mathcal{R}$ and all $\mu \in \varphi(R)$, if for all $i \in N, E s s_{i}\left(L\left(\mu, R_{i}\right), \varphi\right) \subseteq L\left(\mu, \tilde{R}_{i}\right)$, then $\mu \in \varphi(\tilde{R})$. Danilov (1992) and Yamato (1992) show that when there are at least three agents, then essential monotonicity is a sufficient condition for a solution to be implementable. 
Lemma 6.6 For all $R \in \mathcal{R}$, all $k \in N$, and all $\mu \in \Upsilon(R), E s s_{k}\left(L\left(\mu, R_{k}\right), \Upsilon\right)=L\left(\mu, R_{k}\right)$.

Proof. By definition, $\operatorname{Ess}_{k}\left(L\left(\mu, R_{k}\right), \Upsilon\right) \subseteq L\left(\mu, R_{k}\right)$. Let $\bar{\mu} \in L\left(\mu, R_{k}\right)$ and $\bar{R} \in \mathcal{P}$ be such that for all $i \in N \backslash\{k\}$ and all $j \in S_{i} \backslash\{\bar{\mu}(i)\}, \bar{\mu}(i) \bar{P}_{i} j$, and for all $j \in S_{k} \backslash\{k, \bar{\mu}(k)\}$, $j \tilde{P}_{k} \bar{\mu}(k) \vec{R}_{k} k$. Then $\bar{\mu} \in \mathcal{S C}(\bar{R})$ and $\bar{\mu} \in \Upsilon(\bar{R})$. Since $\mu \in \Upsilon(R)$ and $\bar{\mu} \in L\left(\mu, R_{k}\right)$, $L\left(\bar{\mu}, \bar{R}_{k}\right) \subseteq L\left(\mu, R_{k}\right)$. Thus, $\bar{\mu} \in E_{s s_{k}}\left(L\left(\mu, R_{k}\right), \Upsilon\right)$, the desired conclusion.

Lemma 6.6 implies that when there are at least three agents, monotonicity is sufficient for the implementability of the union strong $\mathcal{T}$-core and the weak $\mathcal{T}$-core.

Theorem 6.4 When $|N| \geq 3$, then the union strong $\mathcal{T}$-core and the weak $\mathcal{T}$-core are implementable.

Proof. Note that for all $R \in \mathcal{R}, \Upsilon(R) \subseteq \Upsilon^{\mathcal{T}}(R)$ and, by Proposition 6.3, $\Upsilon^{\mathcal{T}}(R) \subseteq$ $\mathcal{W C}^{\mathcal{T}}(R)$. Since $\Upsilon^{\mathcal{T}}$ and $\mathcal{W} \mathcal{C}^{\mathcal{T}}$ are monotonic, by Lemma 6.6 we obtain that $\Upsilon^{\mathcal{T}}$ and $\mathcal{W} \mathcal{C}^{\mathcal{T}}$ are essentially monotonic. Therefore, by Yamato (1992, Theorem 2), $\Upsilon^{\mathcal{T}}$ and $\mathcal{W C}^{\top}$ are implementable.

Remark 6.1 Yamato (1992) requires that for each preference profile the solution (in his paper a solution is a social choice correspondence) is non-empty. As the reader may check, the proof of Yamato (1992, Theorem 2) even holds when we drop non-emptiness. In particular, the results of Sönmez (1996) hold without this restriction though he makes the assumption that the strong core is always non-empty on his domain of feasible matchings. $\triangleleft$

Remark 6.2 Let $\mathcal{T} \equiv\{\{i\} \mid i \in N\} \cup\{N\}$. Then the weak $\mathcal{T}$-core is equal to the individual rational and weak Pareto solution, i.e., $\mathcal{W} \mathcal{C}^{\top}=\mathcal{I R} \cap \mathcal{W} \mathcal{P O}$. Therefore, by Proposition 6.1 and Theorem 6.4, in non-trivial matching problems the individual rational and Pareto solution is not implementable, whereas the individual rational and weak Pareto solution is implementable.

$\triangleleft$

When $|N|=2$ and $\mathcal{M}^{J} \neq\left\{\mu^{I}\right\}$, the weak core is not implementable. This result is similar to the result that for matching problems when preferences are linear orders and $|N|=2$, the strong core is not implementable (Kara and Sönmez, 1996; Sönmez, 1996).

Proposition 6.4 When $|N|=2$ and $\mathcal{M}^{J} \neq\left\{\mu^{I}\right\}$, then the weak core is not implementable. 
Proof. ${ }^{11}$ Let $N=\{1,2\}$ and $\mathcal{M}^{J}=\left\{\mu^{I}, \mu\right\}$. Suppose that the game form $\Gamma=(X, g)$ implements $\mathcal{W C}$. Let $\vec{R}$ be such that $1 \bar{I}_{1} 2$ and $1 \bar{P}_{2} 2$. Then $\mathcal{W C}(\bar{R})=\left\{\mu^{I} ; \mu\right\}$. Since $\Gamma$ implements $\mathcal{W C}$, there exists $\bar{x} \in N E(\Gamma, \bar{R})$ such that $g(\bar{x})=\mu^{I}$. Thus, for all $x_{2} \in X_{2}$, $g\left(\tilde{x}_{1}, x_{2}\right)=\mu^{I}$.

Let $\tilde{R}$ be such that $2 \tilde{P}_{1} 1$ and $1 \tilde{I}_{2} 2$. Then $\mathcal{W C}(\tilde{R})=\left\{\mu^{I}, \mu\right\}$. Similarly as above, there exists $\tilde{x}_{2} \in X_{2}$ such that for all $x_{1} \in X_{1}, g\left(x_{1}, \tilde{x}_{2}\right)=\mu^{I}$.

Let $R \in \mathcal{R}^{N}$ be such that $2 P_{1} 1$ and $1 P_{2} 2$. Then $\mathcal{W C}(R)=\{\mu\}$. But by the above facts, $\left(\tilde{x}_{1}, \tilde{x}_{2}\right) \in N E(\Gamma, R)$ and $\mu^{I} \in g(N E(\Gamma, R))$, a contradiction.

Remark 6.3 Let $|N|=2$ and $\mathcal{M}^{J}=\left\{\mu^{I}, \mu\right\} \neq\left\{\mu^{I}\right\}$. Proposition 6.4 also applies to the union strong $2^{N} \backslash\{\emptyset\}$-core. When $\mathcal{T}=\{\{1\},\{2\}\}$, then the union strong $\mathcal{T}$-core is the individual rational solution. This solution is implementable. (simply consider the game form $\Gamma \equiv(\{1,2\} \times\{1,2\}, g)$ where for all $x \in\{1,2\} \times\{1,2\}$, if $\left(x_{1}, x_{2}\right)=(2,1)$, then $g(x)=\mu$, and otherwise, $g(x)=\mu^{I}$.)

Given a gane form $\Gamma \equiv(X, g)$ and $R \in \mathcal{R}$, let $S N E(\Gamma, R)$ be the set of strong $N$ ash equilibria in the game $(\Gamma, R) ; S N E(\Gamma, R) \equiv\left\{x \in X \mid\right.$ there exists no $T \in 2^{N} \backslash\{\emptyset\}$ and $\bar{x}_{T} \in$ $\times_{i \in T} X_{i}$ such that for all $i \in T, g\left(\bar{x}_{T}, x_{-T}\right) R_{i} g(x)$ and for some $\left.k \in T, g\left(\bar{x}_{T}, x_{-T}\right) P_{k} g(x)\right\}$. Let $\varphi$ be a solution. We say that the game form $\Gamma$ implements $\varphi$ in strong Nash equilibrium if for all $R \in \mathcal{R}, \varphi(R)=g(\operatorname{SNE}(\Gamma, R))$.

Given a solution $\varphi$, a single-valued correspondence $\phi: \mathcal{P} \rightarrow \mathcal{M}^{f}$ is a selection of $\varphi$ if for all $R \in \mathcal{P}, \phi(R) \in \varphi(R)$ whenever $\varphi(R) \neq \emptyset$.

In marriage problems, Shin and Suh (1996) show that any selection of the strong core implements the strong core in strong Nash equilibrium. Sönmez (1997) generalizes their results and shows that any selection of the individual rational and Pareto solution implements the strong core in strong $N$ ash equilibrium. What mechanisms implement certain solutions when preferences are weak? Typically in practice, each agent is only allowed to report linear orders over his set of possible assignments. The only information that is available for the social planner is a profile of linear orders. Minimally, the social planner chooses for each profile of linear orders a feasible matching that is individual rational and Pareto-optimal. It turns out that any selection of the individual rational and Pareto solution implements the union strong core in strong Nash equilibrium when preferences are weak orders. The union strong core is implemented by a mechanism where agents report only linear orders.

For this theorem we again have to require that $\mathcal{M}^{f}$ is closed under composition.

\footnotetext{
${ }^{11}$ We show in the proof that "condition $\beta "$ of Dutta and Sen (1991) is violated. More precisely, we show that there does not exist a game form from which we could exhaust the sets $C_{i}(R, \mu)$ such that condition $\beta$, part (i.b) is satisfied. We omit the details. See also Moore and Repullo (1990).
} 
Theorem 6.5 Let $\mathcal{M}^{f}$ be closed under composition and $\phi$ be a selection of $\mathcal{I R} \cap \mathcal{P O}$. Then the game form $\Gamma \equiv(\mathcal{P}, \phi)$ implements the union strong core in strong Nash equilibrium.

Proof. Let $R \in \mathcal{R}$. We have to show that $\Upsilon(R)=\phi(S N E(\Gamma, R))$. Let $\mu \in \Upsilon(R)$ and $\bar{R} \in \mathcal{P}$ be such that for all $i \in N$ and all $j \in S_{i} \backslash\{\mu(i), i\}, \mu(i) \bar{R}_{i} i \bar{P}_{i} j$. Then $(\mathcal{I R} \cap \mathcal{P O})(\bar{R})=\{\mu\}$. Thus, $\dot{\phi}(\bar{R})=\mu$. Suppose that $\bar{R} \notin S N E(\Gamma, R)$. Then there exist $T \in \mathcal{T}$ and $\left(\tilde{R}_{T}, \bar{R}_{-T}\right) \in \mathcal{P}$ such that for all $i \in T, \phi\left(\tilde{R}_{T}, \tilde{R}_{-T}\right)(i) R_{i} \mu(i)$ and for some $k \in T, \phi\left(\tilde{R}_{T}, \vec{R}_{-T}\right)(k) P_{k} \mu(k)$. For all $i \in T$, let $T^{i} \subseteq N$ be such that (i) $i \in T^{i}$, (ii) $\phi\left(\tilde{R}_{T}, \bar{R}_{-T}\right)\left(T^{i}\right)=T^{i}$, and (iii) for all $T^{\prime} \subsetneq T^{i}, \phi\left(\tilde{R}_{T}, \bar{R}_{-T}\right)\left(T^{\prime}\right) \neq T^{\prime}$. Since $\dot{\varphi}\left(\tilde{R}_{T}, \bar{R}_{-T}\right)$ is a matching; it follows that for all $i \in T, T^{i}$ is uniquely defined. Let $\bar{T} \equiv \cup_{i \in T} T^{i} . \quad B y$ construction, (i) $T \subseteq \bar{T}$, (ii) $\phi\left(\tilde{R}_{T}, \bar{R}_{-T}\right)(\bar{T})=\bar{T}$, and (iii) for all $i \in \bar{T} \backslash T, \phi\left(\tilde{R}_{T}, \bar{R}_{-T}\right)(i) \neq i$. Hence, by construction of $\bar{R}$, (iii), and individual rationality, for all $i \in \bar{T} \backslash T, \phi\left(\tilde{R}_{T}, \bar{R}_{-T}\right)(i)=\phi(\bar{R})(i) \neq i$. Thus, for all $i \in \bar{T}, \phi\left(\tilde{R}_{T}, \vec{R}_{-T}\right)(i) R_{i} \phi(\bar{R})(i)$ and for some $k \in \bar{T}, \phi\left(\tilde{R}_{T}, \bar{R}_{-T}\right)(k) P_{k} \phi(\bar{R})(k)$. Then for all $R^{\prime} \in S T(R), \bar{T}$ blocks $\mu$ under $R^{\prime}$. Thus, for all $R^{\prime} \in S T(R), \mu \notin \mathcal{S C}\left(R^{\prime}\right)$; a contradiction to $\mu \in \Upsilon(R)$. Hence, $\Upsilon(R) \subseteq \phi(S N E(\Gamma, R))$

To show the converse inclusion relation, let $\mu \in \phi(S N E(\Gamma, R))$ and $\bar{R} \in \operatorname{SNE}(\Gamma, R)$ be such that $\phi(\bar{R})=\mu$. Suppose that $\mu \notin \Upsilon(R)$. Let $\tilde{R} \in S T(R)$ be such that for all $i \in N$ and all $j \in S_{i} \backslash\{\mu(i)\}$, if $j I_{i} \mu(i)$, then $\mu(i) \tilde{P}_{i} j$. Thus, $\mu \notin \mathcal{S C}(\tilde{R})$. Then there exist $T \in 2^{N} \backslash\{\emptyset\}$ and $\tilde{\mu} \in \mathcal{M}^{f}$ such that $\tilde{\mu}(T)=T$, for all $i \in T, \tilde{\mu}(i) \tilde{R}_{i} \mu(i)$, and for some $k \in T, \tilde{\mu}(k) \tilde{P}_{k \mu}(k)$. Let $\bar{R}^{\prime} \in \mathcal{P}$ be such that for all $i \in N \backslash T, \bar{R}_{i}^{\prime} \equiv \bar{R}_{i}$, and for all $i \in T$ and all $j \in S_{i} \backslash\{\tilde{\mu}(i), i\}, \tilde{\mu}(i) \bar{R}_{i}^{\prime} i \bar{P}_{i}^{\prime} j$. By construction of $\bar{R}^{\prime}$ and individual rationality, for all $i \in T, \dot{\phi}\left(\bar{R}^{\prime}\right)(i) \in\{\tilde{\mu}(i), i\}$. Thus, $\phi\left(\tilde{R}^{\prime}\right)(T)=T$. Since $\mathcal{M}^{f}$ satisfies $\left(^{*}\right)$ and $\tilde{\mu}(T)=T$, there exists $\tilde{\mu}^{\prime} \in \mathcal{M}^{f}$ such that for all $i \in T, \tilde{\mu}^{\prime}(i)=\tilde{\mu}(i)$, and for all $i \in N \backslash T, \tilde{\mu}^{\prime}(i)=\dot{\phi}\left(\bar{R}^{\prime}\right)(i)$. Since $\phi\left(\bar{R}^{\prime}\right) \in(\mathcal{I} \mathcal{R} \cap \mathcal{P O})\left(\bar{R}^{\prime}\right)$, it follows that $\phi\left(\bar{R}^{\prime}\right)=\tilde{\mu}^{\prime}$. Then for all $i \in T, \phi\left(\bar{R}^{\prime}\right)(i) \tilde{R}_{i} \phi(\tilde{R})(i)$, and for some $k \in T, \phi\left(\bar{R}^{\prime}\right)(k) \tilde{P}_{k} \phi(\bar{R})(k)$. By our choice of $\tilde{R}$, for all $i \in T, \phi\left(\bar{R}^{\prime}\right)(i) R_{i} \phi(\bar{R})(i)$, and for some $k \in T, \phi\left(\bar{R}^{\prime}\right)(k) P_{k} \phi(\vec{R})(k)$; a contradiction to $\bar{R} \in S N E(\Gamma, R)$. Hence, $\phi(S N E(\Gamma, R)) \subseteq \Upsilon(R)$, the desired conclusion.

The following example shows that the closedness of $\mathcal{M}^{f}$ under composition is indispensable in Theorem 6.5.

Example 6.7 Let $N \equiv\{1,2,3,4\}, S_{1} \equiv\{1,2\}, S_{2} \equiv S_{1}, S_{3} \equiv\{3,4\}, S_{4} \equiv S_{3}, \mathcal{M}^{\}} \equiv$ $\left\{\mu^{l},(2,1,3,4),(1,2,4,3)\right\}$. Note that for all $R \in \mathcal{R},(\mathcal{I R} \cap \mathcal{P O})(R) \neq \emptyset$. We show that there exists no selection from $\mathcal{I R} \cap \mathcal{P O}$ that implements $\Upsilon$ in strong Nash equilibrium. Suppose that $\phi$ is such a selection. Let $\bar{R} \in \mathcal{P}$ be such that $2 \bar{P}_{1} 1,1 \bar{P}_{2} 2,4 \bar{P}_{3} 3$, and $3 \bar{P}_{4} 4$. Then $(\mathcal{I R} \cap \mathcal{P O})(\bar{R})=\{(2,1,3,4),(1,2,4,3)\}$. Without loss of generality, let $\phi(\bar{R})=(2,1,3,4)$. Then $\bar{R} \in S N E(\Gamma, \bar{R})$. However, $\Upsilon(\bar{R})=\mathcal{S C}(\bar{R})=\emptyset$, a contradiction. o 


\subsection{How much Indifference?}

In this section we discuss how much indifference we need for our results to hold. First, all results of Sections 6.3 and Section 6.5 remain true whenever the agents may be indifferent between at most two distinct assignments. Second, Theorem 6.2 remains true on any subdomain of the weak orders that contains all linear orders.

In particular, all proofs use only preferences at which agents are indifferent between at most two distinct assignments. We do not use all weak orders for our results. In real life we cannot exclude the possibility that individuals might be indifferent between two distinct assignments. It is a weak assumption that can be easily supported by empirical research. For example, on the American job market for economists, we could conceive of an applicant being indifferent between several offers, say Boston Lniversity and MIT. Indeed, often an applicant needs several weeks to resolve his indifference between several offers and to accept finally a unique one. This would be an easy decision when each student would have a linear order over the universities. However, the previous fact shows that this is not the case.

\subsection{Applications}

\subsubsection{Marriage Problems}

In a marriage problem (Gale and Shapley; 1962) there are two types of individuals, men and women. Let $M$ denote the set of men and $W$ the set of women. Let $N \equiv M \cup W$, for all $m \in M, S_{m} \equiv W \cup\{m\}$, and for all $w \in W, S_{w} \equiv M \cup\{w\}$. Let

$$
\mathcal{M}^{f} \equiv\left\{\mu \in \mathcal{M} \mid \text { for all } i \in N, \mu(\mu(i))=i \text { and } \mu(i) \in S_{i}\right\}
$$

Let $\mathcal{T} \equiv\{\{i\} \mid i \in N\} \cup\{\{m, w\} \mid m \in M$ and $w \in W\}$. In marriage problems the strong $\mathcal{T}$-core (or equivalently, the strong core) is called the stable solution and the "weak $\tau$-core" (or equivalently, the weak core) the weakly stable solution. By Theorem 6.2, we obtain that the weakly stable solution is the minimal monotonic extension of the stable solution.

Proposition 6.5 In marriage problems, the union strong core is equal to the weakly stable solution.

Proof. By Proposition 6.3, $\Upsilon \subseteq \mathcal{W C}$. Let $R \in \mathcal{R}$ and $\mu \in \mathcal{W C}(R)$. Let $\bar{R} \in S T(R)$ be such that for all $i \in N$ and all $j \in S_{i} \backslash\{\mu(i)\}$, if $\mu(i) I_{i} j$, then $\mu(i) \bar{P}_{i} j$. Suppose that 
$\mu \notin \mathcal{S C}(\bar{R})$. Then there exists $T \subseteq N$ such that $|T| \leq 2$ and $\bar{\mu} \in \mathcal{M}^{f}$ such that $\bar{\mu}(T)=T$, for all $i \in T, \bar{\mu}(i) \bar{R}_{i} \mu(i)$ and for some $j \in T, \bar{\mu}(j) \bar{P}_{j} \mu(j)$. Since $\mu \in \mathcal{W C}(R)$, there exist $m \in M$ and $w \in W$ such that $T=\{m, w\}, \bar{\mu}(m)=w$, and $\bar{\mu}(w)=m$. Suppose that $m \bar{P}_{w} \mu(w)$ and $w \bar{R}_{m} \mu(m)$. If $w \bar{P}_{m} \mu(m)$, then $\mu \notin \mathcal{W C}(R)$, a contradiction. Thus, $w \bar{I}_{m} \mu(m)$. Since $\bar{R} \in \mathcal{P}, w=\mu(m)$. Hence, $n=\mu(w)$ and $m \bar{I}_{w} \mu(w)$, a contradiction.

Corollary 6.3 The weakly stable solution is the minimal monotonic extension of the stable solution.

By Theorem 6.3, if a solution satisfies individual rationality, monotonicity, and Paretoindifference, then any weakly stable matching is chosen by this solution. By Theorem 6.4, the weakly stable solution is implementable in marriage problems when preferences are weak orders.

\subsubsection{Objects Exchange}

Shapley and Scarf (1974) introduce the problem of exchanging indivisible objects. ${ }^{12}$ For these problems we have that for all $i \in N, S_{i} \equiv N$, and $\mathcal{M}^{f} \equiv \mathcal{M}$. Given a preference profile, a matching is called a competitive equilibrium when there exist prices for all $i \in N$ such that each agent maximizes his preference on the set of affordable mates and the price of his assignment at $\mu$ is equal to the price of his endowment assignment. We consider the solution that chooses for each preference profile its set of competitive matchings, called the competitive solution. Formally, $\mu \in \mathcal{M}$ is a competitive equilibrium if there exists a price vector $(p(1), p(2), \ldots, p(n)) \in \mathbb{R}^{N}$ such that for all $i \in N$,

(i) $p(\mu(i))=p(i)$; and

(ii) for all $j \in N$, if $p(j) \leq p(i)$, then $\mu(i) R_{i} j$.

When preferences are linear orders, the strong core contains exactly one matching; which is the unique competitive matching (Roth and Postlewaite, 1977). When preferences are weak orders, the strong core is always a subset of the competitive solution (Wako, 1984). Moreover, for each preference profile, the competitive solution is obtained by computing the strong cores of all its strict transformations (Shapley and Scarf, 1974). This is exactly the union strong core. In objects exchange the following holds: ${ }^{13}$

$$
\mathcal{S C} \subsetneq \Upsilon \subsetneq \mathcal{W C}
$$

\footnotetext{
${ }^{12}$ The literature often calls this model "house exchange" or "house allocation".

${ }^{13}$ Note that in marriage problems, the competitive solution is a strict subsolution of the strong core.
} 
The following example, taken from Roth and Postlewaite (1977) shows the above inclusion relations and that in general, the union strong core violates Pareto-indifference.

Example 6.8 Let $N \equiv\{1,2,3,4\}$ and $R \in \mathcal{R}$ be such that

\begin{tabular}{c|c|c|c}
$R_{1}$ & $R_{2}$ & $R_{3}$ & $R_{4}$ \\
\hline 2,4 & 1 & 1 & 1 \\
1 & 3 & 2,4 & 3 \\
3 & 2 & 3 & 4 \\
& 4 & & 2
\end{tabular}

Then $\mathcal{S C}(R)=\emptyset, \Upsilon(R)=\{(2,1,4,3),(4,3,2,1)\}$, and

$$
\mathcal{W C}(R)=\{(2,1,4,3),(2,3,1,4),(2,3,4,1),(4,1,2,3),(4,2,1,3),(4,3,2,1)\}
$$

Note that $(4,1,2,3)$ is Pareto-indifferent to $(2,1,4,3) \in \Upsilon(R)$, but $(4,1,2,3) \notin \Upsilon(R)$. $\circ$ By Theoren 6.2 we obtain the following corollary.

Corollary 6.4 The competitive solution is the minimal monotonic extension of the strong core.

By Theorem 6.3, if a solution satisfies individual rationality, monotonicity, and Paretoindifference, then this solution is a supersolution of the weak core. By Theorem 6.4, the competitive solution and the weak core are implementable in objects exchange when preferences are weak orders. 


\section{Part III}

\section{Allocation of an Infinitely Divisible Commodity}




\section{Chapter 7}

\section{Resource-Monotonic Allocation when Preferences are Single-Peaked}

\subsection{Introduction}

This chapter, which is based on Ehlers (2000f), considers the problem of allocating some amount of an infinitely divisible commodity among a group of agents who have singlepeaked preferences. Single-peakedness of a preference relation means that there is a most preferred consumption, the "peak", and preference is decreasing in both directions away from the peak. A "rule" associates an allocation with each profile of single-peaked preferences. Examples are the trade of a microeconomic good at fixed prices or the allocation of labor among a group of workers. In the latter example, each worker earns a certain wage for each hour work (then for 10 minutes work he receives one sixth of this wage) and the whole group of workers has to provide a certain amount of working time to finish a project. If they do not finish the project, they are not paid. In this context, each worker has a single-peaked preference relation over all possible amounts of working time. We refer to Moulin (1999) and Thomson $(1994 \mathrm{a}, \mathrm{b})$ for further motivations of this model. In a number of studies a certain rule, the "uniform rule", is essential. Sprumont (1991) was the first to provide an axiomatic characterization of it, but many other characterizations have been established.

An economy consists of a set of agents, a profile of single-peaked preferences, and an endowment. Thomson (1994a) is the first to consider variations in the collective endowment in this model, and he formulates the requirement that if two economies differ only in their collective endowments, then either all agents weakly gain or all agents weakly lose. He 
calls this solidarity property resource-monotonicity ${ }^{1}$ and shows it to be incompatible with other basic requirements such as individual rationality from equal division (every agent weakly prefers his consumption to the equal division consumption) and no-envy (every agent weakly prefers his consumption to any other agent's consumption). He then proposes a weaker version of this property, called weak one-sided resource-monotonicity. Here, solidarity is only required for changes in the endownent such that the economy is either in excess demand before and after the change, or the economy is in excess supply before and after the change. The result here is that the uniform rule is the only rule satisfying weak one-sided resource-monotonicity, Pareto-optimality (only efficient allocations are chosen), and no-envy. Klaus, Peters, and Storcken (1997a) derive a similar characterization when we have to reallocate individual endowments.

Sönmez (1994) proposes a stronger version of weak one-sided resource-monotonicity which we call one-sided resource-monotonicity. Here, if an economy is in excess demand and the collective endowment decreases, then all agents weakly lose. If an economy is in excess supply and the collective endowment increases, then all agents weakly lose. He showed that the uniform rule is the only rule satisfying one-sided resource-monotonicity, individual rationality from equal division, and consistency (if some agents leave with their consumptions, then what remains of the resource should be allocated among the remaining agents in the same way as before). We show that if one-sided resource-monotonicity is replaced by weak one-sided resource-monotonicity, then the same characterization holds. Obviously, the result of Thomson (1994a) holds if we replace weak one-sided resourcemonotonicity by one-sided resource-monotonicity. However, then Pareto-optimality is superfluous. The uniform rule is the only rule satisfying one-sided resource-monotonicity and no-envy.

In a recent paper, Moulin (1999) considers a model where consumptions are bounded for all agents. He introduces physical resource-monotonicity which means that the amount assigned to each agent weakly increases whenever the collective endowment increases and characterizes the fixed-path rationing methods by means of physical resource-monotonicity, Pareto-optimality, strategy-proofness (agents cannot gain by misrepresenting their preferences), and consistency. His condition makes no comparisons in terms of welfare. We point out that under Pareto-optimality all three resource-monotonicity properties are equivalent. ${ }^{2}$ We show that one-sided resource-monotonicity implies Pareto-optimality in Moulin's model. Even if physical resource-monotonicity is replaced by weak one-

\footnotetext{
${ }^{1}$ Resource-monotonicity is also studied in various other models. Thomson and Myerson (1980) investigate its implications in bargaining, and Moulin and Thomson (1988) in classical exchange economies.

${ }^{2}$ Moulin (1999) also makes this observation in Remark 3.
} 
sided resource-monotonicity, then Pareto-optimality is superfluous whenever at least three agents are present. The fixed-path rationing methods are the only rules satisfying weak one-sided resource-monotonicity, strategy-proofness, and consistency. Our characterization is appealing on two accounts. First, the resource-monotonicity condition we use makes comparisons in terms of welfare. We are interested in the changes of the welfares of the agents when the environment of an economy changes. Second, Pareto-optimality is implied by weak one-sided resource-monotonicity, strategy-proofness, and consistency.

Chapter 7 is divided in five sections. Section 7.2 introduces the model and the various resource-monotonicity conditions. Section 7.3 presents the results for fixed-path rationing methods, and Section 7.4 presents the characterizations of the uniform rule. Some proofs are relegated to Section 7.5 .

\subsection{The Model and Resource-Monotonicity}

Our formulation of the model allows variations in the population and the social endowment. There is a finite set $\mathcal{N}=\left\{1, \ldots, n_{0}\right\}$ of potential agents. ${ }^{3}$ For each agent $i \in \mathcal{N}$ there is an a priori fixed amount of maximal consumption $X_{i} \in \mathbb{R}_{++} \cup\{+\infty\}$. Given $X_{i} \in$ $\mathbb{R}_{++} \cup\{+\infty\}$, let $C\left(X_{i}\right)$ denote the set of feasible consumptions of agent $i$. That is, if $X_{i} \neq+\infty$, then $C\left(X_{i}\right) \equiv\left[0, X_{i}\right]$, and $C(+\infty) \equiv[0,+\infty[$. Each agent $i \in \mathcal{N}$ is equipped with a continuous preference relation $R_{i}$ over $C\left(X_{i}\right)$. Let $P_{i}$ denote the strict preference relation associated with $R_{i}$, and $I_{i}$ the indifference relation. The preference relation $R_{i}$ is single-peaked if there is a number $p\left(R_{i}\right) \in C\left(X_{i}\right)$, called the peak of $R_{i}$, such that for all $x_{i}, y_{i} \in C\left(X_{i}\right)$, if $y_{i}<x_{i} \leq p\left(R_{i}\right)$ or $p\left(R_{i}\right) \leq x_{i}<y_{i}$, then $x_{i} P_{i} y_{i}$. Let $\mathcal{R}^{i}$ denote the set of all single-peaked preferences over $C\left(X_{i}\right)$. Given $R_{i} \in \mathcal{R}^{i}$ and $x_{i} \in C\left(X_{i}\right)$, let $r_{i}\left(x_{i}\right) \in C\left(X_{i}\right)$ denote the number for which the following holds: (i) if $x_{i} \geq p\left(R_{i}\right)$, then $r_{i}\left(x_{i}\right) \equiv \min \left\{y_{i} \in\left[0, p\left(R_{i}\right)\right] \mid y_{i} R_{i} x_{i}\right\}$; and (ii) if $x_{i} \leq p\left(R_{i}\right)$, then $r_{i}\left(x_{i}\right) \equiv \sup \left\{y_{i} \epsilon\right.$ $\left[p\left(R_{i}\right),+\infty\left[\cap C\left(X_{i}\right) \mid y_{i} R_{i} x_{i}\right\}\right.$.

A social endowment of an infinitely divisible commodity has to be allocated among a finite set of agents. We allow the set of agents to be any subset $N \subseteq \mathcal{N}$. Let $\Omega \in \mathbb{R}_{+}$ denote the endowment. Given $N \subseteq \mathcal{N}$, a (preference) profile $R$ is a list $\left(R_{i}\right)_{i \in N}$ such that for all $i \in N, R_{i} \in \mathcal{R}^{i}$. Let $\mathcal{R}^{N}$ denote the set of all profiles for $N$. Given $S \subseteq N \subseteq \mathcal{N}$ and $R \in \mathcal{R}^{N}$, let $R_{S} \equiv\left(R_{i}\right)_{i \in S}$ denote the restriction of $R$ to $S$. An economy is a pair $(R, \Omega)$ such that for some $N \subseteq \mathcal{N},(R, \Omega) \in \mathcal{R}^{N} \times C\left(\sum_{i \in N} X_{i}\right)$. The economy $(R, \Omega)$ is in excess demand, if $\Omega \leq \sum_{i \in N} p\left(R_{i}\right)$, and it is in excess supply if $\sum_{i \in N} p\left(R_{i}\right) \leq \Omega$.

For all $N \subseteq \mathcal{N}$, an allocation for $(R, \Omega) \in \mathcal{R}^{N} \times C\left(\sum_{i \in N} X_{i}\right)$ is a vector $z \in \mathbb{R}_{+}^{N}$ such

\footnotetext{
${ }^{3}$ All results remain valid when $\mathcal{N}$ is a countable, infinite set.
} 
that for all $i \in N, z_{i} \in C\left(X_{i}\right)$, and $\sum_{i \in N} z_{i}=\Omega$, i.e., we do not allow free disposal. We call $z_{i}$ the allotment of $i$ at $z$. Let $Z(R, \Omega)$ denote the set of all allocations for $(R, \Omega)$. An allocation rule, or simply a rule, associates with each economy an allocation. Formally, a rule $\varphi$ is a mapping

$$
\varphi: \cup_{N \subseteq \mathcal{N}}\left(\mathcal{R}^{N} \times C\left(\sum_{i \in N} X_{i}\right)\right) \longrightarrow \cup_{N \subseteq \mathcal{N}} \mathbb{R}_{+}^{N},
$$

such that for all $N \subseteq \mathcal{N}$ and all $(R, \Omega) \in \mathcal{R}^{N} \times C\left(\sum_{i \in N} X_{i}\right), \varphi(R, \Omega) \in Z(R, \Omega)$. We are interested in rules satisfying certain axioms. The first one says that a rule only selects efficient allocations.

Pareto-Optimality: For all $N \subseteq \mathcal{N}$, all $(R, \Omega) \in \mathcal{R}^{N} \times C\left(\sum_{i \in N} X_{i}\right)$, and all $z \in$ $Z(R, \Omega)$ : if for some $i \in N, z_{i} P_{i} \varphi_{i}(R, \Omega)$, then for some $j \in N, \varphi_{j}(R, \Omega) P_{j} z_{j}$.

A rule satisfies "sanne-sidedness" when for all $N \subseteq \mathcal{N}$, all agents consume weakly less than their most preferred amounts if $\Omega \leq \sum_{i \in N} p\left(R_{i}\right)$, and weakly more if $\Omega \geq \sum_{i \in N} p\left(R_{i}\right)$. Obviously, as pointed out by Sprumont (1991), Pareto-optimality is equivalent to samesidedness.

Our main property pertains to changes in the environment. It is based on the idea of solidarity among the agents. When the environment changes and no one in particular is responsible for the change, then it would be unfair if the individual welfares of the agents were affected in different directions.

In our model the endowment may vary. If two economies only differ in their endowment, then either all agents weakly gain or they all weakly lose. This property is called resource-monotonicity. Thomson (1994a) shows the incompatibility of it with basic requirements. He proposes a weaker version of this property, which we call weak one-sided resource-monotonicity. It requires the monotonicity condition only for "one-sided changes" in the endowment, meaning that the economy is either in excess demand before and after the change, or it is in excess supply before and after the change. In each of these cases and only in these cases we still require that either all agents weakly gain or they all weakly lose.

Weak One-Sided Resource-Monotonicity: For all $N \subseteq \mathcal{N}$, all $R \in \mathcal{R}^{N}$, and all $\Omega, \Omega^{\prime} \in C\left(\sum_{i \in N} X_{i}\right):$ if $\Omega \leq \Omega^{\prime} \leq \sum_{i \in N} p\left(R_{i}\right)$ or $\Omega \geq \Omega^{\prime} \geq \sum_{i \in N} p\left(R_{i}\right)$, then either [for all $i \in N, \varphi_{i}(R, \Omega) R_{i} \varphi_{i}\left(R, \Omega^{\prime}\right)$ ] or [for all $i \in N, \varphi_{i}\left(R, \Omega^{\prime}\right) R_{i} \varphi_{i}(R, \Omega)$ ].

Sönmez (1994) introduces a stronger condition which we call one-sided resource-monotonicity. At economies in excess demand all agents weakly lose when the endowment 
decreases, and for economies in excess supply all agents weakly lose when the endowment increases.

One-Sided Resource-Monotonicity: For all $N \subseteq \mathcal{N}$, all $R \in \mathcal{R}^{N}$, and all $\Omega, \Omega^{\prime} \in$ $C\left(\sum_{i \in N} X_{i}\right):$ if $\Omega \leq \Omega^{\prime} \leq \sum_{i \in N} p\left(R_{i}\right)$ or $\Omega \geq \Omega^{\prime} \geq \sum_{i \in N} p\left(R_{i}\right)$, then for all $i \in N$, $\varphi_{i}\left(R, \Omega^{\prime}\right) R_{i} \varphi_{i}(R, \Omega)$.

Note that one-sided resource-monotonicity implies weak one-sided resource-monotonicity but not vice versa. Moulin (1999) introduces physical resource-monotonicity, the requirement that the amount assigned to each agent weakly increases whenever the collective endowment increases. No comparisons in terms of welfare are made.

Physical Resource-Monotonicity: For all $N \subseteq \mathcal{N}$, all $R \in \mathcal{R}^{N}$; and all $\Omega, \Omega^{\prime} \in$ $C\left(\sum_{i \in N} X_{i}\right)$ : if $\Omega \leq \Omega^{\prime}$, then for all $i \in N, \varphi_{i}(R, \Omega) \leq \varphi_{i}\left(R, \Omega^{\prime}\right)$.

If we do not impose any other properties, then there is no logical relation between physical resource-monotonicity and the two previous monotonicity conditions.

Remark 7.1 Lnder Pareto-optimality, all three monotonicity conditions are equivalent. $\triangleleft$

Linder consistency, when some agents leave the economy with their allotments, then the rule allocates the remaining amount to the agents who did not leave in the same way as before. Each agent who is still present receives the same allotment as at the original economy.

Consistency: For all $N^{\prime} \subseteq N \subseteq \mathcal{N}$ and all $(R, \Omega) \in \mathcal{R}^{N} \times C\left(\sum_{i \in N} X_{i}\right)$ : for all $i \in N^{\prime}$, $\varphi_{i}\left(R_{N^{\prime}}, \sum_{j \in N^{\prime}} \varphi_{j}(R, \Omega)\right)=\varphi_{i}(R, \Omega)$.

\subsection{Fixed-Path Rationing Methods}

In a recent paper, Moulin (1999) introduces fixed-path rationing methods. In his model, the consumption set of each agent is bounded by a finite amount meaning that for all $i \in \mathcal{N}, X_{i} \neq+\infty$. For each agent $i \in \mathcal{N}$, the set of feasible consumptions is $\left[0, X_{i}\right]$.

Each fixed-path rationing method is an allocation rule that relies on two fixed monotonic paths in the box $\times_{i \in \mathcal{N}}\left[0, X_{i}\right]$. For economies in excess demand, individual consumptions are computed along the first path, except that an agent whose demand is below his path-consumption receives exactly his demand. We apply a similar procedure for 
economies in excess supply by using the other path. We refer to Moulin (1999) for a detailed discussion.

Formally, a path is a mapping $g:\left[0, \sum_{i \in \mathcal{N}} X_{i}\right] \rightarrow \mathbb{R}_{+}^{N}$ such that the following holds:

(a) for all $\lambda \in\left[0, \sum_{i \in \mathcal{N}} X_{i}\right], \sum_{i \in \mathcal{N}} g_{i}(\lambda)=\lambda$, and for all $i \in \mathcal{N}, g_{i}(\lambda) \leq X_{i}$; and

(b) for all $\lambda, \tilde{\lambda} \in\left[0, \sum_{i \in \mathcal{N}} X_{i}\right]$, if $\lambda \leq \tilde{\lambda}$, then for all $i \in \mathcal{N}, g_{i}(\lambda) \leq g_{i}(\tilde{\lambda})$.

Let $\mathcal{G}$ denote the family of all paths.

In the above definition, (a) is feasibility and (b) ensures physical resource-monotonicity of a path.

Fixed-Path Rationing Method, $\phi^{\left(g^{+}, g^{-}\right)}$: Given two paths $g^{+}, g^{-} \in \mathcal{G}$, the fixedpath rationing method $\phi^{\left(g^{+}, g^{-}\right)}$is defined as follows. For all $N \subseteq \mathcal{N}$ and all $(R, \Omega) \in$ $\mathcal{R}^{N} \times\left[0, \sum_{i \in N} X_{i}\right]$

(i) when $\Omega \leq \sum_{j \in N} p\left(R_{j}\right)$, there exists $\lambda \in \mathbb{R}_{+}$such that for all $i \in N, \phi_{i}^{\left(g^{+}, g^{-}\right)}(R, \Omega) \equiv$ $\min \left\{p\left(R_{i}\right), g_{i}^{+}(\lambda)\right\} ;$ and

(ii) when $\Omega \geq \sum_{j \in N} p\left(R_{j}\right)$, there exists $\lambda \in \mathbb{R}_{+}$such that for all $i \in N, \dot{\phi}_{i}^{\left(g^{+}, g^{-}\right)}(R, \Omega) \equiv$ $\max \left\{p\left(R_{i}\right), g_{i}^{-}(\lambda)\right\}$.

The existence of $\lambda$ in (i) and (ii) of the above definition follows from feasibility and physical resource-monotonicity of a path. ${ }^{4}$

Example 7.1 The "uniform" path $g^{u}$ is defined as follows: for all $\lambda \in\left[0, \sum_{i \in \mathcal{N}} X_{i}\right]$, there exists $\gamma \in \mathbb{R}_{+}$such that for all $i \in \mathcal{N}, g_{i}^{u}(\lambda) \equiv \min \left\{X_{i}, \gamma\right\}$. The rule $\phi^{\left(g^{u}, g^{u}\right)}$ is called uniform fixed-path rationing method.

Before we state Moulin's (1999) result, we have to define another property. If a rule satisfies strategy-proofness, then an agent cannot gain by misrepresenting his preference relation.

Strategy-Proofness: For all $N \subseteq \mathcal{N}$, all $i \in N$, and all $(R, \Omega),\left(R^{\prime}, \Omega\right) \in \mathcal{R}^{N} \times$ $\left[0, \sum_{j \in N} X_{j}\right]$ such that $R_{N \backslash\{i\}}=R_{N \backslash\{i\}}^{\prime}, \varphi_{i}(R, \Omega) R_{i} \varphi_{i}\left(R^{\prime}, \Omega\right)$.

Theorem 7.1 (Moulin, 1999) The class of fixed-path rationing methods is characterized by physical resource-monotonicity, Pareto-optimality, strategy-proofness, and consistency.

\footnotetext{
'Our definition of a path is slightly different to Moulin's definition. However, both are equivalent when the potential of agents is finite.
} 
Moulin (1999) establishes the independence of the axioms in Theorem 7.1. By Remark 7.1, Theorem 7.1 remains valid if we replace physical resource-monotonicity by one-sided resource-monotonicity or weak one-sided resource-monotonicity. However, the replacement by one-sided resource-monotonicity does not yield a tight result because one-sided resource-monotonicity implies Pareto-optimality in this model.

Proposition 7.1 If a rule satisfies one-sided resource-monotonicity, then it satisfies Pareto-optimality.

Proof. Let $\varphi$ be a rule satisfying one-sided resource-monotonicity. Let $N \subseteq \mathcal{N}$ and $R \in \mathcal{R}^{N}$. The following notation will be useful. For all $\Omega \in\left[0, \sum_{j \in N} X_{j}\right]$, let

$$
\begin{aligned}
N_{-}(\Omega) & \equiv\left\{i \in N \mid \varphi_{i}(R, \Omega)<p\left(R_{i}\right)\right\} \\
N_{0}(\Omega) & \equiv\left\{i \in N \mid \varphi_{i}(R, \Omega)=p\left(R_{i}\right)\right\} \\
N_{+}(\Omega) & \equiv\left\{i \in N \mid \varphi_{i}(R, \Omega)>p\left(R_{i}\right)\right\} .
\end{aligned}
$$

Suppose that $\varphi$ violates Pareto-optimality at $R$. Then there exists $\Omega \in\left[0, \sum_{j \in N} X_{j}\right]$ such that $N_{-}(\Omega) \neq \emptyset$ and $N_{+}(\Omega) \neq \emptyset$. We consider two cases.

Case 1: $\sum_{i \in N} p\left(R_{i}\right) \leq \Omega$.

Let $\Omega^{1} \equiv \Omega$. We construct an increasing sequence $\left(\Omega^{k}\right)_{k \in N}$ such that for all $k \in \mathbb{N}$, $N_{-}\left(\Omega^{k}\right) \neq \emptyset$. For all $k \in \mathbb{N}$, let $\Omega^{k+1} \equiv \min \left\{\Omega^{k}+\alpha^{k}: \sum_{j \in N} X_{j}\right\}$ where

$$
\alpha^{k} \equiv \min _{i \in N \backslash N_{0}\left(\Omega^{k}\right)}\left\{\left|p\left(R_{i}\right)-\varphi_{i}\left(R, \Omega^{k}\right)\right|,\left|p\left(R_{i}\right)-r_{i}\left(\varphi_{i}\left(R, \Omega^{k}\right)\right)\right|\right\}
$$

Note that $\alpha^{1}>0$.

Claim 1: For all $k \in \mathbb{N}, N_{-}\left(\Omega^{k}\right) \neq \emptyset$.

Proof of Claim 1: We prove the assertion by induction. The induction basis holds since at $\varphi\left(R, \Omega^{1}\right)$ Pareto-optimality is violated. Thus, suppose that the conclusion holds for $\varphi\left(R, \Omega^{k}\right)$. Hence, $\alpha^{k}>0$ and $\Omega^{k} \leq \Omega^{k+1}$. By one-sided resource-monotonicity, for all $i \in N$,

$$
\varphi_{i}\left(R, \Omega^{k}\right) R_{i} \varphi_{i}\left(R, \Omega^{k+1}\right)
$$

Thus, if for some $j \in N, j \in N_{-}\left(\Omega^{k}\right) \cap N_{+}\left(\Omega^{k+1}\right)$, then, by definition of $\Omega^{k+1}, \varphi_{j}\left(R, \Omega^{k+1}\right)>$ $\varphi_{j}\left(R, \Omega^{k}\right)+\alpha^{k}$. Since $\Omega^{k+1}-\Omega^{k} \leq \alpha^{k}$, there must be some $h \in N$ such that $\varphi_{h}\left(R, \Omega^{k+1}\right)<$ $\varphi_{h}\left(R, \Omega^{k}\right)$. Hence, by $(7.1), h \in N_{-}\left(\Omega^{k+1}\right)$.

Note the following facts. By one-sided resource-monotonicity, for all $k \in \mathbb{N}, N_{0}\left(\Omega^{k}\right) \supseteq$ $N_{0}\left(\Omega^{k+1}\right)$, and if $N_{0}\left(\Omega^{k}\right)=N_{0}\left(\Omega^{k+1}\right)$, then

$$
\alpha^{k} \leq \alpha^{k+1}
$$


By Claim 1, for all $k \in \mathbb{N}, \alpha^{k}>0$. Hence, if $\Omega^{k+1}=\Omega^{k}$, then $\Omega^{k+1}=\sum_{j \in N} X_{j}$. Next, we prove that $\left(\Omega^{k}\right)_{k \in \mathbb{N}}$ converges to $\sum_{j \in N} X_{j}$.

Claim 2: For some $K \in \mathbb{N}, \Omega^{K}=\sum_{j \in N} X_{j}$.

Proof of Claim 2: Suppose that the assertion does not hold. Then, by definition of $\left(\Omega^{k}\right)_{k \in \mathbb{N}}$, $\lim _{k \rightarrow \infty} \alpha^{k}=0$. But if for some $k \in \mathbb{N}, \alpha^{k}>\alpha^{k+1}$, then (7.2) implies $N_{0}\left(\Omega^{k}\right) \neq N_{0}\left(\Omega^{k+1}\right)$ and $N_{0}\left(\Omega^{k}\right) \supseteq N_{0}\left(\Omega^{k+1}\right)$. Since $N_{0}\left(\Omega^{1}\right)$ is finite, there are only finitely many such $k$. Hence, we cannot have $\lim _{k \rightarrow \infty} \alpha^{k}=0$.

By Clains 1 and 2, there exists some $K \in \mathbb{N}$ such that $\Omega^{K}=\sum_{j \in N} X_{j}$ and $N_{-}\left(\Omega^{K}\right) \neq$ $\emptyset$, which contradicts the fact that for all $i \in N, \varphi_{i}\left(R, \Omega^{K}\right)=X_{i}$.

Case 2: $\sum_{i \in N} p\left(R_{i}\right) \geq \Omega$.

Since the level of consumption is bounded from below by 0 , using arguments similar to those in Case 1 we obtain a contradiction.

The following rule, defined for two-agent economies will play an important role. Let two agents $i$ and $j$ be ordered by $(i, j)$. If the endowment is $X_{j}$, then $i$ gets 0 and $j$ gets his maximal consumption $X_{j}$. Otherwise, if the endowment is unequal to $X_{j}$, then for economies in excess demand these rules first give agent $j$ priority, i.e., his consumption is his most preferred amount or the whole endowment. For economies in excess supply $i$ is given priority, i.e., his consumption is either his peak or the difference between the endowment and agent $j$ 's maximal consumption. For economies in excess demand, $j$ is given priority above $i$ whereas for economies in excess supply, the rule uses the converse priority ( $i$ above $j$ ) in allocating the endowment. We call this rule a converse-priority rule with respect to $(i, j)$.

Converse-Priority Rule with respect to $(i, j), \varphi^{(i, j)}:$ For all $N=\{i, j\} \subseteq \mathcal{N}$ and all $(R, \Omega) \in \mathcal{R}^{N} \times\left[0, X_{i}+X_{j}\right]$ :

(i) when $\Omega=X_{j}, \varphi_{j}^{(i, j)}\left(R, X_{j}\right) \equiv X_{j}$ and $\varphi_{i}^{(i, j)}\left(R, X_{j}\right) \equiv 0$;

(ii) when $\Omega \neq X_{j}$ and $\Omega \leq p\left(R_{i}\right)+p\left(R_{j}\right), \varphi_{j}^{(i, j)}(R, \Omega) \equiv \min \left\{p\left(R_{j}\right), \Omega\right\}$ and $\varphi_{i}^{(i, j)}(R, \Omega) \equiv$ $\Omega-\varphi_{j}^{(i, j)}(R, \Omega) ;$ and

(iii) when $\Omega \neq X_{j}$ and $\Omega \geq p\left(R_{i}\right)+p\left(R_{j}\right), \varphi_{j}^{(i, j)}(R, \Omega) \equiv \min \left\{\Omega-p\left(R_{i}\right), X_{j}\right\}$ and $\varphi_{i}^{(i, j)}(R, \Omega) \equiv \Omega-\varphi_{j}^{(i, j)}(R, \Omega)$.

Converse-priority rules satisfy weak one-sided resource-monotonicity and strategyproofness. It is easy to see that they satisfy strategy-proofness. Let $i=1$ and $j=2$. 
For weak one-sided resource-monotonicity, we have to consider the one-sided changes of resources from $\Omega \neq X_{2}$ to $X_{2}$. Let $(R, \Omega) \in \mathcal{R}^{N} \times\left[0, X_{1}+X_{2}\right]$ be such that $\Omega, X_{2} \leq$ $p\left(R_{1}\right)+p\left(R_{2}\right)$. If $\varphi^{(1,2)}(R, \Omega)=(0, \Omega)$, then $\varphi_{1}^{(1,2)}(R, \Omega)=0=\varphi_{1}^{(1,2)}\left(R, X_{2}\right)$ and agent 1 is indifferent. Otherwise; if $\varphi^{(1,2)}(R, \Omega)=\left(\Omega-p\left(R_{2}\right), p\left(R_{2}\right)\right)$, then both agents weakly prefer $\varphi^{(1,2)}(R, \Omega)$ to $\varphi\left(R, X_{2}\right)=\left(0, X_{2}\right)$. If $\Omega, X_{2} \geq p\left(R_{1}\right)+p\left(R_{2}\right)$, then similar conclusions can be deduced. Hence, $\varphi^{(1,2)}$ satisfies weak one-sided resource-monotonicity.

For two-agent economies, converse-priority rules are the only rules satisfying weak onesided resource-monotonicity and strategy-proofness but violating Pareto-optimality.

Proposition 7.2 Let $N=\{i, j\}$ and $\varphi$ be a rule on $\mathcal{R}^{N} \times\left[0, X_{i}+X_{j}\right]$ satisfying weak onesided resource-monotonicity and strategy-proofness. Then $\varphi$ violates Pareto-optimality if and only if $\varphi$ is a converse-priority rule.

Converse-priority rules violate Pareto-optimality. Therefore, in general if we replace physical resource-monotonicity by weak one-sided resource-monotonicity, then Theorem 7.1 does not hold. However, if there are at least three agents present, then we obtain a different characterization of the fixed-path rationing methods. The key of the proof is Proposition 7.2 and consistency.

Theorem 7.2 Let $|\mathcal{N}| \geq 3$. The class of fixed-path rationing methods is characterized by weak one-sided resource-monotonicity, strategy-proofness, and consistency.

The proofs of Proposition 7.2 and Theorem 7.2 are relegated to Section 7.5.

Remark 7.2 Moulin's (1999) result applies identically to the allocation of a number of identical indivisible objects. Here, each agent $i$ 's allotment is an integer belonging to $\left[0, X_{i}\right]$ and $i$ 's preference relation is single-peaked over all integers in $\left[0, X_{i}\right]$. It is an open question whether our results apply to the discrete model.

\subsection{The Uniform Rule}

In this section we establish several characterizations of the uniform rule based on resourcemonotonicity conditions. For all $i \in \mathcal{N}$, the maximal consumption is $X_{i} \equiv+\infty$. For all agents, the set of feasible consumptions is $\mathbb{R}_{+}$. First, we define the uniform rule (Benassy, 1982).

Uniform Rule, $U:$ For all $N \subseteq \mathcal{N}$ and all $(R, \Omega) \in \mathcal{R}^{N} \times \mathbb{R}_{4}$, 
(i) when $\Omega \leq \sum_{j \in N} p\left(R_{j}\right)$, there exists $\lambda \in \mathbb{R}_{+}$such that for all $i \in N, U_{i}(R, \Omega)=$ $\min \left\{p\left(R_{i}\right), \lambda\right\} ;$ and

(ii) when $\Omega \geq \sum_{j \in N} p\left(R_{j}\right)$, there exists $\lambda \in \mathbb{R}_{+}$such that for all $i \in N, U_{i}(R, \Omega)=$ $\max \left\{p\left(R_{i}\right), \lambda\right\}$.

When all agents have equal finite maximal consumptions in Moulin's model, the uniform rule is the uniform fixed-path rationing method defined in Example 7.1. We consider two axiomatic characterizations of the uniform rule by Thomson (1994a) and Sönmez (1994).

\subsubsection{No-Envy}

Under no-envy, each agent prefers his consumption to any other agent's consumption.

No-Envy: For all $N \subseteq \mathcal{N}$ and all $(R, \Omega) \in \mathcal{R}^{N} \times \mathbb{R}_{+}:$for all $i, j \in N, \varphi_{i}(R, \Omega) R_{i} \varphi_{j}(R, \Omega)$.

Before we state the result, we introduce a domain restriction on the set of single-peaked preferences. A preference relation $R_{i} \in \mathcal{R}^{i}$ is bounded if $r_{i}(0)<\infty$. In his characterization of the uniform rule, Thomson (1994a) imposes this domain restriction. In fact, neither his nor our characterization of the uniform rule below hold without the restriction. For details we refer to Thomson (1994a).

Theorem 7.3 (Thomson, 1994a) When all agents are only equipped with bounded singlepeaked preferences, then the uniform rule is the only rule satisfying weak one-sided resourcemonotonicity, Pareto-optimality, and nomenvy.

The following example establishes the independence of Pareto-optimality from the other two axioms.

Example 7.2 Let $N \equiv\{1,2,3\}$. Let $\vec{R} \in \mathcal{R}^{N}$ be such that $p\left(\bar{R}_{1}\right) \equiv 2, \bar{R}_{1}$ is symmetric around the peak, $\bar{R}_{2} \equiv \bar{R}_{1}, p\left(\bar{R}_{3}\right)=3$, and $1 \bar{I}_{3} 4$. Then $\varphi(\bar{R}, 6) \equiv(1,1,4)$. For all $(R, \Omega) \in\left(\mathcal{R}^{N} \times \mathbb{R}_{+}\right) \backslash\{(\bar{R}, 6)\}, \varphi(R, \Omega) \equiv U(R, \Omega)$. The rule $\varphi$ satisfies weak one-sided resource-monotonicity and no-envy, but not Pareto-optimality.

If we replace weak one-sided resource-monotonicity by one-sided resource-monotonicityin Theorem 7.3, then the independence does not hold.

\footnotetext{
${ }^{5}$ Proving the other two independences is straightforward.
} 
Theorem 7.4 When all agents are only equipped with bounded single-peaked preferences, then the uniform rule is the only rule satisfying one-sided resource-monotonicity and noenvy.

Proof. Let $\varphi$ be a rule satisfying the properties of Theorem 7.4. Let $N \subseteq \mathcal{N}$ and $(R, \Omega) \in \mathcal{R}^{N} \times \mathbb{R}_{+}$be such that for all $i \in N, r_{i}(0)<+\infty$. Let $\bar{r} \equiv \max _{i \in N} r_{i}(0)$. We prove the following claim.

Claim 1: For all $\Omega \in[0,|N| \bar{r}]$ and all $i \in N, \varphi_{i}(R, \Omega) \in[0, \bar{r}]$.

Proof of Claim 1: Suppose the conclusion does not hold for some $\Omega \in[0,|N| \bar{r}]$. Since $\Omega \leq|N| \vec{r}$, there exist $i, j \in N, i \neq j$, such that $\varphi_{i}(R, \Omega)<\bar{r}$ and $\varphi_{j}(R, \Omega)>\bar{r}$. By $r_{j}(0) \leq \vec{r}$

$$
\varphi_{i}(R, \Omega) R_{j} \bar{T} P_{j} \varphi_{j}(R, \Omega)
$$

Thus, at $(R, \Omega)$ agent $j$ envies $i$, contradicting no-envy.

For all $i \in N$, let $\tilde{X}_{i} \equiv \vec{r}$. By Claim 1, for all $\Omega \in C\left(\sum_{i \in N} \tilde{X}_{i}\right), \varphi_{i}(R, \Omega) \in C\left(\tilde{X}_{i}\right)$. By Proposition 7.1, if $\Omega \in C\left(\sum_{i \in N} \tilde{X}_{i}\right)$, then $\varphi(R, \Omega)$ is Pareto-optimal. Hence, by Theorem 7.3 , for all $\Omega \in[0,|N| \bar{r}], \varphi(R, \Omega)=U(R, \Omega)$. If $\Omega>|N| \bar{r}$, then it is easy to check that $\varphi(R, \Omega)=U(R, \Omega)$.

Chun (1999) characterizes on the domain of bounded single-peaked preferences the uniform rule by a certain "separability" condition and the conditions used in Theorem 7.4. Thus, by Theorem 7.4, we conclude that his characterization is not tight. Theorem 7.4 is a characterization of the uniform rule by only two axioms, i.e., one-sided resourcemonotonicity is a strong requirement which only admits one selection from the no-envy solution.

\subsubsection{Consistency}

Individual rationality from equal division requires that for each economy each agent prefers his allotment to the equal division consumption.

Individual Rationality from Equal Division: For all $N \subseteq \mathcal{N}$ and all $(R, \Omega) \in$ $\mathcal{R}^{N} \times \mathbb{R}_{i}:$ for all $i \in N, \varphi_{i}(R, \Omega) R_{i} \frac{\Omega}{|N|}$.

Sönmez (1994) derives the following result. 
Theorem 7.5 (Sönmez, 1994) The uniform rule is the only rule satisfying one-sided resource-monotonicity, individual rationality from equal division, and consistency.

In Theorem 7.5 it is sufficient to impose weak one-sided resource-monotonicity instead of one-sided resource-monotonicity.

Theorem 7.6 The uniform rule is the only rule satisfying weak one-sided resource-monotonicity, individual rationality from equal division, and consistency.

Proof. Let $\varphi$ be a rule satisfying the properties of Theorem 7.6. We show that $\varphi$ satisfies Pareto-optimality. Then, the assertion follows from Remark 7.1 and Theorem 7.5 .

Suppose that $\varphi$ does not satisfy Pareto-optimality. Then there exists $N \subseteq \mathcal{N}$ and $(R, \Omega) \in \mathcal{R}^{N} \times \mathbb{R}_{+}$such that same-sidedness is violated. Without loss of generality, let $1,2 \in N, \varphi_{1}(R, \Omega)<p\left(R_{1}\right)$ and $\varphi_{2}(R, \Omega)>p\left(R_{2}\right)$. Let $\Omega^{\prime} \equiv \varphi_{1}(R, \Omega)+\varphi_{2}(R, \Omega)$. By consistency, $\varphi\left(R_{\{1,2\}}, \Omega^{\prime}\right)=\left(\varphi_{1}(R, \Omega), \varphi_{2}(R, \Omega)\right)$. Thus,

$$
\varphi_{1}\left(R_{\{1,2\}}, \Omega^{\prime}\right)<p\left(R_{1}\right) \text { and } \varphi_{2}\left(R_{\{1,2\}}, \Omega^{\prime}\right)>p\left(R_{2}\right) .
$$

By individual rationality from equal division,

$$
\varphi_{1}\left(R_{\{1,2\}}, \Omega^{\prime}\right) R_{1} \frac{\Omega^{\prime}}{2} \text { and } \varphi_{2}\left(R_{\{1,2\}}, \Omega^{\prime}\right) R_{2} \frac{\Omega^{\prime}}{2}
$$

We consider two cases.

Case 1: $\varphi_{1}\left(R_{\{1,2\}}, \Omega^{\prime}\right) \leq \varphi_{2}\left(R_{\{1,2\}}, \Omega^{\prime}\right)$.

Hence, $\varphi_{1}\left(R_{\{1,2\}}, \Omega^{\prime}\right) \leq \frac{\Omega^{\prime}}{2} \leq \varphi_{2}\left(R_{\{1,2\}}, \Omega^{\prime}\right)$. Thus, by (7.4) and (7.5),

$$
r_{1}\left(\varphi_{1}\left(R_{\{1,2\}}, \Omega^{\prime}\right)\right) \leq \frac{\Omega^{\prime}}{2} \leq r_{2}\left(\varphi_{2}\left(R_{\{1,2\}}, \Omega^{\prime}\right)\right) .
$$

Hence, $p\left(R_{1}\right) \leq p\left(R_{2}\right)$. We consider two subcases.

Subcase 1.1: $p\left(R_{1}\right)+p\left(R_{2}\right) \geq \Omega^{\prime}$.

Consider the economy $\left(R_{\{1,2\}}, 2 p\left(R_{1}\right)\right)$. Note that $\left(R_{\{1,2\}}, 2 p\left(R_{1}\right)\right)$ and $\left(R_{\{1,2\}}, \Omega^{\prime}\right)$ are one-sided changes. By individual rationality from equal division $\varphi\left(R_{\{1,2\}}, 2 p\left(R_{1}\right)\right)=$ $\left(p\left(R_{1}\right), p\left(R_{1}\right)\right)$. Thus, by $(7.4), p\left(R_{1}\right) P_{1} \varphi_{1}\left(R_{\{1,2\}}, \Omega^{\prime}\right)$. By $(7.4)$ and $(7.6)$, we know that $\varphi_{2}\left(R_{\{1,2\}}, \Omega^{\prime}\right) P_{2} p\left(R_{1}\right)$. The two previous relations contradict weak one-sided resourcemonotonicity.

Subcase 1.2: $p\left(R_{1}\right)+p\left(R_{2}\right) \leq \Omega^{\prime}$.

Similarly, by considering the economy $\left(R_{\{1,2\}}, 2 p\left(R_{2}\right)\right)$, we obtain as in Subcase 1.1 a contradiction to weak one-sided resource-monotonicity.

Case 2: $\varphi_{1}\left(R_{\{1,2\}}, \Omega^{\prime}\right) \geq \varphi_{2}\left(R_{\{1,2\}}, \Omega^{\prime}\right)$.

This case is similar to Case 1 . 
Remark 7.3 Sönmez (1994) also shows that the uniform rule is the only rule satisfying one-sided resource-monotonicity, individual rationality from equal division, and converse consistency. We can show that the replacement of one-sided resource-monotonicity by weak one-sided resource-monotonicity still yields a valid result.

We also establish an example showing that Theorems 7.2, 7.4, and 7.6 do not yield uniqueness when we use physical resource-monotonicity. In Example 7.3 the maximal consumption of each agent may be finite or infinite.

Example 7.3 For all $i \in \mathcal{N}$, let $X_{i} \in \mathbb{R}_{++} \cup\{+\infty\}$. For all $N \subseteq \mathcal{N}$ and all $(R, \Omega) \in$ $\mathcal{R}^{N} \times C\left(\sum_{i \in N} X_{i}\right)$, there exists $\gamma \in \mathbb{R}_{+}$such that for all $i \in N, \bar{\varphi}_{i}(R, \Omega) \equiv \min \left\{X_{i}, \gamma\right\}$. The rule $\bar{\varphi}$ satisfies physical resource-monotonicity, strategy-proofness, and consistency. When for all $i, j \in \mathcal{N}, X_{i}=X_{j}$, then $\bar{\varphi}$ also satisfies individual rationality from equal division and no-envy.

\subsection{Proofs}

\subsubsection{Proof of Proposition 7.2}

Proposition 7.2 Let $N=\{1,2\}$ and $\varphi$ be a rule on $\mathcal{R}^{N} \times\left[0, X_{1}+X_{2}\right]$ satisfying weak onesided resource-monotonicity and strategy-proofness. Then $\varphi$ violates Pareto-optimality if and only if $\varphi$ is a converse-priority rule.

Proof. Let $\varphi$ violate Pareto-optimality. Hence, there exists $(R, \Omega) \in \mathcal{R}^{N} \times\left[0, X_{1}+X_{2}\right]$ such that same-sidedness is violated. Without loss of generality, let

$$
\varphi_{1}(R, \Omega)<p\left(R_{1}\right) \text { and } p\left(R_{2}\right)<\varphi_{2}(R, \Omega)
$$

We show that $\varphi$ is a converse-priority rule with respect to $(1,2)$, i.e., $\varphi=\varphi^{(1,2)}$. We show that without loss of generality, we may suppose that $(R, \Omega)$ possesses the following properties:

(i) $p\left(R_{1}\right)+p\left(R_{2}\right)=\Omega$;

(ii) $p\left(R_{1}\right)<X_{1}$ and $p\left(R_{2}\right)>0$;

(iii) $R_{1}$ and $R_{2}$ are symmetric; and

(iv) $2 p\left(R_{2}\right)-\varphi_{2}(R, \Omega)>0$. 
Suppose that $R$ does not satisfy (i) to (iv). Let $\beta \equiv \min \left\{\frac{1}{2}\left(p\left(R_{1}\right)-\varphi_{1}(R, \Omega)\right), \frac{1}{3}\left(\varphi_{2}(R, \Omega)-\right.\right.$ $\left.\left.p\left(R_{2}\right)\right)\right\}$. By $(7.7), \beta>0$. Let $R_{1}^{\beta} \in \mathcal{R}^{1}$ be such that $p\left(R_{1}^{\beta}\right) \equiv \varphi_{1}(R, \Omega)+\beta$ and $R_{1}^{\beta}$ is symmetric; and let $R^{\beta} \equiv\left(R_{1}^{\beta}, R_{2}\right)$. By strategy-proofness and construction, $\varphi\left(R^{\beta}\right)=$ $\varphi(R)$. Let $R_{2}^{\beta^{\prime}} \in \mathcal{R}^{2}$ be such that $p\left(R_{2}^{\beta^{\prime}}\right) \equiv \varphi_{2}(R, \Omega)-\beta$ and $R_{2}^{\beta^{\prime}}$ is symmetric, and let $R^{\beta^{\prime}} \equiv\left(R_{1}^{\beta}, R_{2}^{\beta^{\prime}}\right)$. By strategy-proofness and construction, $\varphi\left(R^{\beta^{\prime}}\right)=\varphi\left(R^{\beta}\right)$. Note that

$$
p\left(R_{1}^{\beta^{\prime}}\right)+p\left(R_{2}^{\beta^{\prime}}\right)=\left(\varphi_{1}(R, \Omega)+\beta\right)+\left(\varphi_{2}(R, \Omega)-\beta\right)=\Omega .
$$

Thus, (i) holds for $\left(R^{\beta^{\prime}}, \Omega\right)$. Obviously, (ii) and (iii) follow from the construction of $R^{\beta^{\prime}}$. Finally, (iv) follows from the definition of $\beta$. Hence, $\left(R^{\beta^{\prime}}, \Omega\right)$ satisfies (7.7) and (i) to (iv). Without loss of generality, we suppose that $(R, \Omega)$ satisfies (i) to (iv).

Let $\alpha \equiv p\left(R_{1}\right)-\varphi_{1}(R, \Omega)$. Then $\varphi_{2}(R, \Omega)=p\left(R_{2}\right)+\alpha$, and by (i) and (iv), $p\left(R_{2}\right)-\alpha>$ 0 . Let $R_{1}^{\prime} \in \mathcal{R}^{1}$ be such that $0 P_{1}^{\prime}\left(p\left(R_{1}\right)+\alpha\right)$ and $p\left(R_{1}^{\prime}\right) \equiv p\left(R_{1}\right)$, and let $R^{\prime} \equiv\left(R_{1}^{\prime}, R_{2}\right)$. By strategy-proofness, $\varphi\left(R^{\prime}, \Omega\right)=\varphi(R, \Omega)$. Let $R_{2}^{\prime \prime} \in \mathcal{R}^{2}$ be such that $0 P_{2}^{\prime \prime}\left(p\left(R_{2}\right)+\alpha\right)$ and $p\left(R_{2}^{\prime \prime}\right) \equiv p\left(R_{2}\right)$, and let $R^{\prime \prime} \equiv\left(R_{1}^{\prime}, R_{2}^{\prime \prime}\right)$. By strategy-proofness, either $\varphi\left(R^{\prime \prime}, \Omega\right)=\varphi(R, \Omega)$ or $\left[\varphi_{2}\left(R^{\prime \prime}, \Omega\right) \leq p\left(R_{2}\right)-\alpha\right.$ and $\left.\varphi_{1}\left(R^{\prime \prime}, \Omega\right) \geq p\left(R_{1}\right)+\alpha\right]$. We prove the following claim.

Claim 1: $\varphi\left(R^{\prime \prime}, \Omega\right)=\varphi(R, \Omega)=(0, \Omega)$.

Proof of Claim 1: If min $\left\{\varphi_{1}\left(R^{\prime \prime}, \Omega\right), \varphi_{2}\left(R^{\prime \prime}, \Omega\right)\right\}>0$, then $\left(R^{\prime \prime}, \Omega\right)$ and $\left(R^{\prime \prime}, 0\right)$ are one-sided changes. Since $\varphi\left(R^{\prime \prime}, 0\right)=(0,0)$ and by construction, we have either $\left[\varphi_{1}\left(R^{\prime \prime}, 0\right) P_{1}^{\prime \prime} \varphi_{1}\left(R^{\prime \prime}, \Omega\right)\right.$ and $\left.\varphi_{2}\left(R^{\prime \prime}, \Omega\right) P_{2}^{\prime \prime} \varphi_{2}\left(R^{\prime \prime}, 0\right)\right]$ or $\left[\varphi_{1}\left(R^{\prime \prime}, \Omega\right) P_{1}^{\prime \prime} \varphi_{1}\left(R^{\prime \prime}, 0\right)\right.$ and $\left.\varphi_{2}\left(R^{\prime \prime}, 0\right) P_{2}^{\prime \prime} \varphi_{2}\left(R^{\prime \prime}, \Omega\right)\right]$, which contradicts weak one-sided resource-monotonicity. Hence, $\varphi\left(R^{\prime \prime}, \Omega\right) \in\{(0, \Omega),(\Omega, 0)\}$.

Case 1: $\varphi\left(R^{\prime \prime}, \Omega\right)=(\Omega, 0)$.

Let $R^{0} \in \mathcal{R}^{N}$ be such that $p\left(R_{1}^{0}\right) \equiv p\left(R_{2}^{0}\right) \equiv 0$. By weak one-sided resource-monotonicity, $\varphi\left(R^{0}, \cdot\right)$ describes a continuous weakly increasing function in both coordinates. ${ }^{6}$ By applying strategy-proofness twice, $\varphi\left(R^{0}, \Omega\right)=(\Omega, 0)$. By the two previous facts there exists $\left.\bar{\Omega} \in] \Omega, X_{1}+X_{2}\right]$ such that

$$
\varphi_{1}\left(R^{0}, \bar{\Omega}\right) \geq \Omega \text { and } \varphi_{2}\left(R^{0}, \bar{\Omega}\right)=p\left(R_{2}\right)
$$

By $p\left(R_{1}\right)<\Omega$ and applying strategy-proofness twice, $\varphi(R, \bar{\Omega})=\varphi\left(R^{0}, \bar{\Omega}\right)$. Hence,

$$
\begin{aligned}
\varphi_{1}(R, \vec{\Omega}) & \geq \Omega \\
& =\varphi_{1}(R, \Omega)+\varphi_{2}(R, \Omega) \\
& =\varphi_{1}(R, \Omega)+2 \alpha+\left(p\left(R_{2}\right)-\alpha\right) \\
& >\varphi_{1}(R, \Omega)+2 \alpha
\end{aligned}
$$

${ }^{6}$ This is a special case because for all $\Omega \in\left[0, X_{1}+X_{2}\right], \varphi\left(R^{0}, \Omega\right)$ is same-sided. A standard argument yields continuity. Thomson (1994a, Theorem 2 ) gives a formal proof of this statement. 
where the first equality follows from $\varphi_{2}(R, \Omega)=p\left(R_{2}\right)+\alpha$, and the strict inequality follows from the fact $p\left(R_{2}\right)-\alpha>0$. Thus, $\varphi_{1}(R, \Omega) P_{1} \varphi_{1}(R, \bar{\Omega})$ and $\varphi_{2}(R, \bar{\Omega}) P_{2} \varphi_{2}(R, \Omega)$. Since $(R, \Omega)$ and $(R, \tilde{\Omega})$ are one-sided changes, the previous relations contradict weak one-sided resource-monotonicity. Therefore, Case 1 cannot occur.

Case 2: $\varphi\left(R^{\prime \prime}, \Omega\right)=(0, \Omega)$.

Let $R^{0} \in \mathcal{R}^{N}$ be such that $p\left(R_{1}^{0}\right) \equiv p\left(R_{2}^{0}\right) \equiv 0$. By weak one-sided resource-monotonicity, $\varphi\left(R^{0}, \cdot\right)$ describes a continuous weakly increasing function in both coordinates. By applying strategy-proofness twice,

$$
\varphi\left(R^{0}, \Omega\right)=(0, \Omega)
$$

By the two previous facts there exists $\left.\bar{\Omega} \in] \Omega, X_{1}+X_{2}\right]$ such that

$$
\varphi_{1}\left(R^{0}, \bar{\Omega}\right)=p\left(R_{1}\right) \text { and } \varphi_{2}\left(R^{0}, \bar{\Omega}\right) \geq \Omega
$$

By applying strategy-proofness twice, $\varphi(R, \bar{\Omega})=\varphi\left(R^{0}, \bar{\Omega}\right)$. Hence, if $\varphi_{1}(R, \Omega)>0$, then

$$
\varphi_{2}(R, \bar{\Omega}) \geq \Omega=\varphi_{1}(R, \Omega)+\varphi_{2}(R, \Omega)>\varphi_{2}(R, \Omega) .
$$

Thus, $\varphi_{1}(R, \bar{\Omega}) P_{1} \varphi_{1}(R, \Omega)$ and $\varphi_{2}(R, \Omega) P_{2} \varphi_{2}(R, \bar{\Omega})$. Since $(R, \Omega)$ and $(R, \bar{\Omega})$ are one-sided changes, the previous relations contradict weak one-sided resource-monotonicity. Thus, $\varphi_{1}(R, \Omega)=0$ and $\varphi(R, \Omega)=(0, \Omega)=\varphi\left(R^{\prime \prime}, \Omega\right)$, the desired conclusion.

Clain 1 allows us to prove the following.

Claim 2: For all $(\tilde{R}, \tilde{\Omega}) \in \mathcal{R}^{N} \times\left(\left[0, X_{1}+X_{2}\right] \backslash\{\Omega\}\right), \varphi(\tilde{R}, \tilde{\Omega})$ is same-sided.

Proof of Claim 2: Let $(\tilde{R}, \tilde{\Omega}) \in \mathcal{R}^{N} \times\left(\left[0, X_{1}+X_{2}\right] \backslash\{\Omega\}\right)$, and suppose that $\varphi(\tilde{R}, \tilde{\Omega})$ is not same-sided. Thus, by strategy-proofness we may suppose that $(\tilde{R}, \tilde{\Omega})$ possesses the properties corresponding to (i) to (iv) of $(R, \Omega)$. Similarly to Claim 1 it follows that $\varphi(\tilde{R}, \tilde{\Omega}) \in\{(0, \tilde{\Omega}),(\tilde{\Omega}, 0)\}$.

Case 1: $\varphi(\tilde{R}, \tilde{\Omega})=(\tilde{\Omega}, 0)$.

By using the same arguments as in Claim 1, it follows that $\varphi\left(R^{0}, \tilde{\Omega}\right)=(\tilde{\Omega}, 0)$. By Claim 1, $\varphi\left(R^{0}, \Omega\right)=(0, \Omega)$. By weak one-sided resource-monotonicity; $\varphi\left(R^{0}, \cdot\right)$ describes a continuous weakly increasing function in both coordinates. Thus, the two previous facts are in contradiction. Hence, Case 1 cannot occur.

Case 2: $\varphi(\tilde{R}, \tilde{\Omega})=(0, \tilde{\Omega})$.

By using the same arguments as in Clain 1, it follows that $\varphi\left(R^{0}, \tilde{\Omega}\right)=(0, \tilde{\Omega})$. Because $\tilde{\Omega} \neq \Omega$, we may suppose that $\Omega>\tilde{\Omega}$. By Claim $1, \varphi\left(R^{0}, \Omega\right)=(0, \Omega)$. By weak one-sided resource-monotonicity, $\varphi\left(R^{0}, \cdot\right)$ describes a continuous weakly increasing function in both 
coordinates. Thus, there exists $\bar{\Omega} \in\left[0, X_{1}+X_{2}\right]$ such that $\varphi\left(R^{0}, \tilde{\Omega}\right)=\left(p\left(\tilde{R}_{1}\right), \bar{\Omega}-p\left(\tilde{R}_{1}\right)\right)$. Since $\varphi\left(R^{0}, \Omega\right)=(0, \Omega)$, it follows that $\varphi_{2}\left(R^{0}, \bar{\Omega}\right) \geq \Omega>\tilde{\Omega}$. By applying strategyproofness twice, $\varphi(\tilde{R}, \bar{\Omega})=\varphi\left(R^{0}, \bar{\Omega}\right)$. Note that $(\tilde{R}, \tilde{\Omega})$ and $(\tilde{R}, \bar{\Omega})$ are one-sided changes. By the previous facts,

$$
\varphi_{1}(\tilde{R}, \bar{\Omega}) \tilde{P}_{1} \varphi_{1}(\tilde{R}, \tilde{\Omega}) \text { and } \varphi_{2}(\tilde{R}, \tilde{\Omega}) \tilde{P}_{2} \varphi_{2}(\tilde{R}, \tilde{\Omega})
$$

which contradicts weak one-sided resource-monotonicity. Hence, Case 2 cannot occur. $\diamond$

By Clain 2, $\varphi$ is same-sided whenever $\tilde{\Omega} \neq \Omega$. Therefore, strategy-proofness yields the following fact: For all $\tilde{R}, \bar{R} \in \mathcal{R}^{N}$ such that $p\left(\tilde{R}_{1}\right)=p\left(\bar{R}_{1}\right)$ and $p\left(\tilde{R}_{2}\right)=p\left(\bar{R}_{2}\right)$, and all $\tilde{\Omega} \in\left[0, X_{1}+X_{2}\right] \backslash\{\Omega\}$,

$$
\varphi(\tilde{R}, \tilde{\Omega})=\varphi(\bar{R}, \tilde{\Omega})
$$

Claim 3: For all $\tilde{R} \in \mathcal{R}^{N}, \varphi\left(\tilde{R}, X_{2}\right)=\left(0, X_{2}\right)$.

Proof of Claim 3: First, we show that $\Omega=X_{2}$. By Claim 1, $\varphi(R, \Omega)=(0, \Omega)$. Let $R^{\prime} \in \mathcal{R}^{N}$ be such that $X_{1} P_{1}^{\prime} 0, p\left(R_{1}^{\prime}\right) \equiv p\left(R_{1}\right), X_{2} P_{2}^{\prime} 0$, and $p\left(R_{2}^{\prime}\right) \equiv p\left(R_{2}\right)$. By applying strategy-proofness twice, $\varphi\left(R^{\prime}, \Omega\right)$ is not same-sided. Analogous to Claim 1 it follows that $\varphi\left(R^{\prime}, \Omega\right) \in\{(0, \Omega),(\Omega, 0)\}$.

If $\varphi\left(R^{\prime}, \Omega\right)=(\Omega, 0)$, then similarly to Claim 1 it can be shown that $\varphi\left(R^{0} ; \Omega\right)=(\Omega, 0)$, which contradicts $(7.8)$.

If $\varphi\left(R^{\prime}, \Omega\right)=(0, \Omega)$ and $\Omega<X_{2}$, then by definition, $\varphi\left(R^{\prime}, X_{1}+X_{2}\right)=\left(X_{1}, X_{2}\right)$. Since $\left(R^{\prime}, \Omega\right)$ and $\left(R^{\prime}, X_{1}+X_{2}\right)$ are one-sided changes, by construction this contradicts weak one-sided resource-monotonicity. Hence, $\Omega=X_{2}$.

Let $\tilde{R} \in \mathcal{R}^{N}$. If $\varphi\left(\tilde{R}, X_{2}\right)$ is not same-sided, then the previous arguments yield

$$
\varphi\left(\tilde{R}, X_{2}\right)=\left(0, X_{2}\right)
$$

Suppose that $\varphi\left(\tilde{R}, X_{2}\right)$ is same-sided and $\varphi\left(\tilde{R}, X_{2}\right) \neq\left(0, X_{2}\right)$.

We show that without loss of generality, we may suppose that

$$
\left.\varphi_{1}\left(\tilde{R}, X_{2}\right) \in\right] 0, X_{1}\left[\text { and } \varphi_{2}\left(\tilde{R}, X_{2}\right) \in\right] 0, X_{2}[.
$$

Suppose that $\varphi\left(\tilde{R}, X_{2}\right) \in\left\{\left(X_{2}, 0\right),\left(X_{1}, X_{2}-X_{1}\right)\right\}$. If $\varphi\left(\tilde{R}, X_{2}\right)=\left(X_{2}, 0\right), p\left(\tilde{R}_{1}\right) \geq X_{2}$, and $p\left(\tilde{R}_{2}\right) \geq 0$, then let $\gamma \equiv \frac{1}{3} X_{2}$ and $R_{1}^{\gamma} \in \mathcal{R}^{1}$ be such that $p\left(R_{1}^{\gamma}\right) \equiv X_{2}-\gamma$ and $R_{1}^{\gamma}$ is symmetric, and let $R^{\gamma} \equiv\left(R_{1}^{\gamma}, \tilde{R}_{2}\right)$. By strategy-proofness, $\varphi_{1}\left(R^{\gamma}, X_{2}\right)>0$. Hence, by (7.10), $\varphi\left(R^{\gamma}, X_{2}\right)$ is same-sided. If $\left.\varphi_{1}\left(R^{\gamma}\right) \in\right] 0, X_{2}\left[\right.$, then (7.11) holds for $R^{\gamma}$. Suppose that $\varphi\left(R^{\gamma}, X_{2}\right)=\left(X_{2}, 0\right)$. Thus, by same-sidedness, $\nu\left(R_{2}^{\gamma}\right)=0$. Let $R_{2}^{\gamma \prime} \in \mathcal{R}^{2}$ be such that $p\left(R_{2}^{\gamma \prime}\right) \equiv \gamma$ and $R_{2}^{\gamma \prime}$ is symmetric, and let $R^{\gamma^{\prime}} \equiv\left(R_{1}^{\gamma}, R_{2}^{\gamma \prime}\right)$. By strategy-proofness, 
$\varphi_{2}\left(R^{\gamma^{\prime}}, X_{2}\right)<X_{2}$. Hence, by $(7.10), \varphi\left(R^{\gamma^{\prime}}, X_{2}\right)$ is same-sided. Thus, $\varphi\left(R^{\gamma^{\prime}}, X_{2}\right)=\left(X_{2}-\right.$ $\gamma, \gamma)$ and $(7.11)$ holds for $R^{\gamma^{\prime}}$. For all other cases we derive similar conclusions. Hence, without loss of generality, we suppose that (7.11) holds for $\tilde{R}$.

By strategy-proofness we may suppose that $0 \tilde{P}_{1} X_{1}$,

$$
0<p\left(\tilde{R}_{1}\right)=\varphi_{1}\left(\tilde{R}, X_{2}\right)<X_{1} \text { and } 0<\varphi_{2}\left(\tilde{R}, X_{2}\right)=p\left(\tilde{R}_{2}\right)<X_{2}
$$

By Claim 1, $\varphi\left(R^{\prime \prime}, X_{2}\right)=\left(0, X_{2}\right)$. Let $\bar{R}_{1} \in \mathcal{R}^{1}$ be such that $p\left(\bar{R}_{1}\right) \equiv p\left(R_{1}^{\prime \prime}\right)$ and $\tilde{r}_{1}(0)<$ $\bar{r}_{1}(0)<X_{1}$, and let $\bar{R} \equiv\left(\bar{R}_{1}, R_{2}^{\prime \prime}\right)$. By strategy-proofness, $\varphi\left(\bar{R}, X_{2}\right)$ is not same-sided. Hence, by the first part of the proof of Claim $3, \varphi\left(\bar{R}, X_{2}\right)=\left(0, X_{2}\right)$. Let $\bar{R}^{\prime} \equiv\left(\tilde{R}_{1}, R_{2}^{\prime \prime}\right)$. By strategy-proofness and $\tilde{r}_{1}(0)<\bar{r}_{1}(0), \varphi\left(\bar{R}^{\prime}, X_{2}\right)=\left(0, X_{2}\right)$. By $0 P_{2}^{\prime \prime} X_{2}$ and strategyproofness, $\varphi\left(\tilde{R}, X_{2}\right)=\left(0, X_{2}\right)$ which contradicts $(7.12)$.

The final claim proves Proposition 7.2.

Claim 4: $\varphi=\varphi^{(1,2)}$.

Proof of Claim 4: By Claim 3, for all $R \in \mathcal{R}^{N}$,

$$
\varphi\left(R, X_{2}\right)=\left(0, X_{2}\right)=\varphi^{(1,2)}\left(R, X_{2}\right)
$$

Let $(R, \Omega) \in \mathcal{R}^{N} \times\left[0, X_{1}+X_{2}\right] \backslash\left\{X_{2}\right\}$ be such that $\Omega \leq p\left(R_{1}\right)+p\left(R_{2}\right)$. We have to show the following:

(i) $\varphi(R, \Omega)=(0, \Omega)=\varphi^{(1,2)}(R, \Omega)$ or

(ii) $\varphi(R, \Omega)=\left(\Omega-p\left(R_{2}\right), p\left(R_{2}\right)\right)=\varphi^{(1,2)}(R, \Omega)$.

By Claim 2, $\varphi(R, \Omega)$ is same-sided. If $0<\varphi_{1}(R, \Omega) \leq p\left(R_{1}\right)$ and $0 \leq \varphi_{2}(R, \Omega)<p\left(R_{2}\right)$, then by (7.9) we may suppose that $X_{2} P_{2} \varphi_{2}(R, \Omega)$. Let $\bar{R}_{2} \in \mathcal{R}^{2}$ be such that $p\left(\bar{R}_{2}\right)=X_{2}$, and let $\bar{R} \equiv\left(R_{1}, \bar{R}_{2}\right)$. By strategy-proofness, $\varphi(\bar{R})=\varphi(R)$. Note that $X_{2} \leq p\left(\bar{R}_{1}\right)+p\left(\vec{R}_{2}\right)$. By Claim $3, \varphi\left(\bar{R}, X_{2}\right)=\left(0, X_{2}\right)$. Thus,

$$
\varphi_{1}(\bar{R}, \Omega) \bar{P}_{1} 0 \text { and } X_{2} \tilde{P}_{2} \varphi_{2}(\tilde{R}, \Omega) \text {. }
$$

Since $(\bar{R}, \Omega)$ and $\left(\bar{R}, X_{2}\right)$ are one-sided changes, the previous relations contradict weak one-sided resource-monotonicity. Thus, for $\varphi(R, \Omega)$, (i) or (ii) holds. Similar arguments yield the conclusion when $\Omega \geq p\left(R_{1}\right)+p\left(R_{2}\right)$.

\subsubsection{Proof of Theorem 7.2}

By Theorem 7.1 and Remark 7.1 it suffices to show that the axioms in Theorem 7.2 imply Pareto-optimality. 
Lemma 7.1 Let the level of consumption be bounded by $X \in \mathbb{R}_{++}^{\mathcal{V}}$. If a rule satisfies weak one-sided resource-monotonicity, strategy-proofness, and consistency, then it sutisfies Pareto-optimality.

Proof. First, we show the following. If for all $N \subseteq \mathcal{N}$ such that $|N| \geq 3$ and all $(R, \Omega) \in \mathcal{R}^{N} \times\left[0, \sum_{i \in N} X_{i}\right], \varphi(R, \Omega)$ is same-sided, then for all $i, j \in \mathcal{N}$ and all $(R, \Omega) \in$ $\mathcal{R}^{\{i, j\}} \times\left[0, X_{i}+X_{j}\right]$

$$
\varphi(R, \Omega) \text { is same-sided. }
$$

Consider agents 1 and 2 and suppose that (7.13) does not hold. Thus, by Proposition 7.2, $\varphi$ is a converse-priority rule for those two agents. Without loss of generality, we suppose that for all $R \in \mathcal{R}^{\{1,2\}}$,

$$
\varphi\left(R, X_{2}\right)=\left(0, X_{2}\right)
$$

Let $N=\{1,2,3\}$ and $R \in \mathcal{R}^{N}$ be such that $\left.p\left(R_{1}\right)+p\left(R_{2}\right)=X_{2}, p\left(R_{1}\right) \in\right] 0, X_{1}[$, $\left.p\left(R_{2}\right) \in\right] 0, X_{2}\left[\right.$, and $p\left(R_{3}\right)=0$. Since $\varphi$ is same-sided for $N$, it follows that $\varphi\left(R, X_{2}\right)=$ $\left(p\left(R_{1}\right), p\left(R_{2}\right), 0\right)$. By consistency, $\varphi\left(R_{\{1,2\}}, X_{2}\right)=\left(p\left(R_{1}\right), p\left(R_{2}\right)\right)$, which contradicts $(7.14)$. Thus, (7.13) holds.

We complete the proof by showing that $\varphi$ is same-sided whenever $|N| \geq 3$. Let $N \subseteq \mathcal{N}$ be such that $\{1,2,3\} \subseteq N$, and $(R, \Omega) \in \mathcal{R}^{N} \times\left[0, \sum_{i \in N} X_{i}\right]$. Suppose that $\varphi(R, \Omega)$ is not same-sided. Without loss of generality, we suppose that

$$
\varphi_{1}(R, \Omega)<p\left(R_{1}\right) \text { and } p\left(R_{2}\right)<\varphi_{2}(R, \Omega)
$$

By consistency, $\varphi\left(R_{\{1,2\}}, \varphi_{1}(R, \Omega)+\varphi_{2}(R, \Omega)\right)=\left(\varphi_{1}(R, \Omega), \varphi_{2}(R, \Omega)\right)$. Thus, by $(7.15), \varphi$ violates Pareto-optimality for $\{1,2\}$. Hence, by Proposition $7.2, \varphi$ is a converse-priority rule with respect to $(1,2)$ for $\{1,2\}$, and by $(7.15)$,

$$
\varphi\left(R_{\{1,2\}}, \varphi_{1}(R, \Omega)+\varphi_{2}(R, \Omega)\right)=\left(0, X_{2}\right) .
$$

Claim 1: For all $i \in N, \varphi_{i}(R, \Omega) \in\left\{0, p\left(R_{i}\right), X_{i}\right\}$.

Proof of Claim 1: Suppose that for some $j \in N, \varphi_{j}(R, \Omega) \notin\left\{0, p\left(R_{j}\right), X_{j}\right\}$. By (7.15) and (7.16), $j \in N \backslash\{1,2\}$. If $\left.\varphi_{j}(R, \Omega) \in\right] 0, p\left(R_{j}\right)[$, then by consistency and (7.16),

$$
\varphi\left(R_{\{2, j\}}, \varphi_{2}(R, \Omega)+\varphi_{j}(R, \Omega)\right)=\left(X_{2}, \varphi_{j}(R, \Omega)\right) .
$$

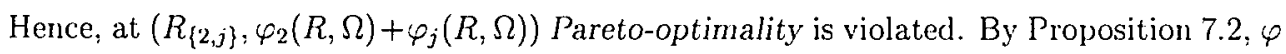
is a converse-priority rule with respect to $(j, 2)$. Therefore, $\varphi\left(R_{\{2, j\}}, \varphi_{2}(R, \Omega)+\varphi_{j}(R, \Omega)\right)=$ $\left(X_{2}, 0\right)$ which contradicts (7.17). 
If $\left.\varphi_{j}(R, \Omega) \in\right] p\left(R_{j}\right), X_{j}\left[\right.$, then by considering $\left(R_{\{1, j\}}, \varphi_{1}(R, \Omega)+\varphi_{j}(R, \Omega)\right)$ we deduce similarly a contradiction.

By consistency, we may suppose that $N=\{1,2,3\}$. By strategy-proofness and consistency, without loss of generality we suppose that $p\left(R_{1}\right)+p\left(R_{2}\right)=X_{2}$. We consider three cases.

Case 1: $\varphi_{3}(R, \Omega)=0$.

Thus, $\Omega=X_{2}$ and $\varphi(R, \Omega)=\left(0, X_{2}, 0\right)$. Let $R_{3}^{\prime} \in \mathcal{R}^{3}$ be such that $p\left(R_{3}^{\prime}\right) \equiv 0$, and let $R^{\prime} \equiv\left(R_{1}, R_{2}, R_{3}^{\prime}\right)$. By strategy-proofness and consistency, $\varphi\left(R^{\prime}, \Omega\right)=\varphi(R, \Omega)$. Let $R_{1}^{\prime \prime} \in \mathcal{R}^{1}$ be such that $p\left(R_{1}^{\prime \prime}\right) \equiv p\left(R_{1}\right)$ and $X_{1} P_{1}^{\prime \prime} 0$, and let $R^{\prime \prime} \equiv\left(R_{1}^{\prime \prime}, R_{2}^{\prime}, R_{3}^{\prime}\right)$.

If $\varphi_{1}\left(R^{\prime \prime}, X_{2}\right)>p\left(R_{1}^{\prime \prime}\right)$, then $\varphi_{2}\left(R^{\prime \prime}, X_{2}\right)<X_{2}$. Let $\bar{\Omega} \equiv \varphi_{1}\left(R^{\prime \prime}, X_{2}\right)+\varphi_{2}\left(R^{\prime \prime}, X_{2}\right)$. By consistency,

$$
\varphi_{1}\left(\left(R_{1}^{\prime \prime}, R_{2}^{\prime \prime}\right), \bar{\Omega}\right)>p\left(R_{1}^{\prime \prime}\right) \text { and } \varphi_{2}\left(\left(R_{1}^{\prime \prime}, R_{2}^{\prime \prime}\right), \bar{\Omega}\right)<X_{2}
$$

By (7.16), $\varphi$ is a converse-priority rule with respect to $(1,2)$ for $\{1,2\}$. Thus, $\bar{\Omega} \neq X_{2}$. Hence, if $\varphi_{1}^{(1,2)}\left(\left(R_{1}^{\prime \prime}, R_{2}^{\prime \prime}\right), \bar{\Omega}\right)>p\left(R_{1}^{\prime \prime}\right)$, then $\varphi_{2}^{(1,2)}\left(\left(R_{1}^{\prime \prime}, R_{2}^{\prime \prime}\right), \bar{\Omega}\right)=X_{2}$. The previous fact contradicts $(7.18)$.

If $\varphi_{1}\left(R^{\prime \prime}, X_{2}\right) \leq p\left(R_{1}^{\prime \prime}\right)$, then by strategy-proofness, $\varphi_{1}\left(R^{\prime \prime}, X_{2}\right)=0$. By consistency, $\varphi\left(R^{\prime \prime}, X_{2}\right)=\left(0, X_{2}, 0\right)$. Since $\sum_{i \in N} p\left(R_{i}^{\prime \prime}\right) \leq X_{2}$, it follows that $\left(R^{\prime \prime}, X_{2}\right)$ and $\left(R^{\prime \prime}, \sum_{i \in N} X_{i}\right)$ are one-sided changes. Because $\varphi\left(R^{\prime \prime}, \sum_{i \in N} X_{i}\right)=\left(X_{1}, X_{2}, X_{3}\right)$, by construction it follows that $X_{1} P_{1}^{\prime \prime} \varphi_{1}\left(R^{\prime \prime}, X_{2}\right)$ and $\varphi_{3}\left(R^{\prime \prime}, X_{2}\right) P_{3}^{\prime \prime} X_{3}$, which contradicts weak one-sided resource-monotonicity. Hence, Case 1 camnot occur.

Case 2: $\varphi_{3}(R, \Omega)=p\left(R_{3}\right) \notin\left\{0, X_{3}\right\}$.

Thus, $\Omega=X_{2}+p\left(R_{3}\right)$ and $\varphi(R, \Omega)=\left(0, X_{2}, p\left(R_{3}\right)\right)$. Let $R_{1}^{\prime} \in \mathcal{R}^{1}$ be such that $p\left(R_{1}^{\prime}\right) \equiv p\left(R_{1}\right)$ and $X_{1} P_{1}^{\prime} 0$, and let $R^{\prime} \equiv\left(R_{1}^{\prime}, R_{2}, R_{3}\right)$.

Suppose that

$$
\varphi_{1}\left(R^{\prime}, \Omega\right)>p\left(R_{1}^{\prime}\right)
$$

By (7.16), $\varphi$ is a converse-priority rule with respect to $(1,2)$ for $\{1,2\}$. Thus, $(7.19)$ and consistency imply $\varphi_{2}\left(R^{\prime}, \Omega\right)=X_{2}$. Hence, $\varphi_{3}\left(R^{\prime}, \Omega\right)<p\left(R_{3}\right)$. By $(7.19)$, consistency, and Proposition $7.2, \varphi$ is a converse-priority rule with respect to $(3,1)$ for $\{1,3\}$. In particular, $\varphi_{1}^{(3,1)}\left(\left(R_{1}^{\prime}, R_{3}\right), p\left(R_{3}\right)\right)>0$. By strategy-proofness, $X_{1} P_{1}^{\prime} 0, \varphi(R, \Omega)=\left(0, X_{2}, p\left(R_{3}\right)\right)$, and consistency,

$$
\varphi_{1}^{(3,1)}\left(\left(R_{1}^{\prime}, R_{3}\right), p\left(R_{3}\right)\right)=\varphi_{1}^{(3,1)}\left(\left(R_{1}, R_{3}\right), p\left(R_{3}\right)\right)=0
$$

which contradicts the previous fact. 
If $\varphi_{1}\left(R^{\prime}, \Omega\right) \leq p\left(R_{1}^{\prime}\right)$, then by strategy-proofness, $\varphi_{1}\left(R^{\prime}, X_{2}\right)=0$. By consistency; $\varphi\left(R^{\prime}, \Omega\right)=\left(0, X_{2}, p\left(R_{3}\right)\right)$. Since $\sum_{i \in N} p\left(R_{i}^{\prime}\right)=\Omega$, the economies $\left(R^{\prime}, \Omega\right)$ and $\left(R^{\prime}, \sum_{i \in N} X_{i}\right)$ are one-sided changes. Because $\varphi\left(R^{\prime}, \sum_{i \in N} X_{i}\right)=\left(X_{1}, X_{2}, X_{3}\right)$, by construction it follows that $X_{1} P_{1}^{\prime} \varphi_{1}\left(R^{\prime}, \Omega\right)$ and $\varphi_{3}\left(R^{\prime}, \Omega\right) P_{3}^{\prime} X_{3}$, which contradicts weak one-sided resource-monotonicity. Hence, Case 2 cannot occur.

Case 3: $\varphi_{3}(R, \Omega)=X_{3}$.

Thus, $\Omega=X_{2}+X_{3}$ and $\varphi(R, \Omega)=\left(0, X_{2}, X_{3}\right)$. Let $R_{3}^{\prime} \in \mathcal{R}^{3}$ be such that $p\left(R_{3}^{\prime}\right) \equiv X_{3}$, and let $R^{\prime} \equiv\left(R_{1}, R_{2}, R_{3}^{\prime}\right)$. By strategy-proofness and consistency, $\varphi\left(R^{\prime}, \Omega\right)=\varphi(R, \Omega)$. Let $R_{2}^{\prime \prime} \in \mathcal{R}^{2}$ be such that $p\left(R_{2}^{\prime \prime}\right) \equiv p\left(R_{2}\right)$ and $0 P_{2}^{\prime \prime} X_{2}$, and let $R^{\prime \prime} \equiv\left(R_{1}^{\prime}, R_{2}^{\prime \prime}, R_{3}^{\prime}\right)$.

If $\varphi_{2}\left(R^{\prime \prime}, \Omega\right)<p\left(R_{2}^{\prime \prime}\right)$, then $\varphi_{3}\left(R^{\prime}, \Omega\right)=X_{3}$ implies $\varphi_{1}\left(R^{\prime \prime}, \Omega\right)>0$. Let $\bar{\Omega} \equiv \varphi_{1}\left(R^{\prime \prime}, \Omega\right)+$ $\varphi_{2}\left(R^{\prime \prime}, \Omega\right)$. By consistency,

$$
\varphi_{1}\left(\left(R_{1}^{\prime \prime}, R_{2}^{\prime \prime}\right), \bar{\Omega}\right)>0 \text { and } \varphi_{2}\left(\left(R_{1}^{\prime \prime}, R_{2}^{\prime \prime}\right), \vec{\Omega}\right)<p\left(R_{2}^{\prime \prime}\right)
$$

By (7.16), $\varphi$ is a converse-priority rule with respect to $(1,2)$ for $\{1,2\}$. Thus, $\bar{\Omega} \neq$ $p\left(R_{1}^{\prime \prime}\right)+p\left(R_{2}^{\prime \prime}\right)$. Hence, if $\varphi_{1}^{(1,2)}\left(\left(R_{1}^{\prime \prime}, R_{2}^{\prime \prime}\right), \bar{\Omega}\right)>0$, then $\varphi_{2}^{(1,2)}\left(\left(R_{1}^{\prime \prime}, R_{2}^{\prime \prime}\right), \bar{\Omega}\right) \geq p\left(R_{2}^{\prime \prime}\right)$, which contradicts $(7.20)$.

If $\varphi_{2}\left(R^{\prime \prime}, \Omega\right) \geq p\left(R_{2}^{\prime \prime}\right)$, then by strategy-proofness, $\varphi_{2}\left(R^{\prime \prime}, \Omega\right)=X_{2}$. By consistency, $\varphi\left(R^{\prime \prime}, \Omega\right)=\left(0, X_{2}, X_{3}\right)$. Since $\sum_{i \in N} p\left(R_{i}^{\prime \prime}\right) \geq \Omega,\left(R^{\prime \prime}, \Omega\right)$ and $\left(R^{\prime \prime}, 0\right)$ are one-sided changes. Because $\varphi\left(R^{\prime \prime}, 0\right)=(0,0,0)$, by construction we have $0 P_{2}^{\prime \prime} \varphi_{2}\left(R^{\prime \prime}, \Omega\right)$ and $\varphi_{3}\left(R^{\prime \prime}, \Omega\right) P_{3}^{\prime \prime} 0$, which contradicts weak one-sided resource-monotonicity. Hence, Case 3 cannot occur. 


\section{Chapter 8}

\section{Strategy-Proof Allocation when Preferences are Single-Plateaued}

\subsection{Introduction}

This chapter, which is based on Ehlers $(2000 \mathrm{~g}, \mathrm{~h})$, considers the problem of allocating some amount of a perfectly divisible good among a finite set of agents. A rule associates an allocation with each profile of preferences. A certain rule has been defined for economies with single-peaked preferences, the uniform rule, and in a number of studies this rule has been shown to play the essential role. ${ }^{1}$ Sprumont (1991) was the first to provide an axiomatic characterization of it. He showed that it is the only rule satisfying strategy-proofness (telling the truth is a weakly dominant strategy), Pareto-optimality (only efficient allocations are chosen) and no-envy (no agent wants to exchange his share with the share of another agent). Ching (1994) showed that in this characterization no-envy can be weakened to equal treatment of equals (when two agents have the same preferences, then they should be indifferent between their shares according to their common preference).

Moulin (1984) extends the domain of single-peaked preferences as follows. Instead of having a unique most preferred consumption, the peak, an agent might have an interval of most preferred consumptions. A preference is single-plateaued if there is an interval such that preference is strictly increasing left of the interval, each amount belonging to the interval is a most preferred consumption, and preference is strictly decreasing right of the interval. It turns out, as shown by Ching and Serizawa (1998), that the domain of single-plateaued preferences is the unique maximal domain including the domain of continuous single-peaked preferences on which Sprumont's requirements are compatible.

\footnotetext{
${ }^{1}$ In most of these papers the uniform rule is the central concept. Herrero and Villar (2000) derive a characterization of the "equal distance rule".
} 
In Chapter 8 each agent is endowed with the domain of single-plateaued preferences. In classical exchange economies, quasi-convex preferences induce single-plateaued preferences on the boundary of a budget set. In applications, an agent might not consider a unique allotment to be his most preferred consumption due to little information. Then his preference relation is single-plateaued.

A solution associates a non-empty set of allocations with each profile of single-plateaued preferences. In general, a solution may recommend arbitrary sets of allocations. Then we face the problem of selection because the solution may recommend two allocations over which agents disagree in terms of welfare. If a solution only recommends sets such that for any two allocations belonging to this set, each agent is indifferent between them, then the previous problem does not arise. Then we can pick any recommendation. Such a solution is called essentially single-valued. A solution satisfies Pareto-indifference when for any two allocations such that all agents are indifferent between them, the solution recommends either both or none of them. Ching (1992) defines the Pareto-indifferent extension of the uniform rule, and characterizes it by Sprumont's requirements, essentially singlevaluedness, and Pareto-indifference. Using the same arguments as in Ching (1994), it can be shown that if no-envy is replaced by equal treatment of equals, then the same uniqueness result holds. However, Ching (1992), Footnote 5, raised the question whether his characterization is tight. We show that Pareto-optimality and essentially single-valuedness are superfuous. The uniform rule is the only rule satisfying Pareto-indifference, no-envy, and strategy-proofness. The proof involves arguments different from former characterizations of the uniform rule. However, it is not yet clear whether the replacement of no-envy by equal treatment of equals would also yield uniqueness.

In the second part of Chapter 8 we drop fairness. Except for two papers the literature focuses on fair allocation of the amount when preferences are single-peaked. Moulin (1999) investigates the class of "fixed-path rationing methods." Barberà, Jackson, and Neme (1997) characterize the class of "sequential-allotment rules" by Pareto-optimality, strategy-proofness, and a certain property called replacement-monotonicity. Their class of rules will be important in our study.

We define strategy-proofness in the second part of Chapter 8 as follows. A solution recommends sets of allocations and we have to extend single-plateaued preferences from allocations to sets of allocations. We say that an agent weakly prefers a set of allocations to another if and only if he weakly prefers each allocation belonging to the former set to each allocation belonging to the latter. We define strict preference in the same manner. Our extension of preferences is unambiguous. A solution is strategy-proof if for all profiles, there does not exist an agent such that he strictly gains by deviation. Instead of 
strategy-proofness we require a weak form of coalitional strategy-proofness. A solution is coalitional strategy-proof if it is strategy-proof and for all profiles there does not exist a pair of two agents such that by joint deviation both agents weakly gain and either (i) one of them strictly gains or (ii) they both strictly prefer some new recommendation to any allocation selected by the solution for the former profile. Part (i) is the usual requirement for any coalitions of size 2. Part (ii) means that both agents can bring about by joint deviation a recommendation which they strictly prefer to any former recommendation. Of particular interest, we do not require that the sets of recommendations made by the solution are comparable in the respect of our extension of preferences.

We call the Pareto-indifferent extensions of the class of rules introduced by Barberà, Jackson, and Nene (1997) sequential-allotment solutions. We show that the class of sequential-allotment solutions is characterized by essentially single-valuedness, Paretoindifference, and coalitional strategy-proofness. We demonstrate by means of examples that our result is robust. If we drop one of the requirements, then other solutions satisfy the remaining requirements. In particular, we include dropping (i) or (ii) of coalitional strategy-proofness. On the domain of single-peaked preferences, our result does not hold. On this donain other solutions may satisfy our properties.

We contrast our result with Barberà, Jackson, and Neme (1997) and Moulin (1999). First of all, on the domain of single-peaked preferences Pareto-optimality implies Paretoindifference. Obviously, the converse is not true. Moreover, they consider only solutions that are single-valued. Both papers impose Pareto-optimality, strategy-proofness, and certain other strong requirements, whereas we do not. As a tradeoff, strategy-proofness is a stronger requirement on the domain of single-plateaued preferences. We impose a weak form of coalitional strategy-proofness in which we do not require comparability of the sets of recommendations made by a solution. Moreover, it is important to understand the allocation problem on the domain of single-plateaued preferences as shown by Ching and Serizawa (1998).

Chapter 8 is divided in four sections. Section 8.2 introduces the model and the basic properties. Section 8.3 presents several characterizations of the uniform rule. Section 8.4 contains the definition and the characterization of sequential allotment solutions.

\subsection{The Model and Basic Properties}

A collective endowment of a perfectly divisible commodity has to be allocated among a finite set of agents. Let $N \equiv\{1, \ldots, n\}$ denote the set of agents and $\Omega$ the collective endowment. A (feasible) allocation is a vector $z \in \mathbb{R}_{+}^{N}$ such that $\sum_{i \in N} z_{i}=\Omega$. We do not allow 
free disposal. Let $Z$ denote the set of all allocations. Each agent $i \in N$ is equipped with a continuous preference relation $R_{i}$ over $[0, \Omega]$. Let $P_{i}$ denote the strict relation associated with $R_{i}$, and $I_{i}$ the indifference relation. The preference relation $R_{i}$ is single-plateaued if there is an interval $\left[\underline{p}\left(R_{i}\right), \bar{p}\left(R_{i}\right)\right] \subseteq[0, \Omega]$ such that for all $x_{i}, y_{i} \in[0, \Omega]$, if $y_{i}<x_{i} \leq \underline{p}\left(R_{i}\right)$ or $\bar{p}\left(R_{i}\right) \leq x_{i}<y_{i}$, then $x_{i} P_{i} y_{i}$, and if $x_{i}, y_{i} \in\left[\underline{p}\left(R_{i}\right), \bar{p}\left(R_{i}\right)\right]$, then $x_{i} I_{i} y_{i}$. A preference relation $R_{i}$ is single-peaked if it is single-plateaued and $\underline{p}\left(R_{i}\right)=\bar{p}\left(R_{i}\right)$. Let $\mathcal{R}$ clenote the set of all single-plateaued preferences and $\mathcal{R}_{s p}$ the set of all single-peaked preferences. Given $R_{i} \in \mathcal{R}$ and $z_{i} \in[0, \Omega]$, we denote by $r_{i}\left(z_{i}\right) \in[0, \Omega]$ the number such that the following holds: (i) if $z_{i} \geq \bar{p}\left(R_{i}\right)$, then $r_{i}\left(z_{i}\right) \equiv \inf \left\{y_{i} \in\left[0, \underline{p}\left(R_{i}\right)\right] \mid y_{i} R_{i} z_{i}\right\}$; (ii) if $\left.z_{i} \in\right] \underline{p}\left(R_{i}\right), \bar{p}\left(R_{i}\right)[$; then $r_{i}\left(z_{i}\right)=z_{i}$; and (iii) if $z_{i} \leq \underline{p}\left(R_{i}\right)$, then $r_{i}\left(x_{i}\right) \equiv \sup \left\{y_{i} \in\left[\bar{p}\left(R_{i}\right), \Omega\right] \mid y_{i} R_{i} z_{i}\right\}$. A preference profile $\left(R_{1}, \ldots, R_{n}\right) \in \mathcal{R}^{N}$ is denoted by $R$. Given $M \subseteq N$, let $R_{M}$ denote the restriction of the profile $R$ to the coalition $M$, i.e., $R_{M} \equiv\left(R_{i}\right)_{i \in M}$. Since the endowment is fixed, an economy is simply denoted by $R \in \mathcal{R}^{N}$. An economy $R$ is in excess demand when $\Omega \leq \sum_{i \in N} \underline{p}\left(R_{i}\right), R$ is balanced when $\sum_{i \in N} \underline{p}\left(R_{i}\right) \leq \Omega \leq \sum_{i \in N} \bar{p}\left(R_{i}\right)$, and $R$ is in excess supply when $\Omega \geq \sum_{i \in N} \bar{p}\left(R_{i}\right)$.

A solution recommends for each economy a non-empty set of allocations. Formally, a solution $\varphi$ is a non-empty correspondence $\varphi: \mathcal{R}^{N} \rightarrow Z$. We are interested in the following axioms and solutions. The first one says that all agents are indifferent between any two recommendations.

Essentially Single-Valuedness: For all $R \in \mathcal{R}^{N}$ and all $z, \bar{z} \in \varphi(R)$ : for all $i \in N$, $z_{i} I_{i} \bar{z}_{i}$

If a solution satisfies essentially single-valuedness, then in terms of welfare it is singlevalued. In classical exchange economies two interesting solutions, the Walrasian solution from equal division and the egalitarian-equivalence solution, are essentially single-valued. Obviously, if a solution makes for each profile only one recommendation, then it is essentially single-valued.

The second axiom says that if all agents are indifferent between a recommendation and some allocation, then the solution also selects the latter allocation.

Pareto-Indifference: For all $R \in \mathcal{R}^{N}$, all $z \in \varphi(R)$, and all $\bar{z} \in Z$ : if for all $i \in N$, $z_{i} I_{i} \bar{z}_{i}$, then $\bar{z} \in \varphi(R)$.

We also consider the following notions of faimess. The first one says that if two agents have the same preference relation, then for each allocation chosen by the solution the al- 
lotments of this two agents are indifferent relative to the common preference relation. The second one says that the names of the agents do not matter. Given $R \in \mathcal{R}^{N}, z \in Z$, and a permutation $\sigma: N \rightarrow N$, let $\sigma(R)$ denote the profile $\left(R_{\sigma(i)}\right)_{i \in N}$, and let $\sigma(z)$ denote the allocation $\left(z_{\sigma(i)}\right)_{i \in N}$.

Equal Treatment of Equals: For all $R \in \mathcal{R}^{N}$, all $z \in \varphi(R)$, and all $i, j \in N$ : if $R_{i}=R_{j}$, then $z_{i} I_{i} z_{j}$.

Anonymity: For all $R \in \mathcal{R}^{N}$ and all permutations $\sigma: N \rightarrow N, \varphi(\sigma(R))=\{\sigma(z) \mid z \in$ $\varphi(R)\}$.

A presolution is a correspondence which may recommend the empty set. The following solution only recommends the allocations such that each agent's allotment is a most preferred consumption.

Unanimity Presolution, $\mathcal{T}$ : For all $R \in \mathcal{R}^{N}: z \in T(R) \Leftrightarrow$ for all $i \in N, \underline{p}\left(R_{i}\right) \leq z_{i} \leq$ $\bar{p}\left(R_{i}\right)$.

The Pareto solution chooses for each economy the set of efficient allocations.

Pareto Solution, $\mathcal{P O}:$ For all $R \in \mathcal{R}^{N}: z \in \mathcal{P O}(R) \Leftrightarrow$ for all $z^{\prime} \in Z$ and all $i \in N$, $z_{i}^{\prime} P_{i} z_{i}$ implies for some $j \in N, z_{j} P_{j} z_{j}^{\prime}$.

It is easy to see that for each economy $R \in \mathcal{R}^{N}$ and each allocation chosen by the Pareto solution, (i) if $R$ is in excess demand, then the consumption of each agent is less than or equal to $\underline{p}\left(R_{i}\right)$; (ii) if $R$ is balanced, then the consumption of each agent belongs to $\left[\underline{p}\left(R_{i}\right), \bar{p}\left(R_{i}\right)\right]$; and (iii) if $R$ is in excess supply, then the consumption of each agent is greater than or equal to $\bar{p}\left(R_{i}\right)$.

The no-envy solution chooses the allocations at which every agent weakly prefers his consumption to any other agent's consumption.

No-Envy Solution, $\mathcal{F}$ : For all $R \in \mathcal{R}^{N}: z \in \mathcal{F}(R) \Leftrightarrow$ for all $i, j \in N, z_{i} R_{i} z_{j}$.

A solution $\varphi$ satisfies (a) unanimity if for all $R \in \mathcal{R}^{N}, T(R) \neq \emptyset$ implies $\varphi(R)=T(R)$, (b) $\varphi$ satisfies Pareto-optimality if for all $R \in \mathcal{R}^{N}, \varphi(R) \subseteq \mathcal{P O}(R)$, and (c) $\varphi$ satisfies no-envy if for all $R \in \mathcal{R}^{N}, \varphi(R) \subseteq \mathcal{F}(R)$. 


\subsection{The Uniform Rule}

\subsubsection{Single-Peaked Preferences}

The allocation problem we consider has been extensively studied on the domain of singlepeaked preferences. The following solution plays the important role. It is a single-valued solution. Given a solution $\varphi$, whenever $\varphi(R)$ is a singleton, we also write $\varphi_{i}(R)$ to indicate the amount of agent $i$ at the unique allocation chosen by $\varphi$ at $R$.

Uniform Rule, $U:$ For all $R \in \mathcal{R}_{\mathrm{sp} \text { ? }}^{N}$

(i) when $\Omega \leq \sum_{j \in N} \underline{p}\left(R_{j}\right)$, there exists $\lambda \in \mathbb{R}_{+}$such that for all $i \in N, U_{i}(R)=$ $\min \left\{\underline{p}\left(R_{i}\right), \lambda\right\} ;$ and

(ii) when $\Omega \geq \sum_{j \in N} \bar{p}\left(R_{j}\right)$, there exists $\lambda \in \mathbb{R}_{+}$such that for all $i \in N, U_{i}(R)=$ $\max \left\{\bar{p}\left(R_{i}\right), \lambda\right\}$.

Again, note that for any single-peaked preference relation $R_{i} \in \mathcal{R}_{s p}$ we have $\underline{p}\left(R_{i}\right)=$ $\bar{p}\left(R_{i}\right)$. This implies that if $R \in \mathcal{R}_{s p}^{N}$ is balanced, then $\mathcal{P O}(R)=\left\{\left(\underline{p}\left(R_{i}\right)\right)_{i \in N}\right\}$.

We give a brief review of some results on the domain of single-peaked preferences. On this domain the literature studies single-valued solutions, meaning that for each economy the solution makes exactly one recommendation.

Benassy (1982) introduces the uniform rule on the domain of single-peaked preferences and shows that it is strategy-proof. Strategy-proofness means that no agent can ever gain by misrepresenting his true preference relation. The next definition only applies to singlevalued solutions.

Strategy-Proofness: For all $R \in \mathcal{R}^{N}$, all $i \in N$, and all $R_{i}^{\prime} \in \mathcal{R}: \varphi_{i}(R) R_{i} \varphi_{i}\left(R_{i}^{\prime}, R_{N \backslash\{i\}}\right)$.

It is easy to check that the uniform rule also satisfies Pareto-optimality and no-envy. It even turns out that the combination of this two properties and strategy-proofness characterizes the uniform rule.

Theorem 8.1 (Sprumont, 1991) On the domain of single-peaked preferences, the uniform rule is the only single-valued solution satisfying Pareto-optimality, no-envy, and strategy-proofness.

When we replace no-envy by anonymity, we also get uniqueness. 
Theorem 8.2 (Sprumont, 1991) On the domain of single-peaked preferences, the uniform rule is the only single-valued solution sutisfying Pareto-optimality, anonymity, and strategy-proofness.

Equal treatment of equals is a weakening of no-envy. For single-valued solutions, anonymity also implies equal treatment of equals. Ching (1994) derives a unification of Theorems 8.1 and 8.2 and shows that equal treatment of equals is sufficient for the above characterizations of the uniform rule.

Theorem 8.3 (Ching, 1994) On the domain of single-peaked preferences, the uniform rule is the only single-valued solution satisfying Pareto-optimality, equal treatment of equals, and strategy-proofness.

\subsubsection{Single-Plateaued Preferences}

It turns out that the domain of single-plateaued preferences is important for Theorem 8.3. On the domain of single-peaked preferences the properties listed in Theorem 8.3 are compatible. Ching and Serizawa (1998) ask the following question: can we expand the domain of single-peaked preferences such that Pareto-optimality, equal treatment of equals, and strategy-proofness remain compatible? In particular what are the maximal expansions that provide a positive answer to the above problem?

Let $\mathcal{C}$ denote the preference relations over $[0, \Omega]$ that are reflexive, complete, transitive, and continuous.

A domain $\mathcal{D}^{N} \subseteq \mathcal{C}^{N}$ is maximal for a list of properties if (i) there is a single-valued solution satisfying these properties on $\mathcal{D}^{N}$; and (ii) for all $\tilde{\mathcal{D}}^{N}$ such that $\mathcal{D}^{N} \subseteq \tilde{\mathcal{D}}^{N} \subseteq \mathcal{C}^{N}$ and $\mathcal{D}^{N} \neq \tilde{\mathcal{D}}^{N}$ there is no single-valued solution satisfying them on $\tilde{\mathcal{D}}^{N}$.

Theorem 8.4 (Ching and Serizawa, 1998) The domain of single-plateaued preferences is the unique maximal domain $\mathcal{D}^{N}$ such that

(i) $\mathcal{R}_{s p}^{N} \subseteq \mathcal{D}^{N} \subseteq \mathcal{C}^{N}$ and

(ii) on $\mathcal{D}^{N}$, Pareto-optimality, equal treatment of equals, and strategy-proofness are compatible.

For part (ii), Ching and Serizawa (1998) provide as an example a single-valued solution that coincides with the uniform rule for single-peaked preferences for all economies in excess demand and excess supply, and for balanced economies the solution chooses some allocation at which for each agent his allotment is a most preferred consumption. 
Ching (1992) extends the uniform rule to the single-plateaued domain as follows. His extension may be not single-valued for balanced economies. For convenience, we call this extension simply uniform rule.

Uniform Rule, $U$ : For all $R \in \mathcal{R}^{N}$,

(i) when $\Omega \leq \sum_{j \in N} \underline{p}\left(R_{j}\right)$, there exists $\lambda$ such that $U(R)=\left\{z \in Z \mid\right.$ for all $i \in N$, $z_{i}=$ $\left.\min \left\{\underline{p}\left(R_{i}\right), \lambda\right\}\right\}$

(ii) when $\sum_{j \in N} \underline{p}\left(R_{j}\right) \leq \Omega \leq \sum_{j \in N} \bar{p}\left(R_{j}\right), U(R)=\left\{z \in Z \mid\right.$ for all $i \in N, \underline{p}\left(R_{i}\right) \leq z_{i} \leq$ $\left.\bar{p}\left(R_{i}\right)\right\} ;$ and

(iii) when $\Omega \geq \sum_{j \in N} \bar{p}\left(R_{j}\right)$, there exists $\lambda$ such that $U(R)=\left\{z \in Z \mid\right.$ for all $i \in N, z_{i}=$ $\left.\max \left\{\bar{p}\left(R_{i}\right), \lambda\right\}\right\}$.

Note that in cases (i) and (iii) $U(R)$ is a singleton. Defining strategy-proofness for solutions is not straightforward. A solution possibly recommends a set of allocations and preferences only compare single allocations. Ching (1992) defines strategy-proofness as follows: for every profile, if an agent deviates, then he weakly prefers each of the former recommendations to every new recommendation. This definition of strategy-proofness requires comparability in the above respect of all recommendations chosen by the solution.

Strong Strategy-Proofness: For all $R \in \mathcal{R}^{N}$, all $i \in N$, and all $R_{i}^{\prime} \in \mathcal{R}$ : for all $z \in \varphi(R)$ and all $z^{\prime} \in \varphi\left(R_{i}^{\prime}, R_{N \backslash\{i\}}\right), z_{i} R_{i} z_{i}^{\prime}$.

The following implication is straightforward. If a solution is strong strategy-proof, then it must be essentially single-valued. This follows from applying the above definition for an agent's true preference relation.

In characterizing the uniform rule on the single-plateaued domain Ching (1992) adds some properties to Theorem 8.1.

Theorem 8.5 (Ching, 1992) The uniform rule is the only solution satisfying essentially single-valuedness, Pareto-indifference, Pareto-optimality, no-envy, and strong strategyproofness.

Ching (1992) raises the question whether the properties in Theorem 8.5 are independent. We show that this is not the case. Essentially single-valuedness and Paretooptimality can be dropped from the above characterization of the uniform rule. 
Theorem 8.6 The uniform rule is the only solution satisfying Pareto-indifference, noenvy, and strong strategy-proofness.

Note that the axioms of Theorem 8.6 imply Pareto-optimality. The following three examples establish the independence of Theorem 8.6.

Example 8.1 For all $R \in \mathcal{R}^{N}$, let $\varphi^{e}(R) \equiv\left\{\left(\frac{\Omega}{n}, \ldots, \frac{\Omega}{n}\right)\right\}$. The solution $\varphi^{e}$ satisfies noenvy and strong strategy-proofness, but not Pareto-indifference.

Example 8.2 For all $R \in \mathcal{R}^{N}$, if $\Omega<\sum_{i \in N} \underline{p}\left(R_{i}\right)$, then $\bar{\varphi}_{1}(R) \equiv \min \left\{\underline{p}\left(R_{1}\right), \Omega\right\}, \bar{\varphi}_{2}(R) \equiv$ $\min \left\{\underline{p}\left(R_{2}\right), \Omega-\bar{\varphi}_{1}(R)\right\}$, and so on. Otherwise, $\bar{\varphi}(R) \equiv U(R)$. The solution $\bar{\varphi}$ satisfies Pareto-indifference and strong strategy-proofness, but not no-envy.

Example 8.3 For all $R \in \mathcal{R}^{N}$, if $\Omega<\sum_{i \in N} \underline{p}\left(R_{i}\right)$, for some $j \in N, \bar{p}\left(R_{j}\right)=\Omega$, and for all $i \in N \backslash\{j\}, 0 P_{i}\left(\Omega-\underline{p}\left(R_{j}\right)\right)$, then $\tilde{\varphi}_{j}(R) \equiv \Omega$ and for all $i \in N \backslash\{j\}, \tilde{\varphi}_{i}(R) \equiv 0$. Otherwise, $\tilde{\varphi}(R) \equiv U(R)$. The solution $\tilde{\varphi}$ satisfies Pareto-indifference and no-envy, but not strong strategy-proofness.

\subsubsection{Proof of Theorem 8.6}

It is easy to verify that the uniform rule satisfies the properties of Theorem 8.6. We prove the converse statement. Strong strategy-proofness implies essentially single-valuedness. The following lemma turns out to be useful for the proof of Theorem 8.6. We show that the properties of Theorem 8.6 imply unanimity.

Lemma 8.1 Let $\varphi$ be a solution satisfying Pareto-indifference and strong strategy-proofness. For all $R \in \mathcal{R}^{N}$, if $T(R) \neq \emptyset$, then $\varphi(R)=T(R)$.

Proof. Let $R \in \mathcal{R}^{N}$ be such that

$$
\sum_{i \in N} \underline{p}\left(R_{i}\right) \leq \Omega \leq \sum_{i \in N} \vec{p}\left(R_{i}\right) .
$$

Let $\bar{R} \in \mathcal{R}^{N}$ be such that for all $i \in N,\left[\underline{p}\left(\ddot{R}_{i}\right), \bar{p}\left(\bar{R}_{i}\right)\right]=[0, \Omega]$. By non-emptiness and Pareto-indifference, $\varphi(\bar{R})=Z=T(\bar{R})$. We show by induction on $|S|$ that for all $S \subseteq N$,

$$
\varphi\left(R_{S}, \bar{R}_{N \backslash S}\right)=T\left(R_{S}, \bar{R}_{N \backslash S}\right)
$$

Applying (8.2) for $S=N$ yields then the desired conclusion.

If $S=\emptyset$, then $\left(R_{\emptyset}, \bar{R}_{N \backslash \emptyset}\right)=\bar{R}$ and $\varphi(\bar{R})=Z=T(\bar{R})$, which is the induction basis.

Induction basis: $\varphi\left(R_{\emptyset}, \ddot{R}_{N \backslash \emptyset}\right)=T\left(R_{\emptyset}, \bar{R}_{N \backslash \emptyset}\right)$. 
By induction, we assume that (8.2) holds for all $S \subseteq N$ such that $|S| \leq t$. For all $t \in N$, let $R^{t} \equiv\left(R_{\{1, \ldots, l\}}, \bar{R}_{N \backslash\{1, \ldots, t\}}\right)$.

Induction step: (8.2) holds for all $S \subseteq N$ such that $|S|=t+1$.

Proof: Without loss of generality, we assume that $S=\{1, \ldots, t+1\}$. By induction, $\varphi\left(R^{\imath}\right)=T\left(R^{\imath}\right)$. By construction, $T\left(R^{l+1}\right) \subseteq T\left(R^{\imath}\right)$. Thus, by $T\left(R^{t+1}\right) \subseteq \varphi\left(R^{t}\right)$, strong strategy-proofness implies that for all $z \in \varphi\left(R^{i+1}\right)$,

$$
z_{t+1} \in\left[\underline{p}\left(R_{t+1}\right), \bar{p}\left(R_{t+1}\right)\right]
$$

Suppose that $\varphi\left(R^{l+1}\right) \neq T\left(R^{l+1}\right)$. Hence, by essentially single-valuedness and Paretoindifference, for some $j \in N$ and some $z \in \varphi\left(R^{t+1}\right), z_{j} \notin\left[\underline{p}\left(R_{j}^{l+1}\right), \bar{p}\left(R_{j}^{l+1}\right)\right]$. By (8.3) and construction of $R^{l+1}, j \in\{1, \ldots, t\}$. By induction, we have $\varphi\left(R_{S \backslash\{j\}}, \bar{R}_{N \backslash(S \backslash\{j\})}\right)=$ $T\left(R_{S \backslash\{j\}}, \bar{R}_{N \backslash(S \backslash j\}))}\right)$, which contradicts strong strategy-proofness.

Next, we prove that a rule satisfying the axioms of Theorem 8.6 coincides with the uniform rule for all profiles at which there is excess demand.

Lemma 8.2 Let $\varphi$ be a rule satisfying Pareto-indifference, strong strategy-proofness, and no-envy. Then for all $R \in \mathcal{R}^{N}$ such that $\Omega<\sum_{j \in N} \underline{p}\left(R_{j}\right), \varphi(R)=U(R)$.

Proof. By Lemma 8.1, $\varphi$ satisfies unanimity. Let $R \in \mathcal{R}^{N}$ be such that $\Omega<\sum_{j \in N} \underline{p}\left(R_{j}\right)$ and let $\lambda \in \mathbb{R}_{i}$ be such that for all $i \in N, U_{i}(R)=\min \left\{\underline{p}\left(R_{i}\right), \lambda\right\}$. Let $\bar{R} \in \mathcal{R}^{N}$ be such that for all $i \in N,\left[\underline{p}\left(\bar{R}_{i}\right), \bar{p}\left(\bar{R}_{i}\right)\right]=\left[U_{i}(R), \Omega\right]$. By unanimity,

$$
\varphi(\vec{R})=U(\bar{R})
$$

Let $S_{\lambda}=\left\{i \in N \mid \underline{p}\left(R_{i}\right) \geq \lambda\right\}$ and for all $t \in N$, let $R^{t} \equiv\left(R_{\{1, \ldots, t\}}, \vec{R}_{N \backslash\{1, \ldots, t\}}\right)$. For all $S \subseteq N$, let us consider the profile $\left(R_{S}, \vec{R}_{N \backslash S}\right)$. By induction on $|S|$ we will show that for all $S \subseteq N$,

$$
\varphi\left(R_{S}, \bar{R}_{N \backslash S}\right)=U(\bar{R})
$$

If $|S|=0$, then $S=\emptyset$. Thus, $\left(R_{\emptyset}, \bar{R}_{N \backslash \emptyset}\right)=\bar{R}$ and the induction basis follows from (8.4). Induction basis: $\varphi\left(R_{\emptyset}, \tilde{R}_{N \backslash \emptyset}\right)=U(\bar{R})$.

By induction, we assume that (8.5) holds for all $S \subseteq N$ such that $|S| \leq t$.

Induction step: (8.5) holds for all $S \subseteq N$ such that $|S|=t+1$.

Proof: Without loss of generality, we may assume that $S=\{1, \ldots, t+1\}$. By induction,

$$
\varphi\left(R^{\iota}\right)=U(\bar{R})
$$


We have to prove that $\varphi\left(R^{l+1}\right)=U(\bar{R})$. Let $z \in \varphi\left(R^{l+1}\right)$. We consider two cases.

Case 1: $t+1 \in S_{\lambda}$.

Strong strategy-proofness and (8.6) imply $z_{t+1} \geq \lambda$. By no-envy, for all $j \in\{t+$ $2, \ldots, n\}, z_{j} \geq \underline{p}\left(R_{j}^{l+1}\right)$. Therefore, if $z \neq U(\bar{R})$, then there exists $j \in\{1, \ldots, t\}$ such that

$$
z_{j}<U_{j}(\bar{R})=\min \left\{\underline{p}\left(R_{j}\right), \lambda\right\}
$$

Consider the profile $\left(R_{\{1, \ldots, t+1\} \backslash\{j\}}, \bar{R}_{N \backslash(\{1, \ldots, t+1\} \backslash\{j\})}\right)=\bar{R}^{t+1}$. Note that $\mid\{1, \ldots, t+$ $1\} \backslash\{j\} \mid=t$. Thus, by induction and $(8.5), \varphi_{j}\left(\tilde{R}^{t+1}\right)=U_{j}(\bar{R})=\min \left\{\underline{p}\left(R_{j}\right), \lambda\right\}$. Because of (8.7), the last statement contradicts strong strategy-proofness.

Case 2: $t+1 \notin S_{\lambda}$.

Suppose that $z \neq U(\vec{R})$. Strong strategy-proofness and (8.6) imply $z_{t+1} \geq U_{t+1}(\bar{R})$. Thus, there exists $j \in N \backslash\{t+1\}$ such that $z_{j}<U_{j}(\bar{R})$. We consider two subcases.

Subcuse 2.1: $j \in\{1, \ldots, t\}$.

Similar to Case 1 , we obtain a contradiction to strong strategy-proofness.

Subcase 2.2: $j \notin\{1, \ldots, t+1\}$.

Hence, $R_{j}^{\ell+1}=\tilde{R}_{j}$. First, suppose that for all $k \in S_{\lambda}, R_{k}^{t+1}=\vec{R}_{k}$. Hence, for all $i \in\{1, \ldots, t+1\}, \underline{p}\left(R_{i}^{t+1}\right)=\underline{p}\left(\vec{R}_{i}\right)$, and

$$
\sum_{i \in N} \underline{p}\left(R_{i}^{l+1}\right)=\sum_{i \in N} \underline{p}\left(\bar{R}_{i}\right)=\Omega
$$

Unanimity implies $\varphi\left(R^{t+1}\right)=U(\bar{R})$. Thus, there exists $k \in S_{\lambda}$ such that $R_{k}^{t+1}=R_{k} \neq \bar{R}_{k}$. Since $R_{j}^{l+1}=\bar{R}_{j}$ and $z_{j}<U_{j}(\bar{R}) \leq \lambda$, no-envy implies

$$
z_{k} \leq z_{j}<\lambda \leq \underline{p}\left(R_{k}\right)
$$

Thus, $k \in\{1, \ldots, t\}$ and $z_{k}<U_{k}(\bar{R})$. By Subcase 2.1, the last inequality cannot occur. $。$

We proved (8.5). Since $U(R)=U(\bar{R})$, applying (8.5) for $S=N$ yields $\varphi(R)=U(R)$, the desired conclusion.

By using the same arguments as in Lemma 8.2 , it is easy to prove that a rule satisfying the axioms of Theorem 8.6 coincides with the uniform rule for all profiles at which there is excess supply.

Lemma 8.3 Let $\varphi$ be a rule satisfying Pareto-indifference, strong strategy-proofness, and no-envy. Then for all $R \in \mathcal{R}^{N}$ such that $\sum_{j \in N} \bar{p}\left(R_{j}\right)<\Omega, \varphi(R)=U(R)$.

Now, Lemmas 8.1, 8.2 and 8.3 imply Theorem 8.6. 


\subsection{Sequential Allotment Solutions}

\subsubsection{Pareto-Indifferent Extensions}

Given a solution we may associate with it the minimal extension of it satisfying Paretoindifference. It is the solution that chooses for each profile the recommendations of the original solution and all allocations that are Pareto-indifferent to some of these recommendations.

Pareto-Indifferent Extension, $\psi:$ Given a solution $\varphi$, the Pareto-indifferent extension $\psi \circ \varphi$ is defined as follows: for all $R \in \mathcal{R}^{N}, \psi \circ \varphi(R) \equiv\{z \in Z \mid$ for some $\bar{z} \in$ $\varphi(R)$ and all $\left.i \in N, z_{i} I_{i} \bar{z}_{i}\right\}$.

Ching (1992) introduces the Pareto-indifferent extension of the uniform rule. Thomson (1999c) discusses Pareto-indifferent extensions in classical exchange economies. In the theory of compensation many authors consider Pareto-indifference to be a weak requirement. We will show that in general this is not true. ${ }^{2}$

Next we define the class of sequential-allotment solutions. Those solutions are the Pareto-indifferent extensions of the class of solutions characterized by Barberà, Jackson, and Neme (1997). They use a slightly different name and call them "sequential allotment rules." For a detailed discussion of these solutions we refer to their paper.

A function $f:\left\{x \in[0, \Omega]^{N} \mid \sum_{i \in N} x_{i} \geq \Omega\right\} \rightarrow Z$ is an allotment function for excess demand when the following holds: for all $j \in N$ and all $\bar{x}, \tilde{x} \in\left\{x \in[0, \Omega]^{N} \mid \sum_{i \in N} x_{i} \geq \Omega\right\}$ such that $\bar{x}_{N \backslash\{j\}}=\tilde{x}_{N \backslash\{j\}}$, (1) for all $i \in N, f_{i}(\bar{x}) \leq \bar{x}_{i}$; (2) if $f_{j}(\bar{x})<\bar{x}_{j}$, then $f_{j}(\tilde{x})=$ $\min \left\{\tilde{x}_{j}, f_{j}(\bar{x})\right\} ;$ and $(3)$ if $f_{j}(\tilde{x}) \leq f_{j}(\bar{x})$, then for all $i \in N \backslash\{j\}, f_{i}(\tilde{x}) \geq f_{i}(\bar{x})$. Let $\mathcal{F}$ denote the set of all allotment functions for excess demand.

A function $g:\left\{y \in[0, \Omega]^{N} \mid \sum_{i \in N} y_{i} \leq \Omega\right\} \rightarrow Z$ is an allotment function for excess supply when the following holds: for all $j \in N$ and all $\bar{y}, \tilde{y} \in\left\{y \in[0, \Omega]^{N} \mid \sum_{i \in N} y_{i} \leq \Omega\right\}$ such that $\bar{y}_{N \backslash\{j\}}=\tilde{y}_{N \backslash\{j\}}$, (1) for all $i \in N, g_{i}(\bar{y}) \geq \bar{y}_{i}$; (2) if $g_{j}(\bar{y})>\bar{y}_{j}$, then $g_{j}(\tilde{y})=$ $\max \left\{\tilde{y}_{j}, g_{j}(\bar{y})\right\}$; and $(3)$ if $g_{j}(\tilde{y}) \geq g_{j}(\bar{y})$, then for all $i \in N \backslash\{j\}, g_{i}(\tilde{y}) \leq g_{i}(\bar{y})$. Let $\mathcal{G}$ denote the set of all allotment functions for excess supply.

Sequential-Allotment Solution, $\phi^{(f, g)}:$ Given $(f, g) \in \mathcal{F} \times \mathcal{G}$, the sequential-allotment solution $\phi^{(f, g)}$ is defined as follows: for all $R \in \mathcal{R}^{N}$,

(i) when $\sum_{i \in N} \underline{p}\left(R_{i}\right) \geq \Omega, \phi^{(f, g)}(R) \equiv\left\{f\left(\left(\underline{p}\left(R_{i}\right)\right)_{i \in N}\right)\right\}$;

\footnotetext{
${ }^{2}$ Classical exchange economies also support this statement. Here, the no-envy solution violates Paretoindifference in economies with quasi-convex preferences (Thomson, 2000).
} 
(ii) when $\sum_{i \in N} \underline{p}\left(R_{i}\right) \leq \Omega \leq \sum_{i \in N} \bar{p}\left(R_{i}\right), \phi^{(f, g)}(R) \equiv\left\{z \in Z \mid\right.$ for all $i \in N, \underline{p}\left(R_{i}\right) \leq$ $\left.z_{i} \leq \bar{p}\left(R_{i}\right)\right\} ;$ and

(iii) when $\sum_{i \in N} \bar{p}\left(R_{i}\right) \leq \Omega, \phi^{(f, g)}(R) \equiv\left\{g\left(\left(\bar{p}\left(R_{i}\right)\right)_{i \in N}\right)\right\}$.

Sequential-allotment solutions select only Pareto-optimal allocations. Since they select for all economies in excess demand and excess supply, respectively, a unique recommendation, they satisfy essentially single-valuedness and Pareto-indifference.

\subsubsection{Coalitional Strategy-Proofness}

In general, different sets of allocations are recommended for different preference profiles. In Section 8.4 we extend preferences over allocations to preferences over sets of allocations as follows. An agent weakly prefers a set of allocations to another, if he weakly prefers any allocation in the first set to any in the second set according to his single-plateaued preference relation. Strict preference over sets is defined as follows. An agent strictly prefers a set of allocations to another, if he strictly prefers any allocation in the first set to any in the second set according to his single-plateaued preference. Manipulation is only profitable if at any chosen allocation of the new recommendations an agent strictly gains to any possible choice of the former set of recommendations. We do not require that the sets of recommendations made by the solution are comparable in the above respect. We say that a solution is strategy-proof if for all profiles there does not exist an agent such that he strictly gains by deviation. A solution is coalitional strategy-proof if it is strategy-proof and for all profiles there does not exist a set consisting of two agents such that by joint deviation both agents weakly gain and either one of them strictly gains or they both strictly prefer some new recommendation to any allocation selected by the solution for the former profile.

Coalitional Strategy-Proofness: For all $R \in \mathcal{R}^{N}$ and all $M \subseteq N$ such that $|M| \leq 2$ : there exists no $\vec{R} \in \mathcal{R}$ such that $\bar{R}_{N \backslash M}=R_{N \backslash M}$, for all $i \in M$, all $z \in \varphi(R)$, and all $\bar{z} \in \varphi(\vec{R}), \bar{z}_{i} R_{i} z_{i}$, and either (i) for some $j \in M$, all $z \in \varphi(R)$, and all $\bar{z} \in \varphi(\bar{R}), \bar{z}_{j} P_{j} z_{j}$; or (ii) when $|M|=2$, for all $i \in M$, all $z \in \varphi(R)$, and some $\bar{z} \in \varphi(\bar{R}), \bar{z}_{i} P_{i} z_{i}$.

It is easy to check that sequential allotment solutions are coalitional strategy-proof. ${ }^{3}$

Theorem 8.7 The sequential-allotment solutions are the only solutions satisfying essentially single-valuedness, Pareto-indifference, and coalitional strategy-proofness.

\footnotetext{
${ }^{3}$ Barberà, Jackson, and Neme (1997) show coalitional strategy-proofness on the domain of single-peaked preferences.
} 
Interestingly, our axioms imply Pareto-optimality. The following three examples establish the proof of independence for Theorem 8.7. These examples also show the weakness of the other axioms when we drop one requirement. In particular, our result does not hold on the domain of single-peaked preferences. Then other solutions like Example 8.7 below satisfy our requirements on this domain.

Example 8.4 Let $\tilde{\varphi}$ be the solution such that for all $R \in \mathcal{R}^{N}, \tilde{\varphi}(R) \equiv Z$. The solution $\tilde{\varphi}$ satisfies Pareto-indifference and coalitional strategy-proofness, but not essentially singlevaluedness.

Example 8.5 For all $R \in \mathcal{R}^{N}, \varphi^{e}(R) \equiv\left\{\left(\frac{\Omega}{n}, \ldots, \frac{\Omega}{n}\right)\right\}$. The solution $\varphi^{e}$ satisfies essentially single-valuedness and coalitional strategy-proofness, but not Pareto-indifference.

The last two examples show that we cannot drop (i) or (ii) of coalitional strategyproofness. Part (i) of coalitional strategy-proofness is a weak requirement. Each Paretoindifferent extension of a constant solution satisfies essentially single-valuedness, Paretoindifference, and (i).

Example 8.6 Let $\tilde{z} \in Z$ and $\varphi^{\bar{z}}$ be the constant solution such that for all $R \in \mathcal{R}^{N}$, $\varphi^{\bar{z}}(R) \equiv\{\tilde{z}\}$. The solution $\psi \circ \varphi^{\bar{z}}$ satisfies essentially single-valuedness, Pareto-indifference, and (i) of coalitional strategy-proofness, but not (ii) of coalitional strategy-proofness. $\circ$

The following solution satisfies essentially single-valuedness, Pareto-indifference, and (ii) of coalitional strategy-proofness, but not (i) of coalitional strategy-proofness. Therefore, each part of coalitional strategy-proofness is independent of the other part of coalitional strategy-proofness together with essentially single-valuedness and Pareto-indifference.

Example 8.7 Let $N=\{1,2,3\}$. For all $R \in \mathcal{R}^{N}$,

(i) if $\Omega<\sum_{i \in N} \underline{p}\left(R_{i}\right)$ and $\underline{p}\left(R_{1}\right)$ is rational, then $\bar{\varphi}(R) \equiv\left\{z \in Z \mid z_{1}=\underline{p}\left(R_{1}\right), z_{2}=\right.$ $\left.\min \left\{\underline{p}\left(R_{2}\right), \Omega-z_{1}\right\}\right\}$

(ii) if $\Omega<\sum_{i \in N} \underline{p}\left(R_{i}\right)$ and $\underline{p}\left(R_{1}\right)$ is irrational, then $\bar{\varphi}(R) \equiv\left\{z \in Z \mid z_{1}=\underline{p}\left(R_{1}\right), z_{3}=\right.$ $\left.\min \left\{\underline{p}\left(R_{3}\right), \Omega-z_{1}\right\}\right\}$

(iii) if $\sum_{i \in N} \underline{p}\left(R_{i}\right) \leq \Omega \leq \sum_{i \in N} \bar{p}\left(R_{i}\right)$, then $\bar{\varphi}(R) \equiv\left\{z \in Z \mid\right.$ for all $i \in N, \underline{p}\left(R_{i}\right) \leq z_{i} \leq$ $\left.\bar{p}\left(R_{i}\right)\right\}$, and

(iv) if $\sum_{i \in N} \bar{p}\left(R_{i}\right)<\Omega$, then $\bar{\varphi}(R) \equiv\left\{z \in Z \mid z_{1}=\bar{p}\left(R_{1}\right), z_{2}=\bar{p}\left(R_{2}\right)\right\}$. 
The solution $\vec{\varphi}$ satisfies essentially single-valuedness, Pareto-indifference, strategy-proofness, and (ii) of coalitional strategy-proofness, but not (i) of coalitional strategy-proofness. To see that $\bar{\varphi}$ violates (i) of coalitional strategy-proofness, let $\Omega=5, R \in \mathcal{R}^{N}$ be such that $\left[\underline{p}\left(R_{1}\right), \bar{p}\left(R_{1}\right)\right]=[2,5]$, and $\underline{p}\left(R_{2}\right)=5=\underline{p}\left(R_{3}\right)$. Also let $\ddot{R} \in \mathcal{R}^{N}$ be such that $\bar{R}_{\{2,3\}}=R_{\{2,3\}}$ and $\left[\underline{p}\left(\bar{R}_{1}\right), \bar{p}\left(\bar{R}_{1}\right)\right]=[\pi, 5]$. Then $\bar{\varphi}(R)=\{(2,3,0)\}$ and $\bar{\varphi}(\bar{R})=$ $\{(\pi, 0,5-\pi)\}$, contradicting (i) of coalitional strategy-proofness. Of particular interest, the rule $\bar{\varphi}$ satisfies coalitional strategy-proofness on $\mathcal{R}_{s p}^{N}$.

Remark 8.1 In the proof of the main result, we use (ii) of coalitional strategy-proofness only once. All other steps require only (i).

\subsubsection{Proof of Theorem 8.7}

Throughout this section let $\varphi$ be a solution satisfying essentially single-valuedness, Paretoindifference, and coalitional strategy-proofness. We prove Theorem 8.7 in several steps. First, we show that $\varphi$ satisfies unanimity. Lsing this result we derive that $\varphi$ is a subsolution of the Pareto solution. With this we prove that $\varphi$ satisfies a very strong invariance property relative to the most preferred consumptions. Then we prove that $\varphi$ satisfies one-sided replacement-domination. Finally, we show that $\varphi$ is a sequential-allotment solution.

Lemma 8.4 For all $R \in \mathcal{R}^{N}, T(R) \subseteq \varphi(R)$, and if $T(R) \neq \emptyset$, then $T(R)=\varphi(R)$.

Proof. For all $R \in \mathcal{R}^{N}$, let

$$
N_{0}(R) \equiv\left\{i \in N \mid \text { for all } z \in \varphi(R), z_{i} \in\left[\underline{p}\left(R_{i}\right), \bar{p}\left(R_{i}\right)\right]\right\} .
$$

Let $R \in \mathcal{R}^{N}$. If $T(R)=\emptyset$, then $T(R) \subseteq \varphi(R)$. Let

$$
\sum_{i \in N} \underline{p}\left(R_{i}\right) \leq \Omega \leq \sum_{i \in N} \bar{p}\left(R_{i}\right)
$$

Suppose that $T(R) \nsubseteq \varphi(R)$. By essentially single-valuedness, $\varphi(R) \cap T(R)=\emptyset$. Thus, $N_{0}(R) \neq N$. We distinguish two cases.

Case 1: $\left|N_{0}(R)\right| \leq n-2$.

Let $\bar{z} \in \varphi(R), N \backslash N_{0}(R) \equiv\left\{i_{1}, \ldots, i_{l}\right\}$, and $\bar{R}^{0} \equiv R$. For all $k \in\{1, \ldots, l\}$, let $\bar{R}^{k} \in$ $\mathcal{R}^{N}$ be such that $\bar{R}_{N \backslash\left\{i_{k}\right]}^{k} \equiv \bar{R}_{N \backslash\left\{i_{k}\right\}}^{k-1}$ and $\left[\underline{p}\left(\bar{R}_{i_{k}}^{k}\right), \bar{p}\left(\tilde{R}_{i_{k}}^{k}\right)\right] \equiv\left[\bar{z}_{i_{k}}, r_{i_{k}}\left(\bar{z}_{i_{k}}\right)\right]$ when $\bar{z}_{i_{k}}<\underline{p}\left(R_{i_{k}}\right)$, and $\left[\underline{p}\left(\bar{R}_{i_{k}}^{k}\right), \bar{p}\left(\bar{R}_{i_{k}}^{k}\right)\right] \equiv\left[r_{i_{k}}\left(\bar{z}_{i_{k}}\right), \bar{z}_{i_{k}}\right]$ when $\bar{z}_{i_{k}}>\bar{p}\left(R_{i_{k}}\right)$. We show by induction that for all $k \in\{1, \ldots, l\}$,

$$
N_{0}\left(\bar{R}^{k}\right)=N_{0}\left(\bar{R}^{k-1}\right) \cup\left\{i_{k}\right\}
$$


Induction basis: $N_{0}\left(\bar{R}^{1}\right)=N_{0}(R) \cup\left\{i_{1}\right\}$.

Without loss of generality, let $\bar{z}_{i_{1}}<\underline{p}\left(R_{i_{1}}\right)$. By essentially single-valuedness, for all $z \in \varphi(R)$

$$
z_{i_{1}} \in\left\{\bar{z}_{i_{1}}, r_{i_{1}}\left(\bar{z}_{i_{1}}\right)\right\}
$$

By essentially single-valuedness and strategy-proofness, for all $z^{\prime} \in \varphi\left(\bar{R}^{1}\right)$,

$$
z_{i_{1}}^{\prime} \in\left[\bar{z}_{i_{1}}, r_{i_{1}}\left(\bar{z}_{i_{1}}\right)\right]
$$

Thus, by contimuity of $R_{i}$, for all $z^{\prime} \in \varphi\left(\bar{R}^{1}\right)$ and all $z \in \varphi(R), z_{i_{1}}^{\prime} R_{i_{1}} z_{i_{1}}$ and $z_{i_{1}} \bar{R}_{i_{1}}^{1} z_{i_{1}}^{\prime}$. Hence, by (i) of coalitional strategy-proofness, $N_{0}(R) \subseteq N_{0}\left(\bar{R}^{1}\right)$. By the previous fact and (8.11), $N_{0}(R) \cup\left\{i_{1}\right\} \subseteq N_{0}\left(\bar{R}^{1}\right)$. By essentially single-valuedness and (i) of coalitional strategy-proofness, for all $j \in N \backslash\left(N_{0}(R) \cup\left\{i_{1}\right\}\right)$, all $z^{\prime} \in \varphi\left(\bar{R}^{1}\right)$, and all $z \in \varphi(R)$,

$$
z_{j}^{\prime} I_{j} \bar{z}_{j}
$$

Hence, $N_{0}\left(\bar{R}^{1}\right)=N_{0}(R) \cup\left\{i_{1}\right\}$, which is the induction basis of (8.9).

Let $k \in\{1, \ldots, l-1\}$.

Induction step: (8.9) holds for $k+1$.

By induction, $N_{0}\left(R^{k}\right)=N_{0}(R) \cup\left\{i_{1}, \ldots, i_{k}\right\}$ and by $(8.12)$, for all $j \in N \backslash N_{0}(R) \cup$ $\left\{i_{1}, \ldots, i_{k}\right\}$ and all $z \in \varphi\left(\bar{R}^{k}\right), z_{j} \bar{I}_{j} \bar{z}_{j}$. Then similarly to the proof of the induction basis it follows that $N_{0}\left(\bar{R}^{k+1}\right)=N_{0}\left(\bar{R}^{k}\right) \cup\left\{i_{k+1}\right\}$.

By (8.9), $N_{0}\left(\bar{R}^{l}\right)=N$. Since $T\left(\bar{R}^{l}\right) \neq \emptyset$, essentially single-valuedness and Paretoindifference imply $\varphi\left(\bar{R}^{l}\right)=T\left(\bar{R}^{l}\right)$. By (8.9) and $l \geq 2, i_{l-1}, i_{l} \notin N_{0}\left(\bar{R}^{l-2}\right)$. Thus, by the previous two facts, for all $z^{\prime} \in \varphi\left(\bar{R}^{l}\right)$ and all $z \in \varphi\left(\bar{R}^{l-2}\right), z_{i_{l-1}}^{\prime} \bar{R}_{i_{l-1}}^{l-2} z_{i_{l-1}}$ and $z_{i_{l}}^{\prime} \bar{R}_{i_{l}}^{l-2} z_{i_{i}}$. By

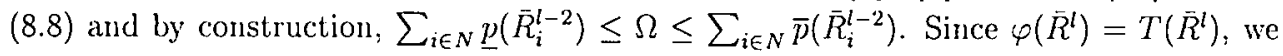
have for some $\tilde{z} \in \varphi\left(\bar{R}^{l}\right)$ and all $z \in \varphi\left(\bar{R}^{l-2}\right), \tilde{z}_{i_{l-1}} \tilde{P}_{i_{l-1}}^{l-2} z_{i_{l-1}}$ and $\tilde{z}_{i_{l}} \bar{P}_{i_{l}}^{l-2} z_{i_{l}}$. The previous facts contradict (ii) of coalitional strategy-proofness. Hence, Case 1 cannot occur.

Case 2: $\left|N_{0}(R)\right|=n-1$.

Without loss of generality, we suppose that $1 \notin N_{0}(R)$. Let $\tilde{z} \in T(R)$ and $\tilde{R} \in \mathcal{R}^{N}$ be such that for all $i \in N, \underline{p}\left(\tilde{R}_{i}\right)=\tilde{z}_{i}=\bar{p}\left(\tilde{R}_{i}\right)$. For all $k \in N$, let $R^{k}=\left(\tilde{R}_{\{1, \ldots, k\}}, R_{N \backslash\{1, \ldots, k\}}\right)$. We show by induction that for all $k \in N, N_{0}\left(R^{k}\right) \neq \emptyset$.

Induction basis: $N_{0}\left(R^{1}\right) \neq \emptyset$.

By essentially single-valuedness and strategy-proofness, $1 \notin N_{0}\left(R^{\mathrm{l}}\right)$.

Let $k \in\{1, \ldots, n-1\}$.

Induction step: $N_{0}\left(R^{k+1}\right) \neq \emptyset$. 
By induction, $N_{0}\left(R^{k}\right) \neq \emptyset$. If $N_{0}\left(R^{k+1}\right)=N$, then by our choice of $\tilde{z}$, for all $z \in$ $\varphi\left(R^{k+1}\right)$ and all $z^{\prime} \in \varphi\left(R^{k}\right), z_{k} R_{k}^{k} z_{k}^{\prime}$. Since $N_{0}\left(R^{k}\right) \neq \emptyset$, this contradicts (i) of coalitional strategy-proofness. Hence, $N_{0}\left(R^{k+1}\right) \neq \emptyset$.

By induction, $N_{0}(\tilde{R}) \neq \emptyset$. By construction, for all $i \in N, \tilde{R}_{i}$ is single-peaked with peak $\tilde{z}_{i}$. Since $N_{0}(\tilde{R}) \neq \emptyset$ and $\sum_{i \in N} \tilde{z}_{i}=\Omega$, it follows that $\left|N_{0}(\tilde{R})\right| \leq n-2$. By Case 1 , this cannot occur. Hence, Case 2 cannot occur.

Next we show that $\varphi$ is a subsolution of the Pareto solution.

Lemma $8.5 \varphi \subseteq \mathcal{P O}$

Proof. For all $R \in \mathcal{R}^{N}$, let

$$
\begin{aligned}
& \left.\left.\tilde{N}_{0}(R) \equiv\left\{i \in N \mid \text { for some } z \in \varphi(R), z_{i} \in\right] \underline{p}\left(R_{i}\right), \bar{p}\left(R_{i}\right)\right]\right\} \text { and } \\
& N_{+}(R) \equiv\left\{i \in N \mid \text { for some } z \in \varphi(R), z_{i}>\bar{p}\left(R_{i}\right)\right\}
\end{aligned}
$$

Suppose that $\varphi \nsubseteq \mathcal{P O}$. Then there exists $R \in \mathcal{R}^{N}$ such that $\varphi(R) \nsubseteq \mathcal{P O}(R)$. By Lemma 8.4, if $T(R) \neq \emptyset$, then $\varphi(R)=T(R)=\mathcal{P O}(R)$. Thus, $T(R)=\emptyset$ and $\Omega \notin$ $\left[\sum_{i \in N} \underline{p}\left(R_{i}\right), \sum_{i \in N} \bar{p}\left(R_{i}\right)\right]$. Without loss of generality, suppose that

$$
\Omega<\sum_{i \in N} \underline{p}\left(R_{i}\right)
$$

First, we show that we may assume that $N_{+}(R)=\emptyset$. Suppose that $N_{+}(R) \neq \emptyset$. Let $j \in N_{+}(R)$ and $\bar{z} \in \varphi(R)$ be such that $\bar{z}_{j}>\bar{p}\left(R_{j}\right)$. Let $R^{\prime} \in \mathcal{R}^{N}$ be such that $R_{N \backslash\{j\}}^{\prime} \equiv R_{N \backslash\{j\}}$ and $\left[\underline{p}\left(R_{j}^{\prime}\right), \bar{p}\left(R_{j}^{\prime}\right)\right] \equiv\left[r_{j}\left(\bar{z}_{j}\right), \bar{z}_{j}\right]$. We show that $N_{0}\left(R^{\prime}\right) \subseteq N_{0}(R) \cup\{j\}$ and $\tilde{N}_{0}\left(R^{\prime}\right) \cup N_{+}\left(R^{\prime}\right) \neq \emptyset$. By essentially single-valuedness and strategy-proofness, for all $z^{\prime} \in \varphi\left(R^{\prime}\right), z_{j}^{\prime} \in\left[\underline{p}\left(R_{j}^{\prime}\right), \bar{p}\left(R_{j}^{\prime}\right)\right]$. If $\sum_{i \in N} \underline{p}\left(R_{i}^{\prime}\right) \leq \Omega \leq \sum_{i \in N} \bar{p}\left(R_{i}^{\prime}\right)$, then by Lemma 8.4, $\varphi\left(R^{\prime}\right)=T\left(R^{\prime}\right)$. Then, by (8.13), for some $i \in N \backslash\{j\}, i \notin N_{0}(R)$, which contradicts (i) of coalitional strategy-proofness. Let $\Omega<\sum_{i \in N} \underline{p}\left(R_{i}^{\prime}\right)$. By (i) of coalitional strategy-proofness, $N_{0}(R) \subseteq N_{0}\left(R^{\prime}\right)$. If $\varphi\left(R^{\prime}\right) \subseteq \mathcal{P O}\left(R^{\prime}\right)$, then by (i) of coalitional strategy-proofness, for all $i \in N$ and all $z^{\prime} \in \varphi(R), z_{i}^{\prime} \leq \min \left\{\bar{z}_{i}, \underline{p}\left(R_{i}^{\prime}\right)\right\}$. Since $j \in N_{+}(R)$, we have $\sum_{i \in N} z_{i}^{\prime}<$ $\sum_{i \in N} \bar{z}_{i}=\Omega$, a contradiction. Thus, $\varphi\left(R^{\prime}\right) \not \mathcal{P O}\left(R^{\prime}\right)$ and $N_{0}(R) \cup\{j\} \subseteq N_{0}\left(R^{\prime}\right)$. Since $N$ is finite, we may assume that $N_{+}(R)=\emptyset$.

Then $N_{+}(R)=\emptyset$ and $\varphi(R) \nsubseteq \mathcal{P O}(R)$ imply $\tilde{N}_{0}(R) \neq \emptyset$. Let $\bar{z} \in \varphi(R)$ and $\alpha \equiv \sum_{i \in N}\left(\max \left\{\bar{z}_{i}, \bar{p}\left(R_{i}\right)\right\}-\bar{p}\left(R_{i}\right)\right)$. By essentially single-valuedness and $N_{+}(R)=\emptyset$, the definition of $\alpha$ is independent from the choice of $\bar{z}$. By $\varphi(R) \not \mathcal{P} \mathcal{O}(R), \alpha>0$. Let $\tilde{R} \in \mathcal{R}^{N}$ be such that $\tilde{R}_{N_{0}(R)}=R_{N_{0}(R)}$ and for all $i \in N \backslash N_{0}(R),\left[\underline{p}\left(\tilde{R}_{i}\right), \bar{p}\left(\tilde{R}_{i}\right)\right]=\left[\bar{z}_{i}+\frac{\alpha}{n}, \bar{p}\left(R_{i}\right)\right]$ and $\tilde{r}_{i}\left(\bar{z}_{i}\right)=r_{i}\left(\bar{z}_{i}\right)$. By successive application of coalitional strategy-proofness it follows that 
for all $i \in N$ and all $z \in \varphi(\tilde{R}), z_{i} I_{i} \bar{z}_{i}$. Since $\sum_{i \in N} \underline{p}\left(\tilde{R}_{i}\right) \leq \Omega \leq \sum_{i \in N} \bar{p}\left(\tilde{R}_{i}\right)$, it follows that $T(\tilde{R}) \neq \emptyset$. Thus, by Lemma 8.4, $\varphi(\tilde{R})=T(\tilde{R})$, contradicting the previous fact.

The next lemma is a straightforward consequence of Lemmas 8.4 and 8.5 together with essentially single-valuedness.

Lemma 8.6 For all $R \in \mathcal{R}^{N}$ such that $\left.\Omega \notin\right] \sum_{i \in N} \underline{p}\left(R_{i}\right), \sum_{i \in N} \bar{p}\left(R_{i}\right)[,|\varphi(R)|=1$.

From now on, for all $R \in \mathcal{R}^{N}$, if $|\varphi(R)|=1$, then $\varphi(R)$ denotes the unique allocation recommended by $\varphi$ for $R$.

Lemma 8.7 For all $R, R^{\prime} \in \mathcal{R}^{N}$, if $\left[\Omega \leq \sum_{i \in N} \underline{p}\left(R_{i}\right)\right.$ and for all $\left.i \in N, \underline{p}\left(R_{i}\right)=\underline{p}\left(R_{i}^{\prime}\right)\right]$ or $\left[\sum_{i \in N} \bar{p}\left(R_{i}\right) \leq \Omega\right.$ and for all $\left.i \in N, \bar{p}\left(R_{i}\right)=\bar{p}\left(R_{i}^{\prime}\right)\right]$, then $\varphi(R)=\varphi\left(R^{\prime}\right)$.

Proof. Let $j \in N$ and $R, \bar{R} \in \mathcal{R}^{N}$ be such that $R_{N \backslash\{j\}}=\bar{R}_{N \backslash\{j\}}, \Omega \leq \sum_{i \in N} \underline{p}\left(R_{i}\right)$, and $\underline{p}\left(R_{j}\right)=\underline{p}\left(\bar{R}_{j}\right)$. It suffices to show that $\varphi(\bar{R})=\varphi(R)$.

By Lemma 8.6, $|\varphi(R)|=1=|\varphi(\bar{R})|$. By strategy-proofness and $\varphi \subseteq \mathcal{P O}, \varphi_{j}(\bar{R})=$ $\varphi_{j}(R)$. Thus, by (i) of coalitional strategy-proofness, for all $i \in N \backslash\{j\}, \varphi_{i}(\bar{R})=\varphi_{i}(R)$. Hence, $\varphi(\bar{R})=\varphi(R)$, the desired conclusion.

Next, we show that $\varphi$ satisfies one-sided replacement-domination, meaning that for economies in excess demand, if an agent increases his left-most most preferred consumption, then the allotment of each other agent weakly decreases, and for economies in excess supply, if an agent decreases his right-most most preferred consumption, then the allotment of each other agent weakly increases. Barberà, Jackson, and Neme (1997), Thomson (1997), and Klaus (2000) investigate the implications of this property together with certain other requirements. ${ }^{4}$ We show that essentially single-valuedness, Pareto-indifference, and coalitional strategy-proofness imply one-sided replacement-domination.

One-Sided Replacement-Domination: For all $j \in N$ and all $R, \bar{R} \in \mathcal{R}^{N}$ such that $R_{N \backslash\{j\}}=\bar{R}_{N \backslash\{j\}}$ and either $\Omega \leq \sum_{i \in N} \underline{p}\left(\bar{R}_{i}\right) \leq \sum_{i \in N} \underline{p}\left(R_{i}\right)$ or $\sum_{i \in N} \bar{p}\left(\bar{R}_{i}\right) \leq$ $\sum_{i \in N} \bar{p}\left(R_{i}\right) \leq \Omega$ : for all $i \in N \backslash\{j\}, \varphi_{i}(R) \leq \varphi_{i}(\bar{R})$.

Lemma $8.8 \varphi$ satisfies one-sided replacement-domination.

\footnotetext{
${ }^{4}$ Barberà, Jackson, and Neme (1997) call it "replacement-monotonicity." Thomson (1997) formulates the requirement in terms of welfare and calls it "one-sided welfare-domination under preference replacement."
} 
Proof. Let $j \in N$ and $R, \tilde{R} \in \mathcal{R}^{N}$ be such that $R_{N \backslash\{j\}}=\bar{R}_{N \backslash\{j\}}$. Without loss of generality, we only show one-sided replacement-domination for the case $\sum_{i \in N} \bar{p}\left(\bar{R}_{i}\right) \leq$ $\sum_{i \in N} \bar{p}\left(R_{i}\right) \leq \Omega$. We show that for all $i \in N \backslash\{j\}, \varphi_{i}(R) \leq \varphi_{i}(\bar{R})$. By Lemma 8.6, $|\varphi(R)|=1=|\varphi(\bar{R})|$. Let $R^{\prime} \in \mathcal{R}^{N}$ be such that $R_{N \backslash\{j\}}^{\prime}=R_{N \backslash\{j\}}$ and $\left[p\left(R_{j}^{\prime}\right), \bar{p}\left(R_{j}^{\prime}\right)\right]=$ $\left[0, \bar{p}\left(R_{j}\right)\right]$. By Lemma 8.7,

$$
\varphi\left(R^{\prime}\right)=\varphi(R)
$$

If $\bar{p}\left(R_{j}^{\prime}\right)<\varphi_{j}(\bar{R})$, then strategy-proofness, $\varphi\left(R^{\prime}\right) \subseteq \mathcal{P O}\left(R^{\prime}\right)$, and $\left|\varphi\left(R^{\prime}\right)\right|=1$ imply $\varphi_{j}\left(R^{\prime}\right)=\varphi_{j}(\bar{R})$. Hence, by (i) of coalitional strategy-proofness, $\varphi\left(R^{\prime}\right)=\varphi(\bar{R})$. Hence, by (8.14), $\varphi(R)=\varphi(\bar{R})$, the desired conclusion.

If $\varphi_{j}(\bar{R}) \leq \bar{p}\left(R_{j}^{\prime}\right)$, then strategy-proofness and $\varphi\left(R^{\prime}\right) \subseteq \mathcal{P O}\left(R^{\prime}\right)$ imply $\varphi_{j}\left(R^{\prime}\right)=\bar{p}\left(R_{j}^{\prime}\right)$. Thus, $\varphi_{j}\left(R^{\prime}\right) I_{j}^{\prime} \varphi_{j}(\bar{R})$. Hence, by (i) of coalitional strategy-proofness, for all $i \in N \backslash\{j\}$, $\varphi_{i}\left(R^{\prime}\right) \leq \varphi_{i}(\bar{R})$. Thus, by (8.14), for all $i \in N \backslash\{j\}, \varphi_{i}(R) \leq \varphi_{i}(\bar{R})$, the desired conclusion.

Now, we could apply the result of Barberà, Jackson, and Neme (1997). For the sake of completeness, we provide a proof.

We construct a pair $(f, g) \in \mathcal{F} \times \mathcal{G}$ and show that $\varphi=\phi^{(f, g)}$.

For all $x \in[0, \Omega]^{N}$ such that $\sum_{i \in N} x_{i} \geq \Omega$, let $R^{x} \in \mathcal{R}^{N}$ be such that for all $i \in N$, $\left[\underline{p}\left(R_{i}^{x}\right), \bar{p}\left(R_{i}^{x}\right)\right]=\left[x_{i}, \Omega\right]$. Define $f(x) \equiv \varphi\left(R^{x}\right)$. By Lemmas 8.5 and 8.6, $\varphi\left(R^{x}\right) \subseteq \mathcal{P O}\left(R^{x}\right)$ and $\left|\varphi\left(R^{x}\right)\right|=1$. Thus, $f$ is well-defined, for all $i \in N, f_{i}(x) \leq x_{i}$, and $\sum_{i \in N} f_{i}(x)=$ $\Omega$, which is condition (1). Condition (2) follows from strategy-proofness and condition (3) from one-sided replacement-domination. Thus, $f$ is an allotment function for excess demand.

Similarly, for all $y \in[0, \Omega]^{N}$ such that $\sum_{i \in N} y_{i} \leq \Omega$, let $R^{y} \in \mathcal{R}^{N}$ be such that for all $i \in N,\left[\underline{p}\left(R_{i}^{y}\right), \bar{p}\left(R_{i}^{y}\right)\right]=\left[0, y_{i}\right]$. Define $g(y) \equiv \varphi\left(R^{y}\right)$. By Lemmas 8.5 and 8.6, $\varphi\left(R^{y}\right) \subseteq \mathcal{P O}\left(R^{y}\right)$ and $\left|\varphi\left(R^{y}\right)\right|=1$. Thus, $g$ is well-defined and for all $i \in N, g_{i}(y) \geq y_{i}$ and $\sum_{i \in N} g_{i}(y)=\Omega$, which is condition (1). Condition (2) follows from strategy-proofness and condition (3) from one-sided replacement-domination. Thus, $g$ is an allotment function for excess supply.

Thus, $(f, g) \in \mathcal{F} \times \mathcal{G}$. By Lemmas 8.4 and $8.7, \varphi=\phi^{(f, g)}$, the desired conclusion of Theorem 8.7. 

CHAPTER 8. STRATEGY-PROOFNESS AND SINGLE-PLATEAUEDNESS 


\section{Chapter 9}

\section{Probabilistic Allocation when Preferences are Single-Dipped}

\subsection{Introduction}

This chapter, based on Ehlers (1998), studies the problem of allocating an infinitely divisible endowment among a group of agents with single-dipped preferences. A preference relation is single-dipped when there is a single worst amount, the dip, and preference increases monotonically in either direction away from the dip. For example, two types of consumptions may have negative cross-effects: combinations of the two consumptions are less preferred than pure one type consumptions. Examples are two types of work like teaching and management in a university, or the allocation of natural resources among countries that can afford only one technology to exploit one of the resources. When in a two-goods exchange economy preferences have convex lower contour sets and prices are fixed, preferences are single-dipped on the budget line. If we allocate some free hours among workers for which they are not paid, then they may prefer consuming all free hours or continuing to work than having only a few hours off.

For each profile of preferences, an allocation rule allocates the social endowment among the agents. A serial dictatorship rule is an allocation rule that serves the agents in a certain order. The first agent whose most preferred consumption is receiving the social endownent consumes it. A rule is Pareto-optimal when it chooses only efficient allocations. When preferences are private information of the agents, strategy-proofness ensures that no agent can benefit from misrepresenting his preference relation. Klaus, Peters, and Storcken (1997b) show that serial dictatorship rules are the only rules satisfying Pareto-optimality, strategyproofness, and a certain normative property of "replacement-domination". Klaus (1999b) characterizes these rules by Pareto-optimality, strategy-proofness, and a certain property 
"weak non-bossiness". Klaus (1999b) also proves the incompatibility of Pareto-optimality with important fairness notions and showed that her results apply to assignment problems of an indivisible object. We extend the deterministic model and allow allocation rules to be probabilistic. A probabilistic rule chooses for each profile of preferences a probability distribution on the set of all allocations. The number assigned by the rule to a (measurable) subset of the set of all allocations is the probability that the final allocation belongs to this set.

The central properties we impose are Pareto-optimality and strategy-proofness. In order to formulate them in our context, we extend preferences to distributions ordinally. Given a single-dipped preference relation, a distribution is (weakly) preferred to another one when the first distribution places on each upper contour set a probability that is at least as large as the probability placed by the second distribution. In other words, preference over distributions is defined in terms of first-order stochastic dominance. This is how Ehlers, Peters, and Storcken (1999), and Bogomolnaia and Moulin (1999) suggest extending preferences over deterministic alternatives to preferences over distributions. A probabilistic rule is strategy-proof if honest reporting always results in a distribution that (weakly) dominates, in the sense just described, any distribution brought about by lying. This condition which is analogous to the one adopted by Gibbard $(1977,1978)$ is quite strong; in particular it implies comparability in the sense of stochastic dominance between the distributions induced by the rule in case of honest and false reporting. It is, however, a natural condition in a franework where the probabilistic rule can only use ordinal information about the agents' preferences because by the familiar characterization of first degree stochastic dominance - it implies that no agent can gain by lying independent of any possible von Neumann-Morgenstem representation of his true preference relation. Nevertheless, our strategy-proofness condition still admits a large class of rules.

We show that in our model Pareto-optimality is equivalent to ex-post efficiency, the requirement that for each profile the chosen distribution places probability 1 on the set of deterministic efficient allocations. In general, this equivalence does not need to hold. For example, Bogonolnaia and Moulin (1999) show that in their model ex-post efficiency does not imply Pareto-optimality of distributions.

We study the solidarity property replacement-domination: when some agent's preference is replaced by another admissible preference, all welfares of the other agents are affected in the same direction. We characterize the class of probabilistic rules satisfying strategy-proofness, Pareto-optimality, and replacement-domination. These rules essentially depend only on the set of agents who strictly prefer receiving the social endownent to receiving nothing and on the set of agents who are indifferent between receiving the 
social endownent and receiving nothing. For each profile they choose a distribution which places probability 1 on the set of allocations at which one agent receives the social endowment. For each subset of agents, they choose randomly an agent belonging to this subset as a dictator. For each profile, when at least one agent strictly prefers receiving the social endowment to receiving nothing, the distribution is the random dictatorship according to the set of agents who strictly prefer receiving the social endowment to receiving nothing. If no agent strictly prefers consuming the social endowment to receiving nothing and some agents are indifferent between receiving the social endowment and receiving nothing, then the rule chooses randomly one of those agents to consume the social endowment. If all agents strictly prefer receiving nothing to receiving the social endowment, then each agent receives with some probability the social endowment. These rules are serial, because they satisfy the probabilistic analogue of serial dictatorships, i.e., if an agent is a random dictator and the set of agents who strictly prefer receiving the social endowment to receiving nothing shrinks, then the probability of this agent being the dictator does not decrease. We call these probabilistic rules serial random dictatorship rules.

In the deterministic model Klaus (1999b) proves the incompatibility of Pareto-optimality with important fairness notions, in particular with no-envy: ${ }^{1}$ no-envy requires that each agent weakly prefers his allotment to any other agent's allotment. In our probabilistic model, no-envy means that each agent weakly prefers his marginal distribution to any other agent's marginal distribution. We derive compatibilities with the important faimess notions. The second main result characterizes all probabilistic rules satisfying Pareto-optimality, strategy-proofness, and no-envy. These rules simply give each agent who strictly prefers receiving the social endowment to receiving nothing the same probability to consume the social endowment. We call these rules uniform random dictatorship rules.

Our results also apply to the probabilistic assignment of one indivisible object. For one indivisible object, the uniform random dictatorship rule coincides with the uniform probabilistic rule defined by Sasaki (1997). ${ }^{2}$ For an illustration consider the problem of assigning an indivisible object among a group of agents. If we require Pareto-optimality, strategy-proofness, and no-envy, then a deterministic assignment cannot ensure all properties. In our probabilistic model, the problem is solvable. First, the agents are asked whether they strictly prefer receiving the social endowment to receiving nothing. Among the agents who do, the one who obtains the object is determined by throwing a fair die

\footnotetext{
${ }^{1}$ No-envy was first introduced by Foley (1967).

${ }^{2}$ Sasaki (1997) calls his probabilistic rule "random uniform allocation mechanism". He considers the probabilistic assignment of a finite number of identical indivisible objects among a group of agents with single-peaked preferences.
} 
(each side corresponding to one of those agents). Of course, a posteriori, after throwing the fair die, no-envy is violated. But, a priori, before throwing the fair die, no-envy is ensured, i.e., no agent has an a priori advantage over any other agent.

For the problem of assigning one indivisible object, Klaus (1999b) shows that Paretooptimality implies strategy-proofness. We show that in the probabilistic model, her conclusion remains valid. Therefore, for one indivisible object, the class of uniform random dictatorship rules is characterized by Pareto-optimality and equal treatment of equals (if two agents have the same preference, then they should be indifferent between their marginal distributions according to their common preference relation). If we consider the problem of allocating randomly a finite number of identical indivisible objects, then we only restrict the set of allocations on which probabilistic rules place positive probability. Therefore, all previous conclusions remain valid. By our first main result, the serial random dictatorship rules are characterized by Pareto-optimality, strategy-proofness, and replacement-domination. Since we extend the deterministic model, the result also applies to the deterministic assignment of finitely many indivisible objects, i.e., for each profile a single agent receives all indivisible objects.

The organization of Chapter 9 is as follows. Section 9.2 presents the probabilistic model and the properties Pareto-optimality and strategy-proofness. Section 9.3 is devoted to replacement-domination. Section 9.4 studies fairness properties and Section 9.5 the probabilistic assignment of finitely many identical indivisible objects. Section 9.6 establishes the independence of our characterizations and Section 9.7 concludes.

\subsection{The Model}

We consider the problem of allocating one unit of an infinitely divisible endowment among a group $N=\{1,2, \ldots, n\}$ of agents. Denote by $e^{1}, \ldots, e^{n}$ the unit vectors in $\mathbb{R}^{N}$, where for each $i \in N, e_{i}^{i}=1$ and for all $j \in N \backslash\{i\}, e_{j}^{i}=0$. Let $E^{N}=\left\{e^{1}, \ldots, e^{n}\right\}$ and denote by $\mathcal{E}^{N}$ the unit simplex in $\mathbb{R}_{+}^{N}$. We call a vector $x \in \mathcal{E}^{N}$ an allocation, and $x_{i}$ the allotment of agent $i$ at $x$. Free disposal is not allowed.

A preference relation is a complete and transitive binary relation $R_{i}$ on $[0,1]$. By $P_{i}$ we denote the strict preference relation associated with $R_{i}$, and by $I_{i}$ the indifference relation.

Each agent $i \in N$ is equipped with a single-dipped preference relation $R_{i}$. Singledippedness of $R_{i}$ means that there exists a number $d\left(R_{i}\right) \in[0,1]$, the dip of $R_{i}$, such that for all $x_{i}, y_{i} \in[0,1]$, if $x_{i}<y_{i} \leq d\left(R_{i}\right)$, or $x_{i}>y_{i} \geq d\left(R_{i}\right)$, then $x_{i} P_{i} y_{i}$. By $\mathcal{D}$ we denote the set of all continuous single-dipped preferences and by $\mathcal{D}^{N}$ the set of profiles $R=\left(R_{i}\right)_{i \in N}$ 
such that for all $i \in N, R_{i} \in \mathcal{D} .^{3}$ Given $R, R^{\prime} \in \mathcal{D}^{N}$ and $i \in N$, we call $R^{\prime}$ an $i$-deviation of $R$ if for all $j \in N \backslash\{i\}, R_{j}^{\prime}=R_{j}$. Given $R_{i} \in \mathcal{D}$ and $x_{i} \in[0,1]$, we define the weak upper contour set of $x_{i}$ at $R_{i}$ by $B\left(x_{i}, R_{i}\right) \equiv\left\{y_{i} \in[0,1] \mid y_{i} R_{i} x_{i}\right\}$, and the strict upper contour set of $x_{i}$ at $R_{i}$ by $B^{o}\left(x_{i}, R_{i}\right) \equiv\left\{y_{i} \in[0,1] \mid y_{i} P_{i} x_{i}\right\}$. A deterninistic allocation rule is a function from $\mathcal{D}^{N}$ to $\mathcal{E}^{N}$.

We consider probabilistic (allocation) rules. Such a rule selects for every profile of preferences a probability distribution on $\mathcal{E}^{N}$. The final allocation is chosen according to that distribution. For every (measurable) subset $X$ of $\mathcal{E}^{N}$ the number assigned to $X$ by the distribution represents the probability that the final allocation belongs to $X$. We consider distributions that are defined on the Borel $\sigma$-algebra $\mathcal{L}^{N}$. Given a probabilistic rule $\Phi$ and $R \in \mathcal{D}^{N}$, we denote by $\Phi(R)$ the probability distribution that $\Phi$ assigns to $R .{ }^{4}$

Given $i \in N$ and $X_{i} \subseteq[0,1]$, we denote by $H\left(X_{i}\right)$ the set of allocations such that agent $i$ 's allotment belongs to $X_{i}$, i.e., $H\left(X_{i}\right) \equiv\left\{x \in \mathcal{E}^{N} \mid x_{i} \in X_{i}\right\}$. By $\mathcal{L}^{1}$ we denote the Borel $\sigma$-algebra on $[0,1]$.

Marginal (Probability) Distribution: For all $i \in N$ and all $R \in \mathcal{D}^{N}, \Phi_{i}(R)$ denotes agent $i$ 's maryinal probability distribution of $\Phi(R)$ on $[0,1]$. It is the function from $\mathcal{L}^{1}$ to $[0,1]$ such that for all $X_{i} \in \mathcal{L}^{1}, \Phi_{i}(R)\left(X_{i}\right) \equiv \Phi(R)\left(H\left(X_{i}\right)\right)$.

It follows from basic measure theory that $\Phi_{i}(R)$ is a well-defined distribution on $[0,1]$.

Next, we discuss our extension of preferences to marginal distributions. Each agent is selfish and only takes in account the narginal distributions that distributions induce on his set of possible allotments. We extend preferences ordinally. There are two ways to determine upper contour sets: weakly and strictly. We define our extension with respect to weak upper contour sets. Given a preference relation $R_{i}$ and two marginal probability distributions $Q_{i}$ and $Q_{i}^{\prime}$ on $[0,1]$, we say that $Q_{i}$ is weakly preferred to $Q_{i}^{\prime}$ at $R_{i}$, if to each weak upper contour set of $R_{i}, Q_{i}$ assigns a probability that is at least as large as the probability assigned by $Q_{i}^{\prime}$. For notational convenience we use the same symbols to define preference relations on distributions.

Ordinal Extension of Preferences: For all $i \in N$, all $R_{i} \in \mathcal{D}$, and two distributions $Q_{i}, Q_{i}^{\prime}$ on $[0,1], Q_{i} R_{i} Q_{i}^{\prime}$ if and only if for all $x_{i} \in[0,1]$,

$$
Q_{i}\left(B\left(x_{i}, R_{i}\right)\right) \geq Q_{i}^{\prime}\left(B\left(x_{i}, R_{i}\right)\right)
$$

\footnotetext{
${ }^{3}$ All our results remain true when continuity is dropped.

${ }^{4}$ All basic results of measure theory, which we use, are given in Halmos (1970).
} 
If for some $x_{i} \in[0,1],(9.1)$ holds with strict inequality, then $Q_{i} P_{i} Q_{i}^{\prime}$.

Because of single-dippedness, each weak upper contour set is the union of one or two intervals. Therefore, each weak upper contour set belongs to the Borel $\sigma$-algebra $\mathcal{L}^{1}$ and our extension is well-defined. Observe that (9.1) is a first-order stochastic dominance condition; in particular it requires comparability with respect to this condition of the distributions $Q_{i}$ and $Q_{i}^{\prime}$. Our extension is not complete on the set of all distributions on $\mathcal{E}^{N}$. But not all distributions have to be in the range of a probabilistic rule. For example if a rule is deterministic, then all the chosen distributions are comparable.

The following lemma shows that we could equivalently extend preferences with respect to strict upper contour sets.

Lemma 9.1 Let $i \in N, R_{i} \in \mathcal{D}$, and $Q_{i}, Q_{i}^{\prime}$ be two distributions on $[0,1]$. Then $Q_{i} R_{i} Q_{i}^{\prime}$ if and only if for all $x_{i} \in[0,1]$,

$$
Q_{i}\left(B^{o}\left(x_{i}, R_{i}\right)\right) \geq Q_{i}^{\prime}\left(B^{o}\left(x_{i}, R_{i}\right)\right)
$$

\section{Proof.}

(Only if) Let $Q_{i} R_{i} Q_{i}^{\prime}$ and $x_{i} \in[0,1]$. We have to show $(9.2)$. Note that $B^{\circ}\left(x_{i}, R_{i}\right)$ is an interval or the union of two disjoint intervals. In each case there exists a sequence $\left(x_{i}^{k}\right)_{k \in \mathbb{N}}$ such that for all $k \in \mathbb{N}, x_{i}^{k} \in[0,1]$ and $B\left(x_{i}^{k+1}, R_{i}\right) \subseteq B\left(x_{i}^{k}, R_{i}\right)$, and $\cap_{k \in \mathbb{N}} B\left(x_{i}^{k}, R_{i}\right)=$ $B^{o}\left(x_{i}, R_{i}\right)$. Because distributions are continuous from above, ${ }^{5}$

$$
Q_{i}\left(B^{o}\left(x_{i}, R_{i}\right)\right)=\lim _{k \rightarrow \infty} Q_{i}\left(B\left(x_{i}^{k}, R_{i}\right)\right)
$$

and

$$
Q_{i}^{\prime}\left(B^{o}\left(x_{i}, R_{i}\right)\right)=\lim _{k \rightarrow \infty} Q_{i}^{\prime}\left(B\left(x_{i}^{k}, R_{i}\right)\right)
$$

By (9.1), for all $k \in \mathbb{N}$,

$$
Q_{i}\left(B\left(x_{i}^{k}, R_{i}\right)\right) \geq Q_{i}^{\prime}\left(B\left(x_{i}^{k}, R_{i}\right)\right)
$$

Combining these inequalities with the previous equalities yields (9.2).

(If) Follows similarly.

From now on, we use (9.1) and (9.2) for our ordinal extension of preferences to marginal probability distributions.

\footnotetext{
${ }^{5}$ Page 39 in Halmos (1970).
} 
Using our extension of preferences we define Pareto-optimality and strategy-proofness. A clistribution is Pareto-optimal at $R \in \mathcal{D}^{N}$ if there exists no other distribution that all agents weakly prefer, and one agent strictly prefers.

Pareto-Optimality: For all $R \in \mathcal{D}^{N}$, there exists no distribution $Q$ on $\mathcal{E}^{N}$ such that for all $i \in N, Q_{i} R_{i} \Phi_{i}(R)$, and for some $j \in N, Q_{j} P_{j} \Phi_{j}(R)$.

Strategy-proofness requires that no agent can benefit from misrepresenting his preference relation. When the probabilistic rule can only use ordinal information about the agents' preferences, the familiar characterization of first degree stochastic dominance implies that no agent can gain by lying independent of any possible von NeumannMorgenstern representation of his true preference relation.

Strategy-Proofness: For all $i \in N$ and all $R, R^{\prime} \in \mathcal{D}^{N}$, if $R^{\prime}$ is an $i$-deviation of $R$, then $\Phi_{i}(R) R_{i} \Phi_{i}\left(R^{\prime}\right)$.

By Lemma 9.2 below, a probabilistic rule satisfies Pareto-optimality if and only if it always assigns a distribution with probability 1 on the set of deterministic Paretooptimal allocations (Klaus, Peters, and Storcken, 1997b). Therefore, in our model Paretooptimality is equivalent to ex-post efficiency. We have to introduce additional notation to describe the set of deterministic Pareto-optimal allocations.

Given $R \in \mathcal{D}^{N}$, let $N_{+}(R) \equiv\left\{i \in N \mid 1 P_{i} 0\right\}$ denote the set of agents who strictly prefer 1 to $0, N_{0}(R) \equiv\left\{i \in N \mid 1 I_{i} 0\right\}$ the set of agents who are indifferent between 0 and 1 , and $N_{-}(R) \equiv\left\{i \in N \mid 0 P_{i} 1\right\}$ the set of agents who strictly prefer 0 to 1 . Given $R_{i} \in \mathcal{D}$, we define the number $r_{i}(0)$ as follows: if there exists $x_{i} \in[0,1]$ such that $d\left(R_{i}\right) \leq x_{i}$ and $0 I_{i} x_{i}$, then $r_{i}(0)=x_{i}$; otherwise, $r_{i}(0)=1$. Analogously, we define the number $r_{i}(1)$ : if there exists $x_{i} \in[0,1]$ such that $d\left(R_{i}\right) \geq x_{i}$ and $1 I_{i} x_{i}$, then $r_{i}(1)=x_{i}$; otherwise, $r_{i}(1)=0$. Single-dippedness and continuity of $R_{i}$ imply $r_{i}(0)<1$ when $i \in N_{+}(R)$, and $r_{i}(1)>0$ when $i \in N_{-}(R)$.

Lemma 9.2 Let $\Phi$ be a probabilistic rule. Then $\Phi$ satisfies Pareto-optimality if and only if for all $R \in \mathcal{D}^{N}$ :

(i) If $N_{+}(R) \neq \emptyset$, then for all $\left.\left.i \in N_{+}(R), \Phi_{i}(R)(\{0\} \cup\} r_{i}(0), 1\right\}\right)=1$, and for all $i \in N \backslash N_{+}(R), \Phi_{i}(R)(\{0\})=1$.

(ii) If $N_{+}(R)=\emptyset$ and $N_{0}(R) \neq \emptyset$, then for all $i \in N_{0}(R), \Phi_{i}(R)(\{0,1\})=1$, and for all $i \in N_{-}(R), \Phi_{i}(R)(\{0\})=1$. 
(iii) If $N_{-}(R)=N$, then for all $i \in N, \Phi_{i}(R)\left(\left[0, r_{i}(1)[\cup\{1\})=1\right.\right.$.

Proof. First, we show Lemma 9.2 for (i).

(Only if) Let $\Phi$ satisfy Pareto-optimality. Suppose that there exists $R \in \mathcal{D}^{N}$ at which $\Phi(R)$ violates $(\mathrm{i})$. Let $Z(R) \equiv\left\{x \in \mathcal{E}^{N} \mid\right.$ for all $\left.\left.i \in N_{+}(R), x_{i} \in\{0\} \cup\right] r_{i}(0), 1\right]$, and for all $\left.i \in N \backslash N_{+}(R), x_{i}=0\right\}$ be the set of ex-post efficient allocations at $R$. Since $\Phi(R)$ violates (i),

$$
\Phi(R)(Z(R))<1
$$

For all $S \subseteq N_{+}(R)$, let

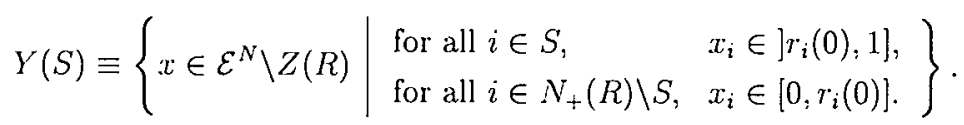

Note the following facts. For all $S \subseteq N_{+}(R), Y(S) \cap Z(R)=\emptyset$ and $Y(S) \in \mathcal{L}^{N}$. By (9.3) and $\cup_{S \subseteq N_{+}(R)} Y(S)=\mathcal{E}^{N} \backslash Z(R)$, for some $S \subseteq N_{+}(R), \Phi(R)(Y(S))>0$. We consider two cases.

Case 1: $S=\emptyset$.

Let $1 \in N_{+}(R)$ and define the distribution $Q$ as follows. For all $X \in \mathcal{L}^{N}, Q(X)=$ $\Phi(R)(X \backslash Y(\emptyset))+\Phi(R)(Y(\emptyset)) 1_{X}\left(e^{1}\right){ }^{6}$ Then by definition, for all $i \in N, Q_{i} R_{i} \Phi_{i}(R)$ and $Q_{1} P_{1} \Phi_{1}(R)$, which contradicts Pareto-optimality.

\section{Case 2: $S \neq \emptyset$.}

Let $g: Y(S) \rightarrow Z(R)$ be the function such that for all $x \in Y(S)$ and all $i \in N$,

$$
g_{i}(x)=\left\{\begin{array}{cl}
x_{i}+\lambda_{x} & \text { when } i \in S, \text { and } \\
0 & \text { when } i \in N \backslash S
\end{array}\right.
$$

where $\lambda_{x}$ solves $\sum_{i \in N} g_{i}(x)=1$. Note that $g$ is continuous and measurable with respect to $\mathcal{L}^{N}$. Define the distribution $Q$ as follows. For all $X \in \mathcal{L}^{N}$;

$$
Q(X)=\Phi(R)(X \backslash Y(S))+\Phi(R)\left(g^{-1}(X \cap g(Y(S)))\right)
$$

Since $g$ is continuous, $Q$ is a well-defined probability distribution. By the definition of $Q$, it follows that for all $i \in N, Q_{i} R_{i} \Phi_{i}(R)$. Because $\Phi(R)(Y(S))>0$, there exists $j \in S$ such that $\left.\left.\left.\left.Q_{j}(] r_{j}(0), 1\right]\right)>\Phi_{j}(R)(] r_{j}(0), 1\right]\right)$. Thus, by Lemma 9.1 and $\left.\left.B^{o}\left(R_{j}, 0\right)=\right] r_{j}(0), 1\right]$, $Q_{j} P_{j} \Phi_{j}(R)$, which contradicts Pareto-optimality.

\footnotetext{
${ }^{6} 1_{X}$ denotes the indicator function for $X$, i.e., $1_{X}\left(e^{1}\right)=1$ when $e^{1} \in X$, and $1_{X}\left(e^{1}\right)=0$ when $e^{1} \notin X$.

${ }^{7} g(Y(S))=\left\{g(x) \in \mathcal{E}^{N} \mid x \in Y(S)\right\}$ and for all $X \subseteq g(Y(S)), g^{-1}(X)=\{x \in Y(S) \mid g(x) \in X\}$.
} 
(If) Let $\Phi$ satisfy (i), and $R \in \mathcal{D}^{N}$ be such that $N_{+}(R) \neq \emptyset$. Let $Q$ be a probability distribution such that for all $i \in N, Q_{i} R_{i} \Phi_{i}(R)$. Suppose that for some $j \in N_{+}(R)$, $Q_{j} P_{j} \Phi_{j}(R)$. By the (Only if)-part we suppose that $Q$ satisfies (i) (otherwise, we construct a distribution $Q^{\prime}$ satisfying (i) that Pareto-dominates $Q$ and $\Phi(R)$ ). Given $i \in N$, the function $f^{i}: \mathcal{E}^{N} \rightarrow[0,1]$ denotes the projection of $\mathcal{E}^{N}$ onto $i$ 's coordinate, i.e., for all $x \in \mathcal{E}^{N}, f^{i}(x)=x_{i}$. Because $Q$ and $\Phi(R)$ satisfy (i), $Q(Z(R))=1=\Phi(R)(Z(R))$. Thus, for all $i \in N_{+}(R)$, the previous fact and $Q_{i} R_{i} \Phi_{i}(R)$ imply

$$
\int_{Z(R)} f^{i} d Q=\int_{\mathcal{E}^{N}} f^{i} d Q=\int_{0}^{1} x_{i} d Q_{i} \geq \int_{0}^{1} x_{i} d \Phi_{i}(R)=\int_{\mathcal{E}^{N}} f^{i} d \Phi(R)=\int_{Z(R)} f^{i} d \Phi(R) .
$$

Thus, for all $i \in N_{+}(R), \int_{Z(R)} f^{i} d Q \geq \int_{Z(R)} f^{i} d \Phi(R)$, and for some $j \in N_{+}(R), \int_{Z(R)} f^{j} d Q>$ $\int_{Z(R)} f^{j} d \Phi(R)$. Hence,

$$
\begin{aligned}
\int_{Z(R)} \sum_{i \in N_{+}(R)} f^{i} d Q & =\sum_{i \in N_{+}(R)} \int_{Z(R)} f^{i} d Q> \\
\sum_{i \in N_{+}(R)} \int_{Z(R)} f^{i} d \Phi(R) & =\int_{Z(R)} \sum_{i \in N_{+}(R)} f^{i} d \Phi(R) .
\end{aligned}
$$

Note that for all $x \in Z(R), \sum_{i \in N_{+}(R)} f^{i}(x)=1$. Hence,

$$
\int_{Z(R)} \sum_{i \in N_{+}(R)} f^{i} d Q=Q(Z(R))=1
$$

and

$$
\int_{Z(R)} \sum_{i \in N_{+}(R)} f^{i} d \Phi(R)=\Phi(R)(Z(R))=1
$$

Now, the previous two facts contradict (9.4).

We showed Lemma 9.2 for (i). Case (ii) is trivial. We outline the proof for (iii). Let $R \in \mathcal{D}^{N}$ be such that $N_{-}(R)=N$.

(Only if) The sets $Z(R)$ and $Y(S), S \subseteq N$, are defined in the same way as in the previous (Only if)-part. Note that $Y(N)=\emptyset$, because otherwise, $x \in Y(N)$ and for all $i \in N, x_{i} \in\left[0, r_{i}(1)[\right.$, and therefore, $x \in Z(R)$ in contradiction to the definition of $Y(N)$. If $\Phi(R)(Y(\emptyset))>0$, then the same argument as in Case 1 of the previous (Only if)-part yields the desired conclusion. If for some $S \subseteq N, S \notin\{\emptyset, N\}, \Phi(R)(Y(S))>0$, then choose $i \in S$ and define $Q$ as in Case 1 of the previous (Only if)-part. Then $Q$ yields the desired conclusion.

(If) The definitions of the projections have to be adjusted. For all $i \in N$, let $\tilde{f}^{i}: \mathcal{E}^{N} \rightarrow$ $[0,1]$ be the function such that for all $x \in \mathcal{E}^{N}, \tilde{f}^{i}(x)=1-x_{i}$. Note that for all $x \in \mathcal{E}^{N}$, $\sum_{i \in N} \tilde{f}^{i}(x)=n-\sum_{i \in N} x_{i}=n-1$. The same argument as in the previous (If)-part yields the desired conclusion. 


\subsection{Replacement-Domination}

This section studies the solidarity property replacement-domination: when an agent replaces his preference relation by another one, then this replacement affects the welfares of all other agents in the same direction, i.e., these agents all weakly gain or they all weakly lose as a result of the replacement.

Replacement-domination: For all $i \in N$ and all $R, R^{\prime} \in \mathcal{D}^{N}$, if $R^{\prime}$ is an $i$-deviation of $R$, then either [for all $\left.j \in N \backslash\{i\}, \Phi_{j}(R) R_{j} \Phi_{j}\left(R^{\prime}\right)\right]$, or [for all $\left.j \in N \backslash\{i\}, \Phi_{j}\left(R^{\prime}\right) R_{j} \Phi_{j}(R)\right]$.

First, we introduce the class of serial random dictatorship rules. For each profile, the following principle underlies these rules:

(i) If there are agents who strictly prefer 1 to 0 , then each agent who strictly prefers 1 to 0 has a certain probability to receive the social endowment, i.e., to become the dictator. These probabilities sum up to 1 , which means that at those profiles the social endowment is allocated through a random dictatorship among the agents who strictly prefer receiving the social endowment to receiving nothing.

(ii) If no agent strictly prefers 1 to 0 and some agents are indifferent between 0 and 1 , then the social endowment is allocated through an arbitrarily chosen random dictatorship among those agents.

(iii) If all agents strictly prefer 0 to 1 then a pre-selected distribution on all unit vectors is assigned. At those profiles, each agent has a certain probability to receive the social endowment.

These rules are called serial when they satisfy the following probabilistic analogue of deterministic serial dictatorships: the probability of being the dictator does not increase when more agents strictly prefer receiving the social endowment to receiving nothing. For a formal description, we introduce the following notation.

Let $\mathcal{G}^{N}$ denote the set of functions $g: \mathcal{D}^{N} \rightarrow \mathcal{E}^{N}$ such that $\sum_{i \in N_{0}(R)} g_{i}(R)=1$ when $N_{+}(R)=\emptyset$ and $N_{0}(R) \neq \emptyset$. By $2^{N^{\prime}}$ we denote the set of all coalitions $S \subseteq N$, including the empty set. Let $\mathcal{A}^{N}$ denote the set of all functions $\alpha: 2^{N} \rightarrow \mathcal{E}^{N}$ such that for all $S \in 2^{N} \backslash\{\emptyset\}$

- $\sum_{i \in S} \alpha_{i}(S)=1$, and

- for all $i \in S$ and all $j \in N, \alpha_{i}(S) \geq \alpha_{i}(S \cup\{j\})$. 
Serial Random Dictatorship Rule, $\Psi_{\alpha, g}$ : For all $\alpha \in \mathcal{A}^{N}$ and all $g \in \mathcal{G}^{N}$, the serial random dictatorship rule $\Psi_{a, g}$ is defined as follows. For all $R \in \mathcal{D}^{N}$,

(i) when $N_{+}(R) \neq \emptyset$, for all $i \in N, \Psi_{\alpha, g}(R)\left(\left\{e^{i}\right\}\right)=\alpha_{i}\left(N_{+}(R)\right)$;

(ii) when $N_{+}(R)=\emptyset$ and $N_{0}(R) \neq \emptyset$, for all $i \in N, \Psi_{\alpha, g}(R)\left(\left\{e^{i}\right\}\right)=g_{i}(R)$; and

(iii) when $N_{-}(R)=N$, for all $i \in N, \Psi_{\alpha, g}(R)\left(\left\{e^{i}\right\}\right)=\alpha_{i}(\emptyset)$.

Note that the distribution $\Psi_{a, g}(R)$ is uniquely defined because for all $S \in 2^{N}, \alpha(S) \in$ $\mathcal{E}^{N}$, and for all $R \in \mathcal{D}^{N}, g(R) \in \mathcal{E}^{N}$.

It is easy to see that the serial random dictatorship rules satisfy Pareto-optimality, strategy-proofness, and replacement-domination.

Theorem 9.1 The serial random dictatorship rules are the only probabilistic rules satisfying Pareto-optimality, strategy-proofness, and replacement-domination.

We prove Theorem 9.1 in 3 steps. First, we discuss non-bossiness conditions, and show that Pareto-optimality and replacement-domination imply weak non-bossiness (in terms of welfare). In the second step we show that every probabilistic rule satisfying the properties of Theorem 9.1 always assigns distributions with probability 1 on the set of unit vectors $E^{N}$. The final step proves the characterization.

Under non-bossiness (in terms of welfare) ${ }^{8}$, if an agent deviates and he is indifferent between his initial marginal distribution and his new marginal distribution, then so should all other agents. In the deterministic model, Klaus (1999b) proves the incompatibility of Pareto-optimality, strategy-proofness, and non-bossiness (in terms of welfare). In our probabilistic model, we show that non-bossiness (in terms of welfare) is not compatible with Pareto-optimality. Note that our result remains valid in the deterministic model. It strengthens the incompatibility of Klaus (1999b). In the proof it is crucial that agents may be indifferent between 0 and 1 .

Non-Bossiness (in Terms of Welfare): For all $i \in N$ and all $R, R^{\prime} \in \mathcal{D}^{N}$, if $R^{\prime}$ is an $i$-deviation of $R$ and $\Phi_{i}(R) I_{i} \Phi_{i}\left(R^{\prime}\right)$, then for all $j \in N \backslash\{i\}, \Phi_{j}(R) I_{j} \Phi_{j}\left(R^{\prime}\right)$.

Proposition 9.1 There exists no probabilistic rule satisfying Pareto-optimality and nonbossiness (in terms of welfare).

\footnotetext{
${ }^{8}$ The concept of non-bossiness was first introduced by Satterthwaite and Sonnenschein (1981).
} 
Proof. Suppose that $\Phi$ satisfies Pareto-optimality and non-bossiness. Let $R_{1} \in \mathcal{D}$ be such that $0 P_{1} \frac{1}{n} I_{1} 1$, and $R=\left(R_{1}\right)_{i \in N} \in \mathcal{D}^{N}$. By Lemma 9.2, (iii), Pareto-optimality implies

$$
\Phi(R)\left(E^{N}\right)=1
$$

Let $i \in N$ be such that $\Phi(R)\left(\left\{e^{i}\right\}\right)<1$, and $R^{\prime} \in \mathcal{D}^{N}$ be an $i$-deviation of $R$ such that $0 I_{i}^{\prime} 1$. By Lemma 9.2, Pareto-optimality implies $\Phi\left(R^{\prime}\right)\left(\left\{e^{i}\right\}\right)=1$. By $B\left(0, R_{i}^{\prime}\right)=\{0,1\}$ and (9.5), $\Phi_{i}\left(R^{\prime}\right) I_{i}^{\prime} \Phi_{i}(R)$. Because $\Phi(R)\left(\left\{e^{i}\right\}\right)<1$ and (9.5), there exists $j \neq i$ such that $\Phi(R)\left(\left\{e^{j}\right\}\right)>0$. By $\Phi\left(R^{\prime}\right)\left(\left\{e^{i}\right\}\right)=1$, for all $x \in[0,1]$,

$$
\Phi_{j}\left(R^{\prime}\right)\left(B\left(x, R_{j}^{\prime}\right)\right)=1 \geq \Phi_{j}(R)\left(B\left(x, R_{j}^{\prime}\right)\right)
$$

and

$$
\Phi_{j}\left(R^{\prime}\right)\left(B\left(0, R_{j}^{\prime}\right)\right)=\Phi_{j}\left(R^{\prime}\right)(\{0\})=1>\Phi_{j}(R)\left(B\left(0, R_{j}^{\prime}\right)\right)
$$

Thus, $\Phi_{j}\left(R^{\prime}\right) P_{j}^{\prime} \Phi_{j}(R)$, which contradicts non-bossiness.

The following weaker version of non-bossiness is compatible with Pareto-optimality. Here, non-bossiness is only required to hold whenever the marginal probability distribution of the deviating agent remains unchanged.

Weak Non-Bossiness (in Terms of Welfare): For all $i \in N$ and all $R, R^{\prime} \in \mathcal{D}^{N}$, if $R^{\prime}$ is an $i$-deviation of $R$ and $\Phi_{i}(R)=\Phi_{i}\left(R^{\prime}\right)$, then for all $j \in N \backslash\{i\}$. $\Phi_{j}(R) I_{j} \Phi_{j}\left(R^{\prime}\right)$.

In the sequel we use the shorter phrase of weak non-bossiness for weak non-bossiness in terms of welfare.

Lemma 9.3 Let $\Phi$ be a probabilistic rule satisfying Pareto-optimality and replacementdomination. Then $\Phi$ satisfies weak non-bossiness.

Proof. Let $i \in N$, and $R, R^{\prime} \in \mathcal{D}^{N}$ be such that $R^{\prime}$ is an $i$-deviation of $R$ and

$$
\Phi_{i}(R)=\Phi_{i}\left(R^{\prime}\right)
$$

We have to show that for all $j \in N \backslash\{i\}, \Phi_{j}(R) I_{j} \Phi_{j}\left(R^{\prime}\right)$. By replacement-domination, there are two cases.

Case 1: For all $j \in N \backslash\{i\}, \Phi_{j}\left(R^{\prime}\right) R_{j} \Phi_{j}(R)$. 
Thus, by (9.6), for all $j \in N, \Phi_{j}\left(R^{\prime}\right) R_{j} \Phi_{j}(R)$. Hence, by Pareto-optimality, for all $j \in N, \Phi_{j}(R) I_{j} \Phi_{j}\left(R^{\prime}\right)$.

Case 2: For all $j \in N \backslash\{i\}, \Phi_{j}(R) R_{j} \Phi_{j}\left(R^{\prime}\right)$.

Similarly as in Case 1 , for all $j \in N \backslash\{i\}, \Phi_{j}(R) I_{j} \Phi_{j}\left(R^{\prime}\right)$.

In the deterministic model, Klaus, Peters, and Storcken (1997b) prove that if an allocation rule satisfies Pareto-optimality, strategy-proofness, and replacement-domination, then it always assigns the social endowment to one agent. A similar result holds for the probabilistic model. If a probabilistic rule satisfies these properties, then it selects only distributions placing probability 1 on the set of unit vectors $E^{N}$. There is never positive probability on broken allocations, i.e., an allocation where no agent receives the social endowment.

Let $\mathcal{B}_{\Phi} \equiv\left\{R \in \mathcal{D}^{N} \mid \Phi(R)\left(E^{N}\right)<1\right\}$ be the set of profiles that assign positive probability to broken allocations. Given $R \in \mathcal{D}^{N}$, let $B_{\Phi}(R) \equiv\left\{i \in N \mid \Phi_{i}(R)(] 0,1[)>0\right\}$ denote the set of agents who have positive probability on broken allotments at $R$.

Remark 9.1 If one agent has positive probability on broken allotments, then at least one other agent has positive probability on broken allotments. Therefore, if $B_{\Phi}(R) \neq \emptyset$, then $\left|B_{\Phi}(R)\right| \geq 2$.

First, we prove a useful tool for the proof of Lemma 9.5 .

Lemma 9.4 Let $\Phi$ be a probabilistic rule satisfying strategy-proofness. Let $i \in N$ and $R, R^{\prime} \in \mathcal{D}^{N}$ be such that $R^{\prime}$ is an $i$-deviation of $R$ and $i \in N_{+}(R)=N_{+}\left(R^{\prime}\right)$. Then for all $x_{i} \in\left[\max \left\{r_{i}(0), r_{i}^{\prime}(0)\right\}, 1\right]$,

$$
\left.\left.\left.\Phi_{i}(R)(] x_{i}, 1\right]\right)=\Phi_{i}\left(R^{\prime}\right)\left(j x_{i}, 1\right]\right)
$$

and for all $\left.\left.x_{i} \in\right] \max \left\{r_{i}(0), r_{i}^{\prime}(0)\right\}, 1\right]$,

$$
\Phi_{i}(R)\left(\left[x_{i}, 1\right]\right)=\Phi_{i}\left(R^{\prime}\right)\left(\left[x_{i}, 1\right]\right)
$$

Proof. First, we prove (9.7). Let $x_{i} \in\left[\max \left\{r_{i}(0), r_{i}^{\prime}(0)\right\}, 1\right]$. Then $B^{o}\left(x_{i}, R_{i}\right)=$ ]$\left.x_{i}, 1\right]=B^{o}\left(x_{i}, R_{i}^{\prime}\right)$. By strategy-proofness, $\Phi_{i}(R)\left(B^{o}\left(x_{i}, R_{i}\right)\right) \geq \Phi_{i}\left(R^{\prime}\right)\left(B^{o}\left(x_{i}, R_{i}\right)\right)$ and $\Phi_{i}\left(R^{\prime}\right)\left(B^{o}\left(x_{i}, R_{i}^{\prime}\right)\right) \geq \Phi_{i}(R)\left(B^{o}\left(x_{i}, R_{i}^{\prime}\right)\right)$. Thus, (9.7) holds. A similar argument, yields (9.8).

Lemma 9.5 Let $\Phi$ be a probabilistic rule satisfying Pareto-optimality, strategy-proofness, and replacement-domination. Then $\mathcal{B}_{\Phi}=\emptyset$. 
Proof. Suppose that $\mathcal{B}_{\Phi} \neq \emptyset$. For all $R \in \mathcal{D}^{N}$, if $N_{+}(R)=\emptyset$ and $N_{0}(R) \neq \emptyset$, then Pareto-optimality implies $R \notin \mathcal{B}_{\mathbb{\Phi}}$. First, we suppose that there exists $R \in \mathcal{B}_{\mathbb{q}}$ such that $N_{+}(R) \neq \emptyset$. The proof for the case $R \in \mathcal{B}_{\Phi}$ and $N_{-}(R)=N$ is outlined after the case $N_{+}(R) \neq \emptyset$.

The first two claims show the existence of profiles satisfying our previous assumption and certain other properties related to the indifferent allotments 0 and $r_{i}(0)$ for the agents who strictly prefer 1 to 0 . Then by certain unilateral deviations the chosen distribution places probability 1 on the set of unit vectors. This yields the desired contradiction in the third claim.

Claim 1: There exists $R \in \mathcal{B}_{\Phi}$ such that $N_{+}(R) \neq \emptyset$ and for all $i \in B_{\Phi}(R), 0<r_{i}(0)<\frac{1}{2}$. Proof of Claim 1: Let $\bar{R} \in \mathcal{B}_{\Phi}$ be such that $N_{+}(\bar{R}) \neq \emptyset$. By Lemma 9.2, Pareto-optimality implies $B_{\Phi}(\bar{R}) \subseteq N_{+}(\bar{R})$. We construct $R \in \mathcal{B}_{\Phi}$ such that for all $i \in B_{\Phi}(\bar{R}), 0<r_{i}(0)<\frac{1}{2}$. Successive application of Cases 1 and 2 yields the desired $R$.

Case 1: For some $i \in B_{\Phi}(\bar{R}), \bar{r}_{i}(0)=0$.

Then there exists an allotment $\left.x_{i} \in\right] 0, \frac{1}{2}\left[\right.$ such that $\Phi_{i}(\bar{R})(] x_{i}, 1[)>0$. Let $R^{\prime} \in \mathcal{D}^{N}$ be an $i$-deviation of $\bar{R}$ such that $1 P_{i}^{\prime} x_{i} I_{i}^{\prime} 0$. Then $i \in N_{+}(\bar{R})=N_{+}\left(R^{\prime}\right)$. Lemma 9.4 implies

$$
\Phi_{i}(\bar{R})\left(B\left(1, \vec{R}_{i}\right)\right)=\Phi_{i}(\bar{R})(\{1\})=\Phi_{i}\left(R^{\prime}\right)\left(B\left(1, R_{i}^{\prime}\right)\right)
$$

and, because $r_{i}^{\prime}(0)=x_{i}>0=\bar{r}_{i}(0)$,

$$
\left.\left.\left.\left.\Phi_{i}(\bar{R})(] x_{i}, 1\right]\right)=\Phi_{i}\left(R^{\prime}\right)(] x_{i}, 1\right]\right)
$$

Therefore, (9.9) and (0.10) together imply $\left.\Phi_{i}\left(R^{\prime}\right)(] x_{i}, 1[)=\Phi_{i}(\bar{R})(] x_{i}, 10\right)>0$. Thus, $B_{\Phi}\left(R^{\prime}\right) \neq \emptyset$ and $0<r_{i}^{\prime}(0)<\frac{1}{2}$.

Case 2: For some $i \in B_{\Phi}(\bar{R}), \bar{r}_{i}(0) \geq \frac{1}{2}$.

Because $\bar{r}_{i}(0)<1$, the same arguments of Case 1 can be applied.

For all $R \in \mathcal{B}_{\Phi}$, such that $N_{+}(R) \neq \emptyset$, let $\gamma_{\Phi}(R) \equiv \min \left\{r_{i}(0) \mid i \in B_{\Phi}(R)\right\}$.

Claim 2: There exists $R \in \mathcal{B}_{\Phi}$ such that $N_{+}(R) \neq \emptyset$ satisfying the following conditions.

(a) For all $i \in B_{\Phi}(R), 0<r_{i}(0)<\frac{1}{2}$; and

(b) for all $i \in N_{+}(R) \backslash B_{\Phi}(R), 1 P_{i} r_{i}(0) R_{i}\left(1-\gamma_{\Phi}(R)\right)$.

Proof of Claim 2: By Claim 1, there exists $\bar{R} \in \mathcal{B}_{\Phi}$ such that for all $i \in B_{\Phi}(\bar{R}), 0<$ $\bar{r}_{i}(0)<\frac{1}{2}$. Suppose that for some $j \in N_{+}(\bar{R}) \backslash B_{\Phi}(\bar{R}), \bar{r}_{j}(0)<1-\gamma_{\Phi}(\bar{R})$. (Otherwise, $\bar{R}$ proves Claim 2) Let $R^{\prime} \in \mathcal{D}^{N}$ be an $j$-deviation of $\bar{R}$ such that $1 P_{j}^{\prime}\left(1-\gamma_{\Phi}(\bar{R})\right) I_{j}^{\prime} 0$. We 
show $B_{\Phi}(\bar{R})=B_{\Phi}\left(R^{\prime}\right)$. Because $j \in N_{+}(\bar{R})=N_{+}\left(R^{\prime}\right)$ and $\bar{r}_{j}(0)<1-\gamma_{\Phi}(\tilde{R})=r_{j}^{\prime}(0)$, Lemma 9.4 implies

$$
\left.\left.\Phi_{j}(\bar{R})\left(\mid r_{j}^{\prime}(0), 1\right]\right)=\Phi_{j}\left(R^{\prime}\right)\left(\mid r_{j}^{\prime}(0), 1\right]\right)
$$

and

$$
\Phi_{j}(\bar{R})(\{1\})=\Phi_{j}\left(R^{\prime}\right)(\{1\}) .
$$

Since $j \in N_{+}(\bar{R}) \backslash B_{\Phi}(\bar{R}), \Phi_{j}(\bar{R})\left(\gamma r_{j}^{\prime}(0), 1[)=0\right.$. Hence, from (9.11) and (9.12) it follows that $\Phi_{j}\left(R^{\prime}\right)(] r_{j}^{\prime}(0), 1[)=0$. By Pareto-optimality, $\left.\left.\Phi_{j}\left(R^{\prime}\right)(] 0, r_{j}^{\prime}(0)\right]\right)=0$. Thus, $\Phi_{j}(\bar{R})=$ $\Phi_{j}\left(R^{\prime}\right)$. By Lemma $9.3, \Phi$ satisfies weak non-bossiness. Hence, by weak non-bosiness, for all $i \in N \backslash\{j\}, \Phi_{i}(\bar{R}) I_{i} \Phi_{i}\left(R^{\prime}\right)$. Thus, $B_{\Phi}(\bar{R})=B_{\Phi}\left(R^{\prime}\right), \gamma_{\Phi}\left(R^{\prime}\right)=\gamma_{\Phi}(\bar{R})$, and $R^{\prime} \in \mathcal{B}_{\Phi}$. Moreover, for all $i \in B_{\Phi}\left(R^{\prime}\right), 0<r_{i}^{\prime}(0)<\frac{1}{2}$. Successive replacement of the preferences $\bar{R}_{k}$, for each $k \in N_{+}(\bar{R}) \backslash B_{\Phi}(\bar{R})$ such that $\bar{r}_{k}(0)<\gamma_{\Phi}(\bar{R})$, by $R_{j}^{\prime}$ yields the desired profile $R$. $。$

The final claim establishes the contradiction.

Claim 3: For all $R \in \mathcal{B}_{\Phi}, N_{+}(R)=\emptyset$.

Proof of Claim 3: By Claim 2 and because $N$ is finite, we may fix $R \in \mathcal{B}_{\Phi}$ satisfying (a) and (b) of Claim 2 such that $\left|B_{\Phi}(R)\right|$ is mininal among the profiles satisfying (a) and (b) of Clain 2. Note that by Remark $9.1,\left|B_{\Phi}(R)\right| \geq 2$. Let $i \in B_{\Phi}(R)$ and $R^{\prime} \in \mathcal{D}^{N}$ be an $i$-deviation of $R$ such that $1 P_{i}^{\prime}\left(1-\gamma_{\Phi}(R)\right) I_{i}^{\prime} 0$. Let $\tilde{\gamma}=\min \left\{r_{j}(0) \mid j \in B_{\Phi}(R) \backslash\{i\}\right\}$. Then $\tilde{\gamma} \geq \gamma_{\Phi}(R)$. We show $B_{\Phi}\left(R^{\prime}\right)=\emptyset$.

Because $R$ satisfies (a) and (b) of Claim 2, for all $j \in N_{+}(R) \backslash B_{\Phi}(R), \tilde{\gamma}<\frac{1}{2}<$ $(1-\tilde{\gamma}) \leq\left(1-\gamma_{\Phi}(R)\right) \leq r_{j}^{\prime}(0)$. Because $\tilde{\gamma}=\min \left\{r_{j}(0) \mid j \in B_{\Phi}(R) \backslash\{i\}\right\}$, the previous fact and Pareto-optimality imply for all $k \in N$,

$$
\Phi_{k}\left(R^{\prime}\right)(] 0, \tilde{\gamma}[)=0
$$

Hence,

$$
\left.\left.\Phi_{i}\left(R^{\prime}\right)(] 1-\tilde{\gamma}, 1\right]\right)=0
$$

because otherwise there exists $k \in N_{+}(R)$ such that $\Phi_{k}\left(R^{\prime}\right)(] 0, \tilde{\gamma}[)>0$, which contradicts (9.13). By Pareto-optimality,

$$
\Phi_{i}\left(R^{\prime}\right)(\{0\} \cup[1-\tilde{\gamma}, 1])=1 .
$$

Thus, $i \notin B_{\Phi}\left(R^{\prime}\right)$. For all $k \in N_{+}(R) \backslash B_{\Phi}(R), r_{k}(0) \geq 1-\tilde{\gamma}$ and $(9.13)$ imply $\Phi_{k}\left(R^{\prime}\right)(] 1-$ $\tilde{\gamma}, 1[)=0$. Hence, $k \notin B_{\Phi}\left(R^{\prime}\right)$. Thus, if $B_{\Phi}\left(R^{\prime}\right) \neq \emptyset$, then $B_{\Phi}\left(R^{\prime}\right) \subseteq B_{\Phi}(R) \backslash\{i\}$ and $\gamma_{\Phi}\left(R^{\prime}\right) \geq \tilde{\gamma}$. In particular, using the same arguments as in Claim 2 we can construct 
$\bar{R} \in \mathcal{D}^{N}$ such that $B_{\Psi}(\bar{R})=B_{\Phi}\left(R^{\prime}\right)$ and $\bar{R}$ satisfies (a) and (b) of Claim 2. But then, by $i \notin B_{\Phi}(\bar{R}),\left|B_{\Phi}(\bar{R})\right|<\left|B_{\Phi}(R)\right|$. By our choice of $R,\left|B_{\Phi}(R)\right|$ is minimal among the profiles satisfying (a) and (b) of Clain 2 . Hence, the previous three facts imply $R^{\prime} \notin \mathcal{B}_{\Phi}$ and $B_{4}\left(R^{\prime}\right)=\emptyset$.

By Lemma 9.4 and $i \in N_{+}(R)=N_{+}\left(R^{\prime}\right)$,

$$
\Phi_{i}(R)(\{1\})=\Phi_{i}\left(R^{\prime}\right)(\{1\}) .
$$

Because $i \in B_{\Phi}(R),(9.14),(9.15)$, and (9.16) imply

$$
\Phi_{i}\left(R^{\prime}\right)(\{0\})=1-\Phi_{i}\left(R^{\prime}\right)(\{1\})=\Phi_{i}(R)\left(\left[0,1[)>\Phi_{i}(R)(\{0\}) .\right.\right.
$$

Therefore, by Pareto-optimality, $\Phi\left(R^{\prime}\right)\left(E^{N_{+}(R)}\right)=1$. Hence, by replacement-domination and (9.17), for all $j \in N \backslash\{i\}$ and all $x_{j} \in[0,1]$,

$$
\Phi_{j}(R)\left(B\left(x_{j}, R_{j}\right)\right) \leq \Phi_{j}\left(R^{\prime}\right)\left(B\left(x_{j}, R_{j}\right)\right) .
$$

By (9.17), (9.18), and $\Phi\left(R^{\prime}\right)\left(E^{N_{+}(R)}\right)=1$, there exists $j \in B_{\Phi}(R) \backslash\{i\}$ such that

$$
\Phi_{j}\left(R^{\prime}\right)(\{1\})>\Phi_{j}(R)(\{1\}) .
$$

Let $R^{\prime \prime} \in \mathcal{D}^{N}$ be a $j$-deviation of $R$ such that $R_{j}^{\prime \prime}=R_{i}^{\prime}$, and $R^{\prime \prime \prime} \in \mathcal{D}^{N}$ be an $i$-deviation of $R^{\prime \prime}$ such that $R_{i}^{\prime \prime \prime}=R_{i}^{\prime}$. Since $j \in N_{+}\left(R^{\prime}\right)=N_{+}\left(R^{\prime \prime \prime}\right)$, Lemma 9.4 implies for the $j$-deviation $R^{\prime \prime \prime}$ of $R^{\prime}, \Phi_{j}\left(R^{\prime}\right)=\Phi_{j}\left(R^{\prime \prime \prime}\right)$. Weak non-bossiness implies $\Phi\left(R^{\prime}\right)=\Phi\left(R^{\prime \prime \prime}\right)$.

Similarly, by interchanging the roles of $i$ and $j$,

$$
\Phi_{j}\left(R^{\prime \prime}\right)(\{0\})=1-\Phi_{j}\left(R^{\prime \prime}\right)(\{1\})=\Phi_{j}(R)\left(\left[0,1[)>\Phi_{j}(R)(\{0\})\right.\right.
$$

and $\Phi\left(R^{\prime \prime}\right)=\Phi\left(R^{\prime \prime \prime}\right)$. Thus,

$$
\Phi_{j}(R)(\{1\})=\Phi_{j}\left(R^{\prime \prime}\right)(\{1\})=\Phi_{j}\left(R^{\prime \prime \prime}\right)(\{1\}),
$$

which contradicts (9.19) and $\Phi\left(R^{\prime}\right)=\Phi\left(R^{\prime \prime \prime}\right)$. Thus, for all $R \in \mathcal{B}_{\Phi}, N_{+}(R)=\emptyset$.

We outline the proof for the case $R \in \mathcal{B}_{\Phi}$ and $N_{-}(R)=N$. First, observe the following fact. For all $i \in N$ and $R, R^{\prime} \in \mathcal{D}^{N}$, when $R^{\prime}$ is an $i$-deviation of $R$ and $i \in N_{-}(R)=N_{-}\left(R^{\prime}\right)$, analogous to Lemma 9.4 it can be proven that for all $x_{i} \in$ $\left[0, \min \left\{r_{i}(1), r_{i}^{\prime}(1)\right\}\right], \Phi_{i}(R)\left(\left[0, x_{i}[)=\Phi_{i}\left(R^{\prime}\right)\left(\left[0, x_{i}[)\right.\right.\right.\right.$, and for all $x_{i} \in\left[0, \min \left\{r_{i}(1), r_{i}^{\prime}(1)\right\}[\right.$, $\Phi_{i}(R)\left(\left[0, x_{i}\right]\right)=\Phi_{i}\left(R^{\prime}\right)\left(\left[0, x_{i}\right]\right)$. We will refer to this fact as Lemma 9.4'. Using Lemma 9.4' and the same arguments as in Claim 1, the following claim can be proven.

Claim 4: There exists $R \in \mathcal{B}_{\Phi}$ such that $N_{-}(R)=N$ and for all $i \in B_{\Phi}(R), \frac{1}{2}<r_{i}(1)<1$.

For all $R \in \mathcal{B}_{\Phi}$ such that $N_{-}(R)=N$, let $\delta_{\Phi}(R) \equiv \max \left\{r_{i}(1) \mid i \in B_{\Phi}(R)\right\}$. Lising similar arguments as in Claim 2, the following claim can be proven.

Claim 5: There exists $R \in \mathcal{B}_{\Phi}$ such that $N_{-}(R)=N$ satisfying the following conditions. 
(a) For all $i \in B_{\Phi}(R), \frac{1}{2}<r_{i}(1)<1$; and

(b) for all $i \in N \backslash B_{\Phi}(R), 0 P_{i} r_{i}(1) R_{i} \min \left\{\frac{1}{n}, \frac{1-\delta_{\Phi}(R)}{n}\right\}$.

We describe how to derive a contradiction. Let $R \in \mathcal{B}_{\Phi}$. By Claims 4 and 5 , we may suppose that $R$ satisfies (a) and (b) of Claim 5. Let $j \in B_{\Phi}(R)$ and $\vec{R}_{j} \in \mathcal{D}$ be such that $1 \bar{P}_{j} \bar{r}_{j}(1) \bar{R}_{j} \min \left\{\frac{1}{n}, \frac{1-\delta_{\Phi}(R)}{n}\right\}$. For $S \subseteq B_{\Phi}(R)$, we consider $\tilde{R}^{S} \in \mathcal{D}^{N}$ such that for all $i \in S, \tilde{R}_{i}=\bar{R}_{j}$, and for all $i \in N \backslash S, \tilde{R}_{i}=R_{i}$. Observe the following facts. First, by our choices of $\delta_{\Phi}(R), R$, and $\bar{R}_{j}$, Pareto-optimality implies $\Phi\left(\tilde{R}^{B_{\phi}(R)}\right)\left(E^{N}\right)=1$. Hence, $B_{\Phi}\left(\tilde{R}^{B_{\Phi}(R)}\right)=\emptyset$, and $\tilde{R}^{B_{\Phi}(R)} \notin \mathcal{B}_{\Phi}$. Second, by our choices of $\delta_{\Phi}(R), R$, and $\bar{R}_{j}$, if for some $S \subseteq B_{\Phi}(R), B_{\Phi}\left(\tilde{R}^{S}\right) \neq \emptyset$, then $\left.\mid B_{\Phi}, \tilde{R}^{S}\right) \cap\left(B_{\Phi}(R) \backslash S\right) \mid \geq 2$.

Hence, by the previous two facts there exists $T \subseteq B_{\Phi}(R), T \neq B_{\Phi}(R)$, such that $B_{\Phi}\left(\tilde{R}^{T}\right) \neq \emptyset$ and $\left|B_{\Phi}\left(\tilde{R}^{T}\right) \cap\left(B_{\Phi}(R) \backslash T\right)\right|$ is minimal. In particular, by the second fact, $\left|B_{\Phi}\left(\tilde{R}^{T}\right) \cap\left(B_{\Phi}(R) \backslash T\right)\right| \geq 2$. By the choice of $T$ and Lemma $9.4^{\prime}$, for all $i \in B_{\Phi}(R) \backslash T$, $i \in B_{\Phi}\left(\tilde{R}^{T}\right)$. Let $j, k \in B_{\Phi}\left(\tilde{R}^{T}\right) \cap\left(B_{\Phi}(R) \backslash T\right)$. Then by our choice of $T$ it follows that $B_{\Phi}\left(\tilde{R}^{T \cup\{j\}}\right)=\emptyset$ and $B_{\Phi}\left(\tilde{R}^{T \cup\{k\}}\right)=\emptyset$. Then the same argument as in Claim 3 yields a contradiction.

Finally, we complete the proof of Theorem 9.1 .

Proof of Theorem 9.1: Let $\Phi$ be a probabilistic rule satisfying Pareto-optimality, strategy-proofness, and replacement-domination. By Lemma 9.3, $\Phi$ satisfies weak nonbossiness. By Lemma 9.5, for all $R \in \mathcal{D}$,

$$
\Phi(R)\left(E^{N}\right)=1
$$

First we define $\alpha \in \mathcal{A}^{N}$. Given $S \subseteq N$, let $R^{S} \in \mathcal{D}^{N}$ be such that for all $i \in S$, $d\left(R_{i}\right)=0$, and for all $i \in N \backslash S, d\left(R_{i}\right)=1$. For all $S \subseteq N$ and all $i \in N$, define $\alpha_{i}(S) \equiv \Phi\left(R^{S}\right)\left(\left\{e^{i}\right\}\right)$. By $(9.20), \sum_{i \in N} \alpha_{i}(\emptyset)=1$. For all $S \in 2^{N} \backslash\{\emptyset\}$, by Paretooptimality and $(9.20), \sum_{i \in S} \alpha_{i}(S)=1$, and by replacement-domination, for all $i \in S$ and all $j \in N, \alpha_{i}(S) \geq \alpha_{i}(S \cup\{j\})$. Hence, the previous three facts imply $\alpha \in \mathcal{A}^{N}$.

Next we define $g \in \mathcal{G}^{N}$. For all $R \in \mathcal{D}^{N}$, when $N_{+}(R)=\emptyset$ and $N_{0}(R) \neq \emptyset$, for all $i \in N, g_{i}(R) \equiv \Phi(R)\left(\left\{e^{i}\right\}\right)$, and otherwise, $g(R)=e^{1}$. By Pareto-optimality, $g \in \mathcal{G}^{N}$.

Weak non-bossiness, strategy-proofness, and (9.20) imply that for all $R \in \mathcal{D}^{N}$, the chosen distribution $\Phi(R)$ depends only on $N_{+}(R)$ when $N_{+}(R) \neq \emptyset$, or on $N_{-}(R)$ when $N_{-}(R)=N$. Therefore, by definition of $\alpha$ and $g, \Phi=\Psi_{\alpha, g}$ and $\Phi$ is a serial random dictatorship rule. 
Remark 9.2 Each probabilistic rule satisfying Pareto-optimality, strategy-proofness, and replacement-domination, except the case when $N_{+}(R)=\emptyset$ and $N_{0}(R) \neq \emptyset$, depends only on the most preferred consumptions of the agents, i.e., the "peaks".

Remark 9.3 Our probabilistic model is an extension of the deterministic model. Every deterministic allocation rule can be defined as a one point probabilistic rule the rule assigns only distributions with probability 1 on a single allocation and vice versa. Then the definitions of all properties coincide with the corresponding definitions in the deterministic setting. Therefore, Theorem 9.1 implies the characterization of Klaus, Peters, and Storcken (1997b). In particular, every convex combination of deterministic serial dictatorship rules is a serial random dictatorship rule.

\subsection{Fairness}

In this section we consider fairness properties. No-envy ${ }^{9}$ requires that each agent should weakly prefer his marginal distribution to any other agent's marginal distribution. In the deterministic model, Klaus (1999b) shows the incompatibility of Pareto-optinality and no-envy. In the probabilistic model, these properties are even compatible with strategyproofness. We characterize the class of probabilistic rules satisfying Pareto-optimality, strategy-proofness, and no-envy.

No-envy: For all $R \in \mathcal{R}^{N}$ and all $i, j \in N, \Phi_{i}(R) R_{i} \Phi_{j}(R)$.

Like in the previous section, we show that a probabilistic rule satisfying these properties never selects a distribution placing positive probability on broken allocations. We use the same notation as in Lemma 9.3.

Lemma 9.6 Let $\Phi$ be a probabilistic rule satisfying Pareto-optimality, strategy-proofness, and no-envy. Then $\mathcal{B}_{\Phi}=\emptyset$.

Proof. Let $R \in \mathcal{D}^{N}$. We show $R \notin \mathcal{B}_{\boldsymbol{\Phi}}$.

Case 1: $\left|N_{+}(R)\right| \geq 2$.

By no-envy, for all $i, j \in N_{+}(R)$,

$$
\Phi_{i}(R)(\{1\})=\Phi_{j}(R)(\{1\}) .
$$

\footnotetext{
${ }^{9}$ For an analysis of no-envy for the problem of fair allocation when preferences are single-peaked, we refer to Sprumont (1991) and Thomson (1997).
} 
It suffices to show that for all $i \in N_{+}(R)$,

$$
\Phi_{i}(R)(\{1\})=\frac{1}{\left|N_{+}(R)\right|} .
$$

Let $i \in N_{+}(R)$ and $R^{\prime} \in \mathcal{D}^{N}$ be an $i$-deviation of $R$ such that $1 P_{i}^{\prime} \frac{3}{4} l_{i}^{\prime} 0$. Since $i \in N_{+}\left(R^{\prime}\right)=$ $N_{+}(R)$, Lemma 9.4 implies $\Phi_{i}\left(R^{\prime}\right)(\{1\})=\Phi_{i}(R)(\{1\})$. Therefore, by no-envy, for all $j \in N_{+}(R)$,

$$
\Phi_{j}\left(R^{\prime}\right)(\{1\})=\Phi_{i}(R)(\{1\})
$$

Thus, we can successively replace all preferences $R_{j}$, for $j \in N_{+}(R)$, by preference $R_{i}^{\prime}$ without affecting the probabilities placed on the unit vectors $e^{j}$. Denote the resulting profile by $\bar{R}$. Then for all $j \in N_{+}(R), \Phi_{j}(\bar{R})(\{1\})=\Phi_{i}(R)(\{1\})$. Note that $N_{+}(\bar{R})=$ $N_{+}(R)$. Pareto-optimality implies $\Phi(\bar{R})\left(E^{N_{+}(\bar{R})}\right)=1$. Thus, by no-envy, for all $i \in N_{+}(\bar{R})$, $\Phi(\bar{R})\left(\left\{e^{i}\right\}\right)=\frac{1}{\left|N_{+}(\bar{R})\right|}$ and $\Phi_{i}(\bar{R})(\{1\})=\frac{1}{\left|N_{+}(R)\right|}$. Therefore, (9.21) holds and $R \notin \mathcal{B}_{\mathbf{\Phi}}$.

Case 2: $N_{-}(R)=N$.

By no-envy, for all $i, j \in N$,

$$
\Phi_{i}(R)(\{0\})=\Phi_{j}(R)(\{0\}) .
$$

It suffices to show that for all $i \in N$,

$$
\Phi_{i}(R)(\{0\})=1-\frac{1}{n} .
$$

Then for all $i \in N, \Phi_{i}(R)([0,1])=\frac{1}{n}$, and the fact that $\Phi(R)$ is a distribution on $\mathcal{E}^{N}$ implies for all $i \in N, \Phi(R)\left(\left\{e^{i}\right\}\right)=\frac{1}{n}$. Let $i \in N$ and $R^{\prime} \in \mathcal{D}^{N}$ be an $i$-deviation of $R$ such that $0 P_{i}^{\prime} \frac{1}{n} I_{i}^{\prime} 1$. Since $N_{-}\left(R^{\prime}\right)=N_{-}(R)$, by applying strategy-proofness twice, $\Phi_{i}\left(R^{\prime}\right)(\{0\})=\Phi_{i}(R)(\{0\})$. Therefore, by no-envy, for all $j \in N$,

$$
\Phi_{j}\left(R^{\prime}\right)(\{0\})=\Phi_{i}(R)(\{0\}) .
$$

Thus, we can successively replace all preferences $R_{j}$, for $j \in N$, by preference $R_{i}^{\prime}$ without affecting the marginal probabilities placed on 0 . Denote the resulting profile by $\bar{R}$. Then for all $j \in N_{+}(R), \Phi_{j}(\bar{R})(\{0\})=\Phi_{i}(R)(\{0\})$. Note that $N_{-}(\bar{R})=N$. Pareto-optimality implies $\Phi(\bar{R})\left(E^{N}\right)=1$. Thus, by no-envy, for all $i \in N, \Phi(\bar{R})\left(\left\{e^{i}\right\}\right)=\frac{1}{n}$. Hence, for all $i \in N, \Phi_{i}(\bar{R})(\{0\})=1-\frac{1}{n}$. Therefore, $(9.22)$ holds and $R \notin \mathcal{B}_{\Phi}$.

For all other cases the desired conclusion follows from Pareto-optimality.

A probabilistic rule satisfying Pareto-optimality, strategy-proofness, and no-envy selects only distributions that place probability one on the unit vectors. No-envy implies 
that these probabilities must be equal among the agents who strictly prefer 1 to 0 . Therefore, it is a serial random dictatorship rule where every agent who strictly prefers 1 to 0 has an equal chance to be the dictator. The same holds if all agents strictly prefer 0 to 1. We call such a rule a uniform random dictatorship rule. It coincides with the uniform probabilistic rule of Sasaki (1997) for one indivisible object.

Let $\gamma: 2^{N} \rightarrow \mathcal{E}^{N}$ denote the function such that for all $S \in 2^{N} \backslash\{\emptyset\}, \gamma(S) \equiv \frac{1}{|S|} \sum_{i \in S} e^{i}$, and $\gamma(\emptyset) \equiv \frac{1}{n} \sum_{i \in N} e^{i}$.

Uniform Random Dictatorship Rule, $U_{g}$ : For all $g \in \mathcal{G}^{N}, U_{g}=\Psi_{\gamma, g}$.

Theorem 9.2 The uniform random dictatorship rules are the only probabilistic rules satisfying Pareto-optimality, strategy-proofness, and no-envy.

Next, we consider the requirement of equal treatment of equals. ${ }^{10}$ This condition says that two agents with the same preference should be indifferent between their marginal distributions according to their common preference. In the deterministic model, Klaus (1999b) shows the incompatibility of Pareto-optimality and equal treatment of equals.

Equal treatment of equals: For all $R \in \mathcal{D}^{N}$ and all $i, j \in N$, if $R_{i}=R_{j}$, then $\Phi_{i}(R) I_{i} \Phi_{j}(R)$.

Equal treatment of equals is a minimal requirement. However, if a probabilistic rule selects only distributions that only place positive probability on unit vectors, then under Pareto-optimality and strategy-proofness, equal treatment of equals implies no-envy.

Proposition 9.2 Let $\Phi$ be a probabilistic rule satisfying Pareto-optimality and strategyproofness. Then $\Phi$ satisfies no-envy if and only if $\Phi$ satisfies equal treatment of equals and $\Phi(R)\left(E^{N}\right)=1$ for all $R \in \mathcal{D}^{N}$.

Proof. The (Only if)-part follows from Theorem 9.2.

Let $\Phi$ satisfy equal treatment of equals and $\Phi(R)\left(E^{N}\right)=1$ for all $R \in \mathcal{D}^{N}$. Suppose that $\Phi$ violates no-envy. Let $R \in \mathcal{D}^{N}$ be a profile for which no-envy is violated. By Pareto-optimality, either $\left|N_{+}(R)\right| \geq 2$ or $N_{-}(R)=N$. Suppose that $\left|N_{+}(R)\right| \geq 2$. (If $N_{-}(R)=N$, then similar arguments can be used to derive the conclusion) Because no-envy

\footnotetext{
${ }^{10}$ Equal treatment of equals was first formulated by Ching (1994) in our model when preferences are single-peaked.
} 
is violated, there exists $i \in N_{+}(R)$ such that $\Phi_{i}(R)(\{1\})<\frac{1}{\left|N_{+}(R)\right|}$. Let $j \in N_{+}(R) \backslash\{i\}$ and $R^{\prime} \in \mathcal{D}^{N}$ be an $i$-deviation of $R$ such that $R_{i}^{\prime}=R_{j}$. Then

$$
\Phi_{i}(R)(\{1\})=\Phi_{i}\left(R^{\prime}\right)(\{1\})=\Phi_{j}\left(R^{\prime}\right)(\{1\})
$$

where the first equality follows from Lemma 9.4 , and the second one from equal treatment of equals. Because $N_{+}\left(R^{\prime}\right)=N_{+}(R)$, it follows that $\Phi_{i}\left(R^{\prime}\right)(\{1\})=\Phi_{j}\left(R^{\prime}\right)(\{1\})<\frac{1}{\left|N_{+}(R)\right|}$. By Pareto-optimality and $\Phi\left(R^{\prime}\right)\left(E^{N}\right)=1$, there exists $k \in N_{+}(R) \backslash\{i, j\}$ such that $\Phi_{k}\left(R^{\prime}\right)(\{1\})>\frac{1}{\left|N_{+}(R)\right|}$. Let $R^{\prime \prime} \in \mathcal{D}^{N}$ be a $k$-deviation of $R^{\prime}$ such that $R_{k}^{\prime \prime}=R_{j}$. Then, similarly, equal treatment of equals and strategy-proofness imply

$$
\Phi_{k}\left(R^{\prime}\right)(\{1\})=\Phi_{k}\left(R^{\prime \prime}\right)(\{1\})=\Phi_{j}\left(R^{\prime \prime}\right)(\{1\})=\Phi_{i}\left(R^{\prime \prime}\right)(\{1\})
$$

Let $\bar{R} \in \mathcal{D}^{N}$ be such that for all $i \in N_{+}(R), \bar{R}_{i}=R_{j}$. Successive replacement of the preferences $R_{i}$, for $i \in N_{+}(R)$, by $R_{j}$ yields either [for all $i \in N_{+}(R), \Phi_{i}(\bar{R})(\{1\})<\frac{1}{\left.\mid N_{+}(R)\right]}$ ], or [for all $\left.i \in N_{+}(R), \Phi_{i}(\bar{R})(\{1\})>\frac{1}{\left|N_{+}(R)\right|}\right]$. Thus, $\Phi(\bar{R})\left(E^{N_{+}(R)}\right) \neq 1$, which contradicts Pareto-optimality and $\Phi(\bar{R})\left(E^{N}\right)=1$.

Remark 9.4 Proposition 9.2 still holds when equal treatment of equals is replaced by anonymity. Anonymity means that the probabilistic rule does not depend on the names of the agents.

Next, we exhibit a probabilistic rule satisfying Pareto-optimality and strategy-proofness but violating no-envy. It also satisfies the weaker fairness properties, anonymity (and equal treatment of equals), and weak non-bossiness. But it selects distributions that place positive probabilities on broken allocations. Therefore, when we replace in Theorem 9.2 no-envy by equal treatment of equals, uniqueness does not hold.

Example 9.1 Let $N=\{1,2,3\}, g \in \mathcal{G}^{N}$, and $R \in \mathcal{D}^{N}$. If $N_{+}(R) \neq N$, then $\Phi(R)=$ $U_{g}(R)$. When $N_{+}(R)=N, \Phi$ is defined as follows. Let $N=\{j, k, l\}$.

(i) If for all $i \in N, r_{i}(0) \geq \frac{1}{2}$, then for all $i \in N, \Phi(R)\left(\left\{e^{i}\right\}\right)=\frac{1}{3}$.

(ii) If $r_{j}(0)<\frac{1}{2}, r_{k}(0) \geq \frac{1}{2}$, and $r_{l}(0) \geq \frac{1}{2}$, then for all $i \in N, \Phi(R)\left(\left\{e^{i}\right\}\right)=\frac{1}{3}$.

(iii) If $r_{j}(0)<\frac{1}{2}, r_{k}(0)<\frac{1}{2}$, and $r_{l}(0) \geq \frac{1}{2}$, then $\Phi(R)\left(\left\{e^{j}\right\}\right)=\frac{1}{3}=\Phi(R)\left(\left\{e^{k}\right\}\right)$ : $\Phi(R)\left(\left\{\frac{1}{2}\left(e^{j}+e^{k}\right)\right\}\right)=\frac{1}{6}$, and $\Phi(R)\left(\left\{e^{l}\right\}\right)=\frac{1}{6}$.

(iv) If for all $i \in N, r_{i}(0)<\frac{1}{2}$, then for all $i \in N, \Phi(R)\left(\left\{e^{i}\right\}\right)=\frac{1}{6}$, and $\Phi(R)\left(\left\{\frac{1}{2}\left(e^{j}+\right.\right.\right.$ $\left.\left.\left.e^{k}\right)\right\}\right)=\Phi(R)\left(\left\{\frac{1}{2}\left(e^{j}+e^{l}\right)\right\}\right)=\Phi(R)\left(\left\{\frac{1}{2}\left(e^{k}+e^{l}\right)\right\}\right)=\frac{1}{6}$. 
Since $\Phi$ places positive probabilities on broken allocations, by Theorem $9.1, \Phi$ violates replacement-domination. This may altematively be seen by considering the change of agent $k$ 's preferences that cause a switch from Case (ii) to Case (iii): agent $j$ is made better off and agent $l$ is made worse off by that change with respect to the ordinal extension. Similarly, $\Phi$ violates no-envy. This may be seen by considering Case (iii): $\Phi_{j}(R)$ and $\Phi_{l}(R)$ are in general not comparable with respect to our extension. Note that when $r_{l}(0)=\frac{1}{2}$ in Case (iii), $\Phi_{j}(R) P_{l} \Phi_{l}(R)$. It is easy to check that the probabilistic rule $\Phi$ satisfies Pareto-optimality, strategy-proofness, anonymity, equal treatment of equals, and weak non-bossiness.

\subsection{Identical Indivisible Objects}

In this section we consider the problem of allocating finitely many identical indivisible objects among a group of agents. For example, teaching courses or jobs having negative cross effects are not infinitely divisible. When the objects are not assigned with certainty; the problem is closely related to probabilistic allocation problem with single-dipped preferences. Some combinations of properties allow only distributions placing probability 1 on the set of unit vectors (Lemmas 9.5 and 9.6 ).

To avoid ambiguity, we briefly introduce the model. A number $M \in \mathbb{N}$ of identical objects has to be assigned among a group $N$ of agents. For all $m \in\{0, \ldots, M\}, \frac{m}{M}$ denotes the event of obtaining $m$ identical objects. Each agent $i \in N$ is equipped with a single-dipped preference relation $R_{i}$ on $\left\{\frac{m}{M} \mid m \in\{0, \ldots, M\}\right\}$. By $\mathcal{D}(M)$ we denote the set of all single-dipped preferences on $\left\{\frac{m}{M} \mid m \in\{0, \ldots, M\}\right\}$. The set of feasible allocations is

$$
E(M)=\left\{\sum_{i \in N} \frac{m_{i}}{M} e^{i} \mid \text { for all } i \in N, m_{i} \in\{0, \ldots, M\}, \text { and } \sum_{i \in N} m_{i}=M\right\}
$$

A probabilistic assignment rule $\phi$ is a function that assigns for every profile $R \in \mathcal{D}(M)^{N}$ a distribution on $E(M)$. Properties of probabilistic assignment rules and further notation are defined as in Sections 9.2, 9.3, and 9.4.

Similar to Lemma 9.2 the following way of formulating Pareto-optimality may be proved. ${ }^{11}$

Lemma 9.7 Let $\phi$ be a probabilistic assignment rule. Then $\phi$ satisfies Pareto-optimality if and only if for all $R \in \mathcal{D}(M)^{N}$ :

\footnotetext{
${ }^{11}$ The proof of Lemma 9.7 is rather trivial since only distributions with finite support are considered.
} 
(i) If $N_{+}(R) \neq \emptyset$, then for all $i \in N_{+}(R), \phi_{i}(R)\left(\{0\} \cup B^{\circ}\left(0, R_{i}\right)\right)=1$, and for all $i \in N \backslash N_{+}(R), \phi_{i}(R)(\{0\})=1$.

(ii) If $N_{+}(R)=\emptyset$ and $N_{0}(R) \neq \emptyset$, then for all $i \in N_{0}(R), \dot{\phi}_{i}(R)(\{0,1\})=1$, and for all $i \in N_{-}(R), \phi_{i}(R)(\{0\})=1$.

(iii) If $N_{-}(R)=N$, then for all $i \in N, \phi_{i}(R)\left(B^{\circ}\left(1, R_{i}\right) \cup\{1\}\right)=1$.

When $M=1$, in the deterministic model Klaus (1999b) shows that Pareto-optimality implies strategy-proofness. It is easy to check that the implication remains true for probabilistic assignment rules.

Lemma 9.8 Let $M=1$ and $\phi$ be a probabilistic assignment rule satisfying Pareto-optimality. Then $\phi$ satisfies strategy-proofness.

Note that when $M=1$, strategy-proofness does not imply Pareto-optimality.

Remark 9.5 When $M=1$, Theorems 9.1 and 9.2 remain valid for the probabilistic assignment model without requiring strategy-proofness. By definition, probabilistic assignment rules always assign probability 1 to the set of unit vectors. Therefore, by Proposition 9.2 and Theorem 9.2, for one indivisible object uniform random dictatorship rules are characterized by Pareto-optimality and equal treatment of equals.

For arbitrary $M$, the set of feasible allocations $E(M)$ is a subset of $\mathcal{E}^{N}$. Therefore, a probabilistic assignment rule is a probabilistic rule that selects only distributions placing probability 1 on $E(M)$. Adapting the proofs of Sections 9.3 and 9.4 to probabilistic assignment rules yields parallel conclusions.

Corollary 9.1 The serial random dictatorship rules are the only probabilistic assignment rules satisfying Pareto-optimality, strategy-proofness, and replacement-domination.

Remark 9.6 Our model extends the deterministic model. Therefore, the result of Klaus, Peters, and Storcken (1997b) also applies to the deterministic allocation of a finite number of identical objects. Hence, a deterministic assignment rule satisfies the combination of properties of Corollary 9.1 if and only if it is a deterministic serial dictatorship rule, i.e., at every profile of preferences all objects are assigned to a single agent.

Remark 9.7 Example 9.1 also applies for the problem of allocating $M=2$ identical objects. Hence, its conclusions remain valid in the probabilistic assignment model. $\triangleleft$ 


\subsection{Independence}

The following four examples establish the independence of the properties in Theorems 9.1 and 9.2 .

Example 9.2 For all $R \in \mathcal{D}^{N}$ and all $i \in N, \tilde{\Phi}(R)\left(\left\{e^{i}\right\}\right)=\frac{1}{n}$. The probabilistic rule $\tilde{\Phi}$ satisfies strategy-proofness, replacement-domination, and no-envy, but not Paretooptimality.

In the following example, given $S \subseteq N$ such that $S \neq \emptyset$, min $S$ denotes the agent belonging to $S$ who has the minimal index among the agents in $S$. Similarly, we define $\max S$.

Example 9.3 Let $g \in \mathcal{G}^{N}$ and $\underline{\alpha}, \bar{\alpha} \in \mathcal{A}^{N}$ be such that for all $S \in 2^{N}$,

(a) when $1 \in S, \underline{\alpha}(S)=e^{1}=\bar{\alpha}(S)$,

(b) when $1 \notin S$ and $S \neq \emptyset, \underline{\alpha}(S)=e^{\min S}$ and $\bar{\alpha}(S)=e^{\max S}$, and

(c) when $S=\emptyset, \underline{\alpha}(\emptyset)=e^{n}=\bar{\alpha}(\emptyset)$.

For all $R \in \mathcal{R}^{N}$, when $d\left(R_{1}\right)$ is rational, $\bar{\Phi}(R)=\Psi_{\underline{\alpha}, g}(R)$, and when $d\left(R_{1}\right)$ is irrational, $\bar{\Phi}(R)=\Psi_{\bar{\alpha}, g}(R)$. The probabilistic rule $\bar{\Phi}$ satisfies Pareto-optimality and strategyproofness, but not replacement-domination and no-envy.

Example 9.4 Let $g \in \mathcal{G}^{N}$. For all $R \in \mathcal{D}^{N}$, let $\underline{S}(R)=\left\{i \in N \mid d\left(R_{i}\right)=\min _{j \in N} d\left(R_{j}\right)\right\}$. For all $R \in \mathcal{D}^{N}$, if $N_{-}(R)=N$, then $\bar{\Phi}(R)\left(\left\{e^{\min \underline{S}(R)}\right\}\right)=1$, and otherwise, $\bar{\Phi}(R)=U_{3}(R)$. The probabilistic rule $\bar{\Phi}$ satisfies Pareto-optimality and replacement-domination, but not strategy-proofness.

Example 9.5 Let $g \in \mathcal{G}^{N}$ and $R \in \mathcal{D}^{N}$. If $N_{-}(R)=N$ and for all $i \in N, \frac{1}{n} P_{i} 1$, then $\hat{\Phi}(R)\left(\left\{\frac{1}{n} \sum_{i \in N} e^{i}\right\}\right)=1$. Otherwise, $\hat{\Phi}(R)=U_{g}(R)$. The probabilistic rule $\hat{\Phi}$ satisfies Pareto-optimality and no-envy, but not strategy-proofness.

Finally, we note the following. Strategy-proofness can be defined alternatively in the following way. Probabilistic rule $\Phi$ is strategy-proof when for all $i \in N$ and all $R, R^{\prime} \in \mathcal{D}^{N}$; if $R^{\prime}$ is an $i$-deviation of $R$, then not $\Phi_{i}\left(R^{\prime}\right) P_{i} \Phi_{i}(R)$. This definition does not require comparability of $\Phi_{i}(R)$ and $\Phi_{i}\left(R^{\prime}\right)$ with respect to the first-order stochastic dominance condition at $R_{i}$. In Example 9.5, $\Phi$ satisfies this requirement. In particular, the class of all probabilistic rules satisfying Pareto-optimality, no-envy, and this weaker strategy-proofness condition is very large. Example 9.5 can be modified in a way such that $\hat{\Phi}(R)\left(\left\{\frac{1}{n} \sum_{i \in N} e^{i}\right\}\right)=1$ may also depend on whether the dip of agent 1 is rational or not. In particular, all our properties can be defined alternatively in the weaker way. 


\begin{tabular}{|c|c|c|c|c|c|c|}
\hline Property & N-E & R-D & WNB & AN & ETE & $E^{N}$ \\
\hline No-Envy (N-E) & Yes & Yes & Yes & Yes & Yes & Yes \\
\hline Replacement-Domination (R-D) & No & Yes & Yes & No & No & Yes \\
\hline Weak Non-Bossiness (WNB) & No & No & Yes & No & No & No \\
\hline Anonymity (AN) & No & No & $?$ & Yes & Yes & No \\
\hline Equal Treatment (ETE) & No & No & $?$ & $?$ & Yes & No \\
\hline
\end{tabular}

Table 9.1: Probabilistic allocation and single-dipped preferences.

\begin{tabular}{|c|c|c|c|c|c|c|c|}
\hline Property & N-E & R-D & WNB & AN & ETE & $E^{N}$ \\
\hline No-Envy (N-E) & \multicolumn{7}{|c|}{$\emptyset$} \\
\hline Replacement-Domination (R-D) & No & Yes & Yes & No & No & Yes \\
\hline Weak Non-Bossiness (WNB) & No & Yes & Yes & No & No & Yes \\
\hline Anonymity (AN) & \multicolumn{7}{|c|}{$\emptyset$} \\
\hline Equal Treatment (ETE) & \multicolumn{7}{|c|}{$\emptyset$} \\
\hline
\end{tabular}

Table 9.2: Deterministic allocation and single-dipped preferences.

\subsection{Conclusion}

In the class of all probabilistic rules satisfying Pareto-optimality and strategy-proofness, Table 9.1 shows the implications we derived and some open problems. Table 9.2 shows the corresponding implications in the deterministic model when preferences are single-dipped (Klaus, Peters, and Storcken, 1997b; Klaus, 1999b), and Table 9.3 in the deterministic model when preferences are single-peaked (Barberà, Jackson, and Neme, 1997; Ching, 1994; Sprumont, 1991). In these tables, e.g., "Yes" in row No-Envy and column $A N$ means that Pareto-optimality, strategy-proofness, and no-envy imply anonymity. "No" means that the implication does not hold and a question mark indicates an open problem. In Table 9.1 " $E^{N "}$ means that the selected distributions place probability 1 on the set of unit vectors. In Table 9.2 " $E^{N}$ " means that the chosen allocations belong to $E^{N}$. In Table 9.2, e.g., " $\emptyset$ " in row Anonymity means that Pareto-optimality, strategy-proofness, and anonymity are not compatible.

Under strategy-proofness and Pareto-optimality, we conclude by comparing our results with the conclusions for the deterministic model:

1. In the deterministic framework none of the important faimess properties is compatible 


\begin{tabular}{|c|c|c|c|c|c|}
\hline Property & N-E & R-D & WNB & AN & ETE \\
\hline No-Envy (N-E) & Yes & Yes & Yes & Yes & Yes \\
\hline Replacement-Domination (R-D) & No & Yes & Yes & No & No \\
\hline Weak Non-Bossiness (WNB) & No & No & Yes & No & No \\
\hline Anonymity (AN) & Yes & Yes & Yes & Yes & Yes \\
\hline Equal Treatment (ETE) & Yes & Yes & Yes & Yes & Yes \\
\hline
\end{tabular}

Table 9.3: Deterministic allocation and single-peaked preferences.

with Pareto-optimality. Pareto-optinality and strategy-proofness are compatible together with all important fairness notions jointly.

2. As in the deterministic model, neither replacement-domination implies anonymity nor the other way round. In the deterministic model with single-peaked preferences, anonymity implies replacement-domination.

3. Klaus (1999b) proves the equivalence of weak non-bossiness and replacement-domination. However, in our probabilistic setting replacement-domination implies weak non-bossiness, but the reverse implication is not true.

4. In the deterministic franework, many desirable properties are not compatible, and when they are compatible, the rules satisfying them always allocate the social endowment to one agent. In our probabilistic model there exist rules that satisfy many desirable properties and also assign distributions placing positive probability on broken allocations.

5. In the deterministic model with single-peaked preferences, Sprumont (1991) characterizes the uniform rule by Pareto-optimality, strategy-proofness, and no-envy. Ching (1994) shows that in this characterization the replacement of no-envy by equal treatment of equals still yields uniqueness. In our probabilistic setting; we characterize the uniform random dictatorship rules by Pareto-optimality, strategy-proofness, and no-envy. In this characterization no-envy camnot be weakened to equal treatment of equals.

6. All our results remain valid for the model of probabilistic allocation of a finite number of identical indivisible objects. In particular, Corollary 9.1 implies that the result of Klaus, Peters, and Storcken (1997b) remains true for deterministic allocation rules since our model extends their model. 


\section{Appendix A}

\section{Some Results from Measure Theory}

In this appendix we collect some basic concepts and results from measure theory that are used in the Chapters 3 and 9. These results can be found for example in Halmos (1970).

Definition A.1 Let $X$ be a nonempty set and let $\mathcal{X}$ be a set of subsets of $X$. Then $\mathcal{X}$ is called a $\sigma$-algebra in $X$ if $\mathcal{X}$ satisfies the following:

(i) $\emptyset \in \mathcal{X}$

(ii) If $A \in \mathcal{X}$, then $X \backslash A \in \mathcal{X}$.

(iii) If $I$ is a countable index set and for all $i \in I, A_{i} \in \mathcal{X}$, then $\cup_{i \in I} A_{i} \in \mathcal{X}$.

The pair $(X, \mathcal{X})$ is called a measure space and the elements of $\mathcal{X}$ are called measurable sets.

Remark A.1 Let $X$ be a nonempty set and let $\mathcal{X}$ be a set of subsets of $X$. Then there exists a unique $\sigma$-algebra $\mathcal{E}$ in $X$ such that $\mathcal{X} \subseteq \mathcal{E}$ and for all other $\sigma$-algebras $\mathcal{E}^{\prime}$ in $X$, if $\mathcal{X} \subseteq \mathcal{E}^{\prime}$, then $\mathcal{E} \subseteq \mathcal{E}^{\prime}$. The $\sigma$-algebra $\mathcal{E}$ in $X$ is said to be generated by $\mathcal{X}$. Notation: $\mathcal{A}\left(\mathcal{X}^{\prime}\right)=\mathcal{E}$.

For example, the Borel $\sigma$-algebra is the unique $\sigma$-algebra in $\mathbb{R}$ generated by the collection of all left half open intervals $\mathcal{J} \equiv\{\mid a, b] \mid a, b \in \mathbb{R}, a \leq b\}$. Hence, $\mathcal{A}(\mathcal{J})=\mathcal{L}$ and $(\mathbb{R}, \mathcal{L})$ is a measurable space. The same holds for the collection of all right half open intervals.

Definition A.2 Let $(X, \mathcal{X})$ be a measure space and $\mu$ a function from $\mathcal{X}$ to $[0,+\infty]$. Then $\mu$ is called a measure on $(X, \mathcal{X})$ if $(1) \mu(\emptyset)=0$ and $(2)$ for all $A_{1}, A_{2}, \ldots$ in $\mathcal{X}$, which are pairwise disjoint, $\mu\left(\cup_{n \in \mathbb{N}} A_{n}\right)=\sum_{n \in \mathbb{N}} \mu\left(A_{n}\right)$ ( $\sigma$-additivity). 
Remark A.2 It is straightforward to prove that each measure $\mu$ on a measure space $(X, \mathcal{X})$ is monotonic, i.e., for all $A, B \in \mathcal{X}$ such that $A \supseteq B, \mu(A) \geq \mu(B)$.

The following theorem is a well-known result from measure theory.

Theorem A.1 Let $\mu$ be a finite valued measure on $(X, \mathcal{X})$, i.e., for all $A \in \mathcal{X}, \mu(A)<$ $+\infty$. Then for all sequences $\left(A_{i}\right)_{i \in \mathbb{N}}$ such that for all $i \in \mathbb{N}, A_{i} \in \mathcal{X}$, and either $\cup_{i \in \mathbb{N}} A_{i}=A$ and for all $i \in \mathbb{N}, A_{i} \subseteq A_{i+1}$, or $\cap_{i \in \mathbb{N}} A_{i}=A$ and for all $i \in \mathbb{N}, A_{i} \supseteq A_{i+1}$, we have $\mu(A)=\lim _{i \rightarrow+\infty} \mu\left(A_{i}\right)$.

The following remark is a useful tool to prove equality of two measures, which are defined on the same measure space.

Definition A.3 Let $X$ be a nonempty set and $\mathcal{X}$ a set of subsets of $X$. Then $\mathcal{X}$ is called a basis of $X$ if (1) for all $A_{1}, A_{2} \in \mathcal{X}, A_{1} \cap A_{2} \in \mathcal{X}$, and (2) for each $A \subseteq X$, there exists a set sequence $\left(A_{i}\right)_{i \in \mathbb{N}}$ with for all $i \in \mathbb{N}, A_{i} \in \mathcal{X}$ and $A_{i} \subseteq A_{i+1}$, such that $\cup_{i \in \mathbb{N}} A_{i}=A$.

Remark A.3 Let $X$ be a nonempty set, $\mathcal{X}$ a set of subsets of $X$, and $\mu_{1}, \mu_{2}$ two measures on $(X, \mathcal{A}(\mathcal{X}))$. Suppose that (1) $\mathcal{X}$ is a basis of $X,(2)$ for all $A \in \mathcal{X}, \mu_{1}(A)=\mu_{2}(A)$, and (3) $\mu_{1}(X)<+\infty$. Then $\mu_{1}=\mu_{2}$.

For example, if $P_{1}$ and $P_{2}$ are two probability measures on $(\mathbb{R}, \mathcal{L})$, then in order to show $P_{1}=P_{2}$ it suffices to prove that for all intervals $\left.\left.\left.] a, b\right] \in \mathcal{J}, P_{1}(] a, b\right]\right)=P_{2}([a, b])$. It also suffices to prove that for all intervals $\left[a, b\left[, P_{1}\left(\left[a, b[)=P_{2}(\{a, b[)\right.\right.\right.\right.$.

Remark A.4 Consider the measure space $(\mathbb{R}, \mathcal{L})$. Then the Borel $\sigma$-algebra in $\overline{\mathbb{R}}$ is defined by

$$
\overline{\mathcal{L}} \equiv\{A, A \cup\{-\infty\}, A \cup\{+\infty\}, A \cup\{-\infty,+\infty\} \mid A \in \mathcal{L}\}
$$

Let $\mu$ be a measure on $(\mathbb{R}, \mathcal{L})$. Define some arbitrary nonnegative weights of $\mu$ for $-\infty$ and $+\infty$. Then $\mu$ can be easily extended to a measure on $(\overline{\mathbb{R}}, \overline{\mathcal{L}})$. Moreover, the $\sigma$-algebra $\overline{\mathcal{L}}$ is generated by the set of intervals $\overline{\mathcal{J}} \equiv\{[x,+\infty] \mid x \in \mathbb{R}\}$. In particular, given two probability measures $P_{1}$ and $P_{2}$ on $(\overline{\mathbb{R}}, \overline{\mathcal{L}})$, we have $P_{1}=P_{2}$ if and only if $x \in \mathbb{R}$, $P_{1}([x,+\infty])=P_{2}([x,+\infty])$. This follows from Remark A.3 and the fact that the last equalities imply that $P_{1}$ and $P_{2}$ coincide on the basis $\{[-\infty, x\} \mid x \in \mathbb{R}\} \cup \mathcal{J} \cup \overline{\mathcal{J}}$ of $\overline{\mathcal{L}}$. $\triangleleft$

Finally, we define setwise convergence in order to formulate the Theorem of VitaliHahn-Saks. 
Definition A.4 Let $(X, \mathcal{X})$ be a measure space, $I$ an infinite index set in $\mathbb{R}$, and for all $i \in I, \mu_{i}$ a measure on $(X, \mathcal{X})$. If $\lim _{i \rightarrow+\infty} \mu_{i}(A)$ exists for every measurable set $A \in \mathcal{X}$, then we say that $\left(\mu_{i}\right)_{i \in I}$ converges setwise.

Theorem A.2 (Vitali-Hahn-Saks) Let $(X, \mathcal{X})$ be a measure space and $\left(\mu_{i}\right)_{i \in I}$ a setwise convergent infinile sequence of measures on $(X, \mathcal{X})$. For all $A \in \mathcal{X}$, let $\mu(A) \equiv$ $\lim _{i \rightarrow+\infty} \mu_{i}(A)$. If $\mu$ is finite valued, then $\mu$ is a measure on $(X, \mathcal{X})$. 


\section{References}

Abdulkadiroğlu, A., and T. Sönmez (1998): "Random Serial Dictatorship and the Core from Random Endownents in House Allocation Problems," Econometrica 66:689 701.

Abdulkadiroğlu, A., and T. Sönmez (1999): "House Allocation with Existing Tenants," Journal of Economic Theory 88:233 260.

Alcalde, J., and S. Barberà (1994): "Top Do minance and the Possibility of StrategyProof Stable Solutions to Matching Problems," Economic Theory 4:417 435.

Bandyopadhyay, T., R. Deb, and P.K. Pattanaik (1982): "The Structure of Coalitional Power under Probabilistic Group Decision Rules," Journal of Economic Theory $27: 366 \cdot 375$.

Barberà, S., and H. Sonnenschein (1978): "Preference Aggregation with Randomized Social Orderings," Journal of Economic Theory 18:244 254.

Barberà, S., H. Sonnenschein, and L. Zhou (1991): "Voting by Committees," Econometrica 59:595 609.

Barberà, S. and M. Jackson (1994): "A Characterization of Strategy-Proof Social Choice Functions for Economies with Pure Public Goods," Social Choice and Welfare 11:241 252.

Barberà, S., M. Jackson, and A. Neme (1997): "Strategy.Proof Allotment Rules," Games and Economic Behaviour 18:1 21.

Barberà, S., A. Bogomolnaia, and H. van der Stel (1998): "Strategy-Proof Probabilistic Rules for Expected Ltility Maximizers," Mathematical Social Sciences 35:89 103.

Barberà, S., and C. Beviá (1999): "Self-Selection Consistent Choices," Working Paper, Universitat Autònoma de Barcelona. 
Benassy, J.P. (1982): The Economics of Market Disequilibrium, San Diego: Academic Press.

Berga, D., and S. Serizawa (2000): "Maximal Domain for Strategy-Proof Rules with One Public Good," Joumal of Economic Theory 90:39 61.

Beviá, C. (1998): "Fair Allocation in a General Model with Indivisible Goods," Review of Economic Design 3:195 213.

Black, D. (1948): "On the Rationale of Group Decision Making," Journal of Political Economy 56:23 34 .

Bogomolnaia, A., and H. Moulin (1999): "A New Solution to the Random Assignment Problem," Journal of Economic Theory, forthcoming.

Border, K.C., and J.S. Jordan (1983): "Straightforward Elections, Lnanimity and Phantom Voters;" Review of Econonic Studies 50:153 170.

Chichilnisky, G., and W. Thomson (1987): "The Walrasian Mechanism from Equal Division is not Monotonic with respect to Variations in the Number of Consumers," Journal of Public Economics 32:119 124.

Ching, S. (1992): "A Simple Characterization of the Lniform Rule," Economics Letters 40:57. 60 .

Ching, S. (1994): "An Alternative Characterization of the Lniform Rule," Social Choice and Welfare 11:131-136.

Ching, S. (1997): "Strategy-Proofness and Median Voters;" International Journal of Game Theory 26:473 490.

Ching, S., and S. Serizawa (1998): "A Maximal Domain for the Existence of StrategyProof Rules," Journal of Economic Theory 78:157-166.

Ching, S., and W. Thomson (1999): "Population-Monotonic Solutions in Public Good Economies with Single-Peaked Preferences;" Social Choice and Welfare, forthcoming.

Chun, Y. (1986): "The Solidarity Axiom for Quasi-Linear Social Choice Problems," Social Choice and Welfare 3:297 320.

Chun, Y. (1999): "The Separability Principle in Economies with Single-Peaked Preferences," Working Paper, Seoul National University. 
Chun, Y., and W. Thomson (1988): "Monotonicity Properties of Bargaining Solutions when Applied to Economics," Mathematical Social Sciences 15:11 27.

Crès, H., and H. Moulin (1998a): "Sequencing with Opting Out: Improving upon Random Priority," Working Paper, HEC Paris and Duke Lniversity.

Crès, H., and H. Moulin (1998b): "Random Priority: A Probabilistic Resolution of the Tragedy of the Commons," Working Paper, HEC Paris and Duke Lniversity.

Danilov, V. (1992): "Implementation via Nash Equilibria," Econometrica 60:43 56.

d'Aspremont, C., and L. Gevers (1977): "Equity and the Informational Basis of Collective Choice," Review of Economic Studies 44:199 209.

Dutta, B. (1980): "Strategic Voting in a Probabilistic Framework," Econometrica 48:447456.

Dutta, B., and A. Sen (1991): "A Necessary and Sufficient Condition of Two Person Nash Implementation," Review of Economic Studies 58:121 128.

Dutta, B., and J. Massó (1997): "Stability of Matchings when Individuals have Preferences over Colleagues," Journal of Economic Theory 75:464 4 475.

Dutta, B., H. Peters, and A. Sen (1999): "Strategy-Proof Probabilistic Mechanisms in Economies with Pure Public Goods," Working Paper, Maastricht Lniversity.

Ehlers, L. (1998): "Probabilistic Allocation Rules and Single-Dipped Preferences," Social Choice and Welfare, forthcoming.

Ehlers, L. (2000a): "Independence Axioms for the Provision of Multiple Public Goods as Options," Mathematical Social Sciences, forthcoming.

Ehlers, L. (2000b): "Multiple Public Goods and Lexicographic Preferences: Replacement Principle," Working Paper, Maastricht Lniversity.

Ehlers, L. (2000c): "Multiple Public Goods and Lexicographic Preferences: PopulationMonotonicity," Working Paper, Maastricht University.

Ehlers, L. (2000d): "Coalitional Strategy-Proof House Allocation," Journal of Economic Theory, forthcoming.

Ehlers, L. (2000e): "Monotonic and Implementable Solutions in Generalized Matching Problems," Working Paper, Maastricht Lniversity. 
Ehlers, L. (2000f): "Resource-Monotonic Allocation when Preferences are Single-Peaked," Economic Theory, forthcoming.

Ehlers, L. (2000g): "Indifference and the Lniform Rule," Economics Letters 67:303 308.

Ehlers, L. (2000h): "Strategy-Proof Allocation when Preferences are Single-Plateaued," Working Paper, Maastricht Lniversity.

Ellers, L., H. Peters, and T. Storcken (1999): "Strategy-Proof Probabilistic Decision Schemes for One-Dimensional Single-Peaked Preferences," Journal of Economic Theory, forthcoming.

Ehlers, L., and B. Klaus (2000a): "Solidarity and Probabilistic Target Rules," Journal of Public Economic Theory, forthcoming.

Ehlers, L., and B. Klaus (2000b): "Coalitional Strategy-Proof and Resource-Monotonic Solutions for Multiple Assignment Problems," Working Paper, Maastricht Lniversity.

Ehlers, L., B. Klaus, and S. Pápai (2000): "Strategy-Proofness and Population-Monotonicity for House Allocation Problems," Working Paper, Maastricht Lniversity.

Ergin, H.I. (2000): "Consistency in: House Allocation Problems," Journal of Mathematical Economics 34:77 97.

Foley, D. (1967): "Resource Allocation and the Public Sector;" Yale Econ. Essays 7, 45. 98.

Freixas, X. (1984): "A Cardinal Approach to Straightforward Probabilistic Mechanisms," Journal of Economic: Theory 34:227 251.

Gale, D., and L. Shapley (1962): "College Admissions and the Stability of Marriage," American Mathematical Monthly 69:9 15.

Gevers, L. (1986): "Walrasian Social Choice: Some Simple Axiomatic Approaches," In: Heller, W., et al. (eds.) Social Choice and Public Decision Making, Essays in Honor of K.J. Arrow. Cambridge Lniversity Press, Cambridge, 97 114.

Gibbard, A. (1973): "Manipulation of Voting Schemes: A General Result," Econometrica 41:587 601.

Gibbard, A. (1977): "Manipulation of Schemes that Mix Voting and Chance," Econometrica 45:665-681. 
Gibbard, A. (1978): "Straightforwardness of Game Forms with Lotteries as Outcomes," Econometrica 46:595 614 .

Halmos, P.R. (1970): Measure Theory, Springer-Verlag New York Heidelberg Berlin.

Herrero, C., and A. Villar (2000): "An Alternative Characterization of the Equal-Distance Rule for Allocation Problems with Single-Peaked Preferences," Economics Letters $66: 311317$.

Hokari, T. (1999): "Population Monotonic Solutions on Convex Games," Working Paper, University of Rochester.

Hotelling, H. (1929): "Stability in Competition," Economic Journal 39:41 57.

Kara, T., and T. Sönmez (1996): "Nash Implementation of Matching Rules," Journul of Economic Theory 68:425 439.

Kim, H. (1999): "Population Monotonic Rules for Fair Allocation Problems," Working Paper, Lniversity of Rochester.

Klaus, B. (1999a): "Status Quo Solutions for Public Good Economies on Tree Networks and in Euclidean Spaces," In: Logic, Game Theory and Social Choice, Proceedings of the International Conference, LGS '99 (ed. H. de Swart).

Klaus, B. (1999b): "Coalitional Strategy-Proofness in Economies with Single-Dipped Preferences and the Assignment of an Indivisible Object," Games and Economic Behaviour; forthcoming.

Klaus, B. (2000): "Lniform Allocation and Reallocation Revisited," Review of Economic Design, forthcoming.

Klaus, B., H. Peters, and T. Storcken (1997a): "Reallocation of an Infinitely Divisible Good," Economic Theory 10:305-333.

Klaus, B., H. Peters, and T. Storcken (1997b): "Strategy-Proof Division of Private Good when Preferences are Single-Dipped," Economics Letters 55:339 346.

Klaus, B., and E. Miyagawa (1999): "Strategy-Proofness, Solidarity, and Consistency for Multiple Assignment Problems," Working Paper, Columbia Lniversity.

Konishi, H., M. Le Breton, and S. Weber (1998): "Equilibrium in a Finite Local Public Goods Economy," Journal of Economic Theory 79:224-244. 
Ma, J. (1994): "Strategy-Proofness and the Strict Core in a Market with Indivisibilities," Intemational Journal of Game Theory 23:75 83.

Maskin, E. (1999): "Nash Equilibrium and Welfare Optimality" Review of Economic Studies $66: 2338$.

May, K.O. (1952): "A Set of Independent, Necessary and Sufficient Conditions for Simple Majority Voting," Econometrica 20:680 684.

Miyagawa, E. (1998a): "Mechanisms for Providing a Menu of Public Goods," Ph.D. dissertation, tniversity of Rochester.

Miyagawa, E. (1998b): "Locating Libraries on a Street," Social Choice and Welfare, forthcoming.

Miyagawa, E. (1998c): "Mechanisms for Providing a Menu of Public Goods: PopulationMonotonicity," Working Paper, Columbia Lniversity.

Miyagawa, E. (1998d): "Strategy-Proof Provision of a Menu," Working Paper, Columbia University.

Miyagawa, E. (2001): "Strategy-Proofness and the Core in House Allocation Problems," Games and Economic Behaviour, forthcoming.

Moore, J., and R. Repullo (1990): "Nash Implementation: A Full Characterization," Econometrica 58:1083 1099.

Moulin, H. (1980): "On Strategy-Proofness and Single-Peakedness," Public Choice 35:437 455.

Moulin, H. (1984): "Generalized Condorcet-Winners for Single-Peaked Preferences and Single-Plateaued Preferences;" Social Choice and Welfare 1:127-147.

Moulin, H. (1987): "The Pure Compensation Problem: Egalitarian versus Laissez-Fairism," Quarterly Joumal of Economics 102:769 783.

Moulin, H. (1990): "Fair Division under Joint Ownership: Recent Results and Open Questions," Social Choice and Welfare 7:149 170.

Moulin, H. (1999): "Rationing a Commodity along Fixed Paths," Joumal of Economic Theory 84:41 $\cdots 2$. 
Moulin, H., and W. Thomson (1988): "Can Everyone Benefit from Growth? Two Difficulties," Journal of Mathematical Economics 17:339 345.

Pápai, S. (2000a): "Strategy-Proof Assignment by Hierarchical Exchange," Econometrica 68:1403 1434 .

Pápai, S. (2000b): "Strategyproof Multiple Assignment using Quotas," Review of Economic Design 5:91 105.

Pápai, S. (2001): "Strategy-Prool and Nonbossy Assignnents," Journal of Public Economic Theory, forthcoming.

Pattanaik, P.K., and B. Peleg (1986): "Distribution of Power under Stochastic Social Choice Rules," Econometrica 54:909 921.

Roth, A.E. (1984): "Misrepresentation and Stability in the Marriage Problem," Joumal of Economic Theory 34:383 387.

Roth, A.E. (1985): "The College Admissions Problem is not equivalent to the Marriage Problem," Journal of Economic Theory 36:277 288.

Roth, A.E., and A. Postlewaite (1977): "Weak versus Strong Domination in a Market with Indivisible Goods," Journal of Mathematical Economics 4:131 137.

Roth, A.E., and M. Sotomayor (1990). Two-Sided Matching: A Study in Game Theoretic Modeling and Analysis, London/New York: Cambridge Lniversity Press.

Samuelson, W., and R.J. Zeckhauser (1988): "Status Quo Bias in Decision Making," Journal of Risk and Uncertainty 1:7-59.

Sasaki, H. (1997): "Randomized Lniform Allocation Mechanism and Single-Peaked Preferences of Indivisible Good," Working Pajer, Waseda Liniversity.

Satterthwaite, M. (1975): "Strategy-Proofness and Arrow's Conditions: Existence and Correspondence Theorem for Voting Procedures and Social Choice Functions," Journal of Economic Theory 10:187-217.

Satterthwaite, M., and H. Sonnenschein (1981): "Strategy-Proof Allocation Mechanisms at Differentiable Points," Review of Economics Studies 48:587 597.

Schummer, J., and R.V. Vohra (2001): "Strategy-Proof Location on a Network," Joumal of Economic Theory, forthcoming. 
Sen, A. (1995): "The Implementation of Social Choice Functions via Social Choice Correspondences: a General Formulation and a Limit Result," Social Choice and Welfare 12:277 292.

Serizawa, S. (1995): "Power of Voters and Domain of Preferences where Voting by Committees is Strategy-Proof," Journal of Economic Theory 67:599 608.

Shapley, L., and H. Scarf (1974): "On Cores and Indivisibility" Journal of Mathematical Economics 1:23 37 .

Shin, S., and S.-C. Suh (1996): "A Mechanism Implementing the Stable Rule in Marriage Problems," Economics Letters 51:185 189.

Sönmez, T. (1994): "Consistency, Monotonicity, and the Uniform Rule," Economics Letlers 46:229 235.

Sönmez, T. (1996): "Implementation in Generalized Matching Problems," Journal of Mathematical Economics 26:429-439.

Sönmez, T. (1997): "Games of Manipulation in Marriage Problems," Gumes and Economic Behaviour 20:169 -176.

Sönnez, T. (1999): "Strategy-Proofness and Essentially Single-Valued Cores," Econometrica 67:677 689.

Sprumont, Y. (1990): "Population Monotonic Allocation Schemes for Cooperative Games with Transferable Utility," Games and Economic Behaviour 2:378 394.

Sprumont, Y. (1991): "The Division Problem with Single-Peaked Preferences: A Characterization of the Liniform Allocation Rule," Econometrica 59:509 519.

Sprumont, Y., and L. Zhou (1999): "Pazner-Schmeidler Rules in Large Societies," Joumal of Mathematical Economics 31:321 339.

Svensson, L.-G. (1994): "Queue Allocation of Indivisible Goods," Social Choice and Welfare 11:323 330 .

Svensson, L.-G. (1999): "Strategy-Proof Allocation of Indivisible Goods," Social Choice and Welfare 16:557 567 .

Tadenuma, K. (1996): "Trade-Off between Equity and Efficiency in a General Economy with Indivisible Goods," Social Choice and Welfare 13:445 450. 
Tadenuma, K., and M. Toda (1998): "Implementable Stable Solutions to Pure Matching Problems," Mathematical Social Sciences 35:121 132.

Thomson, W. (1983a): "The Fair Division of a Fixed Supply among a Growing Population," Mathematics of Operations Research 8:319 326.

Thomson, W. (1983b): "Equity in Exchange Economies," Joumal of Economic Theory $29: 217 \cdot 244$.

Thomson, W. (1987): "The Vulnerability to Manipulative Behaviour of Mechanisms designed to select Equitable and Efficient Allocations," In: Groves T., Radner, R., Reiter, R. (eds.) Information, Incentives and Economic Mechanisms. University of Minnesota Press, Minneapolis, Chap. 14, pp. 395396.

Thomson, W. (1993): "The Replacement Principle in Public Good Economies with Single-Peaked Preferences," Economics Letters 42:31- 36.

Thomson, W. (1994a): "Resource-Monotonic Solutions to the Problem of Fair Division when Preferences are Single-Peaked," Social Choice and Welfare 11:205-223.

Thomson, W. (1994b): "Consistent Solutions to the Problem of Fair Division when Preferences are Single-peaked," Journal of Economic Theory 63:219 245.

Thomson, W. (1995): "Population-Monotonic Solutions to the Problem of Fair Division when Preferences are Single-Peaked," Economic Theory 5:229 246.

Thomson, W. (1997): "The Replacement Principle in Economies with Single-Peaked Preferences," Journal of Economic Theory 76:145 168.

Thomson, W. (1998): "The Replacement Principle in Economies with Indivisible Goods," Social Choice and Welfare 15:57 66.

Thomson, W. (1999a): "Welfare-Domination under Preference-Replacement: a Survey and Open Questions," Social Choice and Welfare 16:373 394.

Thomson, W. (1999b): "Population-Monotonicity: a Survey and Open Questions:" Working Paper, Lniversity of Rochester.

Thomson, W. (1999c): "Monotonic Extensions on Economic Domains," Review of Economic Design 4:13 33.

Thomson, W. (2000): The Theory of Fair Allocation, Book Manuscript. 
Thomson, W., and R. Myerson (1980): "Independence and Monotonicity Axioms," International Journal of Game Theory 9:39 49.

Vohra, R.V. (1999): "The Replacement Principle and Tree Structured Preferences," Economics Letters 63:175 180.

Wako, J. (1984): "A Note on the Strong Core of a Market with Indivisible Goods," Journal of Mathematical Economics 13:189 194.

Wako, J. (1991): "Some Properties of Weak Domination in an Exchange Market with Indivisible Goods," The Economics Studies Quarterly 42:303-314.

Yamato, T. (1992): "On Nash Implementation of Social Choice Correspondences," Gumes and Economic Behaviour 4:484 492.

Zhou, L. (1991): "Impossibility of Strategy-Proof Mechanisms in Economies with Pure Public Goods," Review of Economic Studies 58:107 119. 


\section{Author Index}

Abdulkadiroğlu A., 75, 111, 132, 149

Alcalde J., 149

Bandyopadhyay T., 75

Barberà S., 7, 10, 73, 75, 96, 112, 134, $149,194,195,204,205,210,211$, 237

Benassy J.P., 181, 198

Berga D., 7, 112

Beviá C., 7, 132

Black D., 2

Bogomolnaia A., 7, 73, 75, 76, 132, 214

Border K.C., 7, 21, 75, 80, 94

Chichilnisky G., 74

Ching S., 7 9, 12, 20-22, 27, 53, 57, 73, 74, 94 96, 107, 112, 193 195, 199, $200,204,232,237,238$

Chun Y., 74, 97, 142, 183

Crès H., 75

D'Aspremont C., 160

Danilov V., 163

Deb R., 75

Dutta B., 9, 75, 75, 165

Ehlers L., 7, 10, 53, 72, 75, 111, 113, 131, $147,173,193,213,214$

Ergin H.İ, 111, 131

Foley D., 215

Freixas $\mathrm{X}, 73$

Gale D., 118, 141, 147, 149, 167
Gevers L., 160

Gibbard A., 1, 3, 4, 71, 72, 76, 214

Halmos P.R., 217, 218, 239

Herrero C., 193

Hokari T., 12, 74

Hotelling H., 8

Jackson M., 7, 10, 73, 96, 194, 195, 204, $205,210,211,237$

Jordan J.S., 7, 21, 75, 80, 94

Kara T., 150, 154, 164

Kim H., 12, 74

Klaus B., 10, 12, 53, 72, 74, 113, 131 133, $174,210,213 \cdot 216,219,223,225$, $230,232,235,237,238$

Konishi H., 8

Le Breton M.. 8

Ma J., 113, 131, 149

Maskin E., 149, 153, 157

Massó J., 9

May K.O., 1

Miyagawa E., 2, 7 13, 16 18, 27 29, 31, $35,37,38,53,59,74,111,131$ 133,149

Moore J., 165

Moulin H., 2, 7, 8, 10, 12, 13, 20, 7176 , $87,94,95,97,132,142,173,174$, $177-179,193 \cdot 195,214$

Myerson R., 174 
Neme A., 10, 194, 195, 204, 205, 210, 211, 237

Pápai S., 133, 141, 149

Pattanaik P.K., 75

Peleg B., 75

Peters H., 12, 72, 75, 174, 213, 214, 219, $225,230,235,237,238$

Postlewaite A., 113, 149; 160, 168, 169

Repullo R., 165

Roth A.E., 113, 134, 148, 149, 150, 160, 168,169

Sarmuelson W., 74

Sasaki H., 75, 215, 232

Satterthwaite M., 1, 3, 4, 71, 136, 223

Scarf H., 113, 148, 168

Schummer J., 7

Sen A., 75, 157, 165

Serizawa S., 7, 112 193, 195, 199

Shapley L., 113, 147 149, 167, 168

Shin S., 165

Sönmez T., 75, 111, 113, 132, 147, 149, $150,152,154,161,164,165,174$, $176,182 \cdot 185$

Sonnenschein H., 75, 112, 134, 136, 223

Sotomayor M., 148, 150

Sprumont Y., 10, 12, 74, 173, 176, 193, $194,198,199,230,237,238$

Storcken T.; 12, 72, 174, 213, 214, 219, $225,230,235,237,238$

Svensson L.-G., 111, 131, 141

Suh S.-C., 165

Tadenuma K., 132, 154

Toda M., 154

Thomson W., 7, 10-12, 31, 53, 57, 73, 74, $97,98,104,107,142,160,161$,
$173,174,176,182,186,204,210$, 230

Van der Stel H., 73

Villar A., 193

Vohra R.V., 7, 10, 31

Wako J., 113, 150, 168

Weber S., 8

Yamato T., 163, 164

Zeckhauser R.J., 74

Zhou L., 7, 10, 12, 74, 112, 134 


\section{Summary in Dutch}

Dit proefschrift behandelt enkele onderwerpen uit de sociale keuze-theorie. Wij bestuderen beslissingsproblemen waarbij een groep individuen een collectieve beslissing moet nemen omtrent de keuze van één alternatief uit een gegeven verzameling. In het algemeen zijn de preferenties over de altenatieven niet unaniem en liet is niet duidelijk welk alternatief er gekozen zal worden. Een (beslissings) regel is een systematische procedure die voor elk profiel van preferenties één alternatief uit de gegeven alternatievenverzameling kiest. Behalve deterministische regels bestudeert dit proefschrift ook kansregels. Een kansregel wijst aan elk profiel van preferenties een kansmaat op de alternatievenverzameling toe. Het uiteindelijke alternatief dat gekozen wordt, wordt bepaald door het toegewezen kansmaat. We onderzoeken verschillende economische modellen en karakteriseren regels die wenselijke eigenschappen hebben. Dat betekent dat we in elk model axioma's definieren die sociaal wenselijk zijn, en identificeren voor een combinatie van axioma's de klasse van regels die aan deze combinatie voldoen. Hierbij onderscheiden we tussen twee typen axioma's. In de eerste plaats zijn er "stationaire" axioma's, namelijk axioma's die van toepassing zijn op één profiel van preferenties. Het tweede type axioma's is van toepassing als de eigenschappen van de economie veranderen. Een economie bestaat uit een verzameling van agenten, een verzameling van alternatieven, en een profiel van preferenties. Men kan bijvoorbeeld veronderstellen dat de populatie groter wordt. Dan moeten we een nieuwe beslissing nemen en populatie-monotonie is een voorwaarde op de keuzes voor de oude en de nieuwe populatie.

In Deel I bekijken wij lokatieproblemen. De verzameling van mogelijke lokaties is een gesloten interval. De preferentie van elk individu heeft een piek en aan beide zijden van deze piek zijn alternatieven die dichterbij de piek liggen geprefereerd boven alternatieven die verder weg liggen. Zulke preferenties worden ééntoppig genoemd. In Hoofdstuk 2 onderzoeken wij problemen waarbij we voor twee publieke goederen lokaties moeten kiezen. Een alternatief bestaat uit twee lokaties. Ondat axioma's in termen van preferenties over alternatieven gedefinieerd zijn en preferenties alleen individuele lokaties in het interval kunnen vergelijken, moeten we de preferenties over het interval uitbreiden naar prefer- 
enties over alternatieven. Een mogelijke manier van uitbreiding is de "max-uitbreiding". Hierbij vergelijkt een individu twee alternatieven op basis van de beste lokaties van de alternatieven. De interpretatie is dat iedereen alleen dat publieke goed benut dat zich op de meest geprefereerde lokatie bevindt. Hij zal nooit het goed op de slechtere lokatie gebruiken. De twee lokaties kunnen bijvoorbeeld twee bushaltes van een buslijn zijn. Iemand stapt bij die halte in die het dichtst bij zijn woning ligt. Alternatieven waarbij een lokatie dichtbij de piek ligt worden geprefereerd. Als preferenties met de max-uitbreiding worden uitgebreid, karakteriseren we de "extreme-peaks" regel. Deze regel kiest voor elk profiel als lokaties de kleinste en de grootste top van het profiel. Een andere uitbreiding van preferenties is de lexicografische uitbreiding. Bij deze uitbreiding vergelijkt een individu eerst de twee beste lokaties (zoals bij cle max-uitbreiding). Als hij indifferent is tussen deze lokaties, vergelijkt hij de twee slechtere lokaties. Bij de max-uitbreiding zijn alle alternatieven waarbij een van de twee lokaties op de piek terecht komt beste alternatieven. Bij de lexicografische uitbreiding zijn beide lokaties belangrijk en het beste alternatief is het alternatief waarbij beide lokaties op de piek terecht komen. We karakteriseren de klasse van ééntoppig preferentie-regels middels Pareto-optimaliteit en de solidariteits-eigenschap "replacement-domination". Elke regel in deze klasse wordt bejaald door een ééntoppig preferentie. Verder karakteriseren we de klasse van plateautoppig preferentie-regels middels Pareto-optimaliteit en populatie-monotonie. Deze regels worden vastgelegd door een single-plateaued preferentie relatie.

In Hoofdistuk 3 bestuderen we lokatieproblemen van een publiek goed. Verschillende auteurs hebben dit problem onderzocht voor deterministische regels. Hierbij spelen mediaanregels een grote rol. Voor elke mediaan-regel kiest men eerst $n-1$ ijkpunten. Voor elk profiel van preferenties wijst de mediaan-regel de mediaan van de $n$ pieken van de agenten en de $n-1$ ijkpunten. De mediaan is het punt uit de verzameling van de $n$ pieken en $n-1$ ijkpunten zodat er aan beide zijden van dit punt tenminste $n$ punten uit deze verzameling zijn. Dus het interval tot en met dit punt bevat tenminste $n$ lokaties van de pieken en de ijkpunten. Mediaan regels worden gekarakteriseerd middels Paretooptimaliteit, niet-manipuleerbaarheid en anonimiteit. Een deelklasse van deze regels zijn zogenaande doelregels. Voor deze regels zijn alle ijkpunten gelijk. Deze regels zijn gekarakteriseerd door Pareto-optimaliteit en een solidariteits-eigenschap ("replacementdomination" of populatie-monotonie). We onderzoeken het probleem voor kansregels. Voor elk profiel van preferenties wijst de regel een kansmaat op het interval van lokaties toe. We karakteriseren de "vaste-kans-ballots" regels middels Pareto-optimaliteit, nietmanipuleerbaarheid en anonimiteit. Een dergelijke kansregel wordt vastgelegd door $n-1$ vaste kansmaten op het interval. Een deelklasse van deze regels zijn de kans-doel regels. Hi- 
erbij zijn alle vaste-kans-ballots gelijk. We karakteriseren kans-doel regels middels Paretooptimaliteit en populatie-monotonie. Verder karakterisieren we de deterministische doelregels middels Pareto-optimaliteit en "replacement-domination".

Deel II is gewijd aan allocatie van ondeelbare objecten. Dat kunnen bijvoorbeeld huizen, opgaven of werkplaatsen zijn. In Hoofdstuk 4 verdelen we $n$ objecten onder $n$ agenten waarbij iedereen één object ontvangt en elk object aan een andere agent toegewezen wordt. We zoeken regels die aan Pareto-optimaliteit en niet-manipuleerbaarheid voldoen. We introduceren een sterkere niet-manipuleerbaarheids eigenschap, coalitionele nietmanipuleerbaarheid. Hierbij kan iedere coalitie collectief manipuleren. We bepalen een uniek maximaal domein zodat (i) er een regel bestaat die aan Pareto-optimaliteit en coalitionele niet-manipuleerbaarheid voldoet en (ii) het domein van alle strikte preferenties een deeldomein is van dit domein. Verder karakteriseren we de regels die aan deze eigenschappen voldoen op het unieke maximale domein. In Hoofdstuk 5 verdelen we $k$ objecten onder $n$ agenten. Hierbij ontvangt elke agent een ver'zameling objecten (de lege verzameling word niet uitgesloten) en niet alle objecten hoeven noodzakelijkerwijs toegewezen te worden. We karakteriseren sequentiële dictaturen middels Pareto-optimaliteit en coalitionele manipuleerbaarheid. In Hoofdstuk 6 bestuderen we algemene toewijzingsproblemen. Ieder individu heeft een object en ieder individu kan slechts objecten van een deelverzanneling van agenten krijgen. Dit model omvat bijvoorbeeld het allocatie-probleenn met $n$ objecten en $n$ agenten of het huwelijks problem van vrouwen en mannen (of werknemers en werkgevers). In de literatuur over dit probleem hebben doorgaans alle agenten strikte preferenties over de toewijsbare objecten. We onderzoeken het probleem als individuen indifferent kunnen zijn tussen twee verschillende objecten. We laten zien dat de zogenaamde "union strong core" de minimale monotone uitbreiding is van de "strong core". Een oplossing wijst aan elke profiel van preferenties een of meerdere alternatieven toe. Zo'n oplossing voldoet aan eigenschap $\alpha$ als voor elk profiel waarbij de initiële verdeling Pareto-optimal is, de oplossing alle verdelingen toewijst waarbij iedereen inddifferent is tussen zijn initiële en het toegewezen object. Als een oplossing aan eigenschap $\alpha$, individuele rationaliteit en monotonie voldoet, dan omvat deze oplossing de "union strong core". Als een oplossing aan Pareto-indifferentie, individuele rationaliteit en monotonie voldoet, dan omvat deze oplossing de zwakke kern.

Deel III bestudeert de allocatie van een oneindig deelbaar privaat goed. In Hoofdstuk 7 heeft elk individu een ééntoppige preferentie over de zijn mogelijke consumpties. Als de maximale consumptie van iedereen beperkt is, karakteriseren we de vaste pad verdeelregels middels goed monotonie, niet-manipuleerbaarheid en consistentie. In Hoofdstuk 7 heeft ieder individu een plateautoppig preferentierelatie over de mogelijke consump- 
ties. We karakteriseren de uniforme verdeelregel middels indifferentie (in termen van welvaart), "no-envy" en niet-manipuleerbaarheid. Verder karakterisieren we de klasse van sequentiële verdeelregels met indifferentie en coalitionele niet-nanipuleerbaarheid. In Hoofdstuk 9 heeft ieder individu een ééndippige preferentie over de mogelijke consumpties. Bij zo'n preferentie bestaat een slechtste consumptie (de dip) en aan beide zijden van cle dip worden die consumpties geprefereerd die verder van de dip liggen. Ook hier bekijken we kansregels. We karakteriseren de klasse van seriële random-dictaturen middels Pareto-optimaliteit, niet-manipuleerbaarheid en de solidariteits-eigenschap "replacementdomination". Verder karakteriseren we de uniforme random-dictaturen middels Paretooptimaliteit, niet-manipuleerbaarheid en no-envy. 


\section{Curriculum Vitae}

The author, Lars Ehlers, was born in Roding, Germany, on June 6th, 1971. In November 1992 he began to study mathematics at the Lniversity of Regensburg, Germany. After three years he continued his studies at the Lniversity of Technology Aachen, Germany, in October 1995. In July 1997 he received the master's degree in mathematics with first class honors (German title: "Diplom-Mathematiker (Lniv.)"). Professor Dr. Hans Peters supervised his master's thesis. On July 3rd, 1998, he received the commemorative medal of Springorum from the Lniversity of Technology Aachen.

From October 1st, 1997, he worked as an "assistent in opleiding" (AIO) at the Department of Quantitative Economics at Maastricht Lniversity. He pursued his research in social choice and matching theory under directions of Professor Dr. Hans Peters, Dr. Ton Storcken, and Professor Dr. William Thomson.

From October 1st, 1999, to April 20th, 2000, he stayed as a visiting scholar at the Department of Economics, Lniversity of Rochester, LSA. During this period he received financial support from the Netherlands Organization for Scientific Research. 